System-Wide Water Resources Program

\title{
HEAT - Habitat Evaluation and Assessment Tools for Effective Environmental Evaluations
}

User's Guide

Kelly A. Burks-Copes, Antisa C. Webb, Michael F. Passmore,

December 2012

and Sheila D. McGee-Rosser

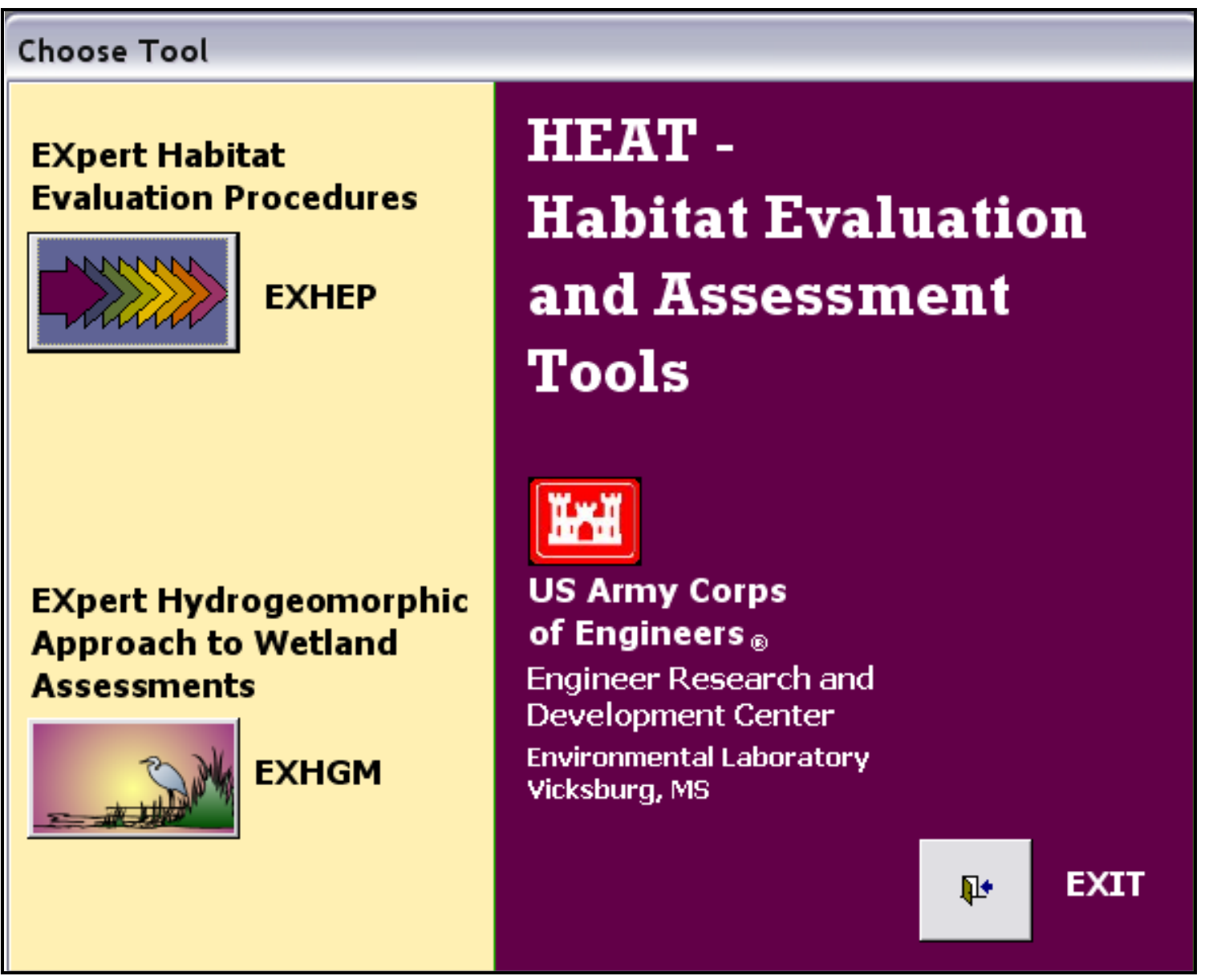




\title{
HEAT - Habitat Evaluation and Assessment Tools for Effective Environmental Evaluations
}

User's Guide

Kelly A. Burks-Copes, Antisa C. Webb, and Michael F. Passmore

Environmental Laboratory

U.S. Army Engineer Research and Development Center

3909 Halls Ferry Road

Vicksburg, MS 39180-6199

Sheila D. McGee-Rosser

Applied Research Associates, Inc.

112 Monument Place, Suite $A$

Vicksburg, MS 39180-5160

Final report

Approved for public release; distribution is unlimited.

\author{
Prepared for U.S. Army Corps of Engineers \\ Washington, DC 20314-1000 \\ Under Work Unit 33143
}




\section{Abstract:}

Rapidly assessing changing habitat conditions and evaluating the effects these changes have on species, communities, and ecosystems must be determined by planners, resource managers, and biologists when comparing environmental design alternatives. Many techniques (e.g., population assessments, qualitative matrices, life-history modeling, and habitat evaluation techniques) have been developed to investigate and predict environmental impacts on ecological systems at numerous scales with varying degrees of success. Advances in technology have led many agencies to automate and distribute environmental evaluation tools to users. The value and validity of these packages depend greatly on their objectivity, repeatability, and efficiency. To guarantee their consistent use, these systems must be easy to apply, cost-effective, and instantly responsive. The Habitat Evaluation and Assessment Tools (HEAT) software was developed to provide a user-friendly (intuitive), flexible, and efficient means to conduct Habitat Evaluation Procedures (HEP) and the Hydrogeomorphic Approach to Wetland Assessments (HGM), using Microsoft ${ }^{\circledR}$ Windows programming capabilities. HEAT operates on a personal computer with a 486 (or better) processor, using readily available commercial software. Two modules within HEAT, namely EXHEP: EXpert Habitat Evaluation Procedures and EXHGM: EXpert HydroGeoMorphic Approach to Wetland Assessments, calculate outputs for with-project conditions (comparing these to baseline and without-project conditions). HEAT accommodates a variety of data input and output files; incorporates a broad range of user-specified index models; allows customization of outputs; and dramatically reduces computation time. This document is a User's Guide, intended to provide both a step-by-step protocol for completing a HEP and/ or an HGM analysis using the software, and an illustration of this software's effectiveness in the evaluation of contemporary index models. In addition, this report includes a brief description of the hardware and software requirements of the system, and provides directions for the installation of the program. 


\section{Contents}

Figures and Tables ............................................................................................................................................ viii

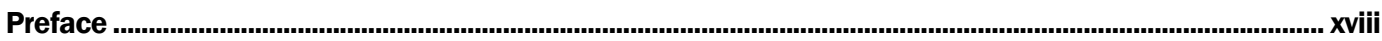

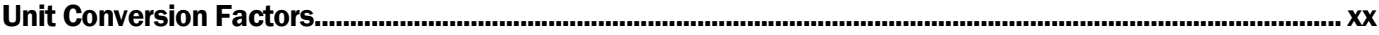

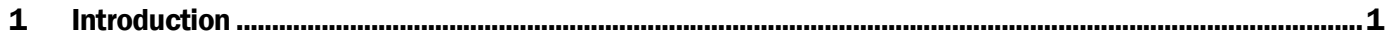

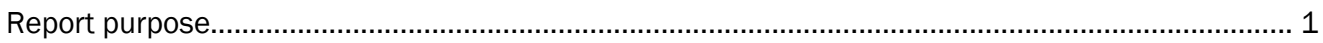

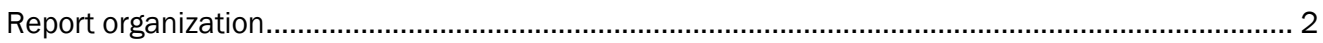

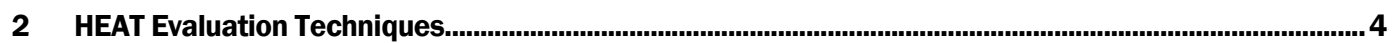

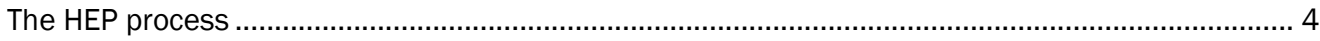

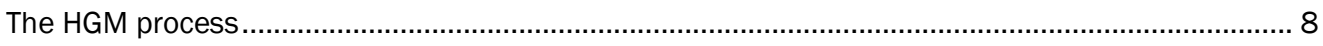

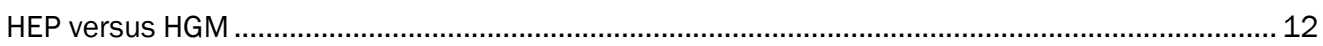

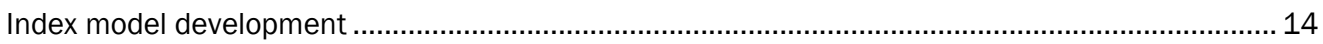

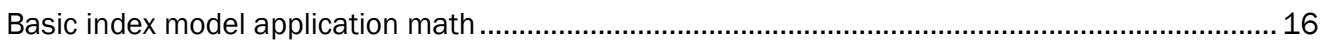

The 12-Step HEP/HGM application strategy .......................................................................... 22

Step 1: Build a multi-disciplinary evaluation team ............................................................ 22

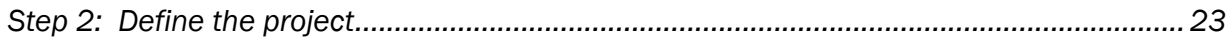

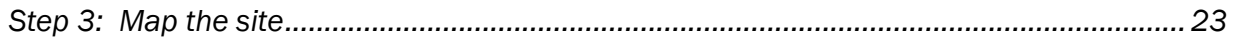

Step 4: Select, modify and/or develop the index models.............................................. 23

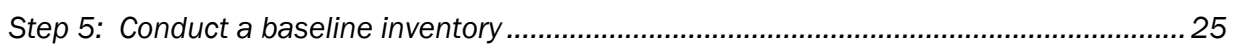

Step 6: Perform data management and statistical analysis ............................................. 25

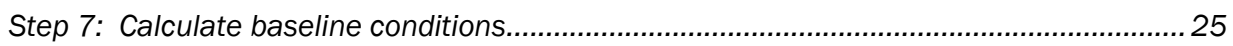

Step 8: Set goals and objectives and establish the assessment's temporal scale .............. 26

Step 9: Generate without-project conditions and calculate the outputs ...............................2 27

Step 10: Generate with-project conditions and calculate the outputs ..................................2 27

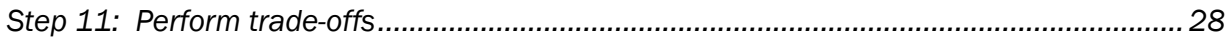

Step 12: Report the results of the analyses .................................................................... 28

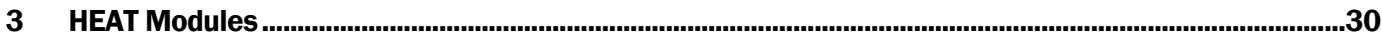

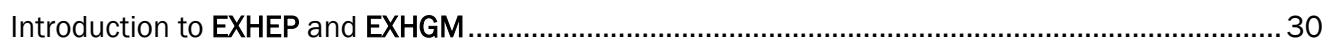

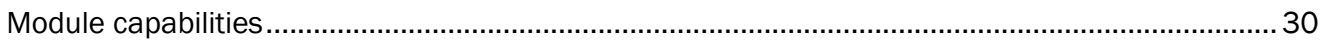

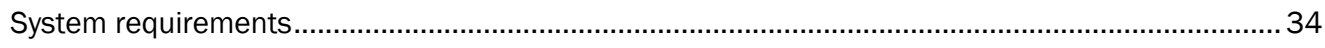

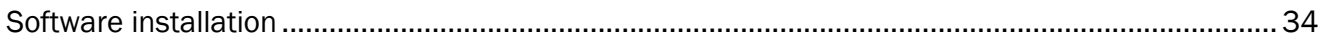

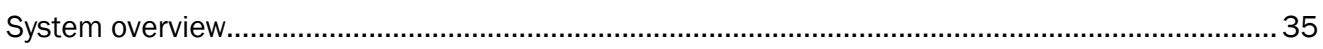

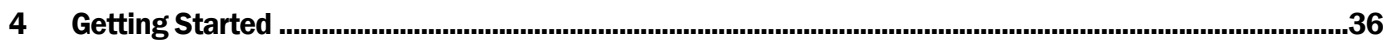

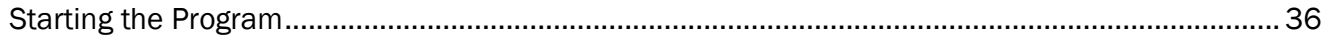

Special instructions for new MS Access 2007 users........................................................ 39

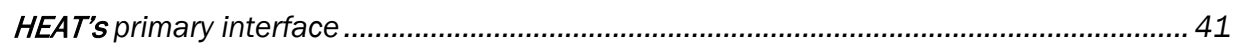

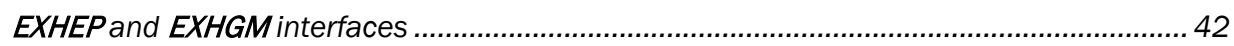

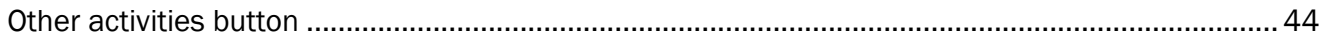




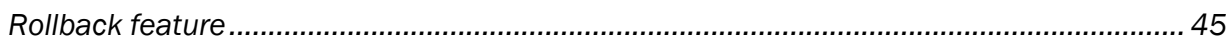

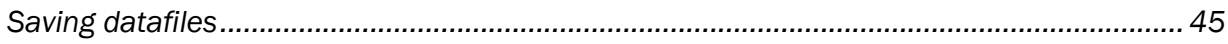

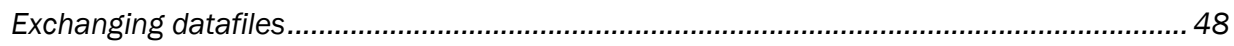

Compacting the current datafile ........................................................................................ 50

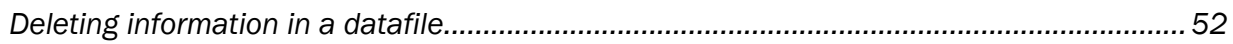

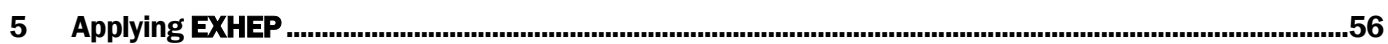

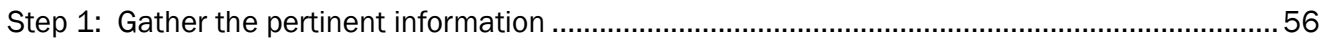

Single versus multiple formula models........................................................................ 57

Data checklist for the EXHEP analyses............................................................................. 58

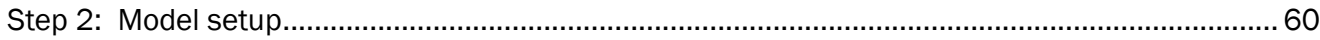

Enter, delete, and/or copy the project methods .............................................................. 61

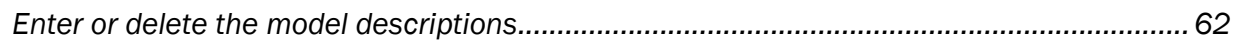

Define or delete the cover types and baseline acres..............................................................64 64

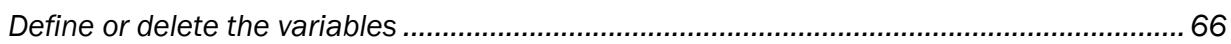

Additional setup actions for multiple formula models ......................................................67

Checking the setup process with EXHEP reports ................................................................... 70

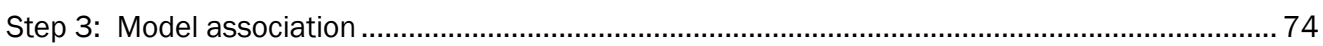

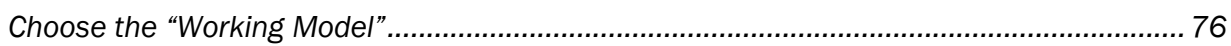

Associate the model parameters ...................................................................................... 77

Additional association actions for multiple formula models .............................................. 90

Checking the association process with EXHEP reports .....................................................106

Step 4: Enter baseline data and generate baseline results ........................................................107

SI graph coordinate entry, deletion, and/or copying ......................................................108

Checking the SI graph coordinates with EXHEP reports ..................................................116

Baseline data entry ....................................................................................................... 118

Checking the baseline data entry with EXHEP reports ..................................................... 128

Perform baseline (TY 0) calculation .................................................................................. 130

Checking the baseline analysis with EXHEP reports ........................................................ 132

Step 5: Enter the without-project conditions and calculate the effects ..........................................136

Without-project forecasting and data entry ................................................................... 137

Perform the without-project calculations............................................................................156

Checking the without-project analysis with EXHEP reports ..............................................160

Step 6: Enter the with-project conditions and calculate the effects ...........................................162

With-project forecasting and data entry ....................................................................... 162

Perform the with-project calculations ........................................................................ 173

Checking the with-project analysis with EXHEP reports.................................................181

Step 7: Recycle the datafile and evaluate alternative designs ....................................................185

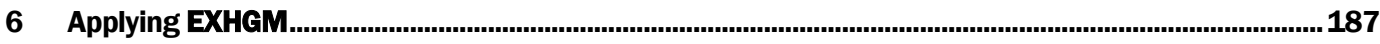

Step 1: Gather the pertinent information ............................................................................... 187

Data checklist for the EXHGM analyses .................................................................. 187

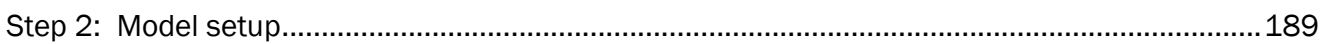

Enter, delete, and/or copy project methods..................................................................... 190

Enter or delete the function descriptions ......................................................................... 192

Define or delete PWAAs and baseline acres ................................................................... 193 
Define or delete the variables ............................................................................................ 195

Checking the setup process with EXHGM reports............................................................ 197

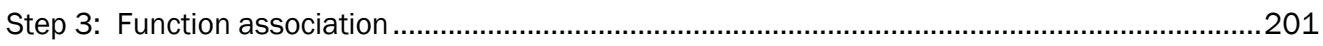

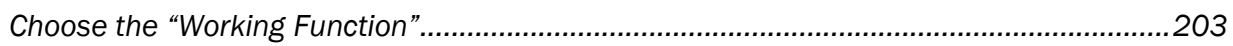

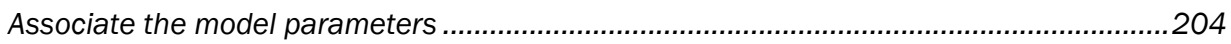

Checking the association process with EXHGM reports ...................................................217

Step 4: Enter baseline data and generate baseline results .........................................................219

VSI graph coordinate entry, deletion, and/or copying .....................................................220

Checking the VSI graph coordinates with EXHGM reports ..............................................226

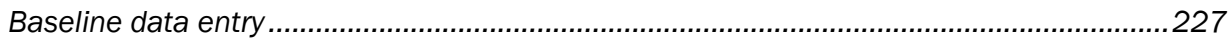

Checking the baseline data entry with EXHGM reports .................................................235

Perform baseline (TY 0) calculation .........................................................................237

Checking the baseline analysis with EXHGM reports.......................................................238

Step 5: Enter the without-project conditions and calculate the effects .......................................240

Without-project forecasting and data entry ....................................................................241

Perform the without-project calculations.............................................................................257

Checking the without-project analysis with EXHGM reports................................................258

Step 6: Enter the with-project conditions and calculate the effects ............................................260

With-project forecasting and data entry ..........................................................................261

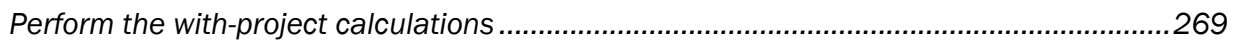

Checking the with-project analysis with EXHGM reports .....................................................275

Step 7: Recycle the datafile and evaluate alternative designs ....................................................279

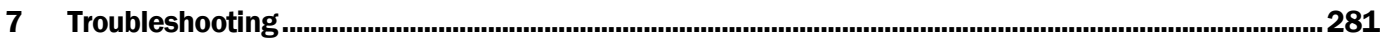

Lesson 1: Get Organized Early (Outside the Software) ................................................................281

Lesson 2: Data Format Incompatibility - Negative Numbers and Modes.........................................282

Lesson 3: Install and Run the Software Locally...........................................................................28

Lesson 4: Make Backups and Organize Datafiles in a Few Key Subdirectories..............................284

Lesson 5: Use the HEAT Engine - Do Not Open the Datafile with MS Access..................................285

Lesson 6: Error Messages and Installation Problems......................................................................286

Lesson 7: Printing and Export Issues...........................................................................................286

Lesson 8. Delete a Model by Working Backwards .......................................................................28

Lesson 9: Change a Model by Working Backwards....................................................................28

Lesson 10: Not Everything Works the Same Way in the Software ..............................................288

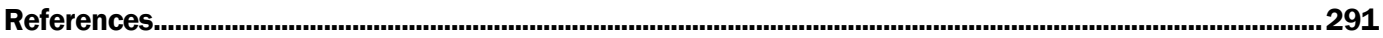

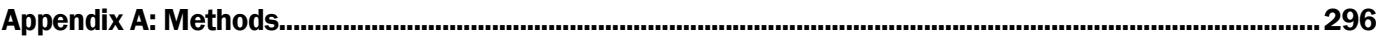

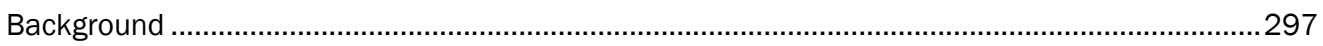

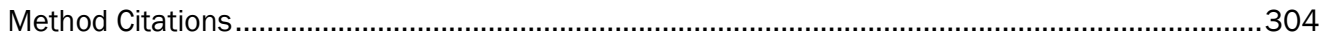

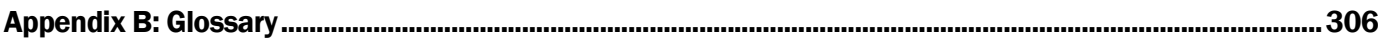

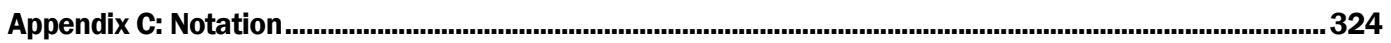

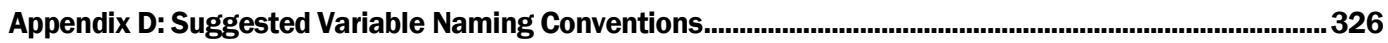

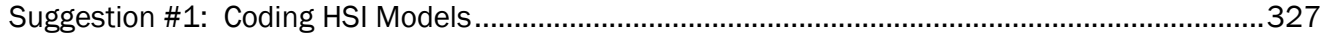




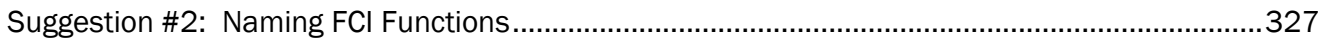

Suggestion \#3: Creating Cover Type (or PWAAs) Codes..............................................................328

Suggestion \#4: Creating Life Requisites Codes ............................................................................329

Suggestion \#5: Generating Variable Codes by Clustering Actions ..................................................330

Suggestion \#6: Generating Variable Codes by Clustering Elements ...............................................331

Suggestion \#7: Generating Variable Codes by Clustering Sources ...............................................332

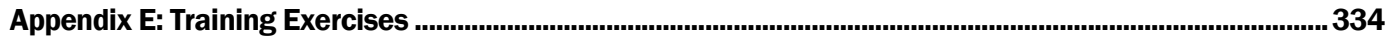

Exercise 1: Project Information and Model Setup in EXHEP........................................................334

Exercise 2: Single Formula Model Association and HSI Formula Writing in EXHEP ........................338

Exercise 3: Multiple Formula Model Association and LRSI/HSI Formula Writing in EXHEP............342

Exercise 4: Entering Model Curves into EXHEP ........................................................................350

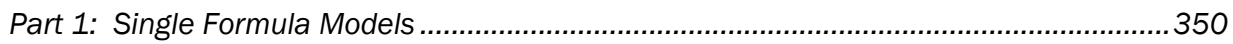

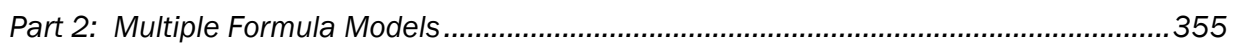

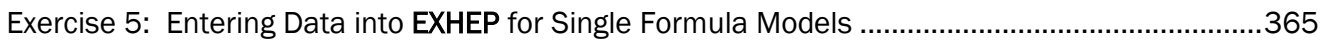

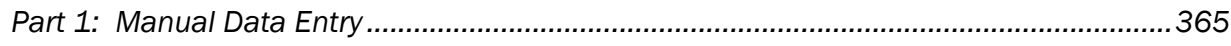

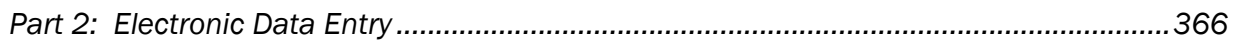

Exercise 6: Entering Data into EXHEP for Multiple Formula Models ................................................368

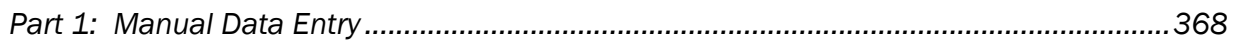

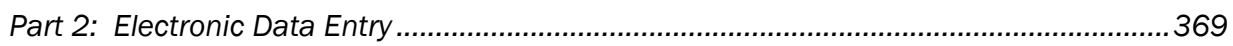

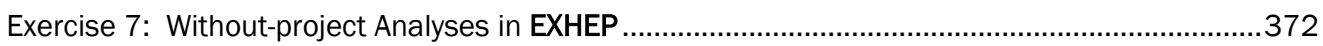

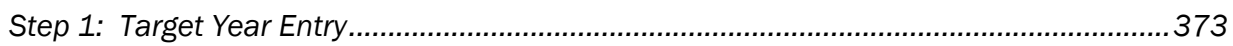

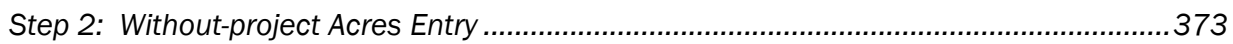

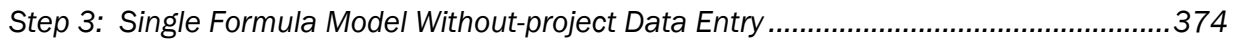

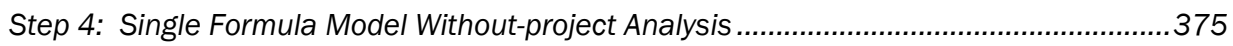

Step 5: Multiple Formula Model Without-project Data Entry .............................................376

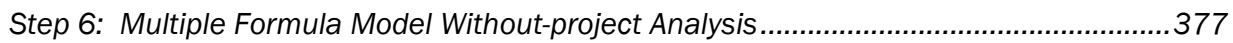

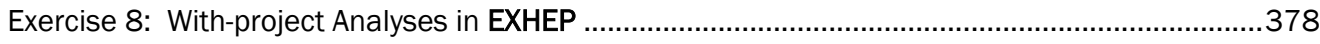

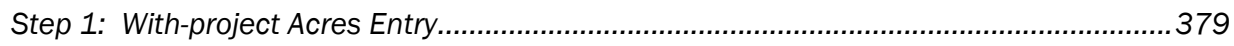

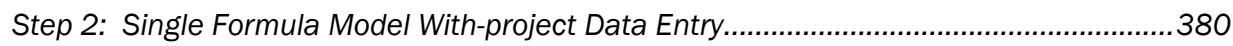

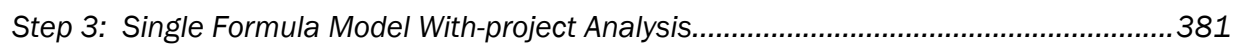

Step 4: Multiple Formula Model With-project Data Entry ..................................................382

Step 5: Multiple Formula Model With-project Analysis ...................................................383

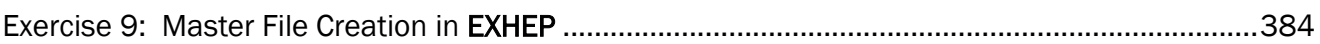

Scenario A: Create a "master file" that can be used for new alternatives at the

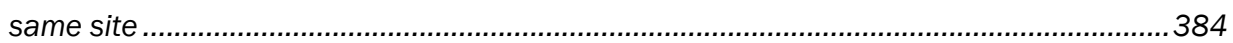

Scenario B: Create a "master file" that can be used for new sites ....................................391

Exercise 10: Project Information and Model Setup in EXHGM .....................................................400

Exercise 11: Formula Association and FCI Formula Writing in EXHGM ..........................................403

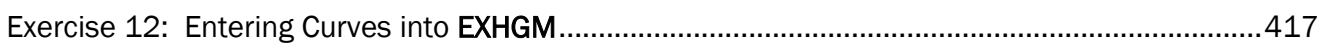

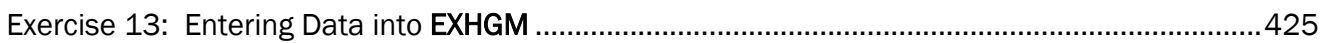

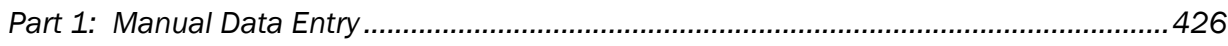

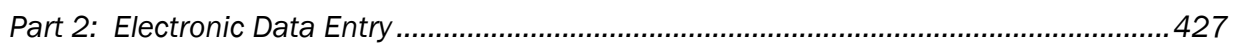

Exercise 14: Without-project Analyses in EXHGM ......................................................................429

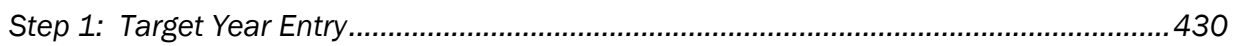

Step 2: Without-project Acres Entry ............................................................................. 430 


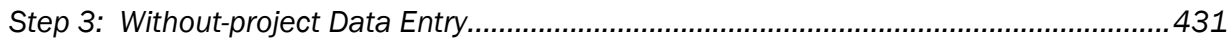

Step 4: Without-project Analysis .............................................................................. 432

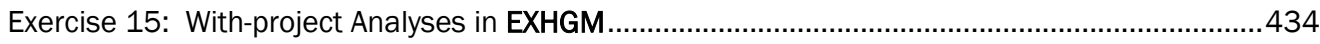

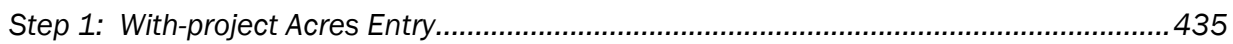

Step 2: With-project Data Entry ...................................................................................436

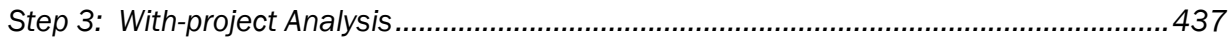

Exercise 16: Master File Creation in EXHGM.............................................................................439

Scenario A: Create a "master file" that can be used for new sites ......................................439

Scenario B: Create a "master file" that can be used for new alternatives at the

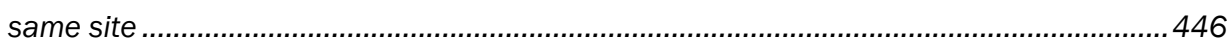




\section{Figures and Tables}

\section{Figures}

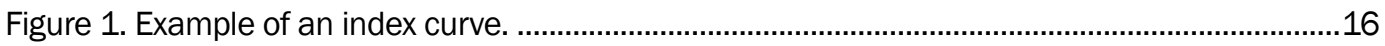

Figure 2. Linking HEAT products to reports. .....................................................................................33

Figure 3. Datafile selection entry screen - blank datafile location......................................................36

Figure 4. Datafile selection entry screen - selection of the blank datafile. ........................................37

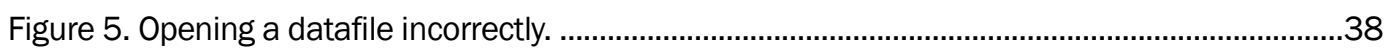

Figure 6. Selecting a previously created datafile........................................................................39

Figure 7. For first-time MS Access 2007 users, a blank screen will appear upon opening HEAT, indicating a security warning and lack of "trusted location" designation................39

Figure 8. Setting HEAT as a "trusted location" in MS Access 2007 - Step 1.....................................40

Figure 9. Setting HEAT as a "trusted location" in MS Access 2007 - Step 2.................................... 41

Figure 10. HEAT's primary interface. .............................................................................................42

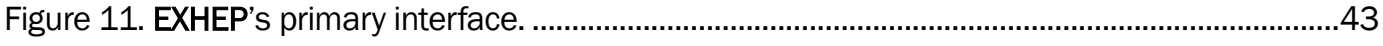

Figure 12. EXHGM's primary interface....................................................................................

Figure 13. Rolling back to the original datafile. …………..................................................................45

Figure 14. Saving datafiles under new names............................................................................46

Figure 15. Overwrite warning in the Save Datafile screen. …….....................................................4

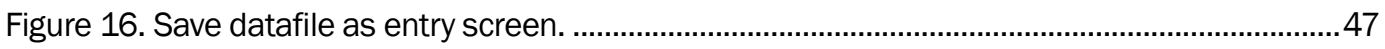

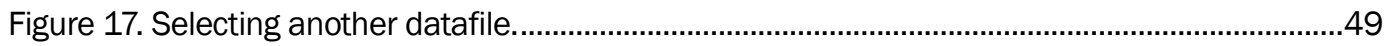

Figure 18. Select a datafile entry screen. .....................................................................................49

Figure 19. New datafile identification in the primary interface. ......................................................50

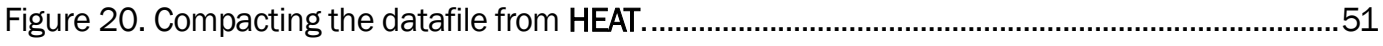

Figure 21. Compacting the datafile in MS Access (outside of HEAT).............................................52

Figure 22. Clearing database information in HEAT......................................................................5

Figure 23. Confirm Data Erasure entry screen..............................................................................54

Figure 24. Click on the Setup button to begin the single formula model setup process in EXHEP 60

Figure 25. Methods and Assumptions entry screen in EXHEP.......................................................61

Figure 26. Model Descriptions entry screen in EXHEP. .....................................................................63

Figure 27. Common EXHEP data entry screen prompts. ..................................................................64

Figure 28. Cover Type Descriptions entry screen in EXHEP............................................................65

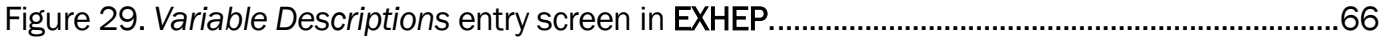

Figure 30. Click on the Setup button to begin the multiple formula model setup process in EXHEP 68

Figure 31. Life Requisite Descriptions entry screen in EXHEP. .......................................................69

Figure 32. Click on the Setup Reports button to review the setup reports in EXHEP.........................70 


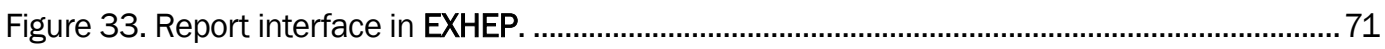

Figure 34. An expanded EXHEP report detailing additional fields. ....................................................72

Figure 35. Export Report entry screen - exporting report information to MS Excel or

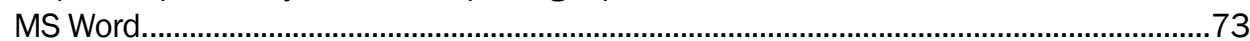

Figure 36. Exported reports in MS Excel and MS Word environments............................................. 74

Figure 37. Click on the Run button to begin the single formula model association process

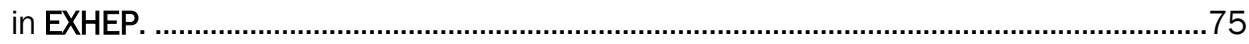

Figure 38. Select a model from the Single Formula Model pull-down list. .........................................76

Figure 39. Identification of the Working Single Formula Model in EXHEP's primary

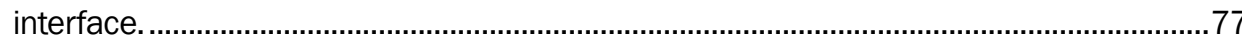

Figure 40. Model Associations window and Variables Available keypad used to associate

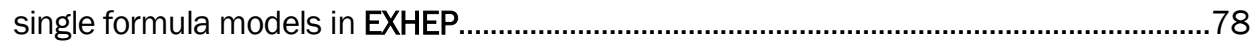

Figure 41. Model Associations entry screen for single formula models............................................78

Figure 42. Select a cover type from the pull-down list. ....................................................................79

Figure 43. Customize the size and location of the floating Variables Available keypad. ...................80

Figure 44. Help and Equation Check buttons provided to assist with HSI formula entry in EXHEP

Figure 45. Model Equation/HSI Calculation Syntax window in EXHEP..............................................82

Figure 46. Use the keypad to correctly enter variable codes into the HSI Model Formula input box.

Figure 47. Begin by entering the variable codes (surrounded by brackets) in the HSI Model

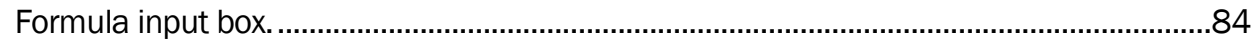

Figure 48. Example of the formula entry process in the Model Associations entry form. ..................85

Figure 49. Completing the HSI formula entry process - removal of the $1^{\star}$ placeholder and

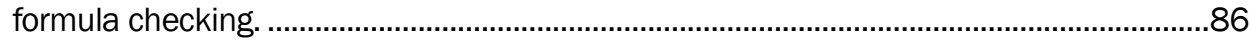

Figure 50. Extra records indicate "phantoms" that must be manually removed................................8

Figure 51. Select the next associated cover type from the pull-down list. .......................................88

Figure 52. Make a new association by duplicating the previous data.............................................89

Figure 53. Show or hide all associated records simultaneously. .....................................................90

Figure 54. Click on the Run button to begin the multiple formula model association

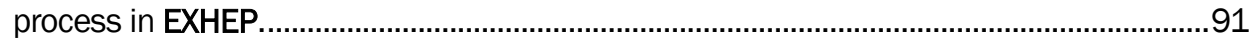

Figure 55. Select a working model from the Multiple Formula Model pull-down list. ......................92

Figure 56. Identification of the Multiple Formula Working Model on EXHEP's primary interface.

Figure 57. Model Associations window and keypads for multiple formula models in EXHEP. .94

Figure 58. Model Associations entry screen for multiple formula models. ......................................95

Figure 59. Select a life requisite from the pull-down list.................................................................96

Figure 60. Select a cover type from the pull-down list. ……...........................................................96

Figure 61. Enter an HSI formula in the space provided using the LR Codes Available keypad.

Figure 62. Enter an LRSI formula in the space provided using the Variables Available keypad. 
Figure 63. An example of an Equivalent Optimum Area (EOA) curve taken from a multiple formula model.

Figure 64. Enter the EOA graph coordinates in the space provided............................................. 100

Figure 65. Extra records indicate "phantoms" that must be manually removed............................ 100

Figure 66. Select additional cover types from the pull-down list.................................................... 102

Figure 67. Make a new association by duplicating previous records (LRSI formula)...................... 103

Figure 68. Make a new association by duplicating previous data (HSI formula).......................... 104

Figure 69. Show or hide all associated records simultaneously. ................................................. 105

Figure 70. Click on the Setup Reports button to review the association reports in EXHEP. .......... 106

Figure 71. Example of EXHEP's formula report for the single formula models. ..............................107

Figure 72. Click on the Run button to begin the single formula model baseline data entry process in EXHEP.

Figure 73. Baseline Data Entry windows for single formula models in EXHEP.............................. 109

Figure 74. Begin data entry by selecting a cover type and a variable from the pull-down

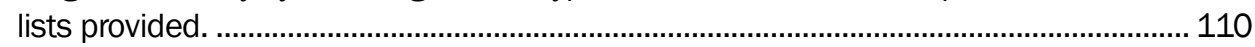

Figure 75. Move to the lower window and enter the SI graph coordinates in the space provided. .............................................................................................................. 111

Figure 76. Example of an SI coordinate entry. ....................................................................... 111

Figure 77. Copying SI graph coordinates from one record to the next............................................ 113

Figure 78. Click on the Run button to begin the multiple formula model SI graph

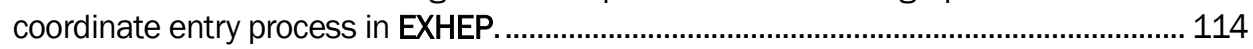

Figure 79. Select a Life Requisite window for multiple formula models in EXHEP........................ 115

Figure 80. Click on the Setup Reports button to review the SI graph coordinate reports in

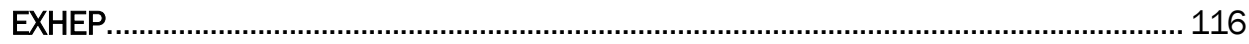

Figure 81. Example of Suitability Index Curves for Single Formula Models report in EXHEP..........117

Figure 82. Click on the Run button to begin the single formula model baseline data entry process in EXHEP.......................................................................................................... 118

Figure 83. An example of the manual data entry process in EXHEP............................................ 120

Figure 84. Importing data from an external spreadsheet into EXHEP.............................................. 121

Figure 85. Exporting data from EXHEP to MS Excel - click the Export Data button........................ 123

Figure 86. Exporting data from EXHEP to MS Excel - assign a directory and path for the

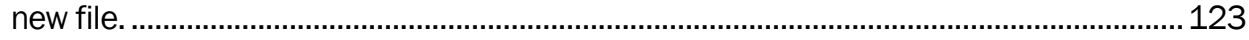

Figure 87. Deleting single data points in EXHEP....................................................................... 124

Figure 88. Click on the Run button to begin the multiple formula model baseline variable data entry process in EXHEP......................................................................................... 126

Figure 89. Select a Life Requisite window for multiple formula models in EXHEP......................... 127

Figure 90. Click on the Setup Reports button to view the variable data reports in EXHEP............ 128

Figure 91. Example of Variable Data for Single Formula Models report in EXHEP.......................... 129

Figure 92. Click on the Run button to begin the baseline calculation process for the single formula models in EXHEP.

Figure 93. Click on the Run button to begin the baseline calculation process for multiple formula models in EXHEP.

Figure 94. Click on the Analysis Reports buttons to review the baseline results in EXHEP. 
Figure 95. Example of the single formula model's Baseline (TY 0) Results report in EXHEP

Figure 96. Example of the multiple formula model's Baseline (TY O) Results report in EXHEP. 135

Figure 97 . Click on the Run button to begin the target year entry process for the single formula models in EXHEP.

Figure 98. Define the target years in EXHEP. 138

Figure 99. Click on the Run button to begin the without-project acres entry process for the single formula models in EXHEP. 140

Figure 100. Without-Project Acres entry window for single formula models in EXHEP. .141

Figure 101. Select a cover type from the pull-down menu, and EXHEP populates the entry form.

Figure 102. Enter the without-project acreage projections into the cells provided to the right of their corresponding target years.

Figure 103. Click on the Setup Reports button to view the Target Year List report in EXHEP

Figure 104. Example of Target Year List and Without-Project TY Acres reports in EXHEP. 145

Figure 105. Click on the Run button to begin the without-project variables entry process for the single formula models in EXHEP.

Figure 106. Describe Future Conditions entry window used to forecast without-project conditions for single formula models in EXHEP. 148

Figure 107. Type the new without-project values into the Mean column. ....................................... 149

Figure 108. Automated data entry for models that share variables. 150

Figure 109. Use the Factor column to enter future projections in terms of percentages of baseline.

Figure 110. Click on the Run button to begin the without-project variables entry process for multiple formula models in EXHEP.

Figure 111. Describe Future Conditions entry window used to forecast without-project conditions for multiple formula models in EXHEP.

Figure 112. Click on the Analysis Reports buttons to review the without-project forecasted entries in EXHEP.

Figure 113. Example of Without-Project Projections reports in EXHEP.

Figure 114. Click on the Run button to begin the without-project calculation process for single formula models in EXHEP.

Figure 115. Click on the Run button to begin the without-project calculation process for multiple formula models in EXHEP.

Figure 116. Click on the Analysis Reports buttons to review the without-project results in EXHEP 160

Figure 117. Examples of Without-Project Results reports in EXHEP.

Figure 118. Click on the Run button to begin the with-project acres entry process for the single formula models in EXHEP...

Figure 119. Enter the with-project acreage projections into the cells provided to the right of their corresponding target years.

Figure 120. Click on the Setup Reports button to view the With-Project TY Acres report in EXHEP. 
Figure 121. Example of With-Project TY Acres reports in EXHEP. 166

Figure 122. Click on the Run button to begin the with-project variables entry process for the single formula models in EXHEP. 166

Figure 123. Describe Future Conditions entry window used to forecast with-project conditions for single formula models in EXHEP. 168

Figure 124. Enter the with-project conditions for the single formula models in EXHEP...... 169

Figure 125. Click on the Run button to begin the with-project variables entry process for multiple formula models in EXHEP.

Figure 126. Describe Future Conditions entry window used to forecast with-project conditions for multiple formula models in EXHEP.

Figure 127. Click on the Analysis Reports buttons to review the with-project forecasted entries in EXHEP.

Figure 128. Example of With-Project Projections reports in EXHEP. 174

Figure 129. Click on the Run button to begin the with-project calculation process for single formula models in EXHEP..

Figure 130. Click on the Analysis Reports buttons to find the AAHU Summary report in EXHEP 176

Figure 131. Example of $A A H U$ Summary reports in EXHEP. 177

Figure 132. Export Report entry screen - exporting report information to MS Word. 178

Figure 133. Exported AAHU summary in the MS Word environment. 179

Figure 134. Click on the Run button to begin the with-project calculation process for multiple formula models in EXHEP. 180

Figure 135. Click on the Analysis Reports buttons to review the with-project results in EXHEP 181

Figure 136. Examples of With-Project Results reports in EXHEP. 182

Figure 137. Examples of With- vs. Without-Project Results reports in EXHEP.............................. 183

Figure 138. Examples of Overall Net Results - All Models report in EXHEP. 184

Figure 139. Recycling datafiles in HEAT to streamline the development of alternatives for a single project. 185

Figure 140. Click on the Setup button to begin the setup process in EXHGM. 189

Figure 141. Methods and Assumptions entry screen in EXHGM. 190

Figure 142. Function Descriptions entry screen in EXHGM. 192

Figure 143. Common EXHGM data entry screen prompts. 193

Figure 144. Partial Wetland Assessment Areas Descriptions entry screen in EXHGM. 194

Figure 145. Variable Descriptions entry screen in EXHGM. 195

Figure 146. Click on the Setup Reports button to view the setup reports in EXHGM................... 197

Figure 147. Report interface in EXHGM. ...................................................................................... 198

Figure 148. An expanded EXHGM report detailing additional fields.............................................. 199

Figure 149. Export Report entry screen - exporting report information to MS Excel or MS Word. 200

Figure 150. Exported reports in MS Excel and MS Word environments. ...................................... 201

Figure 151. Click on the Run button to begin the association process in EXHGM. ....................... 202

Figure 152. Select a function from the pull-down list. 203 
Figure 153. Identification of the Working Function in EXHGM's primary interface. 204

Figure 154. Function Associations window and Variables Available keypad used to associate models in EXHGM. 205

Figure 155. Function Associations entry screen. ............................................................................. 205

Figure 156. Select a partial wetland assessment area from the pull-down list. .............................206

Figure 157. Customize the size and location of the floating Variables Available keypad.............. 207

Figure 158. Help and Equation Check buttons provided to assist with $\mathrm{FCl}$ formula entry in EXHGM. 208

Figure 159. Function Equation/FCl Calculation Syntax window in EXHGM. 209

Figure 160. Use the keypad to correctly enter variable codes into the FCI Function Formula input box. 210

Figure 161. Begin by entering the variable codes (surrounded by brackets) into the $\mathrm{FCl}$ Function Formula input box....................................................................................... 211

Figure 162. Example of the formula entry process in the Function Associations entry form. ....... 212

Figure 163. Completing the $\mathrm{FCl}$ formula entry process - removal of the 1 * placeholder

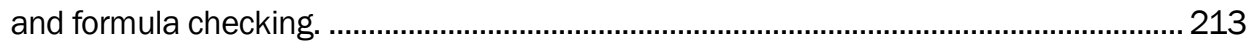

Figure 164. Extra records indicate phantom records that must be manually removed. ................ 214

Figure 165. Select the next associated PWAA from the pull-down list. ........................................... 215

Figure 166. Make a new association by duplicating the previous data. ........................................... 216

Figure 167. Show or hide all associated records simultaneously..................................................217

Figure 168. Click on the Setup Reports button to view the association reports in EXHGM........... 218

Figure 169. Example of EXHGM's formulas report for the functional models................................ 219

Figure 170. Click on the Run button to begin the baseline data entry process in EXHGM...........220

Figure 171. Baseline Data Entry windows in EXHGM. ................................................................. 221

Figure 172. Begin data entry by selecting a partial wetland assessment area and a variable from the pull-down lists provided.

Figure 173. Move to the lower window and enter the VSI graph coordinates in the space provided. 223

Figure 174. Example of a VSI coordinate entry. .............................................................................. 223

Figure 175. Copying VSI graph coordinates from one record to the next.......................................... 225

Figure 176. Click on the Setup Reports button to view the VSI graph coordinate reports in EXHGM. 226

Figure 177. Example of Variable Subindex Curves for Functions Report in EXHGM. ..................... 227

Figure 178. Click on the Run button to begin the baseline data entry process in EXHGM........... 228

Figure 179. An example of the manual data entry process in EXHGM. ..........................................230

Figure 180. Importing data from an external spreadsheet into EXHGM...................................... 231

Figure 181. Exporting data from EXHGM to MS Excel - click the Export Data button................... 233

Figure 182. Exporting data from EXHGM to MS Excel - assign a directory and path for the new file.

Figure 183. Deleting single data points in EXHGM.

Figure 184. Click on the Setup Reports button to view the variable data reports in EXHGM. 235

Figure 185. Example of Variable Data for Functions report in EXHGM. 236 
Figure 186. Click on the Run button to begin the baseline calculation process in EXHGM.......... 237

Figure 187. Click on the Analysis Reports button to review the baseline results in EXHGM.......... 238

Figure 188. Example of Baseline (TY 0) Results report in EXHGM................................................ 239

Figure 189. Click on the Run button to begin the target year entry process in EXHGM. .................241

Figure 190. Define the target years in EXHGM............................................................................... 242

Figure 191. Click on the Run button to begin the without-project acres entry process in EXHGM.

Figure 192. Without-Project Acres entry window in EXHGM...................................................... 245

Figure 193. Select a PWAA from the pull-down menu, and EXHGM populates the entry form.

Figure 194. Enter the without-project acreage projections into the cells provided to the right of their corresponding target years.

Figure 195. Click on the Setup Reports button to view the Target Year List report in EXHGM.

Figure 196. Example of Target Year List and Without-Project TY Acres reports in EXHGM.

Figure 197. Click on the Run button to begin the without-project variables entry process in EXHGM

Figure 198. Describe Future Conditions entry window used to forecast without-project conditions in EXHGM.

Figure 199. Type the new without-project values into the Mean column. 252

Figure 200. Automated data entry for functions that share variables. 253

Figure 201. Use the Factor column to enter future projections in terms of percentages of baseline.

Figure 202. Click on the Analysis Reports button to review the without-project forecasted entries in EXHGM

Figure 203. Example of Without-Project Projections report in EXHGM. 256

Figure 204. Click on the Run button to begin the without-project calculation process in EXHGM.

Figure 205. Click on the Analysis Reports button to review the without-project results in EXHGM.

Figure 206. Examples of Without-Project Results report in EXHGM. 260

Figure 207. Click on the Run button to begin the with-project acres entry process in EXHGM.

Figure 208. Enter the with-project acreage projections into the cells provided to the right of their corresponding target years.

Figure 209. Click on the Setup Reports button to view the With-Project TY Acres report in EXHGM.

Figure 210. Example of With-Project TY Acres reports in EXHGM.

Figure 211. Click on the Run button to begin the with-project variables entry process in EXHGM.

Figure 212. Describe Future Conditions entry window used to forecast with-project conditions in EXHGM.

Figure 213. Enter the with-project conditions in EXHGM. 
Figure 214. Click on the Analysis Reports button(s) to review the with-project forecasted

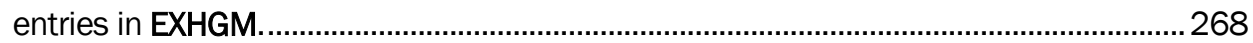

Figure 215. Example of With-Project Projections reports in EXHGM........................................... 269

Figure 216. Click on the Run button to begin the with-project calculation process in

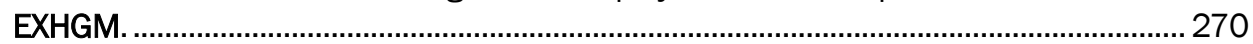

Figure 217. Click on the Analysis Reports buttons to find the AAFCU Summary report in

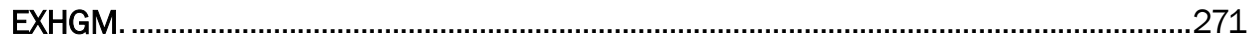

Figure 218. Example of AAFCU Summary reports in EXHGM.................................................... 272

Figure 219. Export Report entry screen - exporting report information to MS Word. .................... 273

Figure 220. Exported AAFCU summary in the MS Word environment. .............................................274

Figure 221. Click on the Analysis Reports button(s) to review the with-project results in

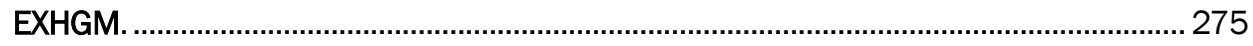

Figure 222. Examples of With-Project Results reports in EXHGM. .............................................276

Figure 223. Examples of With- vs. Without-Project Results reports in EXHGM............................. 277

Figure 224. Examples of Overall Net Results - All Functions report in EXHGM. ............................ 278

Figure 225. Recycling datafiles in HEAT to streamline the development of alternatives for

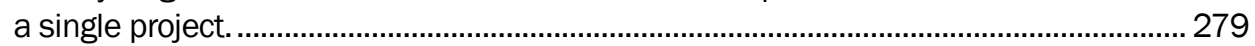

Figure 226. Normalizing negative data for use in the software. ……............................................ 283

Figure 227. Opening a datafile incorrectly. .................................................................................... 285

\section{Tables}

Table 1. Suggested components for community-based HSI models. ................................................

Table 2. Terminology crosswalks between the HEP and HGM methodologies..................................13

Table 3. Interpreting the results of an index assessment. .................................................................20

Table 4. Pertinent information needed to complete a HEP analysis..................................................59

Table 5. Pertinent information needed to complete an HGM analysis.............................................. 188

Table A1. Habitat assessment techniques that can be used to assess ecosystem

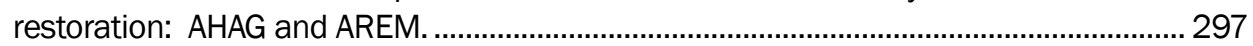

Table A2. Habitat assessment techniques that can be used to assess ecosystem

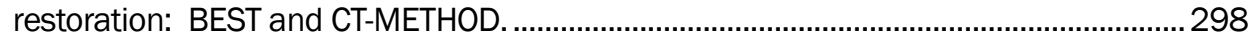

Table A3. Habitat assessment techniques that can be used to assess ecosystem

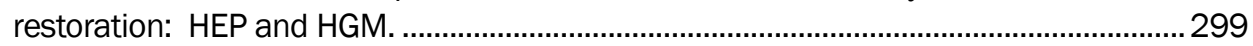

Table A4. Habitat assessment techniques that can be used to assess ecosystem restoration: H-M(N)M, IBI and PAMHEP. ................................................................. 300

Table A5. Habitat assessment techniques that can be used to assess ecosystem

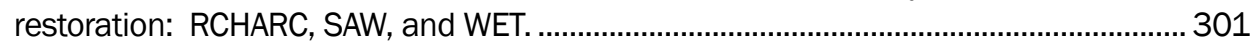

Table A6. Habitat assessment techniques that can be used to assess ecosystem restoration: WRAP.

Table A7. Habitat assessment techniques that can be used to assess ecosystem

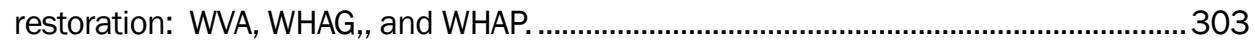

Table E1. Baseline cover types and acreages for the study...........................................................335

Table E2. Record list for Exercise 2 after formula entry................................................................ 341 


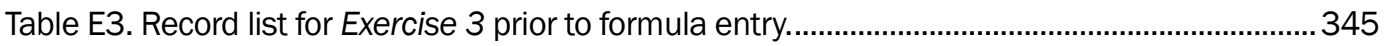

Table E4. Record list for Exercise 3 after HSI formula entry.........................................................346

Table E5. Record list for Exercise 3 after first LRSI formula entries................................................. 347

Table E6. Record list for Exercise 3 after second LRSI formula entries. ..........................................348

Table E7. Record list for Exercise 3 after the third set of LRSI formula entries. ...............................349

Table E8. Record list for Exercise 4 after X,Y coordinate entry into single formula model...............355

Table E9. Record list for Exercise 4 after $\mathrm{X}, \mathrm{Y}$ coordinate entry into the multiple formula

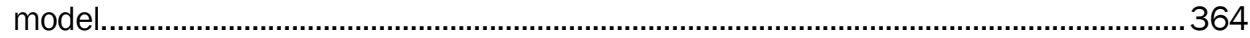

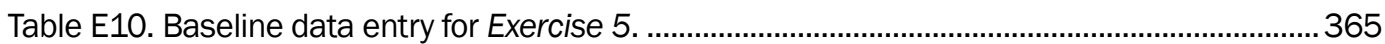

Table E11. Baseline data entry for Exercise 6. .........................................................................368

Table E12. Without-project acre projections for Exercise 7...........................................................372

Table E13. Without-project variable projections for the Marsh Wren HSI model in Exercise 7.

Table E14. Without-project variable projections for the Slider Turtle HSI model in

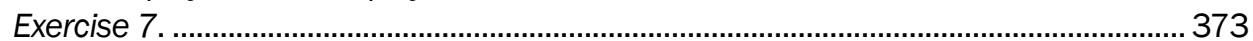

Table E15. With-project acre projections for Exercise 8. ............................................................... 378

Table E16. With-project variable projections for the Marsh Wren HSI model in Exercise 8............ 378

Table E17. With-project variable projections for the Slider Turtle HSI model in Exercise 8............. 378

Table E18. With-project acre projections for "Design 2" in Exercise 9 (Scenario A)........................385

Table E19. With-project variable projections for the Marsh Wren HSI model in "Design 2"

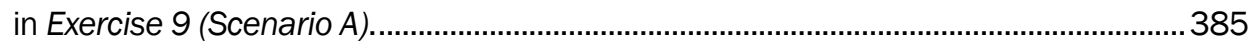

Table E2O. With-project variable projections for the Slider Turtle HSI model in "Design 2"

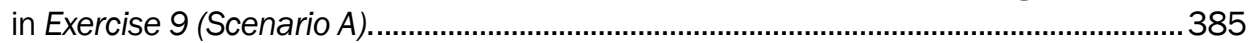

Table E21. Results of the Scenario A Restoration Analyses (“Design 1" vs. "Design 2")...............390

Table E22. With-project acre projections for "Design 1" in Exercise 9 (Scenario B). .......................392

Table E23. With-project variable projections for the Marsh Wren HSI model in "Design 1" in Exercise 9 (Scenario B). 392

Table E24. With-project variable projections for the Slider Turtle HSI model in "Design 1"

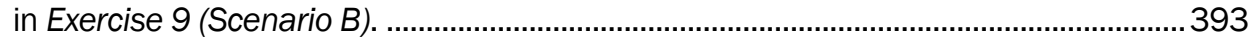

Table E25. Results of the Scenario A Restoration Analyses (“Design 1" vs. "Design 2")............... 399

Table E26. Baseline PWAAs and acreages for Exercise 10............................................................ 400

Table E27. Record list for Exercise 12 after formula entry............................................................416

Table E28. Record list for Exercise 13 after X,Y coordinate entry. .............................................. 424

Table E29. Baseline data entry for Exercise 14 ………….......................................................... 425

Table E30. Without-project acre projections for Exercise 14.................................................... 429

Table E31. Without-project variable projections for Exercise 14 . .................................................. 429

Table E32. With-project acre projections for Exercise 15 ......................................................... 434

Table E33. With-project variable projections for Exercise 15...................................................... 434

Table E34. With-project acre projections for "Scenario $A$ " in Exercise 16...................................... 440

Table E35. With-project variable projections for "Scenario A" in Exercise 16.................................. 440

Table E36. Results of the "Impact” and "Mitigation Analyses" ("Scenario A")................................ 445

Table E37. With-project acre projections for "Scenario B" in Exercise 16 ...................................... 447 
Table E38. With-project variable projections for "Scenario B" in Exercise 16 ............................... 448

Table E39. Results of the "Impact" and "Mitigation Analyses" ("Scenario B")................................ 450 


\section{Preface}

This document is a User's Guide, intended to provide both a step-by-step protocol for completing Habitat Evaluation Procedures (HEP) and/ or Hydrogeomorphic (HGM) assessment of wetlands using newly developed software, and an illustration of the software's effectiveness in evaluation of contemporary index models. In addition, this report briefly describes the hardware and software requirements of the system, and provides directions for installation of the program.

The work described herein was conducted with partial funding from several research programs including the System-Wide Water Resources Program (SWWRP) and the Wetlands Regulatory Assistance Program (WRAP). This report was prepared by Kelly A. Burks-Copes, Antisa C. Webb, and Dr. Michael F. Passmore, U.S. Army Engineer Research and Development Center, Environmental Laboratory (ERDC-EL), Vicksburg, Mississippi; and Sheila D. McGee-Rosser, Applied Research Associates, Inc., also in Vicksburg, Mississippi. At the time of preparation of this report, Ms. Burks-Copes and Ms. Webb were ecologists in the Ecological Resources Branch. Dr. Passmore was Deputy Director, ERDC-EL. Ms. McGee-Rosser was a computer scientist.

The authors wish to thank Susan Herrle, Shannon Langford, and Beattie Williams (Applied Research Associates, Inc.), and J ennifer Emerson (Bowhead Information Technology Services) for their hard work and persistence during the intensive months over which the project was undertaken. Thanks are also extended to J im Henderson, Billy J ohnson, J eff Lin, Rachel Pawlitz, and Andy Casper for their comprehensive review of the user guide and testing of the software. The St. Louis, Los Angeles, Galveston, Mobile, Chicago, Tulsa, Omaha, Albuquerque, and Kansas City USACE Districts have all received training in beta versions ${ }^{1}$ of the software and are currently or have recently used the software to assess feasibility studies in their areas. The authors are extremely grateful to the evaluation teams in these Districts and thank them for their critical feedback and support.

\footnotetext{
${ }^{1}$ Release notes will be provided on the System-Wide Water Resources Depot (https://swwrp.usace .army.mil). All users with beta versions of the software should Uninstall and Reinstall the HEAT software using Control Panel-Add or Remove Programs.
} 
This report was prepared under the general supervision of Antisa C. Webb, Chief, Ecological Resources Branch, EL; and Dr. Edmond J . Russo, Chief, Ecosystem Evaluation and Engineering Division, EL. At the time of publication of this report, Dr. Beth Fleming was Director of EL. COL Kevin J. Wilson was Commander of ERDC. Dr. J effery P. Holland was Director. 


\section{Unit Conversion Factors}

\begin{tabular}{|c|c|c|}
\hline Multiply & By & To Obtain \\
\hline acres & $4,046.873$ & square meters \\
\hline acre-feet & $1,233.5$ & cubic meters \\
\hline angstroms & 0.1 & nanometers \\
\hline atmosphere (standard) & 101.325 & kilopascals \\
\hline bars & 100 & kilopascals \\
\hline British thermal units (International Table) & $1,055.056$ & joules \\
\hline centipoises & 0.001 & pascal seconds \\
\hline centistokes & $1.0 \mathrm{E}-06$ & square meters per second \\
\hline cubic feet & 0.02831685 & cubic meters \\
\hline cubic inches & 1.6387064 E-05 & cubic meters \\
\hline cubic yards & 0.7645549 & cubic meters \\
\hline degrees (angle) & 0.01745329 & radians \\
\hline degrees Fahrenheit & $(F-32) / 1.8$ & degrees Celsius \\
\hline fathoms & 1.8288 & meters \\
\hline feet & 0.3048 & meters \\
\hline foot-pounds force & 1.355818 & joules \\
\hline gallons (U.S. liquid) & 3.785412 E-03 & cubic meters \\
\hline hectares & $1.0 \mathrm{E}+04$ & square meters \\
\hline horsepower (550 foot-pounds force per second) & 745.6999 & watts \\
\hline inches & 0.0254 & meters \\
\hline inch-pounds (force) & 0.1129848 & newton meters \\
\hline kilotons (nuclear equivalent of TNT) & 4.184 & terajoules \\
\hline knots & 0.5144444 & meters per second \\
\hline microinches & 0.0254 & micrometers \\
\hline microns & $1.0 \mathrm{E}-06$ & meters \\
\hline miles (nautical) & 1,852 & meters \\
\hline miles (U.S. statute) & $1,609.347$ & meters \\
\hline miles per hour & 0.44704 & meters per second \\
\hline mils & 0.0254 & millimeters \\
\hline ounces (mass) & 0.02834952 & kilograms \\
\hline ounces (U.S. fluid) & 2.957353 E-05 & cubic meters \\
\hline
\end{tabular}




\begin{tabular}{|c|c|c|}
\hline Multiply & By & To Obtain \\
\hline pints (U.S. liquid) & 4.73176 E-04 & cubic meters \\
\hline pints (U.S. liquid) & 0.473176 & liters \\
\hline pounds (force) & 4.448222 & newtons \\
\hline pounds (force) per foot & 14.59390 & newtons per meter \\
\hline pounds (force) per inch & 175.1268 & newtons per meter \\
\hline pounds (force) per square foot & 47.88026 & pascals \\
\hline pounds (force) per square inch & 6.894757 & kilopascals \\
\hline pounds (mass) & 0.45359237 & kilograms \\
\hline pounds (mass) per cubic foot & 16.01846 & kilograms per cubic meter \\
\hline pounds (mass) per cubic inch & $2.757990 \mathrm{E}+04$ & kilograms per cubic meter \\
\hline pounds (mass) per square foot & 4.882428 & kilograms per square meter \\
\hline pounds (mass) per square yard & 0.542492 & kilograms per square meter \\
\hline quarts (U.S. liquid) & $9.463529 \mathrm{E}-04$ & cubic meters \\
\hline slugs & 14.59390 & kilograms \\
\hline square feet & 0.09290304 & square meters \\
\hline square inches & 6.4516 E-04 & square meters \\
\hline square miles & $2.589998 E+06$ & square meters \\
\hline square yards & 0.8361274 & square meters \\
\hline tons (force) & $8,896.443$ & newtons \\
\hline tons (force) per square foot & 95.76052 & kilopascals \\
\hline tons (long) per cubic yard & $1,328.939$ & kilograms per cubic meter \\
\hline tons (nuclear equivalent of TNT) & 4.184 E+09 & joules \\
\hline tons ( 2,000 pounds, mass) & 907.1847 & kilograms \\
\hline tons ( 2,000 pounds, mass) per square foot & $9,764.856$ & kilograms per square meter \\
\hline yards & 0.9144 & meters \\
\hline
\end{tabular}




\section{Introduction}

Congratulations on the decision to use HEAT $^{\mathbf{1}}$ to assess ecosystem response to proposed planning initiatives! HEAT is well-suited to perform quick assessments of the impacts caused by constructed designs, functional lifts generated by ecosystem restoration activities, quantification of management approaches, and/ or even characterization of stochastic environmental events on your system. This introductory chapter gives a brief background of the techniques upon which HEAT is based, providing valuable context for using the program effectively and interpreting the results correctly. Taking a little bit of time upfront to read the first two chapters of this document will improve the user's ability to apply HEAT to a project's needs.

\section{Report purpose}

Quick assessment of ecosystem response (resulting from proposed construction activities, ecosystem restoration efforts, revised management practices, stochastic events, etc.) is critical to planners when formulating and comparing alternative project designs. Many methods (e.g., population assessments, qualitative matrices, life-history modeling, and habitat evaluation techniques) have been developed to investigate and predict environmental impacts on species, communities, and functions - each with varying degrees of success (Cooperrider 1986; National Biological Service 1995; U.S. Fish and Wildlife Service (USFWS) 1980a). In particular, a series of spatially-dependant species and community modeling systems have been developed to assess the non-monetary value of wildlife (terrestrial and aquatic communities) based on gains and losses in both quantity and quality of critical habitat or functionality (Appendix A). Advances in technology have led many agencies to automate these models and distribute them as computerized tools to planners, resource managers, and wildlife biologists nationwide (Adamus et al. 1987; Hays 1987; National Biological Service 1995; U.S. Army Corps of Engineers (USACE) 1980; USFWS 1980a-c, 1986). The value and validity of these tools depend greatly on their objectivity, repeatability, and efficiency. In order to guarantee their constant support and use by the planner, resource

\footnotetext{
${ }^{1}$ An installation $\mathrm{CD}$ for the Habitat Evaluation and Assessment Tools (HEAT) program can be obtained by contacting Kelly Burks-Copes at 601-634-2290 (Kelly.A.Burks-Copes@usace.army.mil).
} 
manager, or biologist, these tools must be easy to use, cost-effective, and responsive to user concerns.

Many planners, natural resource managers, and regulators have turned to techniques such as Habitat Evaluation Procedures (HEP) and the Hydrogeomorphic (HGM) approach to wetlands assessments to conduct ecological evaluations, only to reject their implementation because the underlying principles were unclear and/ or their application was too costly, dataintensive, and/ or seemingly inflexible. One goal of this User's Guide is to dispel these misconceptions, and present a logical 12-step approach to applying these techniques. ${ }^{1}$ This report provides guidance in the use of automated tools to complete an evaluation using HEP and/ or HGM, for it is hoped that users will find these techniques effective, efficient, and practical.

To that end, a series of exercises have been developed to illustrate the full implementation of HEP and HGM with the support of interactive HEAT Habitat Evaluation and Assessment Tools software (Appendix E). Over the years planners and resource managers have been forced to develop their own custom spreadsheets to compute HEP calculations. However, this approach proved to be inefficient, awkward, and relatively unproductive when handling the critical evaluations necessary to complete studies quickly. Because these spreadsheets were labor-intensive and prone to error, users were often forced to undertake extensive quality control measures to assure accuracy. A single site's HEP analysis (evaluating 15 species, 30 variables, and 25 cover types) required more than $80 \mathrm{hr}$ of technical involvement to complete (in addition to field data collection, photo interpretation, and acreage quantification). Large-scale HEP analyses necessitated the adaptation of traditional technological approaches. HEAT was designed to overcome these obstacles, and deliver outputs in a clear and concise manner, yet provide avenues of adaptation be they modification of model components, the addition of evaluation methodologies, or the incorporation of large-scale ecosystem approaches.

\section{Report organization}

The body of this User's Guide is divided into seven chapters:

- Chapter 1: Introduction

${ }^{1} \mathrm{~A}$ glossary and a complete list of acronyms are provided in Appendix B and Appendix C of this report. 
- Chapter 2: HEAT Evaluation Techniques

- Chapter 3: HEAT Modules

- Chapter 4: Getting Started

- Chapter 5: Applying EXHEP

- Chapter 6: Applying EXHGM

- Chapter 7: Troubleshooting

Chapter 2 (HEAT Evaluation Techniques) introduces the HEP and HGM techniques, describing a 12-step application process in great detail to facilitate application. A crosswalk section has been added to assist users familiar with either method in the deployment of the other technique. Chapter 3 (HEAT Modules) outlines the EXHEP and EXHGM modules discussing system capabilities, basic installation information, and system requirements. Chapters 4 through 6 detail the steps necessary to complete HEP and/ or HGM analyses using the HEAT software. Hands-on exercises are provided to improve the user's skill and highlight system capabilities. Electronic files are available from the installation $\mathrm{CD}^{1}$. Chapter 7 provides frequently encountered problems when using the software, and suggestions to resolve these issues. Together, the seven chapters and appendices describe the HEAT system, its modules, and its application in ecosystem evaluation studies. References follow immediately thereafter. Five appendices (Methods, Glossary, Notation, Suggested Variable Naming Conventions, and Training Exercises) have been included at the end of this report.

${ }^{1}$ An installation CD for the Habitat Evaluation and Assessment Tools (HEAT) program can be obtained by contacting Kelly Burks-Copes at 601-634-2290 (Kelly.A.Burks-Copes@usace.army.mil). 


\section{HEAT Evaluation Techniques}

It is important to understand the basic underlying principles of the HEP and HGM evaluation techniques in order to fully integrate the HEAT system into ongoing studies. It is also important to understand the basic mechanics of the software, acknowledging the data requirements and limitations of the tool. In this chapter, the HEP and HGM processes are described and compared in a crosswalk fashion. To conclude the chapter, a 12-step application strategy has been provided as a template for future studies.

\section{The HEP process}

The USFWS published quantifiable procedures in 1980 for assessing impacts of proposed water-and land-resource developments on fish and wildlife habitats (USFWS 1980 a,b,c). These procedures, referred to collectively as Habitat Evaluation Procedures and known widely as HEP, use a habitat-based approach to ecological assessment and provide a mechanism for predicting changes in habitat quality and quantity over time under alternative future scenarios. Habitat Suitability Indices (HSIs) are deployed within the HEP framework to capture the simple mathematical expressions for calculating each unit-less index of habitat quality as a function of one or more environmental variables that define habitat for a particular species or life history stage. The models are based on suitability indices (normalized on a 0-1 scale) that reflect habitat quality for a particular species or life stage over a range of possible environmental conditions, such as vegetative cover and composition, patch size, or hydrologic regime. The indices for each habitat-defining variable are then combined in an algorithm to yield a composite HSI value. This information is used in turn to compute habitat gains and losses directly attributable to a proposed project and the amount of compensation needed to offset any losses by combining the results with the affected acreage [acres $\times$ HSI $=$ habitat units (HUs), the HEP currency]. Since their inception, results of these assessments have often been applied by natural resource managers and decision-makers (Williams 1988; VanHorne and Wiens 1991; Brooks 1997; Brown et al. 2000; Store and J okimaki 2003; Shifley et al. 2006; Van der Lee et al. 2006; and others). 
Virtually all attempts to use HSI models have been heavily criticized, and many criticisms are well deserved. In most instances, these criticisms have focused on the lack of: (a) identification of the appropriate context (spatial and temporal) for the model parameters, (b) a conceptual framework for what the model is indicating, (c) integration of science and values, and (d) validation of the models (Kapustka 2005; Barry et al. 2006; Hirzel et al. 2006; Inglis et al. 2006; Ray and Burgman 2006; Van der Lee et al. 2006; and others). A fundamental problem with these approaches continues to be the inability to link species presence or relative abundance with relevant aspects of habitat quality (VanHorne and Wiens 1991), such as productivity.

Despite such criticisms, HSI models have played an important role in the characterization of a site's ecological condition. They represent a logical and relatively straightforward process for assessing risks to fish and wildlife habitat (Williams 1988; VanHorne and Wiens 1991; Brooks 1997; Brown et al. 2000; Kapustka 2005). The controlled and economical means of accounting for habitat conditions makes HEP a decision-support process that is superior to techniques that rely heavily upon professional judgment and superficial surveys (Williams 1988; Kapustka 2005). They have proven to be invaluable tools in the development and evaluation of restoration alternatives (Williams 1988; Brown et al. 2000; Store and Kangas 2001; Kapustka 2003; Store and J okimaki 2003; Gillenwater et al. 2006; Schluter et al. 2006; Shifley et al. 2006), managing refuges and nature preserves (Brown et al. 2000; Ortigosa et al. 2000; Store and Kangas 2001; Felix et al. 2004; Ray and Burgman 2006; Van der Lee et al. 2006; and others), and mitigating the effects of human activities on wildlife species (Burgman et al. 2001; National Research Council 2001; Van Lonkhuyzen et al. 2004). These modeling approaches emphasize usability. Efforts are made during model development to ensure that they are biologically valid and operationally robust. Most HSI models are constructed largely as working versions rather than as final, definitive models (VanHorne and Wiens 1991). Simplicity is implicitly valued over comprehensiveness, perhaps because the models need to be useful to field managers with little training or experience in this area. The model structure is therefore simple, and the functions that go into the models are relatively easy to understand. The functions that are included in each model are based on both published and unpublished information that indicates the variables that can influence the potential density of a species through direct or indirect effects on its life requisites. The general approach of the 
Suitability Index graphs is valid, in that the suitability of habitat to a species is likely to exhibit strong thresholds below which the habitat is usually unsuitable and above which further changes in habitat features make little difference. And as such, most HSI models should be seen as quantitative expressions of the best understanding of the relations between easily measured environmental variables and habitat quality available. Habitat suitability models are a compromise between ecological realism and limited available input data (Radeloff et al. 1999; Vospernik et al. 2007).

While empirical HSI models are being continuously produced for new species, it is unlikely that there will ever be production models for all the species of interest. Recent analyses suggest that the restoration and management focus must be broadened beyond species, and the numbers of sites created, restored, and managed must be increased to preserve the full range of the nation's biodiversity (Noss and Cooperrider 1994; Higgins et al. 1999; Groves et al. 2000, 2002; Poiani et al. 2000; Groves 2003; White and Fennessy 2005; and others). For the past 15 years, the study team at ERDC-EL has been called upon by USACE field offices (i.e., Districts) to assist in the development and application of HSI models. Under the auspices of the USACE's System-Wide Water Resources Program (SWWRP), ${ }^{1}$ ERDC has reviewed these activities, identified "lessons learned" from these experiences, and now proposes a new approach to HSI modeling based on characterizing communities as a whole - the intent being to capture the watershed aspects of ecosystem restoration and management activities on a larger scale, which accommodates a federal planning process time scale (1- 2 years) yet still quantifies non-monetary benefits in a manner supportive of the U.S. Army Corps' Strategic Plan for Water Resources ${ }^{2}$ and the USACE's Environmental Operating Principles. ${ }^{3}$ This new model-building paradigm lifts HSI models out of the site-specific approach of yesteryear, allowing for landscape-level accountability regardless of the community assessed.

The basic premise has been to focus community-based index model development on four interrelated components: Diversity, Vegetative Structure,

\footnotetext{
${ }^{1}$ For more information on the USACE's System-Wide Water Resources Program, refer to https://swwrp.usace.army.mil/DesktopDefault.aspx

2 For more information on the USACE's Strategic Plan for Water Resources, refer to http://www.usace.army.mil/inet/functions/cw/hot topics/cw strat.htm

3 For more information on the USACE's Environmental Operating Principles, refer to http://www.hq.usace.army.mil/cepa/envprinciples.htm
} 


\section{Hydrography (including water quality, hydrology, and biogeochemical/ soil characteristics), and Spatial Context (ranging from juxtaposition to distur- bance) (Table 1).}

Table 1. Suggested components for community-based HSI models.

\begin{tabular}{|c|c|c|}
\hline Code & Name & Description \\
\hline DIVERSITY & $\begin{array}{l}\text { Biotic Diversity } \\
\text { of the } \\
\text { Community }\end{array}$ & $\begin{array}{l}\text { Antagonistic and symbiotic interactions among organisms are some of the most } \\
\text { important factors influencing the structure of ecosystems. Because these interactions } \\
\text { have evolved over long periods of time, the deletion of species from or the addition of } \\
\text { species to an ecosystem can dramatically alter its composition, structure, and function. } \\
\text { Biotic interactions are particularly important in maintaining community structure and } \\
\text { ecosystem functions, and are described as "keystone" interactions in the field. These } \\
\text { interactions are a major determinant of the distribution and abundance of species in a } \\
\text { community. In most instances, the vegetative species compositions of living plant } \\
\text { biomass within the systems are thought to dictate the ecological integrity of the } \\
\text { ecosystems and suggest whether the systems can support animal populations and } \\
\text { guilds. The emphasis of the models is therefore placed upon the dynamics of the plant } \\
\text { community as revealed by the vegetative biodiversity and species content of the habitats. }\end{array}$ \\
\hline $\begin{array}{l}\text { VEGETATIVE } \\
\text { STRUCTURE }\end{array}$ & $\begin{array}{l}\text { Biotic Structure } \\
\text { of the } \\
\text { Community }\end{array}$ & $\begin{array}{l}\text { At the local scale, ecosystems possess a natural complexity of physical features that } \\
\text { provide a greater variety of niches and more intricate interactions among species. Local } \\
\text { structural complexity increases with more snags in forests, more woody debris in streams, } \\
\text { and more shrubs in deserts. In most studies, it is thought that each community's physical } \\
\text { characteristics and structures can therefore dictate the ability of the ecosystems to } \\
\text { provide habitat niches for a diverse range of species as well. The emphasis of the models } \\
\text { is therefore placed on characterizing the ecosystem's ability to provide physical space for } \\
\text { its numerous terrestrial and aquatic inhabitants to meet key life requisite requirements } \\
\text { (breeding, feeding, cover, etc.). }\end{array}$ \\
\hline HYDROGRAPHY & $\begin{array}{l}\text { Water Quality } \\
\text { and Hydrology } \\
\text { Characteristics } \\
\text { of the } \\
\text { Community }\end{array}$ & $\begin{array}{l}\text { Ecosystems possess natural hydrologic patterns that provide water for organisms and } \\
\text { physical structure for habitats. This cycle of water is also the vehicle for transfer of abiotic } \\
\text { and biotic materials through the system. Water is essential as sustenance for organisms, } \\
\text { and is a driving force for physical changes to the environment. It serves to transport } \\
\text { energy, nutrients, and species themselves. Wetlands are completely dependent upon } \\
\text { hydrology, and the adjacent terrestrial ecosystems in watershed (particularly riparian } \\
\text { forests) are heavily influenced by pulses of water infiltrating their boundaries throughout } \\
\text { the year. Therefore, wetness and adjacent land use conditions are thought to dictate the } \\
\text { ecosystem's ability to support terrestrial and aquatic inhabitants as well as support the } \\
\text { diverse plant communities indicative of health in the region. }\end{array}$ \\
\hline $\begin{array}{l}\text { SPATIAL } \\
\text { CONTEXT }\end{array}$ & $\begin{array}{l}\text { Landscape- } \\
\text { Level } \\
\text { Components } \\
\text { Both Within } \\
\text { and Adjacent to } \\
\text { the Community }\end{array}$ & $\begin{array}{l}\text { At the landscape level, natural ecosystems have a characteristic pattern and connectivity } \\
\text { of habitat patches. The amount and juxtaposition of these patches support the } \\
\text { movement of species and the transfer of materials (energy and nutrients) among } \\
\text { habitats. Woodland, prairie, and wetland ecosystems all have characteristic patterns of } \\
\text { habitat patches, and at a larger landscape level, these patterns can be viewed as a } \\
\text { mosaic of adjacent ecosystems. To adequately characterize ecosystem functions, one } \\
\text { must capture both the system's "place" in the landscape, as well as identify key corridors } \\
\text { and the processes that "shape" the system (i.e., habitat fragmentation). Therefore, } \\
\text { landscape-level characteristics (i.e., patch size, core size, edge size, and distribution as } \\
\text { well as the levels of disturbance immediately adjacent to the systems) are thought to } \\
\text { dictate whether flora and fauna would find the ecosystem serviceable. In general, high } \\
\text { levels of disturbance are thought to "drive off" sensitive species and reduce the system's } \\
\text { ecological integrity. }\end{array}$ \\
\hline
\end{tabular}


This approach to model development synthesizes the diffuse literature, identifies knowledge and data gaps to guide future research, and provides a framework for assimilating new information acquired in the future to facilitate adaptive management. Current efforts aim to improve ecosystem assessments by providing a framework that couples conceptual modeling, community-based HSI modeling, and Geographic Information Systems (GIS)-based data processing/ spatial analysis under a unified planning environment. Key to this approach is the ability of the model to utilize expert knowledge in a transparent fashion, and the ability to characterize communities across the system in a reasonable manner using GIS as a support tool.

Several advantages of this approach are evident. First the technique provides a logically consistent ordering of relations among environmental factors, as defined by the nature of their influences on organisms and their habitat. Second, these relations can be supported by formal logical expressions (mathematical equations) couched in terms of ecosystem integrity. Third, the approach provides a standard framework that can be applied to all manner of ecosystems, facilitating model consolidation across large studies with multiple species or communities of concern. And finally, in the process of constructing alternative designs and assessing these with landscape-level community models, attention may be drawn to variables that might otherwise be overlooked.

\section{The HGM process}

In the past 30 years or so, there has been increased public awareness of the values and benefits of wetlands to society (Conservation Foundation 1988; Rheinhardt et al. 1999; National Research Council 2001; Thiesing 2001). In the United States, this has produced changes in national policy, which include increased regulation of wetlands as well as both public and private conservation efforts to protect, acquire, enhance, and restore these precious resources. At the same time, wetland areas are under increasing pressure from development and urbanization across entire systems. Resource management concerns, as well as regulatory needs, force choices among the different, often conflicting, uses. The need to make decisions about wetlands has thus created a need for information on the value, both from an ecological and a societal standpoint, of these wetland resources; hence the need for wetland assessment. 
To date, the most common use of wetland assessment has focused on the evaluation of impacts due to development. The placement of fill material into wetlands and other waters, which often results in both acreage and functional loss of wetlands, is regulated by $\S 404$ of the Clean Water Act (49 CFR 230), and requires a permit from USACE. The decisions made by regulators under the $\$ 404$ Program increasingly require not only some means of quantifying areal losses, but also a means of addressing functional loss. Brinson (1993) began to address this need with the development of the Hydrogeomorphic (HGM) Classification Method, which has since evolved into a technique akin to HEP, but is focused on wetland functions rather than terrestrial functions (Brinson 1995, 1996; Rheinhardt et al. 1999; Smith et al. 1995; Smith and Bartoldus 1995). HGM involves a phased application - each task plays a different role in the overall approach. Three distinct activities comprise an HGM investigation: (1) wetland classification, (2) reference wetland identification and model calibration, and (3) the actual wetland functional assessment. This last phase (wetland assessment) is of particular interest for this report. Readers interested in details regarding the phased HGM process should refer to the extensive writings of Drs. Mark Brinson, Ronald (Dan) Smith, Ellis (Buddy) Clairain, and others posted on ERDC-EL's website http://el.erdc.usace.army.mil/wetlands/guidebooks.html, Accessed 27J une 2007) and the Environmental Law Institute's National Wetlands Newsletter archived articles found online at http://www2.eli.org/nwn/login/index.cfm (click on "Complete NWN Article Index," Accessed 27J une 2007).

According to Smith et al. (1995) the HGM methodology can be used to measure a large suite of wetland functions in a consistent manner across a large geographic region. HGM is a reference-based process, which uses a series of index models (normalized on a 0-1 scale) that capture the functionality of a wetland calibrated on reference standard wetlands in the adjacent landscape. As mentioned above, the first phase requires that users classify the wetlands of concern based on hydrology and geomorphic setting - the result is considered a "hydrogeomorphic subclass." The assessment protocol is then formulated - variables (indicative of function) are devised to characterize the aquatic condition across a suite of wetlands of the same subclass within a geographic region (i.e., reference domain) to determine the range of performance, for those functions in wetlands within the landscape. These variables are combined through mathematical algorithms to generate Functional Capacity Indices (FCIs), which estimate the capacity of a wetland to perform a function relative to other wetlands 
of the same hydrogeopmorphic type in the reference domain. These comparisons are based on reference stands, which are defined as the conditions under which the highest sustainable level of function is achieved across the suite of functions performed by wetlands of that subclass. This information is used in turn to compute functional gains and losses directly attributable to a proposed project and/ or the amount of compensation needed to offset any losses by combining the results with the affected acreage [acres $\times$ FCI $=$ functional capacity units (FCUs), the HGM currency]. The models are developed by a multidisciplinary (and often multiagency) team of experts referred to as the Assessment Team or A-Team, and are subjected to both peer review and public comment before the model is finalized. Thus, HGM provides an objective means by which functional performance can be measured, objectively compared across geographic areas, and evaluated in an effective and comprehensive manner.

Critics of the HGM approach abound (Hruby 1997, 2001; Kusler and Niering 1988; Rheinhardt et al. 1999; Thiesing 2001; Cole and Kooser 2002). Most point out that USACE proposed a National Action Plan for the adoption of HGM as the national standard for wetland assessment for use within the regulatory program (USACE 1996), which indicated their goal would be to develop sufficient models over the subsequent two years so that HGM could be used in $80 \%$ of the permit cases. As of this date, there are neither a sufficient number of models available to support implementation of HGM as a standard assessment method in most Districts, nor is the protocol for its use sufficiently documented to allow the development of consistent functional models (Thiesing 2001). Research programs have focused on aspects of HGM, but in recent years the methodology seems to have faded from active view, at least from the regulatory and scientific perspectives (Cole and Kooser 2002). The cost of model development is considered to be quite high and the effort to develop models can span the course of several years (2- 7+years). Other problems stem from ambiguous outputs. The numerous functional outputs are both complex and somewhat obscure - neither reflecting nor quantifying the extensive array of decision-making criteria involved in the planning and management of wetland ecosystems (Hruby 2001; Thiesing 2001; Cole and Kooser 2002). Finally, HGM does not adequately evaluate highly impacted wetlands such as those wetlands in urban settings without modifications (Hruby 1997, 2001; Kusler and Niering 1988). Reference standards (key to the development and calibration of the HGM models) are based on the 
highest sustainable level of functional performance (Brinson 1995, 1996). Owing to their location within the landscape, urban wetlands might be functioning at a level higher than the reference standard (as is the case with sediment removal and floodwater attenuation) and would have a significant benefit to the watershed because of their location within it. The application of HGM would result in a low functional rating for such a wetland because both positive and negative deviations from the reference standard are set lower than the standard. Such performance is not likely to be sustainable in the long term, but nonetheless would result in much greater benefits. Unfortunately wetlands that receive low functional indices do not receive the same priority for protection as those that receive high functional scores; as such, they may be at greater risk of destruction. This challenge is currently being researched by ERDC-EL.

Contrary to these criticisms, HGM has a number of fundamental strengths that set it apart from other assessment techniques. Perhaps its greatest strength is the iterative model development process, which allows users to refine and validate the model(s) based on data and expert review (a noted strength of HEP as well). As an approach, HGM is both objective and quantitative. It uses reference wetlands to provide objective bases for standards of comparison - something missing from most other assessment techniques (again, HEP is the exception). Considerable up-front effort (data synthesis, field collections, model derivations and calibration, etc.) is invested in developing an HGM model. Field testing, a critical effort undertaken during the initial model development, improves both precision (repeatability among users) and accuracy (via validation). Of course, this intensive up-front effort is iterative and thus a costly investment in managers' time over the long term. However, once the models are developed, the HGM assessment is expected to be relatively rapid, consistent, and reproducible (Smith et al. 1995). Ultimately, the development of HGM models provides an opportunity to bridge the gap between science and regulation - the model development process incorporates the best information available on a wetland's structure and functional attributes and captures the effects of proposed impacts prior to regulation. In response, functional assessment of prospective mitigation sites will help establish the design and the monitoring and assessment procedures for the wetland to be created or restored. Ultimately, HGM effectively incorporates reliable indicators of important wetland processes (hydrology, sedimentation, and primary production) into the dynamic planning paradigm - reporting continuous, parametric scores that quantify the effects of designs in 
relation to study objectives over a broad range of wetland ecosystems (National Research Council 2001).

\section{HEP versus HGM}

Ecosystems are generally characterized in terms of their structural components and the processes that link these components (Bormann and Likens 1969). Structural components of the ecosystem and the surrounding landscape (e.g., plants, animals, detritus, soil, the atmosphere, etc.) interact through a variety of physical, chemical, and biological processes. Understanding how these structural components interact or flow provides the basis for assessing ecosystem functions. HEP has been used in past studies (Williams 1988; VanHorne and Wiens 1991; Brooks 1997; Brown et al. 2000; Store and J okimaki 2003; Shifley et al. 2006; Van der Lee et al. 2006; and others), and HGM is likely to be used in future studies (Rheinhardt et al. 1999; National Research Council 2001; Thiesing 2001); therefore, the authors believe it is important to address the similarities between these two approaches when measuring ecosystem integrity, and discuss the use of multiple tools in evaluations of any significant magnitude. It is also important to validate the use of these two tools in an ecosystem setting, to assure users that the success of ecosystem restoration studies in the future can be assessed effectively and efficiently using a combination of the HEP and HGM methodologies.

As one might expect, the HEP and HGM approaches are quite similar, varying in matters of terminology and assessment focus. Probably one of the most important issues to address is the communication of results using the appropriate verbiage. To that end, Table 2 has been included here to demonstrate crosswalks between terms used in HEP and analogous terms used in the HGM application process.

The distinguishing difference between the HEP and HGM methodologies is the physical and ecological components or processes they each were developed to assess. HEP was designed to interpret the effects of environmental change through a species or community-based habitat suitability relationship across the landscape - a habitat maintenance function in the ecosystem setting. Although the HEP technique was not initially developed to assess additional ecosystem functions, combinations of HSI models in the HEP methodology indirectly measure ecosystem functionality across terrestrial and aquatic systems. In other words, HSI model parameters 
Table 2. Terminology crosswalks between the HEP and HGM methodologies.

\begin{tabular}{|c|c|c|}
\hline Parameters & $\begin{array}{l}\text { HEP } \\
\text { Terminology }\end{array}$ & HGM Terminology \\
\hline $\begin{array}{l}\text { An interdisciplinary group of regional and local scientists responsible for } \\
\text { determining significant resources, identification of reference sites, } \\
\text { construction of assessment models, definition of reference standards, } \\
\text { and calibration of assessment models }\end{array}$ & $\begin{array}{l}\text { Evaluation Team } \\
\qquad(\text { E-Team) }\end{array}$ & $\begin{array}{l}\text { Assessment Team } \\
\quad(\text { A-Team) }\end{array}$ \\
\hline $\begin{array}{l}\text { Measurable parcel of land defined by its vegetative cover, soils, and } \\
\text { topography }\end{array}$ & $\begin{array}{l}\text { Cover Type } \\
\quad(\mathrm{CT})\end{array}$ & $\begin{array}{l}\text { Partial Wetland } \\
\text { Assessment Area } \\
\text { (PWAA) }\end{array}$ \\
\hline $\begin{array}{l}\text { A measurable parameter that can be quantitatively described, with some } \\
\text { degree of repeatability, using standard field sampling and/or mapping } \\
\text { techniques }\end{array}$ & Variable & Variable \\
\hline $\begin{array}{l}\text { The index that rates the variable relative to optimum conditions. Both } \\
\text { Indices are, by definition, scaled from } 0.0 \text { to } 1.0\end{array}$ & Suitability Index (SI) & $\begin{array}{l}\text { Variable Subindex } \\
\qquad \text { (VSI) }\end{array}$ \\
\hline $\begin{array}{l}\text { A mathematical aggregation of the variable indices used to describe the } \\
\text { interrelationships among variables that define the suitability or } \\
\text { functionality of the site }\end{array}$ & $\begin{array}{l}\text { Habitat Suitability } \\
\text { Index } \\
\text { (HSI) }\end{array}$ & $\begin{array}{l}\text { Functional Capacity } \\
\text { Index } \\
\text { (FCl) }\end{array}$ \\
\hline $\begin{array}{l}\text { The product of the quality of the site (determined by the HSI or FCl) } \\
\text { multiplied by the quantity of the site } \\
\text { Unit = Quality Index X Quantity }\end{array}$ & $\begin{array}{l}\text { Habitat Unit } \\
\qquad(\mathrm{HU})\end{array}$ & $\begin{array}{l}\text { Functional Capacity } \\
\text { Unit } \\
\text { (FCU) }\end{array}$ \\
\hline $\begin{array}{l}\text { Units of time measurement that allow users to anticipate and direct } \\
\text { significant changes (in area or quality) across the system }\end{array}$ & $\begin{array}{l}\text { Target Year } \\
\quad \text { (TY) }\end{array}$ & $\begin{array}{l}\text { Target Year } \\
\quad \text { (TY) }\end{array}$ \\
\hline $\begin{array}{l}\text { The annualized change in the product of the quality and quantity of the } \\
\text { site's resources (species, communities or wetlands) over the life of the } \\
\text { project }\end{array}$ & $\begin{array}{l}\text { Average Annual } \\
\text { Habitat Unit (AAHU) }\end{array}$ & $\begin{array}{l}\text { Average Annual } \\
\text { Functional Capacity } \\
\text { Unit (AAFCU) }\end{array}$ \\
\hline $\begin{array}{l}\text { Value judgments captured through simple weighting or complex multi- } \\
\text { criteria decision analysis to account for the myriad of differing (but } \\
\text { relevant) criteria when comparing outputs }\end{array}$ & Tradeoffs & Tradeoffs \\
\hline
\end{tabular}

correlate closely with measures of ecosystem integrity such as improved water quality (i.e., turbidity, $\mathrm{pH}$, salinity, and temperature - factors in many fish HSI models), patchiness and/ or disturbance (i.e., distance to cover and water, riparian zone widths, human disturbance - factors of many bird and mammal HSI models), and both plant community and wildlife habitat maintenance (a factor of all the HSI models developed). Of course, HSI models are limited because they define only ecological communities as they pertain to physical and chemical characteristics of the landscape. HSI models do not, for example, include geomorphic setting, water source, and hydrodynamics - features that directly relate to aquatic ecosystem integrity. But a combination of well-chosen index models can be deployed to capture and reflect change in ecosystem functions across a site or a system. 
HGM, on the other hand, was specifically designed to assess wetland functions rather than individual species or community requirements. Strictly speaking, HGM applications are limited to wetlands defined as areas with less than $1 \mathrm{~m}$ of standing water present. Thus, HGM was not designed to evaluate all systems across the system or watershed. However, HGM is a powerful tool that can define the normal, or characteristic, activities that take place in a wetland ecosystem setting. As wetlands perform a wide variety of simple and complex activities based on their physical, chemical, and biological attributes, HGM has been designed to measure functional capacity. The combination of HGM, with its functional assessment approach, and HEP, with its coverage of both aquatic and terrestrial settings, can blanket an entire study area, capturing changes in ecosystem activities across the landscape. Maintenance of ecological integrity, the function that encompasses all of the structural components and processes in an aquatic and/ or terrestrial ecosystem, might therefore be assessed using a combination of HEP and HGM.

\section{Index model development}

Regardless of the approach taken to assess a study (HEP, HGM, or any other index-based methodology), index models are predominantly couched in terms of regional conditions and local system dynamics, and therefore users beware - these models must be adjusted to capture local conditions prior to their application outside their documented ranges. A workshop setting seems to provide the most efficient and effective forum to develop or modify/ adapt index models. Under the direction of knowledgeable facilitators, participants would familiarize themselves with the evaluation process; review the study's problems, opportunities and constraints; identify significant resources within the study boundaries; develop lists of species, communities, and functions critical to the system; associate and describe the physical settings integral to ecosystem integrity and health; establish goals and objectives; and ultimately build new models or modify existing models to meet their needs. Ideally, the development of index models would involve a close linkage between scientists and decision makers, in which the quantitative results of the index model would inform decision makers and the public by characterizing the response of ecological conditions under particular management regimes, and support those decision makers in making choices that reflect desired societal values, including issues of economics, politics, and culture. A systematic approach to model development is recommended, where drivers (water and land management) are tethered to stressors (e.g., 
hydrologic alteration, exotic species introductions, etc.) that affect significant components of the ecosystem, namely water, soils, habitat, and the landscape context. The effects of change (i.e., degradation) on these components would vary, and would potentially be quite diverse (e.g., altered surface flow, fragmentation, loss of structural complexity, etc.). Ideally, the model-building activity would include the following five tasks:

1. Compile all available information that can be used to characterize the ecosystem(s) of concern.

2. Convene the expert panel in the workshop setting to examine this material and generate a list of significant resources and common characteristics (land cover classes, topography, hydrology, physical processes) of the system that could be combined in a meaningful manner to "model" the community. In the workshop, it is important to outline study goals and objectives and then identify the desired model endpoints (e.g., outputs of the model). It is also critical that participants identify the limiting factors present in the project area relative to the model endpoints and habitat requirements. The outcome of the workshop is a series of mathematical formulas that can be divided into components or functions (e.g., hydrology, water quality, vegetative structure, diversity, connectivity, disturbance, etc.) comprised of variables that are: (a) biologically, ecologically, or functionally meaningful for the subject; (b) easily measured or estimated; (c) able to have scores assigned for past and future conditions; (d) related to an action that can be taken or a change that can occur;

(e) able to be influenced by planning and management actions; and (f) independent from other variables in the model.

3. Develop field, GIS, and hydrology data collection protocols, and use these strategies to collect all neoessary data, as well as apply these data to the model(s) in both the "reference" setting and on the proposed project area.

4. Present the model's results to an E-Team and revise/ recalibrate the model based on their experiences, any additional and relevant regional data, and application directives.

5. Submit the model to both internal ERDC-EL/District/ E-Team review and then request review from the original expert panel who participated in the original workshop, as well as solicit review from independent regional experts who were not included in the model development and application process.

This process can take many years to complete, or can be simplified and collapsed into short workshops dependent upon study application. 


\section{Basic index model application math}

The math involved in the application of any index model is fairly straightforward and can be summarized as follows:

1. BaselineAnalysis:

a. Calculate the means or mode for each variable data on a cover type (or PWAA) basis.

Water Depth Data $(\mathrm{cm})=10,10,20,20,5,0,12,8,10,5$

Mean $=10$

b. Normalize the mean/mode of the variables by locating their values on theX-axis of the index curve, and solving for the index score on theYaxis (using rise/ run). For example, if the mean water depth at Site $\mathrm{X}$ is on average $10 \mathrm{~cm}$, the value "10" is entered into the X-axis on the index curve below (Figure 1), and the resultant index score (Y-axis) is recorded (SI $=0.667)$.

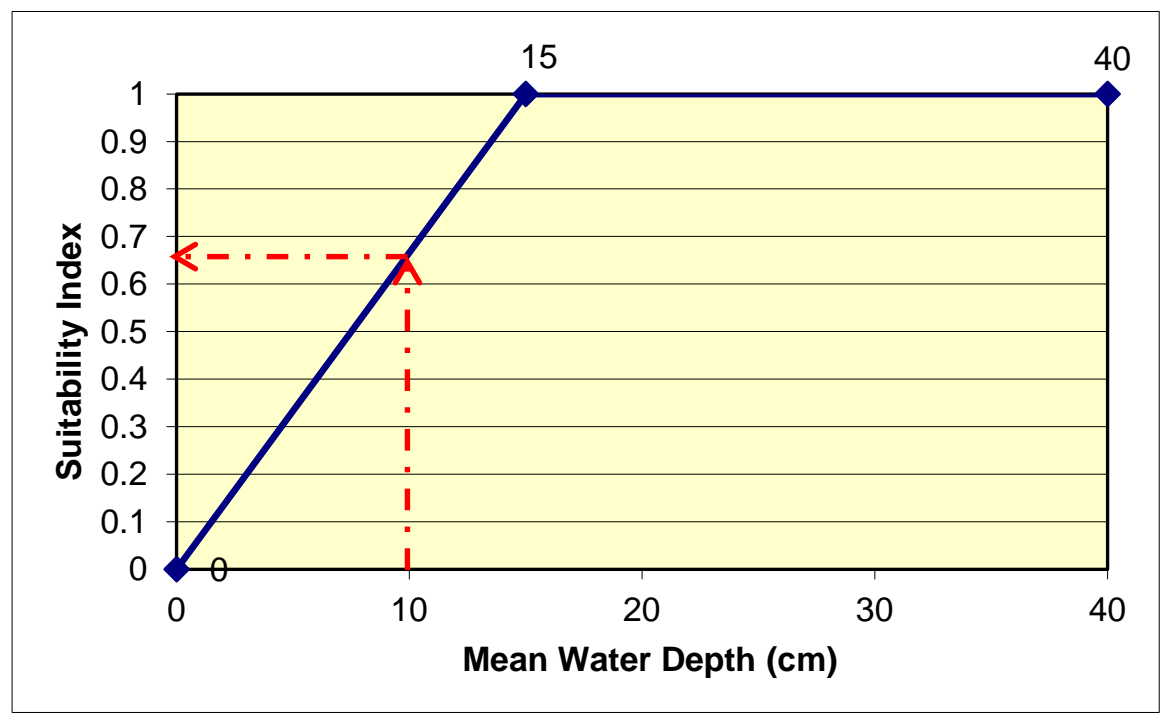

Figure 1. Example of an index curve.

c. For each cover type, plug the index scores into the overall index algorithm and generate cover type indices. For example, assume that there are two cover types associated with a marsh model (existing and newly restored). Further assume that the variables have been measured and normalized, and thus it is time to "plug" them into the index model(s): 
d. Calculate the relative area of each cover type associated with each life requisite. ${ }^{1}$

\begin{tabular}{|c|c|c|}
\hline \multicolumn{3}{|c|}{ Applicable Cover Types = } \\
\hline Cover Type & & Acres \\
\hline Restored Marsh & & 50.0 \\
\hline Existing Marsh & & 100.0 \\
\hline TOTALS & & 150.0 \\
\hline \multicolumn{3}{|c|}{ Relative Area $=$ Cover Type Area $\div$ Total Area } \\
\hline Cover Type & Acres & $\begin{array}{c}\text { Relative } \\
\text { Area }\end{array}$ \\
\hline Restored Marsh & 50.0 & 0.333 \\
\hline Existing Marsh & 100.0 & 0.667 \\
\hline TOTALS & 150.0 & 1.00 \\
\hline
\end{tabular}

e. Sum the weighted index scores to generate an overall index for the model. ${ }^{2}$

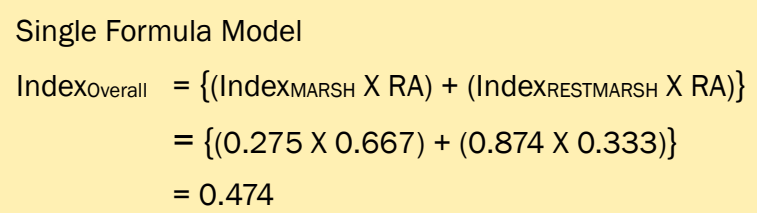

1 Relative Area (RA) is a mathematical process used to "weight" the various applicable cover types on the basis of quantity. To derive the relative area of a model's cover type for the study, the following equation was utilized:

Relative Area $\quad=\quad$ Cover Type Area

Total Area

where:

Cover Type Area = only those acres assigned to the cover type of interest

Total Area $=$ the sum of the acres utilized in the model

$2 \mathrm{HSI}_{\text {Model }}=\sum(\text { Cover Type Index x RA })_{\mathrm{X}}$

where :

CT Index = Results of the cover type index calculation

$X=$ Number of cover types associated with the model

$\mathrm{RA}=$ Relative area of each cover type 
2. Without-project Analysis:

a. Repeat steps a- $\mathrm{f}$ above for each target year.

b. Annualize the outputs using the following formula:

Annualized Units $=$

$\sum$ Cumulative Units $\div$ Number of years in the life of the project

where:

$$
\text { Cumulative Units }=\sum\left(\mathrm{T}_{2}-\mathrm{T}_{1}\right)\left[\frac{\left(\mathrm{A}_{1} \mathrm{I}_{1}+\mathrm{A}_{2} \mathrm{I}_{2}\right)}{3}+\frac{\left(\mathrm{A}_{2} \mathrm{I}_{1}+\mathrm{A}_{1} \mathrm{I}_{2}\right)}{6}\right]
$$

and where:

$\mathrm{T}_{1}=$ First Target Year time interval

$\mathrm{T}_{2}=$ Second Target Year time interval

$\mathrm{A}_{1}=$ Ecosystem area at beginning of $\mathrm{T}_{1}$

$\mathrm{A}_{2}=$ Ecosystem area at end of $\mathrm{T}_{2}$

$\mathrm{I}_{1}=$ Index score at beginning of $\mathrm{T}_{1}$

$\mathrm{I}_{2}=$ Index score at end of $\mathrm{T}_{2}$.

3. Repeat the process for each proposed alternative (with-project analysis), subtracting without-project annualized units from the with-project results to generate net gains or losses per alternative.

4. Interpret and present the results (Table 3).

Table 3. Interpreting the results of an index assessment.

\begin{tabular}{|l|l||}
\hline Index Score & Interpretation \\
\hline 0.0 & $\begin{array}{l}\text { Non-functional or not suitable - the ecosystem is not functioning at a measurable level and will not recover } \\
\text { through natural processes }\end{array}$ \\
\hline Above 0.0 to 0.19 & $\begin{array}{l}\text { Extremely low or very poorly functioning - the ecosystem processes and/or functions cannot be recovered } \\
\text { through natural processes }\end{array}$ \\
\hline 0.2 to 0.29 & Low or poorly functioning system with good potential for rehabilitation or restoration \\
\hline 0.3 to 0.39 & $\begin{array}{l}\text { Fair to moderate functioning capacity (again a good candidate for restoration and/or mitigation } \\
\text { compensation with action) }\end{array}$ \\
\hline 0.4 to 0.49 & Moderately functioning ecosystem \\
\hline 0.5 to 0.59 & Moderately high functional capacity \\
\hline 0.6 to 0.79 & $\begin{array}{l}\text { High or good functional capacity (areas to be avoided, and/or their impacts minimized under impact } \\
\text { scenarios) }\end{array}$ \\
\hline 0.8 to 0.99 & Very high or excellent functional capacity (again, these areas should be avoided under impact scenarios) \\
\hline 1.0 & Indicates optimal conditions (functionally speaking) \\
\hline
\end{tabular}


For those users interested in the derivation of the annualization formula, cumulative units are computed by summing the area under a plot of units versus time. This is equivalent to mathematical integration of the unit relationship over time, or

$$
\text { Cumulative_Units }=\int_{0}^{T} U d t
$$

But $U=A \times I$

where:
$\mathrm{A}=$ Area area
I = Quality index.

Also, over any time interval of length $T\left(=T_{2}-T_{1}\right)$ within which $A$ and I either change linearly or not at all, the values of $A$ and I are given by:

$$
\begin{aligned}
& A=A_{1}+m_{1} t \\
& I=I_{1}+m_{2} t
\end{aligned}
$$

where :
$\mathrm{t}=$ time
$A_{1}=$ the area at the beginning of the time interval
$\mathrm{I}_{1}=$ the quality index at the beginning of the time interval
$\mathrm{m}_{1}=$ the rate of change of area with time
$\mathrm{m}_{2}=$ the rate of change of quality with time.

Thus,

$$
\begin{aligned}
\int_{0}^{T} U d t & \equiv \int_{0}^{T}\left(A_{1}+m_{1} t\right)\left(I_{1}+m_{2} t\right) d t \\
& \equiv \int_{0}^{T} A_{1} I_{1} d t+\int_{0}^{T} m_{1} I_{1} t d t+\int_{0}^{T} m_{2} A_{1} t d t+\int_{0}^{T} m_{1} m_{2} t^{2} d t \\
& \equiv A_{1} I_{1} T+\frac{m_{1} I_{1} T^{2}}{2}+\frac{m_{2} A_{1} T^{2}}{2}+\frac{m_{1} m_{2} T^{3}}{3}
\end{aligned}
$$

Substitute the following equations for the slopes, $\mathrm{m}_{1}$ and $\mathrm{m}_{2}$

$$
\begin{aligned}
& m_{1}=\frac{A_{2}-A_{1}}{T} \\
& m_{2}=\frac{I_{2}-I_{1}}{T}
\end{aligned}
$$

into the above formula to generate the following:

$$
\int_{0}^{T} U d t \equiv A_{1} I_{1} T+\frac{\left(A_{2}-A_{1}\right) I_{1} T}{2}+\frac{\left(I_{2}-I_{1}\right) A_{1} T}{2}+\frac{\left(A_{2}-A_{1}\right)\left(I_{2}-I_{1}\right) T}{3}
$$

Collecting terms, substituting $\left(\mathrm{T}_{2}-\mathrm{T}_{1}\right)$ for $\mathrm{T}$, and simplifying yields:

$$
\int_{0}^{T} U d t \equiv\left(T_{2}-T_{1}\right)\left[\left(\frac{A_{1} I_{1}+A_{2} I_{2}}{3}\right)+\left(\frac{A_{2} I_{1}+A_{1} I_{2}}{6}\right)\right]
$$




\section{The 12-step HEP/HGM application strategy}

Based on the USFWS's Ecological Service Manual series on HEP (USFWS 1980 a,b,c), and a series of protocols for HGM application developed by ERDC-EL (Brinson 1993; Smith et al. 1995), 12 steps are involved in the application of HEP or HGM when assessing an environmental project:

1. Build a multi-disciplinary evaluation team.

2. Define the project.

3. Map the site's cover types or PWAAs.

4. Select, modify, and/or createindex model(s).

5. Conduct a baseline inventory.

6. Perform data management and statistical analyses.

7. Calculate baseline conditions.

8. Set goals and objectives, and establish the assessment's temporal scale.

9. Generate without-project conditions and calculate outputs.

10. Generate with-project conditions and calculate outputs.

11. Perform trade-offs.

12. Report the results of the analyses.

The following sections describe these steps in further detail and discuss their various applications to a planning study.

\section{Step 1: Build a multi-disciplinary evaluation team}

In HEP and HGM, an interagency, interdisciplinary team (E-Team/ ATeam) must be formed to lead both the model selection/ development phase of the project, and to establish the baseline and future conditions of the site(s). Participants often include representatives from the USACE, the U.S. Environmental Protection Agency (USEPA), the USFWS, the Natural Resources Conservation Service (NRCS), state fish and game offices, and other federal, state, and local governments, as well as tribes, as is deemed necessary. The technical expertise necessary to support planning efforts should include, but is not restricted to, representatives from botany, soils, hydrology, and wildlife ecology disciplines. The team should also include those individuals responsible for project design and management [i.e., engineers, project managers, NEPA (National Environmental Policy Act) consultants, cost-share sponsors, university professors, etc.]. 


\section{Step 2: Define the project}

Geographic location, project purpose, pre-settlement conditions, and the overall restoration/ mitigation/ compensation approach should be assembled to define the overall project. Identification and description of the affected counties, watersheds, primary water sources, and topography are particularly helpful. Artificial (man-made) structures should be described, and transportation networks should be identified. The project evolution and purpose should be addressed at this time (e.g., flood damage reduction, wetland restoration, mitigation, etc.). One might focus on the presettlement conditions of the region, concentrating on the cultural, ecological, and hydrological setting of the site. Ongoing problems to be addressed by the proposed project should be described at this point as well.

\section{Step 3: Map the site}

To evaluate the study site conditions, the area is divided into manageable and homogenous sections and quantified in terms of hectares or acres. This process is known as cover typing. A cover type in HEP (PWAA in HGM) is a parcel of land (or water) that has similar physical, chemical, and biological characteristics contained within its borders. Cover typing includes defining the differences between vegetative covers, soils, and hydrology (e.g., tall grass prairie, forested wetlands, shrub lands, lakes, and streams, etc.), and clearly delineating these distinctions on a map (preferably in a GIS). Once the team identifies the various vegetative communities/ systems on the site, and associates cover types with these systems, maps are generated to fully document the location and size of cover type patches within the study area (again, preferably in a GIS).

\section{Step 4: Select, modify, and/or develop the index models}

The model development and/ or selection process can "make" or "break" a study, and it is extremely important that the process focuses on the study's performance measures (i.e., success criteria), ecosystem incidence and architecture, and model parameters directly contributing to the ecosystem function. To do this, it has been suggested that teams select guild representative models or develop comprehensive community indices rather than focus on a series of independent species-based index models. A guild representative is, by definition, an animal (or plant) that belongs to a group of functionally similar species with comparable habitat 
requirements whose members interact strongly with one another. If results indicate a decline in a guild representative's habitat (or a decline in the overall ecosystem's health), it is assumed that associated flora and fauna will be subject to the same decline in conditions, and the system as a whole will degrade. Thus, index models should be selected as representative of identifiable guilds (or better yet, entire communities). In addition, model selection should be based on sensitivity of the indices to the proposed changes. Thus, identification of proposed actions and limiting factors within a model must be considered during the model selection and development process.

The number of HSI models available has increased dramatically in the last several years, and many now have MS Windows-based software and training to facilitate their application. More than 300 HSI models were initially developed by the USFWS in conjunction with other Federal agencies, including the USACE, to describe habitat quality for selected fish and wildlife species in the HEP arena. USFWS Ecological Services Manual 103 describes more about the models (USFWS 1980c). Although they were developed for assessing impacts of large water resource projects, their utility and flexibility in other areas has been proven. These models can also be accessed from the U.S. Geologic Survey National Wetlands Research Center page: http://www.nwrc.usgs.gov/wdb/pub/hsi/hsiindex.htm (Accessed on 27J une 2007). In addition to those published by the USFWS, several HSI models have been constructed and published in other formats http://el.erdc.usace.army.mil/emrrp/emris/ (Accessed on 27J une 2007).

In HGM, Regional Guidebooks serve as the primary source of documentation for each of the subclasses nationwide. Guidebooks include thorough characterizations of the regional wetland's subclass in terms of its geomorphic setting, water sources, hydrodynamics, vegetation, soil, and other features that were taken into consideration during the classification process. Classifying wetlands based on how they function narrows the focus of attention to a specific type or subclass of wetland, the functions that wetlands within the subclass are most likely to perform, and the landscape/ ecosystem factors that are most likely to influence how wetlands in the subclass function. This increases the accuracy of the assessment, allows for repeatability, and reduces the time needed to conduct the assessment. Unlike HSI models, FCI models for HGM subclasses are only just emerging from the research arena. At this time, few HGM subclass models have been published for distribution. ERDC-EL is leading a 
research effort under the Ecosystem Management and Restoration Research Program (EMRRP) to develop HGM subclass models (http://el.erdc.usace.army.mil/wetlands/guidebooks.html, accessed on 27J une 2007).

\section{Step 5: Conduct a baseline inventory}

Baseline characterization or "inventorying" includes gathering data on water quality, geochemistry, hydrology, fluvial geomorphology, substrate conditions, flora, and fauna, and to the greatest extent possible, identifying underlying stressors in the region. In particular, land-use activities, physical habitat alterations, and invasive species should be identified. In addition to the physical and chemical characteristics of the study area, land ownership and regulatory jurisdictions play an important role in determining opportunities for action. Much of this information is spatially based, and can be developed and stored in a GIS. As part of the basic site characterization, historical data on landscape-scale habitat conditions, land-use characteristics, and ownership patterns should be collected as well. A structured (yet flexible) sampling protocol with comprehensive documentation is required to collect data, while assuring easy replication. And, of course, an effort should be made to generate adequate sample sizes with minimal variation.

\section{Step 6: Perform data management and statistical analysis}

Some limits to the each assessment's data should be acknowledged in a data management step. In some instances, variables are sampled incorrectly or not measured at all in some settings, and extrapolations or corrections must be made after sampling has been concluded. Often, ground-truthing of cover type maps leads to data management actions (i.e., some data might be discarded due to incompatibility with the new classification). In those instances where data are discarded or absent, extrapolations are often made on the basis of overall site means or watershed means. When data management problems arise, it is important to consult with the team prior to data handling, so that solutions are devised with their full knowledge and consent.

\section{Step 7: Calculate baseline conditions}

Once the baseline inventory is complete, the variable means/modes are generated, then normalized with index curves, and finally weighted by contributing relative areas. Next, an algorithm is used to combine these 
weighted values into an overall site HSI/ FCI score that is then multiplied by the baseline acreages to generate units. Refer to the section on "Basic Index Model Application Math" for details surrounding these aspects of the application process. The results (indices, acres, and units) of this step are often presented in tabular displays or GIS maps.

\section{Step 8: Set goals and objectives and establish the assessment's temporal scale}

To measure an alternative's success, the team must set goals and objectives to capture the ecosystem return on investment. A goal by definition is considered an "end" or a final purpose (USACE 2000). In ecosystem restoration and mitigation activities, the goals are often focused on the restoration of significant ecosystem(s) function, structure, and dynamic processes that have been degraded. In impact studies, the goal is often to minimize, avoid, and compensate for losses of ecosystem(s) function, structure, and dynamic processes.

Objectives are smaller (in scale) and more obtainable endpoints that quantify the effectiveness of the proposed alternatives. Often objectives focus on the outputs of the models - namely, the generation of set units, indices, or acres on a specific schedule. One suggestion is to list the existing cover types/ PWAAs and estimate both the desired lift in terms of indices and desired amount of acreage change. Another suggestion is to quantify the pre-settlement conditions across the system and set objectives to meet this standard at some time in the future with action.

Given these goals and objectives, the team must designate a "Project Life" in terms of years, and develop a series of Target Years (TYs) (think of these as "snapshots in time") for which both without- and with-project conditions must be assessed. In HEP and HGM, there can be no less than three TYs, and they are by definition:

- $\mathrm{TY}_{0}$ - baseline

- $\mathrm{TY}_{1}$ - first year of construction/ restoration

- $\mathrm{TY}_{\mathrm{X}}$ - last year of project life or evaluation period. 
Between $\mathrm{TY}_{1}$ and $\mathrm{TY}_{\mathrm{X}}$, it is not uncommon for the team to add additional TYs to capture changes in vegetative cover and structure or other parameters resulting from anticipated impacts or proposed designs. ${ }^{1}$

\section{Step 9: Generate without-project conditions and calculate the outputs}

No single element is more critical to the planning process than predicting the most likely future condition(s) anticipated if no action is undertaken (aka the "without-project" condition). The process of developing these predictions (i.e., forecasting), is undertaken to identify patterns in natural systems and human behavior, and to discover relationships among variables and systems, so that the timing, nature, and magnitude of change in future conditions can be estimated. Though many forecasting methods can be used in an HEP or HGM assessment, a judgment-based method (supported by the scientific and professional expertise of the evaluation team) is often relied upon to forecast the site's future conditions.

Once changes in variable means/ modes have been predicted, the values are again normalized with the index curves on a TY-by-TY basis. These 0-1 scores are then weighted by contributing relative areas and applied to the algorithm to generate the overall site HSI/ FCI score for each TY. Next, this index is multiplied by the acreages (per TY) to generate units, which in turn are summed across all TYs to generate cumulative outputs over the life of the project. Finally, the cumulative units are annualized (again over the life of the project), in order to display and compare costs of the "No Action" plan with environmental repercussions (i.e., positive or negative annualized outputs). Refer to the section on "Basic Index Model Application Math" for details surrounding the aspects of this application process. The results of this step (i.e., cumulative and annualized units) are often presented in line graphs, tabular displays, and GIS maps.

\section{Step 10: Generate with-project conditions and calculate the outputs}

Identifying problems, opportunities, constraints, baseline conditions, and the without-project scenario leads the evaluation team into the plan formulation process, wherein scenarios are developed to address the

\footnotetext{
1 The authors wish to offer a precautionary note - the selection of too few TYs can result in a coarse analysis insensitive to key ecological/physical changes in the environment. The selection of too many TYs can lead to excessive data handling, often with no return on investment. Five TYs spread over the course of 50 years offers a reasonable expectation of return for the level of data analysis and management required.
} 
goals and objectives efficiently. Some alternatives are dropped if their approaches are too costly, if their undertaking or rationales are incongruous with the overall study concept, if their designs are impossible to achieve due to conflicting relationships, or if the results are thought to be ecologically unproductive. As in the without-project setting, the team generates a list of general trends for the overall study - incorporating changes in hydrology, biodiversity, connectivity, disturbance, water quality, biochemistry, topography, etc. into projected variable trends on a TY-by-TY basis. At this point, various construction and operation/ maintenance activities should be discussed in great detail - the outcomes of each action must be quantified and incorporated into the forecasting effort. Annualized outputs are generated in the same fashion as the without-project scenario. The net change in annualized units determines each alternative's productivity (i.e., with - without = net change). Once again, the results (i.e., cumulative, annualized, and net gains/losses), are presented in line graphs, tabular displays, and GIS maps.

\section{Step 11: Perform trade-offs}

In instances where multiple indices are used to assess ecosystem change, the comparison of alternative productivity is concluded with the ranking and combination of model outputs. These rankings (i.e., "trade-offs") can be as simple, or as complex, as necessary to afford the highest level of accounting. In a simple application, trade-offs are frequently based on professional judgments made by the team (i.e., the team trades off model contributions based on their own accumulated technical expertise, general experience, and specific knowledge of the study area). In the overall scheme of project design, trade-offs serve as prisms to concentrate attention on those changes that will impact the area's significant resources. Determining "value" is a somewhat subjective exercise in the assessment process, but HEP and HGM provide avenues of documentation and justification necessary to support decisions in this arena.

\section{Step 12: Report the results of the analyses}

The success of any ecosystem assessment lies in the ability to communicate assessment strategies and findings to the public. Underlying the HEP and HGM processes is the concept of "repeatability." To assure that the assessments are reasonable and reliable, the reader should be able to follow the descriptions of approach and applications presented, and repeat the analyses just as the team did. To assure the repeatability aspects of the 
assessments, it behooves the team to document, to the fullest extent, the evaluation in its entirety. Most often this is done through an assessment report medium. Typically, depending on the type of planning effort undertaken, five or more chapters are provided in every assessment report: Introduction, Methods, Results, Trade-offs, and Summary/ Conclusions. In addition, the report typically carries a Literature Cited section and an appendix documenting the models used in the assessment. Further reporting of the assessment results can include (but is not limited to) the production of interactive graphics (maps, graphs, tables, etc.) that visually depict the conditions (both without- and with-project) of the study area under evaluation. In HEP and HGM, it is important to document the results in terms of units, quality (i.e., indices), and quantity (acres). In addition, any factors that significantly affected the outcome of the study (e.g., minutes of team meetings, data extrapolations, etc.) should be documented, either in the report itself, or in an appendix attached to the report. 


\section{HEAT Modules}

The sheer number of calculations necessary to conduct a HEP or HGM evaluation in a study necessitates the use of automated systems to complete the assessments in a timely manner. ERDC-EL has developed HEAT - Habitat Evaluation and Assessment Tools to address this need. Currently comprised of two evaluation modules (i.e., EXHEP: EXpert Habitat Evaluation Procedures and EXHGM: EXpert Hydrogeormorphic Approach to Wetland Assessments), the system provides a fully automated interface to facilitate simultaneous HEP and HGM assessments. The basic HEAT architecture is described in this chapter, and a discussion of its application to various studies follows immediately thereafter. The chapter concludes with the basic system requirements and installation instructions.

\section{Introduction to EXHEP and EXHGM}

EXHEP and EXHGM are Microsoft Access ${ }^{\circledR}$ 2003-2007 software modules developed by ERDC-EL to automate standard HEP and HGM calculations in an attempt to facilitate large-scale ecological assessments efficiently and effectively. EXHEP and EXHGM use Microsoft Windowscompatible programming to: (1) solve complex mathematical calculations quickly, and (2) provide a highly intuitive, visual interface to facilitate communication between the system and the user. As with any sophisticated mathematical evaluation, a well-tested, efficiently written, standard software package is a critical tool that saves time, and improves the reliability and repeatability of the results. However, these modules cannot replace a sound understanding of the conceptual basis of HEP and HGM, or their application to the decision-making process. EXHEP and EXHGM should not be viewed as the end-all means to providing the only predictive environmental response to project development. Rather, the modules should be viewed as tools that can provide rational, supportable, focused, and traceable evaluations of environmental effects.

\section{Module capabilities}

The EXHEP and EXHGM modules were designed to process large quantities of data quickly and efficiently, handling a large number of index 
models simultaneously. Each model can incorporate any number of cover types (or PWAAs). Each model can include any number of variables, and the user can incorporate as many components or functions into each model as demanded. These capabilities support the examination of complex studies with large numbers of permutations. In some studies, it is not unusual to evaluate 10 to 15 index models (with more than 25 cover types) in an attempt to describe complex interdependencies (i.e., interrelationships) within the ecosystem. The large number of tedious mathematical calculations necessary to compute HEP and HGM at this level requires a powerful tool to evaluate environmental output. EXHEP and EXHGM, enhanced by their abilities to communicate these activities in an organized fashion, can quickly accomplish this task. The number of permutations, processing speed, and program performances are limited only by the capacity of the user's hardware, where data storage becomes the limiting factor.

The EXHEP and EXHGM modules allow users to evaluate a large number of projected changes (future conditions) across numerous years for each alternative design. The modules allow users to assign Future Factors (FFs) to the variables with the index models for each year considered across the life of the project (i.e., each TY). This capability allows users to manage forecasts across the long-term planning horizon. Again, the number of permutations is limited only by computer storage capacity. EXHEP evaluates any species or community HSI model. In most instances, a species or community can be described based upon its single cover type dependence. A standard HEP software package must complete these computations, regardless of whether the model utilizes a single cover type or multiple cover types. EXHEP can be used to calculate suitability for any single or multiple cover type models whether they are single- or multiformula models. ${ }^{1}$ EXHGM evaluates any FCI-based model. In most instances, a wetland cannot be described using a single PWAA and a standard HGM software package must complete these computations,

1 Single formula models by definition are based on the existence of a single life requisite requirement, and thus a single index formula is used to depict the relationship between quality and carrying capacity for the site. Multi-formula models employ more than one index formula to assess the suitability of the habitat for a species or a community. If an ecosystem's integrity is limited by the existence of more than one life requisite (aka components such as food, cover, hydrology biodiversity, connectivity, water quality, biogeochemistry, etc.), and the quality of the site is dependent on a minimal level of each component, then the model is considered a "Life Requisite" model in HEP. To calculate the overall HSI for any Life Requisite Model in HEP, the value of a Life Requisite Suitability Index (LRSI) must be derived for each component in the model - a process requiring the user to calculate multiple indices and then combine these in a second algorithm. This multi-formula processing has led to the name "Multiple Formula Model" in EXHEP. 
regardless of whether the model utilizes a single PWAA or multiple PWAAs. EXHGM can be used to calculate suitability for any single or multiple PWAA model, whether the wetlands functionality is based on one or more multi-faceted functions.

The HEAT modules are capable of reevaluating index models as the user adapts previously created alternative designs to fit new situations. It is not necessary to reinvent indices, cover type interdependencies, or life requisite interrelationships once a standard evaluation configuration has been created. The modules allow access to previously created configurations and introduce change (e.g., adding field data, future factors, TYs, indices, cover types, acreages, etc.). This capability supports the software's utilization in a wide range of agency activities over the long term. For example, an alternative design developed to evaluate project impacts for a stream restoration study in the past can be adapted to evaluate stream restoration projects throughout the region in the future. By simply altering the cover type composition of a previously developed EXHEP/ EXHGM datafile, the software can characterize and assess for regional variations, and quickly quantify impacts and/ or benefits resulting from these changes. Thus, as projects are funded or evolve, these assessments can be easily implemented with little effort devoted to modeling "setup" in the HEAT system.

HEAT has a series of automated utilities designed to make the HEP and HGM processes more user-friendly. HEAT is programmed to produce pre-designed database reports, but supports the export of these reports for user customization. If a report must be adapted to meet the needs of several different audiences (e.g., construction crews, environmental compliance agencies, administrative boards, decision-making teams, etc.), these products can be quickly exported and redesigned to display outcomes in a meaningful manner to suit the occasion. This feature ensures rapid reportability and supports decision-making at all levels.

HEAT can also generate result tables documenting the outcome of each alternative design in a clear, concise manner. These database tables (generated in Microsoft Access 2003-2007 and exported to Microsoft Excel ${ }^{\circledR} 2003-2007$ format) can be directly embedded or linked to Microsoft Windows-compatible word processing packages (Figure 2). 


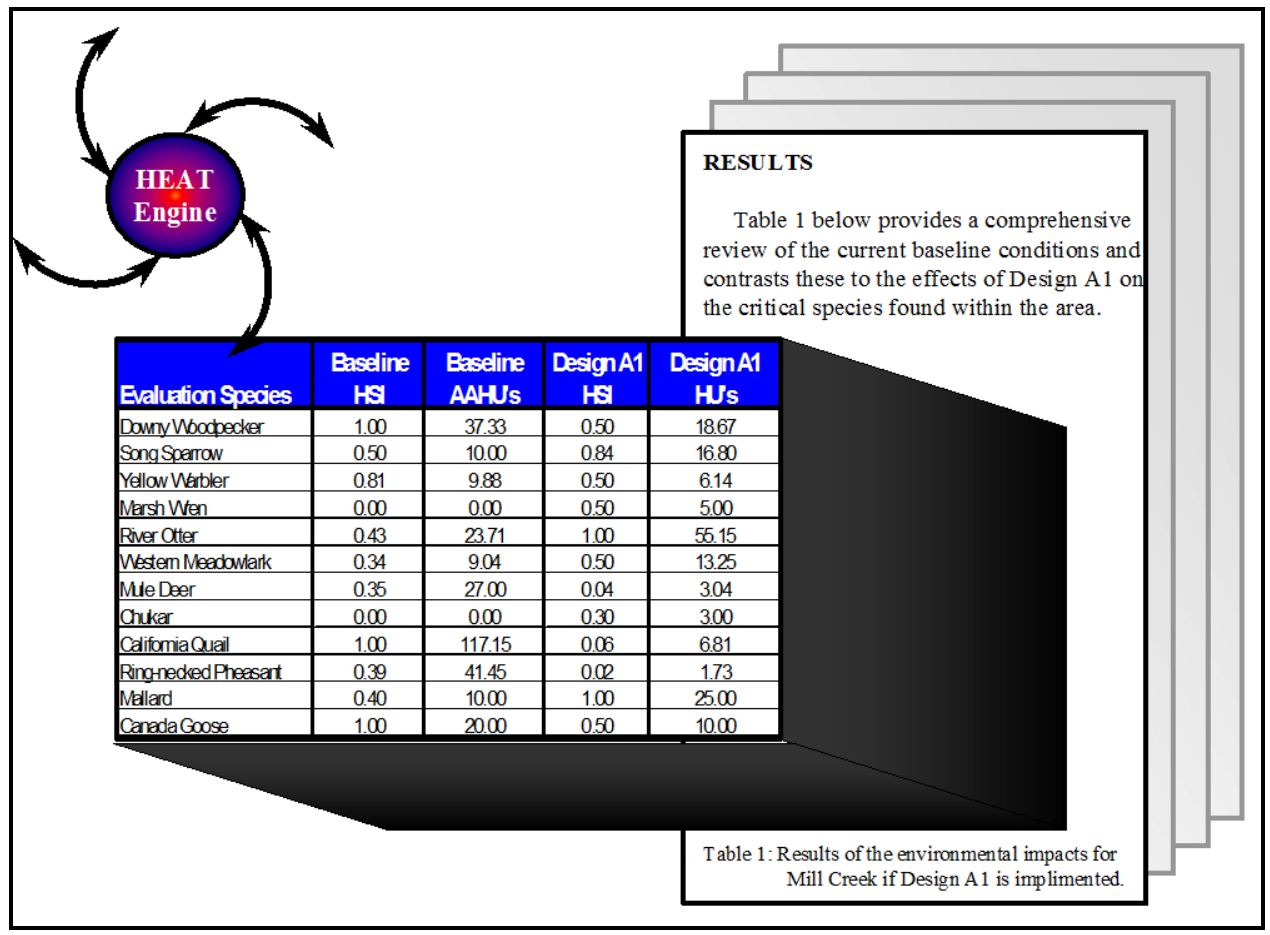

Figure 2. Linking HEAT products to reports.

If files are linked to a document, alterations in the HEAT datafiles are automatically transferred to the word processing document, reducing the effort and time spent updating documents during "what-if" alternative design analysis.

Throughout the analysis process, HEAT offers comprehensive help windows and messages to produce a user-friendly atmosphere. For instance, HEAT provides online syntax assistance to users via interactive information windows, demonstrations, and equation testing buttons. These interfaces, in particular, help users develop models effectively in the setup process. HEAT also provides online messages throughout the evaluation process via pop-up messages and standard Microsoft

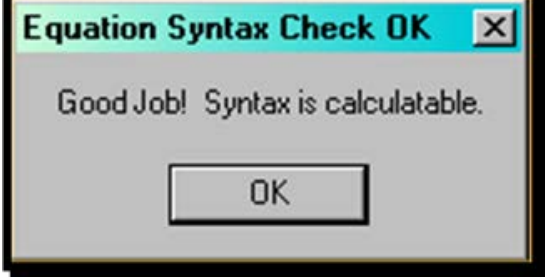

Windows status bars at the bottom of the computer screen. These features "prompt" users to complete activities following a specific protocol and provide critical information concerning data entry processes at pivotal moments throughout the interactive process. Furthermore, HEAT provides pop-up windows that display the status of automated processes (i.e., time-elapsed displays and record-counting devices) to keep users apprised of the evaluation's progress. These innovations create a 
user-friendly environment for index model setup and provide insight into model analysis.

\section{System requirements}

HEAT was developed for a personal computer with a 486 (or better) processor. Calculation times vary based upon file size. At least $4 \mathrm{MB}$ of random access memory (RAM) are needed for installation and operation. Hard drive space requirements (necessary for file storage and processing) will be dependent on the size and scope of the analysis required. Large files may require over $8 \mathrm{MB}$ of hard disc storage. A video graphics adapter (VGA) monitor (or better) with 256 color graphics capabilities and a laser or ink jet printer with at least 300 dots per inch (dpi) resolution are also required. HEAT's database and user interface were developed in Microsoft Access 2003-2007; therefore, HEAT requires that this software package be installed on the computer. HEAT can accept data from many Microsoft ${ }^{\circledR}$ Windows-compatible software packages (e.g., Microsoft Excel 2003-2007, Microsoft Access 2003-2007, Lotus ${ }^{\circledR} 123$, Corel's ${ }^{\circledR}$ Quatro Pro 6.0+, etc.). HEAT will export data to Microsoft Excel 2003 spreadsheets and Lotus 123 spreadsheets (*.wk1 and *.wk2 versions). HEAT was developed to run under a Microsoft Windows ${ }^{\circledR}$ '98 or better operating system.

\section{Software installation}

HEAT is distributed on an installation CD. ${ }^{1}$ This CD can be obtained by contacting Kelly Burks-Copes at 601-634-2290 (Kelly.A.BurksCopes@usace.army.mil).The program can be installed on more than one computer, in any directory. ${ }^{2}$ To successfully install HEAT, insert the installation disk into the $\mathrm{CD}$ drive. The installation program will start automatically. ${ }^{3}$ Restart the computer after this process is complete prior to running the HEAT software.

\footnotetext{
1 The user must have Administrator permissions/access for the computer being used in order to install the HEAT software.

2 The HEAT program and associated datafiles must be installed and stored locally on the hard drive network installation and operation have proven unsuccessful to date.

3 If the Install Shield does not begin immediately, left-click on the Start button. Move the cursor up to the Run listing. Select the CD drive and find Setup.exe. Double-click on the file and allow the installation process to complete itself.
} 


\section{System overview}

The entire HEAT system has been assembled in two Microsoft Access 2003-2007 databases: the analysis engine (USACE_HEAT.mdb) and the datafile (datafile.mdb). Three separate processes are performed by the system: model setup and data input, mathematical processing, and reporting outputs. To complete an analysis using HEAT, provide the following data:

1. Index models

2. Variables and methods of data collection

3. Project life span and TYs of interest

4. Baseline and design acreages per TY

5. Ecosystem quality for baseline and future conditions across the time intervals.

If quality data are generated in a Microsoft Windows-compatible application (e.g., Microsoft Excel, Microsoft Access, Lotus 123, Corel's Quatro Pro, etc.), the data can be imported into HEAT's data entry screens early in the process. The mathematical processor combines these inputs and generates the baseline and development output values for each model. The results are reported using a customized report generator built into the HEAT program. HEAT also provides an option to save tabular results to Microsoft Excel spreadsheets, allowing findings of the analysis to be reported in a professional context.

The remaining chapters offer step-by-step protocols to complete HEP and HGM analyses using the HEAT modules. Exercises have been provided to instruct users in the system's capabilities. Appendix D contains a suggested naming convention protocol to support development of a standardized and easily understood approach to model building in HEAT. 


\section{Getting Started}

This chapter is dedicated primarily to the basic functions of the HEAT software and the interfaces encountered upon entering and moving within the software. Primary buttons on the system's main interface are defined, and suggestions regarding file management and logistics are provided.

\section{Starting the program}

To begin an analysis using HEAT, use the mouse to click on the Start button in the bottom left-hand corner of the screen. Move the cursor up to the All Programs option and scroll the menu to HEAT-Habitat Evaluation and Assessment Tools. Hold down the mouse button, and move across to HEAT and click on this option. HEAT will now ask for a datafile name and location (Figure 3 ).

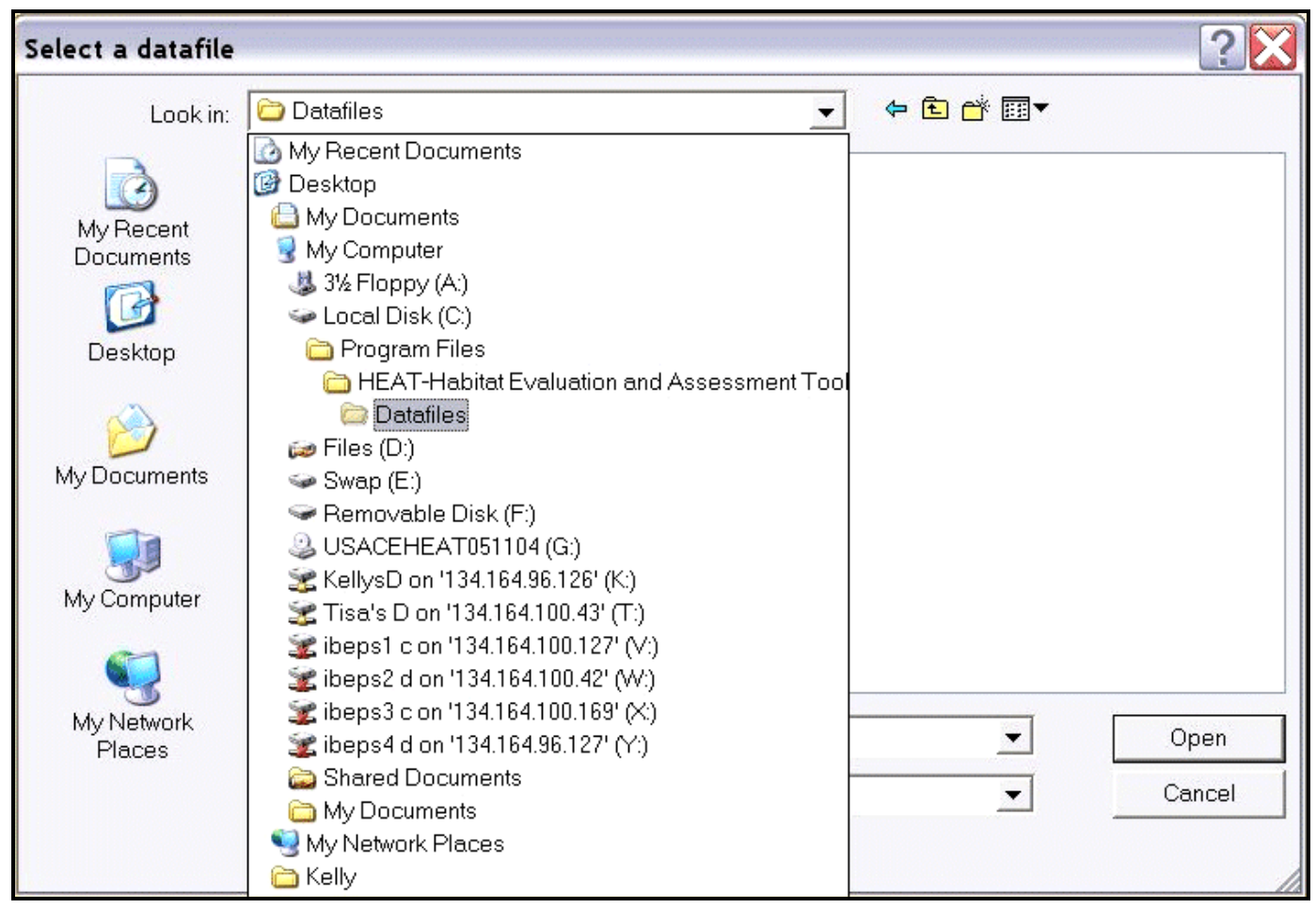

Figure 3. Datafile selection entry screen - blank datafile location. 
If working on a new project (with a new index model), model specifics and initial "baseline" site conditions must be entered into a blank datafile. Once users have installed the HEAT program, the blank datafile can be viewed/ accessed from the following path:

\section{- Program Files/HEAT-Habitat Evaluation and Assessment Tools/Datafiles/ Blank Datafile.mdb.}

Move through the paths in the Look in: window to guide HEAT to the location of the blank datafile. Select Datafile.mdb in the File Name: window and press Open to begin (Figure 4).

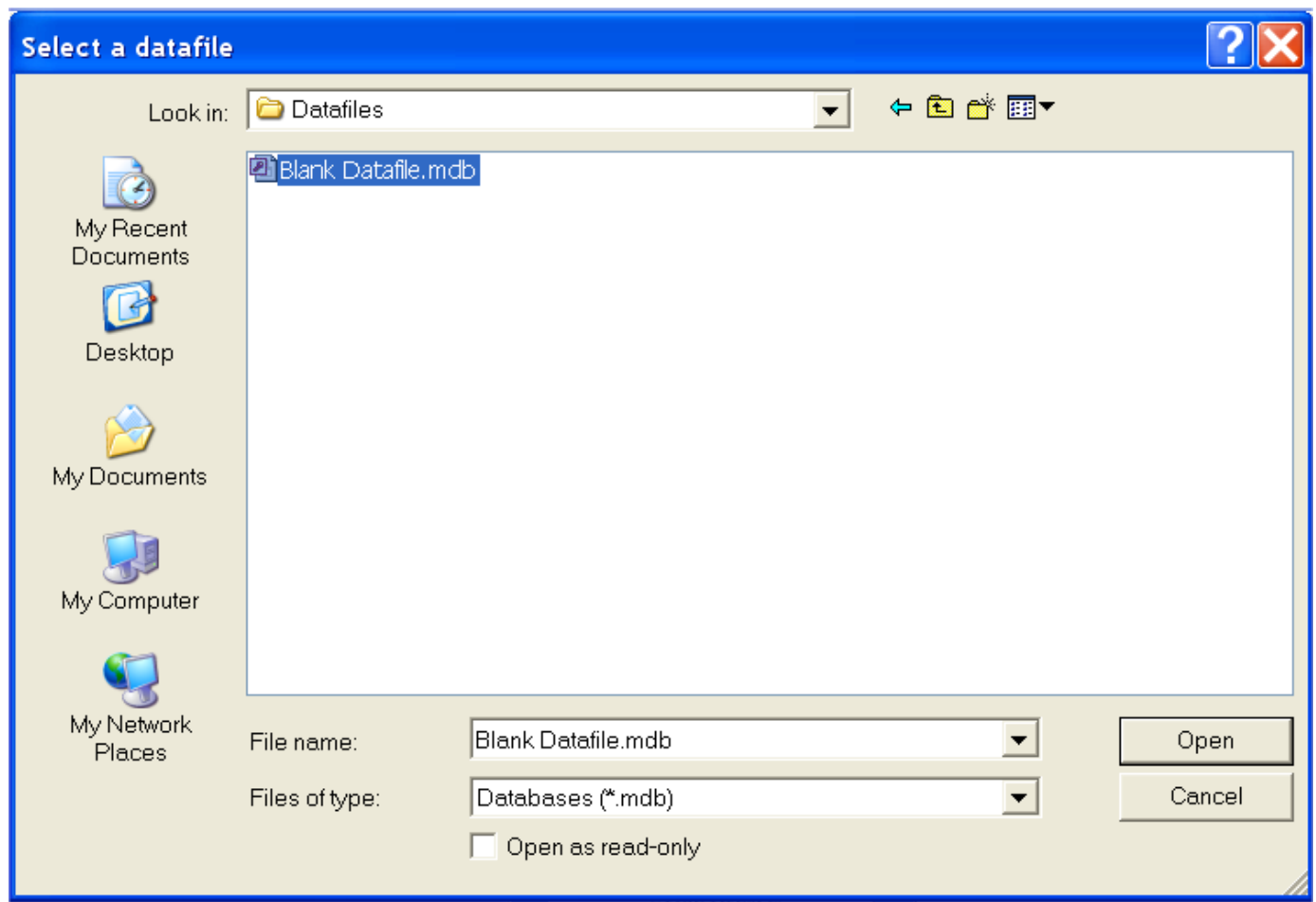

Figure 4. Datafile selection entry screen - selection of the blank datafile.

HEAT will attach the empty datafile to the HEAT analysis engine as soon as this selection is made. A loading message on the bottom of the screen will indicate the status of the attachment process. It is imperative that all datafiles be opened in this manner. If a datafile

USER'S NOTE:

You must attach a datafile to continue. If you choose to click the "Cancel" button here, HEAT will stop all actions, close, and return you to your desktop. is opened without the HEAT analysis engine, a database window will open, but the interface screens will be inaccessible (Figure 5). 


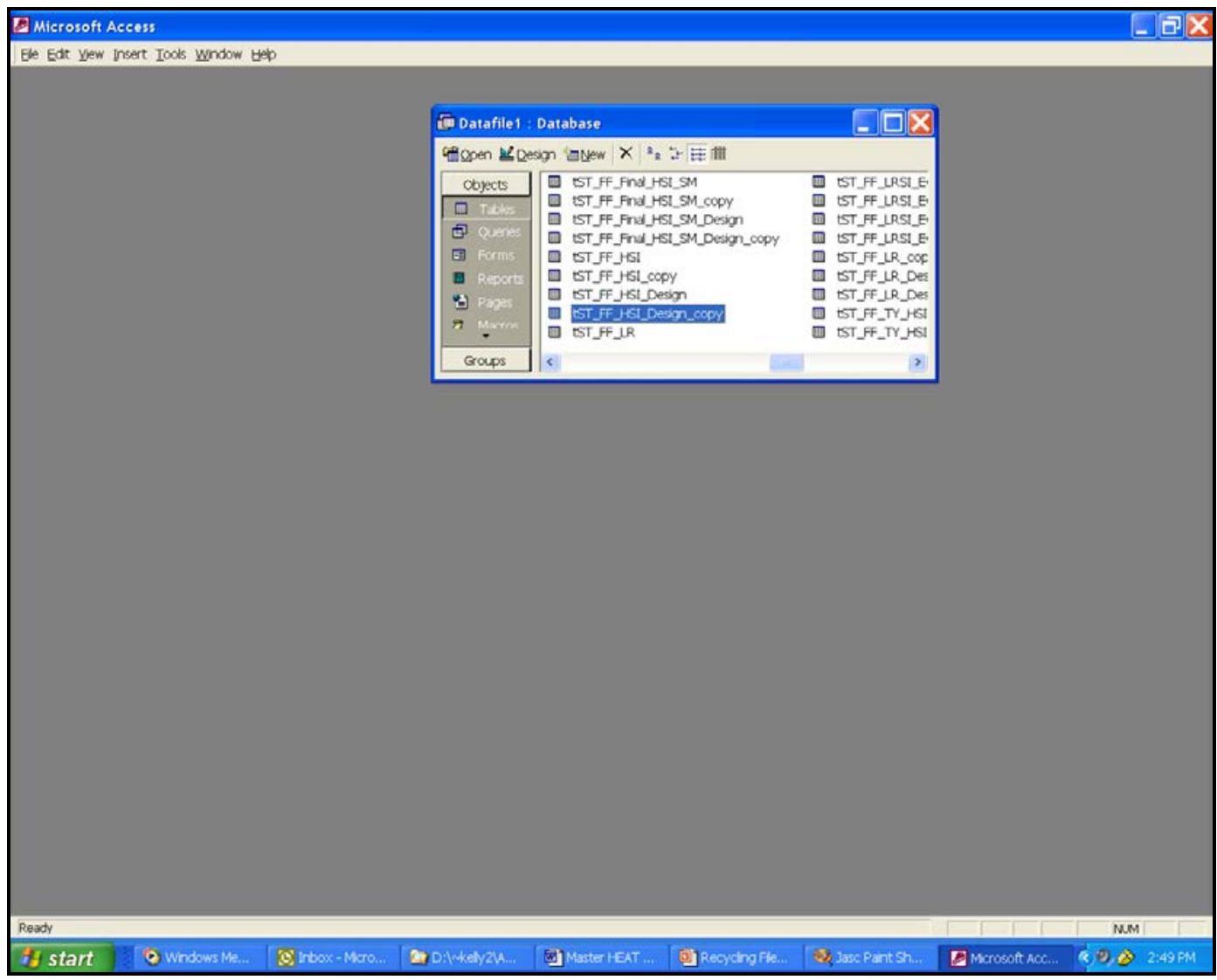

Figure 5. Opening a datafile incorrectly.

If this happens, close MS Access and return to the directions above to start the HEAT engine correctly.

If HEAT has been used to develop a model or project datafile in the past, users can quickly and easily review or alter a previous file for a new study. Often, this situation occurs when a previously developed datafile is a good starting point for project re-assessment. Select both the relevant path in the Look in: window and the name of the previously saved datafile in the File Name: window of the screen provided (Figure 6). 


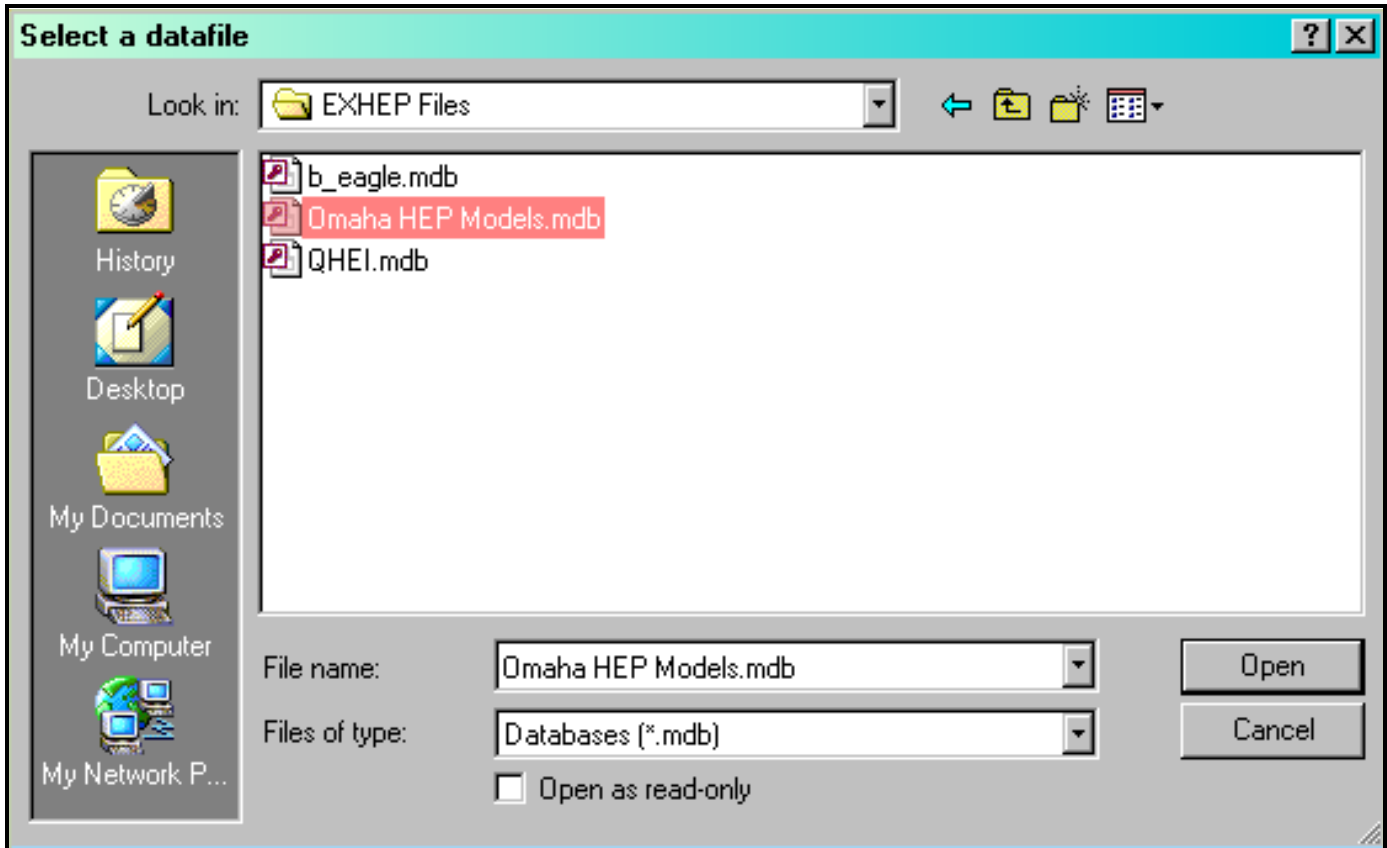

Figure 6. Selecting a previously created datafile.

\section{Special instructions for new MS Access 2007 users}

If an attempt is made to open HEAT with MS Access 2007 and a blank screen appears (Figure 7), added security in the new MS Access 2007 software requires that users first identify the HEAT program as a "trusted location" on their system.

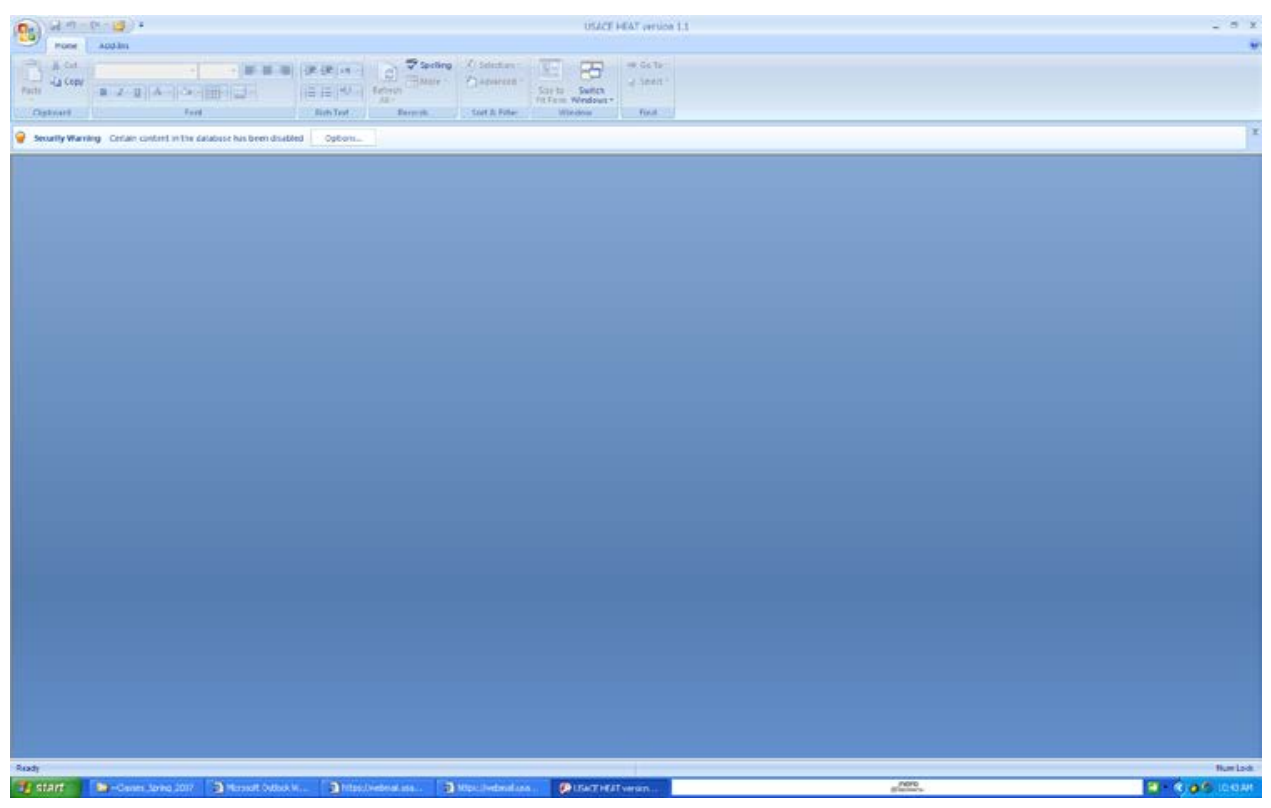

Figure 7. For first-time MS Access 2007 users, a blank screen will appear upon opening HEAT, indicating a security warning and lack of "trusted location" designation. 
To complete this step, close HEAT and simply re-open MS Access 2007. Click first on the MS Office 2007 button, and then on the Access Options button in the bottom right-hand corner of the screen to open the Access Options Screen. Click on the

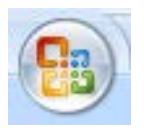
Trust Center option in the Table of Contents (left-hand side), and then click on the Trust Center Settings button (Figure 8).

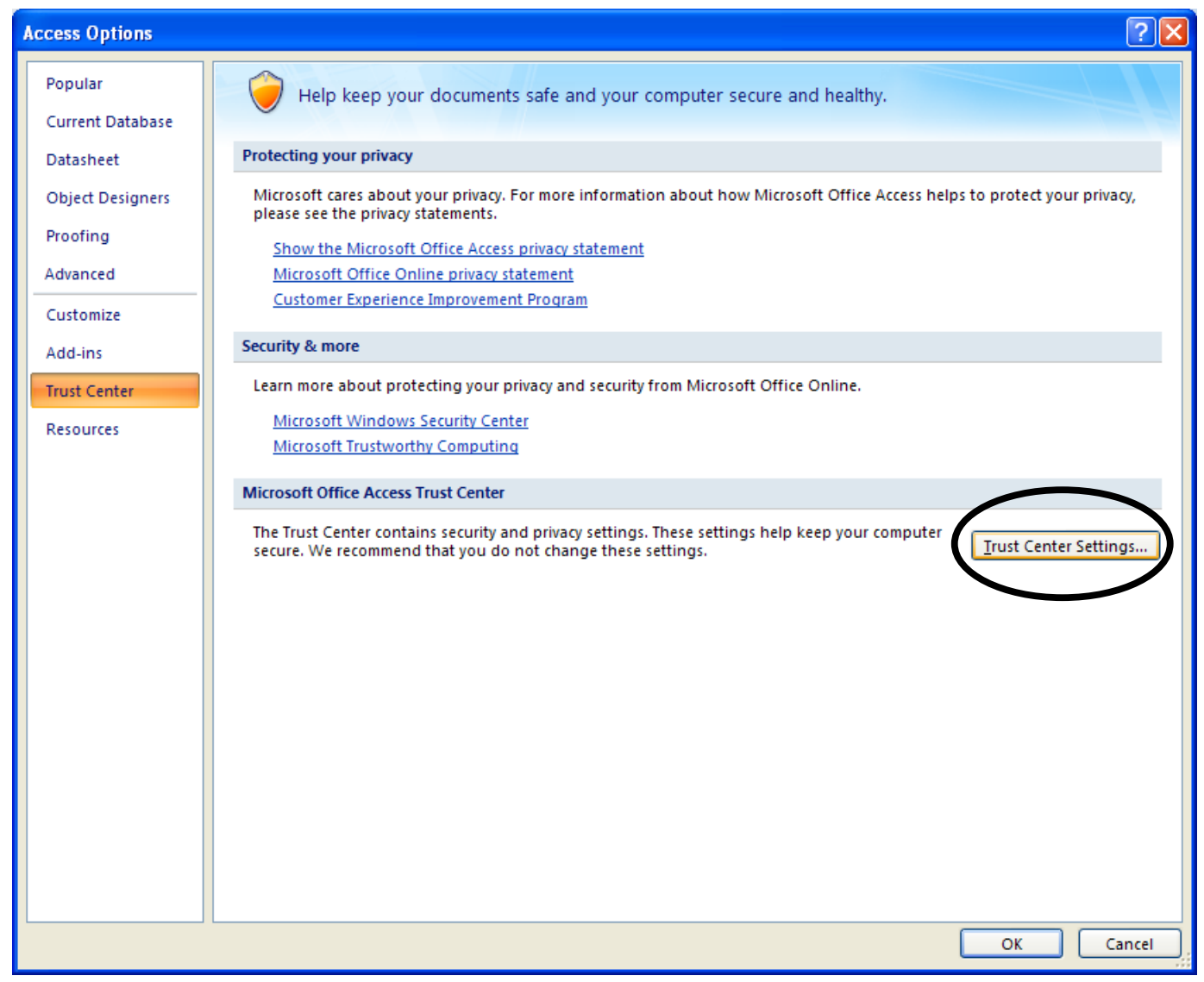

Figure 8. Setting HEAT as a "trusted location" in MS Access 2007 - Step 1.

Now click the Add new location and browse to the HEAT folder on the C: drive (path = C: $\backslash$ Program Files $\backslash$ HEAT-Habitat Evaluation and Assessment Tools) and click "OK" (Figure 9). 


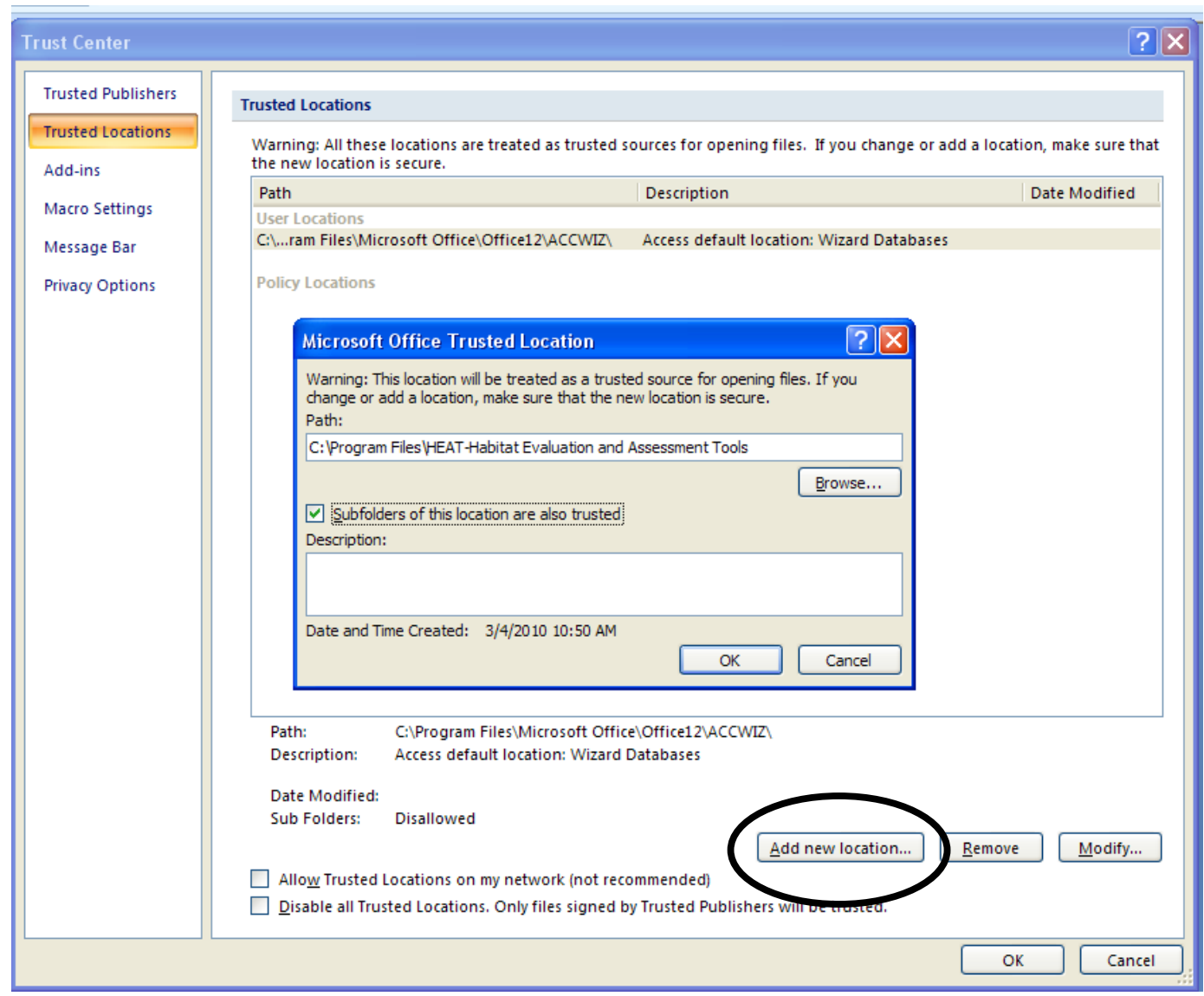

Figure 9. Setting HEAT as a "trusted location" in MS Access 2007 - Step 2.

The HEAT folder will be added as a trusted location. Close MS Access 2007 and use the steps described previously in the "Starting the Program" section above to proceed.

\section{HEAT's primary interface}

Once a datafile has been located and its tables have been attached to the HEAT analysis engine, HEAT's primary interface is opened (Figure 10). 


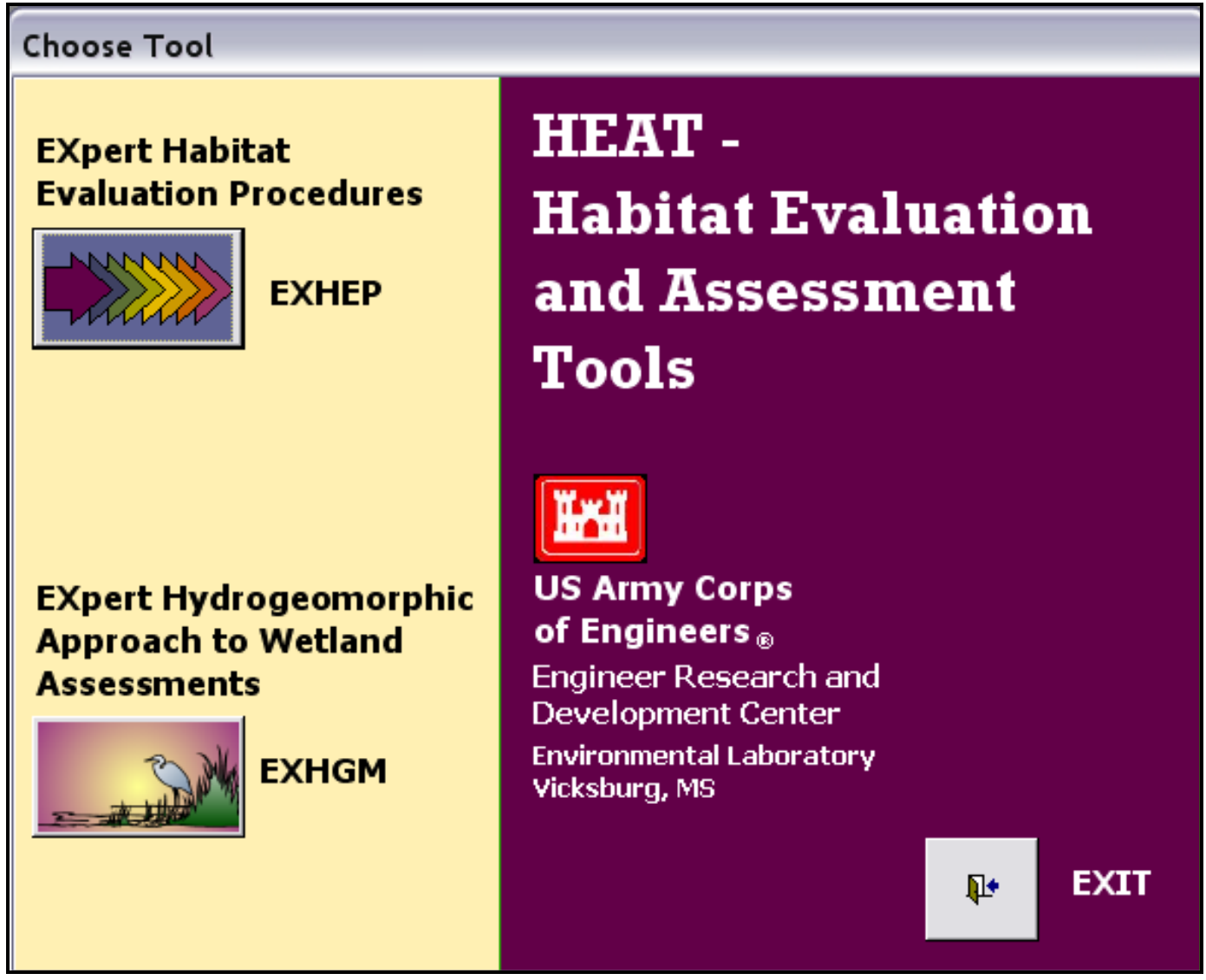

Figure 10. HEAT's primary interface.

The primary interface allows users to choose between the available environmental evaluation modules within its toolbox. At this time, EXHEP: EXpert Evaluation Procedures and EXpert HydroGeoMorphic Approach to Wetland Assessments are available. Potential research could lead to module additions in the near future. If conducting a HEP analysis, click on the EXHEP button now. If conducting an HGM analysis, click on the EXHGM button now. If planning to conduct both a HEP and an HGM analysis, choose either button. Once that assessment has been completed, return to this screen and move into the second technique's module.

\section{EXHEP and EXHGM interfaces}

Once the EXHEP button has been selected on the HEAT screen, the primary EXHEP interface is opened (Figure 11). 


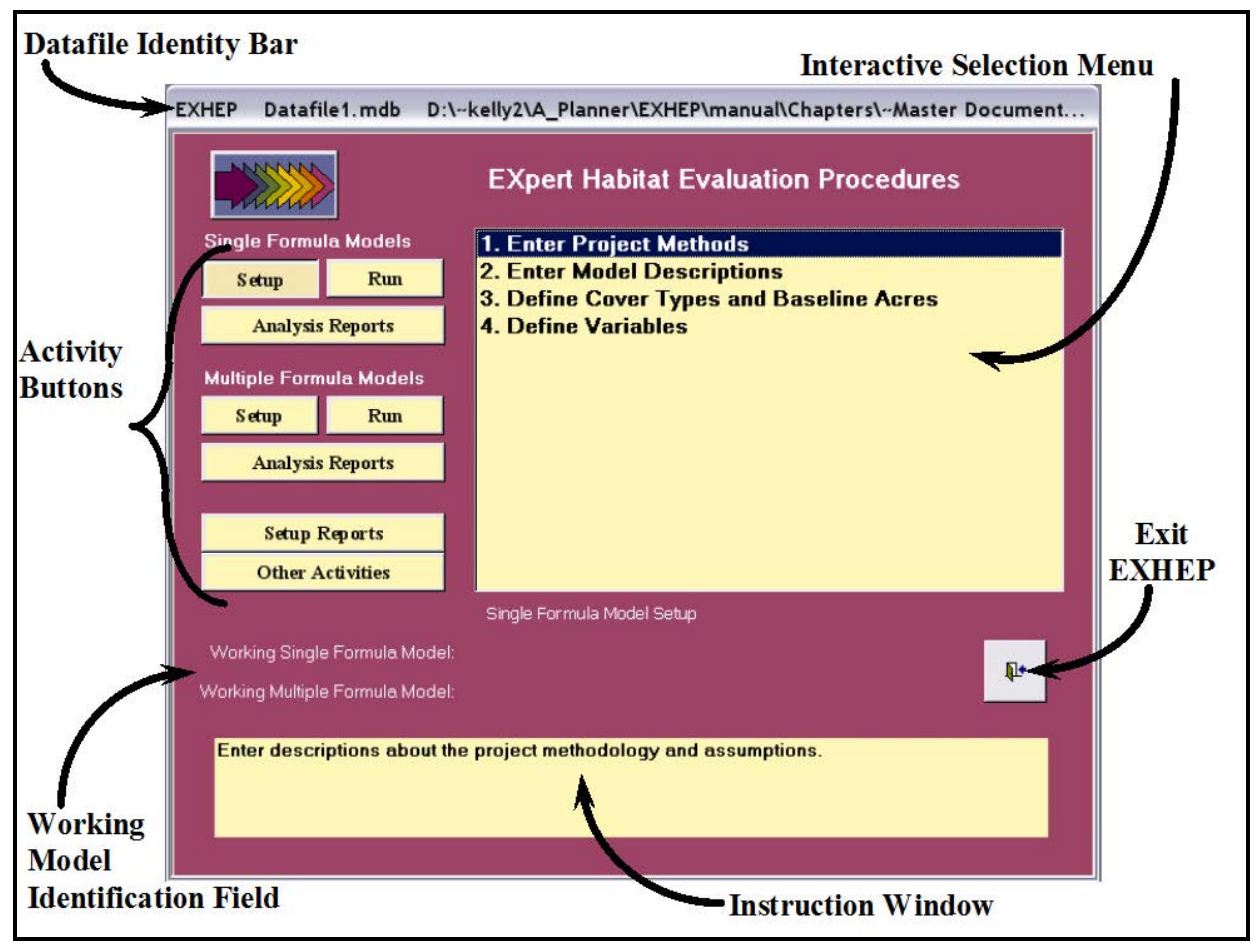

Figure 11. EXHEP's primary interface.

This main interface was designed to promote quick and easy access to pertinent information. This EXHEP primary interface contains the following functions:

Datafile Identity Bar. At the top of the window, the path and filename of the datafile currently in use are shown.

Activity Buttons. Use these buttons, along the left-hand side of the screen, to set up models, run analyses, and generate reports. Clicking on these buttons provides additional options that are activated and displayed to the right, in the Selection Menu.

Selection Menu. This interactive menu provides detailed functions and configuration options related to the activity buttons. The menu options change as users click on different activity buttons to the left, and this is where users also access many of the software's key functions. To select an option from this menu, highlight the option and double-click.

Working Model Identification Field. This area, just below the Activity Buttons and Selection Menu, displays information about the evaluation's progress and system status. 
Instruction Window. At the bottom of the screen, this text box clarifies actions that need to be executed in conjunction with functions that have been selected.

The EXHGM primary interface looks very similar to the EXHEP interface, although some minor variations can be found (Figure 12).

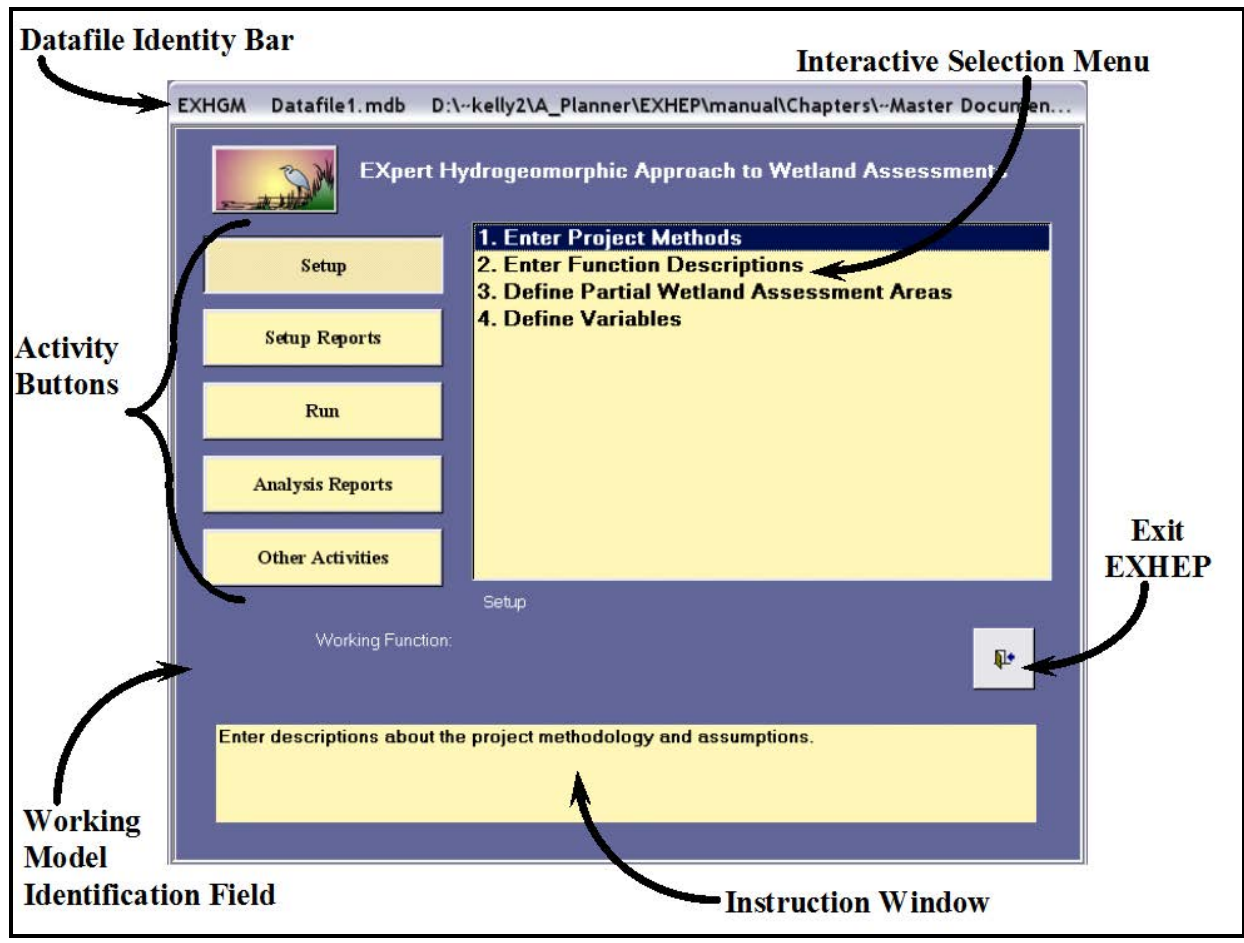

Figure 12. EXHGM's primary interface.

\section{Other activities button}

A series of file maintenance actions have been developed for the HEAT modules and stored under the Other Activities button on the primary interface. Options to save datafiles, compact datafiles, clear the database, and open new datafiles are all activated by this button. Below, these actions are described in greater detail (Figure 13). 


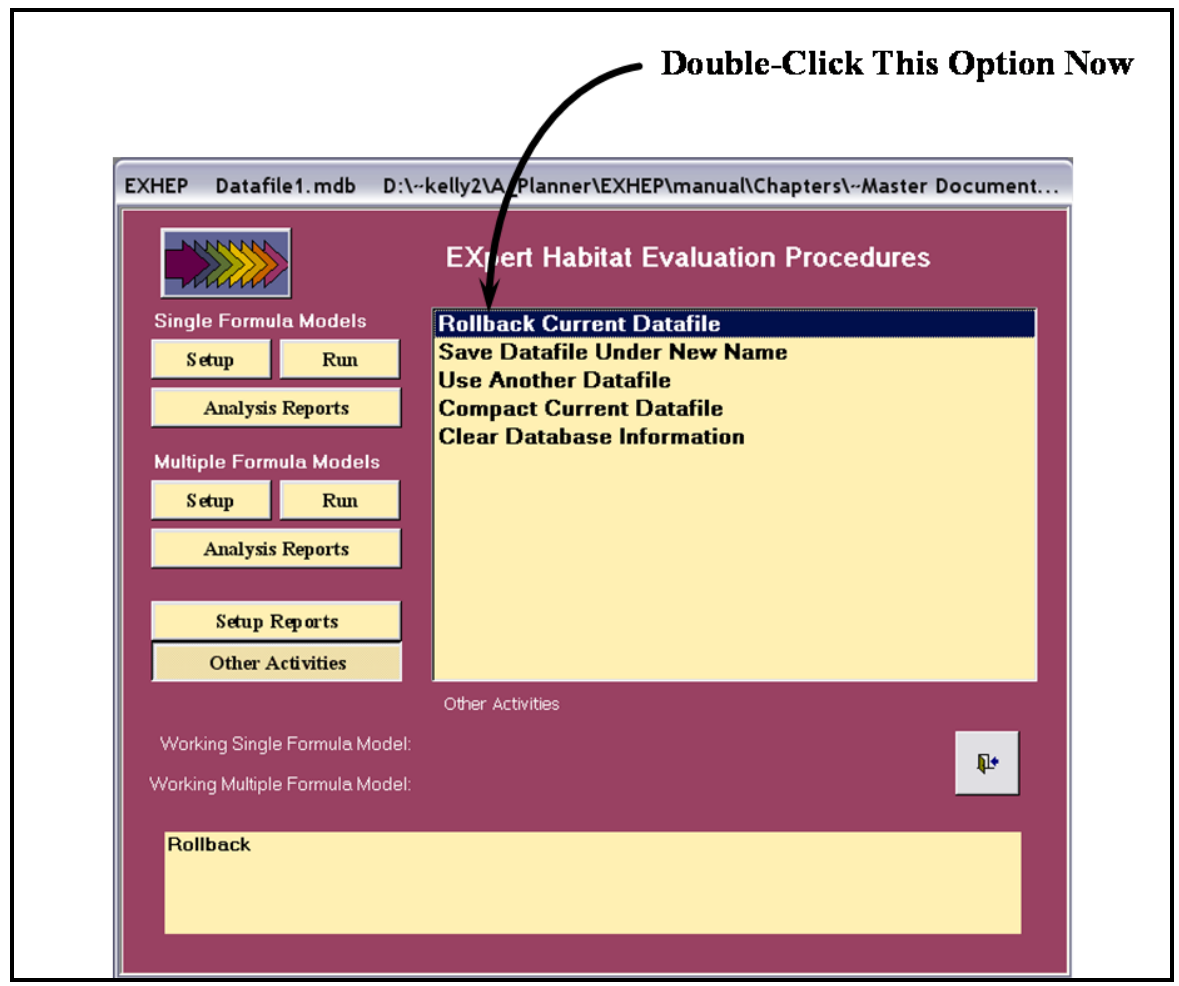

Figure 13. Rolling back to the original datafile.

\section{Rollback feature}

The first option (Rollback Current Datafile) is a global erasing action that should be used only if every action taken since the datafile was opened is incorrect, and users want to start anew from the beginning of the session. This action is irreversible, but allows users to return to the previous file's information when extensive errors are made during the session. For example, if a user

USER'S NOTE:

The Rollback Feature is re-set upon HEAT software shutdown. If you realize you have been working in the wrong file, DO NOT close the HEAT software. Proceed directly to the Other Activities Screen, and click on Rollback Current Datafile. mistakenly selects the wrong datafile, and proceeds to make changes to the database without realizing the error, double-clicking on this option will return the database to its original content.

\section{Saving datafiles}

HEAT was designed with flexibility in mind. Each time users move between data entry screens and the primary directive screens (i.e.,

USER'S NOTE:

Save files prior to alteration - unless you intend to overwrite existing information.

primary interface, model entry interface, acres entry interface, etc.), Microsoft Access 2003-2007 automatically saves the datafile. The newest version of a datafile can be quickly and easily saved under another name. 
By utilizing the "Save As" opportunity prior to alteration, quality control can be assured (data are not overwritten or lost) and datafile/ project library organization and maintenance can be improved. To save the datafile under a new filename, click on the Other Activities button on the primary interface and then double-click the Save Datafile Under New Name option in the list of tasks offered in the window (Figure 14).

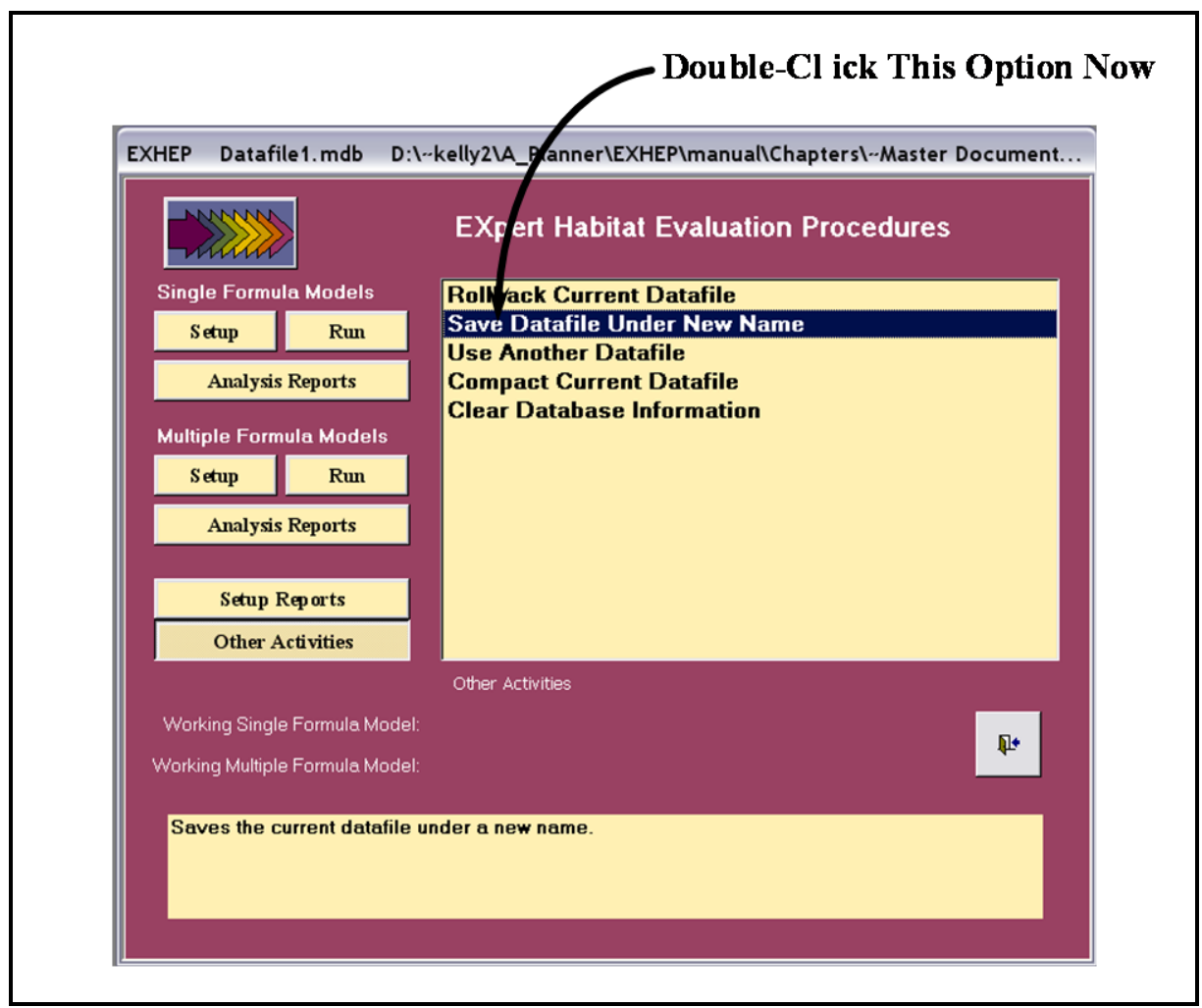

Figure 14. Saving datafiles under new names.

HEAT provides a warning that an irreversible change to the original datafile is about to occur (Figure 15). Click the Datafile Control Checkbox to proceed with this file after the saving process. 


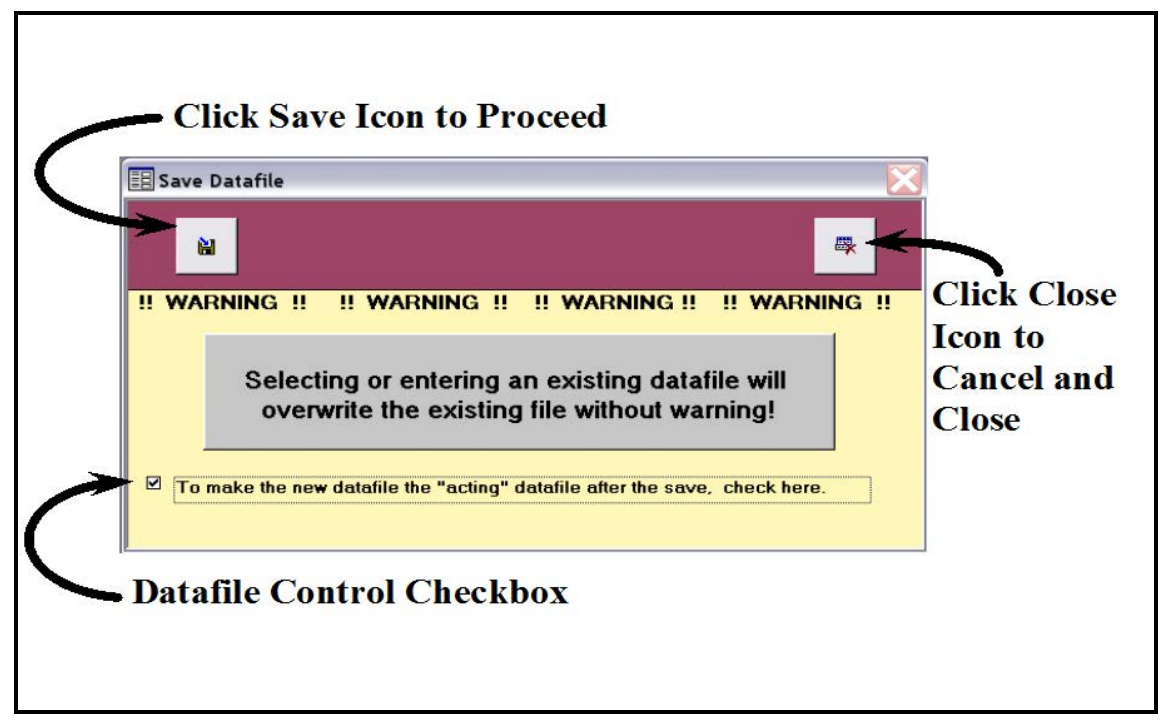

Figure 15. Overwrite warning in the Save Datafile screen.

Click the Save button in the upper left-hand corner to proceed. HEAT provides a new parameters screen (Save datafile as) to assist in the assignment of the new datafile's name and path (Figure 16).

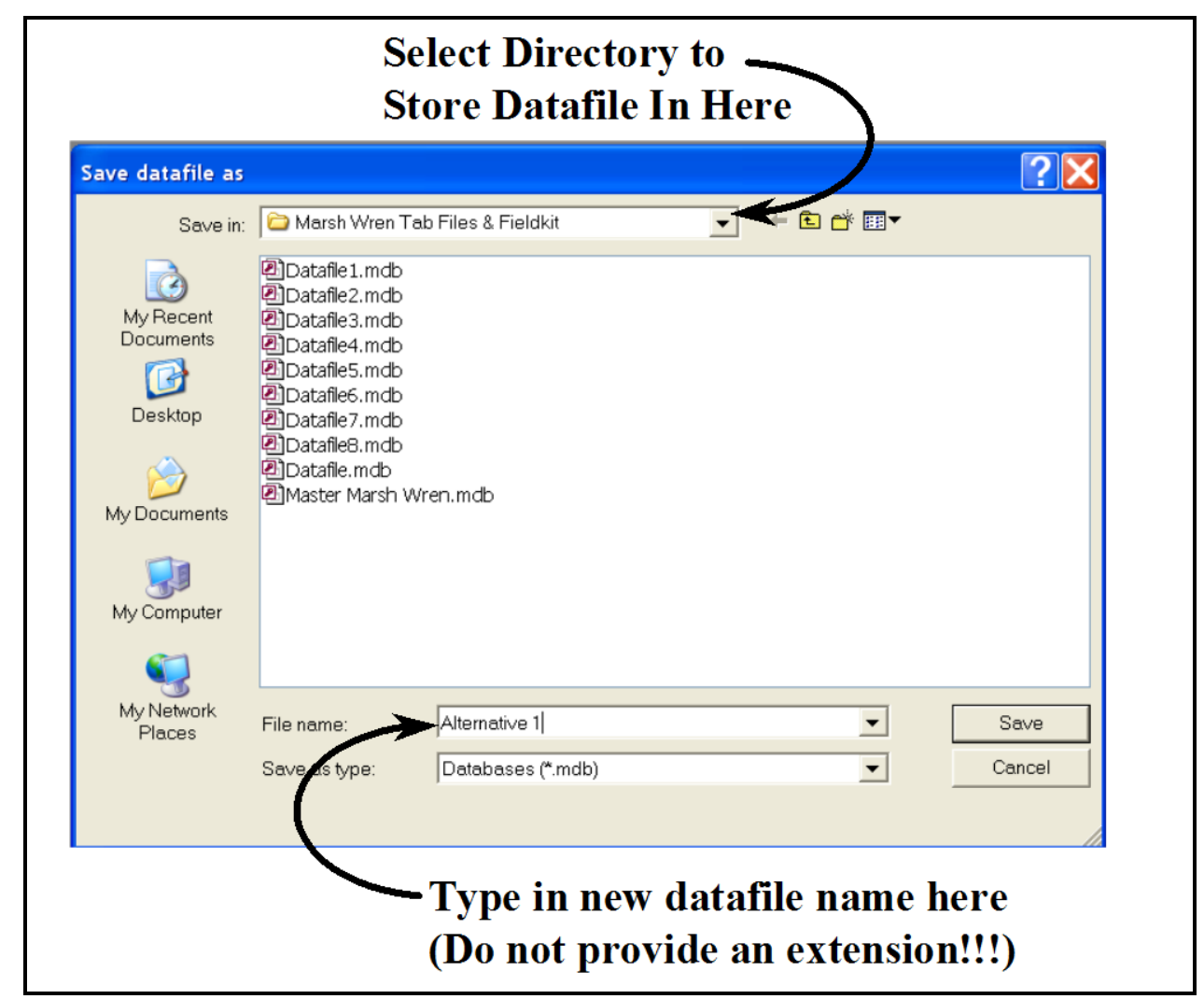

Figure 16. Save datafile as entry screen. 
Identify the storage location via the Save in: window at the top of the interface; provide a new name ${ }^{1}$ for the datafile in the File name: window; and press Save. HEAT will close the current datafile and then open the new datafile. The status bar (at the bottom of the screen) reports the progress of the table attachment once this selection is made. As soon as this process is completed, HEAT will provide a pop-up window

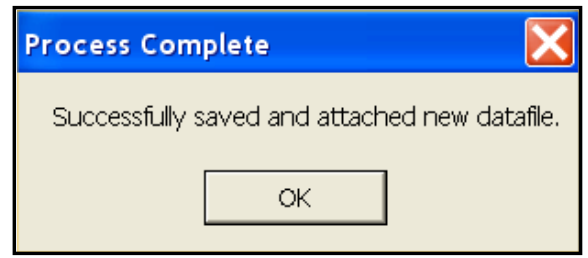
indicating the system is ready to proceed. Click OK, and HEAT will return to the primary interface.

If users choose to ignore the Datafile Control Checkbox (i.e., they simply wish to save the file as a "spare" copy for backup purposes), continue by clicking the Save button in the upper left-hand corner of the Save Datafile screen. HEAT provides the same parameters screen described in Figure 16. Select the storage location; provide a new name; and press Save. HEAT will save the file and return to the Save Datafile screen. Click on the Close button to complete the process.

Users who do not wish to proceed with this saving process can click the Close button in the upper right-hand corner of the Overwrite Warning Screen. This cancels the action and returns the user to the primary interface (Figure 15 above).

\section{Exchanging datafiles}

To switch to another datafile, click on the Other Activities button and double-click on the Use Another Datafile option (Figure 17).

\footnotetext{
1 It is not necessary to provide an extension for the datafile in the File name: - MS Access pre-determines the extension and assigns an *.mdb to the file name provided.
} 


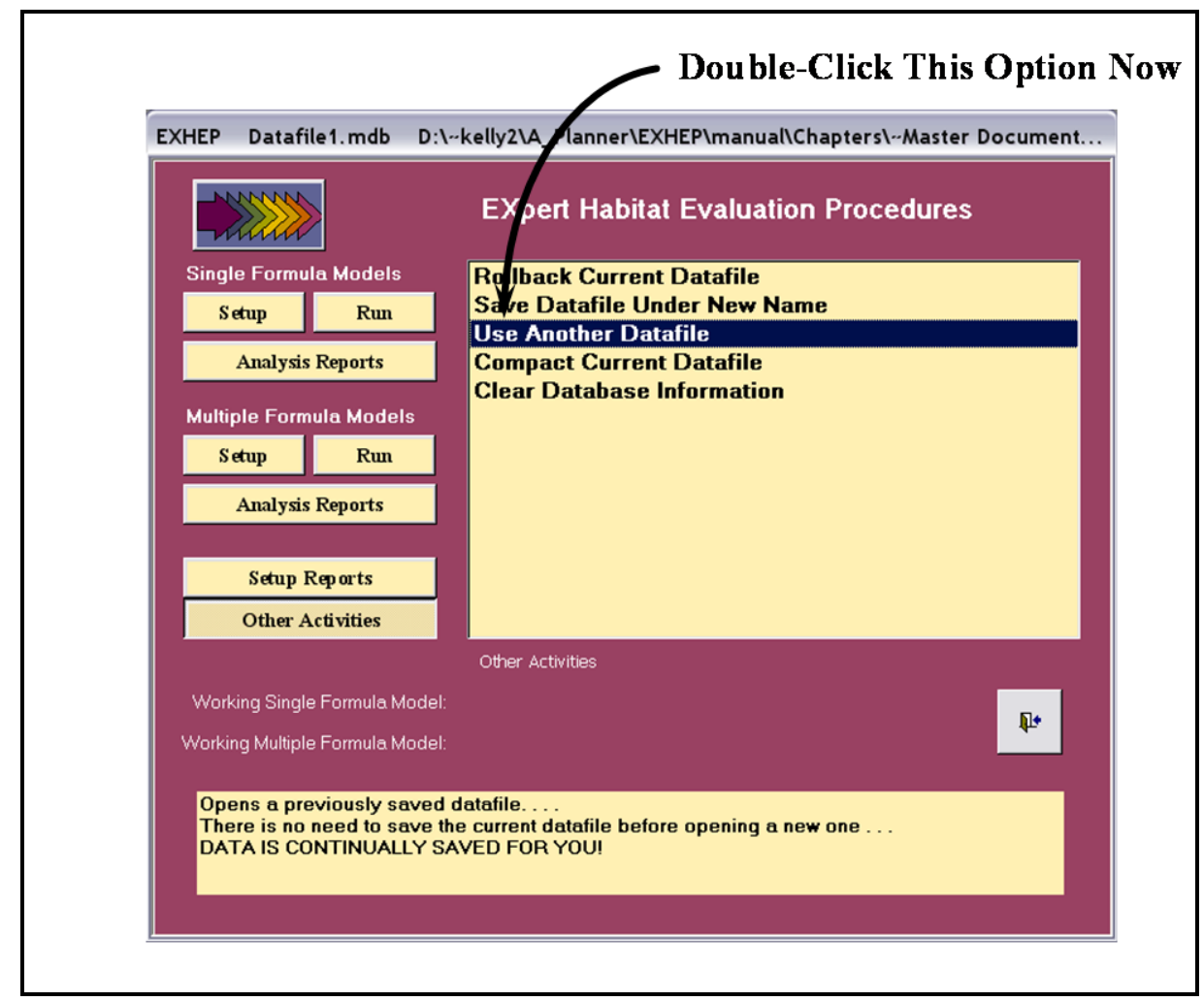

Figure 17. Selecting another datafile.

HEAT provides a new parameters screen (Select a datafile) to assist in identifying the new datafile's name and path (Figure 18).

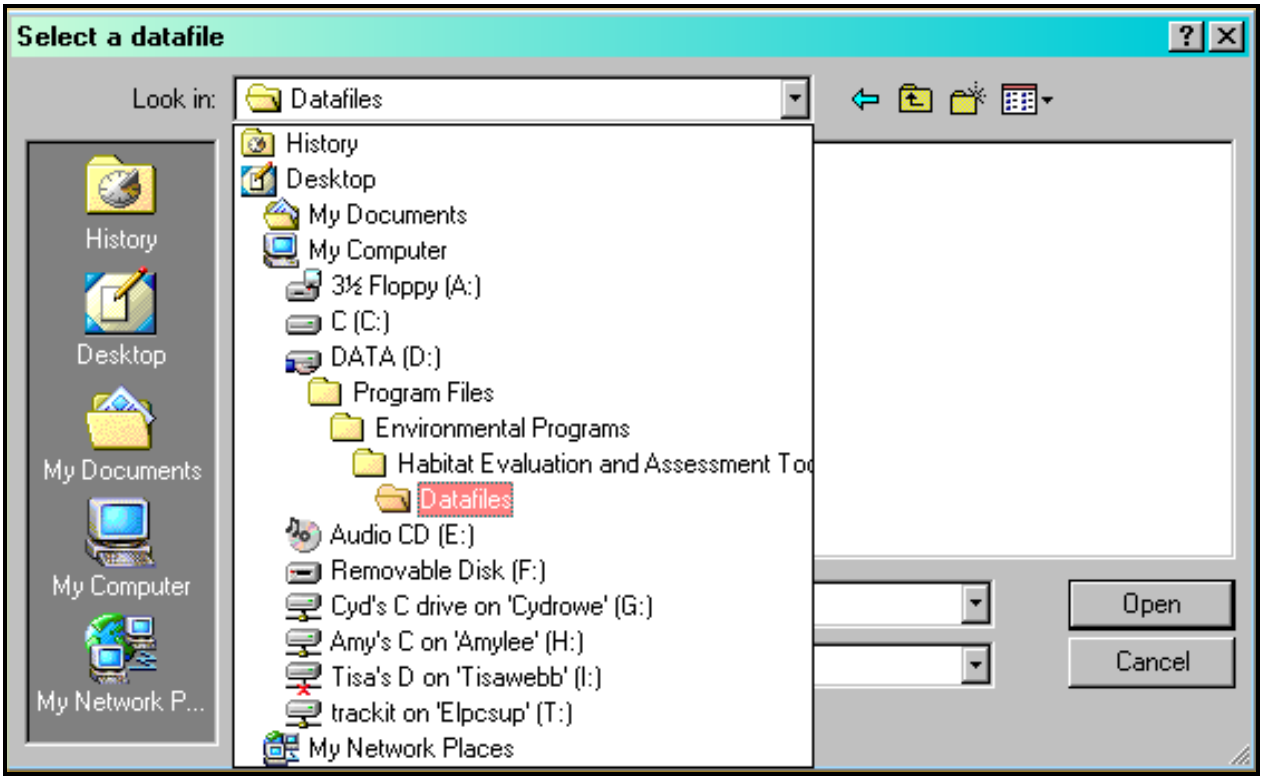

Figure 18. Select a datafile entry screen. 
Select the path for the new datafile in the Look in: window, the name of the previously saved datafile in the File name: window, and press Open. HEAT will close the current datafile and then open the new datafile. The status bar (on the bottom of the screen) reports the progress of the table attachment once this selection is made. As soon as this attachment is completed, HEAT will return to the primary interface. At the top of the screen, the Datafile Identity Bar will show the new path and filename of the datafile selected (Figure 19).

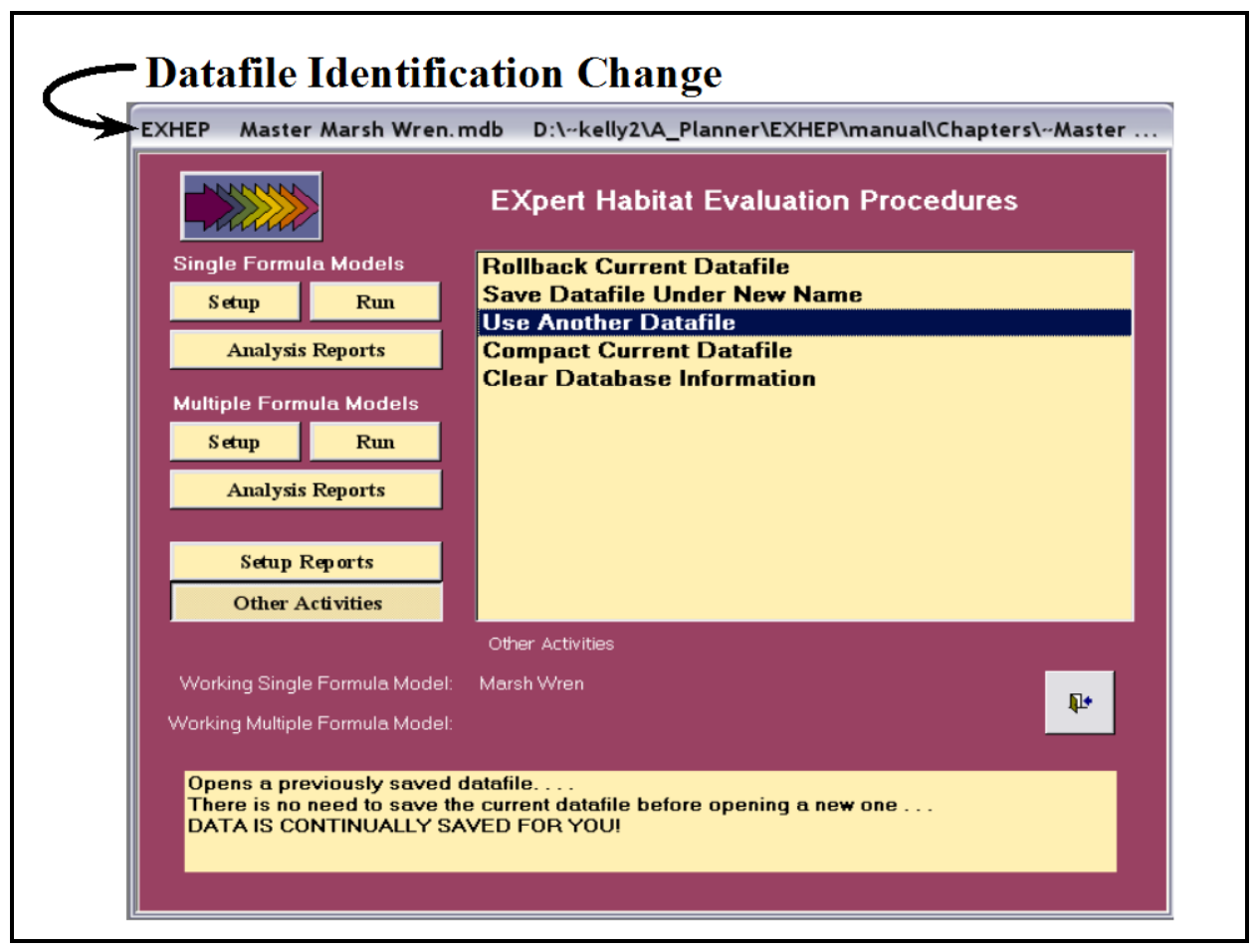

Figure 19. New datafile identification in the primary interface.

\section{Compacting the current datafile}

To build a datafile for an ongoing study, the file will expand incrementally to capture change. If data or objects are deleted in the HEAT software, the database can become fragmented and use disk space inefficiently. Compacting the datafile generates a copy of the file and rearranges how the file is stored on a disk. Thus, "compacting" optimizes software performance and reduces storage space demands for ongoing projects. A datafile can be directly compacted from within the HEAT software by double-clicking the Compact Current Datafile option (Figure 20). 


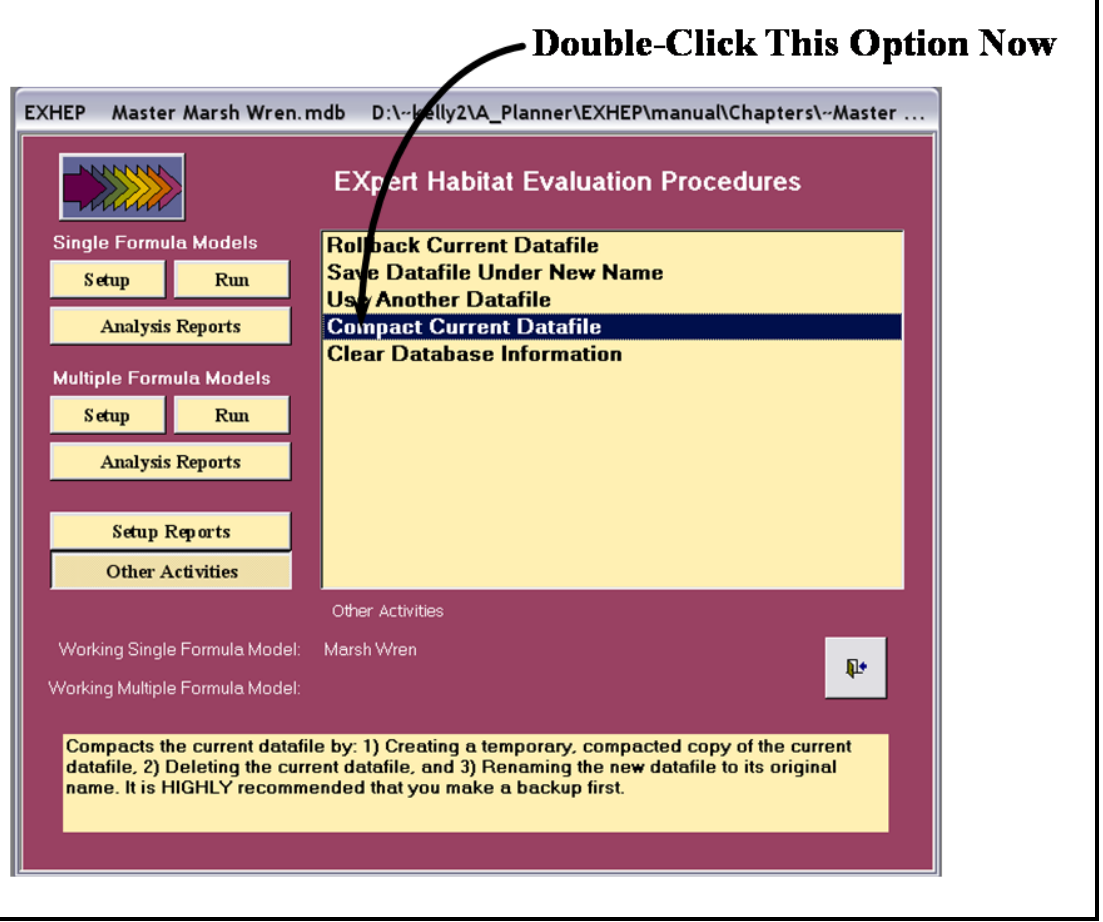

Figure 20. Compacting the datafile from HEAT.

A warning will appear instructing users to save the datafile before continuing - users are strongly encouraged to follow this directive. To save, close the software entirely and make a copy (or use the Save

\section{Datafile Under New} Name option), then

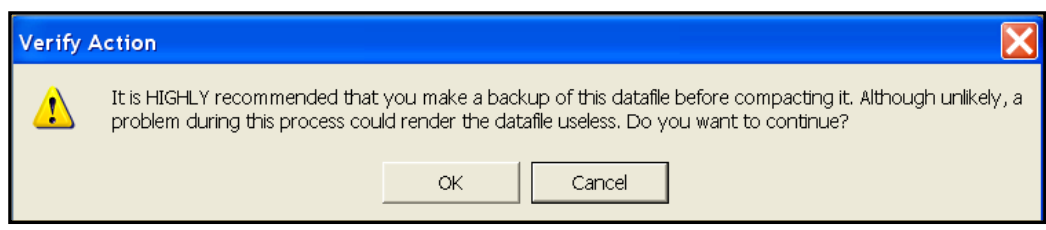
return to this screen. Click OK when ready to proceed. HEAT will generate a message saying the action was successful, or indicating problems that arose during the compaction process. Click OK once again to proceed.

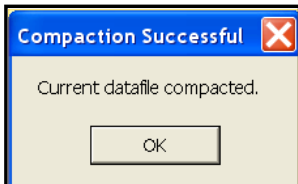

Alternatively, a datafile can be compacted from outside the HEAT software by opening MS Access 2003-2007, opening the datafile itself, and clicking on the Tools pull-down menu (Figure 21). Select Database Utilities, and Compact and Repair Database. Again, the software will generate a message indicating success or failure of the action. 


\section{Microsoft Access}

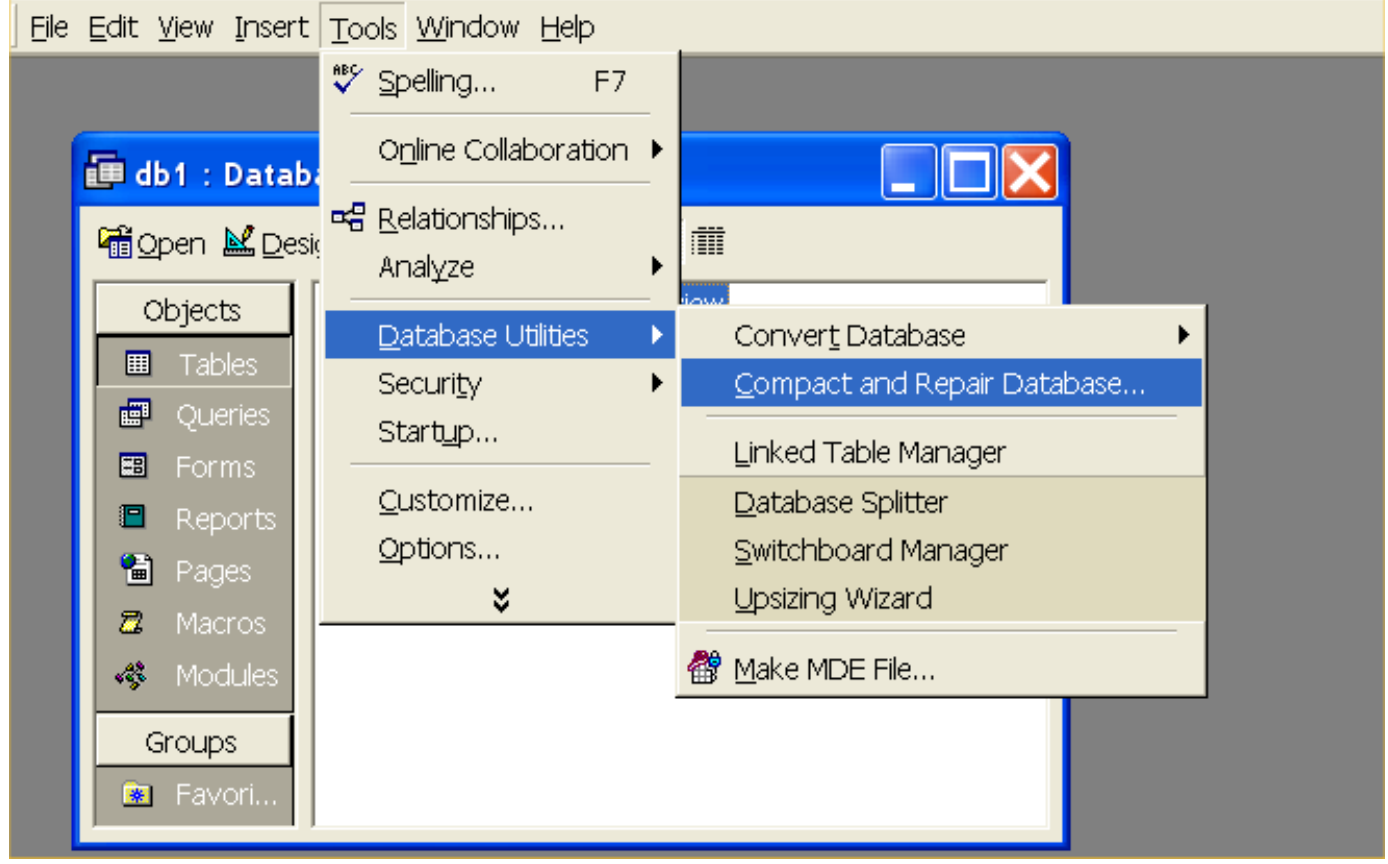

Figure 21. Compacting the datafile in MS Access (outside of HEAT).

\section{Deleting information in a datafile}

As users move through the setup and run processes inside the HEAT modules, situations may arise that force a user to delete or modify information in the originating datafile. To delete an index model, a variable, a cover type, or a target year from the datafile, proceed to Chapters $5 \mathrm{and} /$ or 6 , and follow the directions therein. In some instances, it may be necessary to completely erase the entire datafile and begin anew. Or, the file can be recycled for a new study by removing the baseline data of the originating study, and replacing it with newly gathered information. In these instances, use the Clear Database Information option found under the Other Activities button on this screen (Figure 22). 


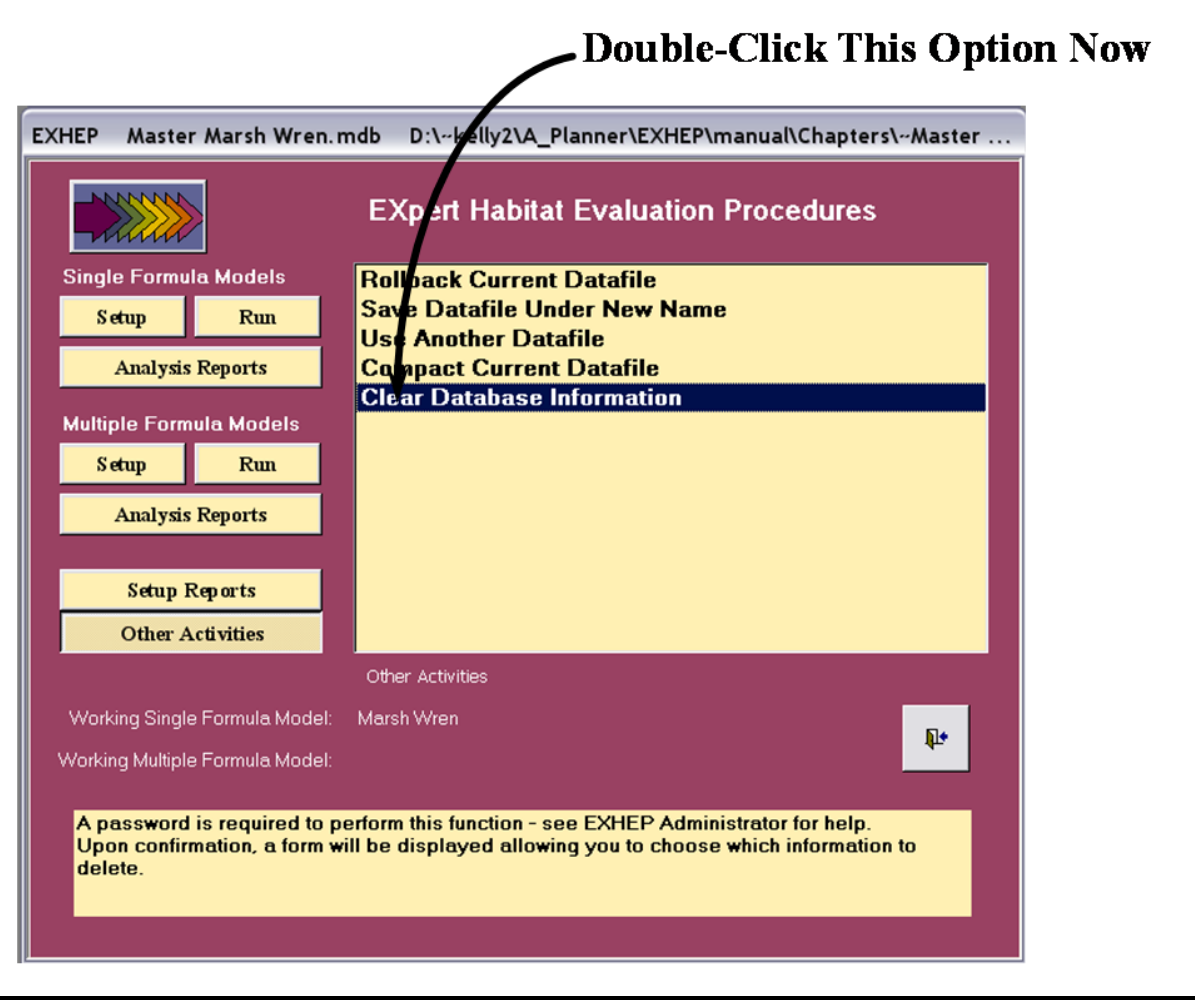

Figure 22. Clearing database information in HEAT.

Users will be prompted to provide a password at this time - this is provided strictly to protect data, and a default password (EXHEP) has been assigned to the blank datafile. Please contact the authors to customize this password. Type EXHEP (all lower case, one word) into the screen and click OK. HEAT provides a list of possible actions to take at this time (Figure 23).

\begin{tabular}{|l|r|}
\hline Provide Password & Xn \\
\hline Enter administrative password. & OK \\
\hline & Cancel \\
\hline Exhep & \\
\hline
\end{tabular}




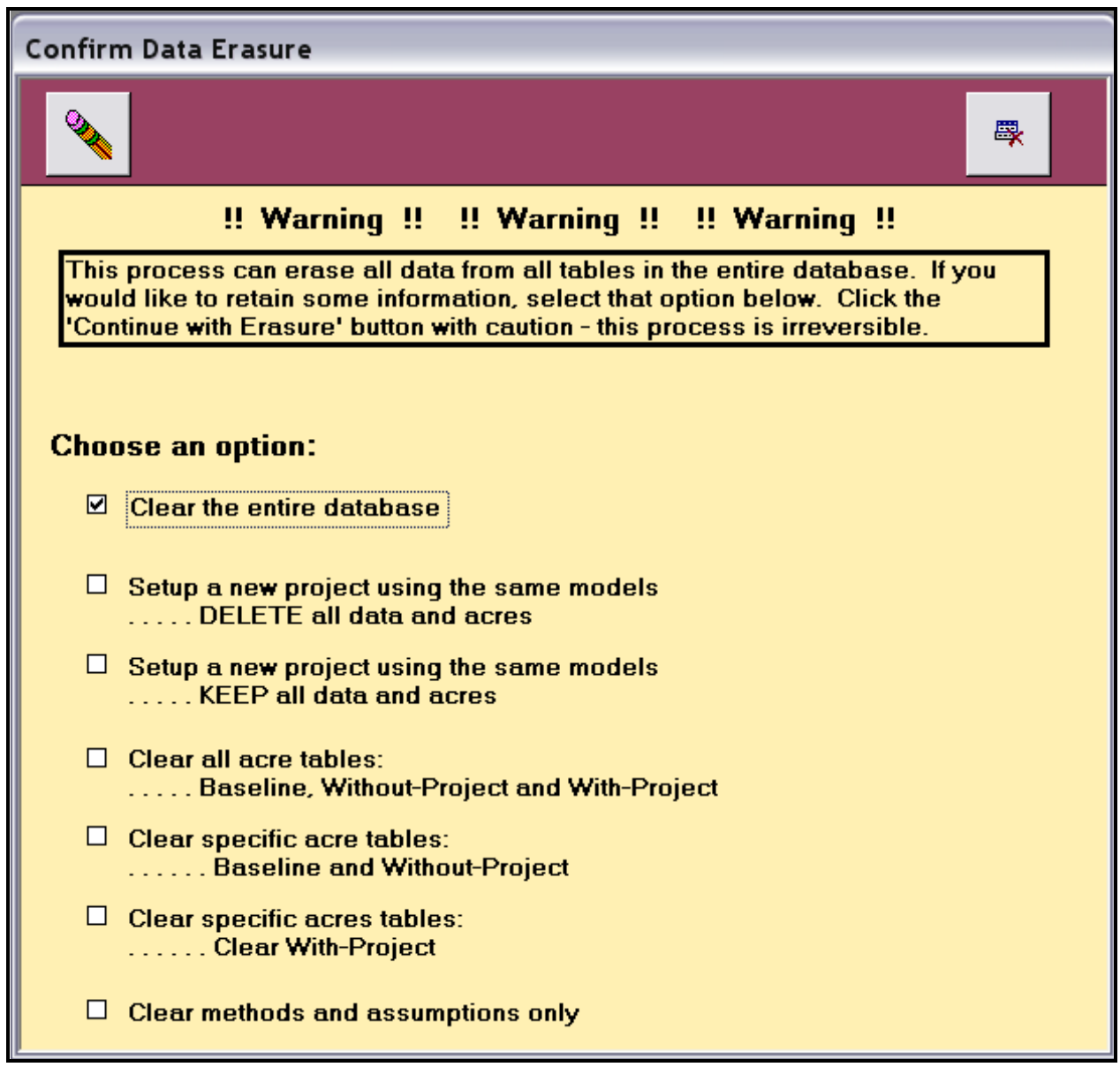

Figure 23. Confirm Data Erasure entry screen.

Seven different erasing options are offered here.

1. Clear the entire database - all models, data, and projections will be cleared from the database, and users can start from a blank datafile after selecting this option. Use this option to begin the process of building a new study datafile from an existing study datafile.

2. Set up a new project using the same models ... DELETE all data and acres - this option allows users to retain the models from the originating file. Information and data regarding methods, baseline data, baseline acres, target years, and projections of future conditions are cleared from the database. Use this option to recycle the models for a new study from an existing study datafile.

3. Set up a new project using the same models ... KEEP all data and acres - this option allows users to retain the models, as well as the baseline data and acres, from the originating file. Information and data regarding methods, target years, and projections of future conditions are 
cleared from the database. Use this option to recycle the models and the data for a new alternative in an existing study.

4. Clear all acre tables: ... Baseline, Without-Project, and WithProject - only the acres in the baseline, without-project, and with-project projection screens are cleared. The models, variables, and future variable projections are retained. Hypothetically speaking, this option might be used if new mapping requires changes in the baseline, without-project, AND with-project acreages after the analysis has been run at least one time before - this option allows users to quickly clear the old acres out, and insert the new acreage estimates.

5. Clear specific acre tables: . . . Baseline and Without-Project only the acres in the baseline and without-project screens are cleared; the models, variables, and future variable projections are retained. Again, this option would be used if new mapping generates new acreages, but the with-project scenario is unaffected - this option allows users to quickly clear the old acres out, and insert the new acreage estimates.

6. Clear specific acre tables: ... Clear With-Project - only the acres in the with-project screens are cleared; the models, variables and future variable projections are retained. This option allows users to develop a new alternative by modifying acreage use on the site (maintaining variable data and their future projections).

7. Clear methods and assumptions only - as this option implies, only the methods and assumptions are cleared from the database.

\section{USER'S NOTE:}

HEAT reports will not print if the methods and assumptions are left blank - therefore be sure to return to the Methods section and provide the new information.

After selecting one of the seven options by clicking the check box to the left of the option, the Erase button in the upper left-hand

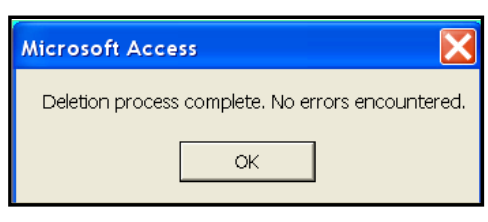
corner of the screen must be clicked to proceed. HEAT will confirm the success or indicate problems with the action with a popup message box. Click $\mathbf{O K}$ to proceed.

To abort the process and return to the primary interface, click on the Cancel button in the upper right-hand corner of the screen. 


\section{Applying EXHEP}

In Chapters 3 and 4, the HEAT architecture was introduced, datafile management was discussed, and the software's primary interfaces were illustrated. With this framework in place, it is time to focus on using HEAT to evaluate ecosystems using HEP (refer to Chapter 6 for HGM applications).

As discussed in earlier chapters, there are 12 steps to complete when applying HEP in an ecosystem evaluation. Once a model or models have been selected/ developed (Step 4), and the evaluation team has inventoried the site using the model's parameters (i.e., Step 5), it becomes necessary to generate outputs (i.e., Habitat Units or HUs). At this point, the HEAT software can be fully deployed. A series of steps have been devised to move through this process quickly and cleanly:

1. Gather the pertinent information

2. Set up the models in EXHEP

3. Associate the models

4. Enter the baseline data and generate baseline results

5. Enter the without-project conditions and calculate the effects

6. Enter the with-project conditions and calculate the effects

7. Recycle the datafile and evaluate alternative designs.

\section{Step 1: Gather the pertinent information}

Basic information regarding the study's goals, objectives, opportunities, and constraints is always useful to capture early on in the analysis process. EXHEP allows users to store this information iteratively, and provides access to this data at the touch of a button. Issues surrounding model selection, modification, and creation can also be addressed and documented in the software. Model architecture can have a profound effect on the analysis strategy, and should be recorded and incorporated into this data-gathering process as well. 


\section{Single versus multiple formula models}

Several questions must be asked prior to setting up the software and running the analysis. For example, are the evaluation models based upon single or multiple index algorithms? The HEAT software has different data requirements for the two types of models, so it is important to classify the models in the beginning of model setup. An example of a single formula model is Gutzwiller and Anderson's (1987) marsh wren model developed for the USFWS in 1987. ${ }^{1}$ In this model, the habitat suitability of the marsh wren is predicated by the dominant vegetative type (in this case cattails, cordgrasses, and bulrushes are favored by the species), presence of a significant amount of emergent canopy cover ( $>80 \%)$, adequate water depth ( $>15 \mathrm{~m})$, and minimal woody canopy cover ( $0 \%$ is optimal). These parameters are combined in a single formula to capture habitat suitability for the marsh wren:

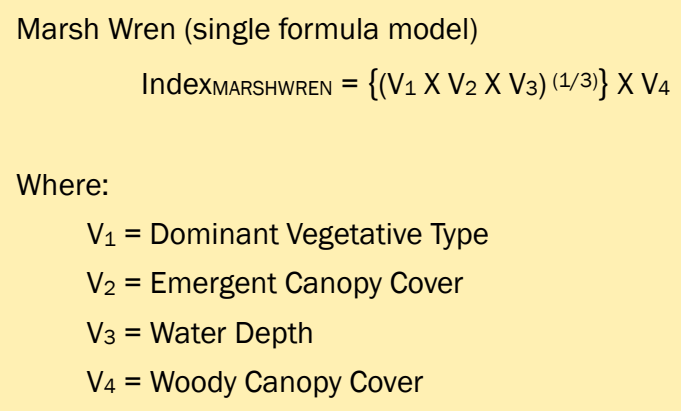

An example of a multiple formula model is Morreale and Gibbon's (1986) slider turtle model developed for the USFWS in 1986. ${ }^{2}$ Habitat suitability for the turtle is dependent on three factors: (1) availability of food/ cover, (2) water quality, and (3) water temperature. For each of the components or life requisites, a separate set of parameters is quantified. For example, the suitability of the Food/ Cover Life Requisite is based upon the amount of submerged and emerged aquatic vegetation on site (100\% cover is optimal). The Water Quality Life Requisite focuses on the absence of water velocity, water depth (1 to $2 \mathrm{~m}$ is optimal), and overall water regime (a permanently flooded condition must exist to maximize habitat suitability). The Water Temperature Life Requisite is based on a single parameter mean surface water temperature in April through September ( 25 to $30{ }^{\circ} \mathrm{C}$ ). Each life requisite has its own formula:

1 The marsh wren HSI model has been provided on the installation CD (Marsh Wren Model).

2 The slider turtle HSI model has been provided on the installation CD (Slider Turtle Model). 


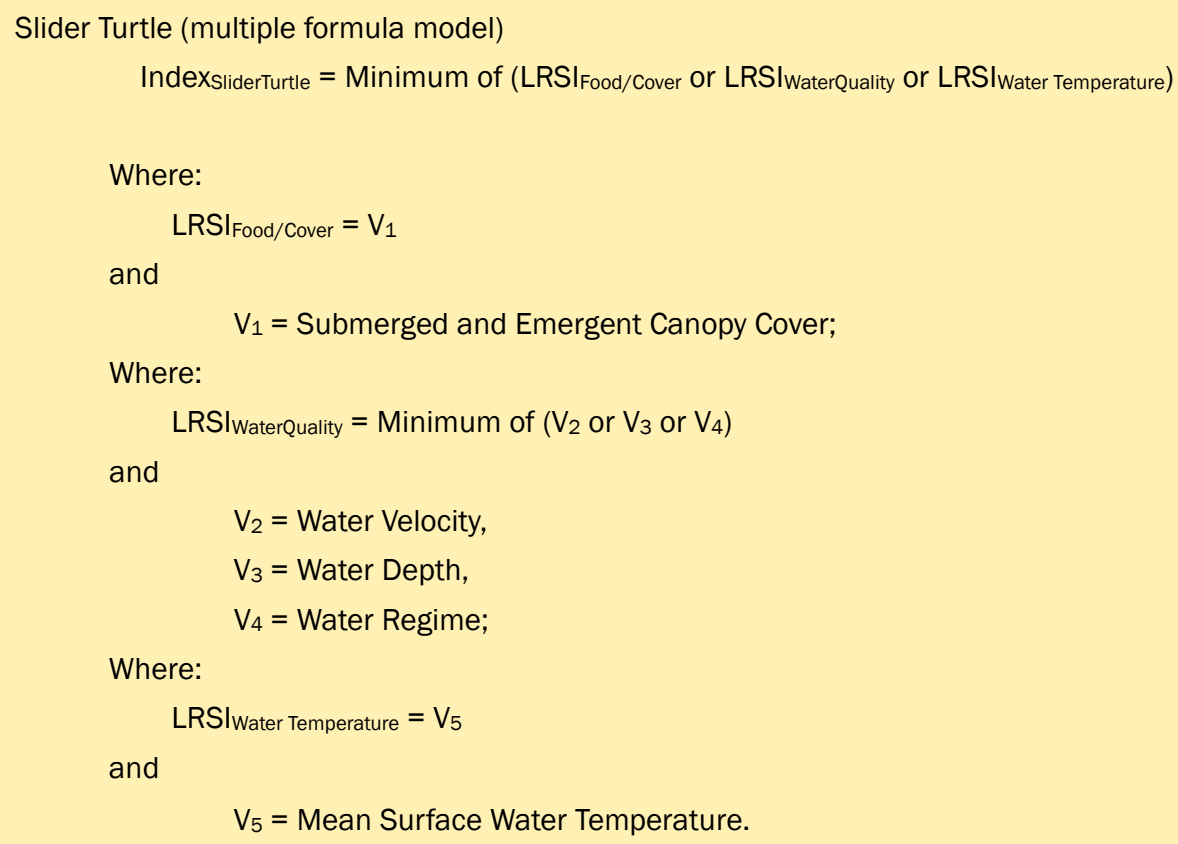

Because two groups of algorithms must be calculated before the site's habitat suitability can be determined for the model, the model is classified as a "multiple formula model" in HEAT. For purposes of this User's Guide, the single formula model set-up and analysis activities are demonstrated first; differences in the multiple formula model processing are presented immediately thereafter.

\section{Data checklist for the EXHEP analyses}

It is also important to gather all information supporting the application of the models prior to setting up the software. Collect basic information (e.g., references, cover types, variables, sampling protocols, SI curves, HSI formulas, etc.) early on. The software can be set up incrementally as this information becomes available, but analysis cannot commence without these basics. A checklist has been provided here to help organize the necessary information and data (Table 4). 
Table 4. Pertinent information needed to complete a HEP analysis.

\begin{tabular}{|c|c|}
\hline Items & Example \\
\hline \multicolumn{2}{|r|}{ Background Information } \\
\hline Project Name & Mill Creek Ecosystem Restoration Study \\
\hline Alternative Name & Design 1 \\
\hline Methods & $\begin{array}{l}\text { Model References and Support Documentation } \\
\text { Model Modifications } \\
\text { List of Evaluation Team Members } \\
\text { Goals and Objectives } \\
\text { Data Management Strategies } \\
\text { Evaluation Strategies (including tradeoff approaches) } \\
\text { Field Sampling Team and Metadata (include locations, assumptions, dates, etc.) }\end{array}$ \\
\hline \multicolumn{2}{|r|}{ Model Specifics } \\
\hline $\begin{array}{l}\text { Model(s) and Life } \\
\text { Requisite Names }\end{array}$ & $\begin{array}{l}\text { Species/Community and life requisites (both short-hand names or codes and detailed descriptions). } \\
\text { Examples: } \\
\quad \text { Model: Slider Turtle } \\
\text { Life Requisite: Food and Cover }\end{array}$ \\
\hline Cover Type(s) & $\begin{array}{l}\text { Short-hand names or codes and detailed descriptions. } \\
\text { Examples: } \\
\text { Deciduous Forested Wetlands } \\
\text { Herbaceous Wetlands } \\
\text { Freshwater Lakes } \\
\text { Riverine } \\
\text { Deciduous Scrub-Shrub Wetland }\end{array}$ \\
\hline Variables & $\begin{array}{l}\text { Short-hand names or codes, detailed descriptions, sampling protocols, and data management } \\
\text { (statistical) activities. } \\
\text { Examples: } \\
\text { Emergent and submerged vegetation } \\
\text { Water depth } \\
\text { Water regime } \\
\text { Water temperature } \\
\text { Velocity }\end{array}$ \\
\hline SI Curves & $\begin{array}{l}X, Y \text { coordinates for all variables included in the model(s). } \\
\text { For example: } 0,0.2,90,1,100,1\end{array}$ \\
\hline $\begin{array}{l}\text { LRSI and HSI } \\
\text { Formulas }\end{array}$ & $\begin{array}{l}\text { Mathematical algorithms for each function in each wetland subclass. } \\
\text { Example: } \\
\text { IndexsliderTurtle }=\text { Minimum of (LRSIFoodCover or } \\
\qquad \text { LRSIwQ or LRSIWaterTemp) }\end{array}$ \\
\hline \multicolumn{2}{|r|}{ Evaluation Data } \\
\hline Target Years & $\begin{array}{l}\text { Baseline, end of construction, and life of the project (including additional years when needed). } \\
\text { Example: } \\
\begin{array}{ll}\frac{\text { TY }}{0} & \frac{\text { Calendar Year }}{2002} \\
1 & 2003 \\
51 & 2053\end{array}\end{array}$ \\
\hline Acres & $\begin{array}{l}\text { Baseline acres per cover type } \\
\text { Without-project acres per cover type } \\
\text { With-project acres per cover type for each alternative }\end{array}$ \\
\hline Variables & $\begin{array}{l}\text { Baseline means/modes per cover type } \\
\text { Without-project means/modes per cover type } \\
\text { With-project means/modes per cover type for each alternative }\end{array}$ \\
\hline
\end{tabular}




\section{Step 2: Model setup}

To begin the setup process, open the HEAT software, select the EXHEP module, and load a blank datafile (Chapter 4). Name this file immediately (Other Activities button), and store it in a local folder of the user's preference (refer to Chapter 4 for details). When finished, click on the Setup button under the Single Formula Models section of the primary interface (upper left-hand corner under the EXHEP logo) (Figure 24). ${ }^{1}$

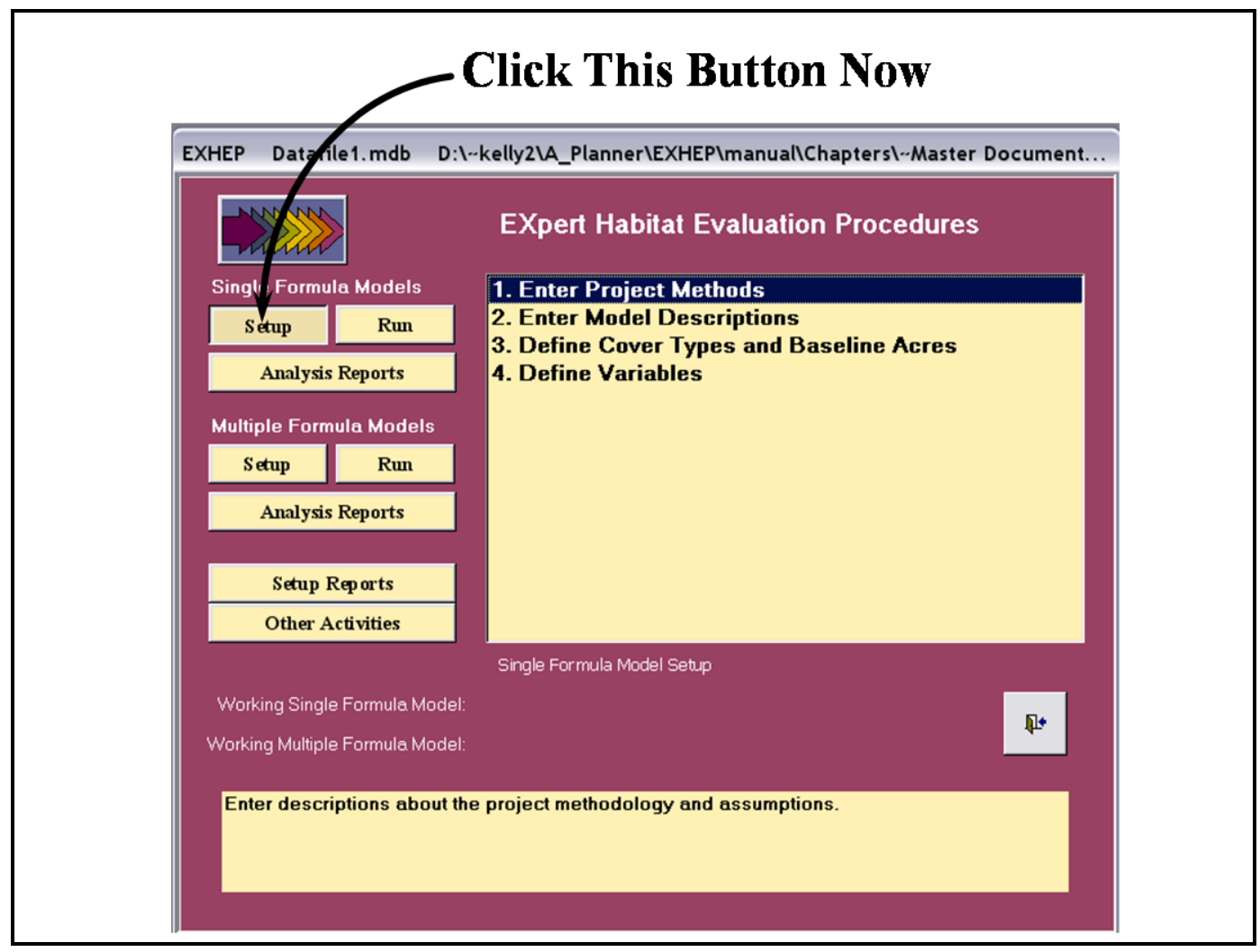

Figure 24. Click on the Setup button to begin the single formula model setup process in EXHEP.

A screen appears in the center of the primary interface with four options:

\section{Enter Project Methods}

2. Enter Model Descriptions

3. Define Cover Types and Baseline Acres

4. Define Variables

\footnotetext{
1 To assist in the software application, a series of training exercises have been included in Appendix Erefer to Exercise 1 for relevant training in model setup in EXHEP.
} 
Moving through the process in a step-by-step fashion, systematically double-click each option from top to bottom to enter the methods, models, cover types, acres, and variables as described below.
USER'S NOTE:

If at any time new information is acquired that affects the analysis, return to these options and update the data.

\section{Enter, delete, and/or copy the project methods}

Double-click on the first option in the primary interface (Enter Project Methods), to open the following window (Figure 25).

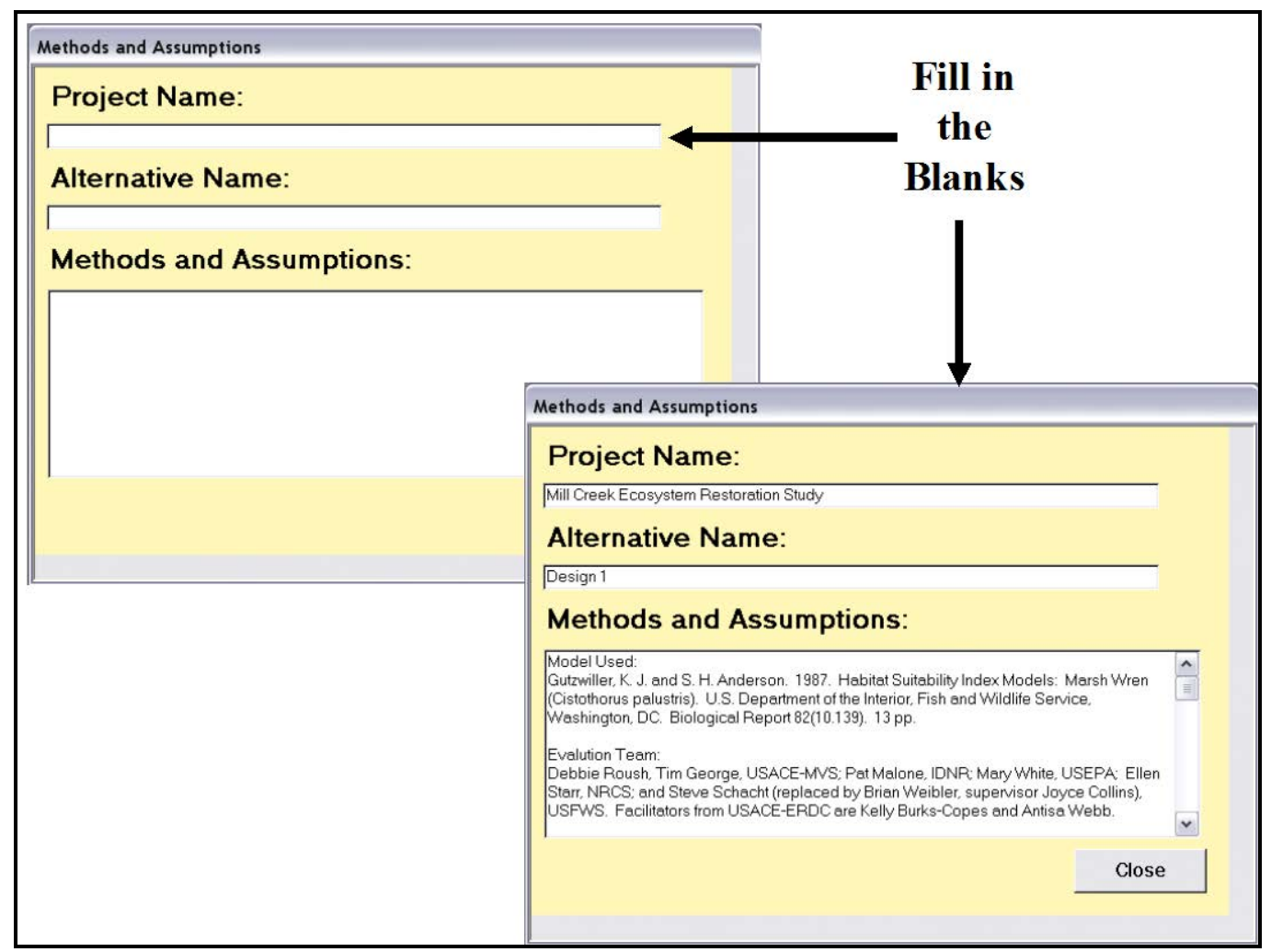

Figure 25. Methods and Assumptions entry screen in EXHEP.

Fill in the blanks with all the information available. Return to this screen as often as necessary to adequately document the analyses and applications. The key to success in HEP applications is documentation. At the absolute minimum, provide a Project Name (limit 255 characters) and an Alternative Name (limit 20 characters) in the spaces provided. The EXHEP module has no restrictions on the number of characters for the Methods and Assumptions section - there should be adequate space to fully document the study activities. Include any references for the model(s) used, and list the evaluation team participants with their 
associated affiliations. Consider including items such as cover type codes and definitions, model modifications, dates of field collections, sources on historically gathered data (including GIS-based information), a list of the target years with their associated calendar years, sources for acreage estimates, descriptions of the future condition, forecasting strategies and decisions, information regarding who was responsible for the overall analysis and results, and points of contact for further information and support. Once this information has been entered, click on the Close button at the bottom of the screen and EXHEP returns to the primary interface.

If an entry must be entirely deleted from the datafile, click on the Other Activities button on EXHEP's primary interface, choose the Clear Database Information option, and proceed through the process as indicated in Chapter 4 of this guide. If details regarding the study's methods have been stored in an MS Word or MS Excel file, these details can be cut and pasted into this screen now. Highlight the information in the external file. Hold down the CTRL key and type $\mathbf{C}$ on the keyboard to copy the information. Return to EXHEP and click on the Methods and Assumptions section and click once. Hold down the CTRL key and type $\mathbf{V}$ on the keyboard to paste the information into this space. When finished with these

USER'S NOTE:

Ctrl $+\mathbf{C}$ is the universal Microsoft shortcut key to copying text and numbers onto the clipboard and works well in these instances.

Ctrl $+\mathbf{V}$ is the universal Microsoft shortcut key to paste text and numbers from the clipboard.

Ctrl $+\mathbf{Z}$ is the universal Microsoft shortcut key to undo an action.

F7 is the universal Microsoft shortcut key to check spelling.

Esc is an additional universal Microsoft shortcut key to undo an action.

These shortcut keys operate on a cell-bycell basis and cannot be used to copy entire spreadsheet rows or records in the data entry screens. activities, click on the Close button at the bottom of the screen and EXHEP returns to the primary interface.

\section{Enter or delete the model descriptions}

Double-click on the second option in the primary interface (Enter Model Descriptions), to open the following window (Figure 26). 


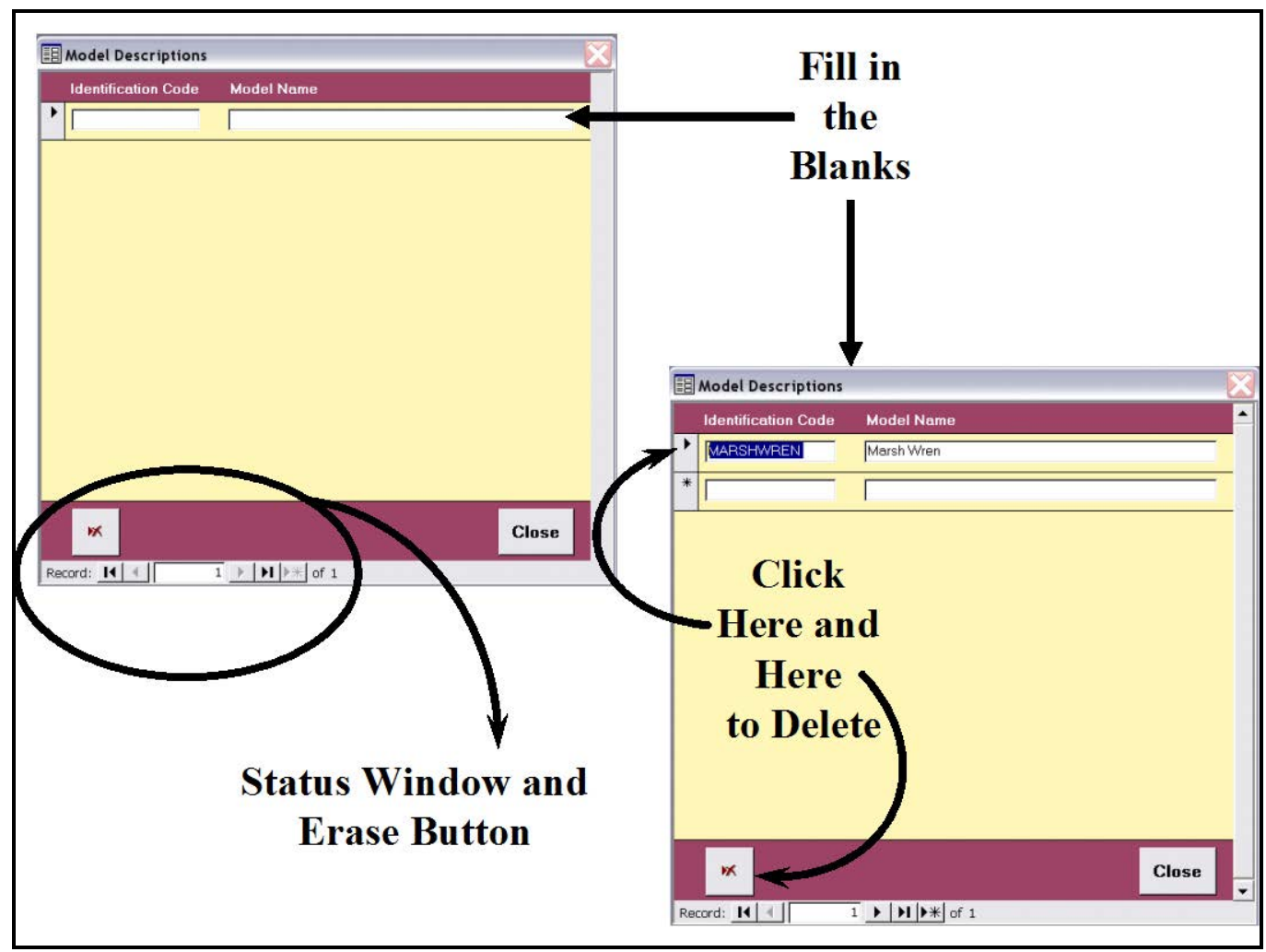

Figure 26. Model Descriptions entry screen in EXHEP.

Provide an Identification Code for the model (limit 10 characters) and a Model Name (limit 100 characters) for each index model in the analysis. A new row is assigned to each model. EXHEP has set no limits on the number of models that can be used for any analyses. A status window at the bottom of the form indicates the number of records (i.e., models) entered into the database. Restrictions on the data entry requirements of this form are offered at the bottom left-hand corner of the screen (Figure 27).

This is a common feature of every EXHEP screen. If a model must be removed, click on the gray block immediately to the left of the model's Identification Code and click on the erase button in the lower left-hand corner of this form (see Figure 26).

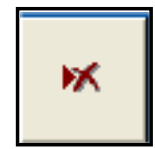
When finished with these activities, click on the Close button at the bottom of the screen and EXHEP returns to the primary interface. 


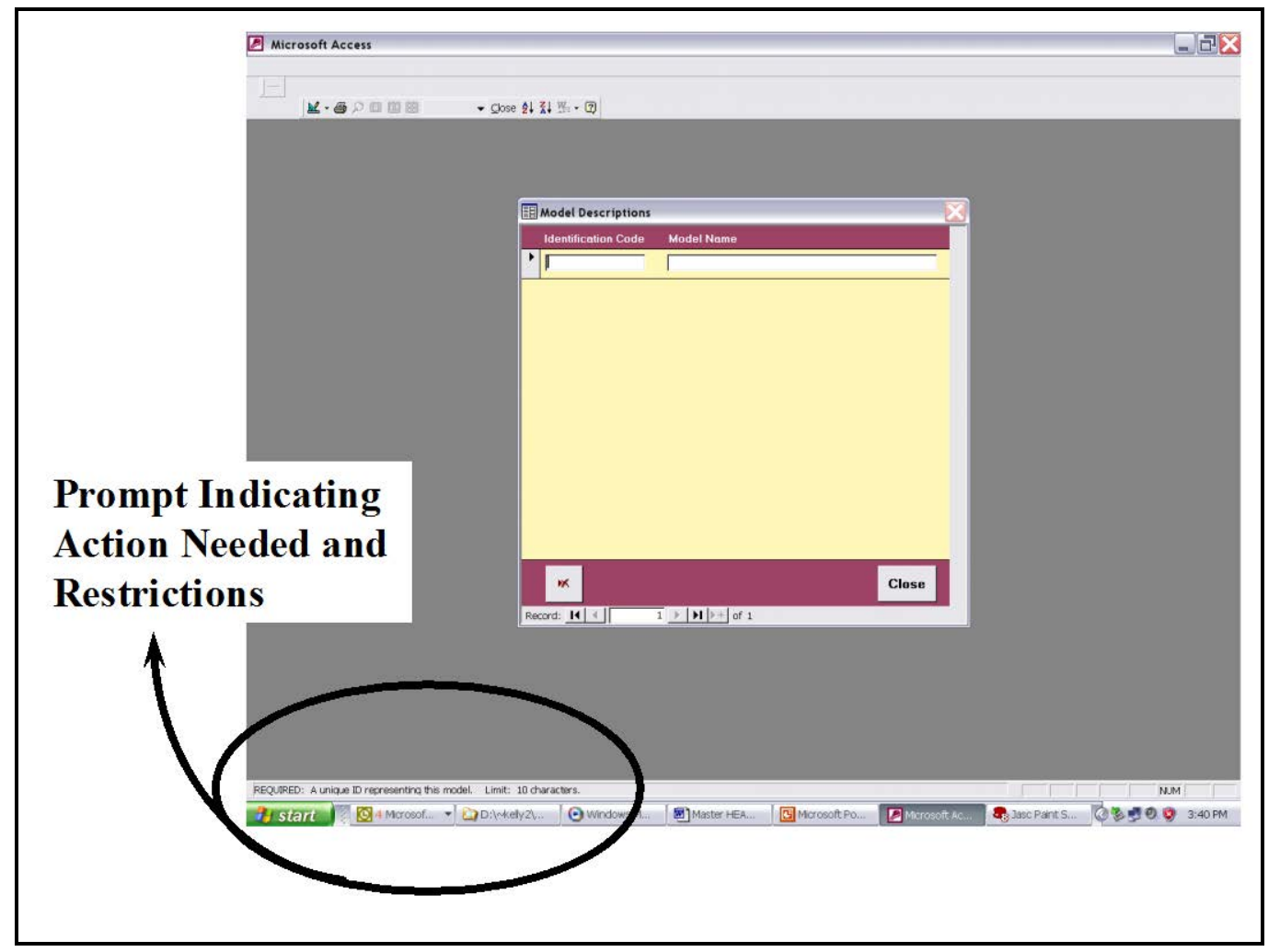

Figure 27. Common EXHEP data entry screen prompts.

\section{Define or delete the cover types and baseline acres}

Double-click on the third option in the primary interface (Define Cover Types and Baseline Acres), to open the following window (Figure 28).

Provide a Numeric Code (1 - $\infty$ ), a shorthand Identification Code (limit 10 characters), a cover type Description (limit 100 characters), and the number of Baseline Acres for each cover type on the site. A new

\section{USER'S NOTE:}

Each cover type's Numeric Code and Identification Code must be unique and contain only alphanumeric characters.

row is assigned to each cover type. There are no limits to the number of cover types EXHEP can process within the analysis. Again, a status window at the bottom of the form indicates the number of records (i.e., cover types) entered into the database, and restrictions on the data entry requirements of this form are offered at the bottom left-hand corner of the screen. 


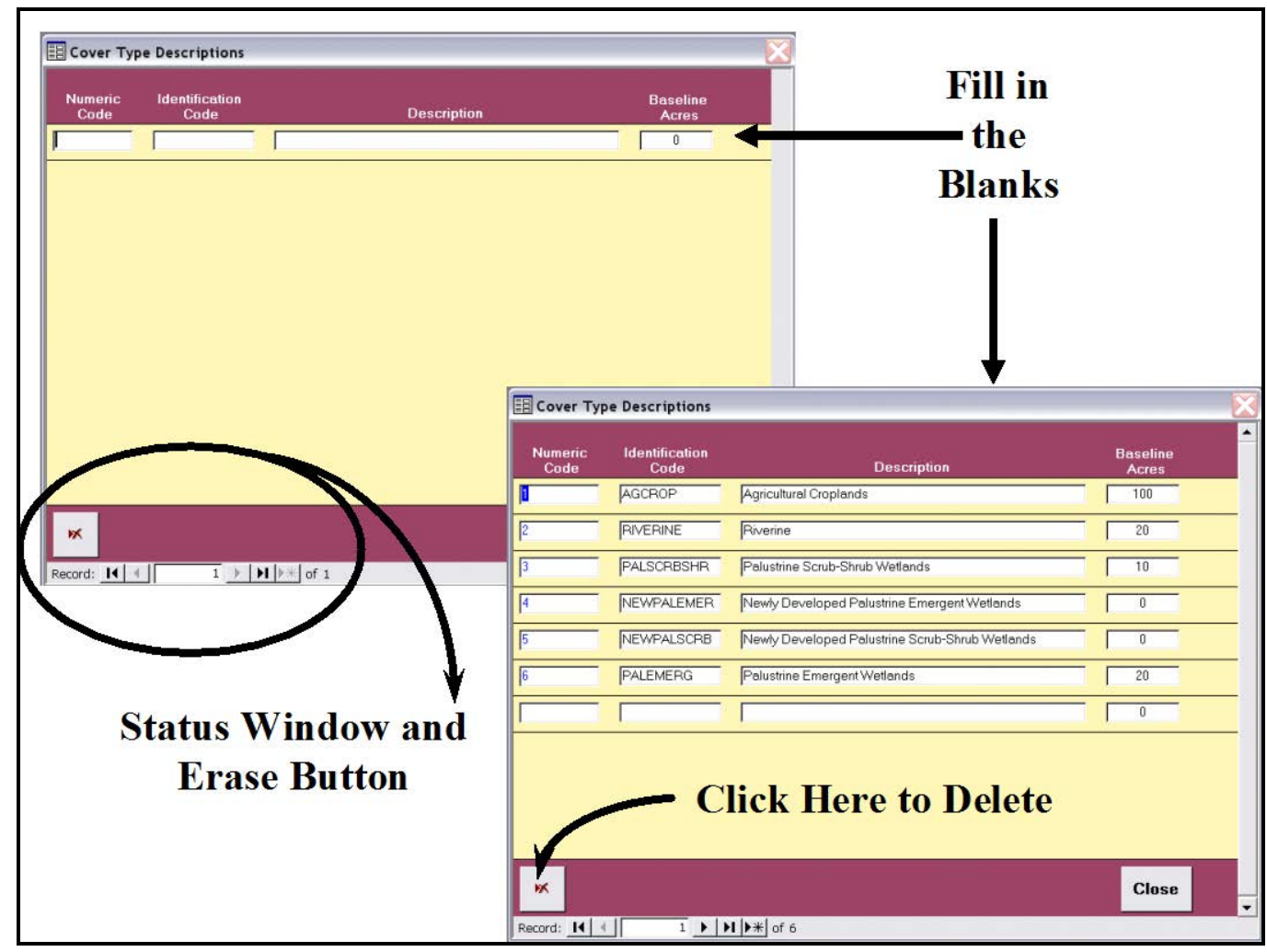

Figure 28. Cover Type Descriptions entry screen in EXHEP.

Account for every acre within the project boundary, even if the cover type is not associated with a specific model. In some instances, these unassociated cover types are not considered habitat today, but could be restored to useable habitat in the future, and thus the current acres should be tracked (i.e., AGCROP $=100$ acres of soybeans that could be replaced with forested wetlands under a proposed withproject scenario). Create "placeholder" cover types for future development options. Thus, for every existing cover type that is associated with the model, create a "with-project" cover type (e.g., Palustrine Emergent Wetlands and Newly Developed Palustrine Emergent Wetlands). If a cover type must be removed, left-click once anywhere on the cover type's row and click on the erase button in the lower lefthand corner of this form. When finished with these activities, click on the Close button at the bottom of the screen and EXHEP returns to the primary interface.

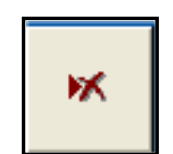

USER'S NOTE:

If the baseline acreages for the cover types are unavailable at this time, EXHEP assumes zero acres. Once the mapping is complete, return to this screen and refine the acreage estimates. 


\section{Define or delete the variables}

Double-click on the fourth option in the primary interface (Define Variables), to open the following window (Figure 29).

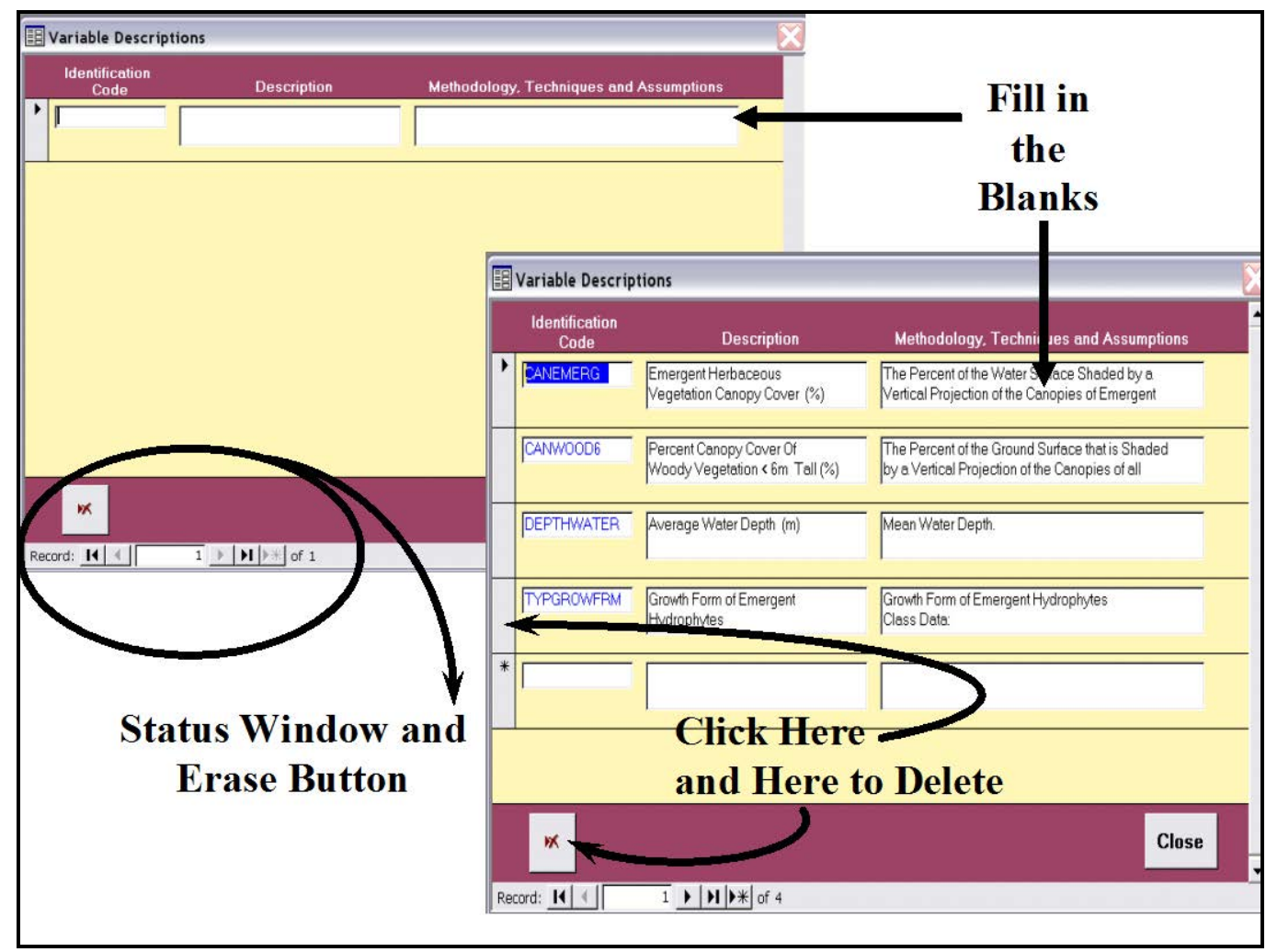

Figure 29. Variable Descriptions entry screen in EXHEP.

Provide an Identification Code (limit 10 characters), a variable Description (limit 100 characters), and a brief description of the

\section{Methodology, Techniques and}

Assumptions used to gather/ generate the analyses variable data (limit 255 characters including periods and spaces). A new row is assigned to each variable. There are no limits to the number of variables EXHEP can process in the analysis. When creating variable codes, begin with the action to be measured, and follow this with specifics. For example:

- Shrub canopy cover $=$ CANSHRUB

- Tree canopy cover $=$ CANTREE

- Water depth = DEPTHWATER
USER'S NOTE:

Each variable's Identification Code must be unique and contain only alphanumeric characters. The variable Description and Methodology, Techniques and Assumptions can contain symbols (i.e., $\%, \&, \#)$. 
- Percent of the area covered by open water =AREAVEGOPW

- Ratio of wet to total prairie acreage =AREAWETDRY

To shorten the codes (to meet the 10-character limit), try removing spaces, periods, conjunctions and vowels, and then use acronyms where possible (e.g., species $=$ SPP or vegetation $=$ VEG). This approach allows EXHEP to cluster "like" variables together, and will help position variables in lists throughout the process. ${ }^{1}$

Remember, if variables share the same sampling protocol, use the $\mathbf{C t r l}+\mathbf{C}$ and $\mathbf{C t r l}+\mathbf{V}$ shortcut keys to copy the sampling methodology information from one variable to the next. This can only be used on a cell-by-cell basis - an entire row cannot be copied using this technique. Once again, a status window at the bottom of the form indicates the number of records (i.e., variables) entered into the

USER'S NOTE:

The 255-character limit in the Methodology, Techniques and Assumptions cell is strictly enforced. To shorten the amount of information entered in this field, drop periods and use acronyms and symbols whenever possible.

\section{USER'S NOTE:}

Use Ctrl + Enter to insert hard returns into the Description and Methodology, Techniques and Assumptions fields of this form.

The Esc key on the keyboard jumps the cursor out of cells - use when a mistake is made during the data entry process. database. Restrictions on the data entry requirements of this form are offered at the bottom left-hand corner of the screen. If a variable must be removed, click on the gray block immediately to the left of the variable's Identification Code and click on the erase button in the lower left-hand corner of this form (see Figure 29). Initial model setup is now complete. If there are no multiple formula models to set up, click on the Close button at the bottom of the screen and in EXHEP (returning to the primary interface), and proceed to the "Checking the setup process with EXHEP reports" section later in this guide. If there are multiple formula models in the analysis, proceed to the next section below.

\section{Additional setup actions for multiple formula models}

If multiple formula models have been included in the analysis, these models must now be entered into the datafile using the second set of buttons on EXHEP's primary interface (Figure 30).

\footnotetext{
${ }^{1}$ Appendix D provides a general naming convention protocol the authors have developed to simplify and standardize this process.
} 


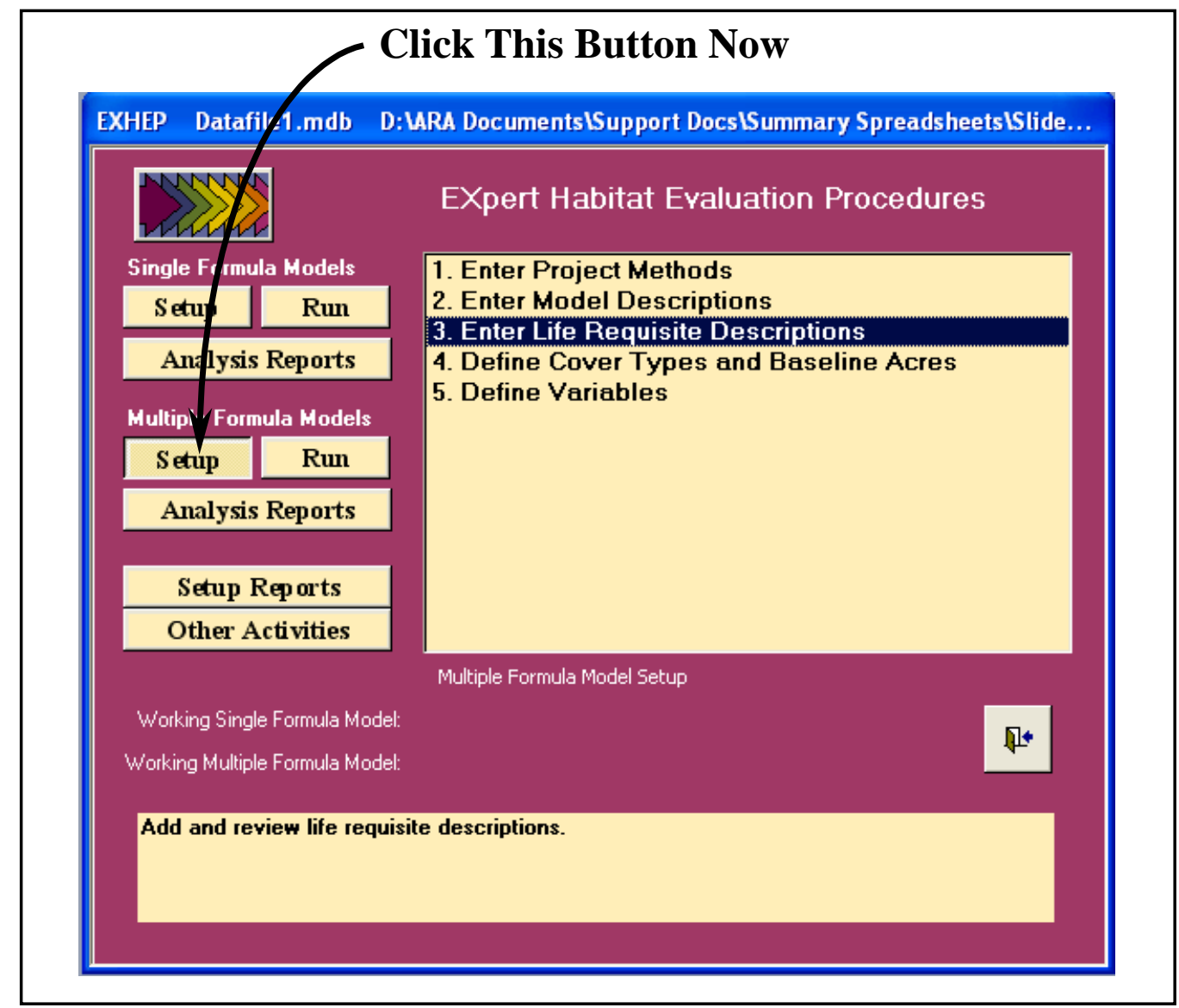

Figure 30. Click on the Setup button to begin the multiple formula model setup process in EXHEP.

A new screen with the following five options appears in the center of the primary interface:

\section{Enter Project Methods}

2. Enter Model Descriptions

3. Enter Life Requisite Descriptions

4. Define Cover Types and Baseline Acres

5. Define Variables

Again, in systematic fashion, start at the top of the options list, click on each option, and provide the data requested in each interface screen. EXHEP uses the same interfaces to register the multiple formula models as it did

USER'S NOTE:

If at any time new information is acquired that affects the analysis, return to these options and update the data. in the single formula model example with the exception of the third option (Enter Life Requisite Descriptions). This option is described below. 
Enter life requisite descriptions

Click on the third option in the primary interface (Enter Life Requisite Descriptions) to open the following window (Figure 31).

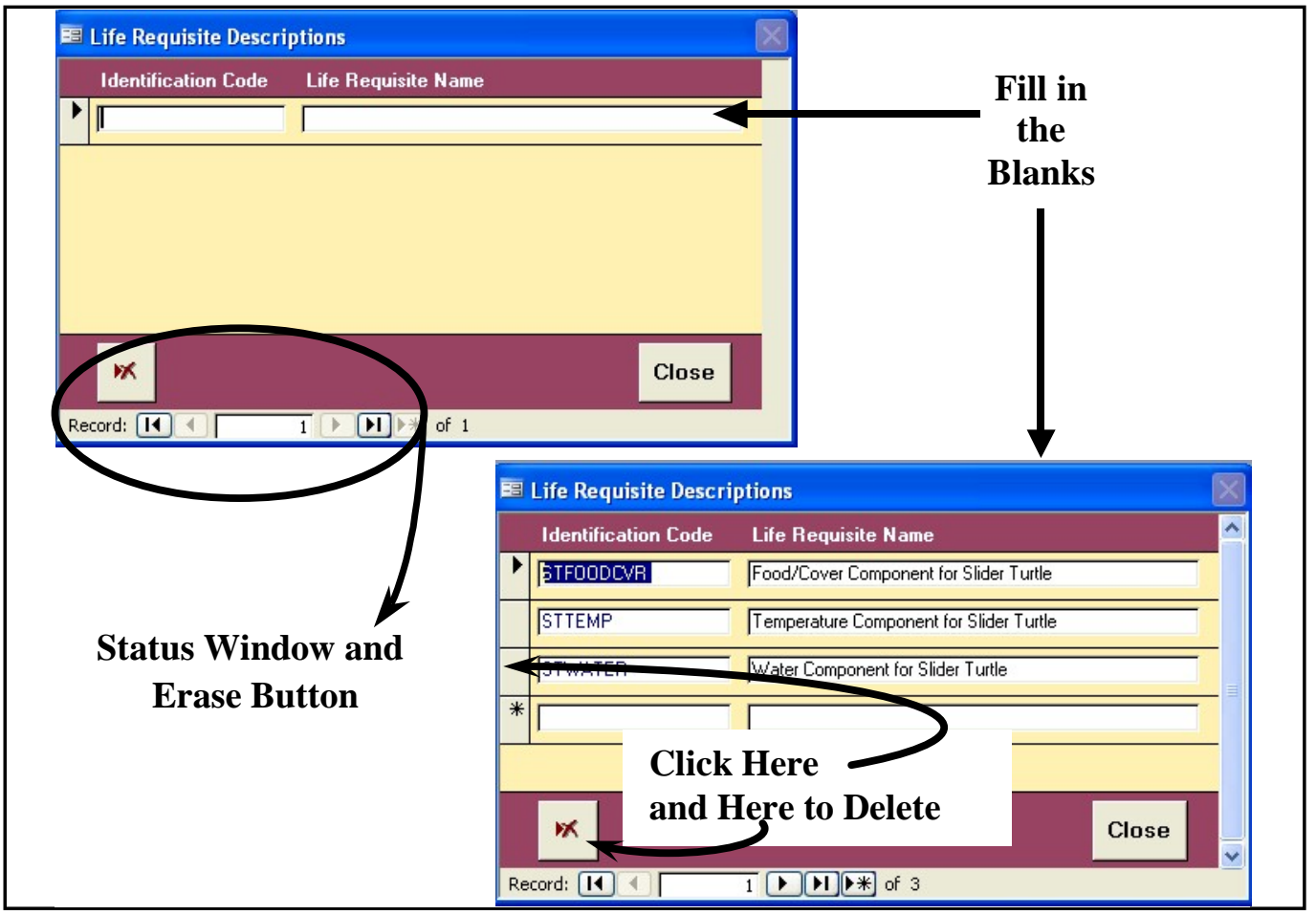

Figure 31. Life Requisite Descriptions entry screen in EXHEP.

Provide an Identification Code (limit 10 characters) and a Life Requisite Name (limit 50 characters) for each life requisite in the analysis. A new row is assigned to each life requisite for every model. EXHEP places no

USER'S NOTE:

Each life requisite's Identification Code must be unique and contain only alphanumeric characters.

limits on the number of life requisites that can be used for these analyses. Again, a status window at the bottom of the form indicates the number of records (i.e., life requisites) entered into the database, and restrictions on the data entry requirements of this form are offered at the bottom lefthand corner of the screen. If a life requisite must be removed, click on the gray block immediately to the right of the life requisite's Identification Code and click on the erase button in the lower left-hand corner of this form (see Figure 31 above). Once this information has been entered, click on the Close button at the bottom of the screen and EXHEP returns to the primary interface. Continue through the remaining list of options as indicated above in the single formula model's section to complete initial model setup for multiple 
formula models. Initial model setup is now complete. Proceed to the "Checking the setup process with EXHEP reports" section below.

\section{Checking the setup process with EXHEP reports}

EXHEP provides a series of summary reports as each step in the process above is completed. To review and check the setup information entered thus far, click on the Setup Reports button (Figure 32).

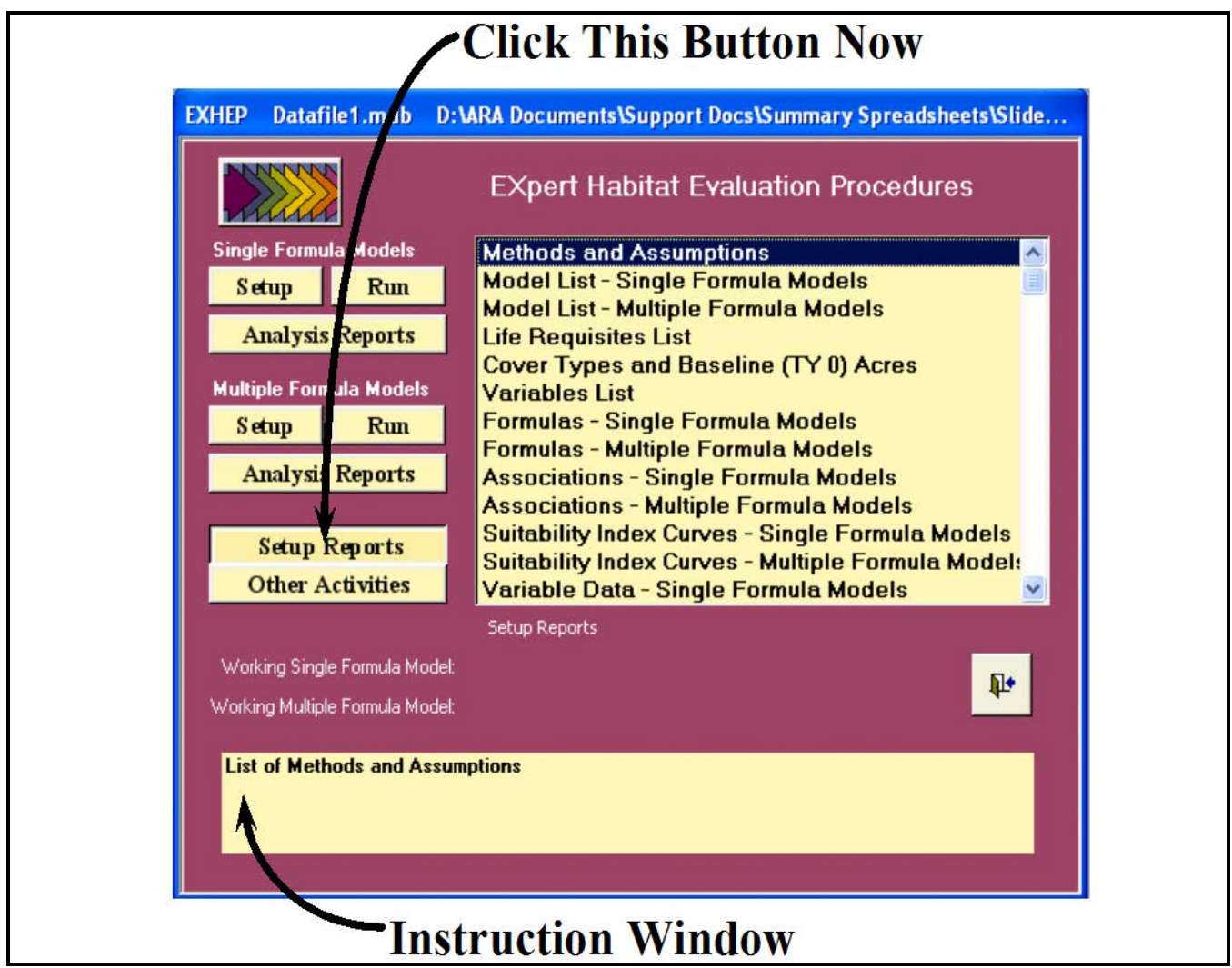

Figure 32. Click on the Setup Reports button to review the setup reports in EXHEP.

A list of available reports appears in the center of the primary interface. The first six reports should be populated at this point in the analysis:

- Methods and Assumptions

- Model List - Single Formula Models

- Model List - Multiple Formula Models

- Life Requisites List

- Cover Types and Baseline (TY 0) Acres

- Variables List 
Single-click on any report and look to the Instruction Window to preview the contents of the report. Double-click on any report, and EXHEP opens a new window with the report inside (Figure 33).
USER'S NOTE:

A printer driver must be installed in order to view any of these reports.

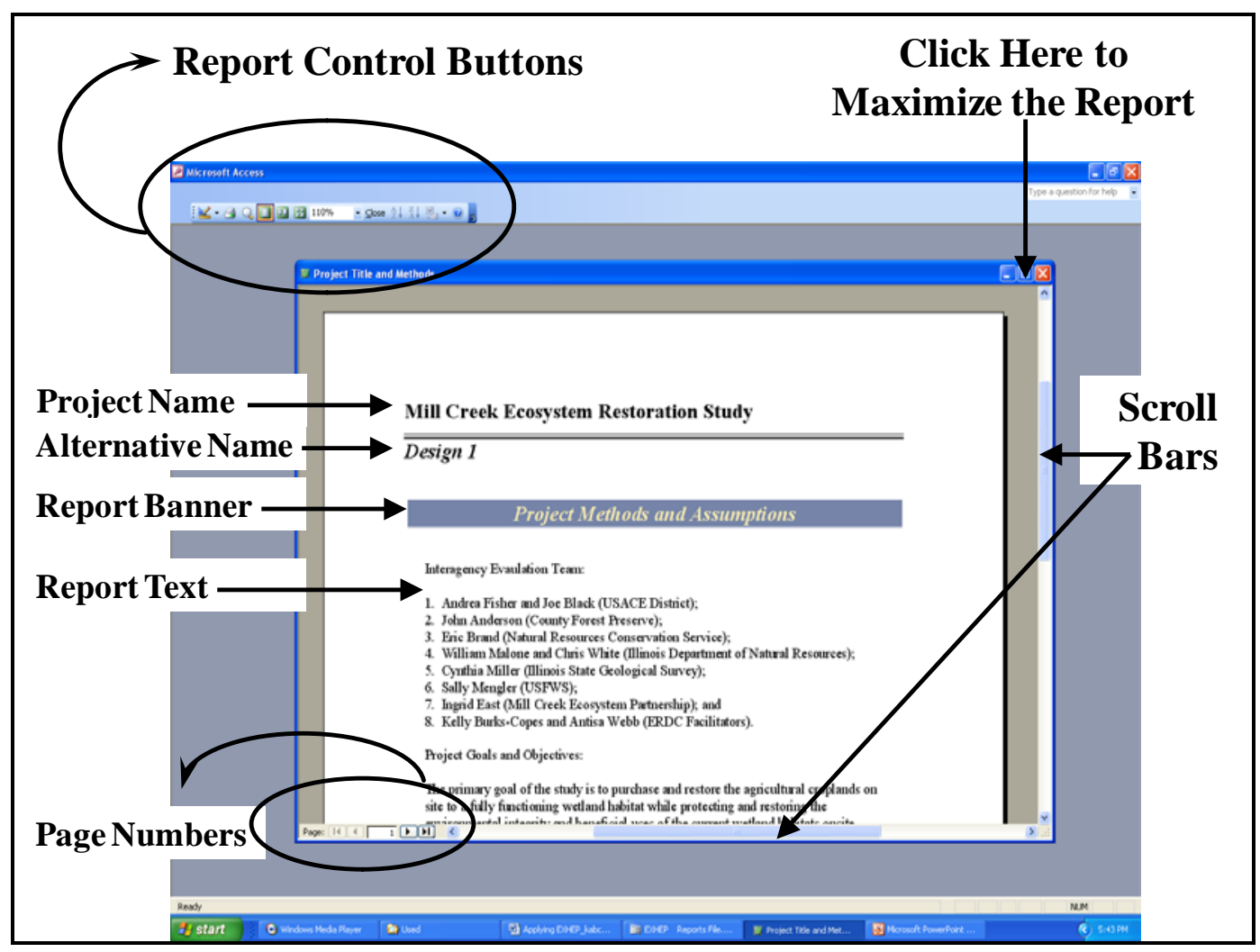

Figure 33. Report interface in EXHEP.

EXHEP reports are somewhat standardized to present the datafile's information in a clear and concise manner. The header of each report contains the project and alternative names separated by a heavy line. A banner below these fields indicates the name of the report (in this case Project Methods and Assumptions). The report's contents are presented immediately below the banner. Standard MS Access scroll bars are provided on the bottom and right-hand edges of the report itself to aid in navigation. Page numbers are presented in the lower left-hand corner of the report to help move through the report as well. Standard Minimize/ Maximize/ Close buttons are located in the upper right-hand corner of the report. Outside of the report, on the toolbar in the upper left-hand corner of the screen, a series of report control buttons on the Print Preview toolbar can be found that allows users to review, print, close, and export the report as needed. 
Some default printer settings may cause an EXHEP report to span multiple pages if the margins are set too narrowly. To solve this problem, rightclick on the report, and select Page Setup from the list of options. Reset the margins, column widths, and page sizes as necessary using the Margins, Page, and Columns tabs to customize

\begin{tabular}{|l|}
\hline Zoom: $100 \%$ \\
圆 One Page \\
四 Multiple Pages \\
\hline 口 Page Set..... \\
曷 Print... \\
\hline Save As... \\
Export... \\
Send To
\end{tabular}
the report for the printing environment.

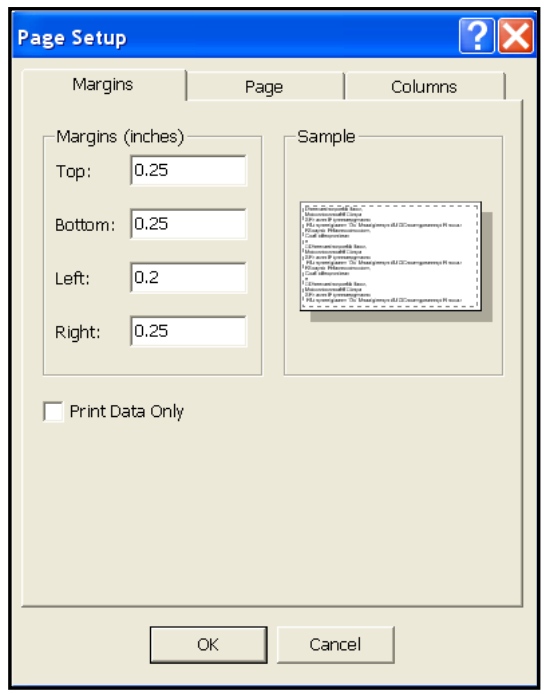

Click on the Maximize button and select Fit from the Zoom pull-down box on the toolbar. The footer of the report is revealed. Here, EXHEP provides two additional pieces of information that will assist in assessment logistics - the date and time the report was run, and the datafile's storage path (Figure 34).
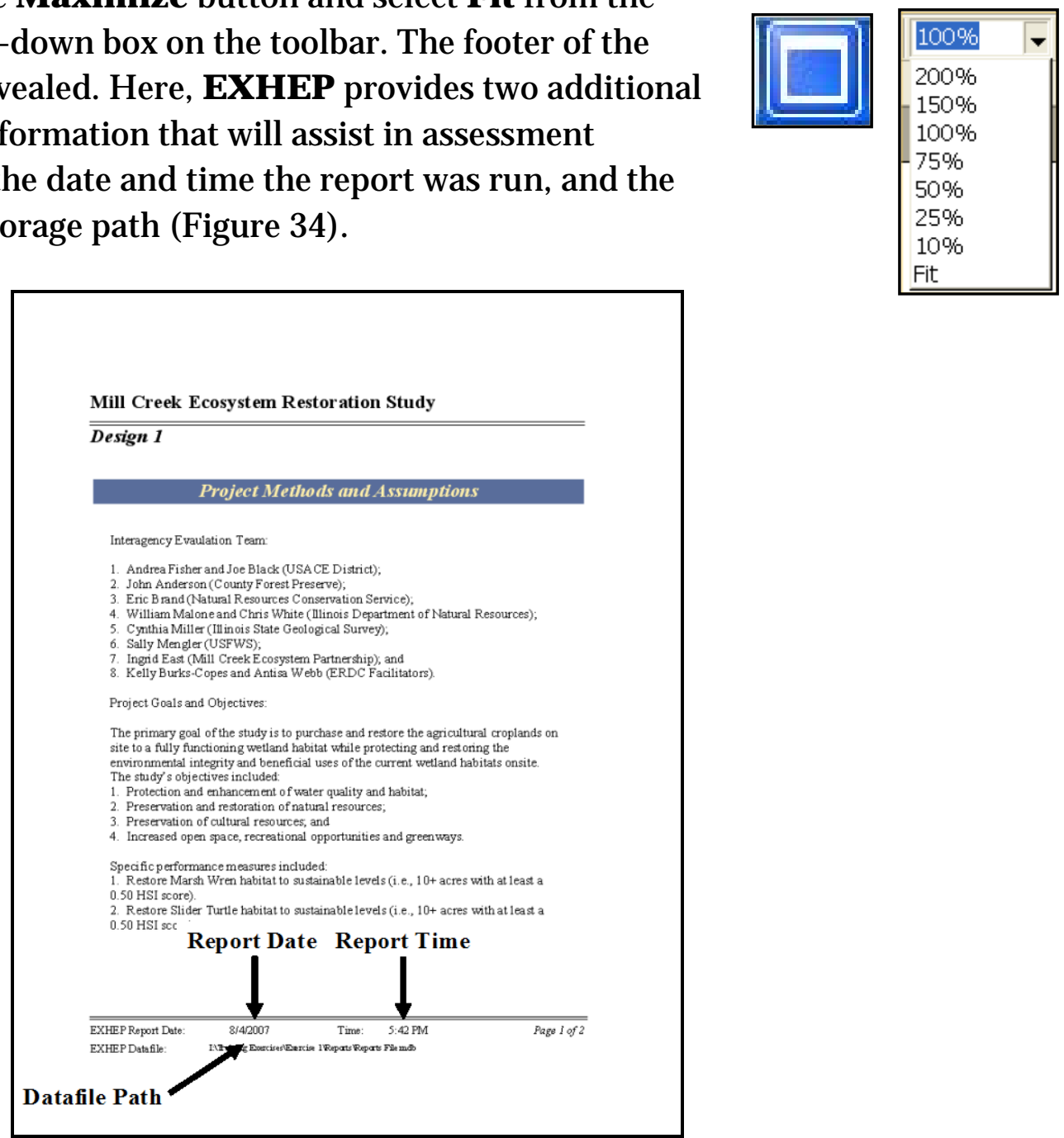

Figure 34. An expanded EXHEP report detailing additional fields. 
One convenient advantage of using the EXHEP software is its compatibility with other Microsoft software packages (namely MS Word and MS Excel). By simply clicking on the Officelinks

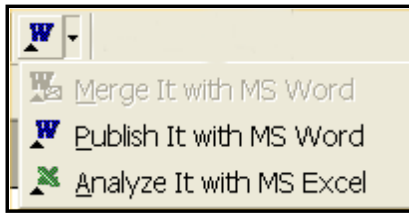
pull-down box, and clicking on either option, the report is exported out to the new medium, allowing customization to meet documentation needs.

If the Print Preview toolbar is not available in this screen, right-click on the report, and select the Export option from the list of choices presented. An Export entry screen will open in response to this action (Figure 35).

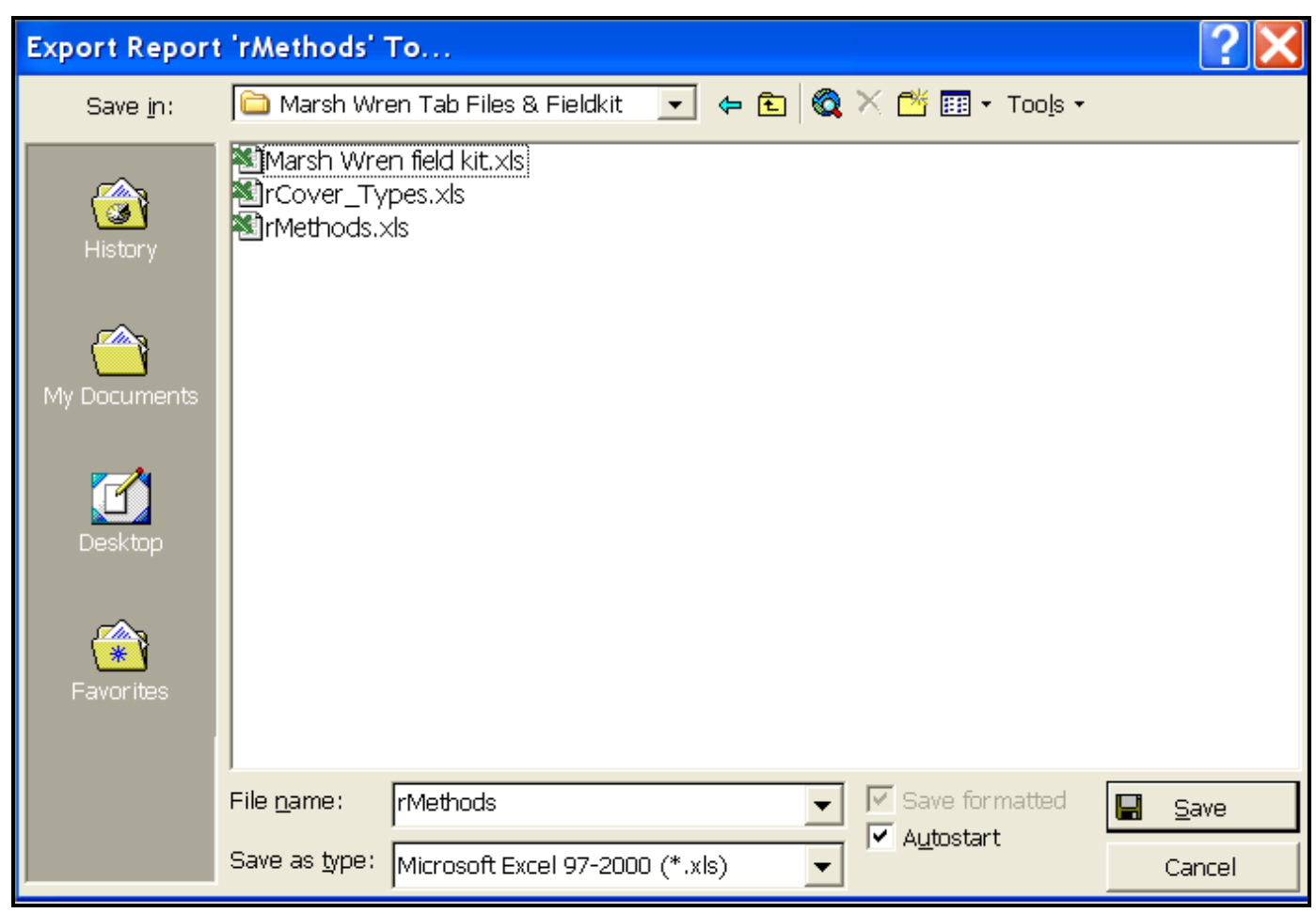

Figure 35. Export Report entry screen - exporting report information to MS Excel or MS Word.

Use the Save in: window at the top of the entry screen to select a destination for report exportation. Provide a name for the report in the File name: window in the bottom of the entry screen. Use the Save as type: pull-down list to select the type of file to be exported [i.e., choose either MS Excel 97-2007 (*.xls) for a spreadsheet format or Rich Text Format (*.rtf) for a word-processing format]. To associate the software (i.e., MS Excel for spreadsheets or MS Word for word processing documents), such that the medium opens and displays the information in the new environment upon exportation, click the Autostart checkbox 
immediately to the right of the Save as type: pull-down list. Press the Save button in the lower right-hand corner of the screen. EXHEP will export the data displayed in the report to the new file, and if the Autostart option was clicked, MS Excel/ MS Word will open and display the report in the new environment (Figure 36).

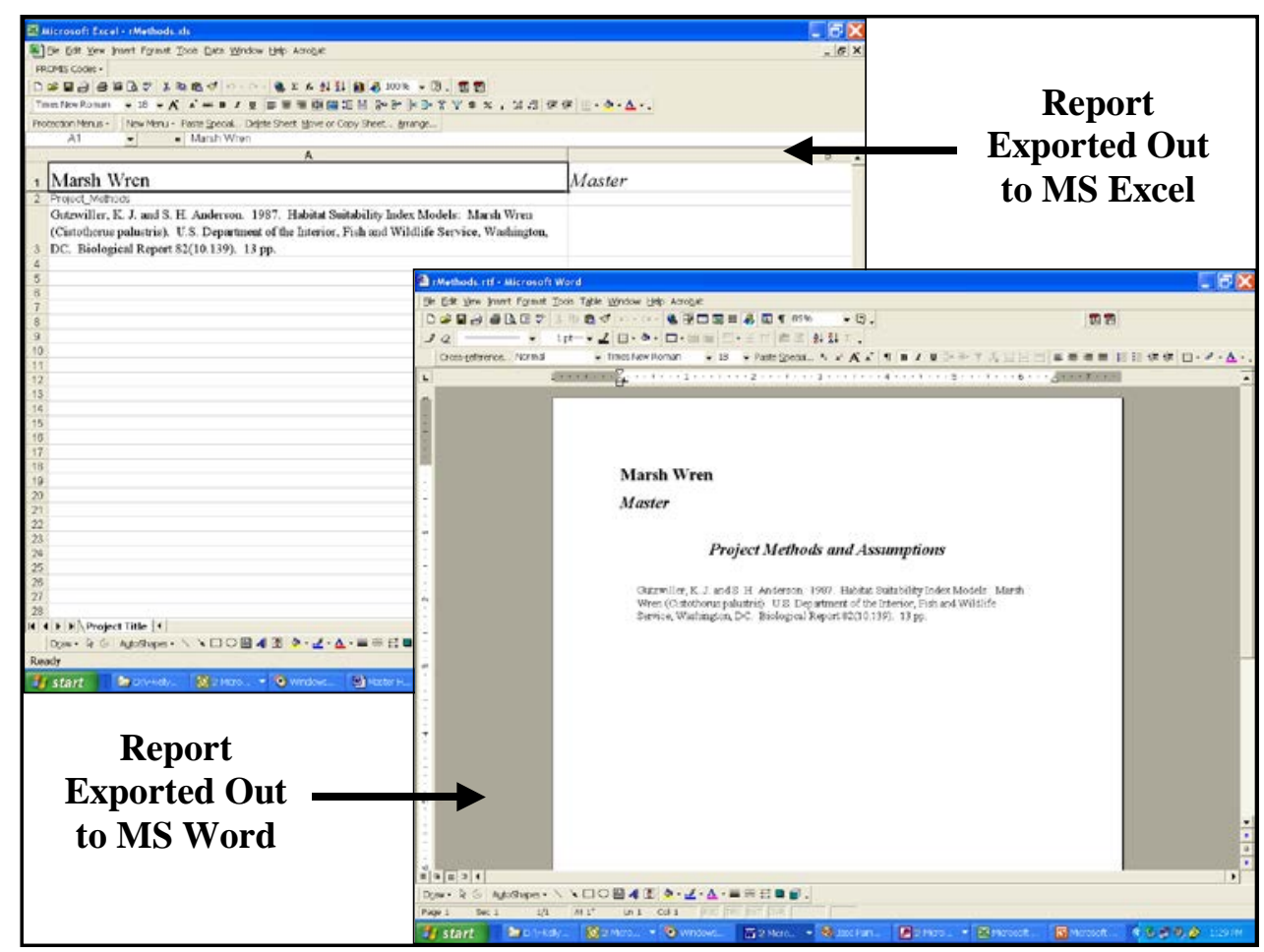

Figure 36. Exported reports in MS Excel and MS Word environments.

Format and save the files as needed, close the external applications (i.e., MS Excel or MS Word), and return to EXHEP. Click on the Close button from the suite of buttons offered in the Print Preview toolbar - EXHEP returns to the primary interface. Repeat the process as often as necessary to check the products developed thus far in the setup activities. Proceed to "Step 3: Model Association" section to continue.

\section{Step 3: Model association}

When the HSI models are set up in EXHEP, assess the models by clicking on the Run button under the Single Formula Models section of the primary interface (Figure 37). ${ }^{1}$

\footnotetext{
1 To assist in the software application, a series of training exercises have been included in Appendix E refer to Exercises 2 and 3 for relevant training in model association in EXHEP.
} 


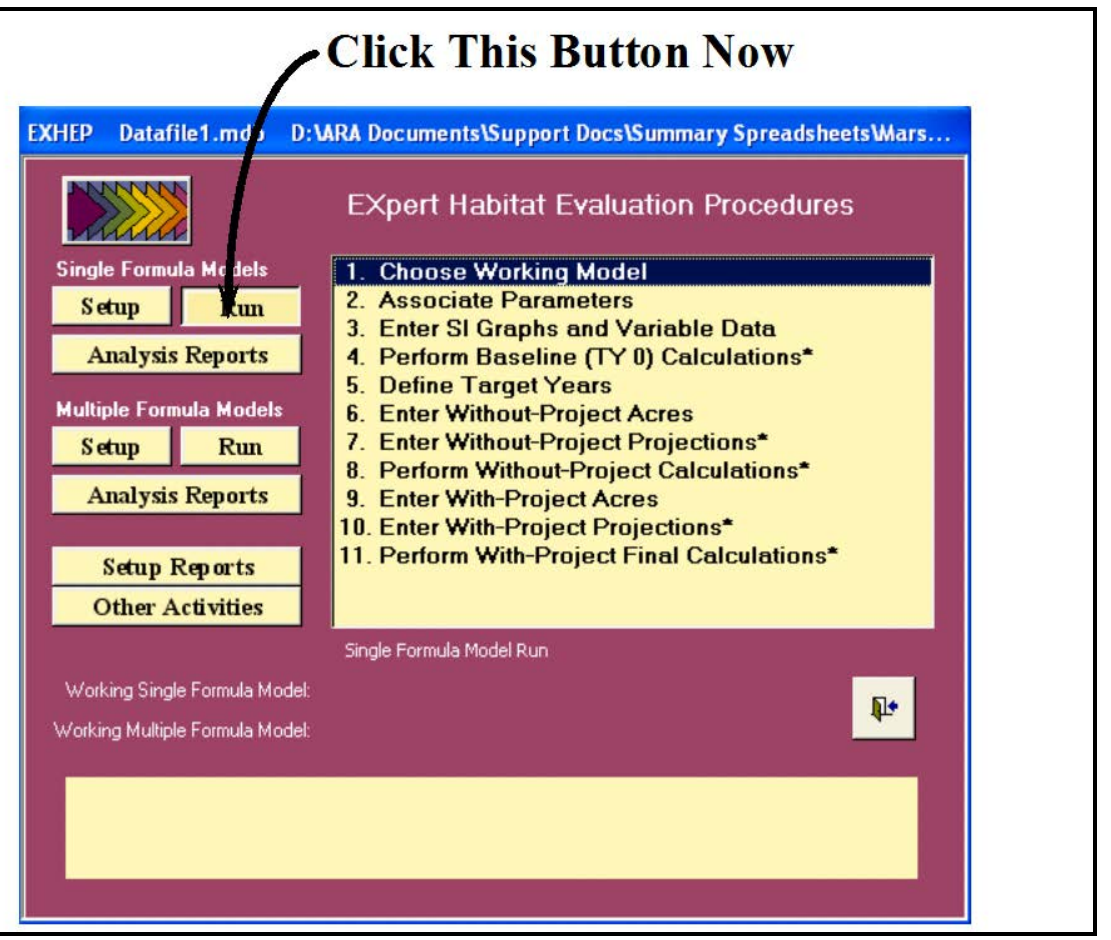

Figure 37. Click on the Run button to begin the single formula model association process in EXHEP.

A screen appears in the center of the primary interface with 11 options:

\section{Choose Working Model}

2. Associate Parameters

3. Enter SI Graphs and Variable Data

4. Perform Baseline (TY 0) Calculations*

5. Define Target Years

6. Enter Without-Project Acres

7. Enter Without-Project Projections*

8. Perform Without-Project Calculations*

9. Enter With-Project Acres

10. Enter With-Project Projections*

11. Perform With-Project Final Calculations*

In systematic fashion, start at the top of the options list, click on each option, and provide the data or perform the action requested in each interface screen.

\section{USER'S NOTE:}

If at any time new information is acquired that affects the analysis, return to these options and update the data. 


\section{Choose the "Working Model"}

Initially, EXHEP was developed to associate and compute all index models simultaneously. Although this provided answers in a single-step fashion, the rigorous processing cycles tied up computers for long periods of time. In addition, alteration of the information within any model made it necessary to re-process the entire database. This proved to be an inefficient mode of operation. EXHEP was re-designed to

USER'S NOTE:

Once a "Working Model" has been identified, EXHEP will focus solely on the data entry and evaluation of this model. To enter data for another model, a new "Working Model" must be identified. incorporate a means to directly process models in a more focused approach. EXHEP's new evaluation strategy requires users to focus the EXHEP analysis engine on a single "Working Model" for model association and processing. Thus, to begin the run process, a "Working Model" must first be identified. To do this, click on the first option (Choose Working Model) - the following window opens (Figure 38).

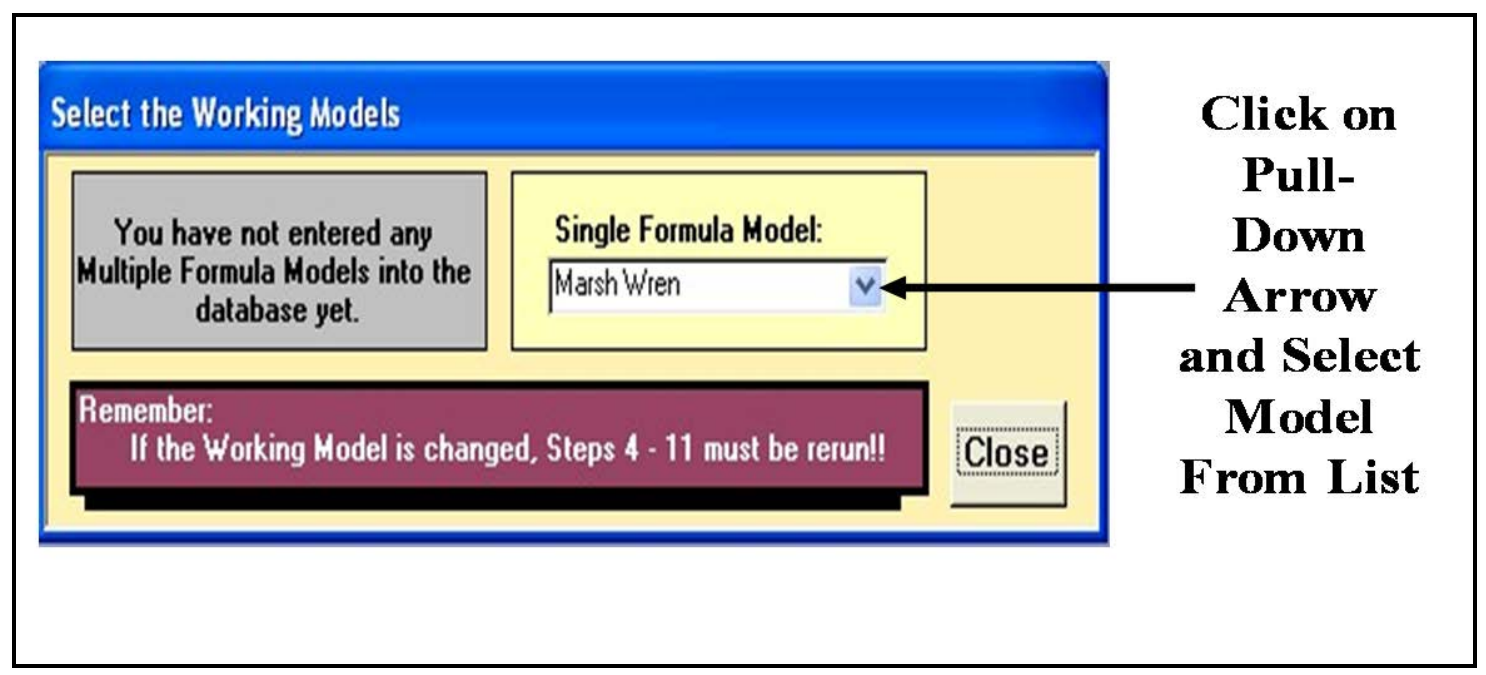

Figure 38. Select a model from the Single Formula Mode/pull-down list.

Simply choose the assessment model from the pull-down list on the righthand box in this screen and click Close. EXHEP returns to the primary interface. At the bottom of the screen, the "Working Model" is now identified (Figure 39). Proceed to the next option. 


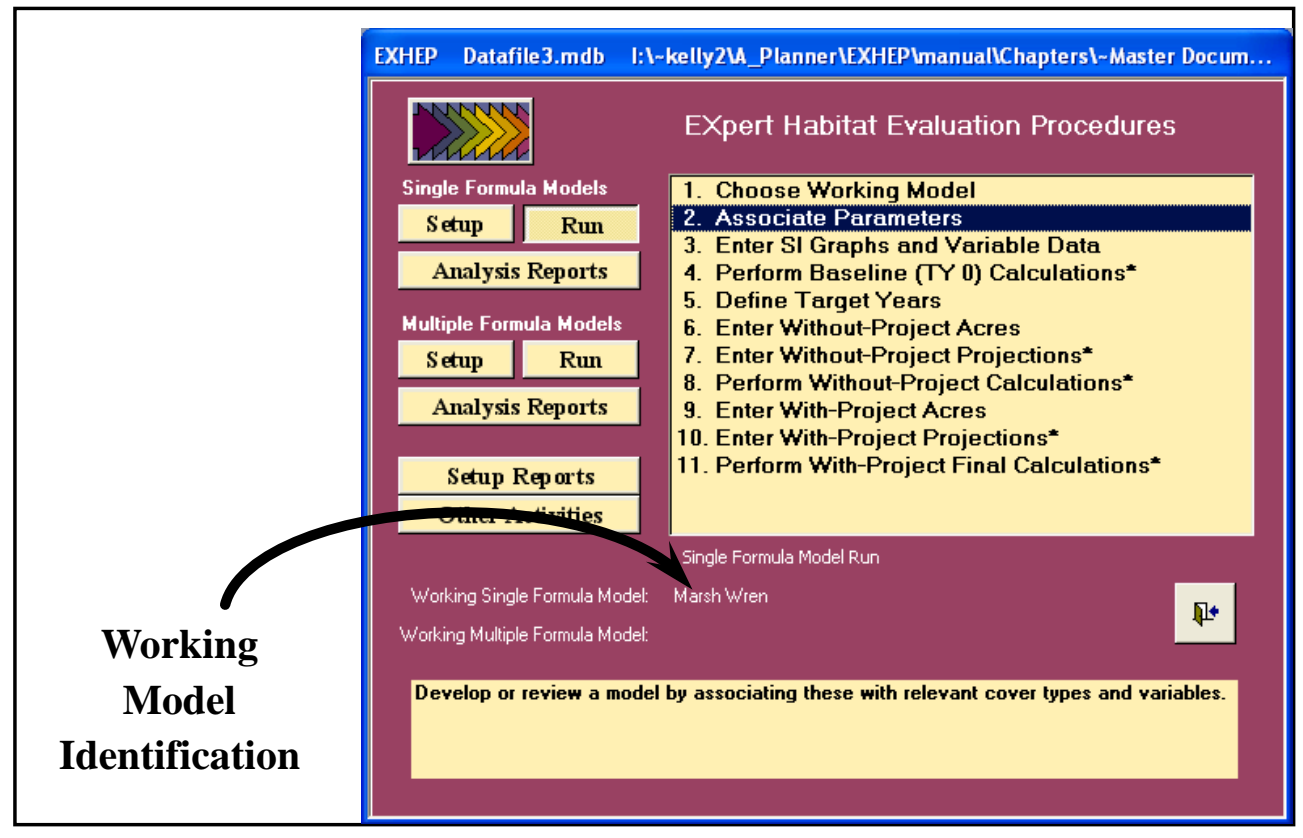

Figure 39. Identification of the Working Single Formula Model

in EXHEP's primary interface.

\section{Associate the model parameters}

In EXHEP, association is the process of combining model cover types and variables in a meaningful manner. In other words, association provides an interface with the EXHEP analysis engine and guides it in model definition and application. In this process, model definition is the translation of mathematical equations (e.g., HSI = Variable $1 *$ Variable $2 *$ Variable 3 , etc.), often derived from the USFWS model publications, into MS Access compatible language. Model application refers to the act of tying these models to specific habitat conditions (i.e., cover types). The association activity should be viewed as an opportunity to define and communicate the sensitivity of HSI models to the EXHEP program. Click on the second option (Associate Parameters), to open the following windows (Figure 40).

The Model Associations window is the activity platform for the model association process. Indicate which cover types are evaluated in the model, and what formula will be used to determine the cover type's suitability. The current Working Model is provided as the first item in the yellow box in the center of this form (Figure 41). 


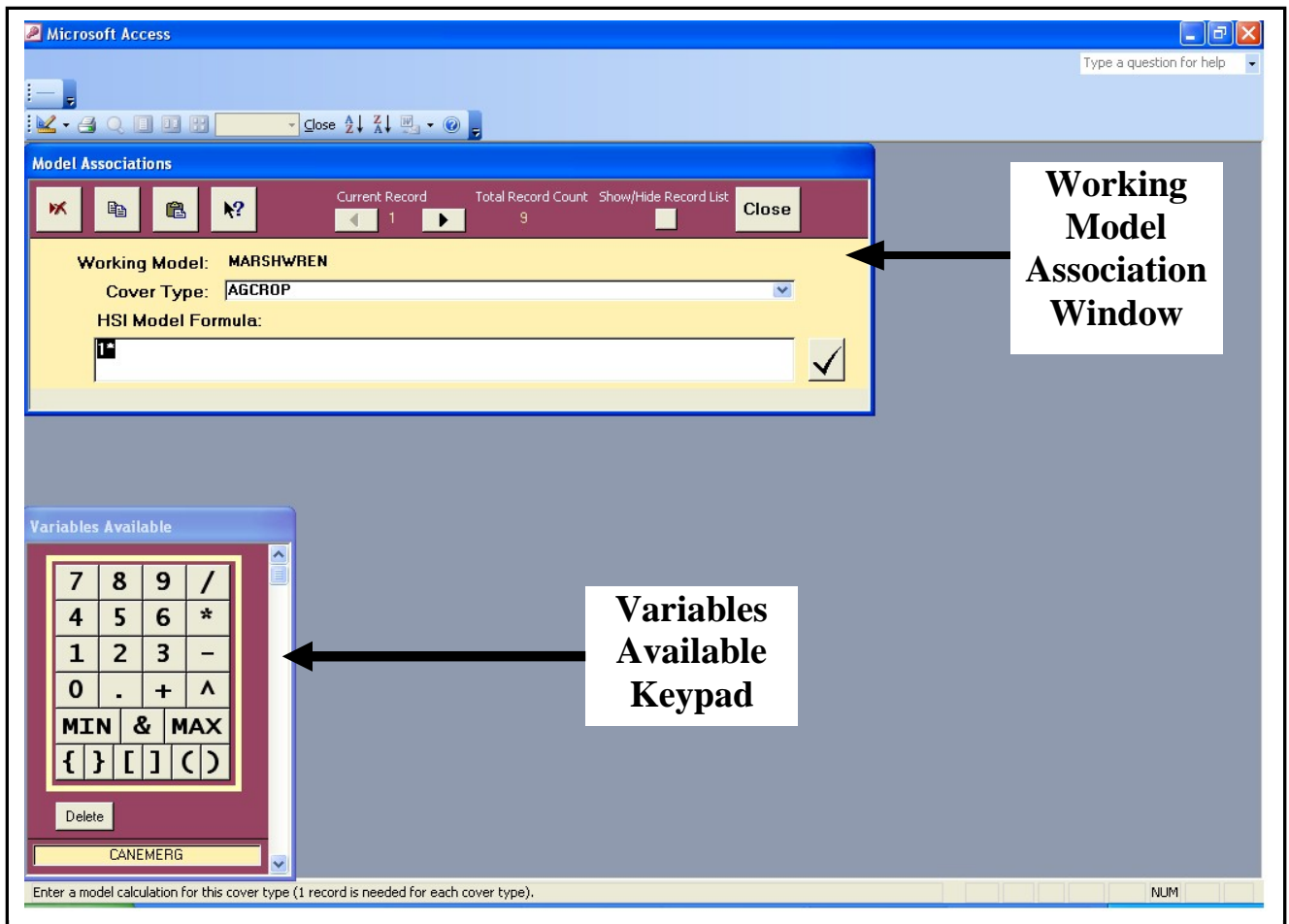

Figure 40. Model Associations window and Variables Available keypad used to associate single formula models in EXHEP.

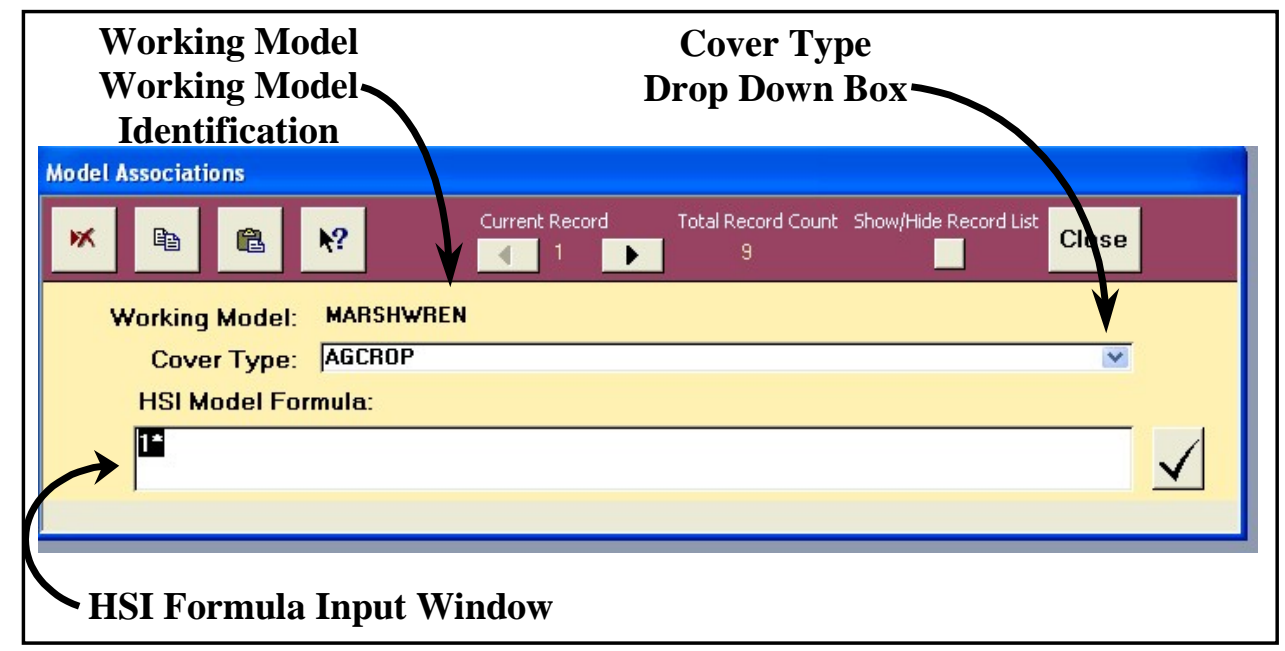

Figure 41. The Model Associations entry screen for single formula models.

Immediately below the Working Model line, a pull-down box for Cover Type identification and an HSI Model Formula input box are provided. Model association for single formula models is a two-step process - select a cover type first, and then enter an HSI formula. For details regarding these two steps, read below. 
Select a cover type

To begin, click on the Cover Type drop-down box, and choose the first cover type that will be associated with the model (Figure 42).

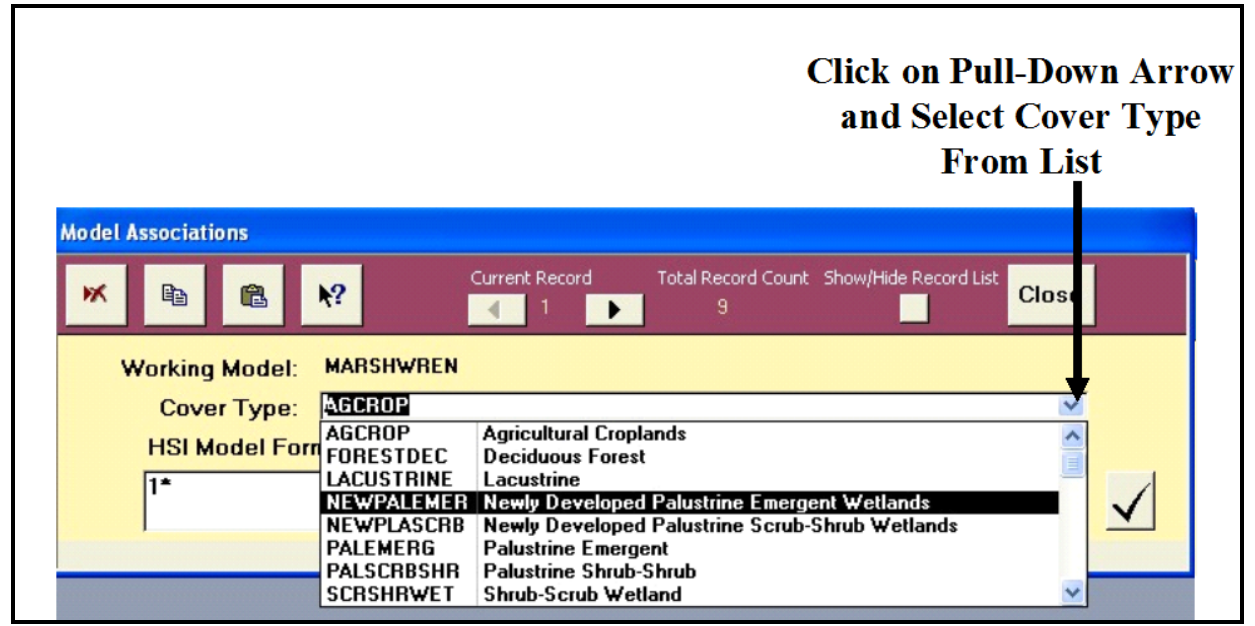

Figure 42. Select a cover type from the pull-down list.

Enter an HSI formula

Now left-click once in the HSI Model Formula input box in the lower portion of the screen. Define the combination of variables that together formulate the HSI model for the ecosystem. In other words, in mathematical equations, provide the HSI model in a syntax that MS Access will understand. There are five basic syntax rules in EXHEP.

1. Every open parenthesis (or bracket) must have a corresponding closing parenthesis (or bracket).

2. Capitalize all letters in the variable codes.

3. Surround all variable codes with brackets (i.e., [VARIABLE]).

4. Use only the operators provided in the Model Equation/HSI Calculation Syntax window.

5. If the MIN/MAX function is used in a formula, braces ( \{\} ) are used to surround the statement and the variables themselves are separated with an ampersand $(\boldsymbol{\&})$. For example:

MIN $\{$ [VARIABLE_A] \& [VARIABLE_B] \}. 
The equation entry task is undoubtedly the hardest step to complete in the EXHEP analysis process. Although this task can be tedious and somewhat technical, a series of equation development support tools have been included in the EXHEP package to ease the process. First, EXHEP offers a keypad and list of potential variables in an interactive floating window (Figure 43).

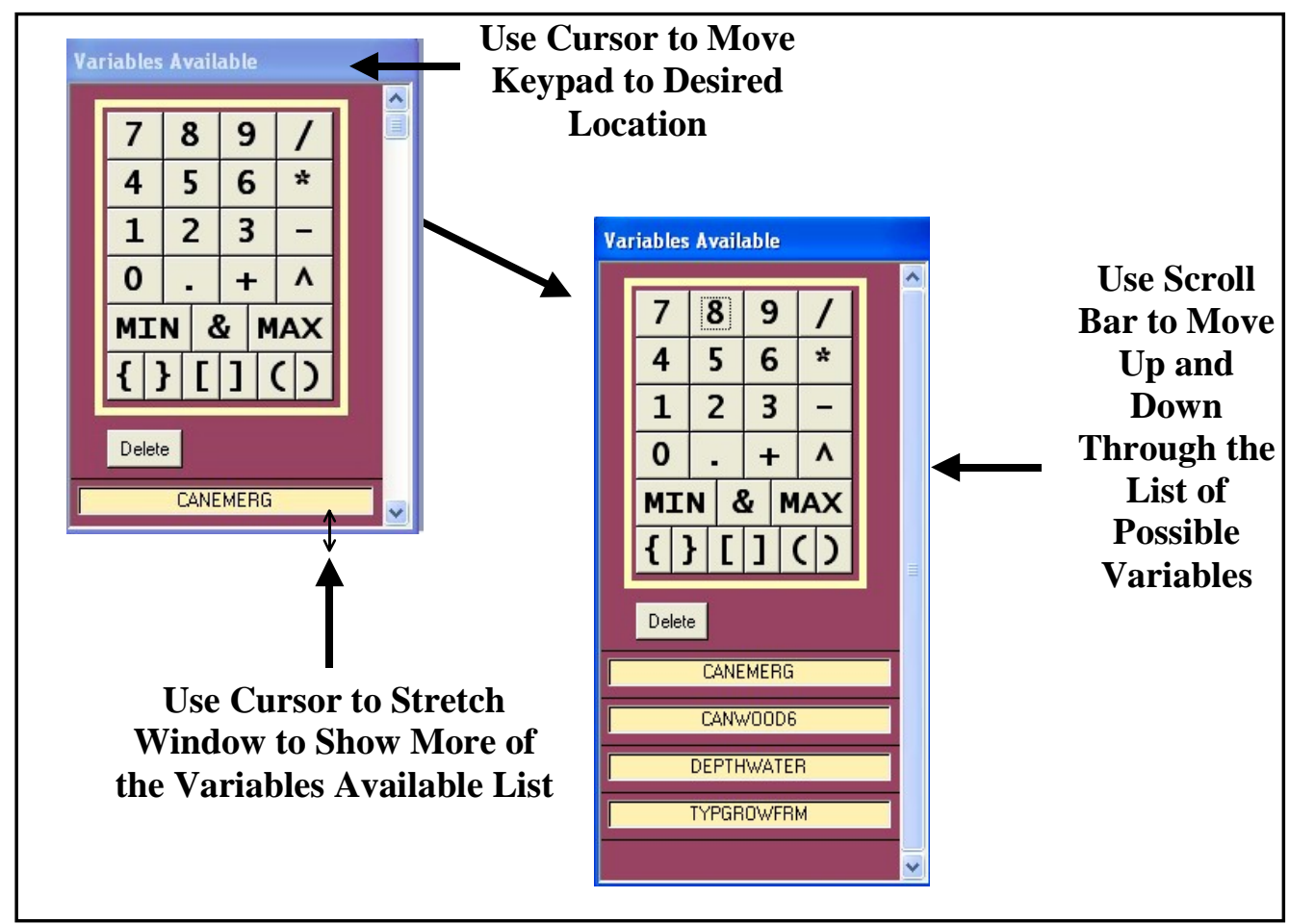

Figure 43. Customize the size and location of the floating Variables Available keypad.

By left-clicking on the banner at the top of the keypad and dragging it across the screen, the keypad can be moved anywhere on the desktop. Use the cursor to stretch the window - showing more of the available list of variables. To do this, float the cursor near the bottom of the window until it converts to a double-ended arrow. Left-click and drag the window down to the desired size. Use the scroll bar on the right-hand side of the keypad to scroll down through the variable list as well. All variables entered during the setup process are listed in alphabetical order in this list to simplify the search. If a variable is missing from this list, then it was not entered correctly in the variable setup process. To correct this problem return to Step 2 above and complete the variable setup process, then return here and find the variable in the list. 
A help window has been developed to provide assistance during the equation entry process. This window is opened by clicking the Help button in the toolbar at the top of the Model Associations screen (Figure 44).

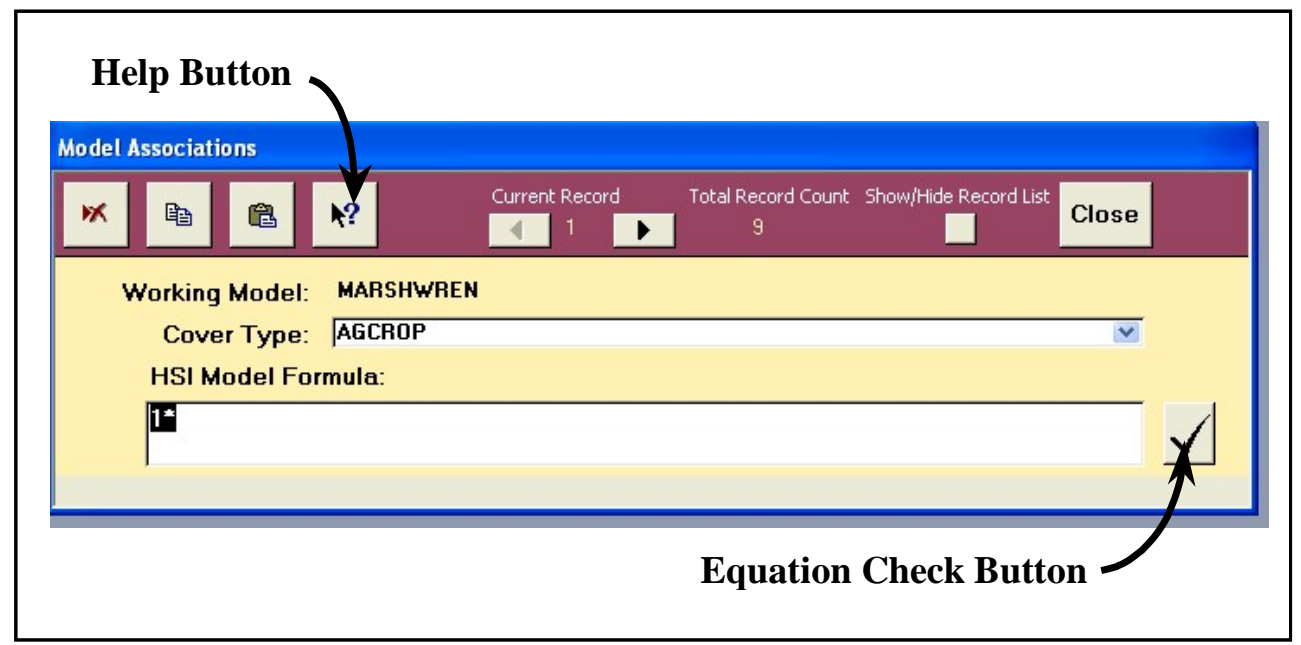

Figure 44. Help and Equation Check buttons provided to assist with HSI formula entry in EXHEP.

Once opened, a list of operands, basic equation development criteria, and several equation techniques (i.e., helpful examples) are displayed to further demonstrate proper equation syntax in EXHEP (Figure 45).

To close the Model Equation/HSI Calculation Syntax window, click on the " $\mathbf{X}$ " in the upper right-hand corner of the screen. 


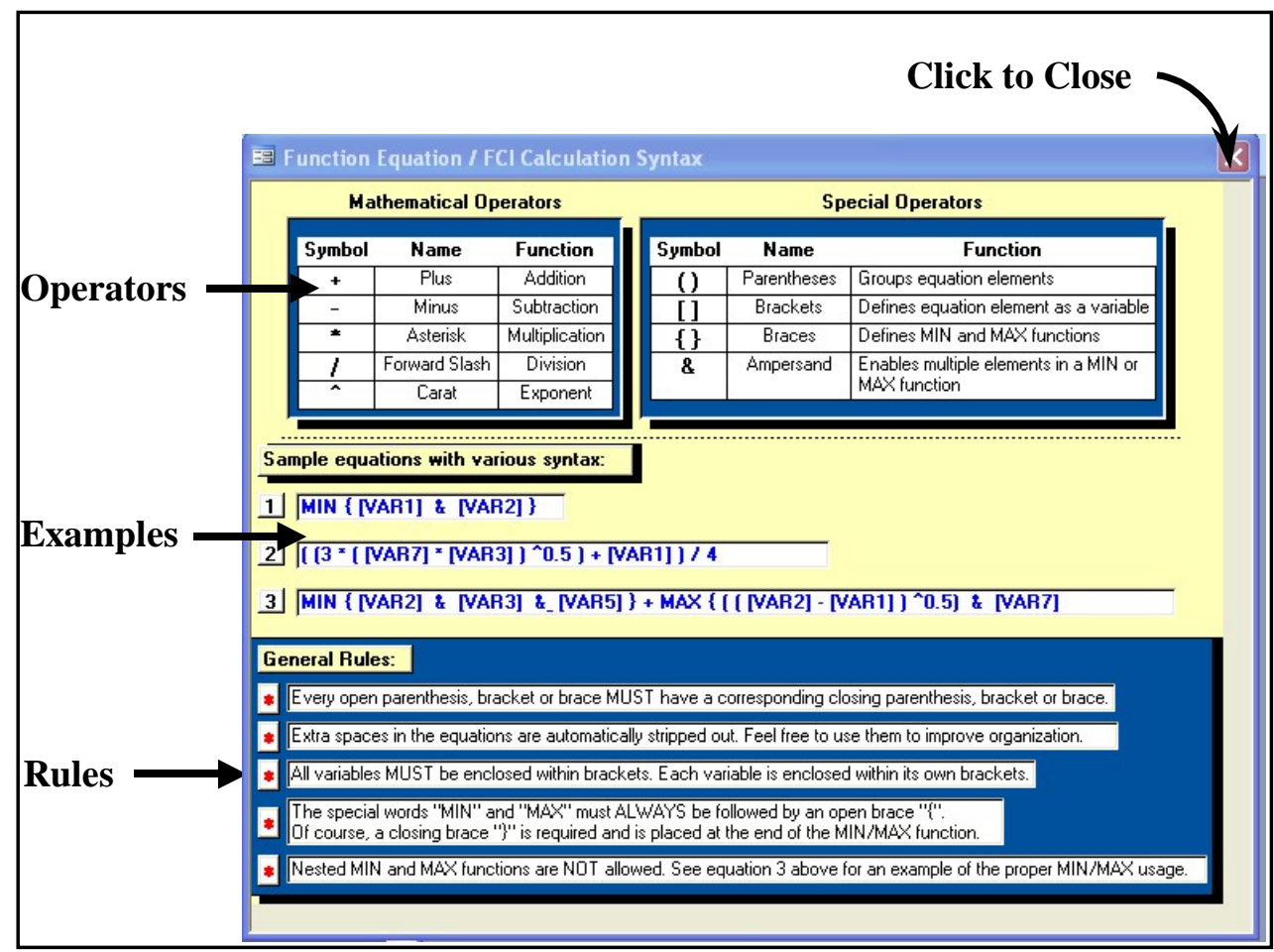

Figure 45. Model Equation/HSI Calculation Syntax window in EXHEP.

To further assist in the equation entry process, a testing button has been installed in the Model Associations screen to the immediate right of the HSI Model Formula input box, and should help verify each equation entry as it is supplied. Once the formula has been entered, click on this button and receive either confirmation that the formula is mathematically sound, or helpful suggestions to modify the formula. The syntax verification process in EXHEP is a substitution process. EXHEP verifies the

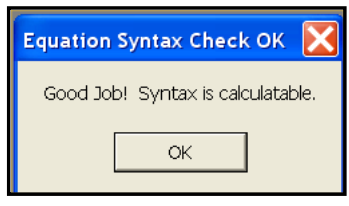
equation syntax by substituting the value of 1.0 for each variable and calculating \begin{tabular}{l} 
Invalid Equation \\
An open bracket '[' was found without a closing bracket ']'. Every variable MUST be completely enclosed. verify equation and \\
try again. \\
$\qquad \mathrm{OK}$ \\
\hline
\end{tabular} the result. If the equation is written correctly, a message box will indicate that the entry is acceptable. If there is an error in the equation's syntax, EXHEP will provide message windows with suggestions on how to correct the situation.

USER'S NOTE:

EXHEP can only review the accountability of the HSI formula entered - it cannot verify that the model is biologically correct - i.e., the software is unable to determine whether the limiting resources have been captured in the model. 
Input the variable codes

To begin the equation entry task, left-click once on the HSI Model Formula input box and place the cursor to the immediate right of the "1*" placeholder. Now move the cursor to the floating Variables Available keypad and scroll down through the list of variables. Singleclick on the variable to be entered into the formula. EXHEP automatically adds the variable code (fully capitalized) to the HSI Model Formula input box, and places "[ ]" characters around the variable's code (Figure 46).

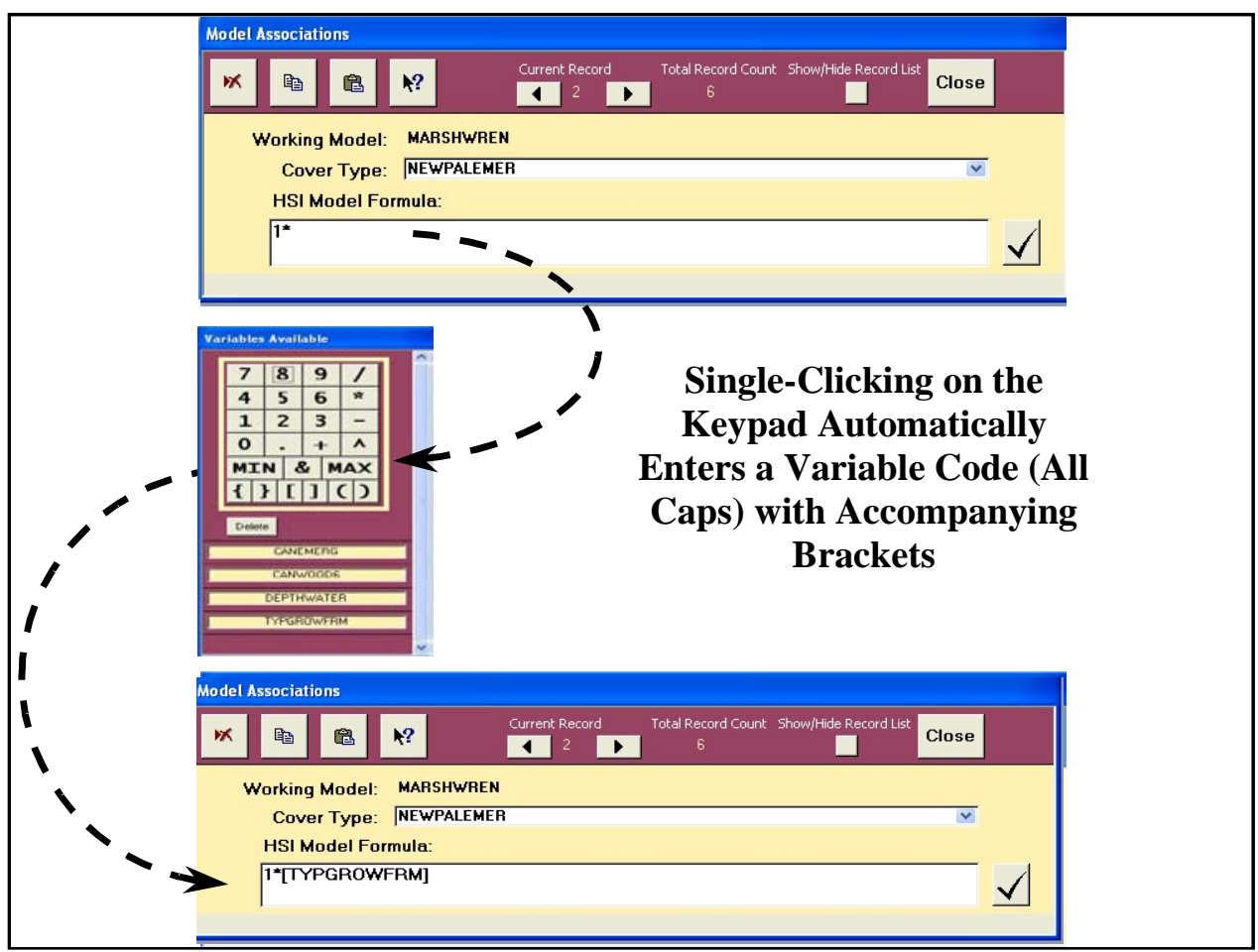

Figure 46. Use the keypad to correctly enter variable codes into the HSI Model Formula input box.

Repeat the process as often as necessary to populate the entry window with all variables required to complete the HSI algorithm for this model (Figure 47). Alternatively, formulas may be manually typed into the space provided.

Remember to fully capitalize the variable codes and place square brackets around each code. Spelling is critical - the database must be able to find the code in the list of available variables, so be sure to spell-check the entries.

\section{USER'S NOTE:}

Users who leave this screen for any reason and return to complete the formula entry process, will need to click on the HSI Model Formula before using the keypad. 


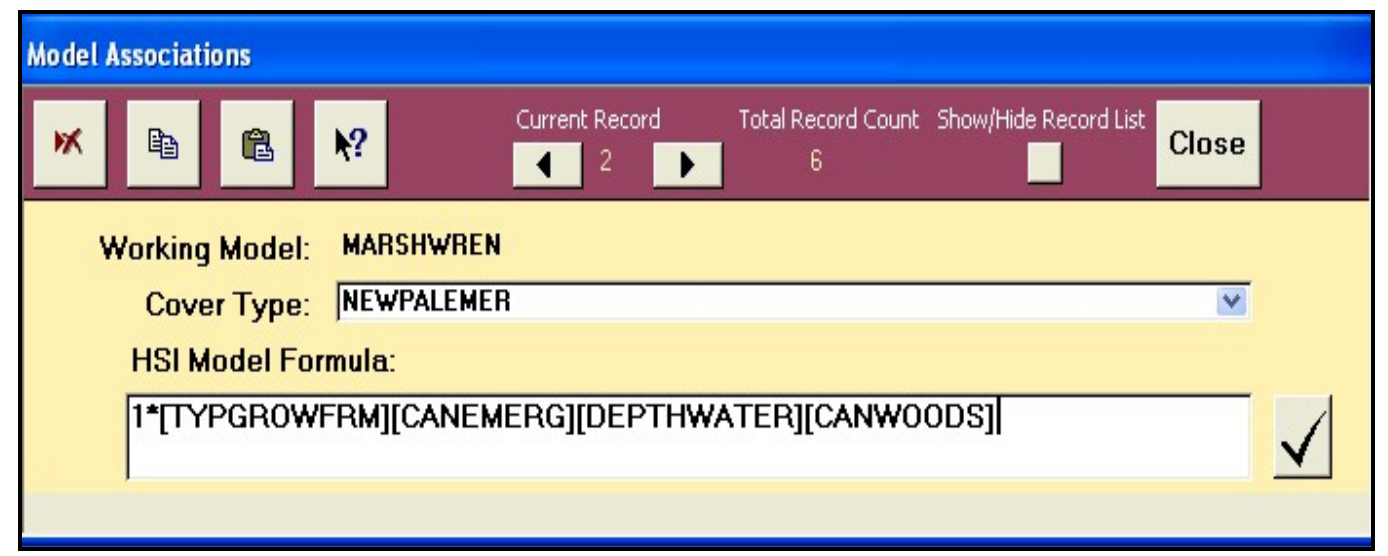

Figure 47. Begin by entering the variable codes (surrounded by brackets) in the HSI Model Formula input box.

Add the mathematical operators

Now enter the mathematical operators that combine the variables in a meaningful manner. There are five basic mathematical operators in EXHEP: 1) plus (+) for additions; 2) minus (-) for subtractions;

3) asterisks (*) for multiplications; 4) forward slashes (/) for divisions; and 5) carets $(\wedge)$ for exponential expressions. Four additional operators define special relationships inside the equation: 1) parentheses ( ( )) to group or cluster equation elements; 2) brackets ([ ]) to compartmentalize variable codes; 3) braces ( \{\} ) to distinguish minimum (or maximum) arguments; and 4 ) ampersands ( $\boldsymbol{\&}$ ) to separate the variables within the minimum (or maximum) arguments.

To proceed, systematically place the cursor between each of the variable codes in the HSI Model Formula entry box, and insert an operator using either the operator keys on the floating keypad or the keys on the keyboard (Figure 48). 


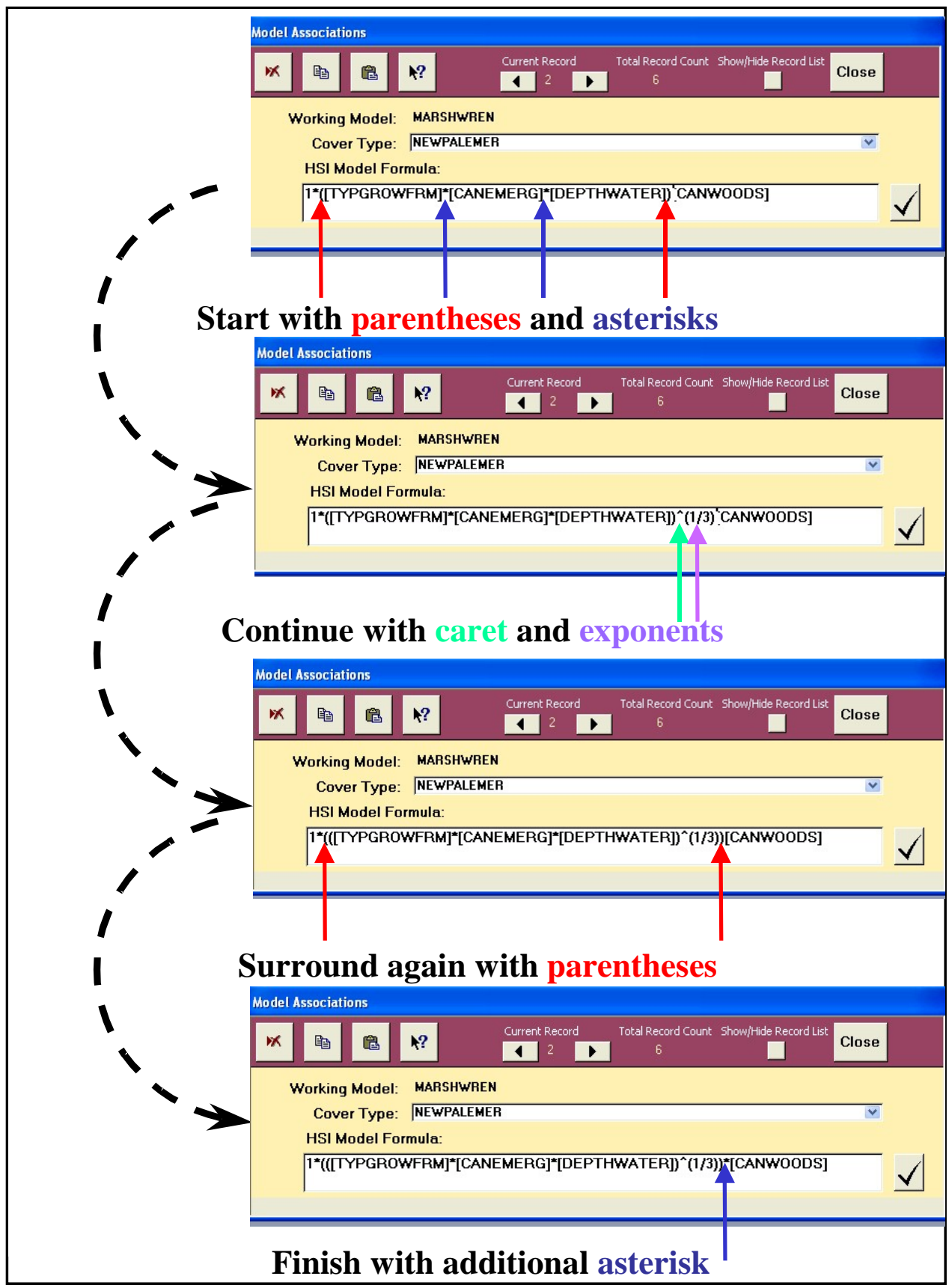

Figure 48. Example of the formula entry process in the Model Associations entry form.

At this point, it is acceptable to delete the "1*" placeholder. To do this, put the cursor between this first variable and the "1*" and backspace twice or put the cursor immediately before the " $\mathbf{1}$ " and hit the Delete button on the floating keypad (Figure 49).

\section{USER'S NOTE:}

The Delete button on the floating Variables Available keypad can be used at any time to delete errors in the formula - note that this button removes items to the immediate right of the cursor. 


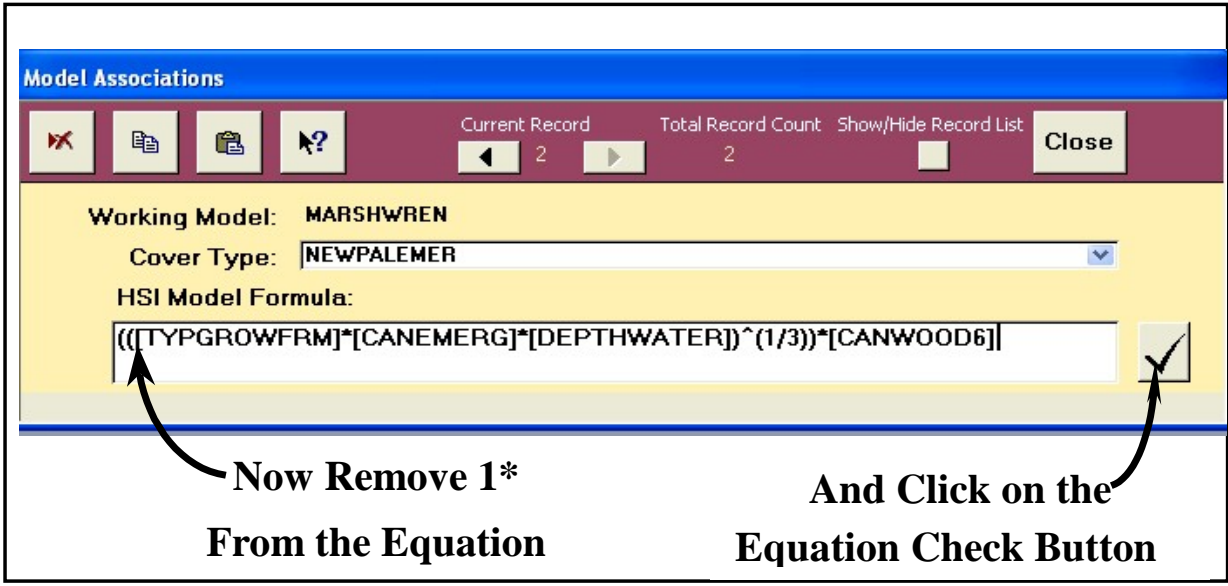

Figure 49. Completing the HSI formula entry process - removal of the $1^{*}$ placeholder and formula checking.

Check the formula

To check the formula, click on the check button immediately to the right of the HSI Model Formula input box. If the model is mathematically calculable, a confirmation will be received. If there are problems with the formula entry, the system will provide direction on the best possible solutions.

Remove any phantom records

If, as is the case in the example, the first cover type in the list of cover types is not associated with the model, EXHEP assigns the first cover type in the list a default association that must now be removed (i.e., it is considered a "phantom record"). To determine if a phantom record exists, look to the top of the Model Associations window and note the number of records showing. If only one association has been made thus far, and the record counter shows that there are two records instead, assume that a phantom record exists (Figure 50). 


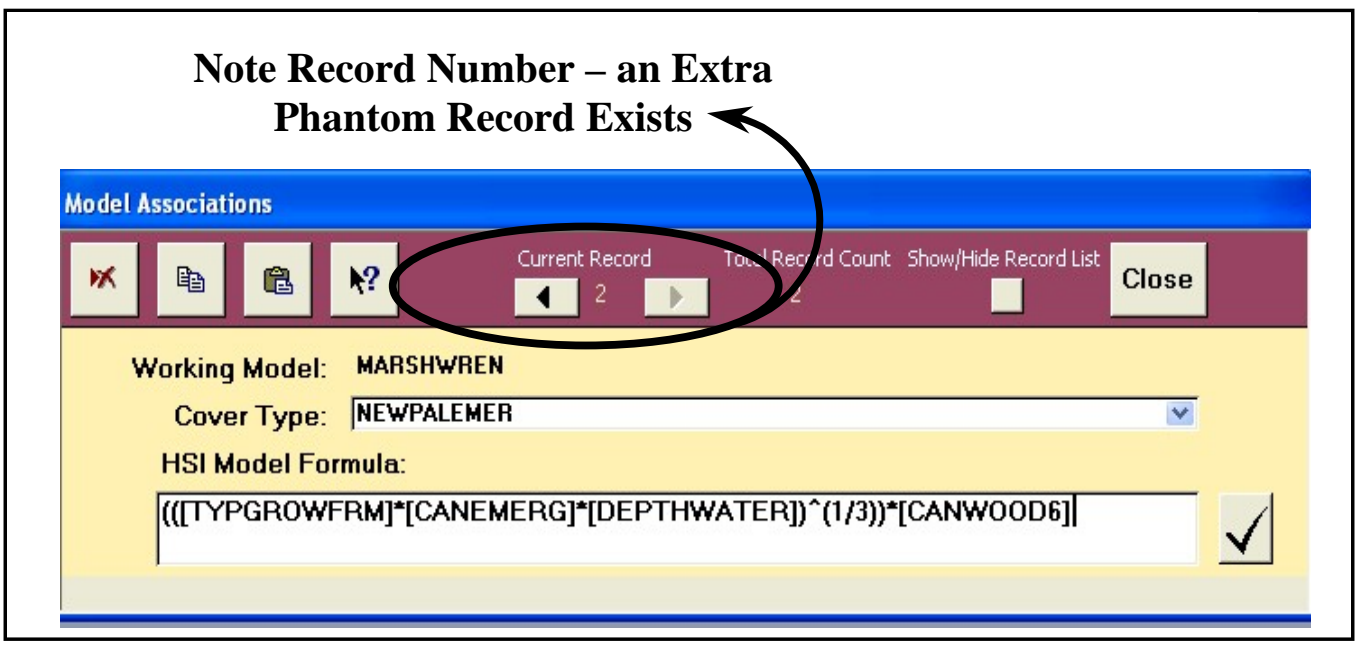

Figure 50. Extra records indicate "phantoms" that must be manually removed.

To remove this phantom record, complete the following steps: 1) click on the Back Record button, and 2) click on the Delete Record button.

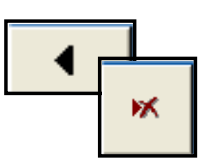

Duplicate or delete the records for multiple cover types

As a general rule, each record in the Model Associations window represents a single application of the model. Single formula models with one cover type require one record of associative information (i.e., one record represents one species with one cover type associated with it). Single formula models with multiple cover types require an individual record for each cover type associated with the model. In most instances, the same formula applies across multiple cover types in a model. EXHEP

USER'S NOTE:

A new record must be created for every cover type utilized in a model. To capture the potential for restorative or rehabilitative actions in the future condition, it is essential to associate both an existing and a newly developed cover type with each model (e.g., two records would be associated for marsh - existing and newly restored marsh). provides a copy feature in this window to expedite the formula entry process. To enter a multi-cover type model into EXHEP, follow the directions above to enter the first cover type. Now click on the Cover Type drop-down box, and choose the next cover type to associate with the model (Figure 51). 


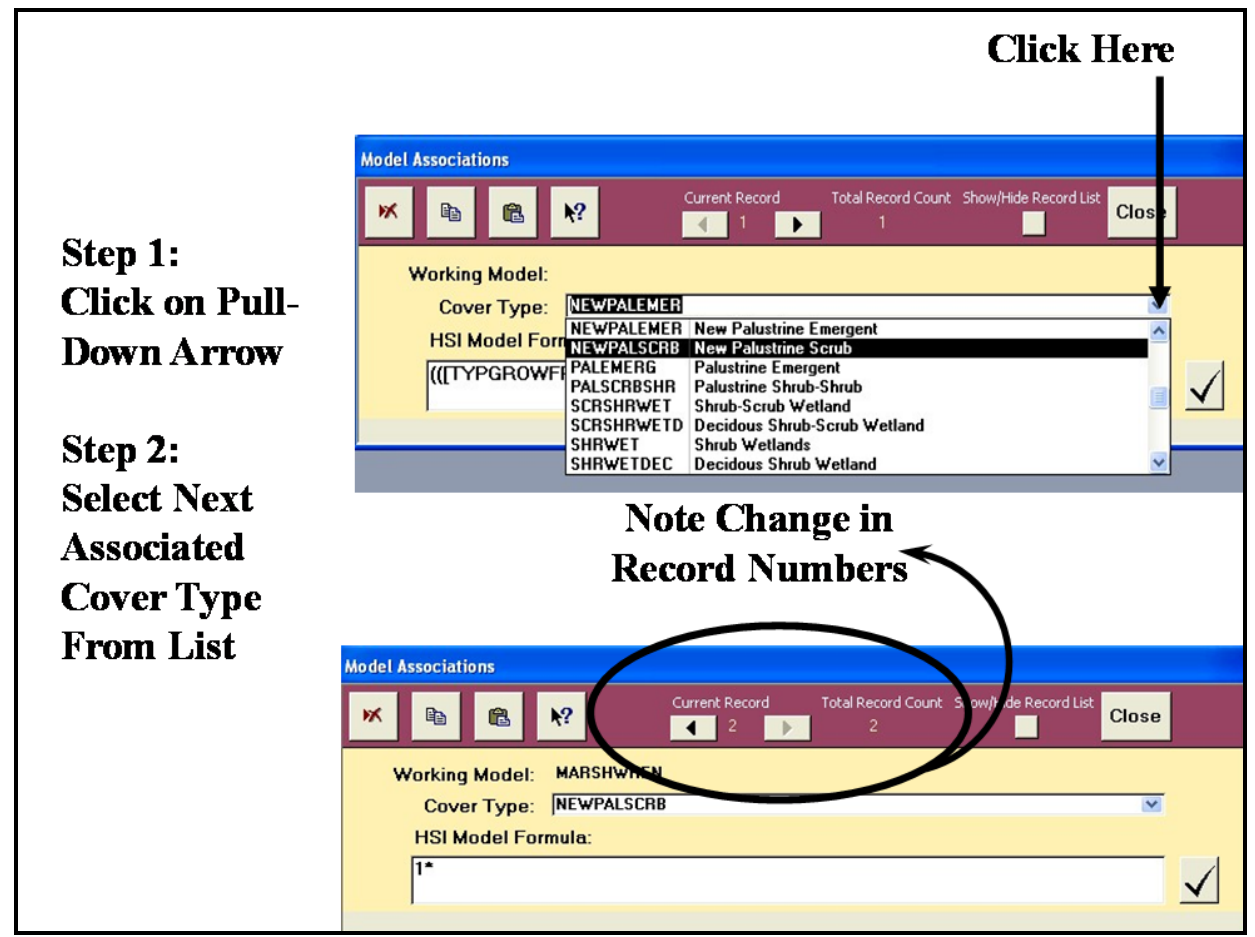

Figure 51. Select the next associated cover type from the pull-down list.

EXHEP creates a new record and assigns the cover type a formula of "1*." In stepwise fashion, complete the following quick steps:

1. Click on the Back Record button.

2. Click on the Copy button.

3. Click on the Forward Record button.

4. Click on the Paste button (Figure 52).

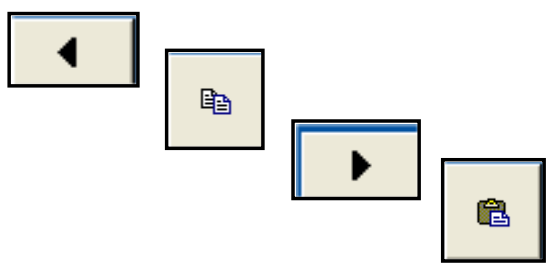




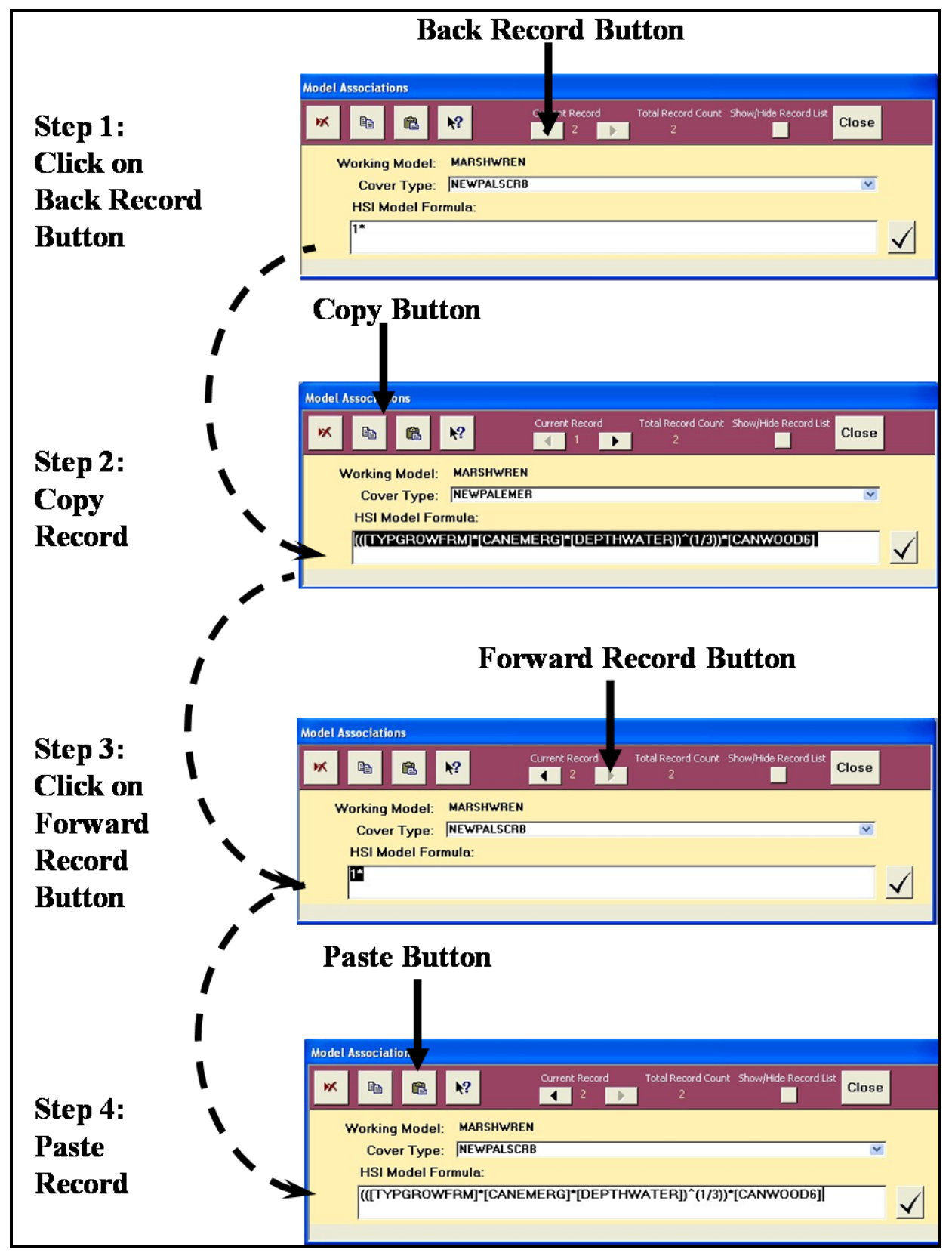

Figure 52. Make a new association by duplicating the previous data.

Repeat this process as many times as necessary to fully associate the various cover types (and HSI formulas) with the "Working Model." If at any time a model's associated information must be removed, open the
USER'S NOTE:

There is one exception to this rule - if the targeted record is the only cover type association for the model, EXHEP will not allow this deletion using this protocol. Instead, simply overwrite the HSI formula with " 1 *" and click on the Close button. This will remove the one and only association for the model.

Model Associations window, left-click once on the HSI Model Formula entry box, and click on the Delete button. 
Reviewing the associations

To review the list of associated records entered thus far, click on the Show/ Hide Records List toggle button. In response, EXHEP opens the listing window (Figure 53).

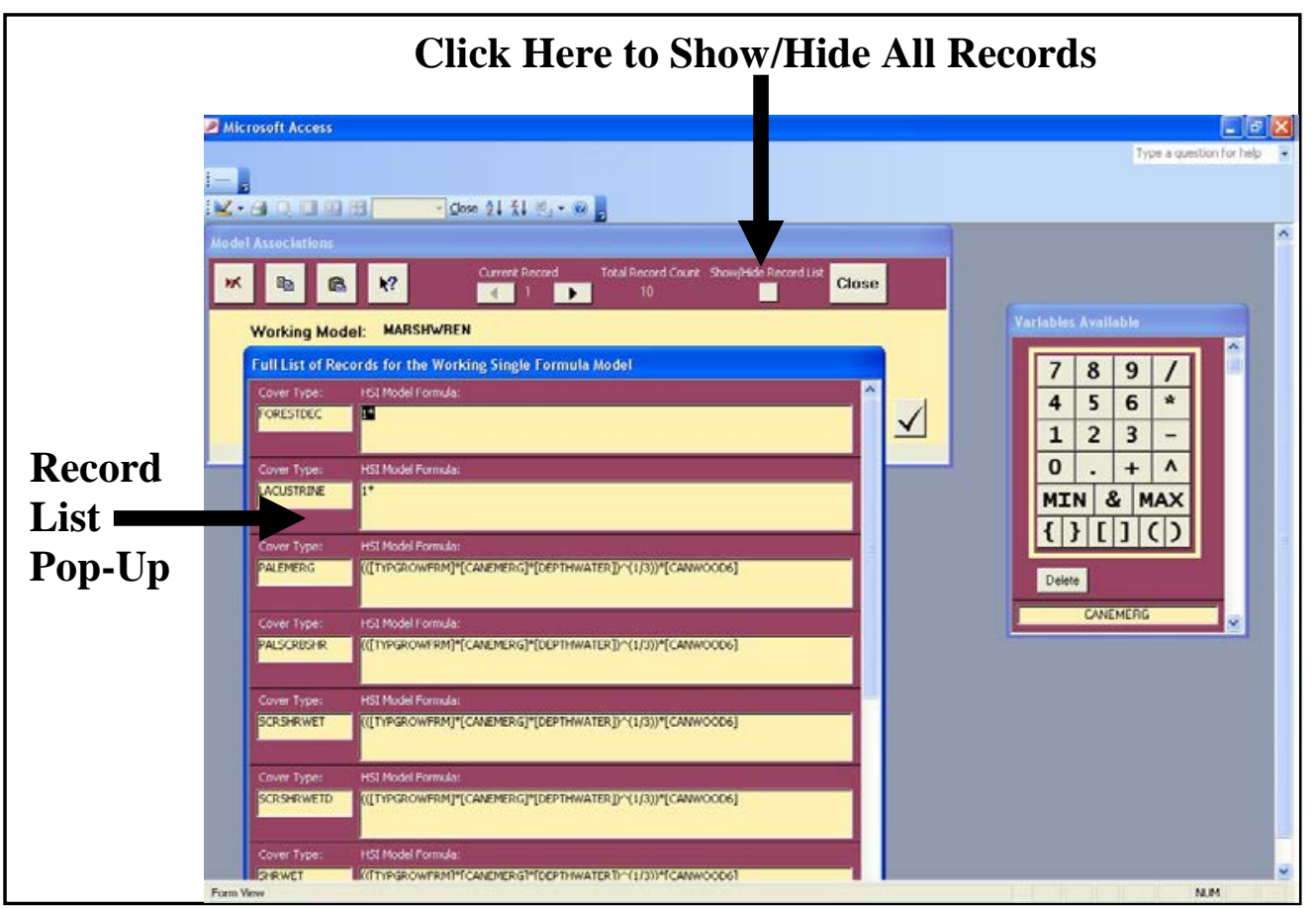

Figure 53. Show or hide all associated records simultaneously.

Scroll down this list with the scroll bar on the far right-hand side of this window. To close the window, click the toggle button a second time. When finished with the associations, click on the Close button in the upper right-hand corner of the toolbar and EXHEP returns to the primary interface. Repeat Step 3 (i.e., Model Association) for each "Working Model" in the analysis.

\section{Additional association actions for multiple formula models}

If multiple formula models are called for in the analysis, the model associations must be completed using the second set of buttons on EXHEP's primary interface (Figure 54). 


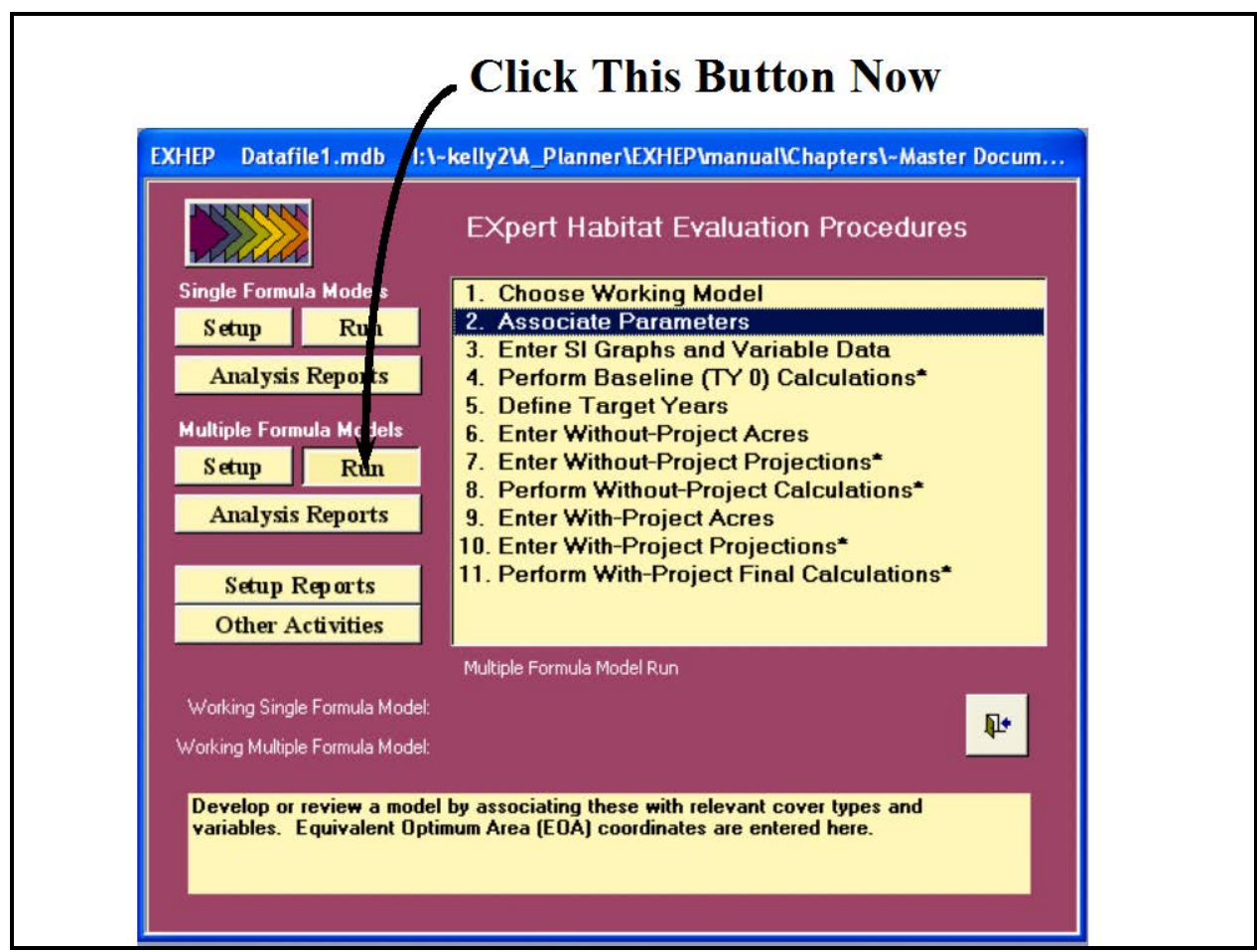

Figure 54. Click on the Run button to begin the multiple formula model association process in EXHEP.

A screen appears in the center of the primary interface with 11 options:

\section{Choose Working Model}

2. Associate Parameters

3. Enter SI Graphs and Variable Data

4. Perform Baseline (TY 0) Calculations*

5. Define Target Years

6. Enter Without-Project Acres

7. Enter Without-Project Projections*

8. Perform Without-Project Calculations*

9. Enter With-Project Acres

10. Enter With-Project Projections*

11. Perform With-Project Final Calculations* 
Again, in systematic fashion, start at the top of the options list, click on each option, and provide the data requested in each interface screen. EXHEP uses the same process to associate the multiple formula models as it did in the single formula model example, with one exception - it now asks for life requisite information. For more details see the sections below.

Choose the Working Model

To identify a "Working Model," click on the first option (Choose Working Model) - the following window opens (Figure 55).

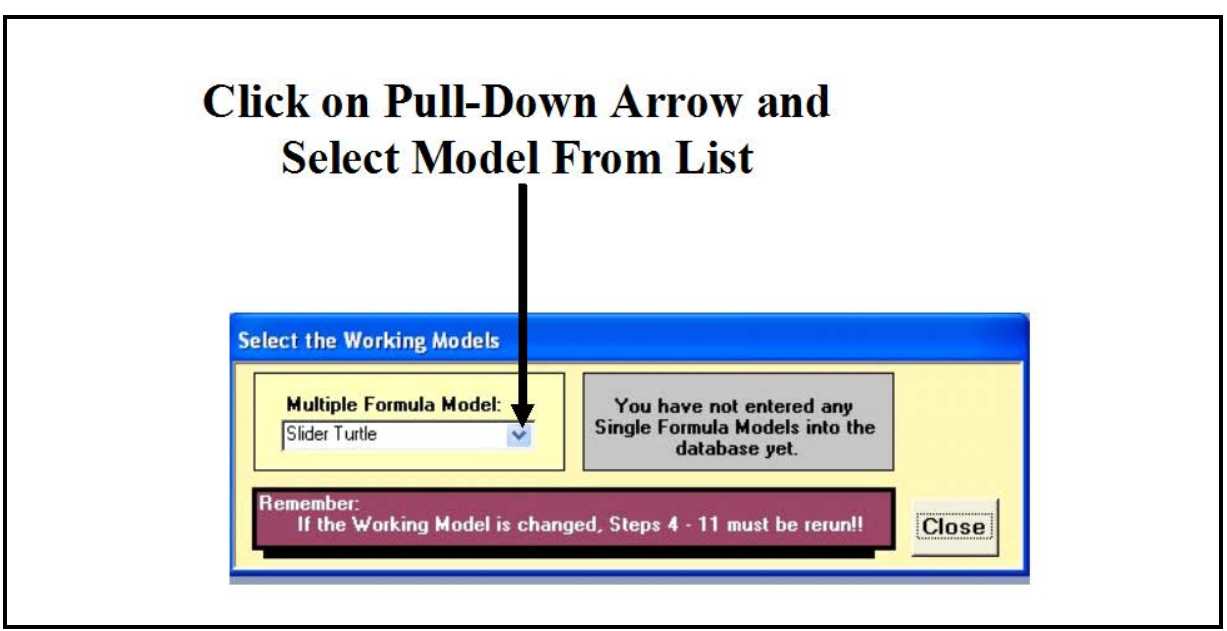

Figure 55. Select a working model from the Multiple Formula Model pull-down list.

Select the model from the pull-down list in the left-hand box on this screen and click Close. EXHEP returns to the primary interface. At the bottom of this screen, the model is now identified as the "Working Model" (Figure 56). 


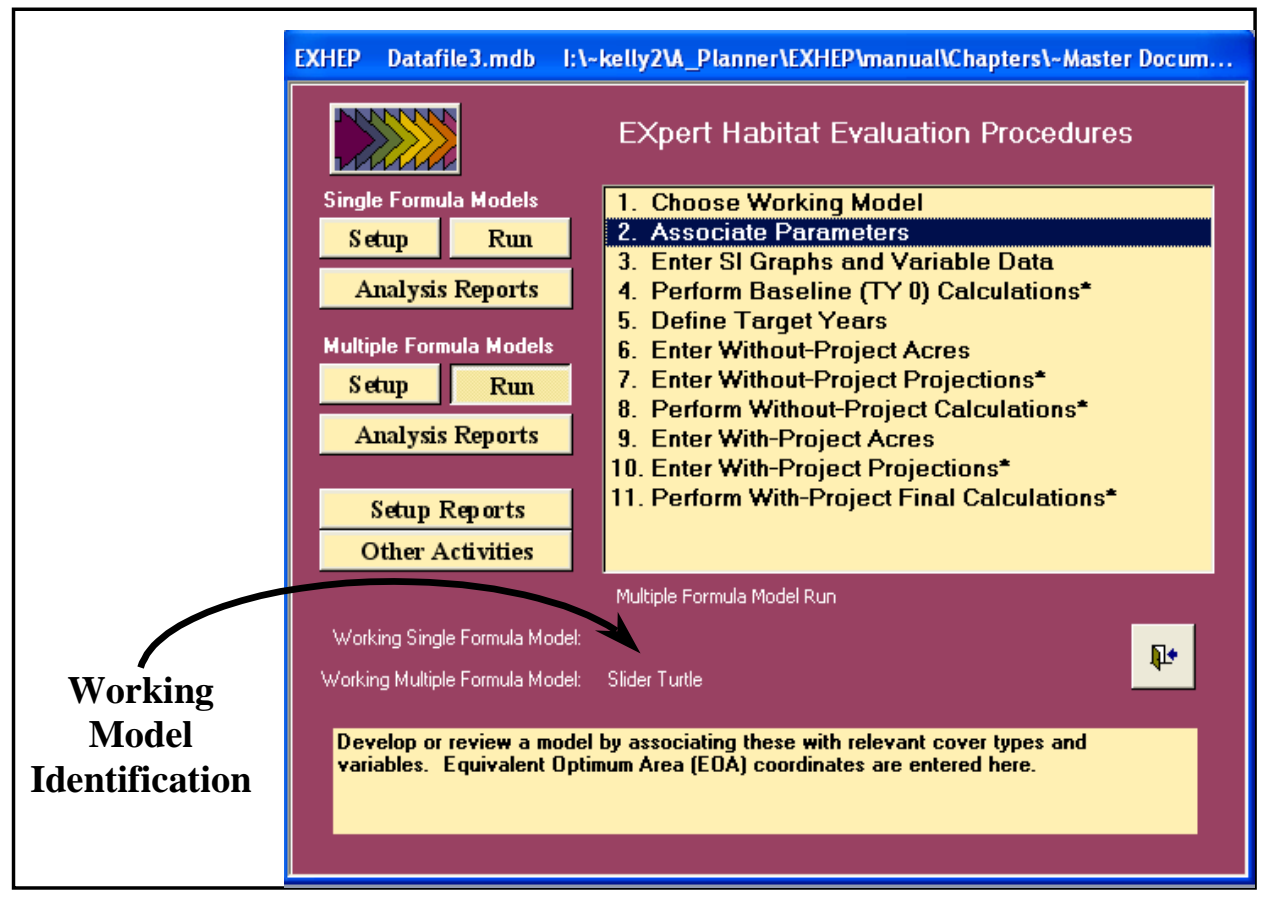

Figure 56. Identification of the Multiple Formula Working Model on EXHEP's primary interface.

Now click on the second option (Associate Parameters) and the following windows will open (Figure 57).

The Model Associations window and the Variables Available keypad are similar to the single formula model's interfaces presented earlier. A second keypad (LR Codes Available) is provided to assist with life requisite associations. Note that three

USER'S NOTE:

The Delete button on the floating LR Codes Available keypad can be used at any time to delete errors in the LRSI formula - note that it removes items to the immediate right of the cursor. additional entry fields have been created in the Model Associations window - a pull-down menu for life requisite identification (directly below the Working Model field); a second formula input window just below the Cover Type field (one is provided for the HSI Model Formula, and one for the LRSI Model Formula); and an EOA Graph Coordinates $\mathbf{X}, \mathbf{Y}$ input field (at the bottom of the window) (Figure 58). 


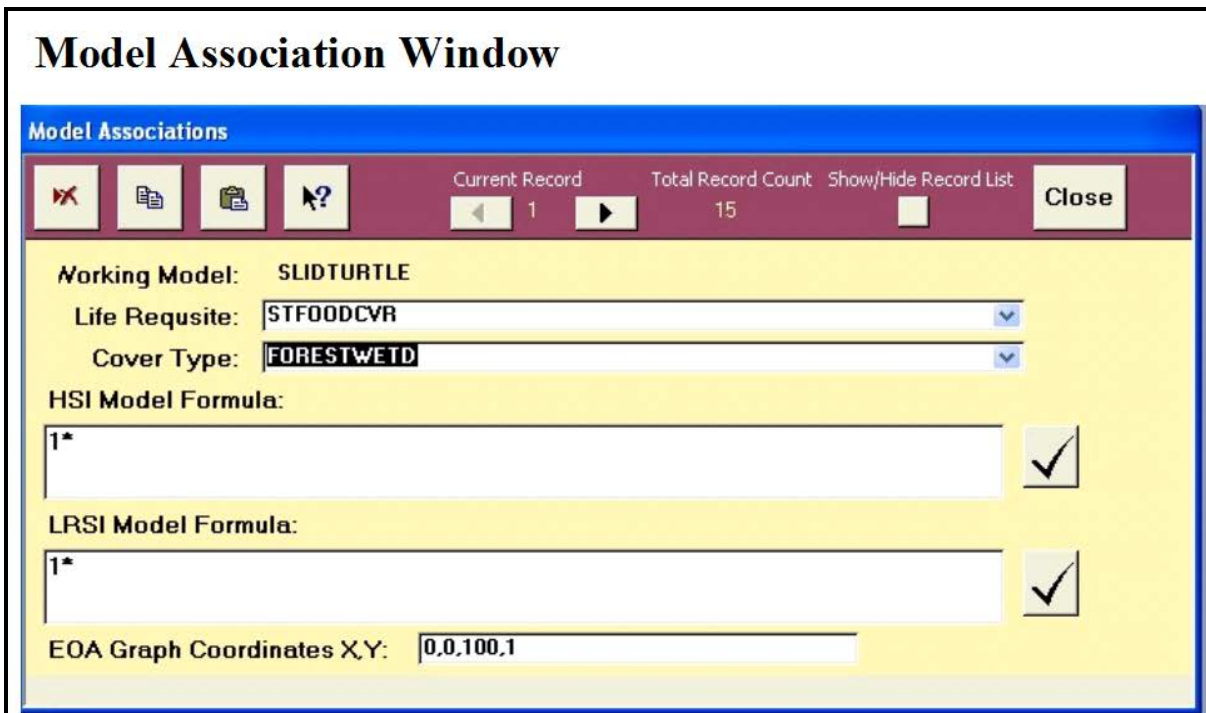

Variables Available Keypad

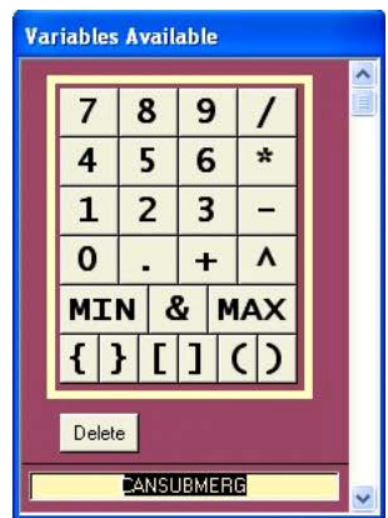

LR Codes Available Keypad

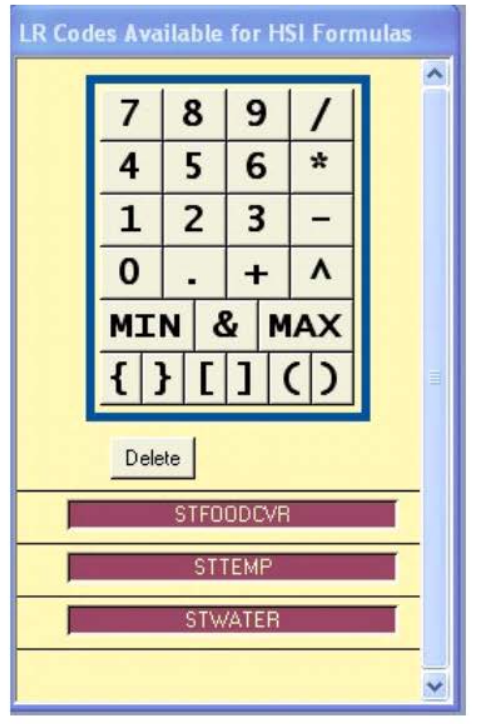

Figure 57. Model Associations window and keypads for multiple formula models in EXHEP. 


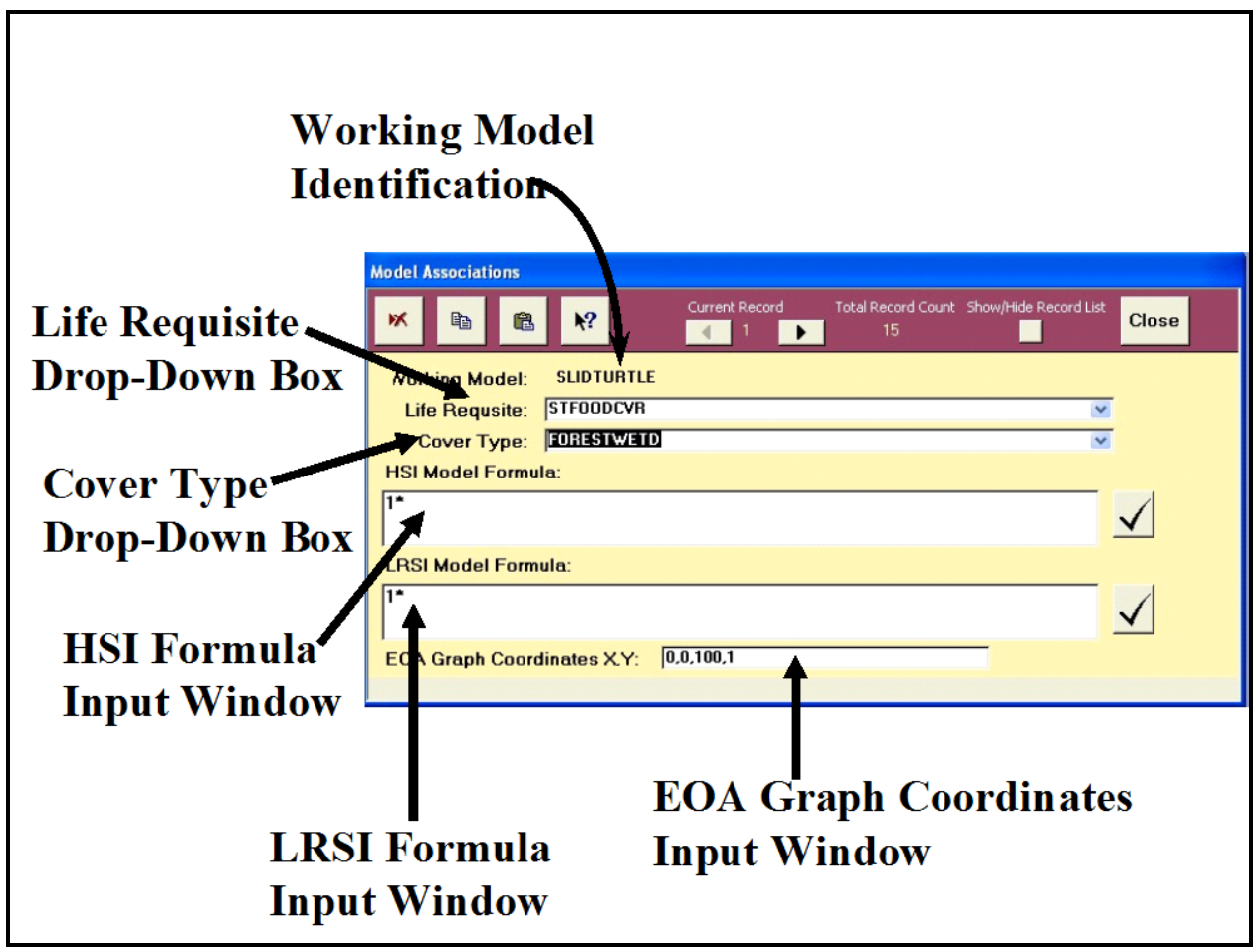

Figure 58. Model Associations entry screen for multiple formula models.

Model association for multiple formula models is a five-step process: 1) select a life requisite, 2) select a corresponding cover type, 3) enter the HSI formula for the model, 4) enter the LRSI formula for the model, and 5) provide the EOA graph coordinates in X,Y syntax. For

\section{USER'S NOTE:}

If a life requisite is not on this list, return to the setup process and enter the missing life requisite, as described above. The newly added life requisite will appear in this floating window (alphabetically) upon returning to the Model Associations screen.

details regarding these steps, read below.

Select a life requisite-cover type combination

To begin, click on the Life Requisite drop-down box, and choose the first life requisite to associate with the model (Figure 59).

Now click on the Cover Type drop-down box, and choose the first cover type to associate with the life requisite in the model (Figure 60). 


\section{Click on Pull-Down Arrow and Select a Life Requisite From the List}

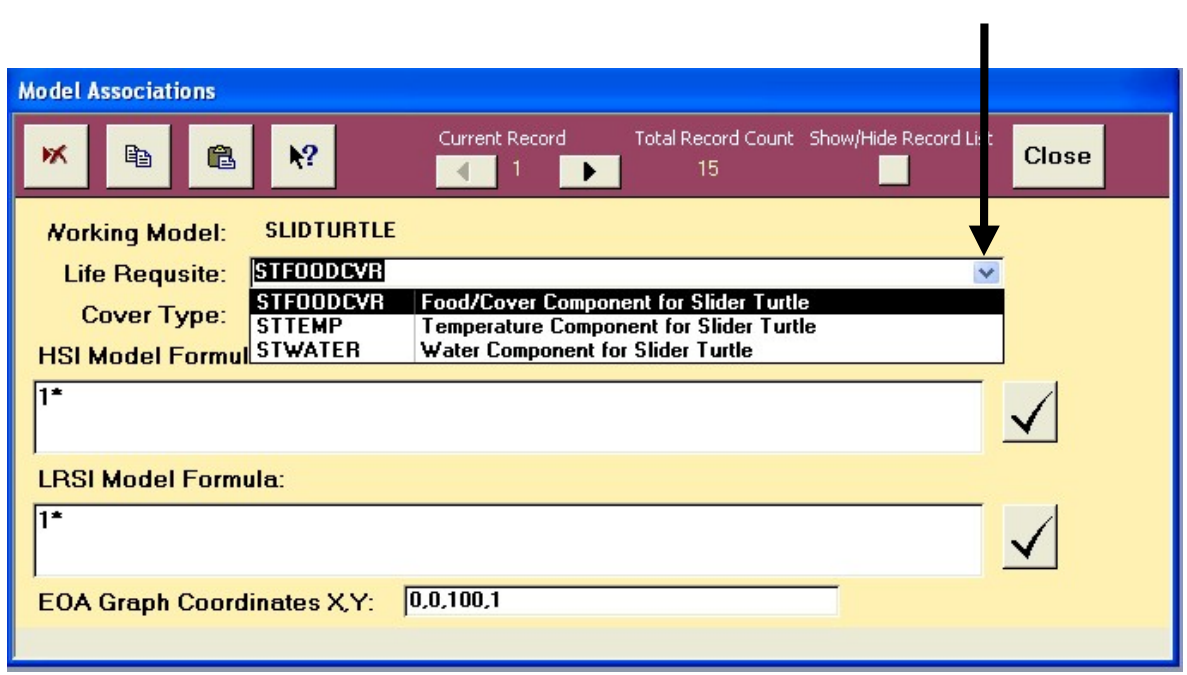

Figure 59. Select a life requisite from the pull-down list.

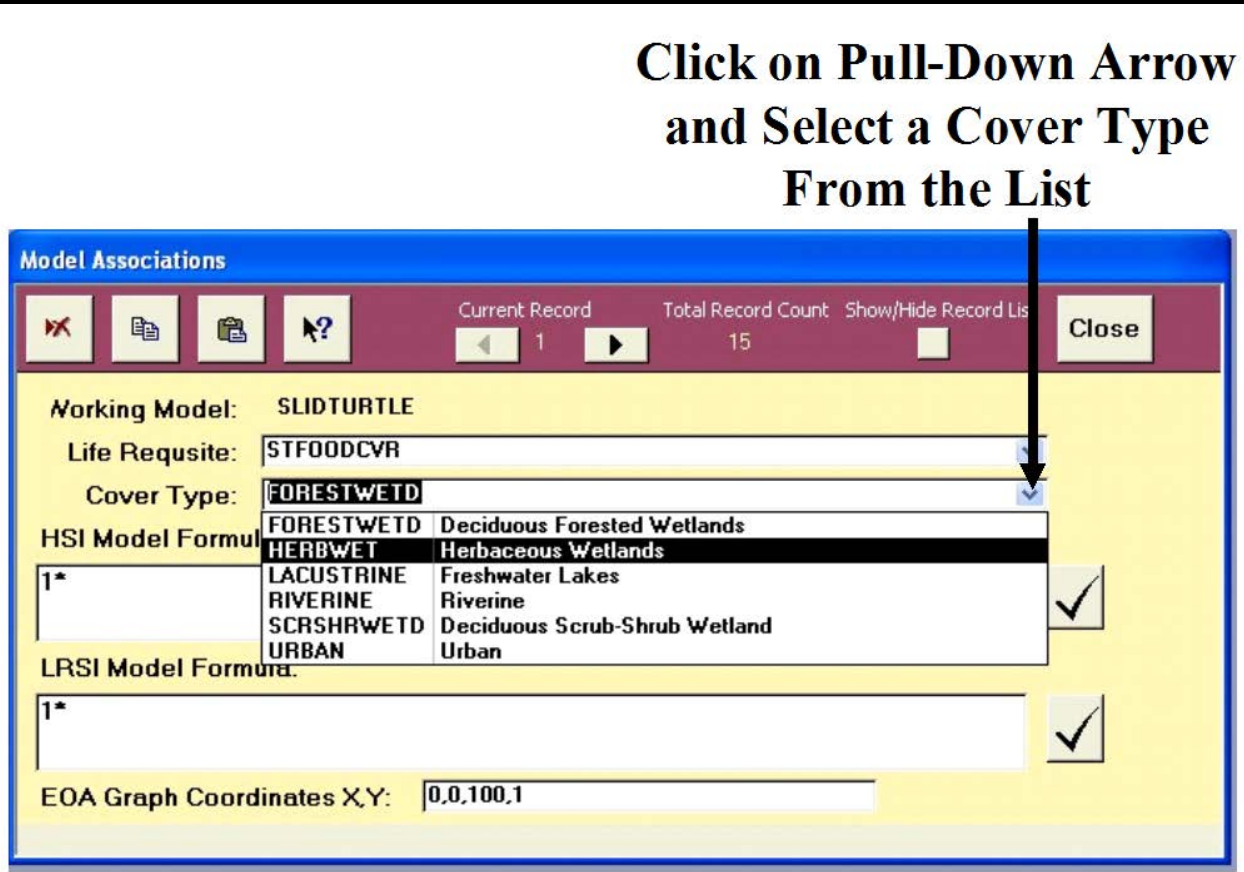

Figure 60. Select a cover type from the pull-down list. 


\section{Enter an HSI formula}

Left-click once on the HSI Model Formula input box. Define the combination of life requisites that together formulate the HSI model for the ecosystem (emulating the single formula models association process above), but this time use the LR Codes Available keypad to generate codes for the formula (Figure 61).

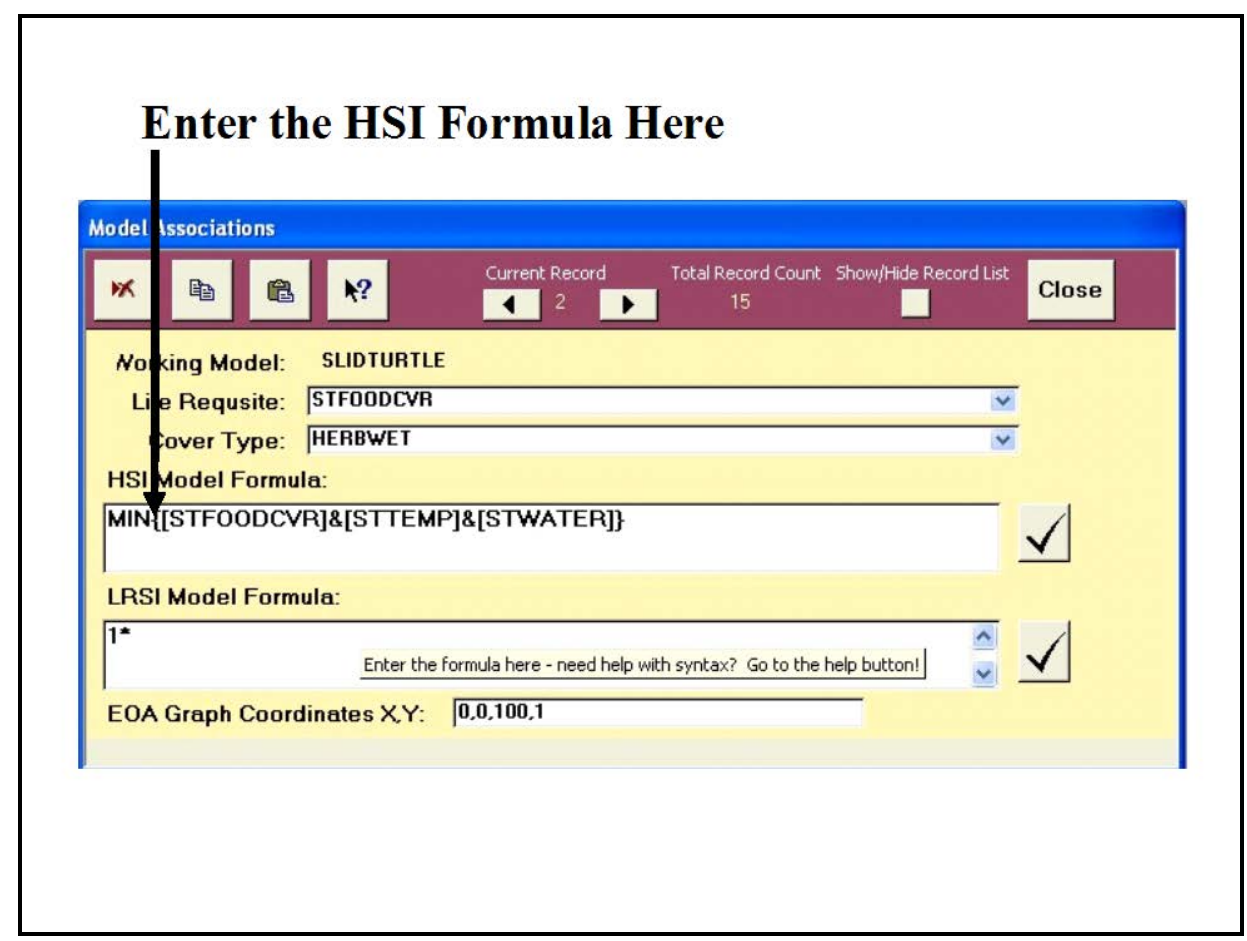

Figure 61. Enter an HSI formula in the space provided using the $L R$ Codes Available keypad.

First add the life requisite codes using the keypad - they must be capitalized and surrounded with brackets (e.g., [LIFEREQUISITE]). Then insert separation operators (parentheses) and math operators (e.g., $+,-, /, *, \uparrow)$. Finish with Minimum/ Maximum functions (e.g., MIN or MAX), and place the ampersands between these arguments. Be sure to check the work using the Help and Equation Check buttons to assure that the formula is calculable.

\section{Enter an LRSI formula}

Left-click once on the LRSI Model Formula input box. Define the combination of variables that together formulate the LRSI model for the model, but this time use the Variables Available keypad (Figure 62). 


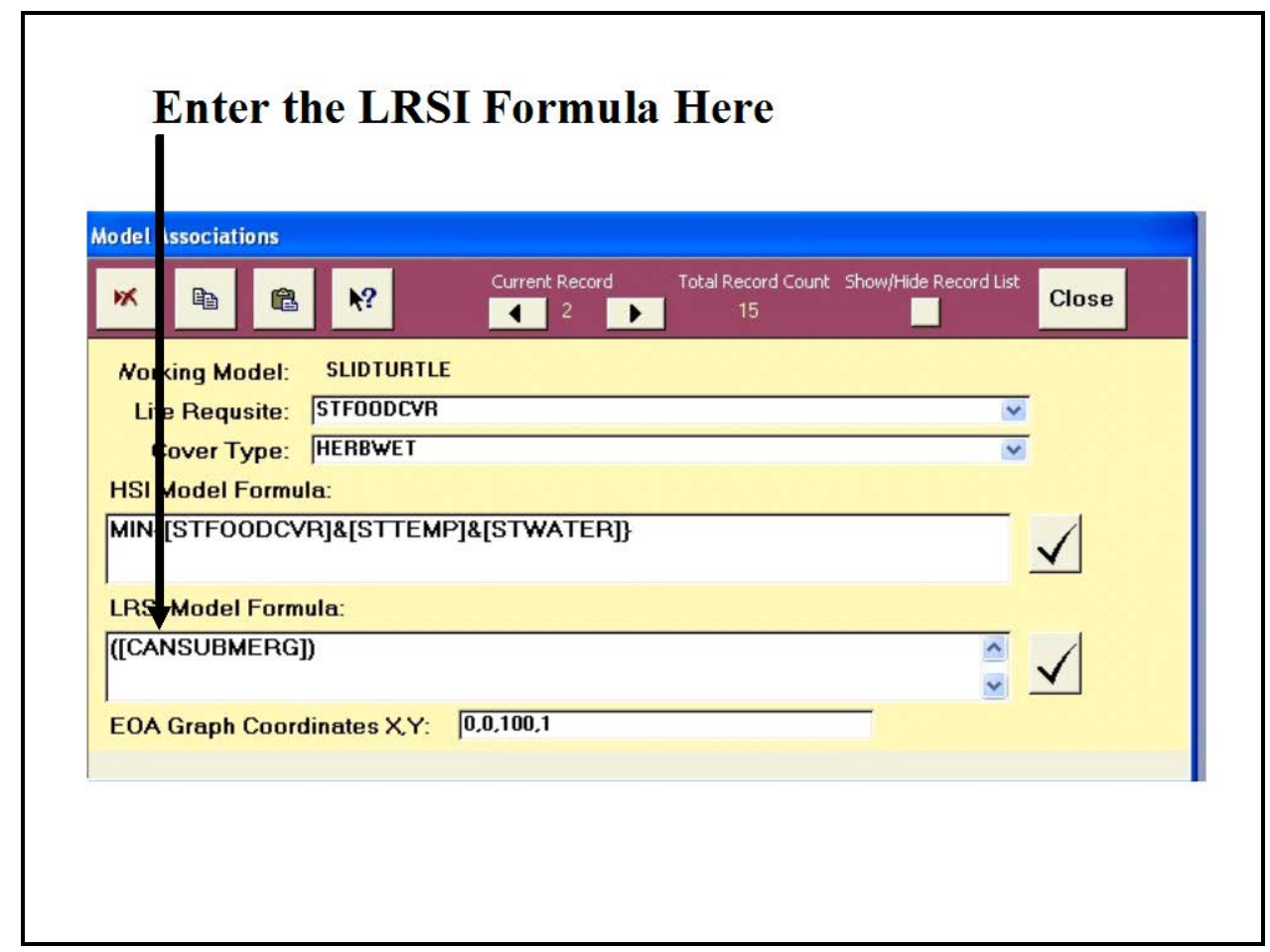

Figure 62. Enter an LRSI formula in the space provided using the Variables Available keypad.

Add the variable codes using the keypad - they must be capitalized and surrounded with brackets (e.g., [VARIABLE]). Then insert separation operators (parentheses) and math operators (e.g., +, -, / , *, ^). Finish with Minimum/ Maximum functions (e.g., MIN or MAX), and place the ampersands between arguments. Now Remove the "1*" placeholder. Be sure to check the formula again using the Help and Equation Check buttons to assure that the formula is calculable.

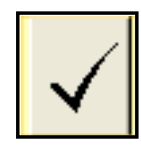

\section{Provide the EOA graph coordinates}

In introducing multiple formula models in previous sections of this guide, the importance of capturing landscape level conditions that could affect the overall Life Requisite Suitability Index (LRSI) score was noted (e.g., by weighting these values when less than optimum conditions exist). For purposes of the evaluation, this can be accomplished by entering an Equivalent Optimum Area (EOA) weighting factor into the system. An example of an EOA has been presented in graphical format below (Figure 63), and must be provided to EXHEP using appropriate graph coordinate syntax. 


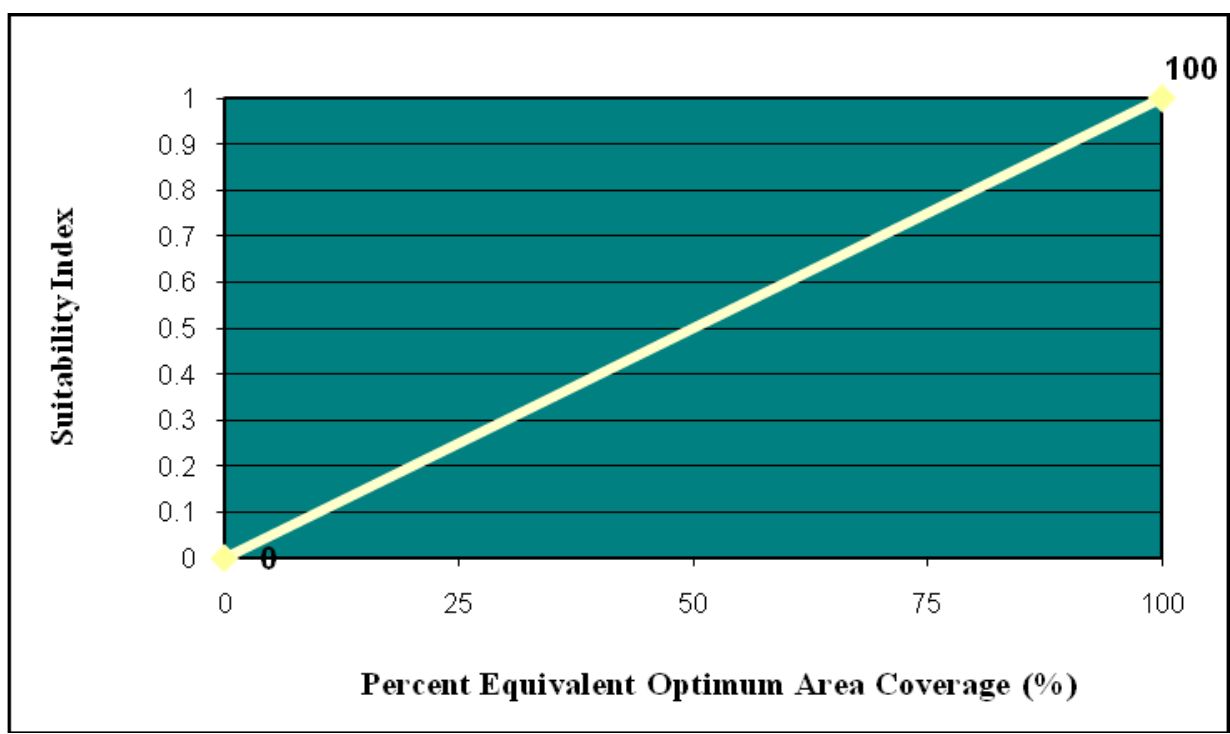

Figure 63. An example of an Equivalent Optimum Area (EOA) curve taken from a multiple formula model.

The correct syntax is: X1 , Y1 , X2 , Y2 , X3 , Y3, .... . Xn , Yn. Given the example curve's coordinates:

$\begin{array}{cc}\text { X-Values } & \text { Y-Values } \\ 0 & 0 \\ 100 & 1\end{array}$

And the EOA coordinates in EXHEP-sensitive syntax would be:

$$
\mathbf{0 , 0 , 1 0 0 , 1}
$$

Left-click on the EOA Graph

Coordinates X,Y input box in the Model Associations window and enter the correct EOA coordinates for the model (Figure 64).

\section{USER'S NOTE:}

The majority of HSI models use these same EOA coordinates to capture the landscape conditions. EXHEP defaults to this coordinate system $(0,0,100,1)$. Feel free to overwrite these values with EOAs from the models in the current assessment. If the model documentation does not provide EOA graph coordinates, these values should be left as a default. 


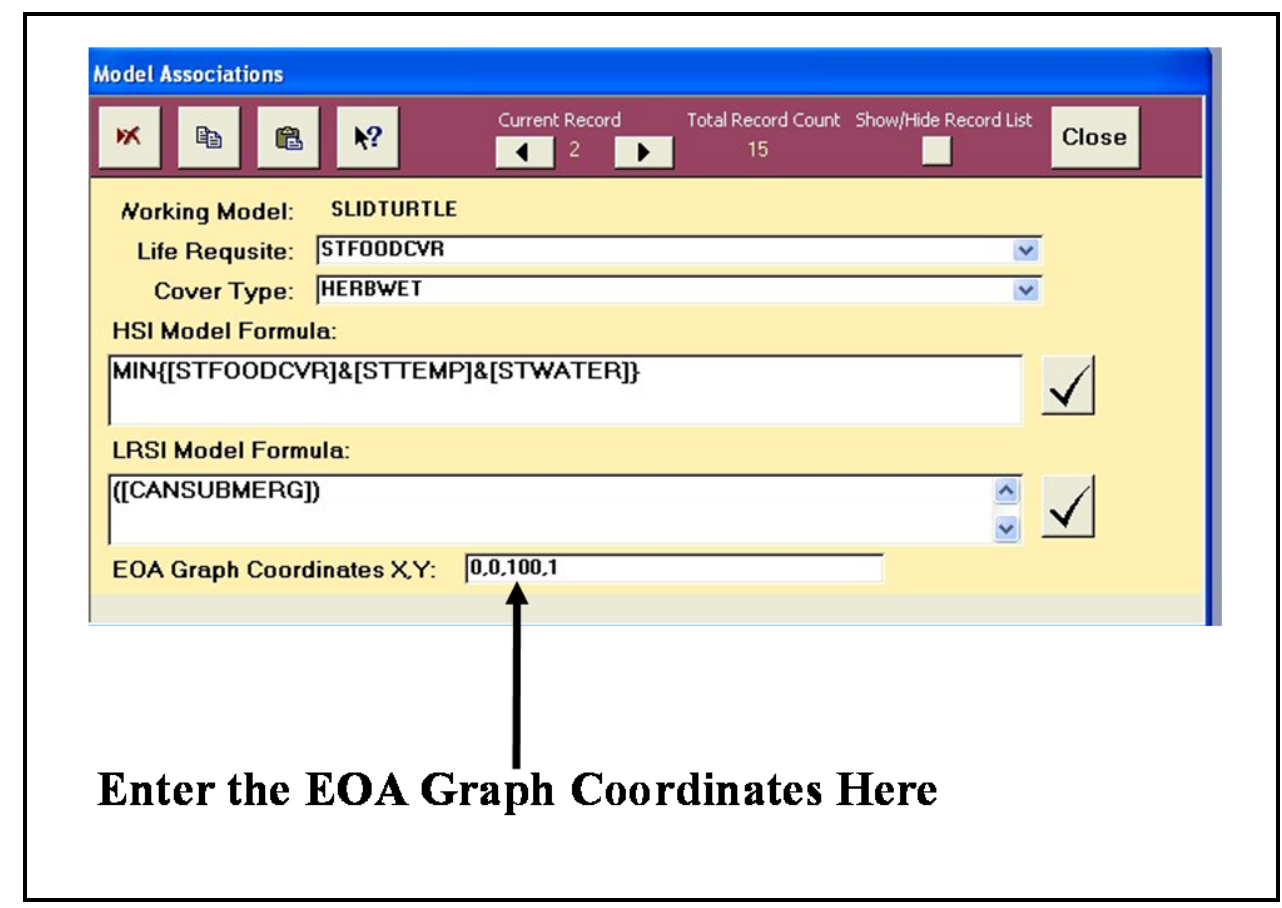

Figure 64. Enter the EOA graph coordinates in the space provided.

Remove any phantom records

Again, check the record numbers at the top of the screen to determine whether phantom records exist (Figure 65).

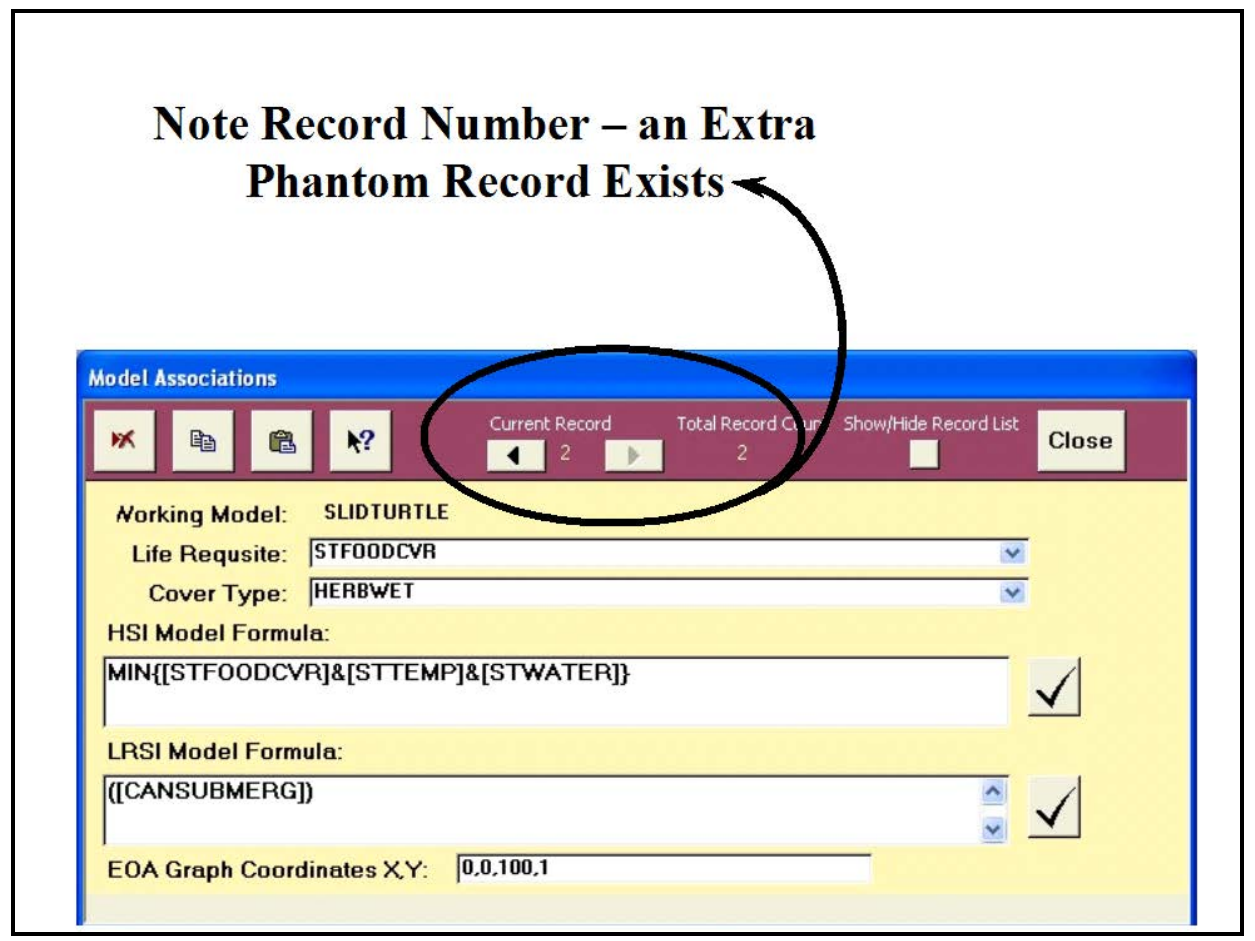

Figure 65. Extra records indicate "phantoms" that must be manually removed. 
To remove this phantom record, complete the following steps: 1) click on the Back Record button, and 2) click on the Delete Record button.

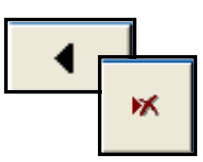

Duplicate or delete the records for multiple cover types and life requisites

As a general rule, each record in the Model Associations window represents a single application. Multiple formula models with one cover type require one record of associative information for each life requisite in the model. Multiple formula models with multiple cover types require individual records for every unique combination of life requisite and cover type. The same HSI formula applies to all life requisites, but there could be different LRSI formulas, and these could vary by cover type. Follow the directions above to associate the USER'S NOTE:

A new record must be created for every cover type-life requisite combination utilized in a model. To capture the potential for restorative or rehabilitative actions in the future condition, it is essential that the user associates both an existing and a newly developed cover type for each model (e.g., two records would be associated for marsh - existing and newly restored marsh).

first life requisite, cover type, and formulas in the Model Associations screen. Complete one life requisite at a time. Now click on the Cover Type drop-down box, and choose the next cover type to associate with this life requisite (Figure 66).

EXHEP creates a new record and assigns the cover type HSI and LRSI formulas a "1*" placeholder. In stepwise fashion, complete the following quick steps:

1. Click on the Back Record button.

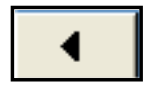

2. Highlight the LRSI Formula and click on the Copy button.

3. Click on the Forward Record button.

4. Click on the Paste button (Figure 67).

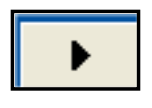




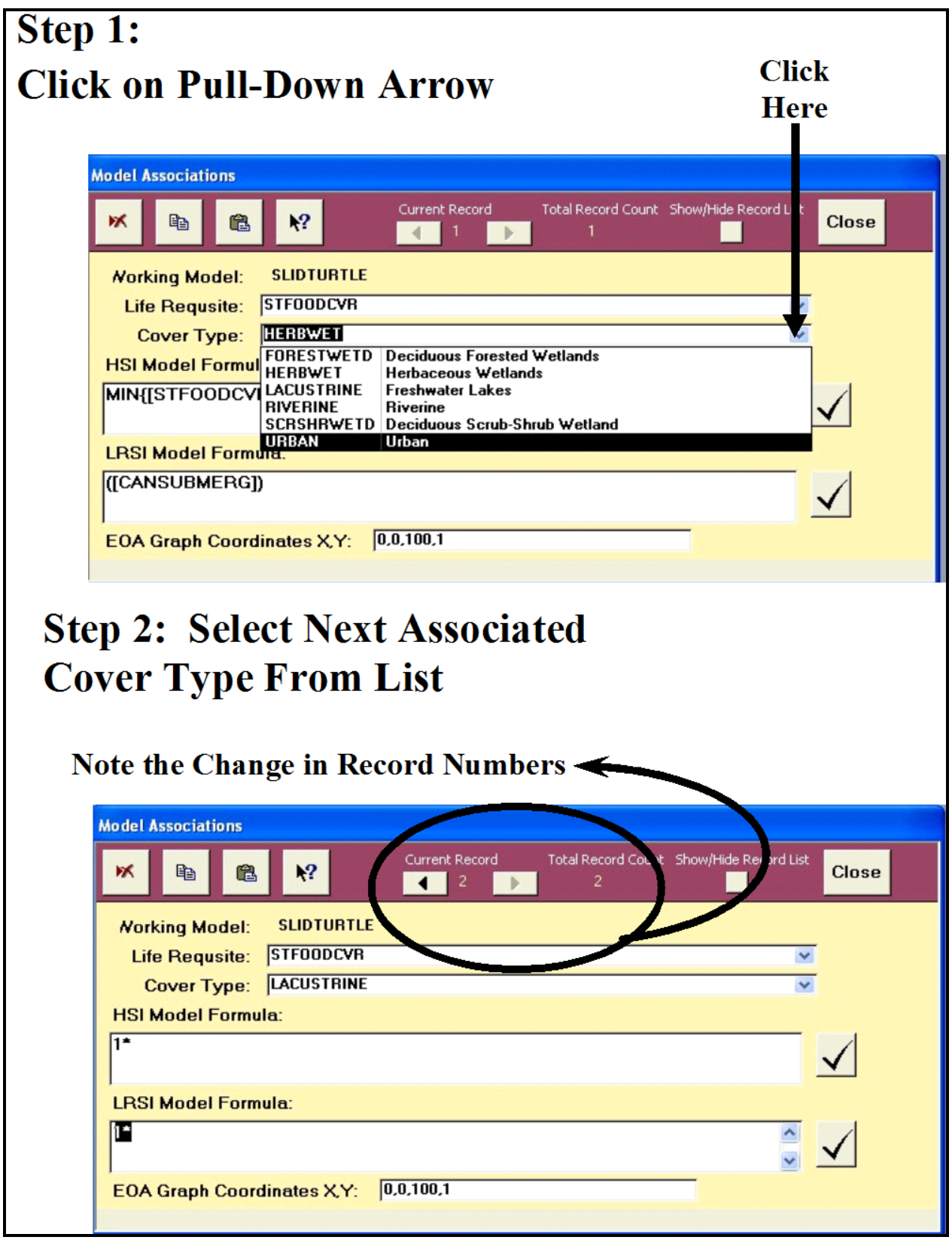

Figure 66. Select additional cover types from the pull-down list. 


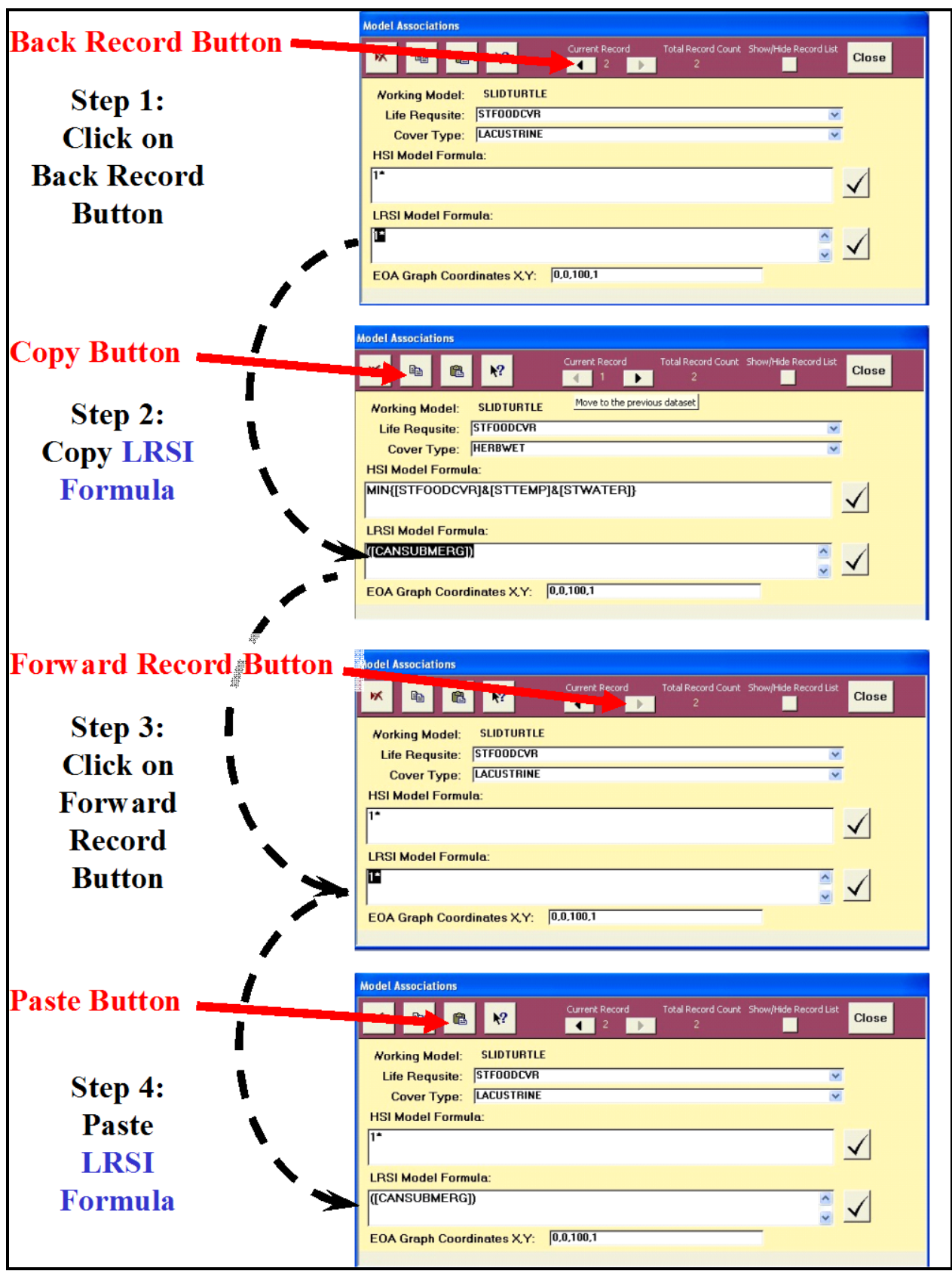

Figure 67. Make a new association by duplicating previous records (LRSI formula).

Now return to the first record to pick up the HSI formula by following these steps:

5. Click on the Back Record button.

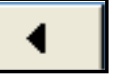

6. Highlight the HSI Formula, hold down the CTRL key, and type $\mathbf{C}$ on the keyboard to copy the formula.

7. Click on the Forward Record button.

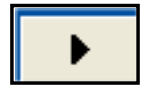


8. Highlight the $\mathbf{1}$ in the HSI Formula entry space and hold down the CTRL key while typing $\mathbf{V}$ on the keyboand to paste the formula (Figure 68). ${ }^{1}$

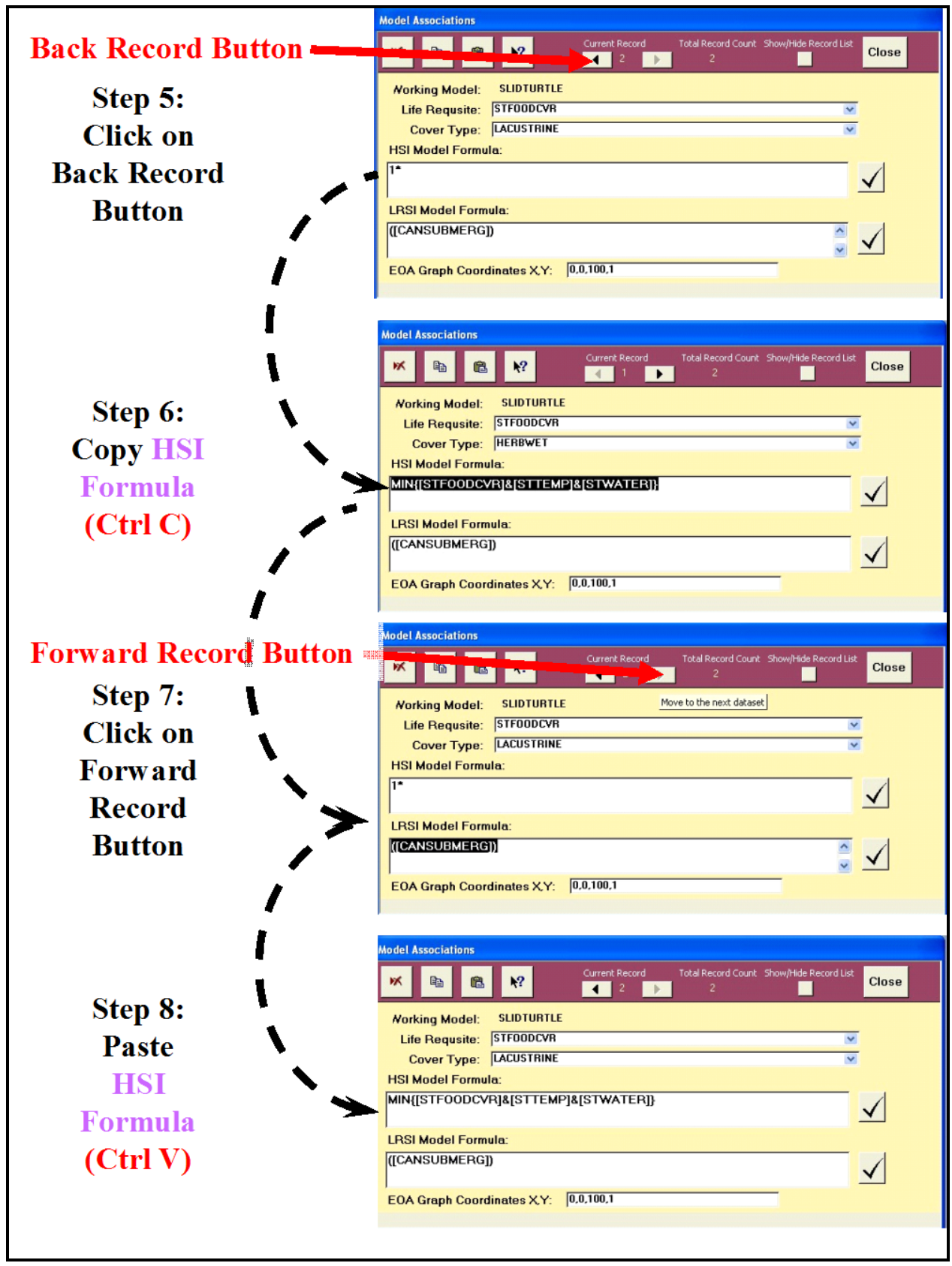

Figure 68. Make a new association by duplicating previous data (HSI formula).

${ }^{1} \mathrm{Ctrl}+\boldsymbol{C}$ is the universal Microsoft shortcut key to copying text and numbers onto the clipboard and works well in these instances. $C t r l+V$ is the universal Microsoft shortcut key to paste text and numbers from the clipboard. $C t r l+Z$ is the universal Microsoft shortcut key to undo an action. 
Repeat this process as many times as necessary to associate the cover type, LRSIs, and HSIs with the "Working Model." If at any time, a model's associated information must be removed, open the Model

USER'S NOTE:

There is one exception to this rule - if the targeted record is the only cover type association for the model, EXHEP will not allow this deletion using this protocol. Instead, simply overwrite the HSI formula with " 1 *" and click on the Close button. This will remove the one and only association for the model.

Associations window, left-click once on the HSI Model Formula entry box, and click on the Delete button.

Reviewing the associations

To review the list of associated records entered thus far, click on the Show/ Hide Records List toggle button. In response, EXHEP opens the listing window (Figure 69).

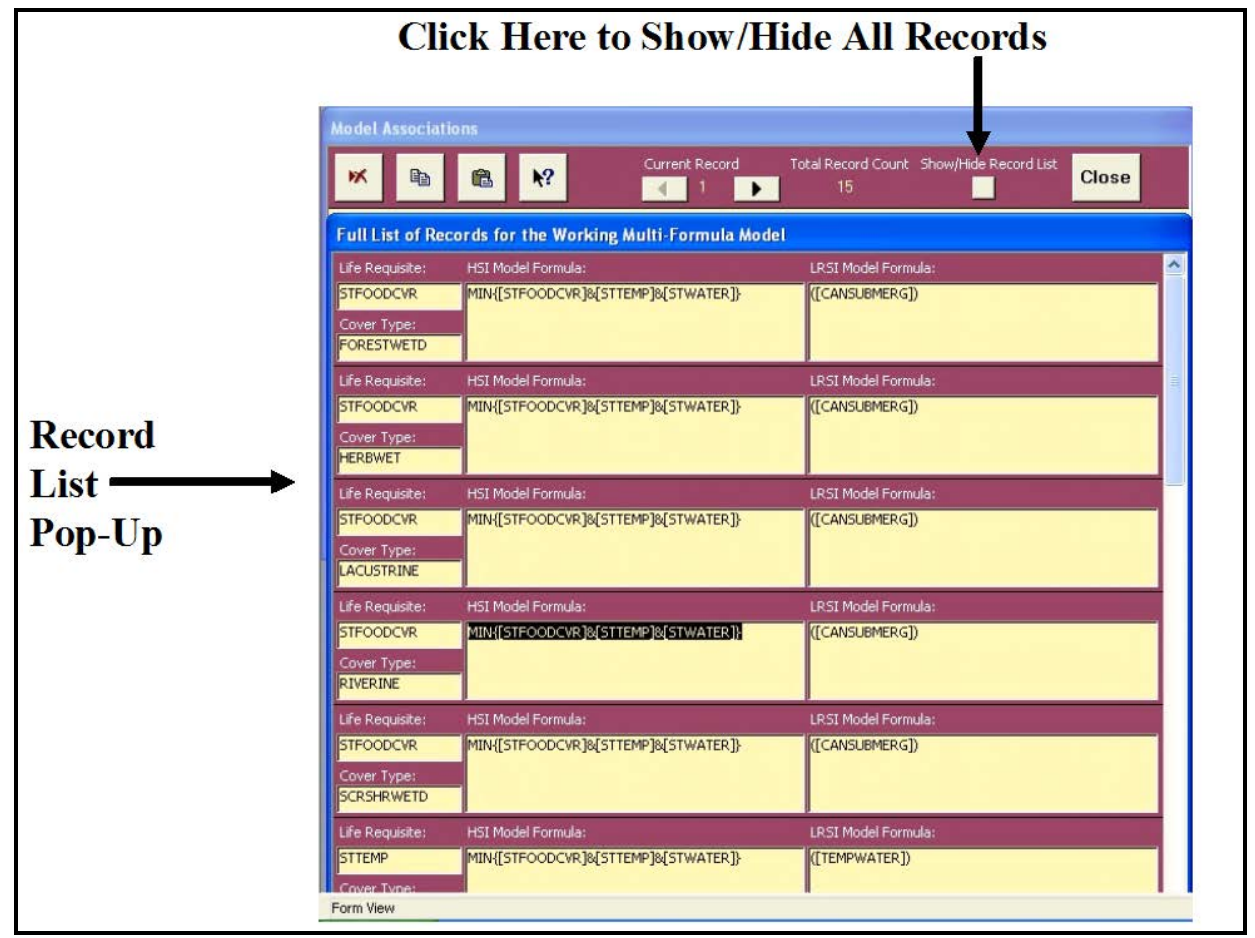

Figure 69. Show or hide all associated records simultaneously.

Scroll down this list with the scroll bar on the far right-hand side of this window. To close the window, click the toggle button a second time. When finished with the associations, click on the Close button in the upper right-hand corner of the toolbar and EXHEP returns to the primary interface. Repeat Step 3 (i.e., Model Association) for each "Working Model" in the analysis. 


\section{Checking the association process with EXHEP reports}

EXHEP provides a series of summary reports as each step in the process above is completed. To review and check the associations made thus far, click on the Setup Reports button in the center of the primary interface (Figure 70).

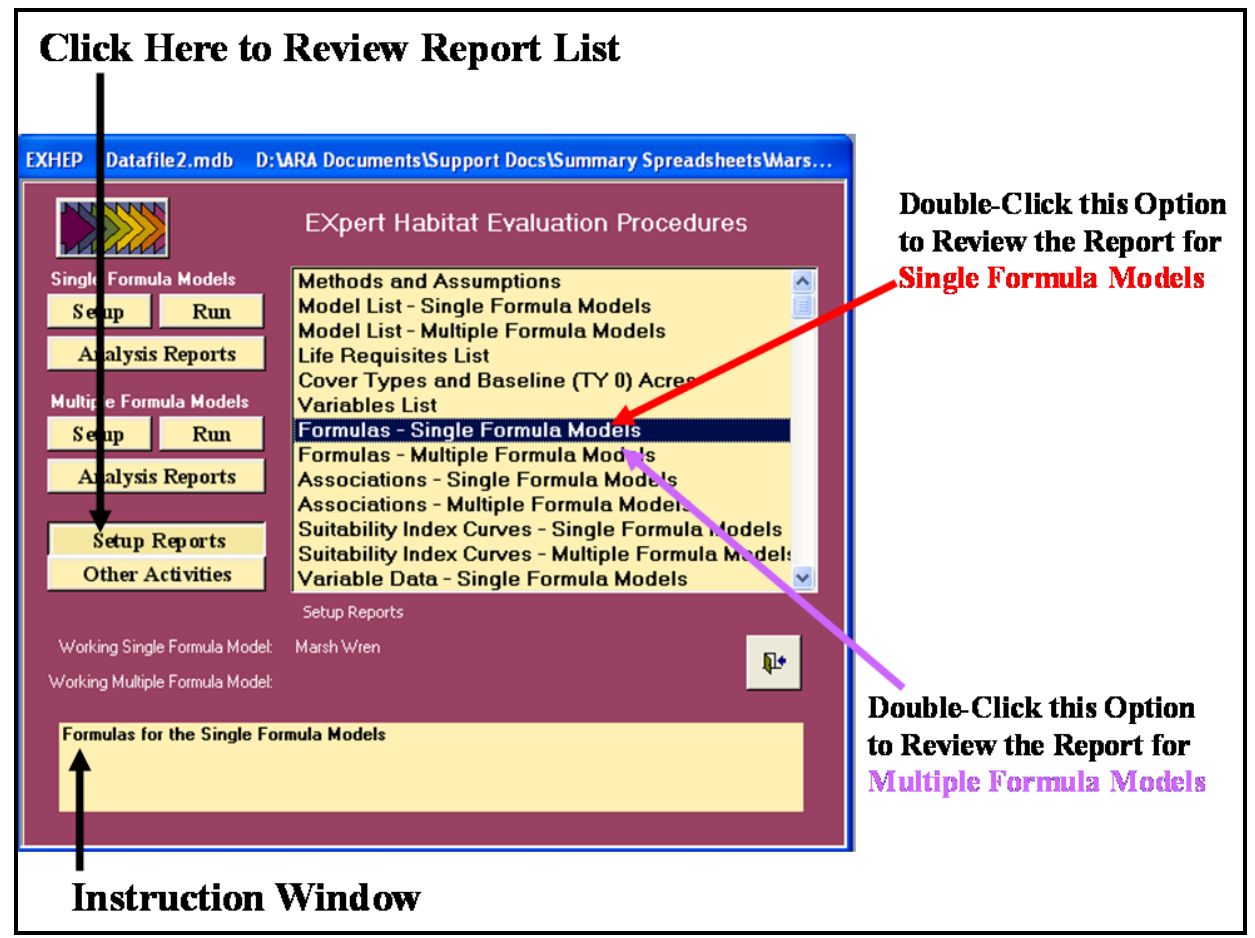

Figure 70. Click on the Setup Reports button to review the association reports in EXHEP.

A list of available reports appears in the center of the primary interface. Four additional reports should be populated by the time the model association process is complete:

\section{- Formulas - Single Formula Models}

- Formulas - Multiple Formula Models

- Associations - Single Formula Models

- Associations - Multiple Formula Models

Single-click on any of these reports and look to the Instruction Window to check the contents of the report. Double-click on any report, and EXHEP opens a new window with the report inside (Figure 71). 


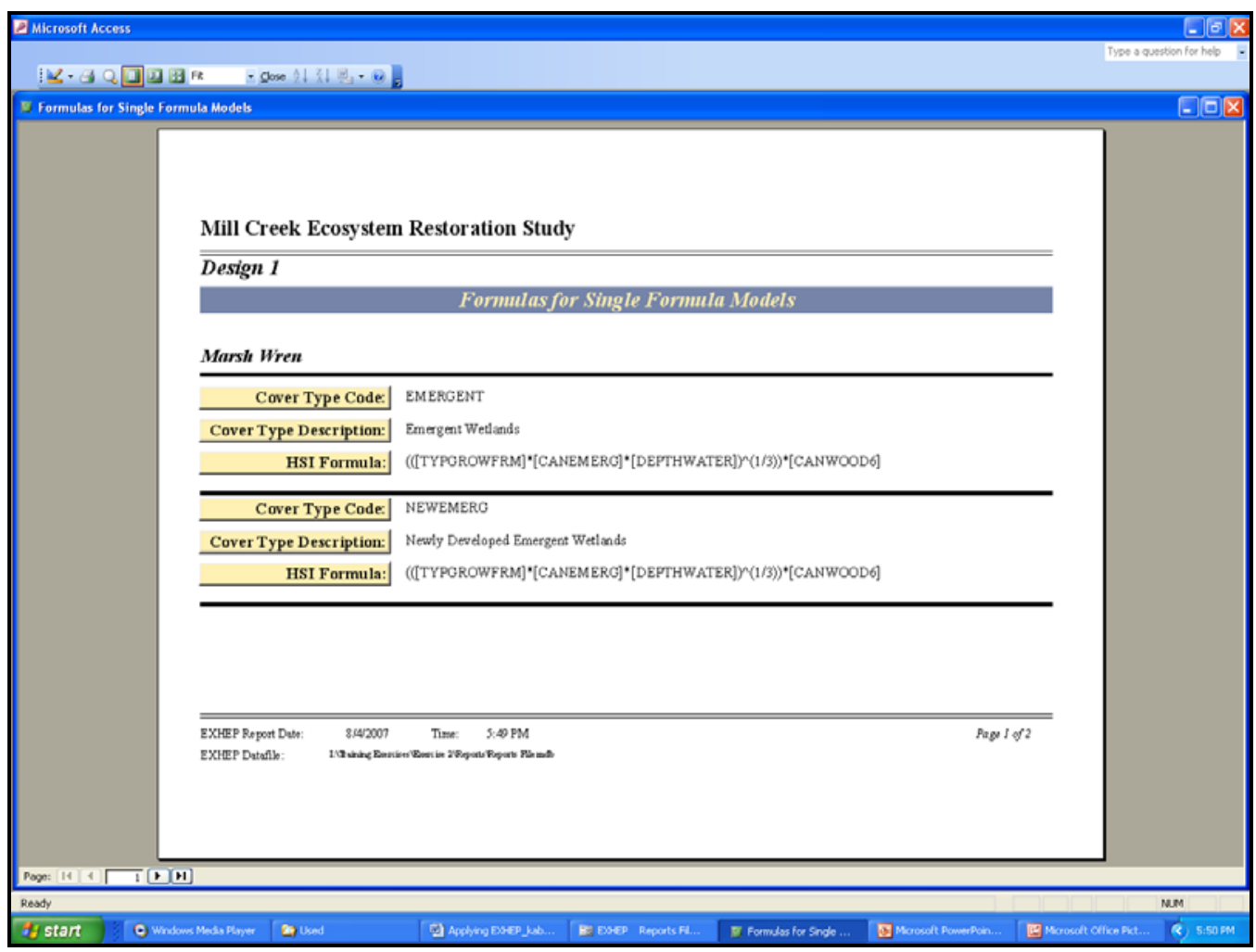

Figure 71. Example of EXHEP's formula report for the single formula models.

Click on the Close button from the suite of buttons offered in the Print Preview toolbar - EXHEP returns to the primary interface. Repeat the process as often as necessary to check the products developed thus far in the association activities. Proceed to Step 4: Enter Baseline Data and Generate Baseline Results to continue.

\section{Step 4: Enter baseline data and generate baseline results}

Once the HSI models are set up and associated in EXHEP, the baseline data can be entered into the system. In some studies, several models are constructed with similar variables in an attempt to characterize the quality of the site in an efficient manner. EXHEP provides entry screens to import variable data, automating the data entry task. This capability reduces the amount of labor required to complete the analysis, while increasing the system's evaluation efficiency. Although EXHEP has segregated the input of single formula models and multiple formula models thus far, it is not uncommon for these two types of models to share similar variables and their accumulated data. Automated data input into EXHEP streamlines access to this data by importing it from a single source regardless of its application to the single or multiple formula models. This 
capability further enhances the system's efficiency and reduces the amount of time necessary to input the data. ${ }^{1}$

\section{SI graph coordinate entry, deletion, and/or copying}

Prior to entering data into EXHEP, a normalized relationship (scaled from 0 - 1) must be defined between each variable and the suitability of the habitat it represents to the community or species. To begin the process, click on the Run button under the Single Formula Models section of the primary interface (Figure 72).

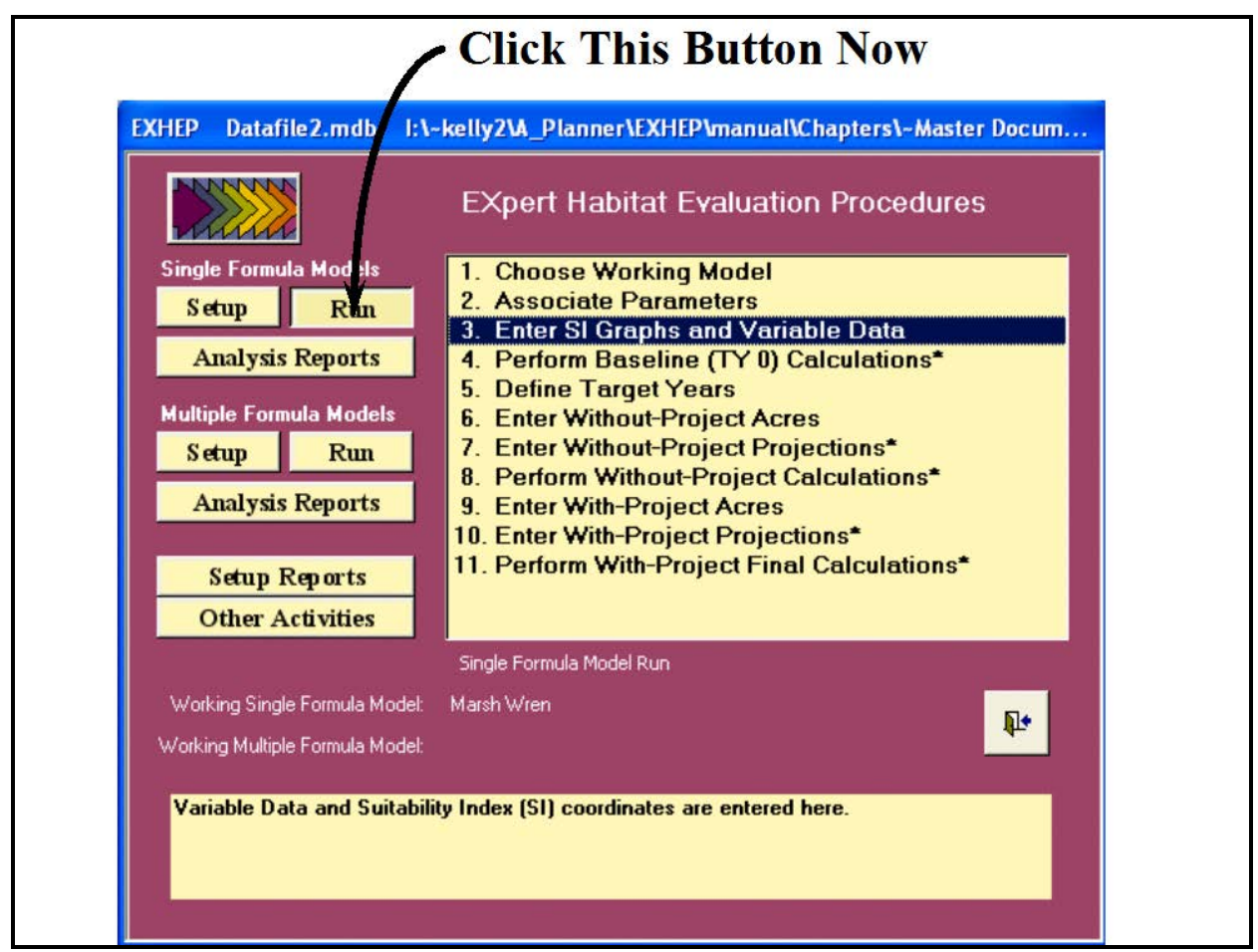

Figure 72. Click on the Run button to begin the single formula model baseline data entry process in EXHEP.

As it did before, a screen appears in the center of the primary interface with 11 options:

\section{Choose Working Model}

2. Associate Parameters

3. Enter SI Graphs and Variable Data

4. Perform Baseline (TY 0) Calculations*

5. Define Target Years

1 To assist in the software application, a series of training exercises have been included in Appendix E refer to Exercises 4 - 6 for relevant training in baseline data entry in EXHEP. 
6. Enter Without-Project Acres

7. Enter Without-Project Projections*

8. Perform Without-Project Calculations*

9. Enter With-Project Acres

10. Enter With-Project Projections*

11. Perform With-Project Final Calculations*

By now, the first two steps in this options list have been completed. However, the software may not be ready to accept coordinates for the models in the analysis - particularly if several models were defined earlier in the setup process. A systematic approach works best in this instance choose a "Working Model" (refer to Step 2: Model Association, Choose the Working Model above for assistance) to proceed. Now double-click on step 3, Enter SI Graphs and Variable Data to open the following windows (Figure 73).

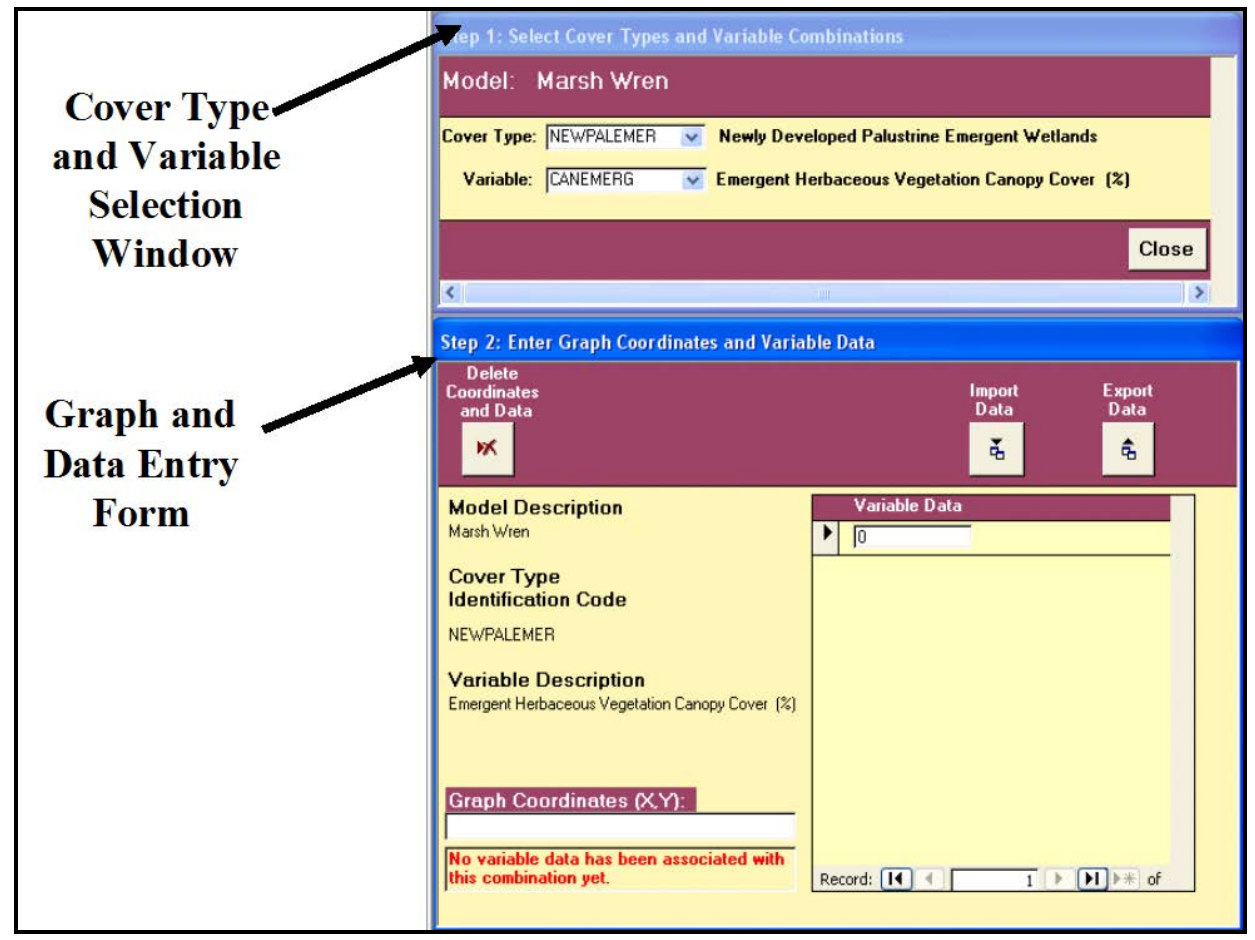

Figure 73. Baseline Data Entry windows for single formula models in EXHEP.

The Step 1: Select Cover Types and Variable Combinations window focuses the system on a cover type-variable combination. The Step 2: Enter Graph Coordinates and Variable Data form is the interface that will accept the coordinates for the variable's SI curve and the data points collected by the evaluation team. 
Step 1: Select a cover type and a variable

To begin, select a cover type and a variable from the pull-down menus in the upper window (Step 1: Select Cover Types and Variable Combinations) (Figure 74).

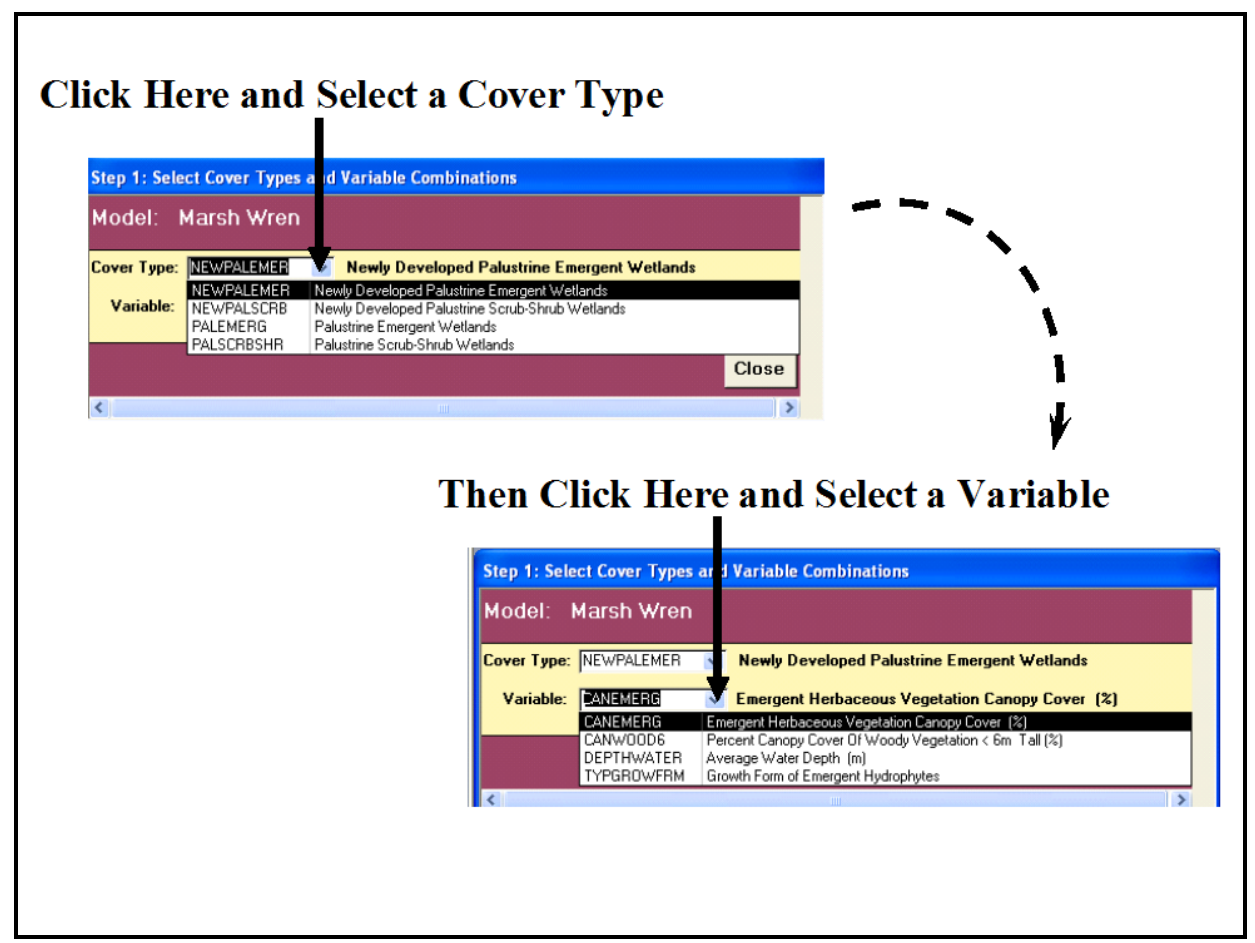

Figure 74. Begin data entry by selecting a cover type and a variable from the pull-down lists provided.

Step 2: Enter the SI graph coordinates

Now move to the lower window (Step 2: Enter Graph Coordinates and Variable Data) and provide the SI graph coordinates for the selected variable in the selected cover type (Figure 75). 


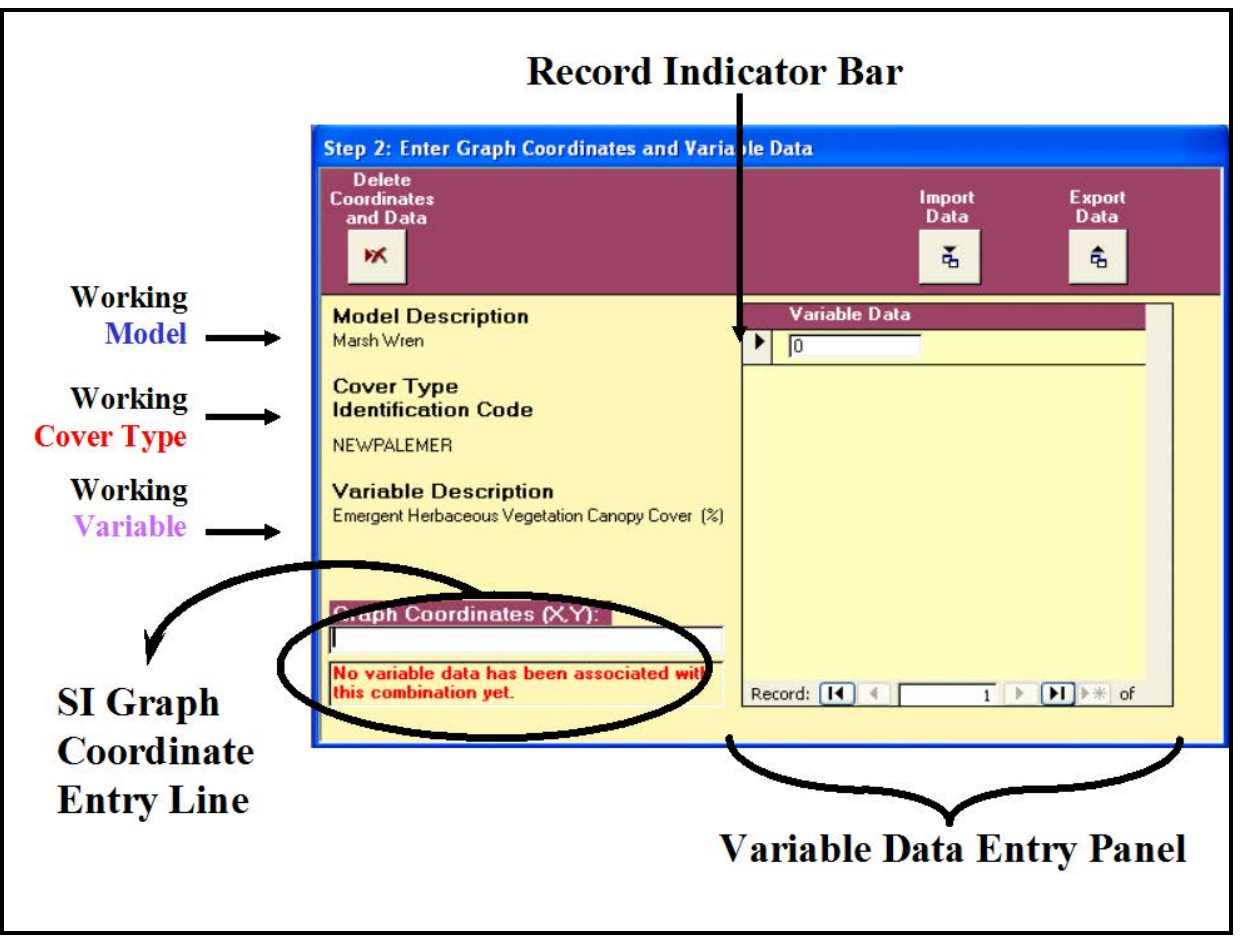

Figure 75. Move to the lower window and enter the SI graph coordinates in the space provided.

To do this, enter sets of $X$ and $Y$ coordinates (separated by commas - $X_{1}, Y_{1}$, $\mathrm{X}_{2}, \mathrm{Y}_{2}, \mathrm{X}_{3}, \mathrm{Y}_{3}, \ldots \mathrm{X}_{\mathrm{n}}, \mathrm{Y}_{\mathrm{n}}$ ) into the lower left-hand space provided [i.e., Graph Coordinates $(\mathbf{X}, \mathbf{Y})$ and hit the Tab key on the keyboard (Figure 76).

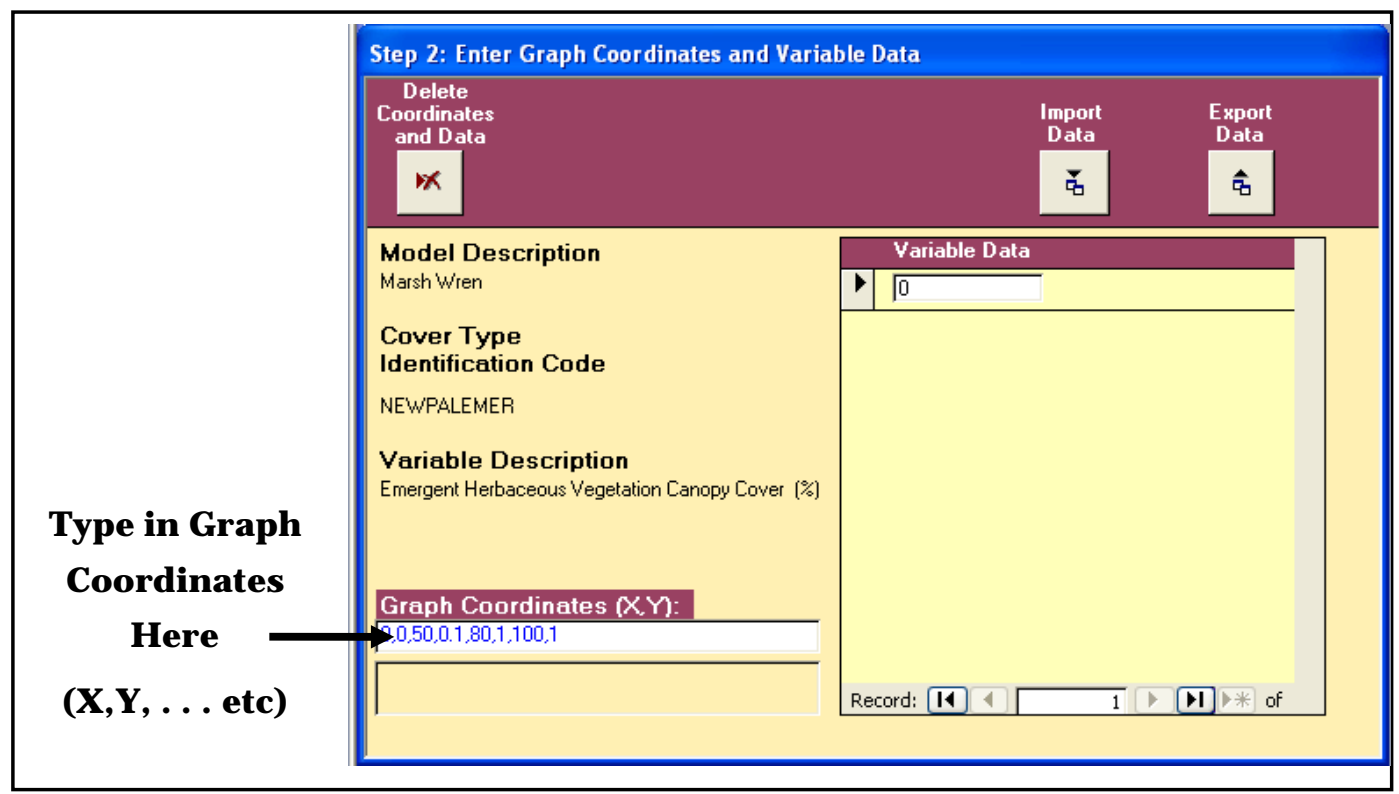

Figure 76. Example of an SI coordinate entry. 
EXHEP ignores spaces in these entries, so use spaces within the entry to clarify and organize the $\mathrm{X}$ and $\mathrm{Y}$ values entered. As soon as the coordinates are entered, hit Enter on the keyboard. EXHEP will check the numbers if an entry is missing commas, or has too many commas, EXHEP will flash a warning and directions on the best way to proceed. Once the coordinates have been checked

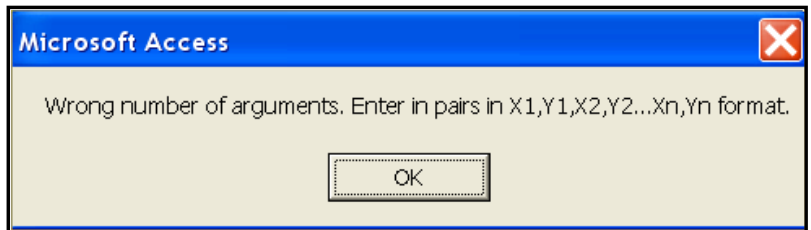
by the program, EXHEP will move the cursor to the first entry of the Variable Data entry panel on the right-hand side of the screen.

Continue the process of SI coordinate entry for all cover type/ variable combinations in the model before proceeding to the data entry phase of the process. If the need should arise, the SI graph coordinates can be deleted by highlighting the string of ' $\mathrm{X} / \mathrm{Y}$ " coordinates and hitting the Delete button on the keyboard. As is often the case, cover types may share the same SI curve. In this instance, save time by copying the SI graph coordinates to the clipboard and pasting them back down into the next record. To do this, follow these quick steps:

1. Highlight the coordinates and press Ctrl C on the keyboard.

2. Now moveup to the upper form (Step 1: Select Cover Types and Variable Combinations) and pull down the next cover type. Leave the variable selection as it is.

3. Move down to the lower form (Step 2: Enter Graph Coordinates and Variable Data) and left-click once in the Graph Coordinates $(\mathbf{X}, \mathbf{Y})$ box.

4. Press Ctrl V on the keyboard and Tab to accept the coordinates (Figure 77). 


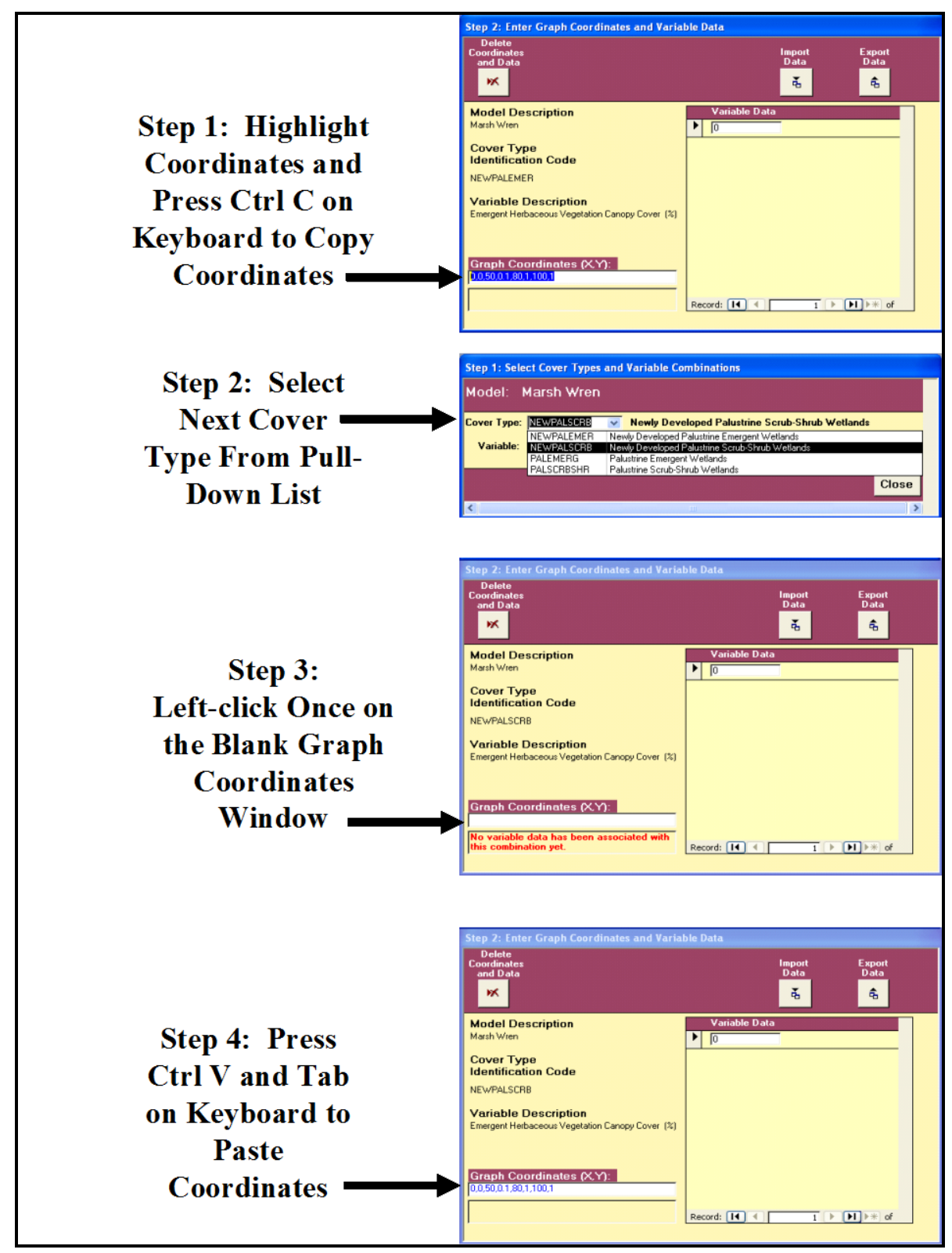

Figure 77. Copying SI graph coordinates from one record to the next.

If there were no errors in the process, EXHEP will move the cursor up to the first entry of the Variable Data entry panel on the right-hand side of the screen. Repeat this process for every variablecover type combination for every model in the analysis. Return to the top of the cover type and variable lists, and double-check each record to assure that the SI graph coordinates have been entered for all

USER'S NOTE:

Although this process may seem tedious at first, the flexibility of the EXHEP software allows users to sensitize the model to conditions in their region. In other words, because the software asks for these graphical coordinates on a variable-by-variable basis for every cover type, the system will allow users to develop highly specialized relationships between the model's various components. records. Click Close to return to the primary interface. Choose the next 
"Working Model" and repeat the process as often as necessary to fully set up the datafile for all applicable models. ${ }^{1}$

\section{Additional actions necessary to input SI graph coordinates for multiple} formula models

If multiple formula models are used in the analysis, the SI graph coordinates must be entered using the second set of buttons on EXHEP's primary interface (Figure 78).

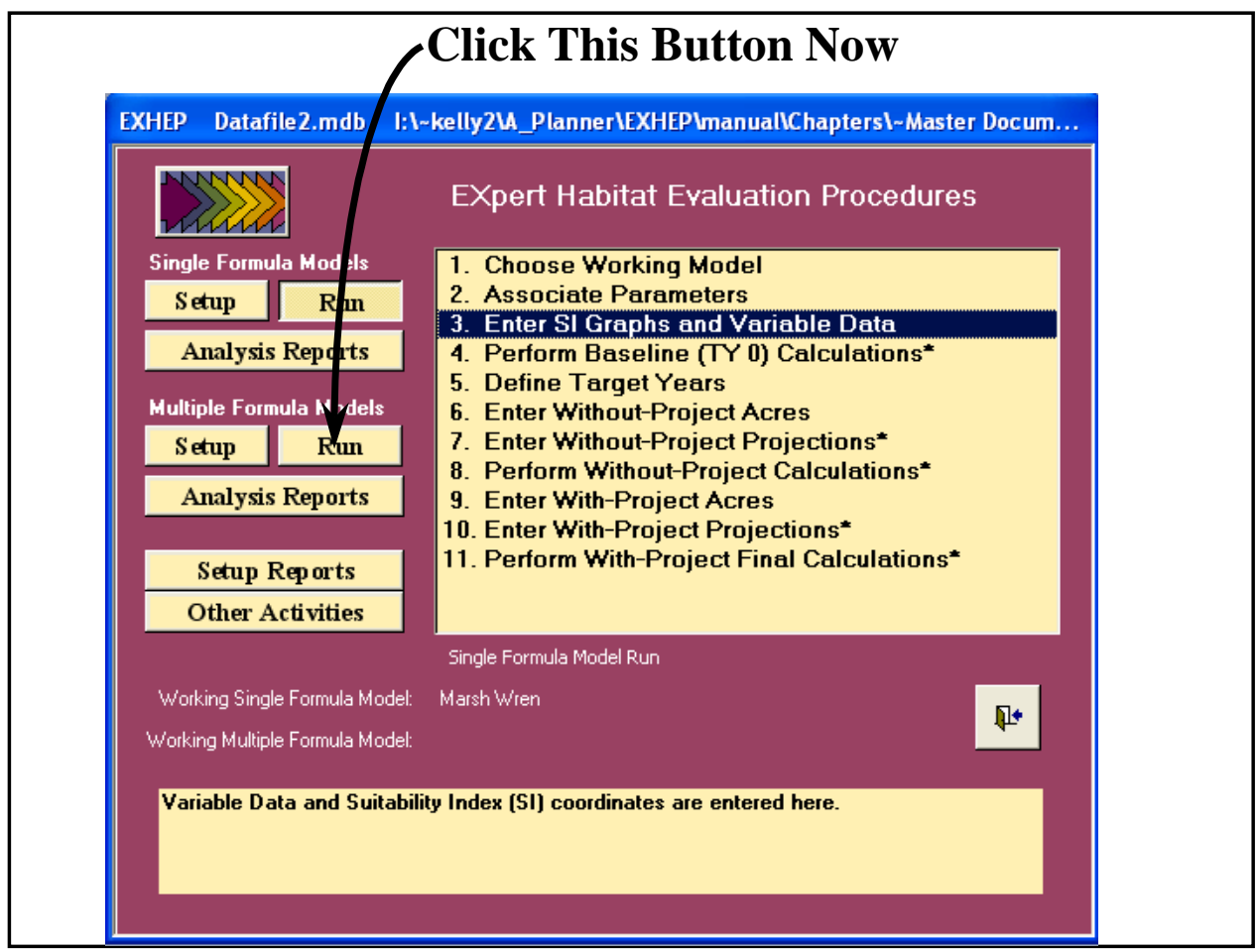

Figure 78. Click on the Run button to begin the multiple formula model SI graph coordinate entry process in EXHEP.

As it did before, the screen appears in the center of the primary interface with 11 options:

\section{Choose Working Model}

\section{Associate Parameters}

\section{Enter SI Graphs and Variable Data}

1 Once the suite of SI coordinates have been entered into the file for the models, the authors suggest saving the file as a "master." This is a particularly useful approach if the models are to be used at multiple sites (with unique baseline conditions such as an impact site and a subsequent mitigation site or perhaps multiple restoration sites under the premise of a watershed study). By opening and saving the "master" as a new file, the setup information is retained, and the data entry process for the new site can begin immediately thereafter. 


\section{Perform Baseline (TY 0) Calculations*}

5. Define Target Years

6. Enter Without-Project Acres

7. Enter Without-Project Projections*

8. Perform Without-Project Calculations*

9. Enter With-Project Acres

10. Enter With-Project Projections*

11. Perform With-Project Final Calculations*

By now, the first two steps in this options list have been completed. However, the software may not be ready to accept coordinates for the models in the analysis - particularly if several models were defined earlier in the setup process. A systematic approach works best in this instance choose a "Working Model" (refer to Step 2: Model Association, Choose the Working Model above for assistance) to proceed. EXHEP uses the same process to link the SI graph coordinates to the model's cover types and variables as it did in the single formula model example, with one exception - it now asks for life requisite information. Click on the third option (Enter SI Graphs and Variable Data) and the following window will open (Figure 79).

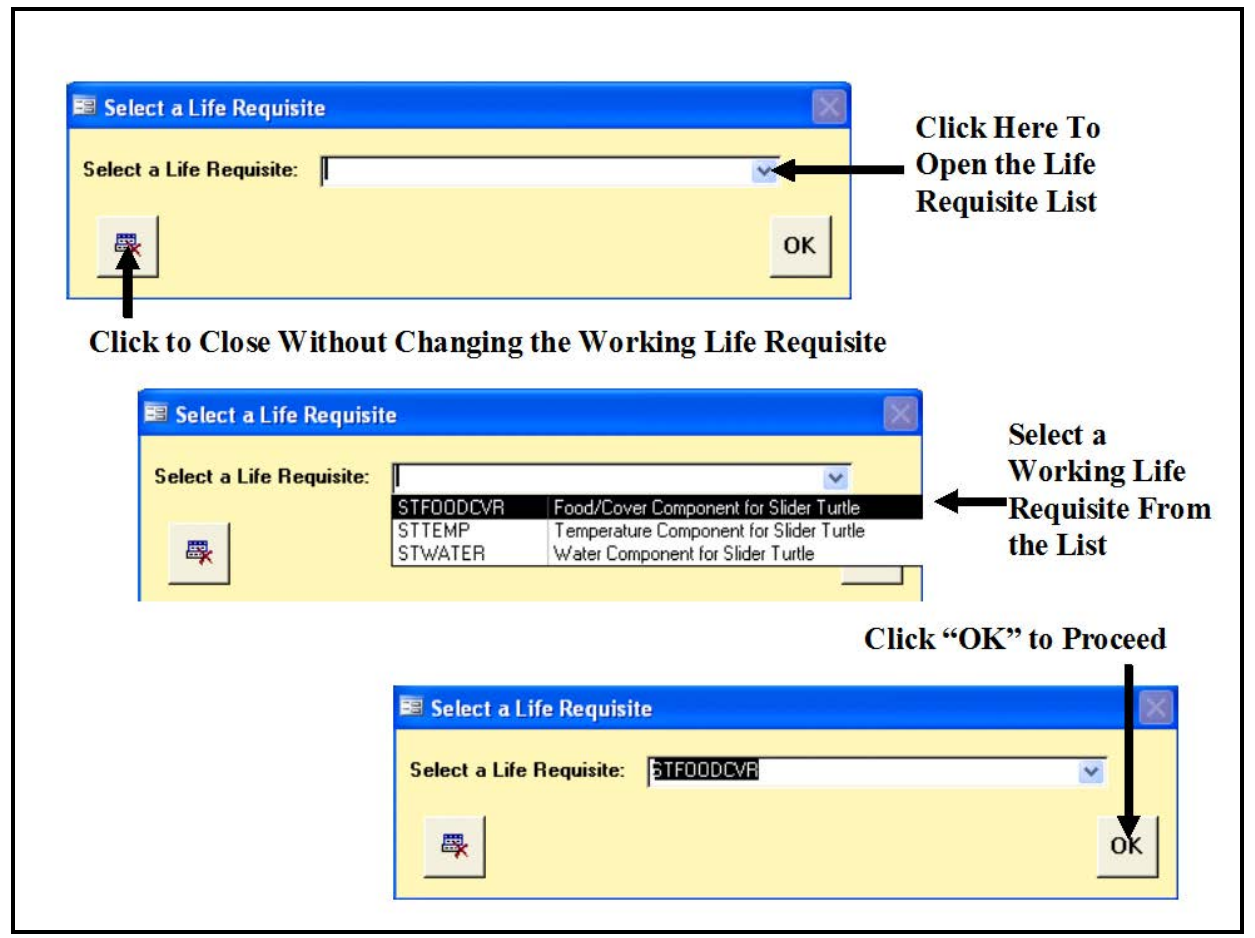

Figure 79. Select a Life Requisite window for multiple formula models in EXHEP. 
The Select a Life Requisite window focuses the EXHEP engine on the working life requisite for model setup. Use the pull-down menu to the immediate right of the Select a Life Requisite window and select the first life requisite in the model. Click OK, and EXHEP will open the Step 1: Select Cover Types and Variable Combinations and Step 2: Enter Graph Coordinates and Variable Data forms just as it did for the single formula models. Use the same protocol described above in the single formula models section to enter SI graphical coordinates for the cover types and variables associated with this life requisite. When finished, close the forms and return to the primary interface. Repeat the process for each life requisite in every multiple formula model.

\section{Checking the SI graph coordinates with EXHEP reports}

To review and check the entries made thus far, click on the Setup Reports button (Figure 80).

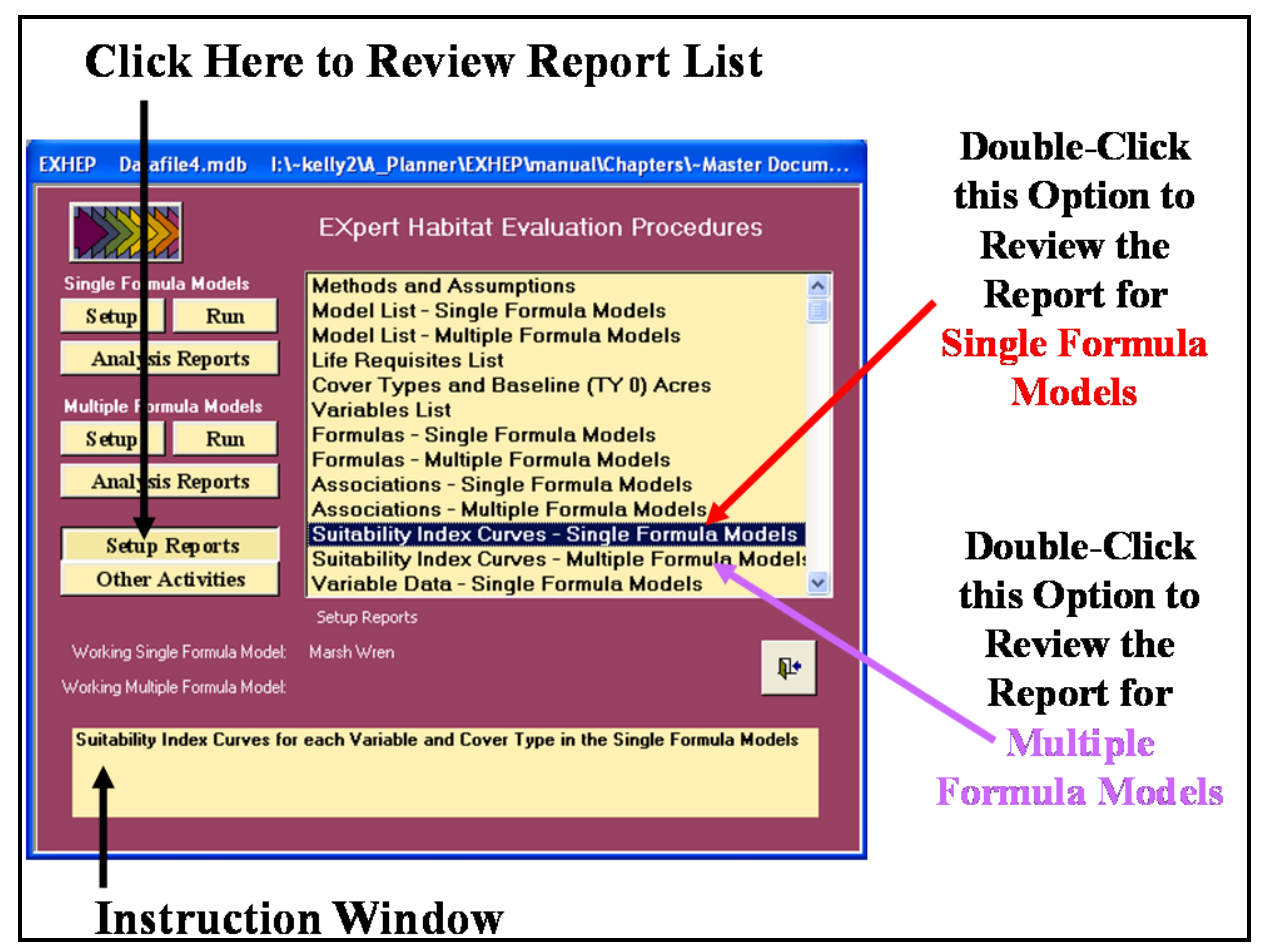

Figure 80. Click on the Setup Reports button to review the SI graph coordinate reports in EXHEP.

A list of available reports appears in the center of the primary interface. Two additional reports should be populated by the time the graph coordinates entry process is complete: 


\section{- Suitability Index Curves - Single Formula Models.}

- Suitability Index Curves - Multiple Formula Models

Single-click on either report and look to the Instruction Window to check the contents of the report. Double-click on any report, and EXHEP opens a new window with the report inside (Figure 81).

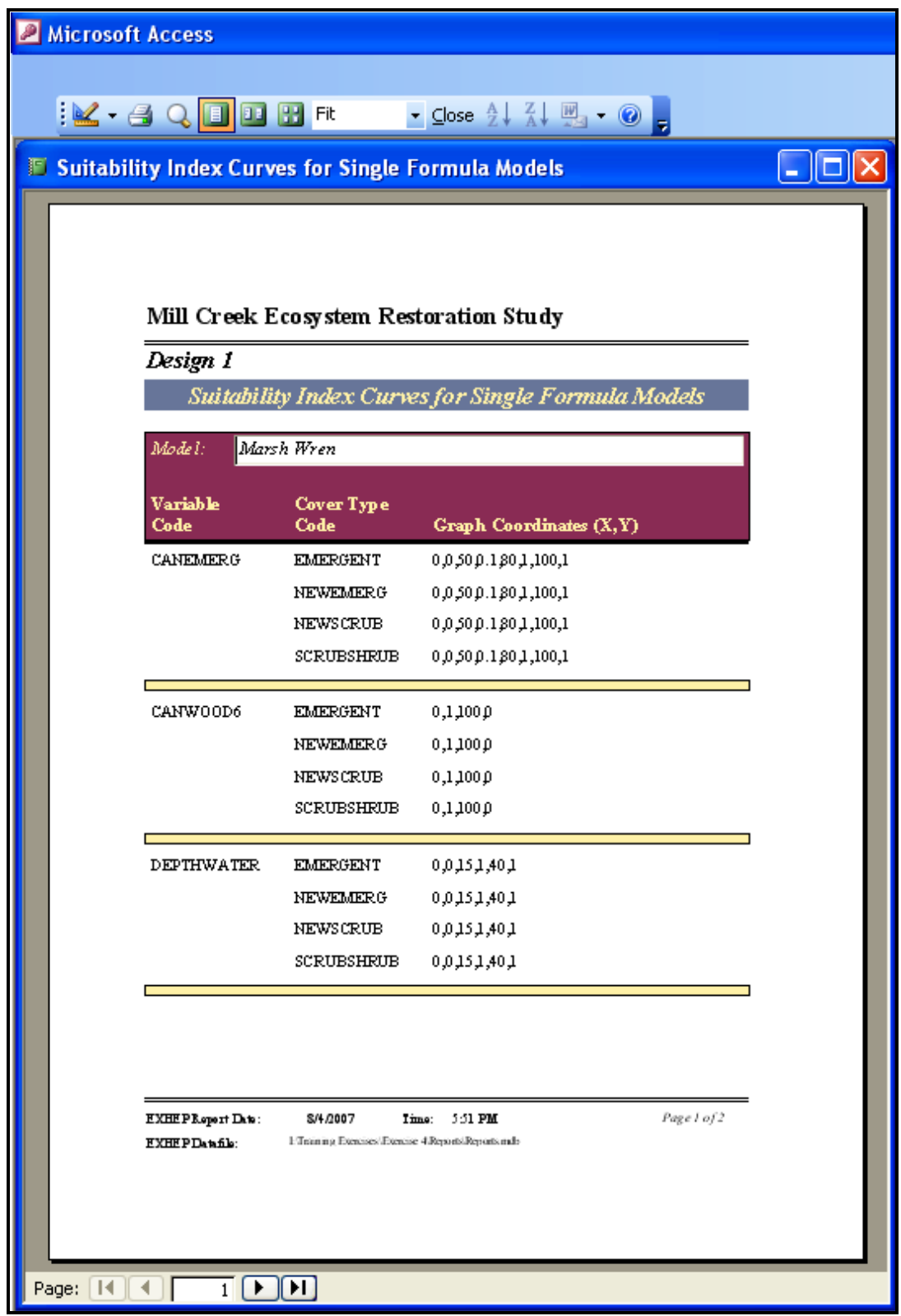

Figure 81. Example of Suitability Index Curves for Single Formula Models report in EXHEP.

Click on the Close button from the suite of buttons offered on the Report Control toolbar, and EXHEP returns to the primary interface. Now that 
the SI graph coordinates entry process is complete, and the information has been confirmed, proceed to the next section to learn how to enter variable data into EXHEP.

\section{Baseline data entry}

It is often useful to organize the cover types and their associated variables in some fashion or manner before beginning the process of entering data. In most HEP studies, several data sets are generated for each variable (in each applicable cover type). In its original form, this information is often unwieldy and prone to disorganization. One option is to store the data in a spreadsheet - EXHEP was designed to import data from such electronic formats (specifically Microsoft-compatible formats such as MS Excel). ${ }^{1}$

Now, begin the data entry process by clicking on the Run button under the Single Formula Models section of the primary interface (Figure 82).

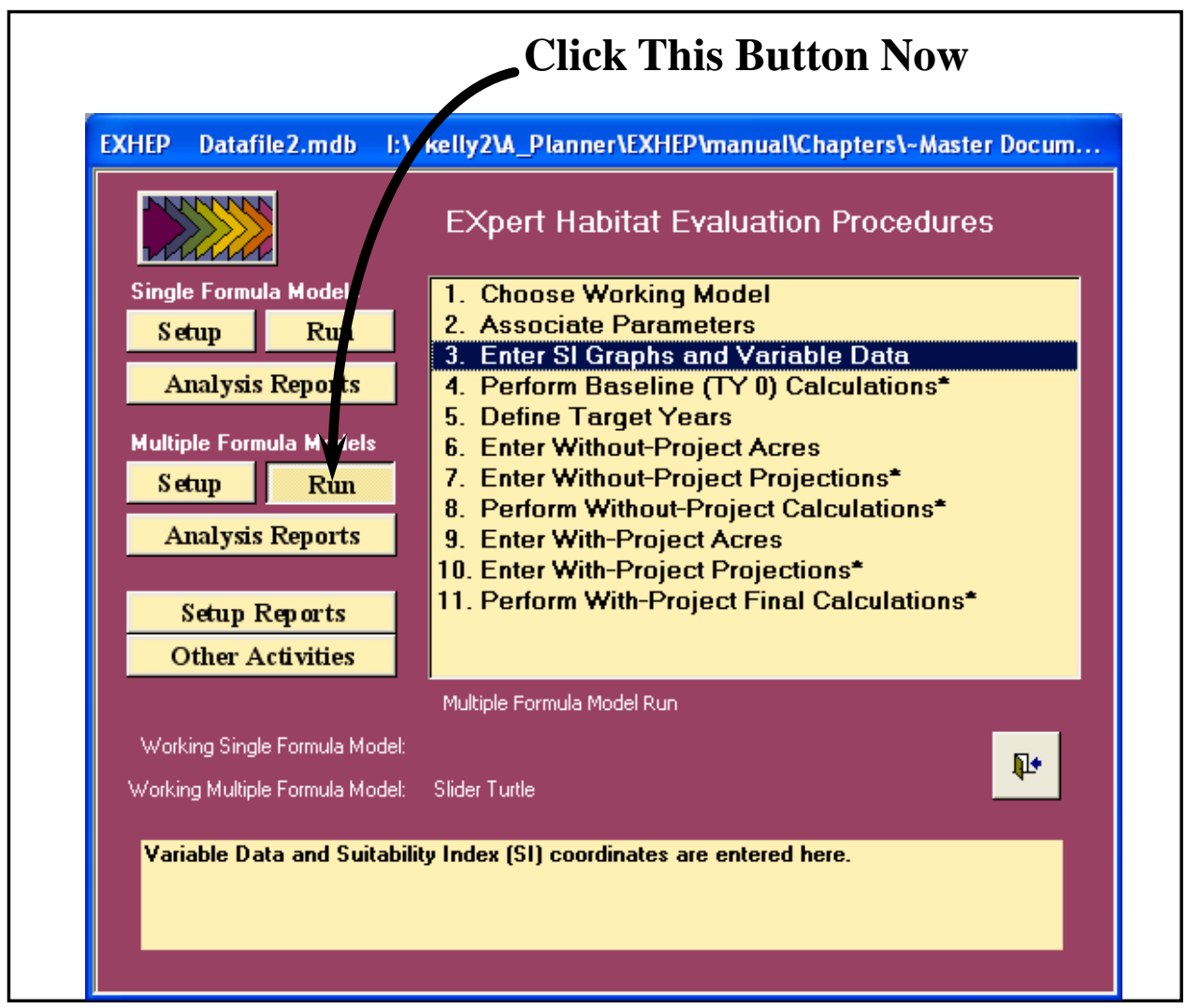

Figure 82. Click on the Run button to begin the single formula model baseline data entry process in EXHEP.

\footnotetext{
${ }^{1}$ Example spreadsheets have been provided on the installation CD (Marsh Wren Field Kit and Slider Turtle Field Kit).
} 
As it did before, a screen appears in the center of the primary interface with 11 options:

1. Choose Working Model

2. Associate Parameters

3. Enter SI Graphs and Variable Data

4. Perform Baseline (TY 0) Calculations*

5. Define Target Years

6. Enter Without-Project Acres

7. Enter Without-Project Projections*

8. Perform Without-Project Calculations*

9. Enter With-Project Acres

10. Enter With-Project Projections*

11. Perform With-Project Final Calculations*

Before continuing, verify that the correct "Working Model" has been selected (refer to Step 2: Model Association, Choose the Working Model above for assistance). Now click on the third option (Enter SI Graphs and Variable Data) to continue. EXHEP will again open the Step 1: Select Cover Types and Variable Combinations and Step 2: Enter Graph Coordinates and Variable

Data forms. Select the first cover type and variable combination for which there are data, and click on the " 0 " space of the Variable Data entry panel in the bottom window. Enter the data (either manually or electronically) in the space provided. ${ }^{1}$

Manual data entry

To enter data manually, type in the first value and press Enter on the keyboard (Figure 83).

\footnotetext{
1 In this first edition of the EXHEP software, it is assumed that each variable's index was developed in relation to the standard means of the data gathered. Thus all data are summed and averaged across the suite of data points collected, and the graph is plotted using direct linear configuration. If for any reason the data must be statistically analyzed in a different fashion (e.g., modes taken instead of means), perform this calculation outside the EXHEP software and provide the result(s) in the Variable Data entry panel.
} 


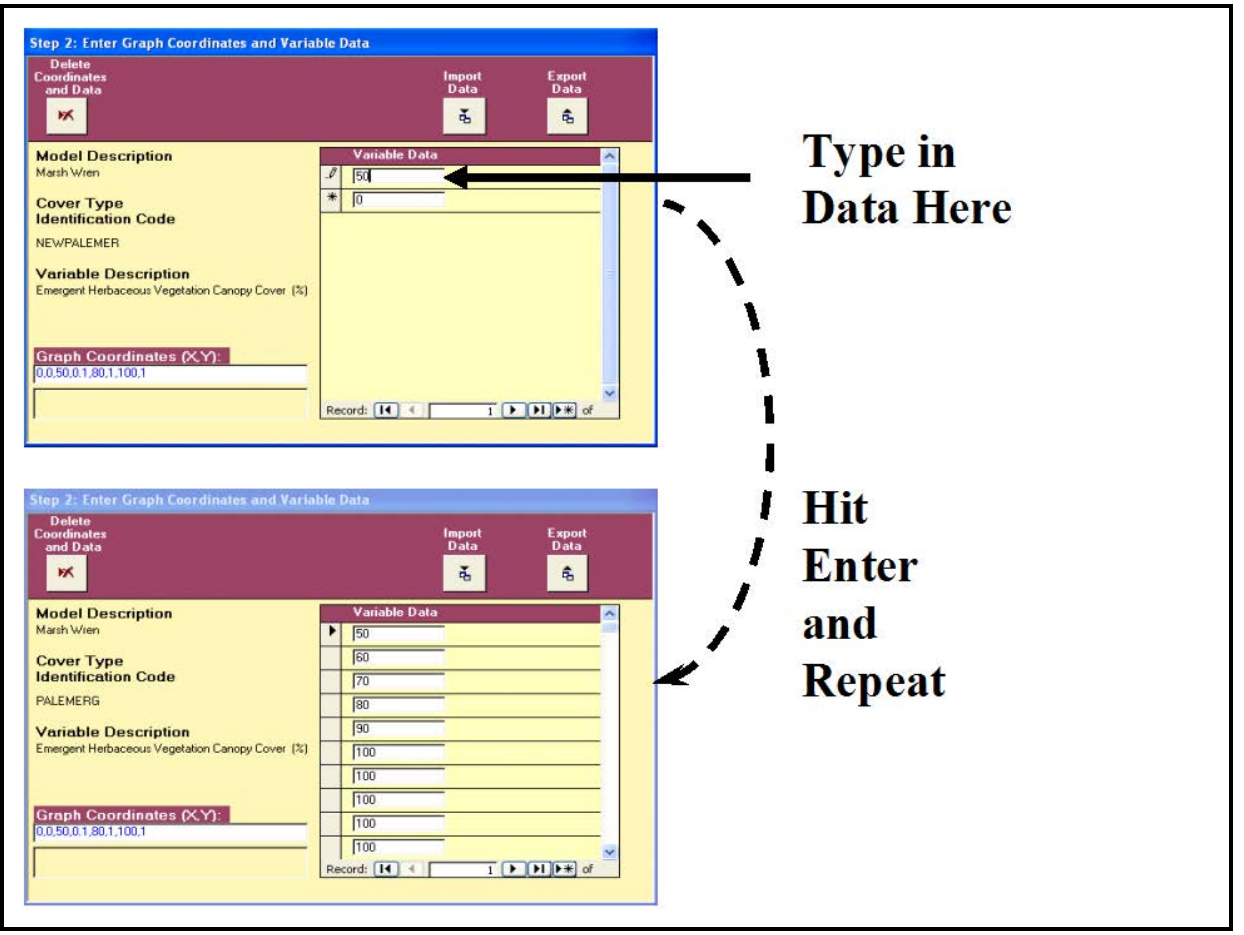

Figure 83. An example of the manual data entry process in EXHEP.

With this action, EXHEP will save the data point to the datafile and proceed to the next line. Repeat the process, entering all of the data for this variable and cover type combination. Use the scroll bar on the righthand side of this panel (or the arrow keys on the keyboard) to move up and down through the entries. Return to the upper form (Step 1: Select Cover Types and Variable Combinations) and choose the next combination of variables and cover types to continue the process. Repeat as often as necessary to fully populate the datafile.

\section{Electronic data entry}

If data are stored electronically in a spreadsheet, they can be imported into the datafile relatively easily. The data in the external spreadsheet must be organized in a variable per cover type manner, and they must be stored in columns. Now, import the data by following these steps:

1. Open the external spreadsheet.

2. Highlight a single column of data.

3. Press Ctrl C on the keyboard to copy the data to the clipboard.

4. Return to the EXHEP software (press ALT + Tab on the keyboard until the Microsoft Access icon appears in the viewer or click on the Microsoft Access icon on the taskbar at the bottom of the screen). 
5. Click the Import Data button above the Variable Data entry panel (Figure 84).

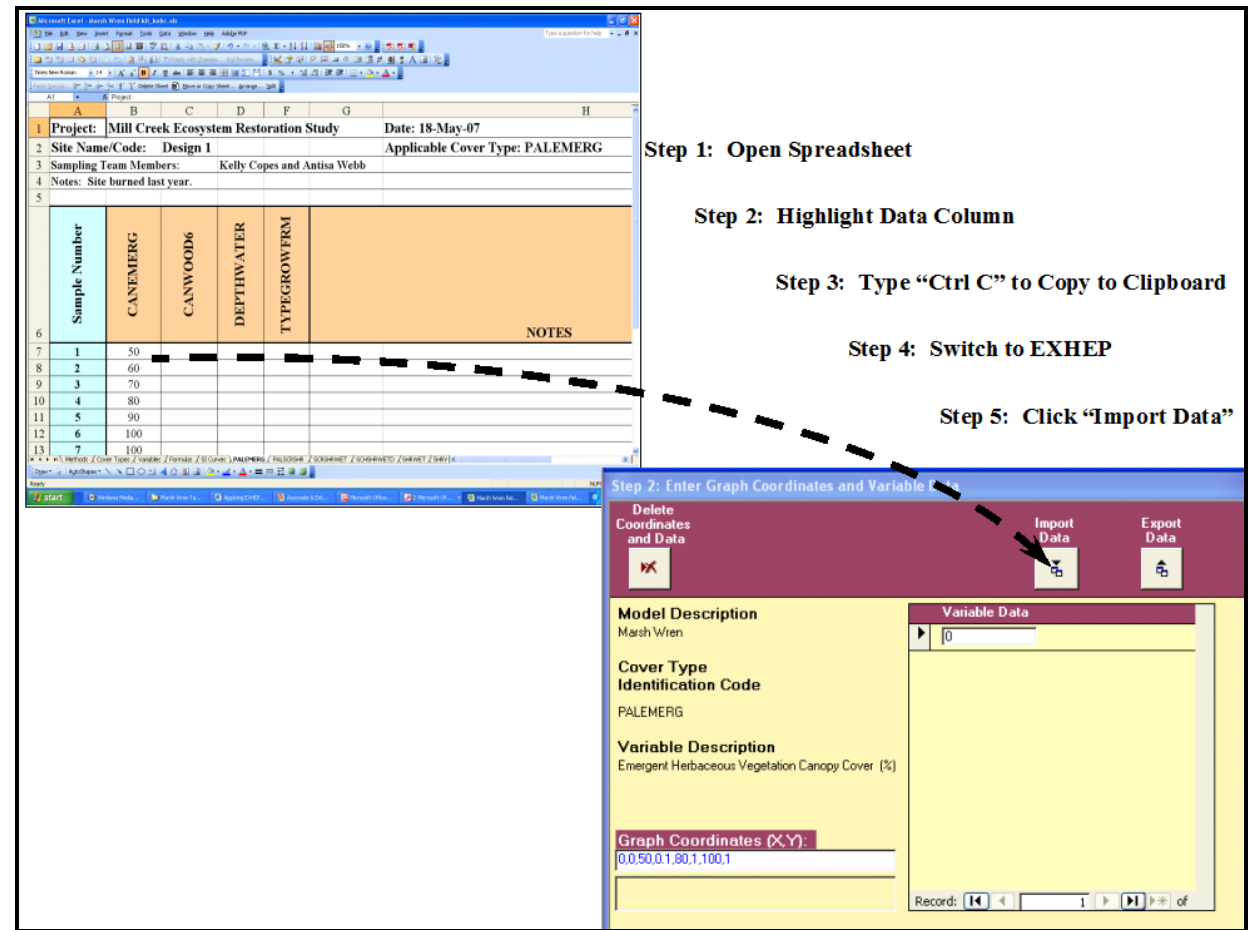

Figure 84. Importing data from an external spreadsheet into EXHEP.

6. Click on the Import Data button in the lower left-hand corner of the Field Data Import Process screen to continue.

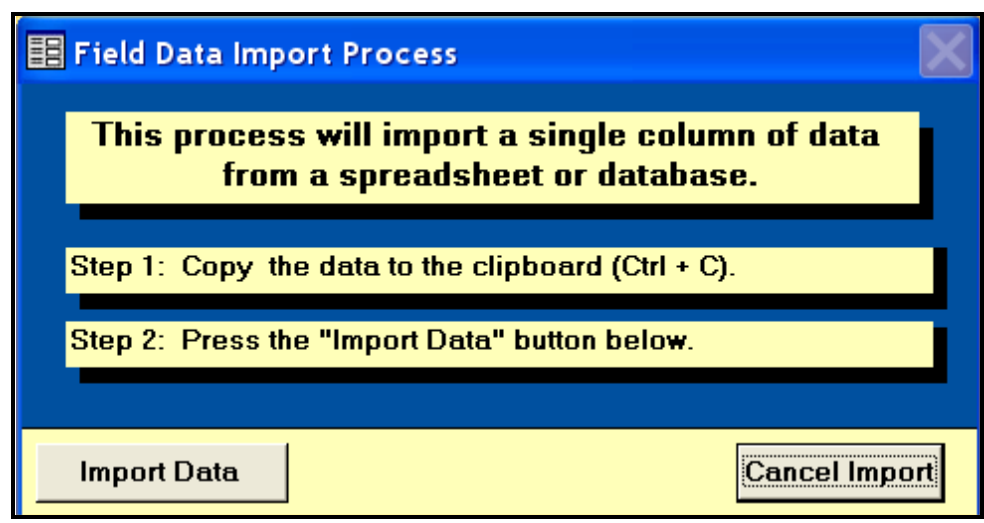


EXHEP pastes each cell from the copied dataset into the Variable Data entry panel. The Cancel Import button (lower right-hand corner) will halt the importation process and return to the Variable Data entry panel.

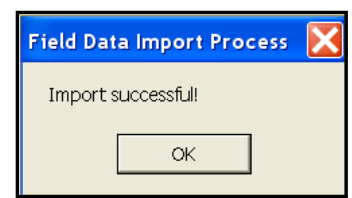
When the importation process has finished, EXHEP provides a message indicating that the import action was completed successfully. Press OK to proceed. If the

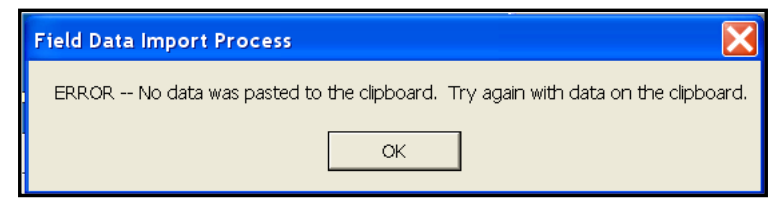
process failed, EXHEP will display an error message. To correct the error, empty the clipboard and try copying the data once again. Repeat the process, entering all of the data for the variable-cover type combinations in the model.

\section{Exporting data to MS Excel}

Whether entering the data manually or electronically, every variable per cover type in each model must be entered into the software systematically. Often, variables are shared across models or even across cover types. Rather than re-entering the same data multiple times, a process has been devised to save this information in a compatible spreadsheet file for importation via the protocol described above. To do this, follow these quick steps:

1. Manually enter the data for the variable into the software following the directions above.

2. Save the data to an MS Excel file by clicking the Export

Data button above the Variable Data entry panel (Figure 85).

3. Select the location to save the datafile via the Save in: window, providea new name (only) for the datafile in the File name: window, and press Save $^{1}$ (Figure 86).

\footnotetext{
1 It is not necessary to provide an extension for the datafile in the File name: - MS Access pre-determines the extension and assigns an *.xls to the file name provided.
} 


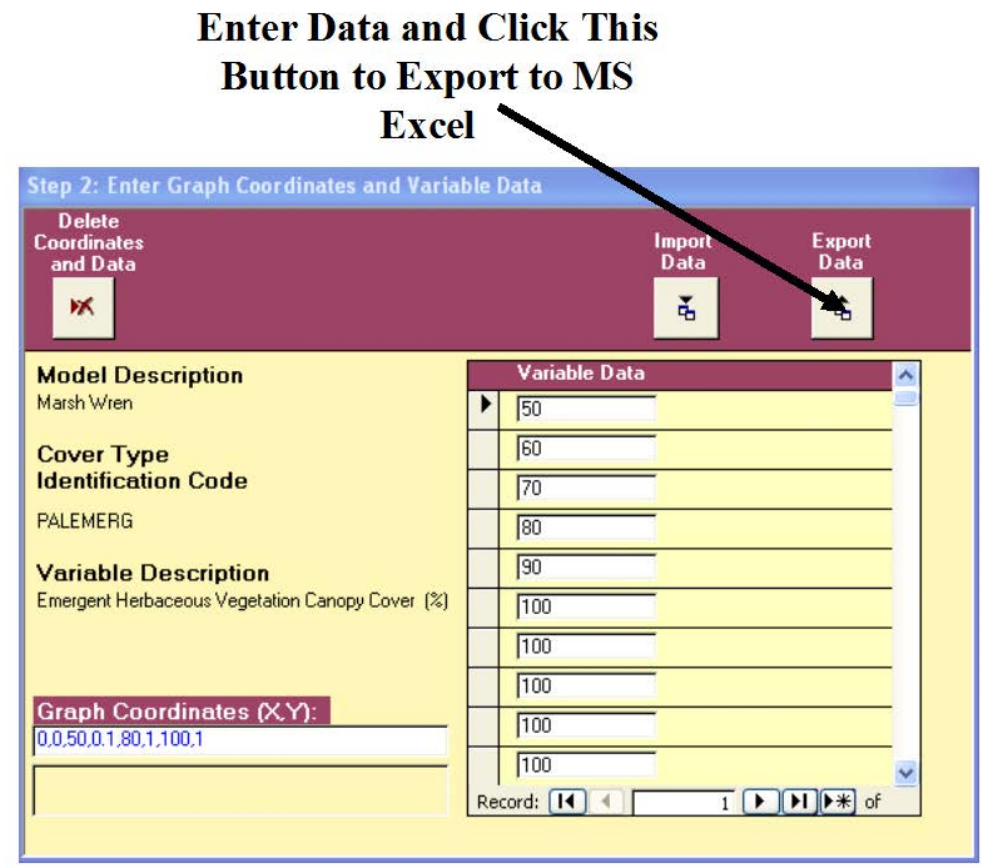

Figure 85. Exporting data from EXHEP to MS Excel - click the Export Data button.

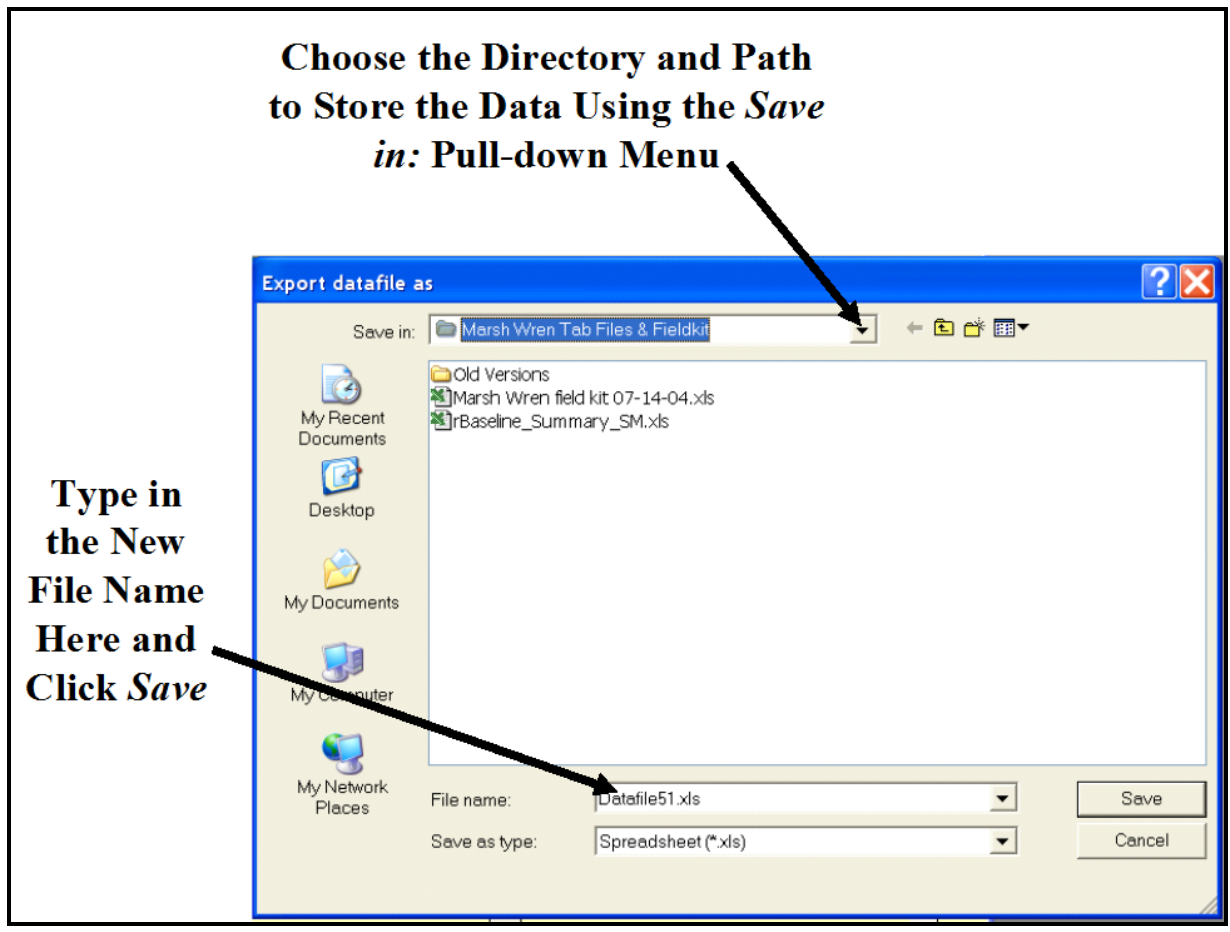

Figure 86. Exporting data from EXHEP to MS Excel - assign a directory and path for the new file. 
If EXHEP was able to export the data to MS Excel, a confirmation message is displayed. Click OK to close the message. EXHEP will return to the entry screens when finished. Select the next variable and cover type combination, and repeat the process until all field

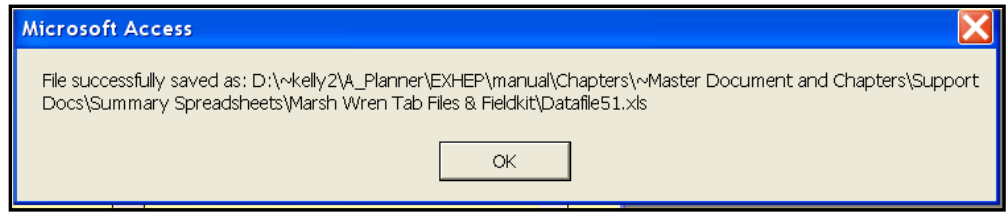
data have been entered, exported, and saved.

If the need should arise, delete an individual data point by clicking on the Record Indicator Bar to the immediate left of the data point and pressing Delete on the keyboard (Figure 87).

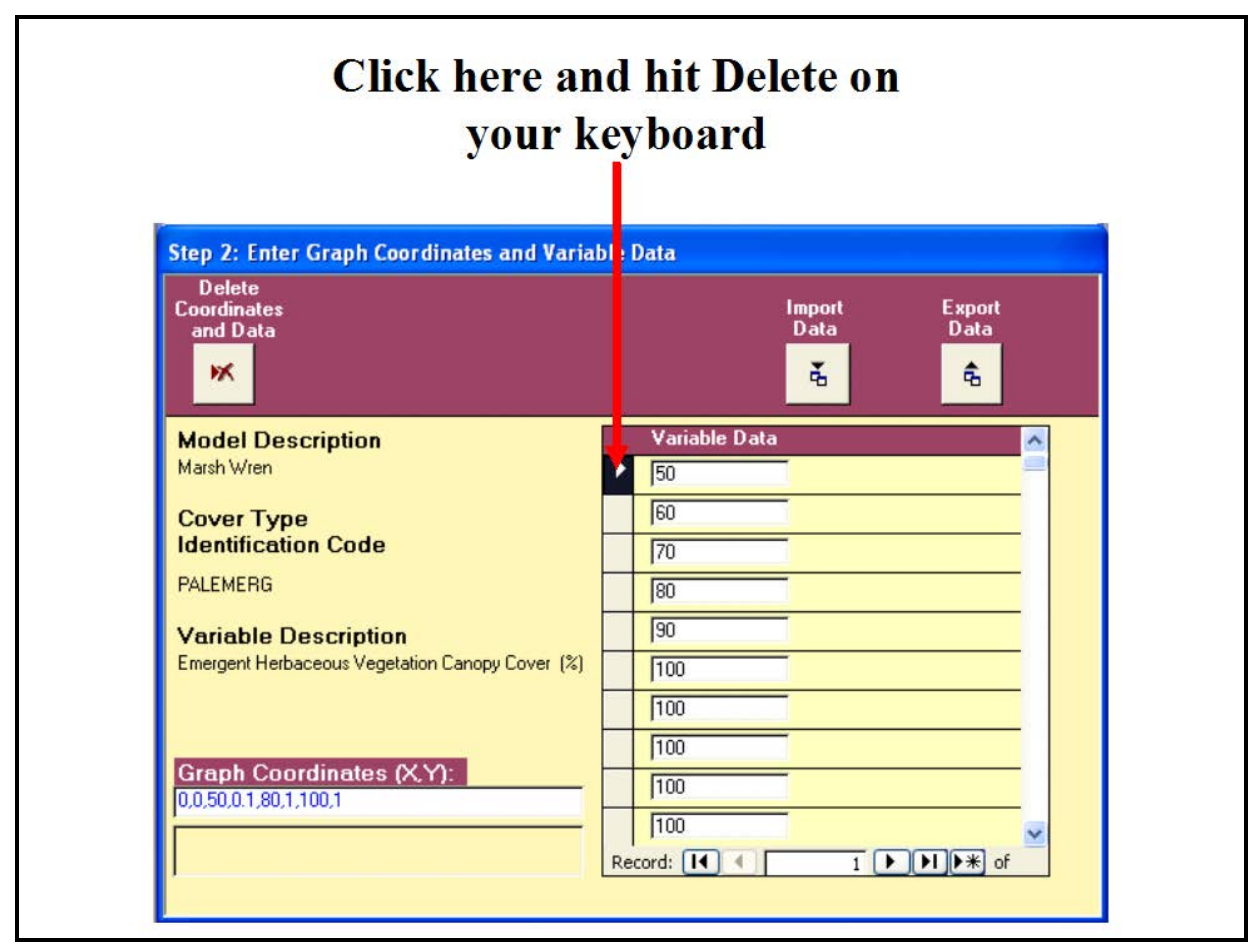

Figure 87. Deleting single data points in EXHEP. 
To delete the entire data set (if for example, a mistake was made and data were entered against the wrong variable or cover type), press the Delete Coordinates and Data button in the upper left-hand corner of the Step 2: Enter Graph Coordinates and Variable Data screen. Before deleting the entire data set and SI coordinates, EXHEP explains the implications of this action

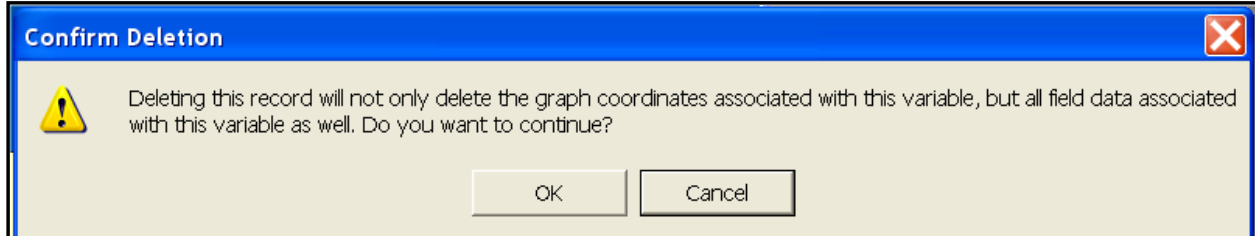

and provides an avenue to cancel the action or proceed. Click on $\mathbf{O K}$ to proceed with the deletion or Cancel to stop the deletion process. Once deleted, the information cannot be recovered from the EXHEP datafile, and will need to be reentered. When the data have been removed from the database, a status message appears. Click on $\mathbf{O K}$ to proceed with the data entry process.

Additional actions necessary to input data for multiple formula models

If multiple formula models are used in the analysis, the variable data must be entered using the second set of buttons on EXHEP's primary interface (Figure 88). 


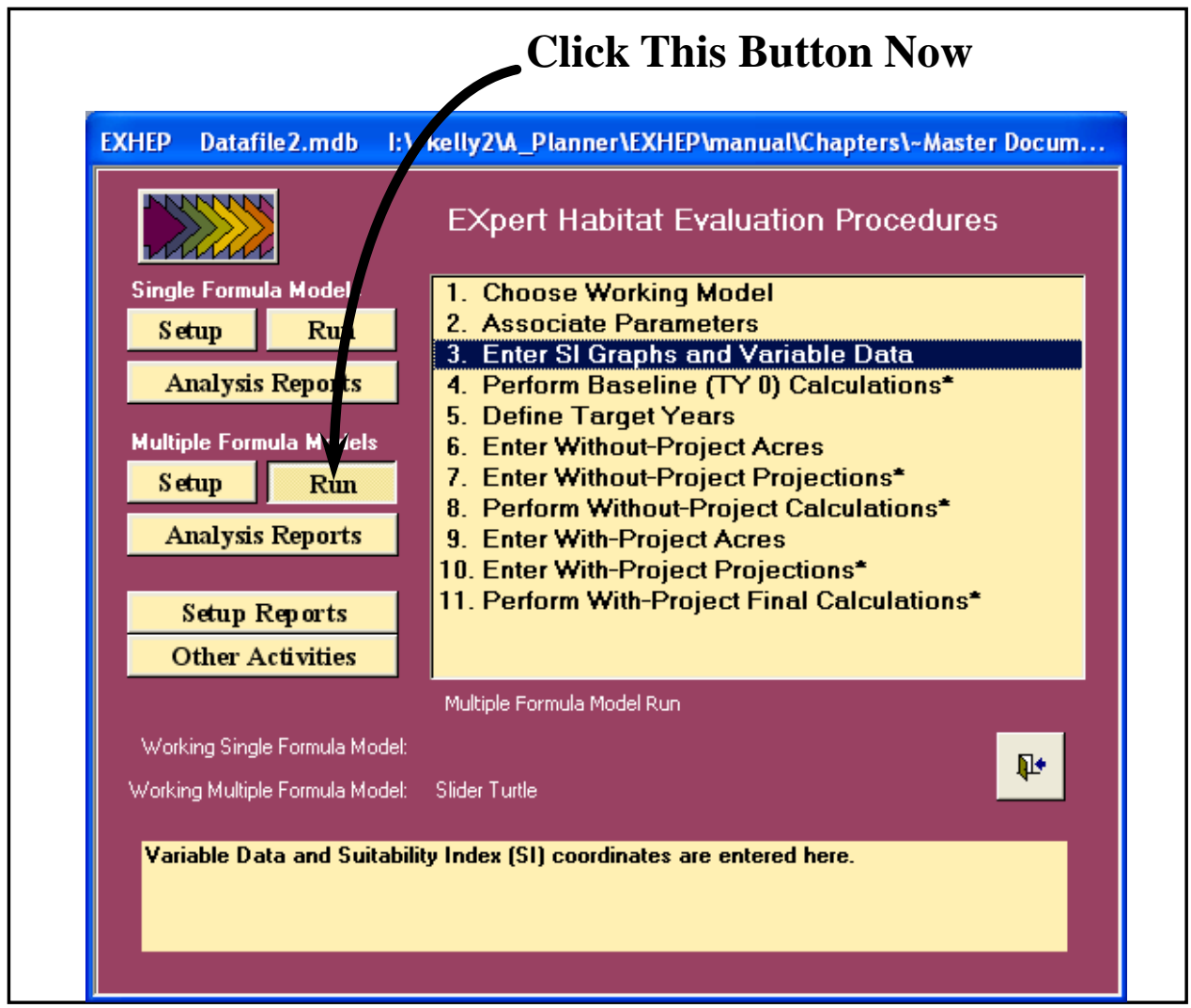

Figure 88. Click on the Run button to begin the multiple formula model baseline variable data entry process in EXHEP.

As it did before, a screen appears in the center of the primary interface with 11 options:

\section{Choose Working Model}

2. Associate Parameters

3. Enter SI Graphs and Variable Data

4. Perform Baseline (TY 0) Calculations*

5. Define Target Years

6. Enter Without-Project Acres

7. Enter Without-Project Projections*

8. Perform Without-Project Calculations*

9. Enter With-Project Acres

10. Enter With-Project Projections*

11. Perform With-Project Final Calculations*

Before continuing, verify that the correct "Working Model" has been selected (refer to Step 2: Model Association, Choose the Working Model above for assistance). Now click on the third option (Enter SI Graphs and Variable Data) and the following window will open (Figure 89). 


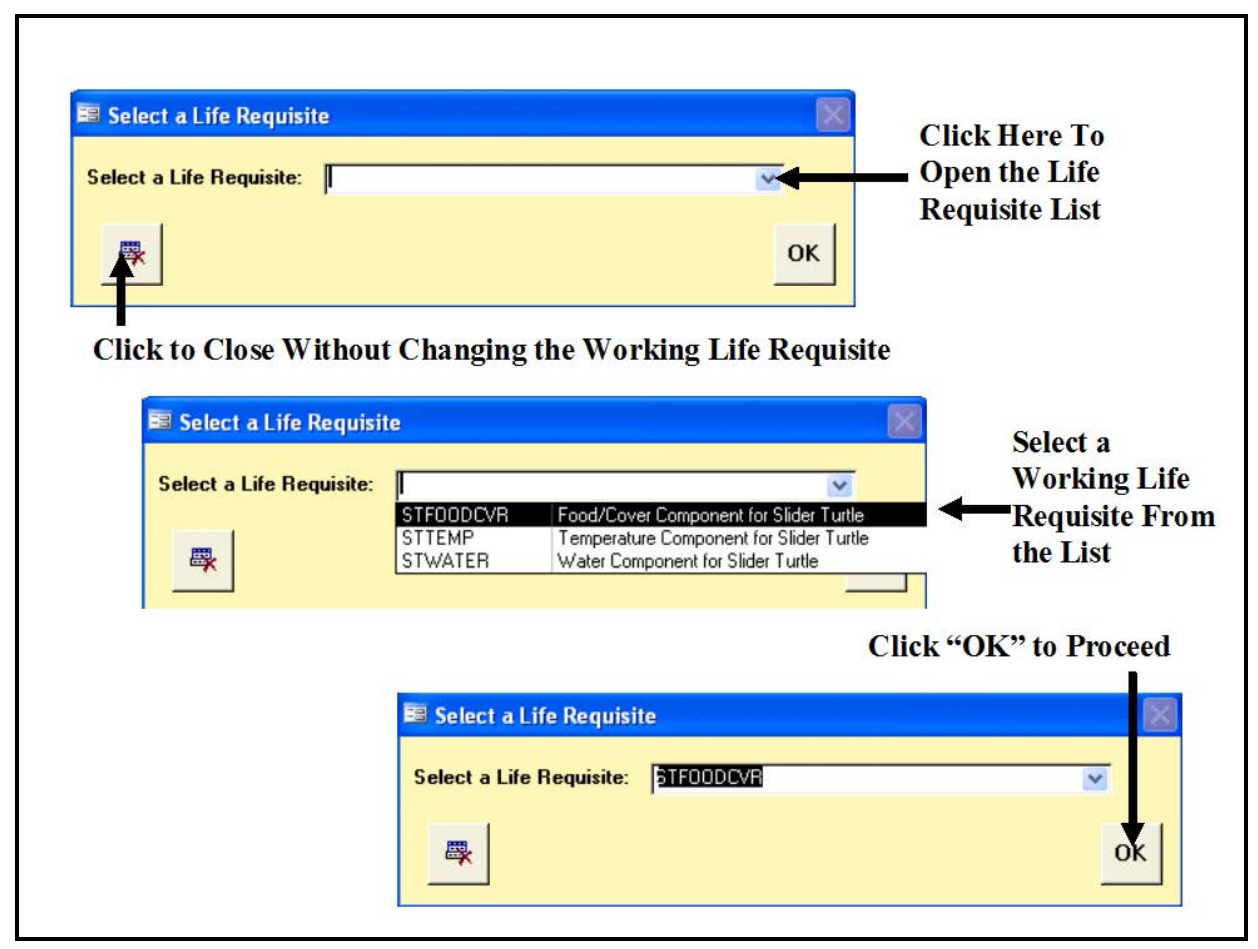

Figure 89. Select a Life Requisite window for multiple formula models in EXHEP.

The Select a Life Requisite window focuses the EXHEP engine on the working life requisite for model setup. Use the pull-down menu to the immediate right of the Select a Life Requisite window and select the first life requisite in the model. Click OK, and EXHEP will open the Step 1: Select Cover Types and Variable Combinations and Step 2: Enter Graph Coordinates and Variable Data forms just as it did for the single formula models. Use the same protocol described above in the single formula models section to enter data for the cover types and variables associated with this life requisite. When finished, close the forms and return to the primary interface. Repeat the process for each life requisite in every multiple formula model. 


\section{Checking the baseline data entry with EXHEP reports}

To review and check the entries made thus far, click on the Setup

Reports button on the primary interface (Figure 90).

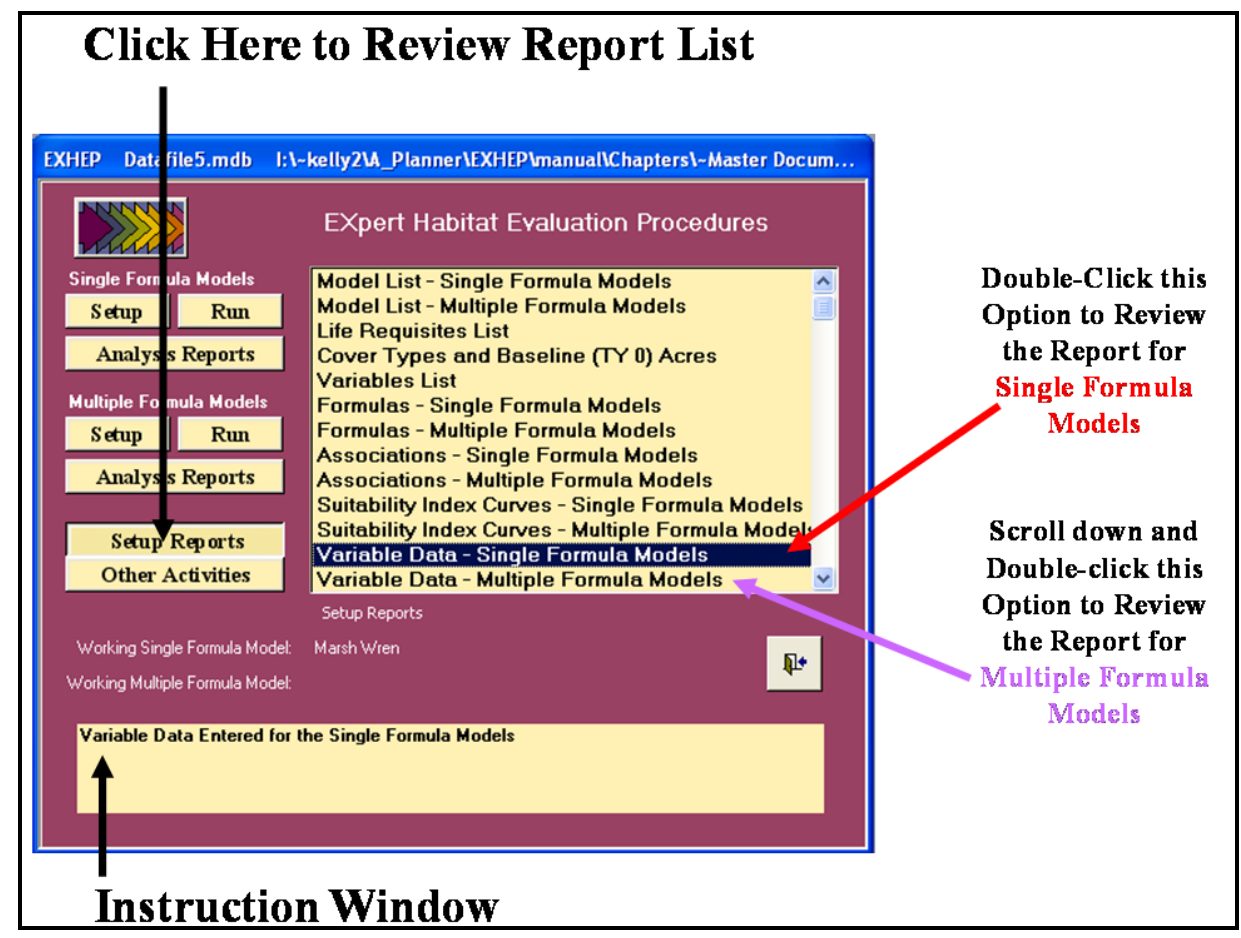

Figure 90. Click on the Setup Reports button to view the variable data reports in EXHEP.

A list of available reports appears in the center of the primary interface. Two additional reports should be populated by the time the data entry process is complete:

\section{- Variable Data - Single Formula Models - Variable Data - Multiple Formula Models}

Single-click on either report and look to the Instruction Window to check the contents of the report. Double-click on any report, and EXHEP opens a new window with the report inside (Figure 91). 


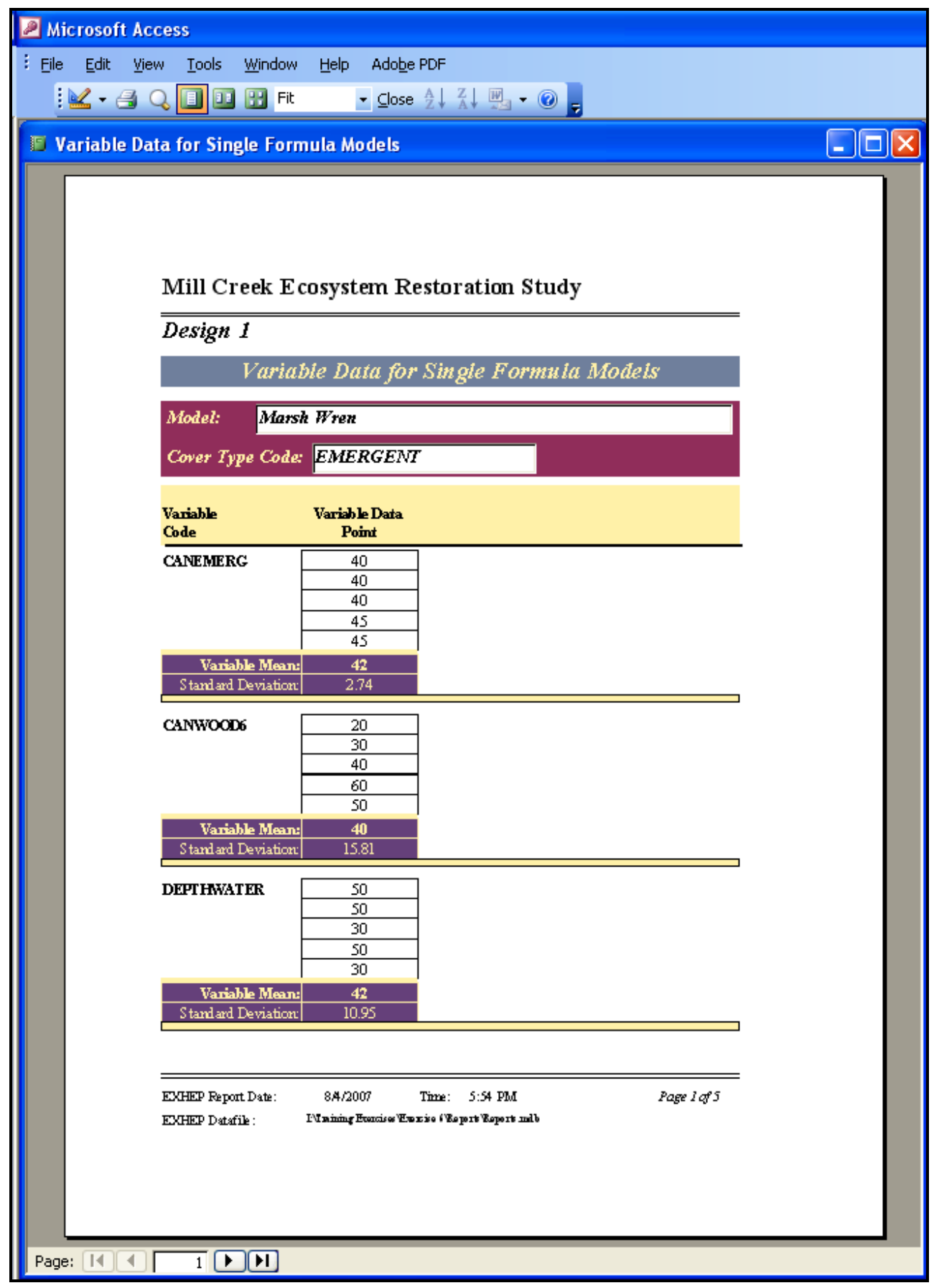

Figure 91. Example of Variable Data for Single Formula Models report in EXHEP.

Click on the Close button from the suite of buttons offered on the Report Control toolbar, and EXHEP returns to the primary interface. Now that the data entry process is complete, and the information has been confirmed, proceed to the next section to learn how to perform the baseline analyses in EXHEP. 


\section{Perform baseline (TY 0) calculation}

EXHEP will calculate the baseline results on a model-by-model basis for both single and multiple formula models. To proceed, click on the Run button under the Single Formula Models section of the primary interface (Figure 92).

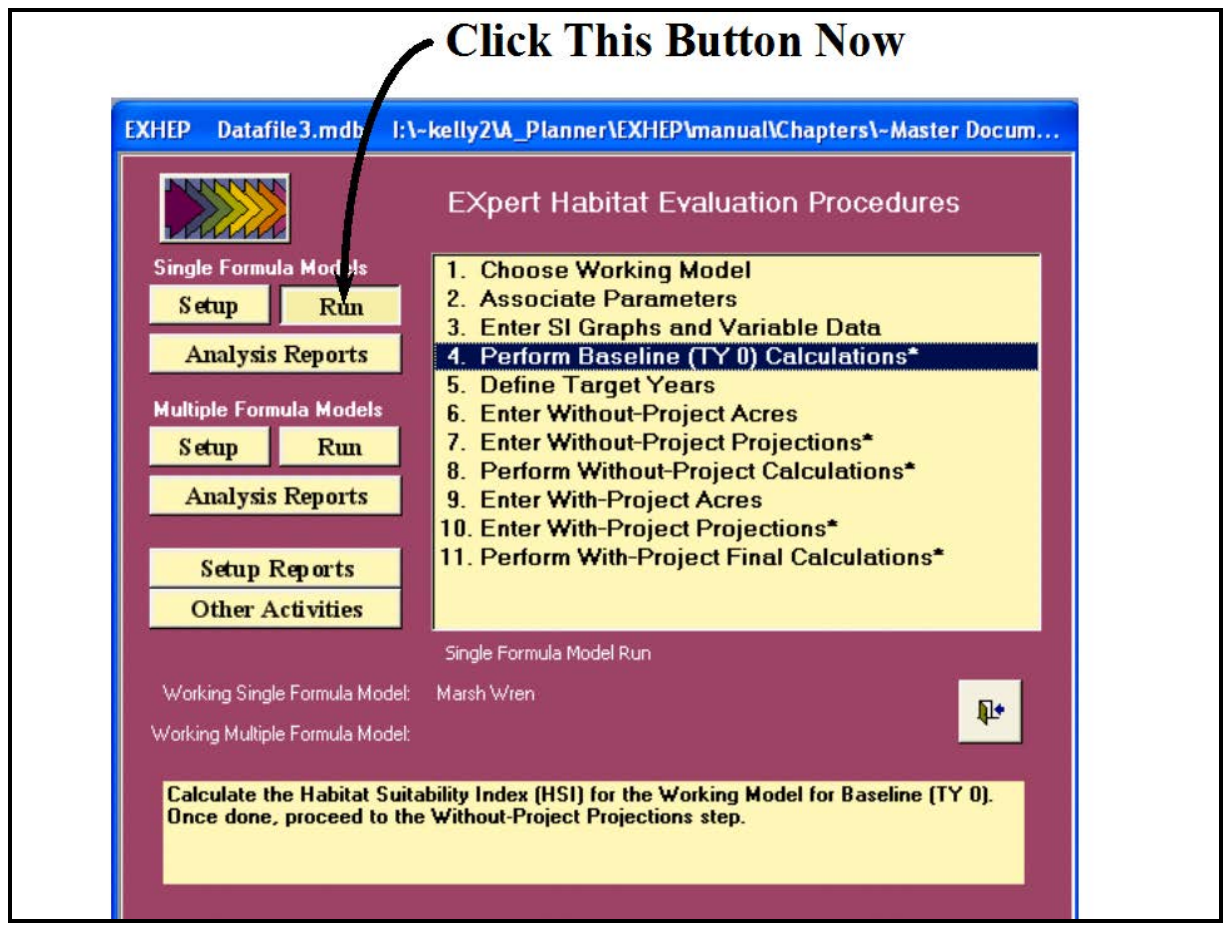

Figure 92. Click on the Run button to begin the baseline calculation process for the single formula models in EXHEP.

As it did before, a screen appears in the center of the primary interface with 11 options:

\section{Choose Working Model}

2. Associate Parameters

3. Enter SI Graphs and Variable Data

4. Perform Baseline (TY 0) Calculations*

5. Define Target Years

6. Enter Without-Project Acres

7. Enter Without-Project Projections*

8. Perform Without-Project Calculations*

9. Enter With-Project Acres

10. Enter With-Project Projections*

11. Perform With-Project Final Calculations* 
Check to assure that the "Working Model" is set to the correct model, and click on the fourth option [Perform Baseline (TY 0) Calculations*] to continue. In response, EXHEP will begin the calculation process. As the calculations con-

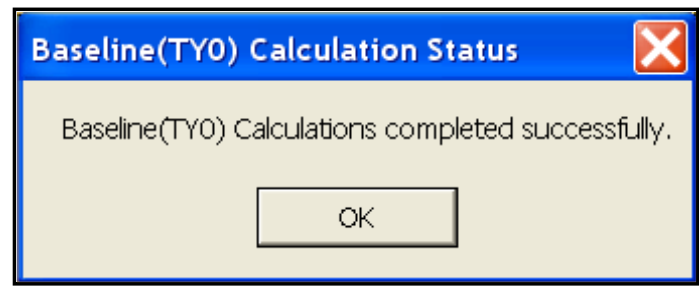
tinue, a Progress Indicator window will open in the screen. This progress window will track the status of the analysis (number of records to assess versus number of records processed) and provide a tentative estimate of Elapsed Time. When completed, EXHEP returns a message that the calculations are

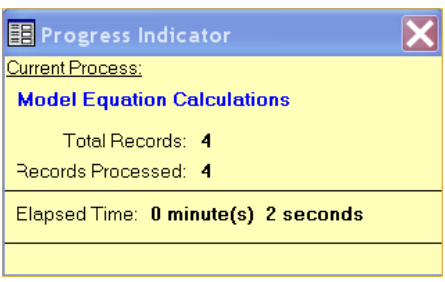
concluded.

Additional baseline entry/analysis actions for multiple formula models

If multiple formula models are used in the analysis, calculate baseline results for the models using the second set of buttons on EXHEP's primary interface (Figure 93).

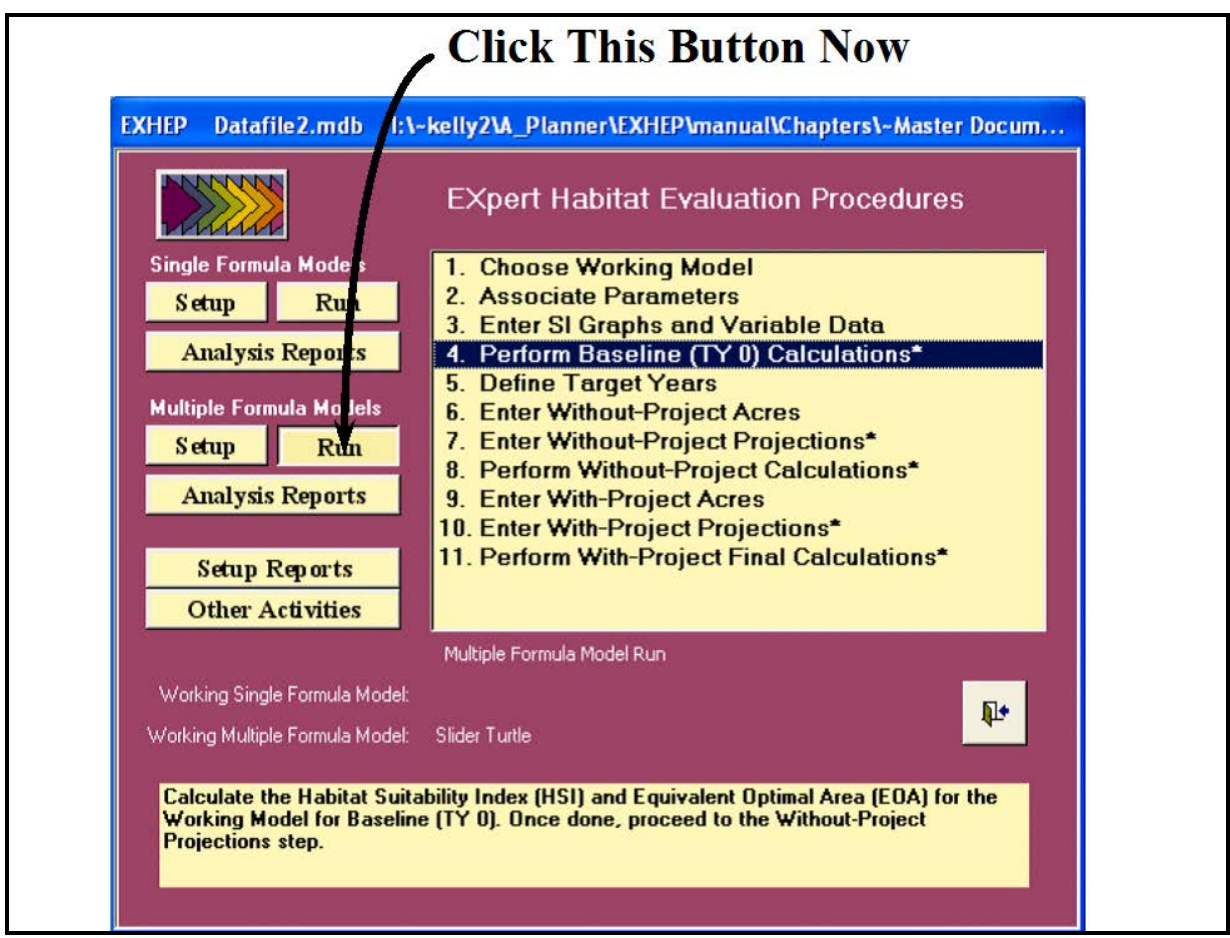

Figure 93. Click on the Run button to begin the baseline calculation process for multiple formula models in EXHEP. 
As it did before, the screen appears in the center of the primary interface with 11 options:

\section{Choose Working Model}

2. Associate Parameters

3. Enter SI Graphs and Variable Data

4. Perform Baseline (TY 0) Calculations*

5. Define Target Years

6. Enter Without-Project Acres

7. Enter Without-Project Projections*

8. Perform Without-Project Calculations*

9. Enter With-Project Acres

10. Enter With-Project Projections*

11. Perform With-Project Final Calculations*

Check to assure that the "Working Model" is set to the correct model, and click on the fourth option

[Perform Baseline (TY 0) Calculations*] to continue. In response, EXHEP will begin the calculation process. As the calculations continue, a

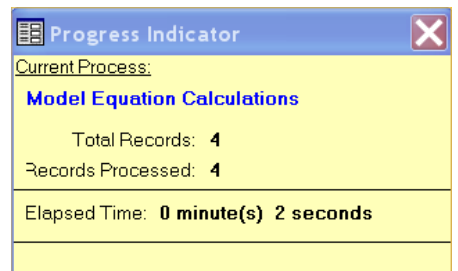
Progress Indicator window will open in the screen.

This progress window will track the status of the analysis

(number of records to assess vs. number of records processed) and provide a tentative estimate of Elapsed Time. When completed, EXHEP returns a message that the calculations are concluded.

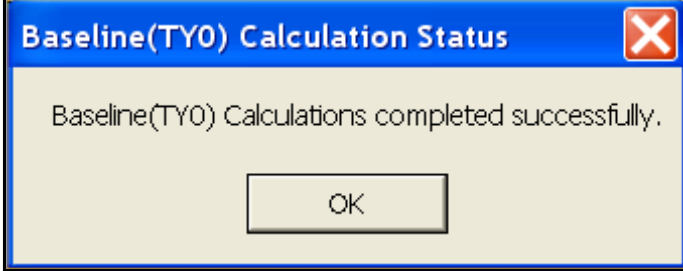

Checking the baseline analysis with EXHEP reports

To review and check the results, click on the Analysis Reports button(s) on the primary interface (Figure 94). 


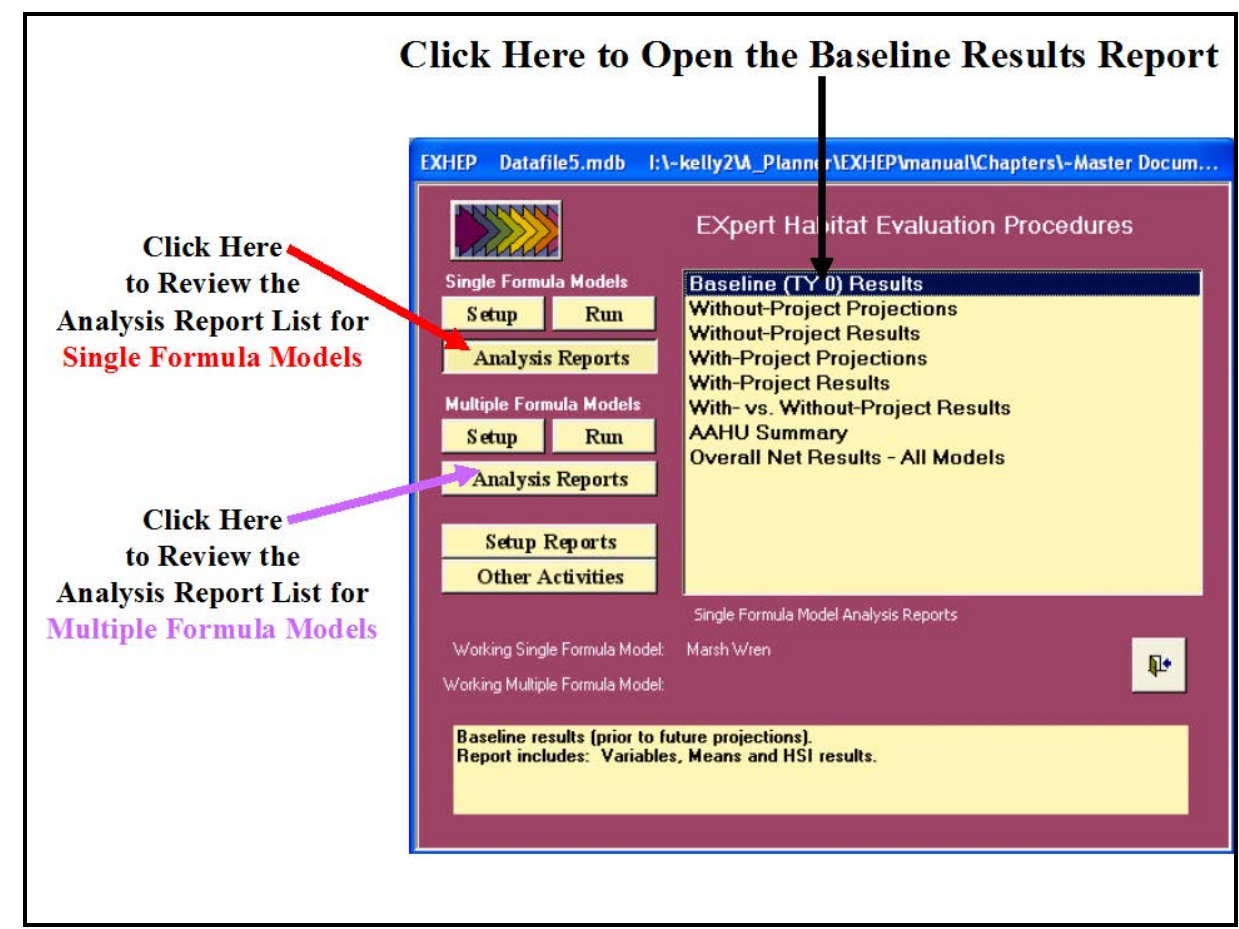

Figure 94. Click on the Analysis Reports buttons to review the baseline results in EXHEP.

A list of available reports appears in the center of the primary interface. The first report listed, Baseline (TY0) Results, is ready for inspection. Double-click on this report to review the results (Figure 95). 


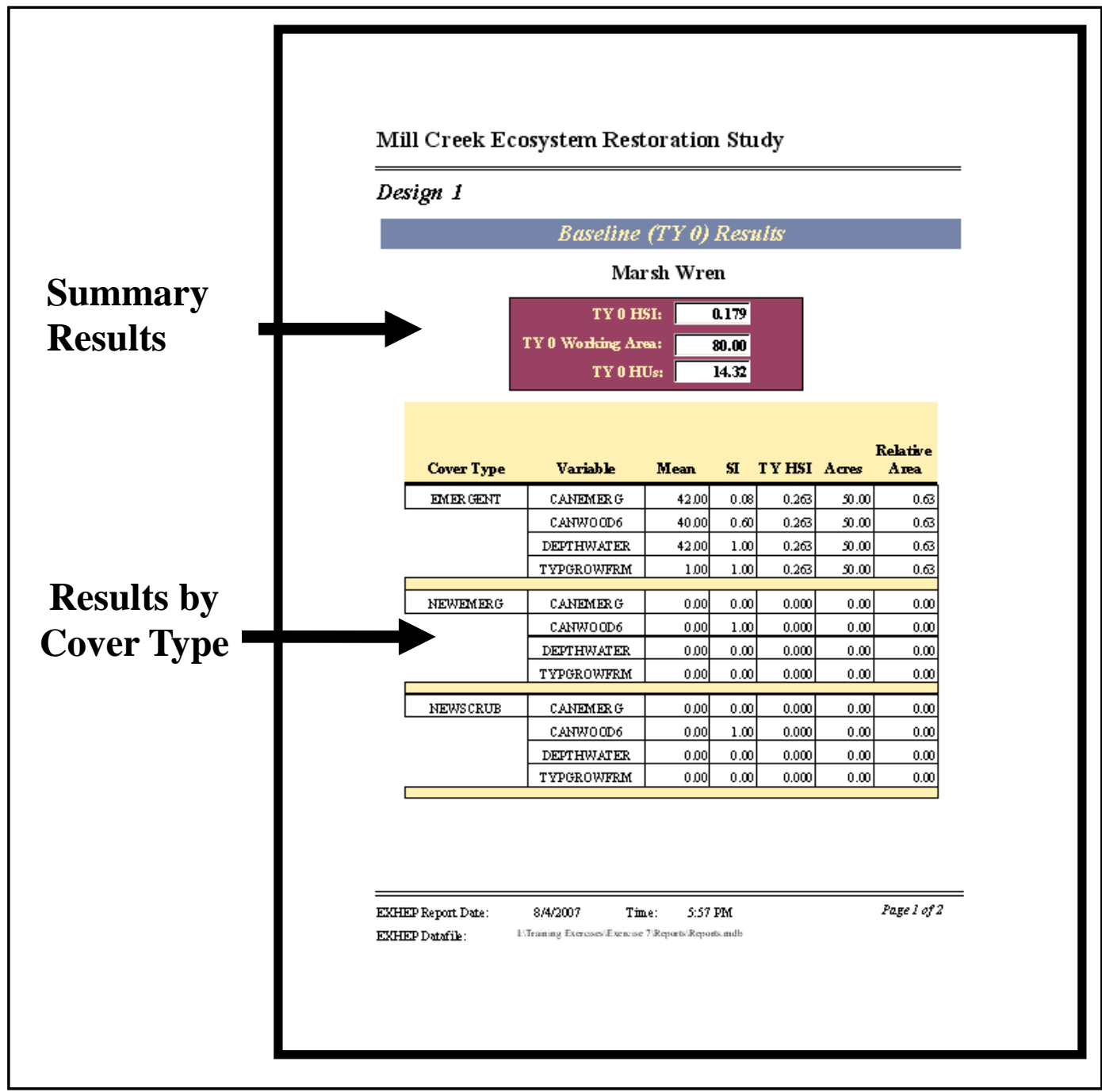

Figure 95. Example of the single formula model's Baseline (TYO) Results report in EXHEP.

The site's overall HSI score (i.e., TY 0 HSI), associated acres (i.e., TY 0 Working Area), and the resulting HU score (i.e., TY 0 HUs derived from the multiplication of the HSI score and the associated acres) are reported under the model name. The specifics used to generate the overall scores are reported in the tables below this summary. These details are sorted alphabetically by cover type and variable. This report is cumulative; thus, all single formula models assessed in the EXHEP analyses are presented in this report.

The multiple formula model's Baseline (TY 0) Results report looks similar to the earlier report (Figure 96). 


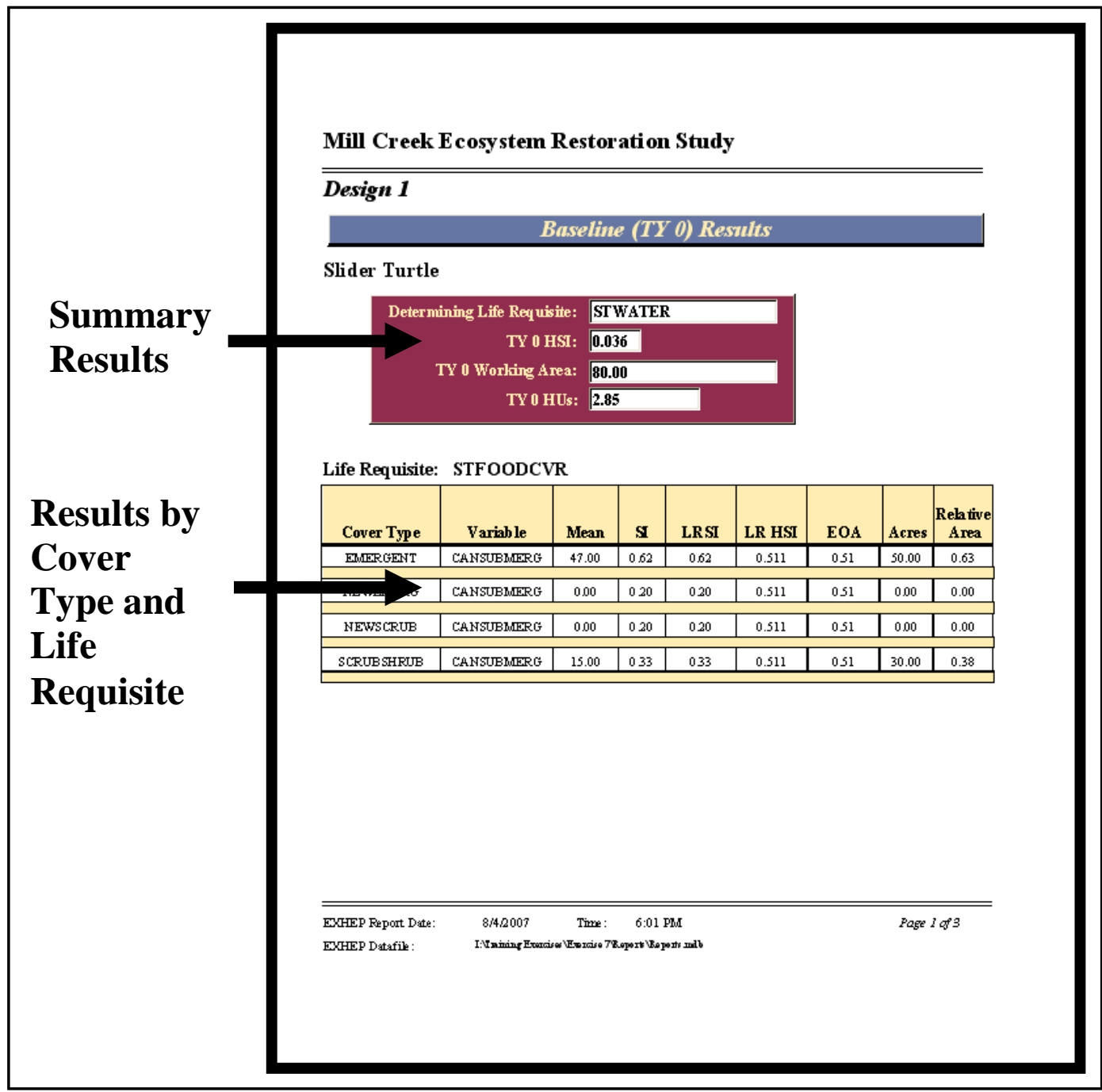

Figure 96. Example of the multiple formula model's Baseline (TYO) Results report in EXHEP.

Again, the site's overall HSI score (i.e., TY 0 HSI), associated acres (i.e., TY 0 Working Area), and the resulting HU score (i.e., TY 0 HUs derived from the multiplication of the HSI score and the associated acres) are reported under the model name. In addition, EXHEP indicates which life requisite was the determining factor in the generation of these scores (i.e., Determining Life Requisite). ${ }^{1}$ Again, the specifics used to generate the overall scores are reported in the tables below this summary. These details are sorted alphabetically by life requisite, cover type, and then by variable. This report is cumulative; therefore it includes all multiple formula models assessed in the EXHEP analyses.

\footnotetext{
1 If the HSI formula for the multiple formula model is not a discriminating formula (i.e., minimum or maximum of the LRSIs), then EXHEP has a default for this field - ALL EQUAL.
} 
Click on the Close button from the suite of buttons offered in the Report Control toolbar, and EXHEP returns to the primary interface. Now that the baseline assessment is complete, proceed to the next step to learn how to assess the without-project conditions of the site in EXHEP.

\section{Step 5: Enter the without-project conditions and calculate the effects}

Often confused with the terms "Baseline Condition" or "Existing Condition," the Without-Project Condition (WOP) is the forecasted change in site conditions over the life of the project when no action is taken to counteract or change the current situation, and is also referred to as the "No Action Alternative" or the "No Action Plan" in traditional planning studies. In order to capture the WOP conditions in EXHEP, significant points in time (i.e., target years) must be defined to compartmentalize and focus the evaluation on critical temporal dimensions across the life of the project. These "target years" (TYs) then become the focus of the forecasting effort - predictions of site conditions are scaled to these snapshots in time. In HEP there can be no less than three TYs, and they are by definition:

- $\mathrm{TY}_{0}$ - baseline

- $\mathrm{TY}_{1}$ - first year of construction/ restoration

- $\mathrm{TY}_{\mathrm{X}}$ - last year of project life or evaluation period

Between $\mathrm{TY}_{1}$ and $\mathrm{TY} \mathrm{X}$, it is not uncommon for the team to add additional TYs to capture changes in vegetative cover and structure or other parameters resulting from anticipated impacts or proposed designs. ${ }^{1}$

To quantify the projected change to the site if no action is taken, both without-project acreage and habitat quality must be documented and incorporated into the evaluation. "No Action" might imply that the site will not change over time - this is not necessarily the case. Often, vegetation matures and succeeds over time. Or artificial constructs (e.g., dams, levees, ditches, etc.) degrade the habitat over time. The timing, nature, and

1 The authors wish to offer a precautionary note - the selection of too few TYs can result in a coarse analysis insensitive to key ecological/physical changes in the environment. The selection of too many TYs can lead to excessive data handling, often with no return on investment. Five TYs spread over the course of 50 years offers a reasonable expectation of return for the level of data analysis and management required. 
magnitude of these changes must be quantified before the team can devise an alternative future for the site. ${ }^{1}$

\section{Without-project forecasting and data entry}

In EXHEP, the projected without-project changes to the site are undertaken first by defining target years and forecasting alterations of aerial extent given this timeframe. These changes in space and time are then coupled with changes in physical and biological conditions along this space-time continuum to characterize the quality and magnitude of change for the site under the without-project paradigm.

Enter, change, or delete the target years

To proceed, first click on the Run button under the Single Formula Models section of the primary interface (Figure 97).

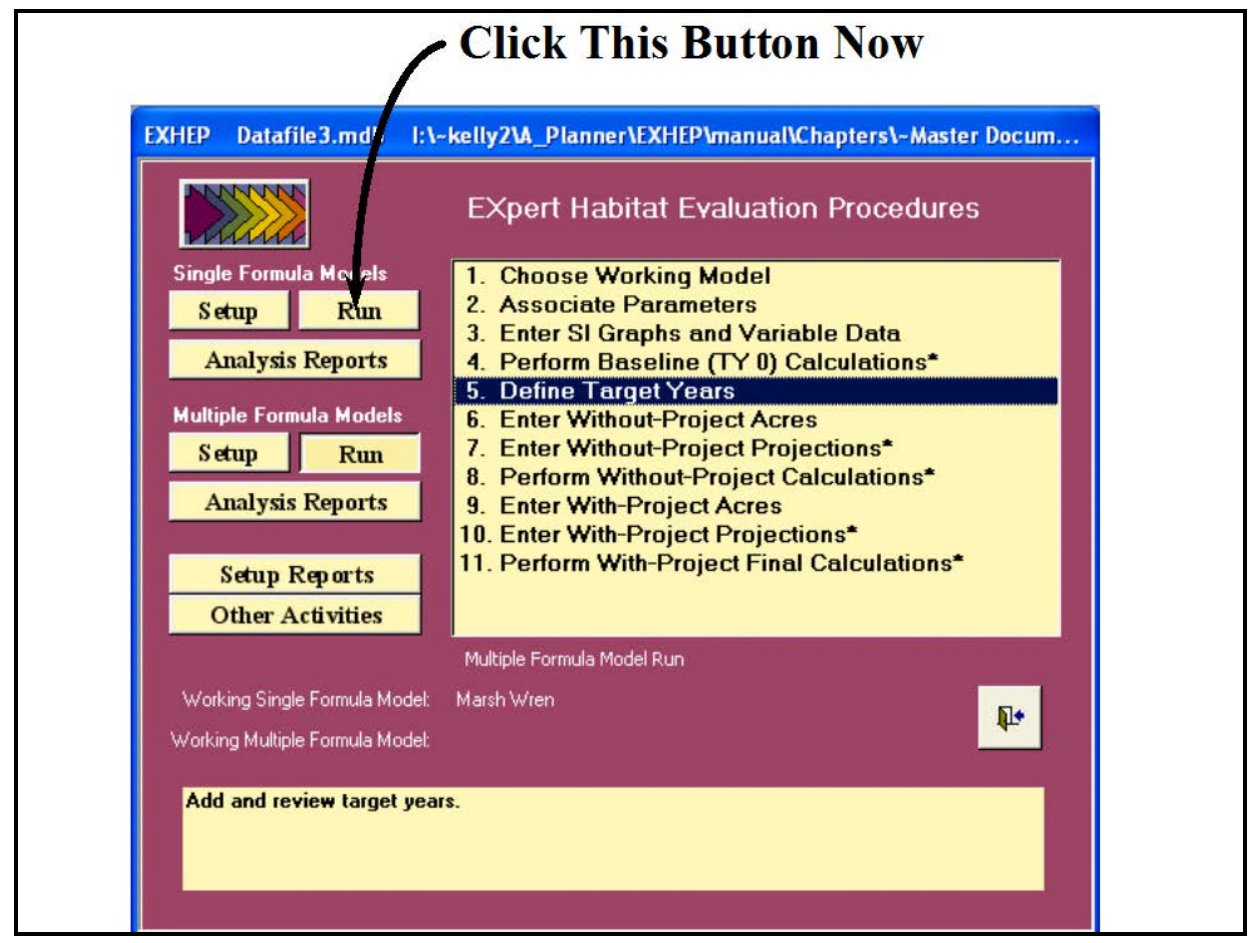

Figure 97. Click on the Run button to begin the target year entry process for the single formula models in EXHEP.

As it did before, a screen appears in the center of the primary interface with 11 options:

\footnotetext{
1 To assist in the software application, a series of training exercises have been included in Appendix E refer to Exercise 7 for relevant training in without-project data entry and analysis in EXHEP.
} 
1. Choose Working Model

2. Associate Parameters

3. Enter SI Graphs and Variable Data

4. Perform Baseline (TY 0) Calculations*

5. Define Target Years

6. Enter Without-Project Acres

7. Enter Without-Project Projections*

8. Perform Without-Project Calculations*

9. Enter With-Project Acres

10. Enter With-Project Projections*

11. Perform With-Project Final Calculations*

Check to assure that the "Working Model" is set to the correct model, and click on the fifth option (Define Target Years) to continue. In response, EXHEP opens the following window (Figure 98).

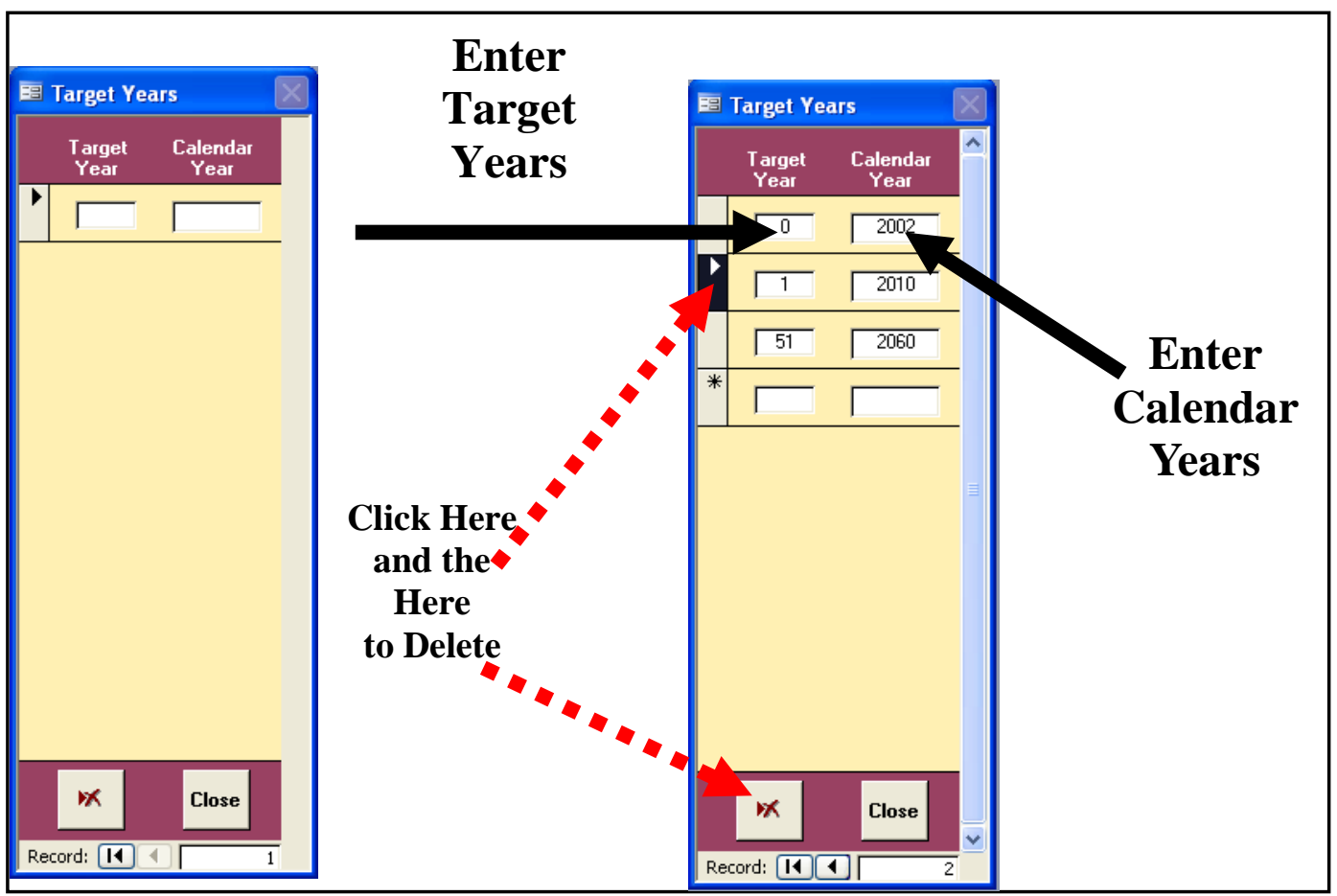

Figure 98. Define the target years in EXHEP.

The cursor is automatically placed in the first entry space in the left-hand column (Target Years).

Type in the first target year (i.e., this should be “0”) and hit the Tab button on the keyboard to move to

USER'S NOTE:

The EXHEP software uses the TYs to calculate annualized outputs - not the calendar years. the right-hand column. Now enter the corresponding calendar year in this 
column. Hit Enter on the keyboard to accept the entry. Repeat the process for each set of TYs and calendar years. ${ }^{1}$

If for some reason a TY must be modified, it must be completely erased from the database and re-entered. To do this, click once on the Record Indicator Bar (i.e., the square to the immediate left of the TY) and press the Delete button at the bottom of the form (left-hand corner). A warning message is displayed asking to continue or cancel the action.

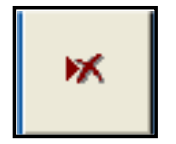

\section{Microsoft Office Access}

This will delete all data related to this target year from the database. Do you wish to continue?

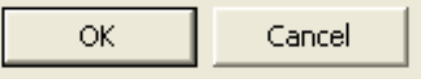

The removal of a TY is a very serious undertaking in EXHEP - one click of the OK key removes every record associated with the TY throughout the database. Click OK to continue, or Cancel to halt the process. Either way, the software returns to the Target Year Description entry screen, where the TY correction can be entered and/ or the data entry can be completed.

When finished, click on the Close button. EXHEP offers a reminder to enter future conditions into the system to support the TY identification,

You have added target years - be sure to forecast future conditions (variables and acres) for each Target Year.

\section{OK}

so users can click the $\mathbf{O K}$ button and proceed to the next section (i.e., Enter and/ or modify without project acres) to focus on the withoutproject forecasting task. But first, it is important to note that the TYs assigned to the "Working Model" apply to all models in the software (both single and multiple formula model types). Therefore it is only necessary to

\footnotetext{
1 As is evidenced in the examples above, a period of construction activity is often needed to implement the designs, and the sites are not ready for assessment for several calendar years (i.e., 8 years in the example). In HEP, it is as if those years do not count - they are condensed between $\mathrm{TY}_{0}$ and $\mathrm{TY}_{1}$. The period of analysis pertinent to HEP begins at the end of the construction phase and ends with the end of the project life (i.e., oftentimes 50 years later). Thus it is not unusual to find TYs and calendar years "out-of-sync" so to speak.
} 
enter the TYs once - they will be carried into the analysis for the remaining models. If there are no single formula models in the analysis, the TYs may be entered via the Run button under the Multiple Formula Models section of the primary interface. Simply follow the steps described above to complete the entries as needed, and proceed to the next section to continue with the forecasting activities.

Enter, change, or delete the without-project acres

Now click on the Run button under the Single Formula Models section of the primary interface (Figure 99).

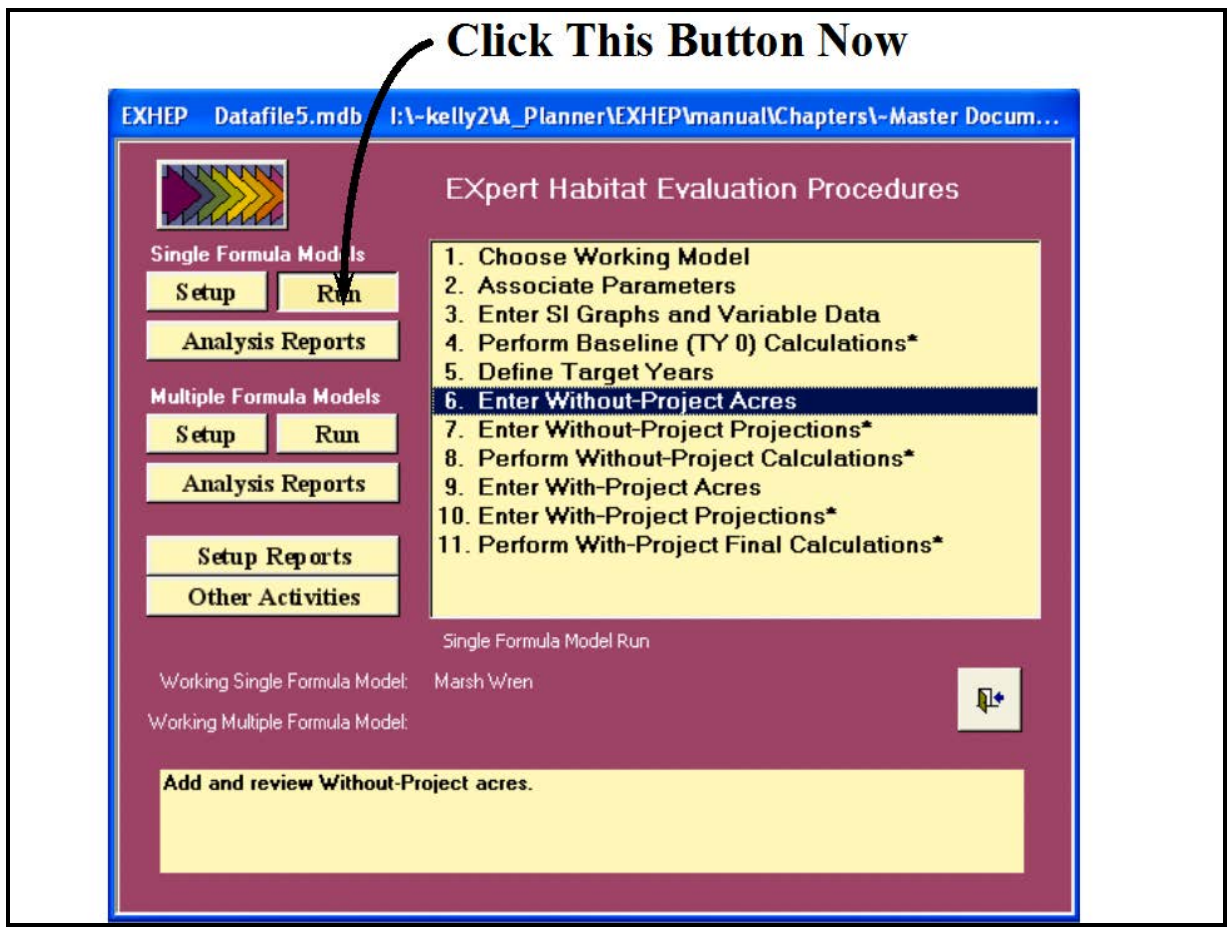

Figure 99. Click on the Run button to begin the without-project acres entry process for the single formula models in EXHEP.

As it did before, a screen appears in the center of the primary interface with 11 options:

\section{Choose Working Model}

2. Associate Parameters

3. Enter SI Graphs and Variable Data

4. Perform Baseline (TY 0) Calculations*

5. Define Target Years

6. Enter Without-Project Acres 


\section{Enter Without-Project Projections*}

8. Perform Without-Project Calculations*

9. Enter With-Project Acres

10. Enter With-Project Projections*

11. Perform With-Project Final Calculations*

Check to assure that the "Working Model" is set to the correct model, and click on the sixth option (Enter Without-Project Acres) to continue. In response, EXHEP opens the following window (Figure 100).

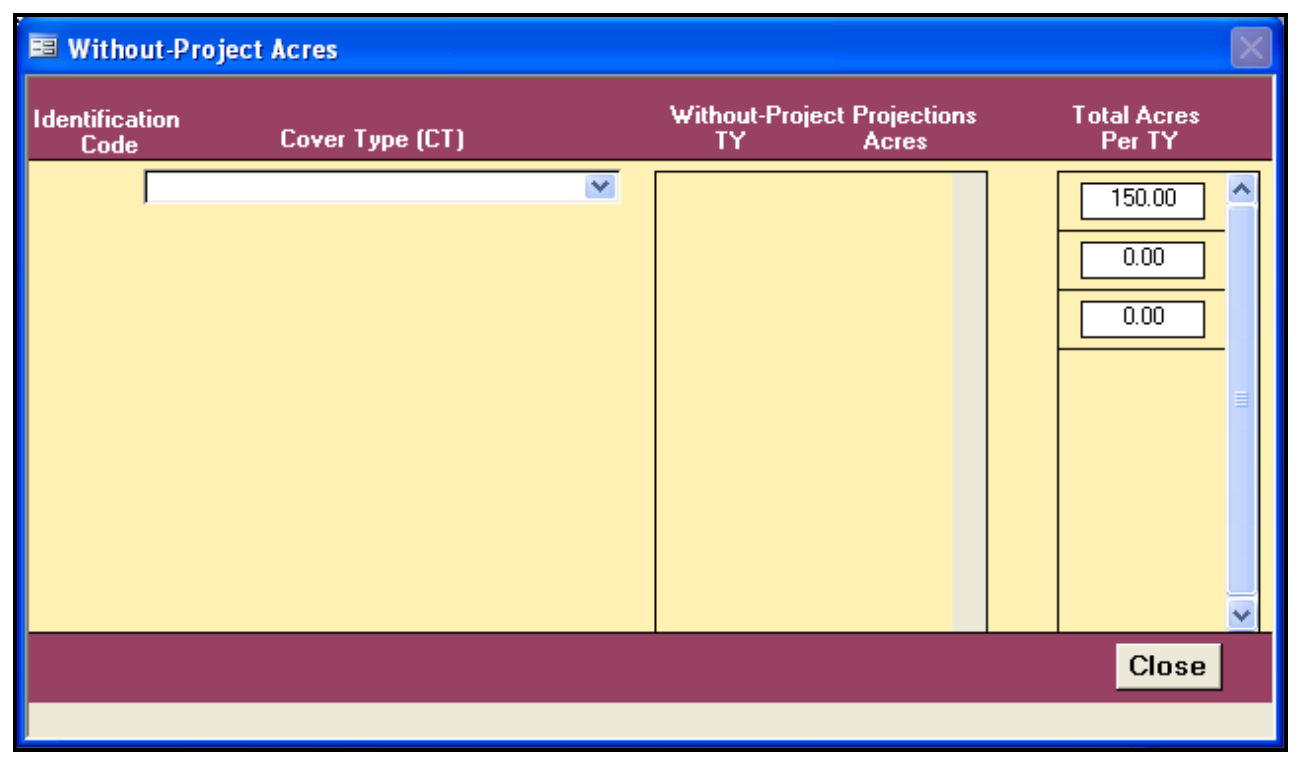

Figure 100. Without-Project Acres entry window for single formula models in EXHEP.

Click once on the Cover Type (CT) pull-down menu and select the first cover type from the list of available habitat types. EXHEP queries the database for target years and baseline acres (based on the entries made in the steps above), and presents these in a tabular format in anticipation of projected future without-project conditions (Figure 101). 


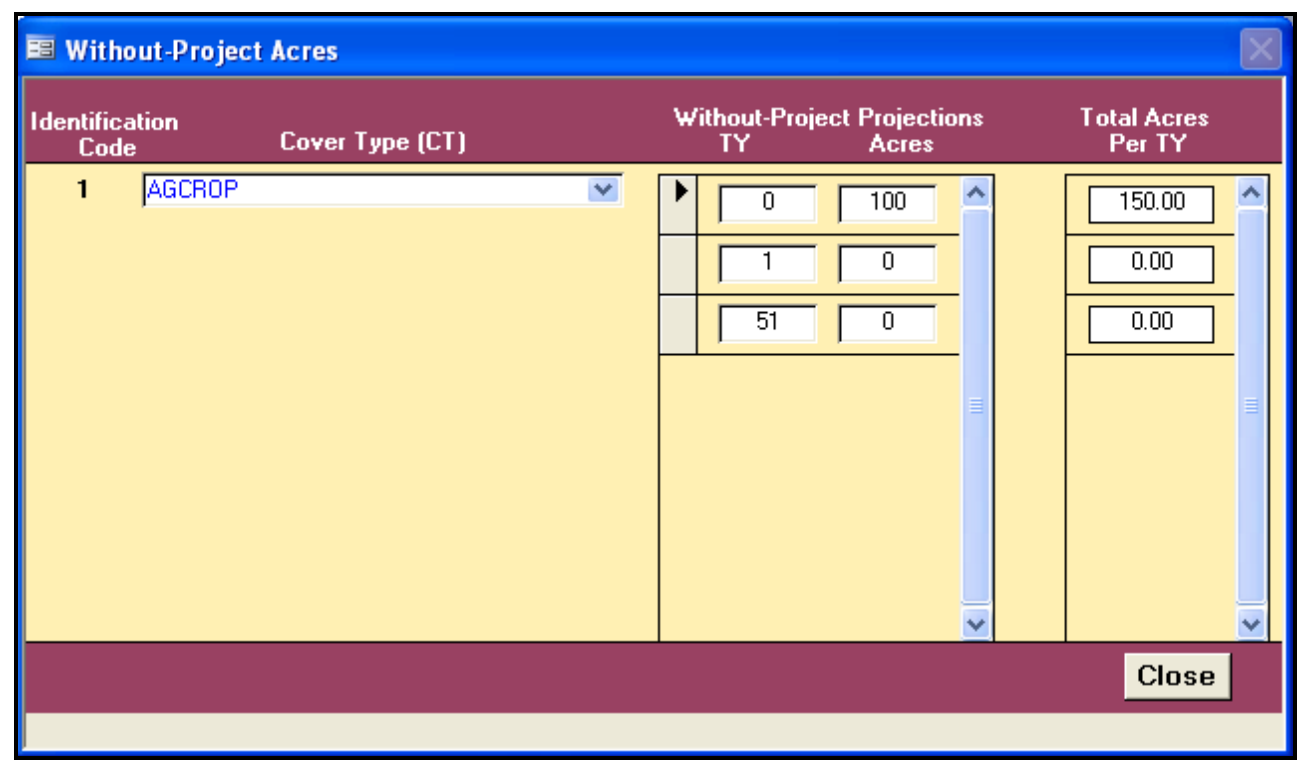

Figure 101. Select a cover type from the pull-down menu, and EXHEP populates the entry form.

Note that target years themselves cannot be modified in this interface. To make a target year change, return to option 5. Define Target Years and follow the directions above to modify these values. It is also important to note that the system's query automatically populated the acreages in the rows of this table. Modifications to the baseline (TY $\mathbf{0}$ ) acreages cannot be made in this interface. Return to the setup interface to make these acreage changes (refer to the "Step 2: Model setup" section above for details). Zeroes are used as defaults in all outlying years for the acreages of this habitat.

Now forecast and enter the number of without-project (WOP) acres for this habitat in the cells to the right of the corresponding target years by highlighting and typing in the correct acres for that timeframe (Figure 102).

Hit Enter once on the keyboard to accept the change, and hit Enter a second time to move down to the next row. Continue to the end of the column, at which point hitting Enter a final time on the keyboard will complete the process for this cover type. Systematically "select" the remaining cover types one by one from the pull-down menu, forecast their acreages, and enter these values into the software. All cover types must be accounted for in this activity - even if they are not associated with the models in the assessment. 


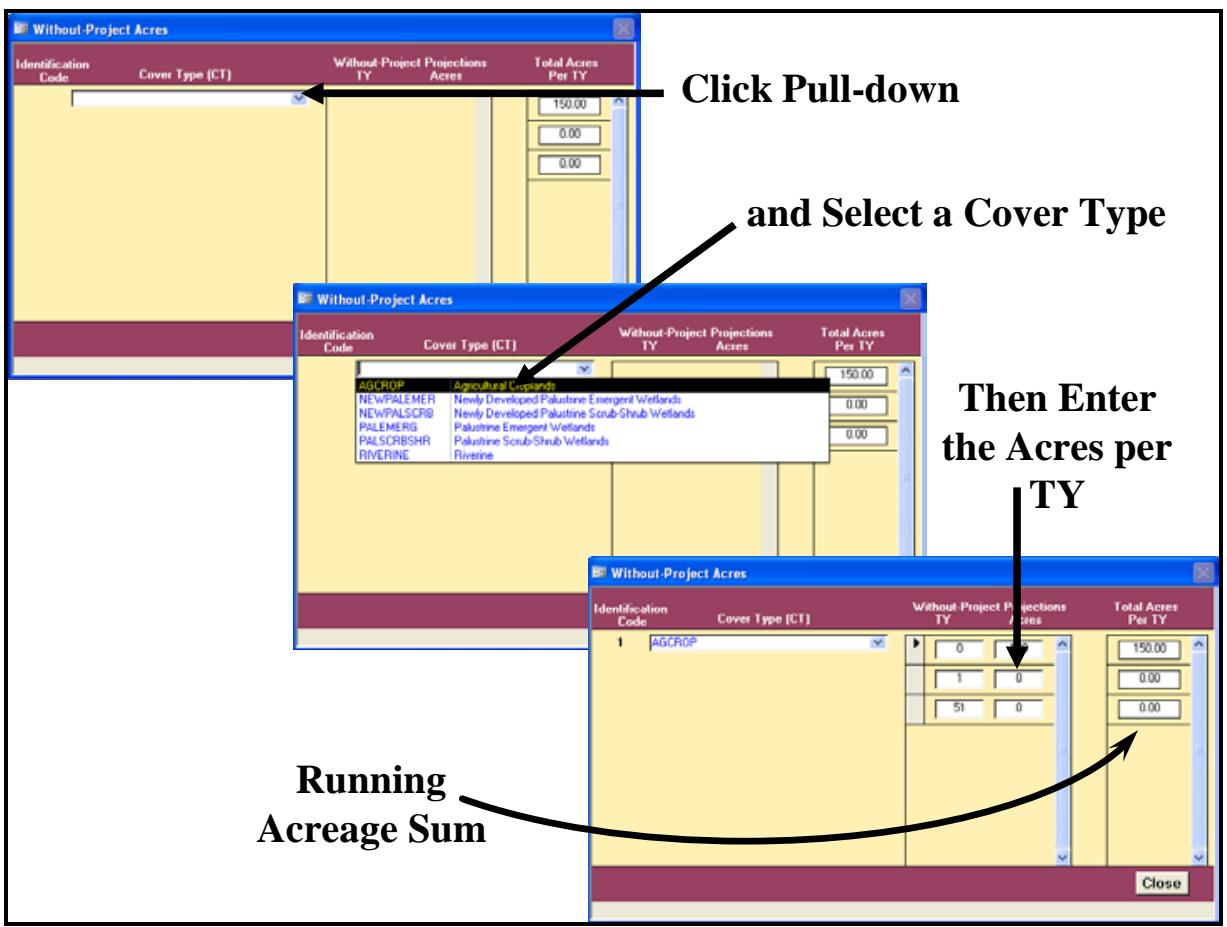

Figure 102. Enter the without-project acreage projections into the cells provided to the right of their corresponding target years.

A running sum of acres on the site is calculated by TY and presented in the third column running along the right-hand side of this form (Total Acres per TY). At the end of this exercise, the numbers in each row of this column should be the same (i.e., if there are 100 acres at baseline, there are 100 acres at the end of the study), or a problem has occurred in the entry process. Revisit and check the entries in each cover type to find and correct any errors. If at any time acreages must be modified, simply return to this interface, highlight the acreage that must be changed, type in the new values, and hit Enter twice on the keyboard to accept the change.

\section{USER'S NOTE:}

Inevitably, acreages and/or data are modified throughout the planning of a study. Moving in and amongst models can lead to a break in the system's focus on a particular "Working Model." If the focus has changed, and the data have been changed (i.e., baseline acres, target tears, WOP acres, etc.), it is prudent to re-run the starred $(*)$ options in the primary interface [i.e., in particular 4. Perform Baseline (TY 0) Calculations* at this point in time].

\section{USER'S NOTE:}

WOP acres assigned to the "Working Model" apply to all models in the software (both single and multiple formula model types). Therefore it is only necessary to enter the WOP acres once - they will be carried into the analysis for the remaining models. 
When finished, click on the Close button, and EXHEP returns to the primary interface. If there are no single formula models in the analysis, the without-project acres may be entered via the Run button under the Multiple Formula Models section of the primary interface. Simply follow the steps described above to complete the entries as needed. Now proceed to the next section to check the entries and focus on the withoutproject forecasting task.

Checking the without-project target years and without-project acres with EXHEP reports

To review and check the target year and acreage entries made thus far, click on the Setup Reports button on the primary interface (Figure 103).

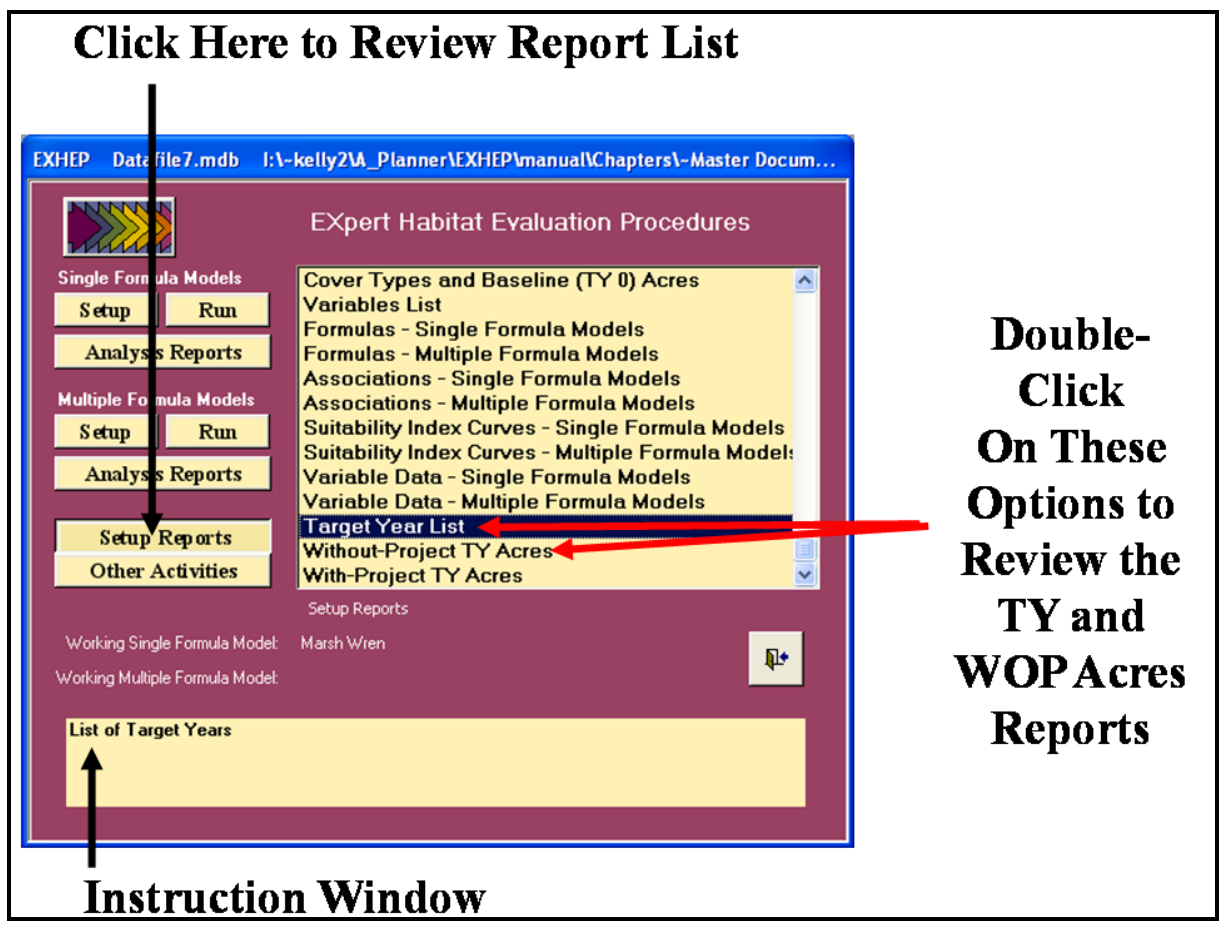

Figure 103. Click on the Setup Reports button to view the Target Year List report in EXHEP.

Two additional "setup" reports should be populated at this point:

- Target Year List

- Without-Project TY Acres 
Single-click on either report and look to the Instruction Window to check the contents of the report. Double-click on either report, and EXHEP opens a new window with the report inside (Figure 104).

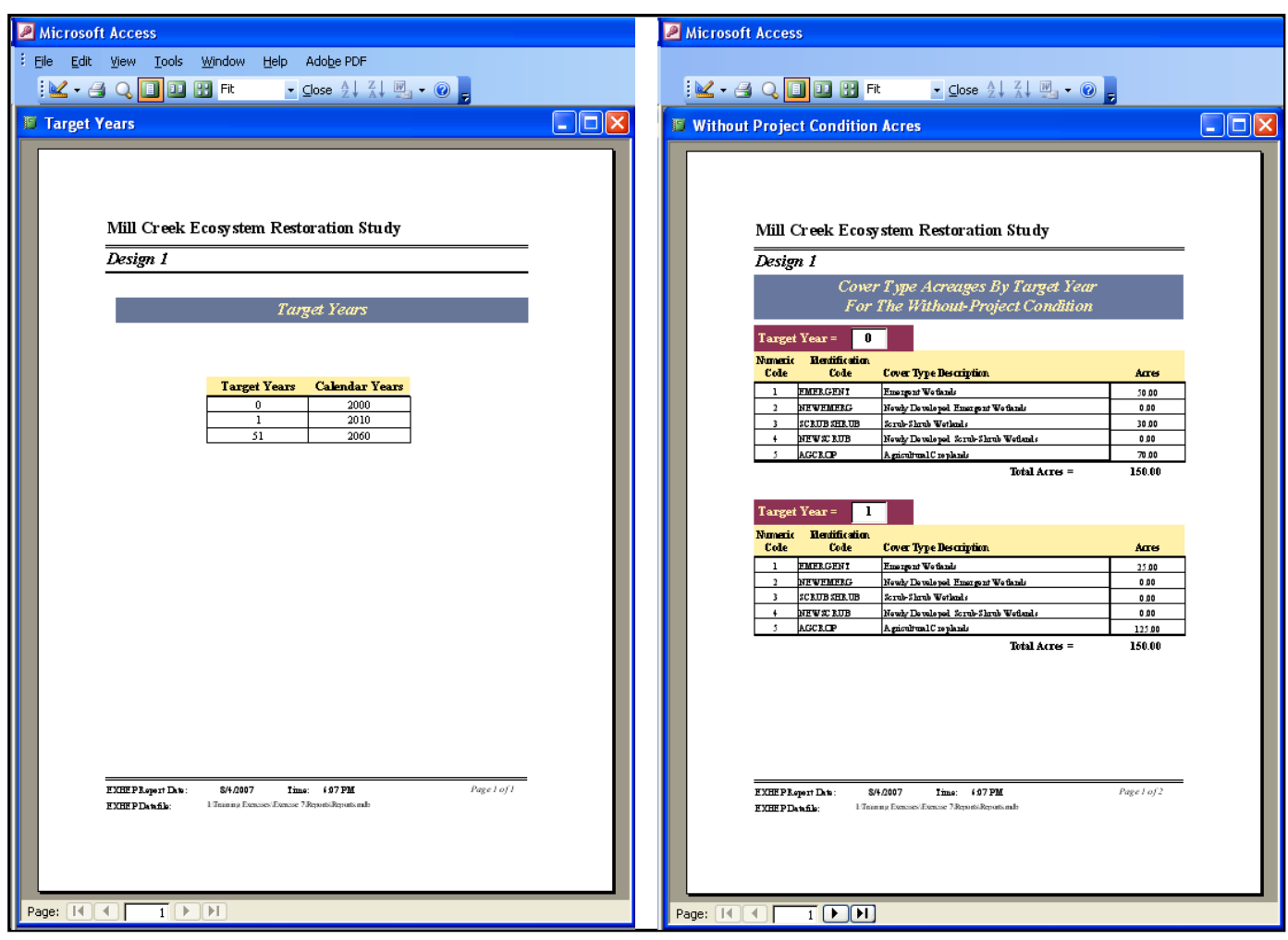

Figure 104. Example of Target Year List and Without-Project TY Acres reports in EXHEP.

Click on the Close button from the suite of buttons offered on the Report Control toolbar, and EXHEP returns to the primary interface.

Enter the without-project variable projections

Now click on the Run button under the Single Formula Models section of the primary interface (Figure 105). 


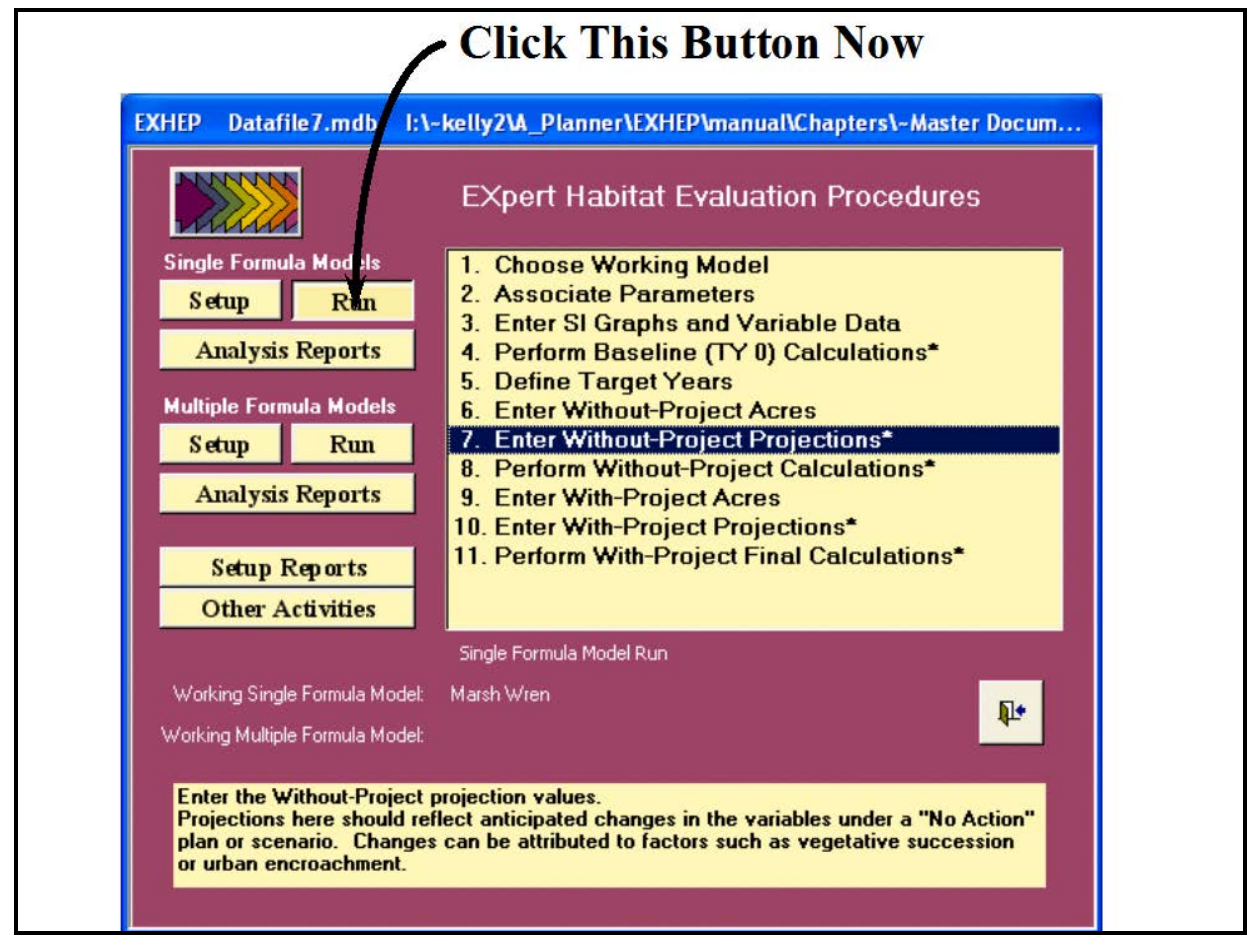

Figure 105. Click on the Run button to begin the without-project variables entry process for the single formula models in EXHEP.

As it did before, a screen appears in the center of the primary interface with 11 options:

\section{Choose Working Model}

2. Associate Parameters

3. Enter SI Graphs and Variable Data

4. Perform Baseline (TY 0) Calculations*

5. Define Target Years

6. Enter Without-Project Acres

7. Enter Without-Project Projections*

8. Perform Without-Project Calculations*

9. Enter With-Project Acres

10. Enter With-Project Projections*

11. Perform With-Project Final Calculations*

Check to assure that the "Working Model" is set to the correct model, and click on the seventh option (Enter Without-Project Projections*) to continue. In response, EXHEP asks the question, "Do you want to initialize the future factors first?" 


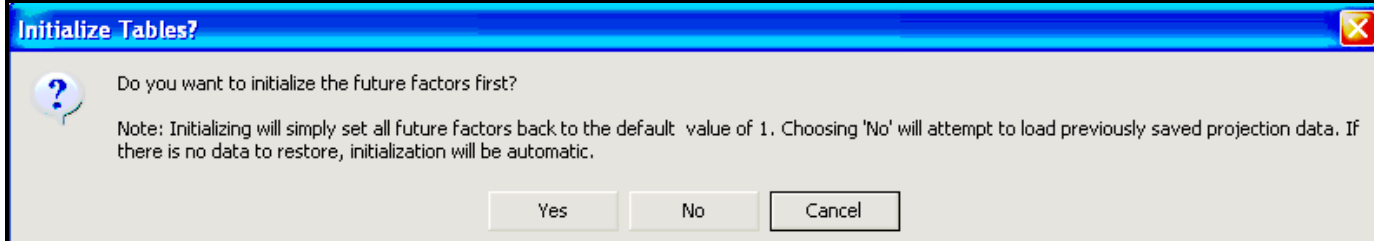

Initialization is EXHEP's attempt to save some time by automating the forecast conditions of the variables. In short, the program queries the database, finds the baseline means for each variable in each cover type, and inputs this value into the projected forecast across all target years in the life of the project. For example, if the mean of a Variable $\mathrm{X}$ is 50 at baseline, EXHEP simply takes this value, copies it into every target year, and presents these values for inspection and modification. The key here is to only initialize

\section{USER'S NOTE:}

The initialization process is irreversible. If the Yes button is clicked in error, all the variables must be re-entered by hand.

\section{USER'S NOTE:}

The initialization process focuses on the "type" of model (single vs. multiple formula models), and initializes the database across all models within that "type." If the "Working Model" is changed, and this question is asked a second time, answer NO to proceed.

the without-project conditions once. Since this is the first time to enter

\begin{tabular}{|l|}
\hline 盽 Progress Indicator \\
Current Process: \\
Initializing Future Factors for single \\
Formula Models \\
Total Records: $\mathbf{4}$ \\
Records Processed: 4 \\
\hline Elapsed Time: $\mathbf{0}$ minute(s) 2 seconds \\
\hline
\end{tabular}

this process, click Yes to proceed. As the initialization commences, a Progress

Indicator window will open in the screen tracking the status of the process, providing a tentative estimate of Elapsed Time.

USER'S NOTE:

Say "Yes" to initialization if:

1. Variable projections have not been made for any model.

2. Variable projections have been made for single formula models, but multiple formula models are now the focus (i.e., each "type" must experience initialization once).

3. Baseline data have been modified after an initialization.

4. Target years have been modified after an initialization.

5. Model associations have been modified after an initialization.

6. Models, cover types, life requisites and/or variables have been added/deleted/modified post-initialization (basically any change in Setup necessitates re-initialization).

Say "No" to initialization when:

1. Variable projections have been made for at least one model in the "type."

2. Modifying current projection trends - post-initialization.

3. Running models through the without- and/or with-project calculations in series.

4. Modifying acreages in the baseline, without- and/or with-project interfaces. 
When complete, EXHEP opens the following window (Figure 106).

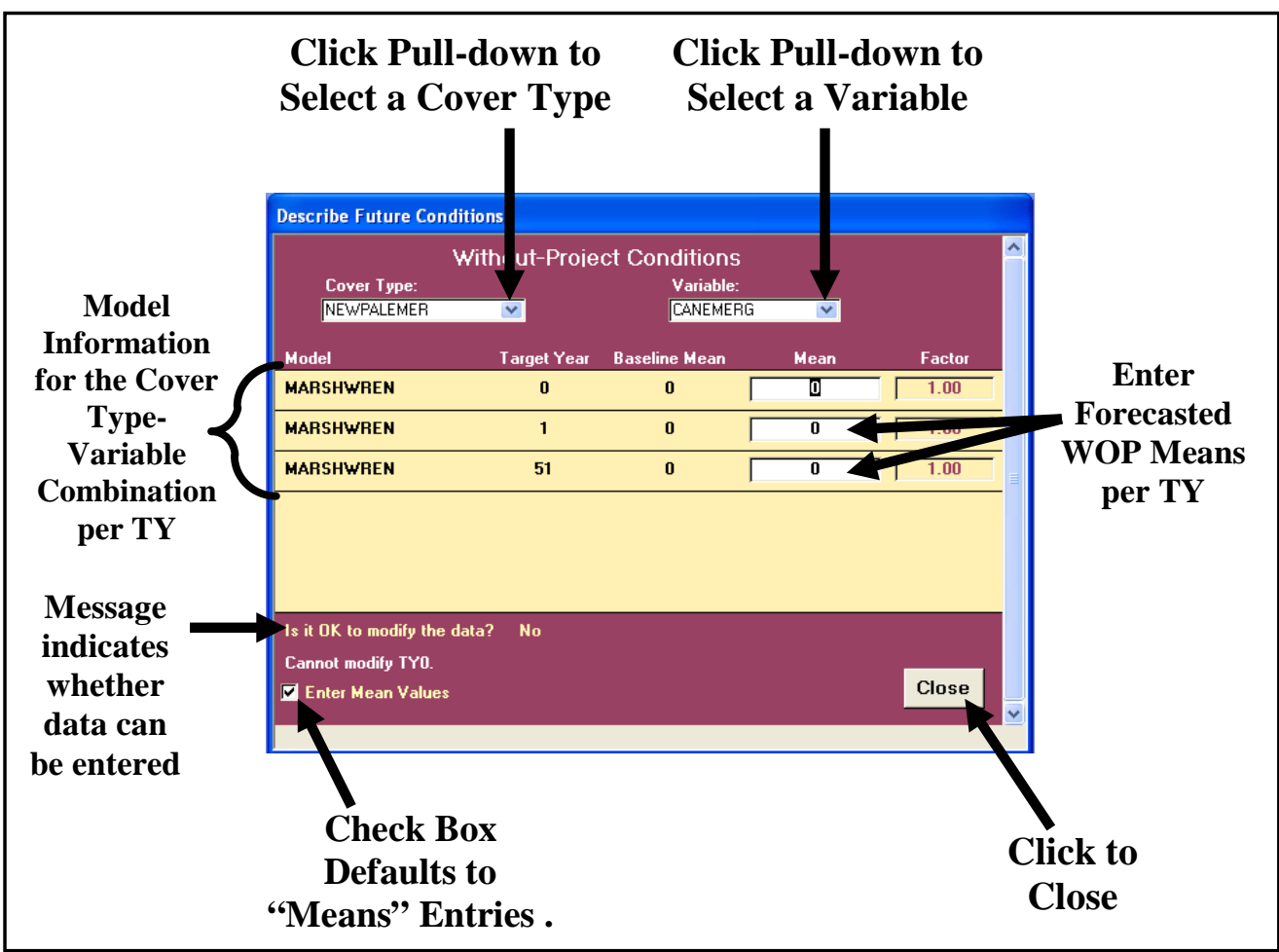

Figure 106. Describe Future Conditions entry window used to forecast without-project conditions for single formula models in EXHEP.

Click on the Cover Type pull-down menu and select the first cover type from the list. Now click on the Variable pull-down menu and select the first variable from the list. EXHEP queries the database for target years and baseline data (based on the entries made in the steps above), and presents these in a tabular format. Note that outlying years are set to equal the baseline $\left(\mathrm{TY}_{0}\right)$ value.

Now make changes to the data where necessary to capture the withoutproject conditions of this variable in this cover type over the course of the target years by highlighting the value and typing in the corrected version in the space provided. Press the Tab button on the keyboard to "accept" the change and move to the next row (Figure 107). 


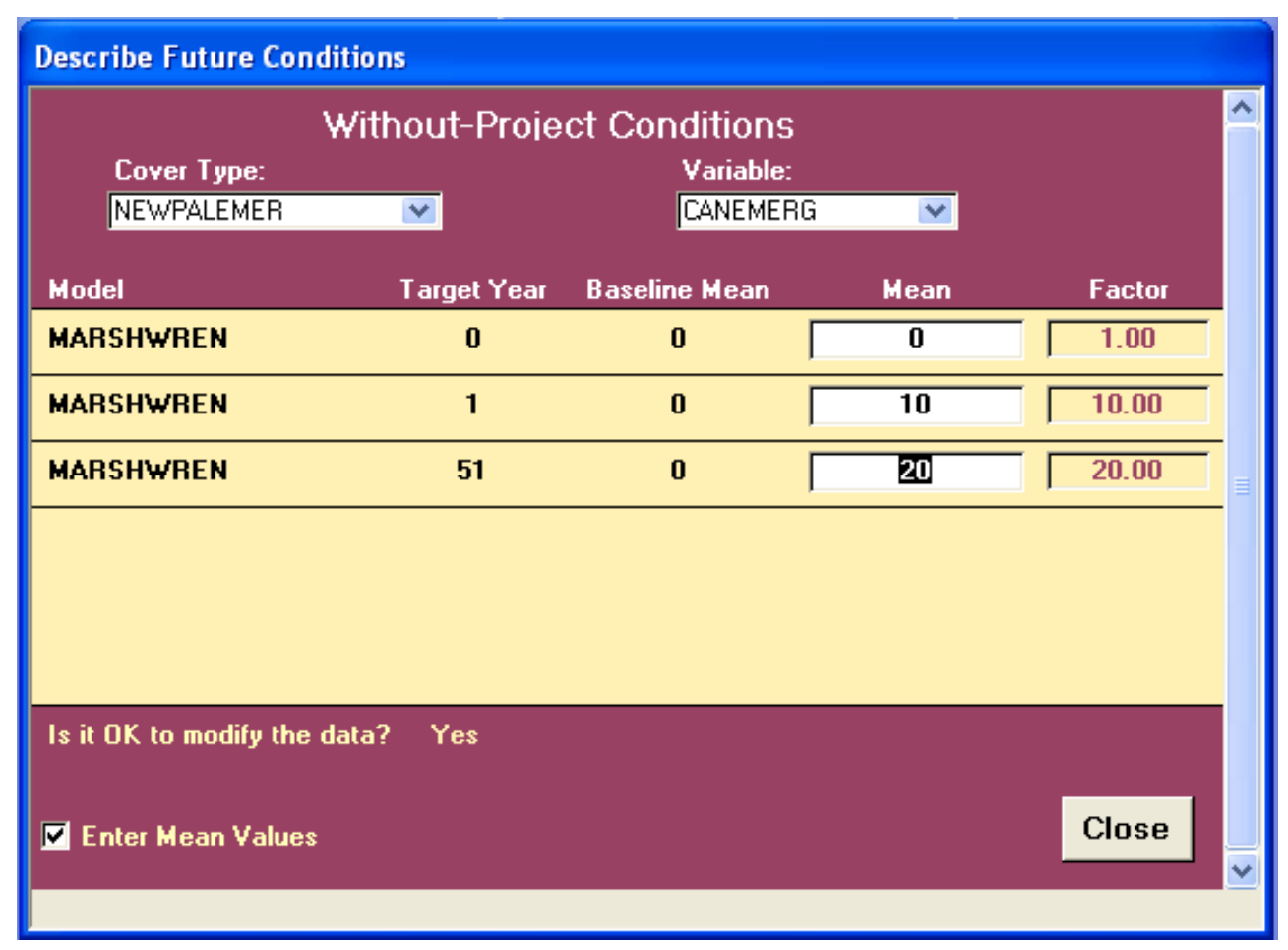

Figure 107. Type the new without-project values into the Mean column.

Note that the baseline means cannot be modified in this interface - a message in the lower left-hand corner of the screen indicates whether or not a field can be altered. To make changes to the baseline conditions, new data must be entered elsewhere (i.e., return to option 3. Enter SI

Graphs and Variable Data and follow the directions above to modify these values). It is also important to note that the system's query filters the cover types and variables presented in this interface - only variables associated inside model formulas will be presented in this screen.

Modifications to the associations cannot be made in this interface. Return to the model association interface to make these acreage changes (refer to the "Step 3: Model association" section above for details).

Single formula models that share similar variables will be presented simultaneously and sorted alphabetically by Model code in this interface. Changes to shared variables are automatically carried down into the subsequent models, eliminating the need to enter the information into the system multiple times (Figure 108). 


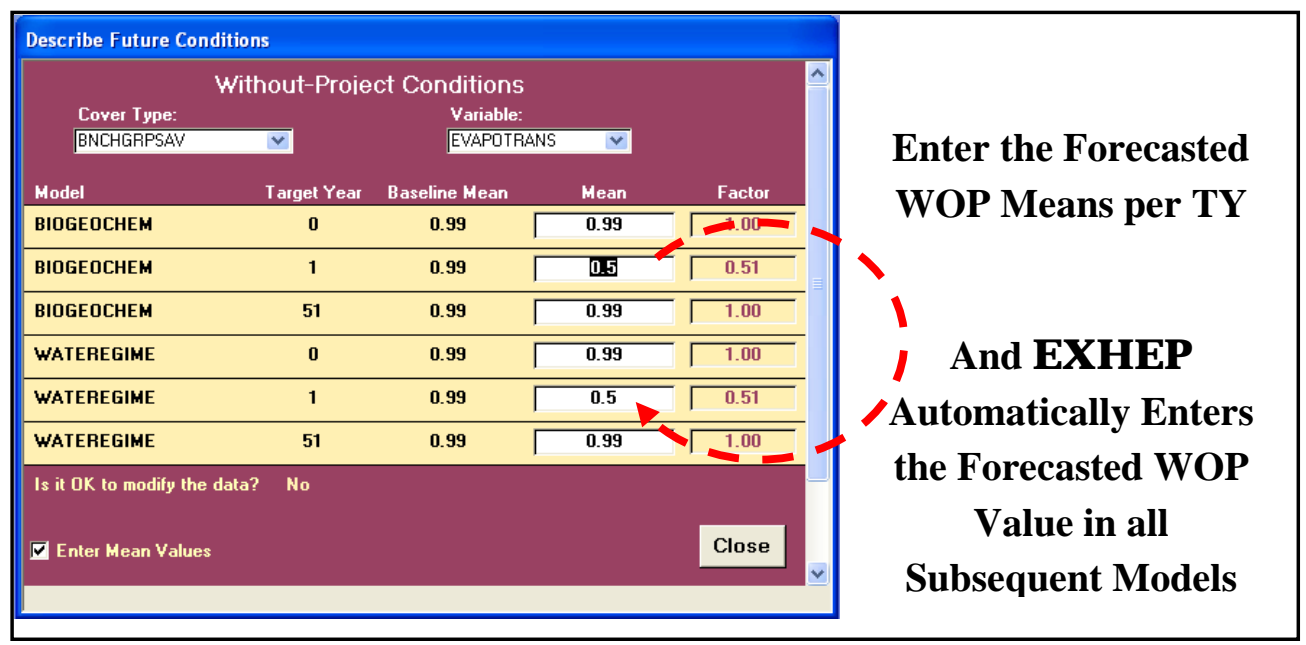

Figure 108. Automated data entry for models that share variables.

Systematically pull down the cover type and variable combinations and repeat the process as many times as necessary to fully document the without-project conditions at the site. When finished, click on the Close button, and EXHEP returns to the primary interface.

Although the system defaults are set to capture forecasted information documentation on a mean-by-mean basis, it is sometimes useful to forecast future conditions on the basis of percentages of the baseline mean. If this is a desired protocol, un-check the box to the immediate left of the Enter Mean Values label in the bottom left-hand corner of this form, and EXHEP will move the cursor to the Factor column (Figure 109).

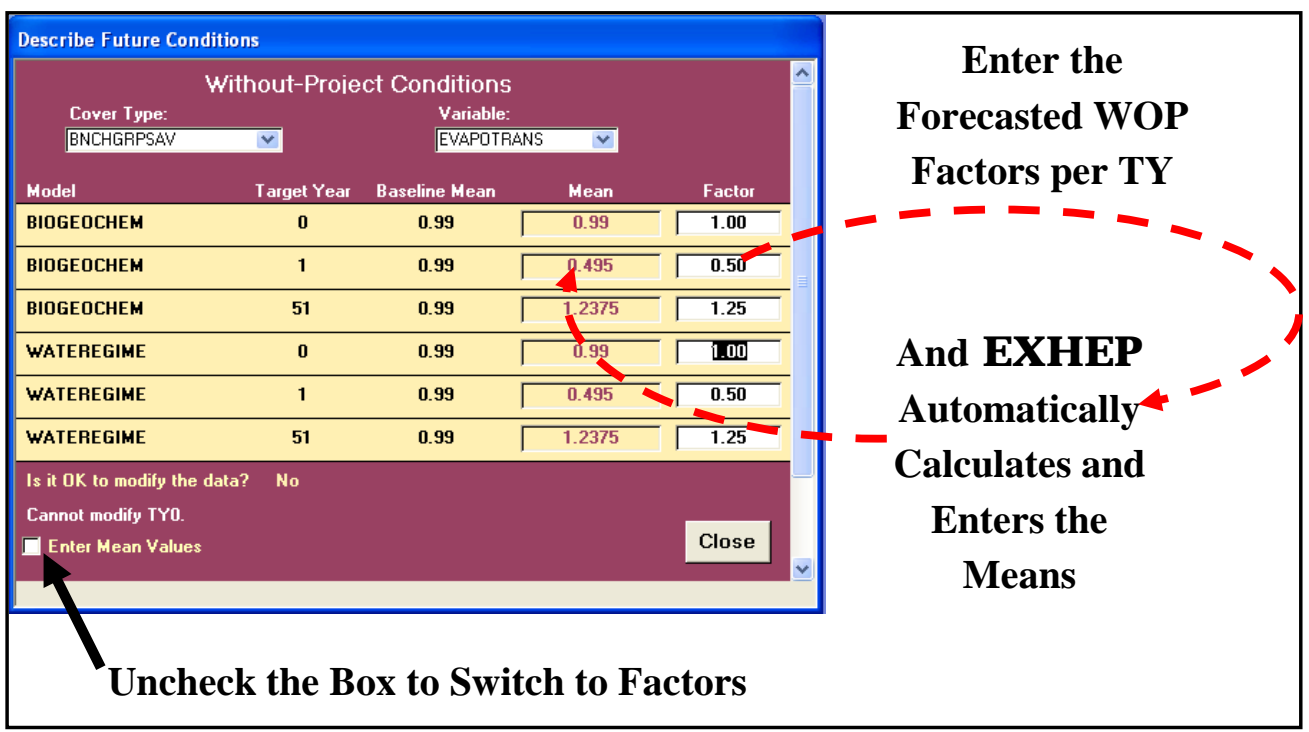

Figure 109. Use the Factor column to enter future projections in terms of percentages of baseline. 
Enter the without-project values for the variable in this cover type by setting weights here (i.e., $50 \%=0.5 ; 125 \%=1.25$, etc.). EXHEP multiplies the factor against the baseline mean and generates the new mean for the TY. Systematically pull down the cover type and variable combinations and repeat the process as many times as necessary to fully document the without-project conditions at the site.

When the without-project data entry task is complete, click on the Close button, and EXHEP asks about saving the data:

Would you like to save the projection data for possible use at a later time?

If you choose to save, you may use this data each time you enter projections by choosing 'No' when asked to initialize the projections table.

\begin{tabular}{|l|l|l|}
\hline Yes & No \\
\hline
\end{tabular}

If an error was made, click the No button, and all the changes will be discarded. To correct the error immediately, click the Cancel button and EXHEP will return to the data entry interface. If the data were entered correctly, click on the Yes button, and EXHEP affirms that the information was saved successfully. Click $\mathbf{O K}$ and EXHEP returns to the primary interface.

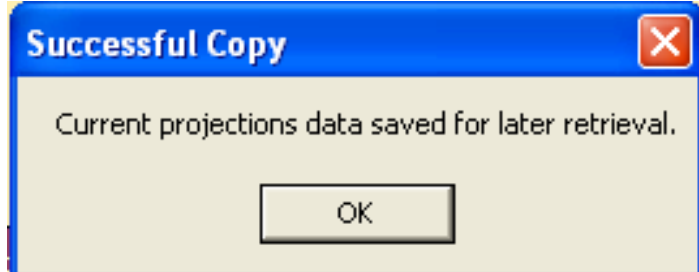

Additional actions for the without-project projection entries in multiple formula models

If multiple formula models are used in the analysis, the without-project variable projections must be completed using the second set of buttons on EXHEP's primary interface (Figure 110). 


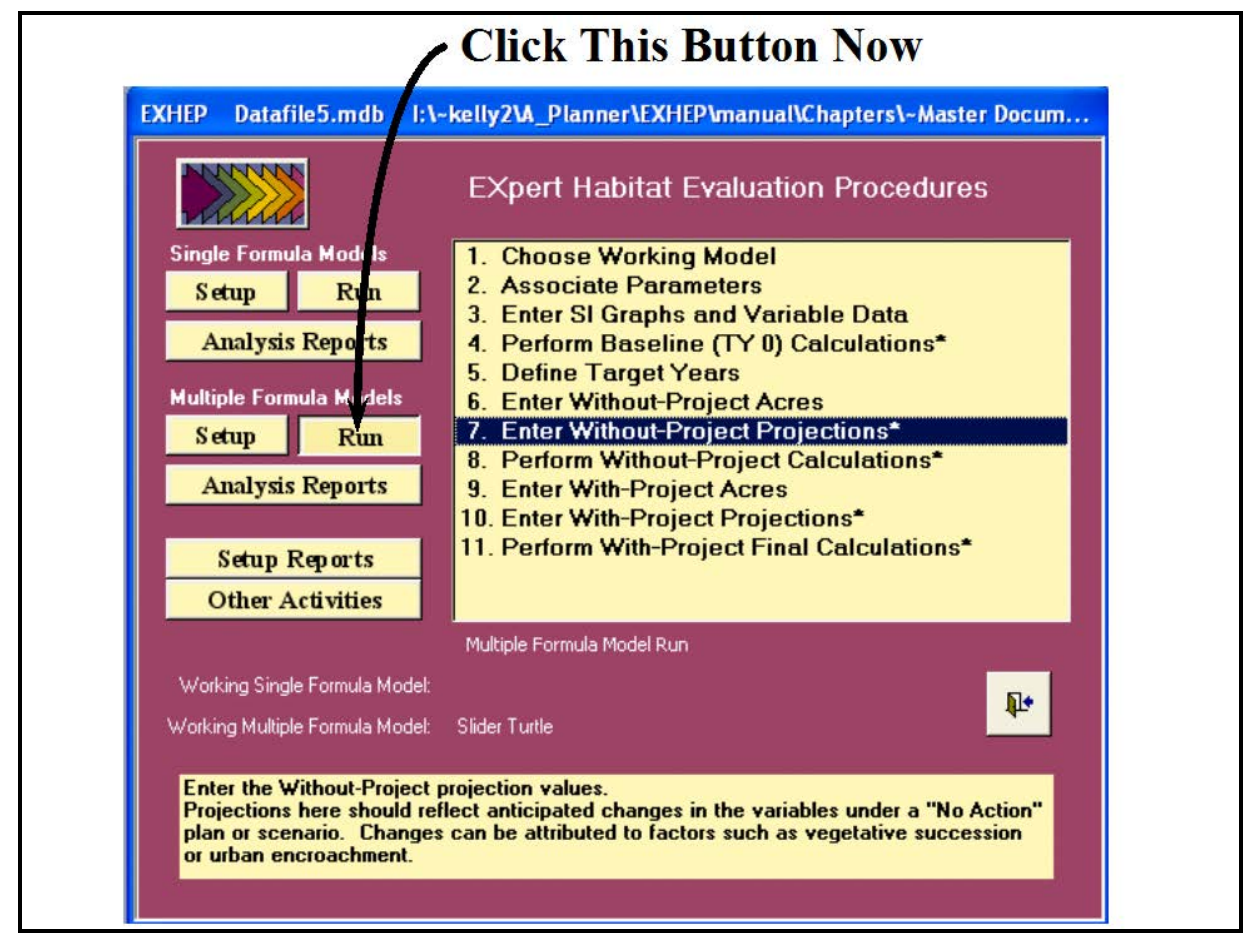

Figure 110. Click on the Run button to begin the without-project variables entry process for multiple formula models in EXHEP.

As it did before, a screen appears in the center of the primary interface with 11 options:

\section{Choose Working Model}

2. Associate Parameters

3. Enter SI Graphs and Variable Data

4. Perform Baseline (TY 0) Calculations*

5. Define Target Years

6. Enter Without-Project Acres

7. Enter Without-Project Projections*

8. Perform Without-Project Calculations*

9. Enter With-Project Acres

10. Enter With-Project Projections*

11. Perform With-Project Final Calculations*

Check to assure that the "Working Model" is set to the correct model, and click on the seventh option (Enter Without-Project Projections*) to continue. In response, EXHEP asks the question, "Do y ou want to initialize the future factors first?" 
? Do you want to initialize the future factors first?

Note: Initializing will simply set all future factors back to the default value of 1 . Choosing 'No' will attempt to load previously saved projection data. If there is no data to restore, initialization will be automatic.

No Cancel

Click Yes to proceed - initialization here will not affect the data entered above in the single formula model exercise. As the initialization commences, a Progress Indicator window will open in the screen tracking the status of the process, providing a

\begin{tabular}{l}
\hline 圆 Progress Indicator \\
\hline Current Process: \\
Initializing Future Factors for Multiple \\
Formula Models \\
Total Records: 4 \\
Zecords Processed: 4 \\
\hline Elapsed Time: 0 minute(s) 2 seconds \\
\hline
\end{tabular}

tentative estimate of Elapsed Time.

\section{USER'S NOTE:}

The initialization process is irreversible. If the Yes button is clicked in error, all the variables must be re-entered by hand.

\section{USER'S NOTE:}

The initialization process focuses on the "type" of model (single vs. multiple formula models), and initializes the database across all models within that "type." If the "Working Model" is changed, and this question is asked a second time, answer NO to proceed.

USER'S NOTE:

Say "Yes" to initialization if:

1. Variable projections have not been made for any model.

2. Variable projections have been made for single formula models, but multiple formula models are now the focus (i.e., each "type" must experience initialization once).

3. Baseline data have been modified after an initialization.

4. Target years have been modified after an initialization.

5. Model associations have been modified after an initialization.

6. Models, cover types, life requisites and/or variables have been added/deleted/modified post-initialization (basically any change in Setup necessitates re-initialization).

Say "No" to initialization when:

1. Variable projections have been made for at least one model in the "type."

2. Modifying current projection trends - post-initialization.

3. Running models through the without- and/or with-project calculations in series.

4. Modifying acreages in the baseline, without- and/or with-project interfaces. 
When complete, EXHEP opens the following window (Figure 111).

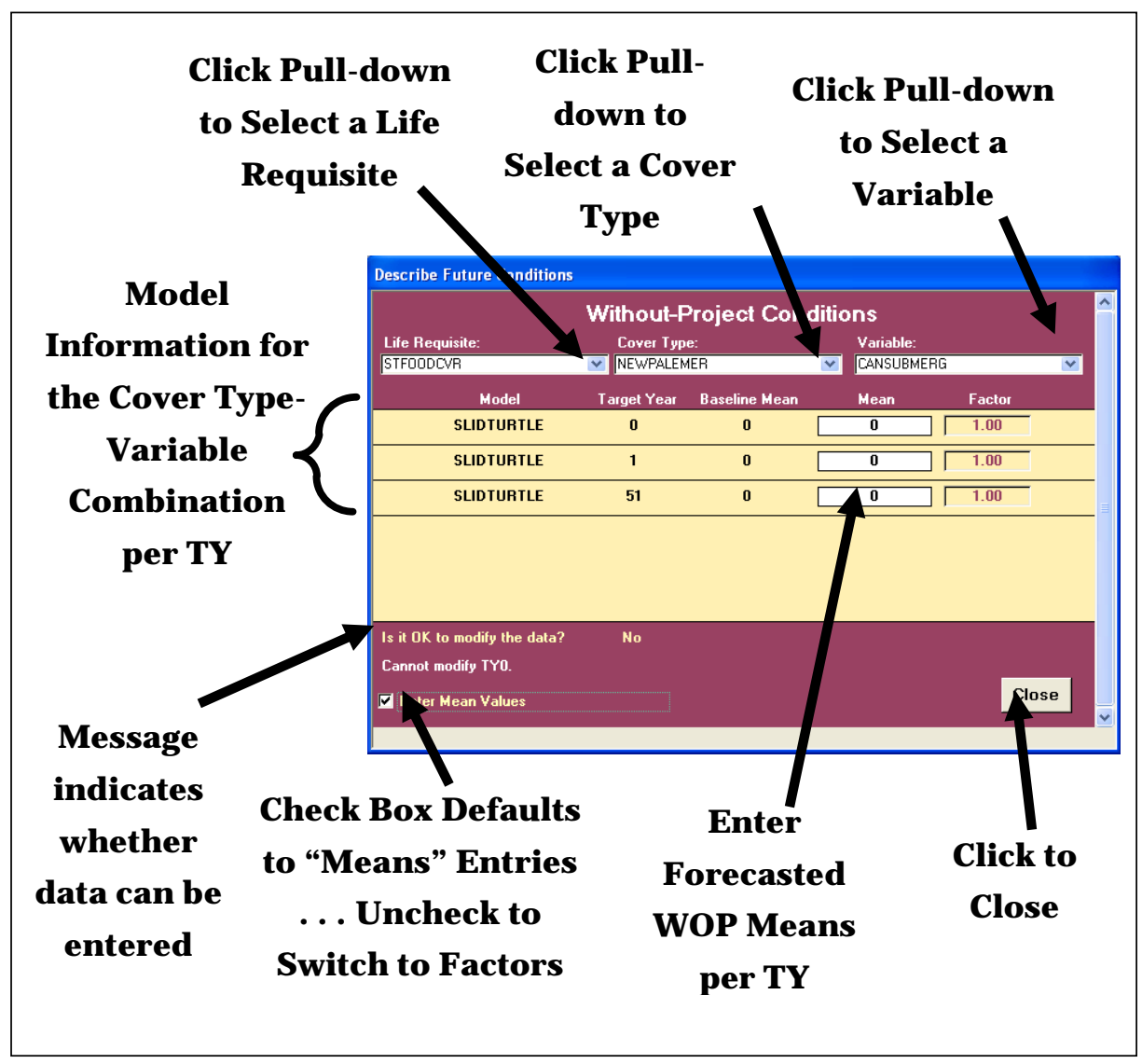

Figure 111. Describe Future Conditions entry window used to forecast without-project conditions for multiple formula models in EXHEP.

Click on the Life Requisite pull-down menu and select the first life requisite from the list. Click on the Cover Type pull-down menu and select the first cover type from the list. Now click on the Variable pulldown menu and select the first variable from the list. EXHEP queries the database for target years and baseline data (based on the entries made in the steps above), and presents these in a tabular format. Note that outlying years are set to equal the baseline $\left(\mathrm{TY}_{0}\right)$ value. Use the same protocol described above in the single formula models section to enter data for the cover types and variables associated with the life requisite. Repeat the process for each life requisite in every multiple formula model. When finished, close the forms and return to the primary interface. 
Checking the without-project variable entries with EXHEP reports

To review and check the results, click on the Analysis Reports button(s) on the primary interface (Figure 112).

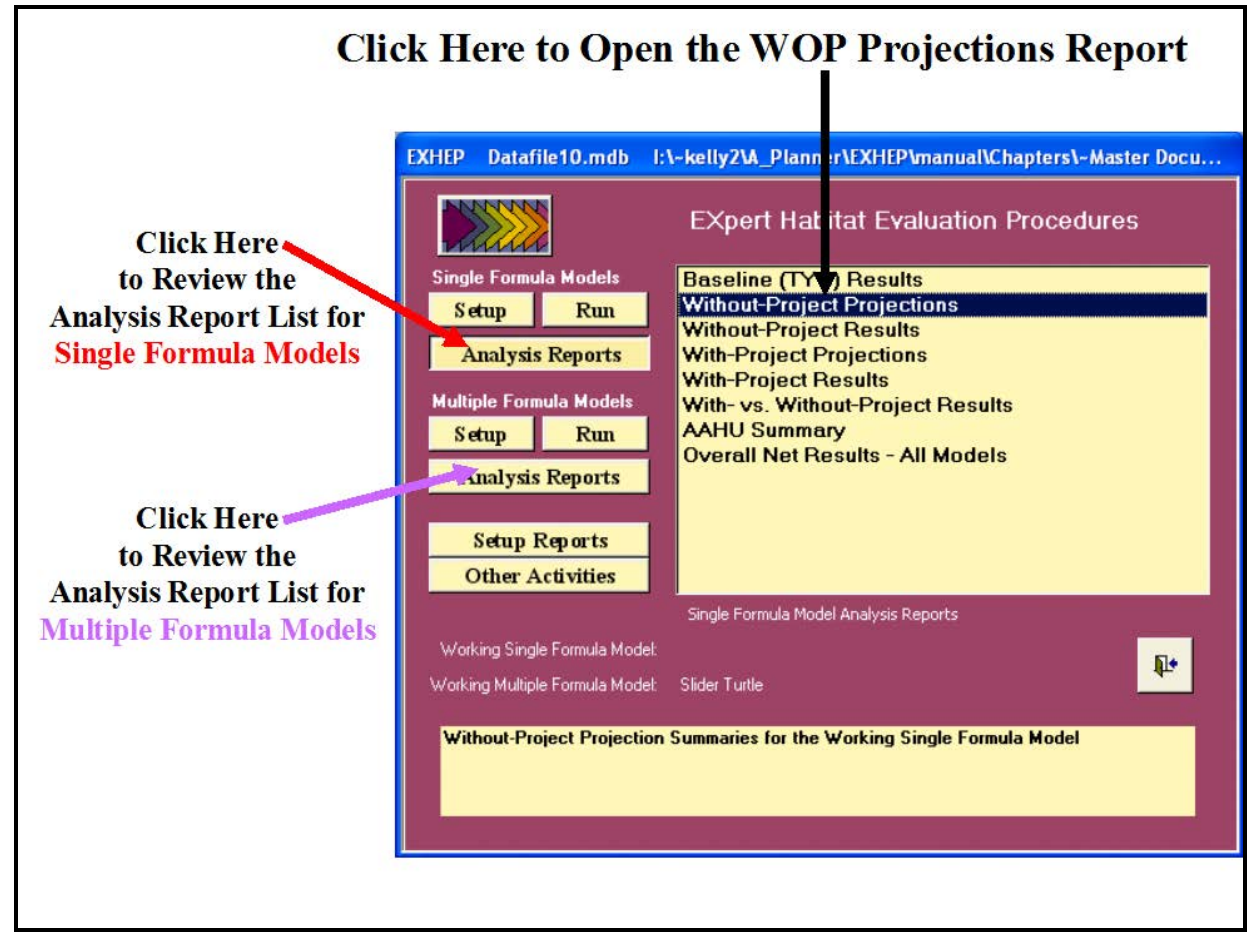

Figure 112. Click on the Analysis Reports buttons to review the without-project forecasted entries in EXHEP.

A list of available reports appears in the center of the primary interface. The second report listed, Without-Project Projections, is ready for inspection. Double-click on this report to review the results (Figure 113). 


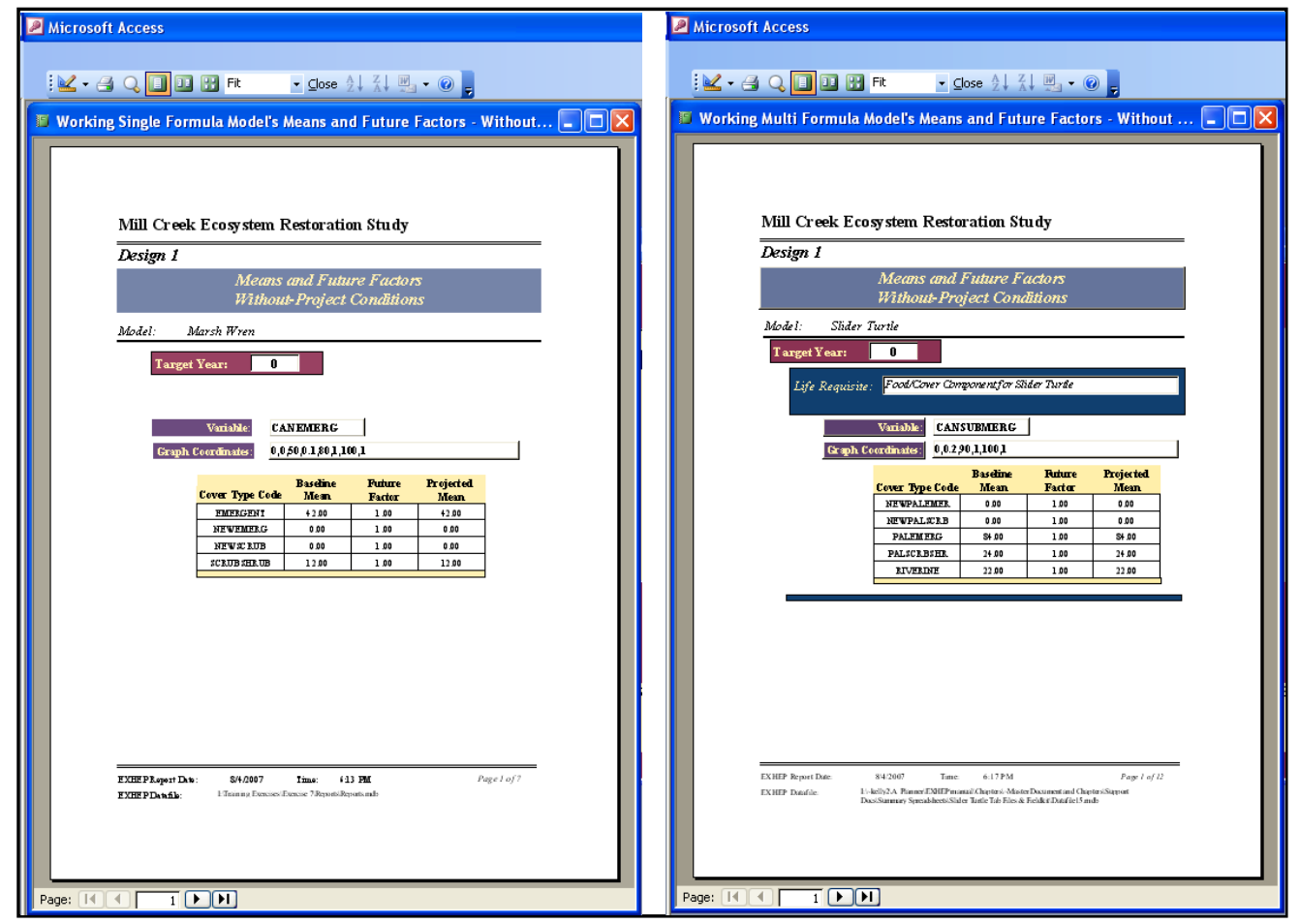

Figure 113. Example of Without-Project Projections reports in EXHEP.

Click on the Close button from the suite of buttons offered in the Report Control toolbar, and EXHEP returns to the primary interface. Now that the without-project forecasts have been entered into the system, proceed to the next step to learn how to assess the without-project conditions of the site in EXHEP.

\section{Perform the without-project calculations}

EXHEP will calculate the without-project results on a model-by-model basis for both single and multiple formula models. To proceed, click on the Run button under the Single Formula Models section of the primary interface (Figure 114). 


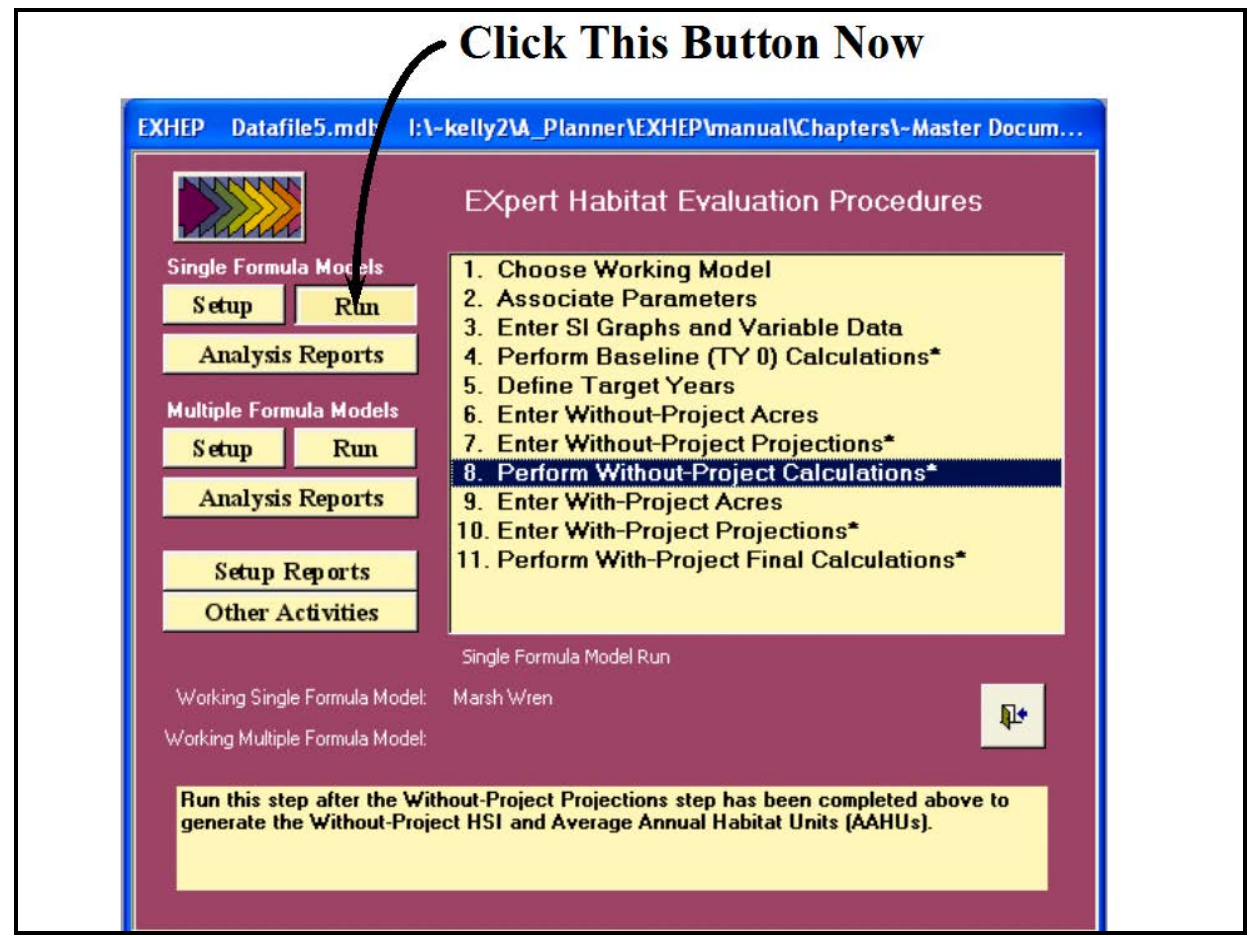

Figure 114. Click on the Run button to begin the without-project calculation process for single formula models in EXHEP.

As it did before, a screen appears in the center of the primary interface with 11 options:

\section{Choose Working Model}

2. Associate Parameters

3. Enter SI Graphs and Variable Data

4. Perform Baseline (TY 0) Calculations*

5. Define Target Years

6. Enter Without-Project Acres

7. Enter Without-Project Projections*

8. Perform Without-Project Calculations*

9. Enter With-Project Acres

10. Enter With-Project Projections*

11. Perform With-Project Final Calculations*

Check to assure that the "Working Model" is set to the correct model, and click on the eighth option (Perform Without-Project Calculations*) to continue. In response, EXHEP will begin the calculation process. As the calculations continue, a Progress Indicator window will open in the screen.

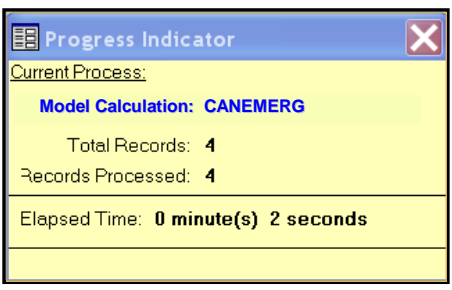


This progress window will track the status of the analysis (number of records to assess versus number of records processed) and provide a tentative estimate of Elapsed Time. When completed, EXHEP returns a message that the calculations are concluded. This

\section{Final Calculation Status}

The Working Model's Final Without-Project Calculations completed successfully.

process must be

completed for each

model in the analysis.
OK

Each time a "Working Model" is selected, the options on the primary interface that have stars next to their titles must be re-run to focus the database. The "quick steps" are as follows:

1. Click on 1. Choose Working Model and select the next model for processing.

2. Click on 4. Perform Baseline (TY 0) Calculations*, let the system run, and click $\mathbf{O K}$ when it is finished.

3. Click on 7. Enter Without-Project Projections*, click on the No button for initialization, click the Close button at the bottom of the interface, click Yes to save the data, and click $\mathbf{O K}$ when that save is affirmed.

USER'S NOTE:

REMEMBER, when asked about initializing the data in Option 7, Enter Without-Project Projections*, answer No or the data inside will be overwritten.

4. Click on 8. Perform Without-Project Calculations*, let the system run, and click $\mathbf{O K}$ when it is finished.

Additional actions for the without-project calculations in multiple formula models

If multiple formula models are used in the analysis, calculate withoutproject results for the models using the second set of buttons on EXHEP's primary interface (Figure 115). 


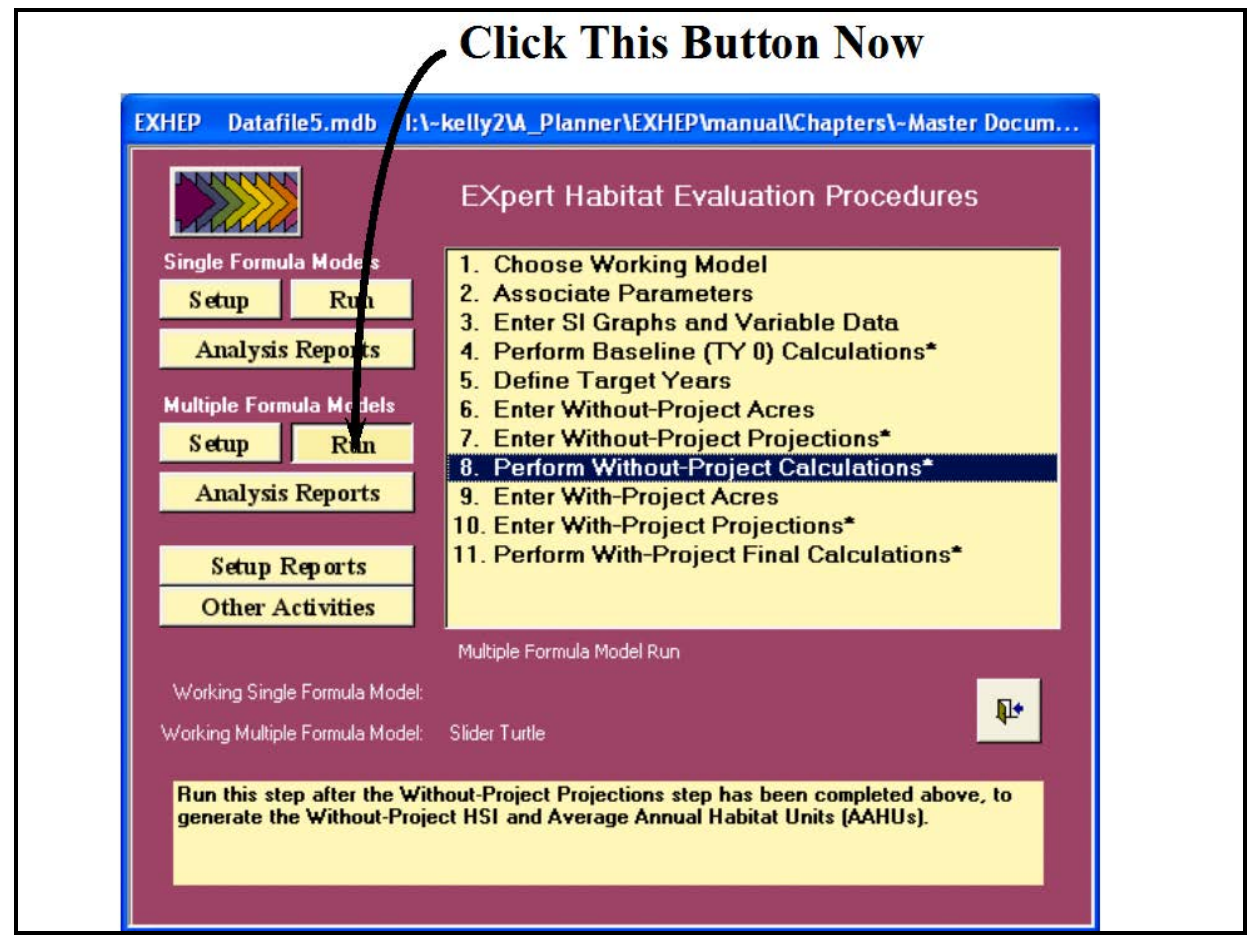

Figure 115. Click on the Run button to begin the without-project calculation process for multiple formula models in EXHEP.

As it did before, the screen appears in the center of the primary interface with 11 options:

\section{Choose Working Model}

2. Associate Parameters

3. Enter SI Graphs and Variable Data

4. Perform Baseline (TY 0) Calculations*

5. Define Target Years

6. Enter Without-Project Acres

7. Enter Without-Project Projections*

8. Perform Without-Project Calculations*

9. Enter With-Project Acres

10. Enter With-Project Projections*

11. Perform With-Project Final Calculations*

Use the same protocol described above in the single formula models section to run the without-project analysis for each of the models in the analysis. 


\section{Checking the without-project analysis with EXHEP reports}

To review and check the results, click on the Analysis Reports button(s) on the primary interface (Figure 116).

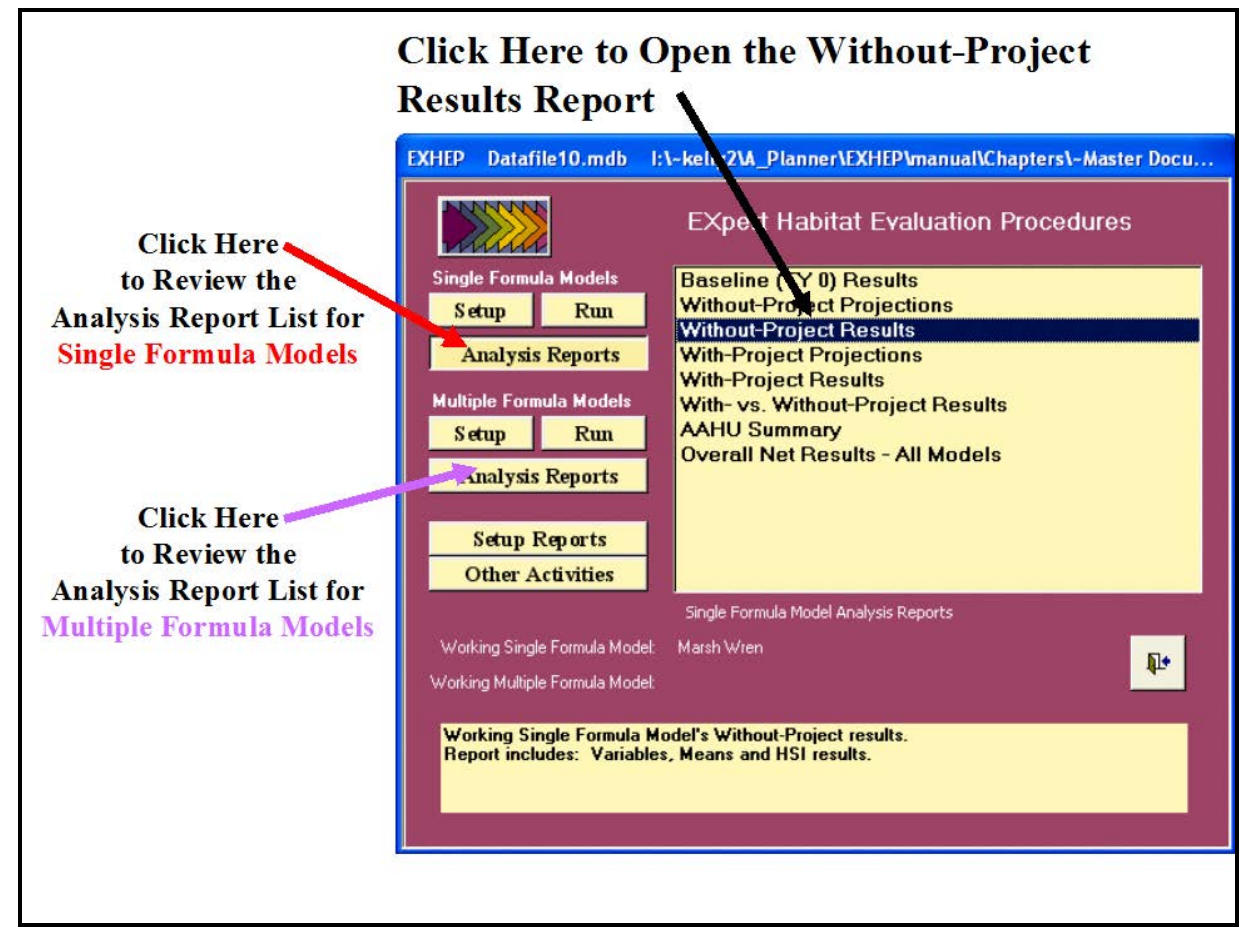

Figure 116. Click on the Analysis Reports buttons to review the without-project results in EXHEP.

A list of available reports appears in the center of the primary interface. The third report listed, Without-Project Results, is ready for inspection. Double-click on this report to review the results (Figure 117). 


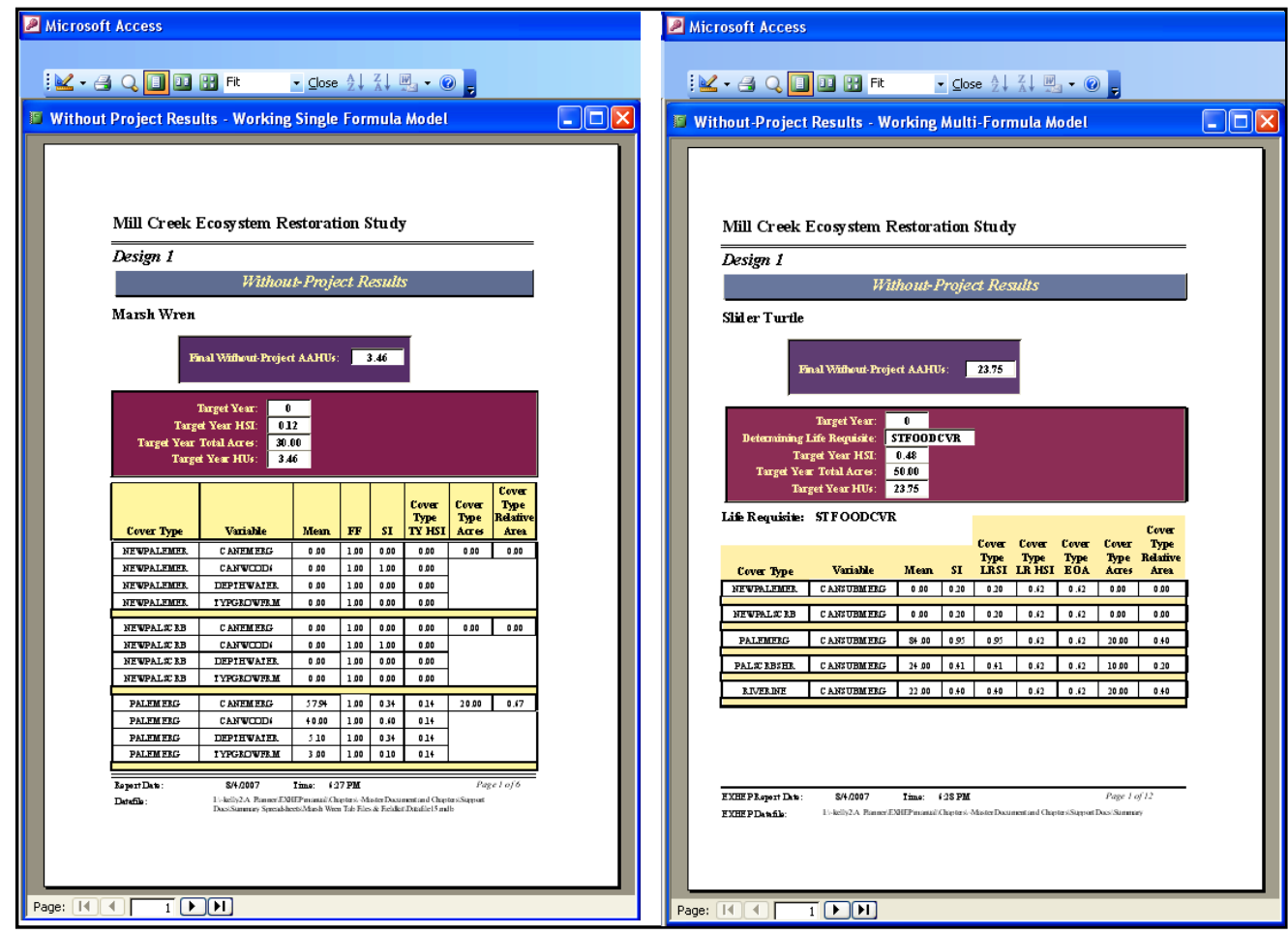

Figure 117. Examples of Without-Project Results reports in EXHEP.

The site's overall average annual outputs (i.e., AAHUs) are reported just under each model's name. The HSI scores, associated acreages, and the resulting HU scores are then reported on a target year-by-target year basis below the AAHU values. The specifics used to generate the overall scores are reported in the tables below this summary. These details are sorted alphabetically by life requisite (where applicable), cover type, and variable. These reports are cumulative; thus, all models assessed in the EXHEP analyses are presented herein.

Click on the Close button from the suite of buttons offered in the Report Control toolbar, and EXHEP returns to the primary interface. Now that the without-project assessment is complete, proceed to the next step to learn how to assess the with-project conditions of the site in EXHEP. 


\section{Step 6: Enter the with-project conditions and calculate the effects}

Once the without-project has been conceptualized, the evaluation team begins the iterative process of developing action plans (i.e., alternatives) to either counteract the degraded conditions (the goal of restoration or mitigation projects) or modify the existing site with activities that degrade the habitat (the focus of impact studies). To quantify the projected change to the site if one of these actions is taken, both with-project acreage and habitat quality must be documented and incorporated into the evaluation. The timing, nature, and magnitude of these changes must be quantified for each alternative proposed for the site. These values in turn must be entered into the software (one alternative at a time) and results are generated on an alternative-by-alternative basis. ${ }^{1}$

\section{With-project forecasting and data entry}

In EXHEP, the target years defined in the without-project condition are the same target years used in the with-project assessment, so it is not necessary to re-enter these milestones. Instead, the data entry efforts focus on the prediction of acreage and quality of that acreage over time with the proposed alternative in place.

USER'S NOTE:

The interfaces EXHEP uses to capture and organize this information will look very similar to those in the without-project section - only subtle differences in form titles indicate the with-project focus.

Enter, change, or delete the with-project acres

To begin, click on the Run button under the Single Formula Models section of the primary interface (Figure 118).

\footnotetext{
1 To assist in the software application, a series of training exercises have been included in Appendix E -
} refer to Exercise 8 for relevant training in with-project data entry and analysis in EXHEP. 


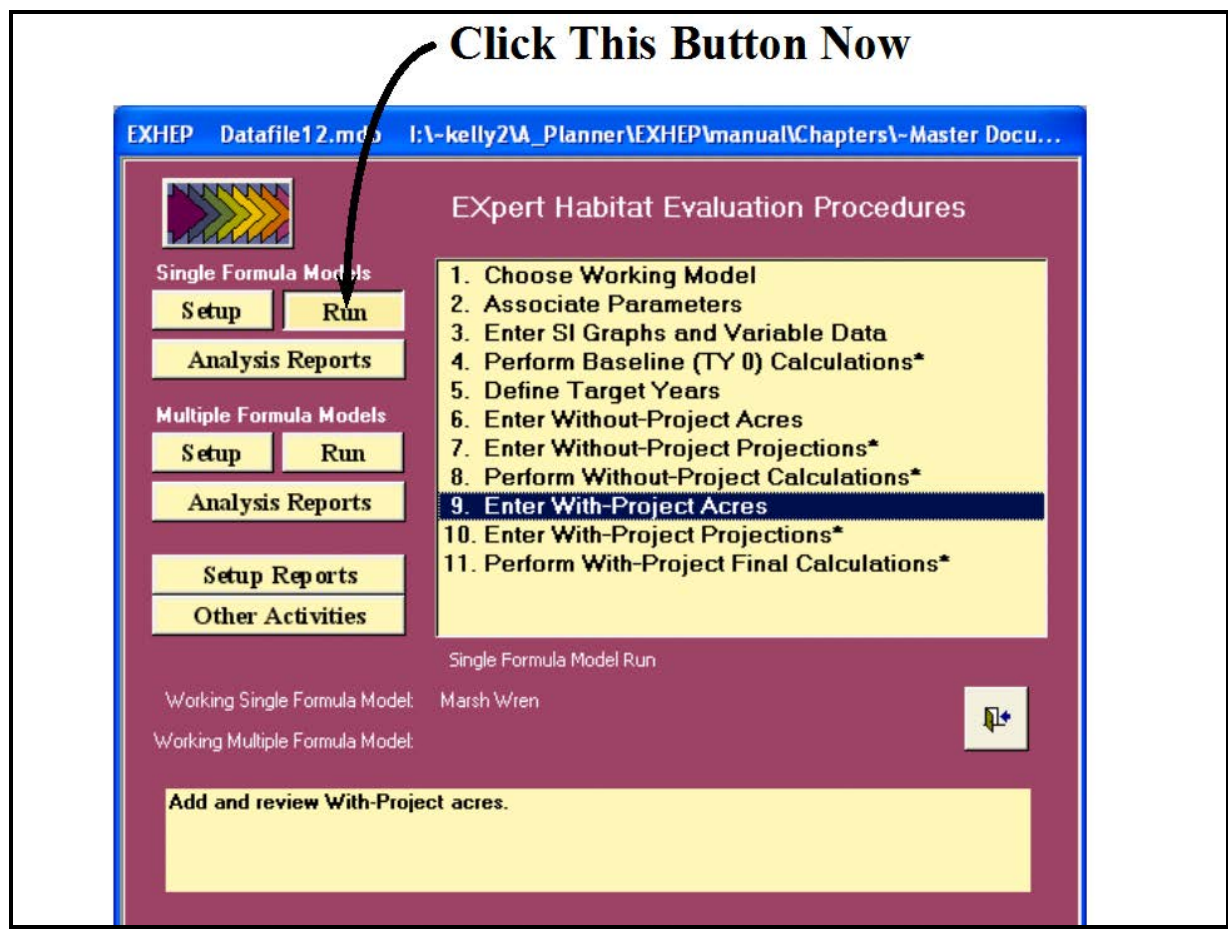

Figure 118. Click on the Run button to begin the with-project acres entry process for the single formula models in EXHEP.

As it did before, a screen appears in the center of the primary interface with 11 options:

\section{Choose Working Model}

2. Associate Parameters

3. Enter SI Graphs and Variable Data

4. Perform Baseline (TY 0) Calculations*

5. Define Target Years

6. Enter Without-Project Acres

7. Enter Without-Project Projections*

8. Perform Without-Project Calculations*

9. Enter With-Project Acres

10. Enter With-Project Projections*

11. Perform With-Project Final Calculations*.

Check to assure that the "Working Model" is set to the correct model, and click on the ninth option (Enter With-Project Acres) to continue. Use the same protocol described above in the without-project
USER'S NOTE:

With Project (WP) acres assigned to the "Working Model" apply to all models in the software (both single and multiple formula model types). Therefore it is only necessary to enter the WP acres once - they will be carried into the analysis for the remaining models. 
section to enter acres for the cover types on the site under the with-project scenario (Figure 119).

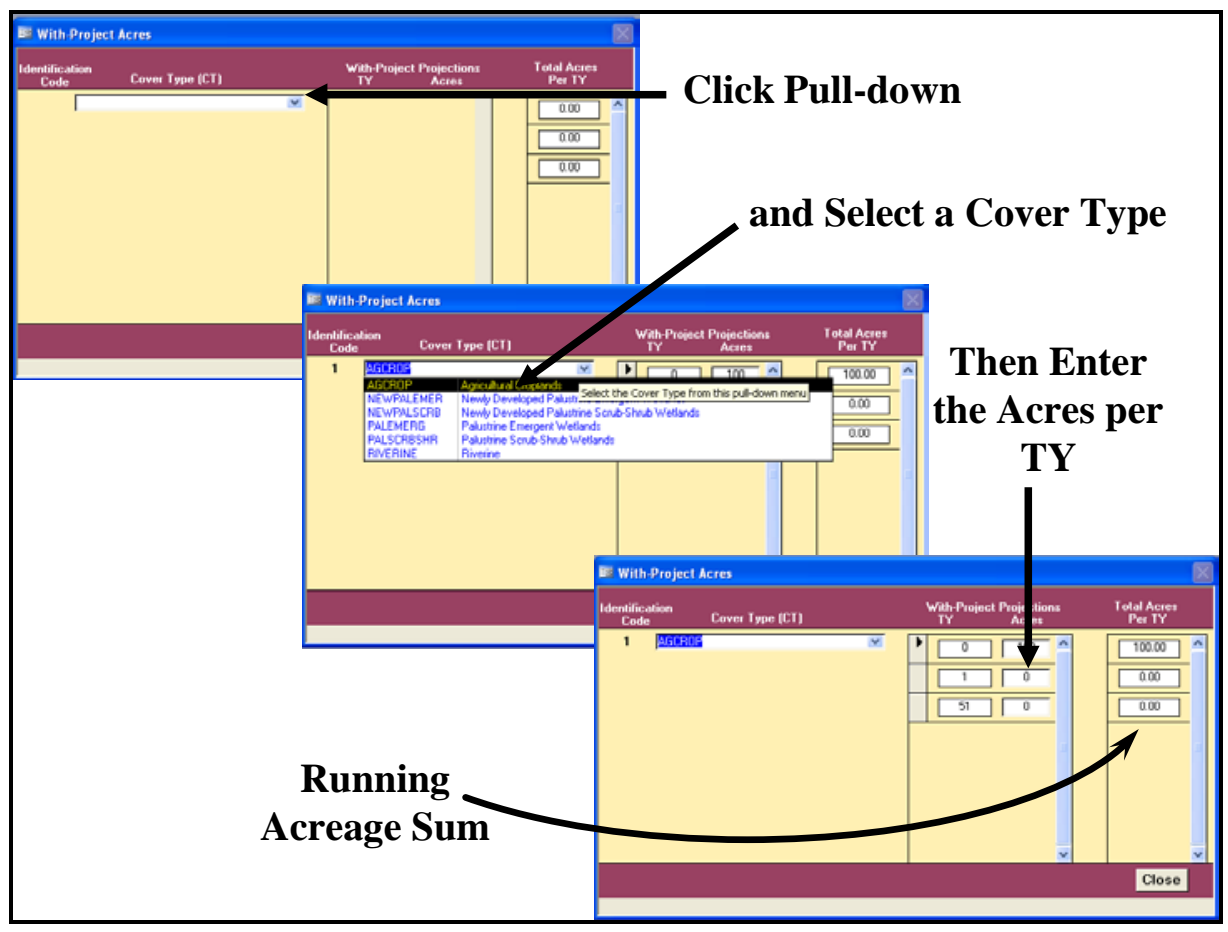

Figure 119. Enter the with-project acreage projections into the cells provided to the right of their corresponding target years.

When finished, click on the Close button, and EXHEP returns to the primary interface. If there are no single formula models in the analysis, the with-project acres may be entered via the Run button under the Multiple Formula Models section of the primary interface. Simply follow the steps described above to complete the entries as needed. Now proceed to the next section to check the entries and focus on the with-project forecasting task.

Checking the with-project acres with EXHEP reports

To review and check the target year and acreage entries made thus far, click on the Setup Reports button on the primary interface (Figure 120). 


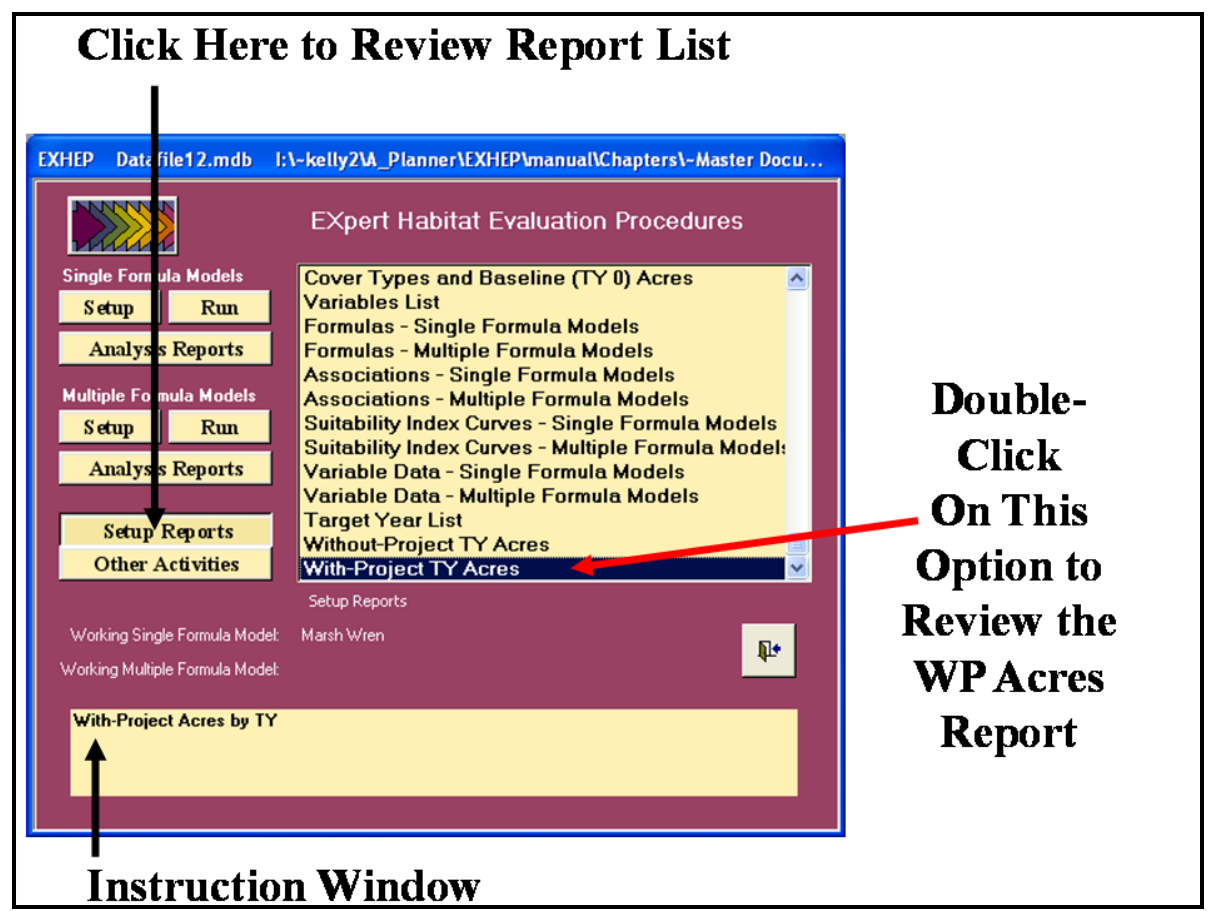

Figure 120. Click on the Setup Reports button to view the With-Project TYAcres report in EXHEP.

The With-Project TY Acres report should be populated at this point. Single-click on the report and look to the Instruction Window to check the contents of the report. Double-click on the report, and EXHEP opens a new window with the report inside (Figure 121).

Click on the Close button from the suite of buttons offered on the Report Control toolbar, and EXHEP returns to the primary interface.

Enter the with-project variable projections

Now click on the Run button under the Single Formula Models section of the primary interface (Figure 122). 


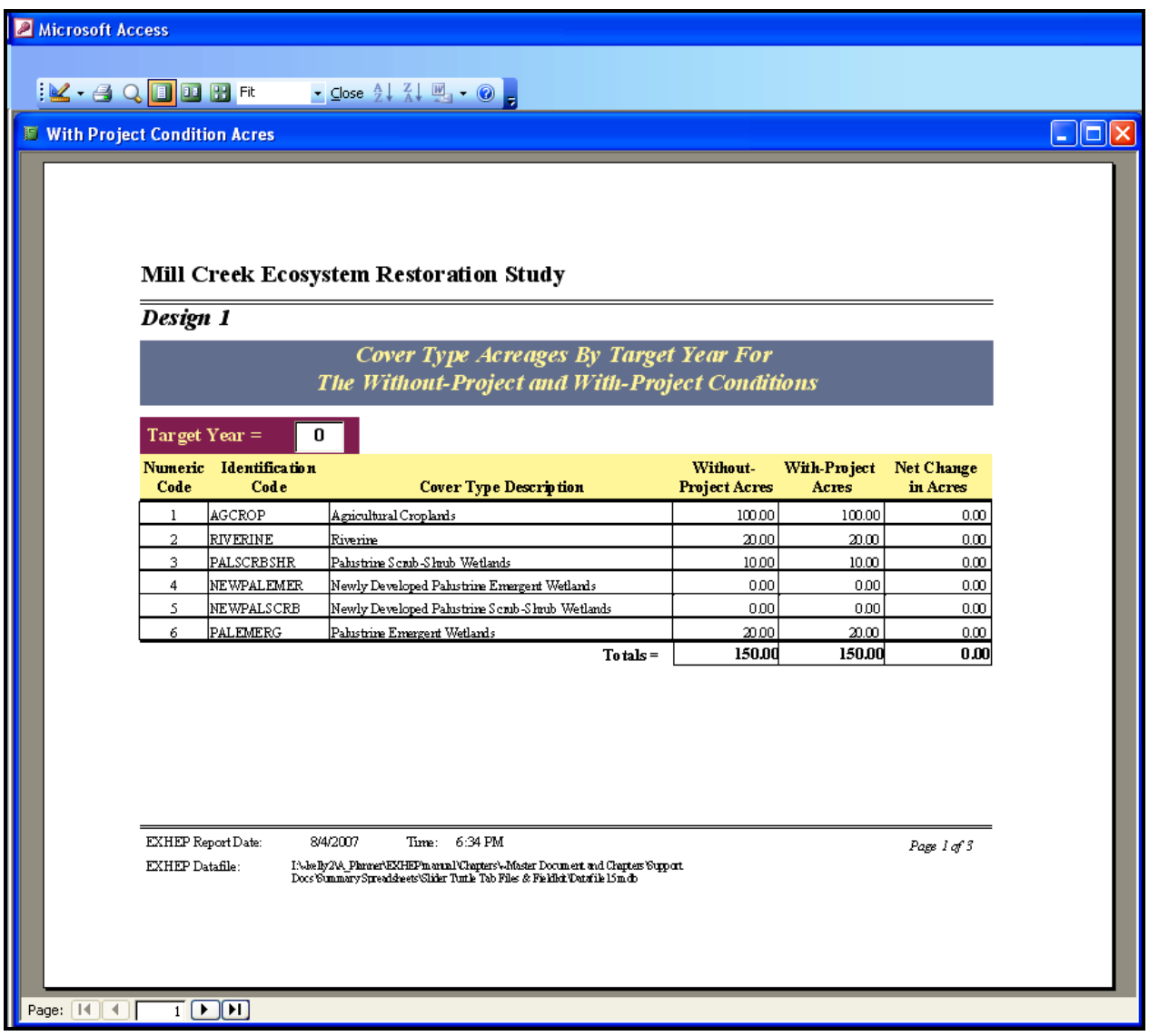

Figure 121. Example of With-Project TY Acres reports in EXHEP.

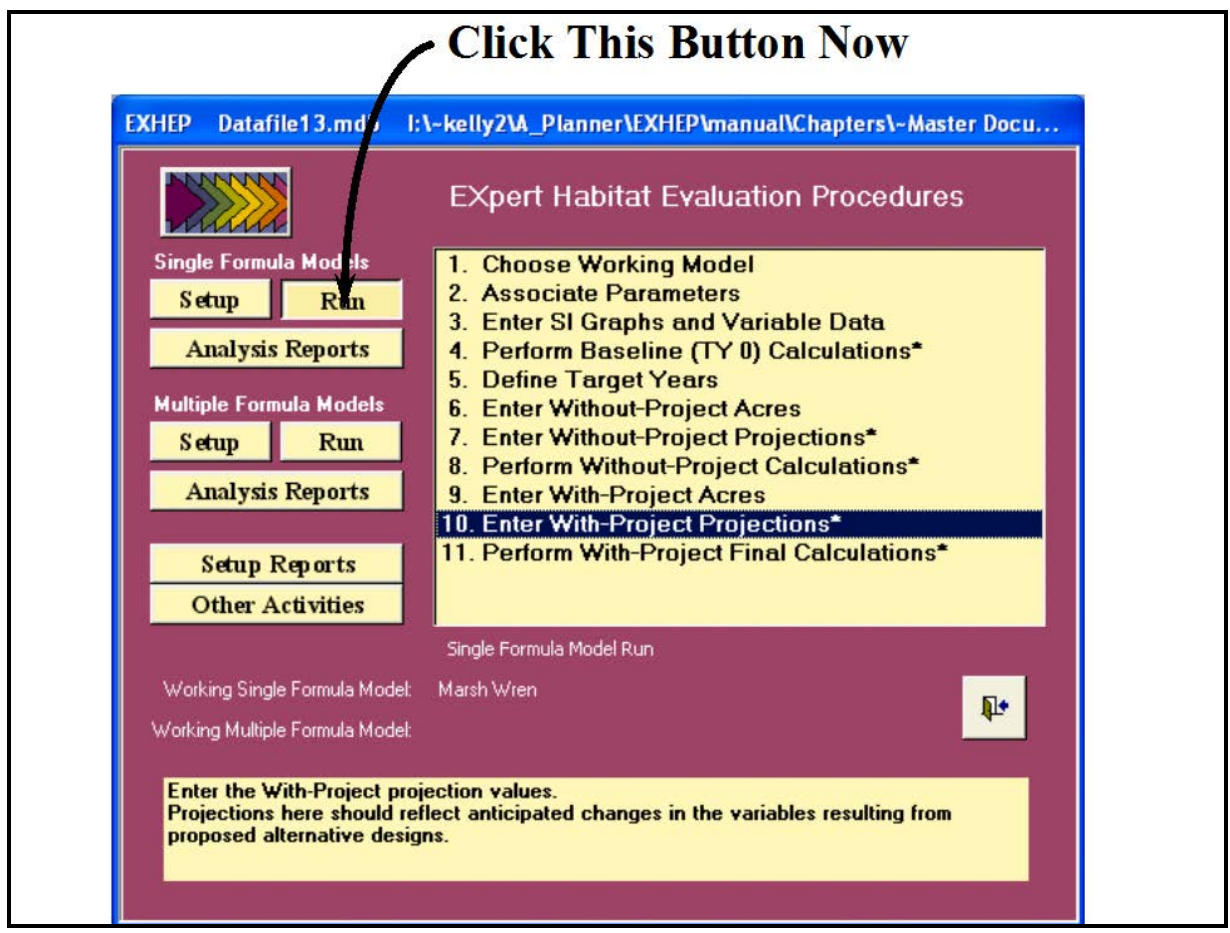

Figure 122. Click on the Run button to begin the with-project variables entry process for the single formula models in EXHEP. 
As it did before, a screen appears in the center of the primary interface with 11 options:

\section{Choose Working Model}

2. Associate Parameters

3. Enter SI Graphs and Variable Data

4. Perform Baseline (TY 0) Calculations*

5. Define Target Years

6. Enter Without-Project Acres

7. Enter Without-Project Projections*

8. Perform Without-Project Calculations*

9. Enter With-Project Acres

10. Enter With-Project Projections*

11. Perform With-Project Final Calculations*

Check to assure that the "Working Model" is set to the correct model, and click on the tenth option (Enter With-project Projections*) to continue. In response, EXHEP asks the question, "Do you want to initialize the future factors first?"

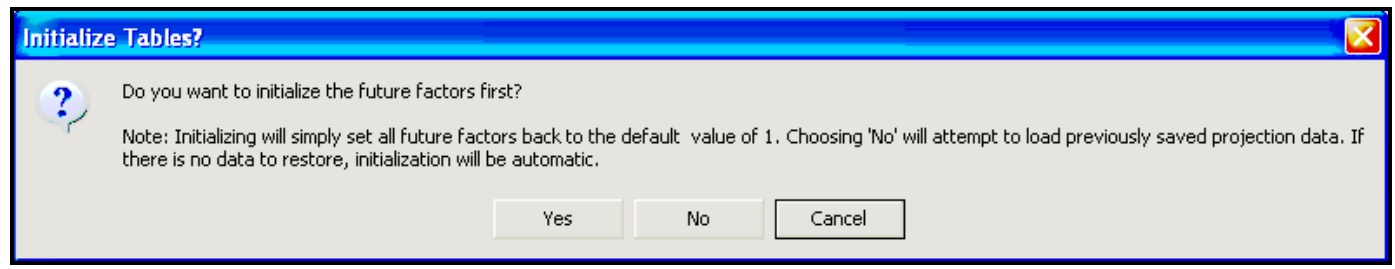

The key here again is to only initialize the WP conditions once. Since this is the first time to enter this process, click Yes to proceed. As the

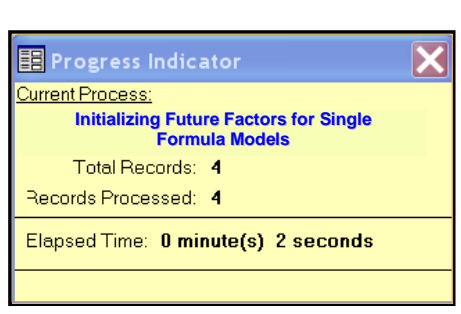
initialization commences, a Progress Indicator window will open in the screen tracking the status of the process, providing a tentative estimate of Elapsed Time.
USER'S NOTE:

The initialization process is irreversible. If the Yes button is clicked in error, all the variables must be re-entered by hand.

\section{USER'S NOTE:}

The initialization process focuses on the "type" of model (single vs. multiple formula models), and initializes the database across all models within that "type." If the "Working Model" is changed, and this question is asked a second time, answer NO to proceed. 
USER'S NOTE:

Say "Yes" to initialization if:

1. Variable projections have not been made for any model.

2. Variable projections have been made for single formula models, but multiple formula models are now the focus (i.e., each "type" must experience initialization once).

3. Baseline data have been modified after an initialization.

4. Target years have been modified after an initialization.

5. Model associations have been modified after an initialization.

6. Models, cover types, life requisites, and/or variables have been added/deleted/modified post-initialization (basically any change in Setup necessitates re-initialization).

Say "No" to initialization when:

1. Variable projections have been made for at least one model in the "type."

2. Modifying current projection trends - post-initialization.

3. Running models through the without- and/or with-project calculations in series.

4. Modifying acreages in the baseline, without- and/or with-project interfaces.

When complete, EXHEP opens the following window (Figure 123).

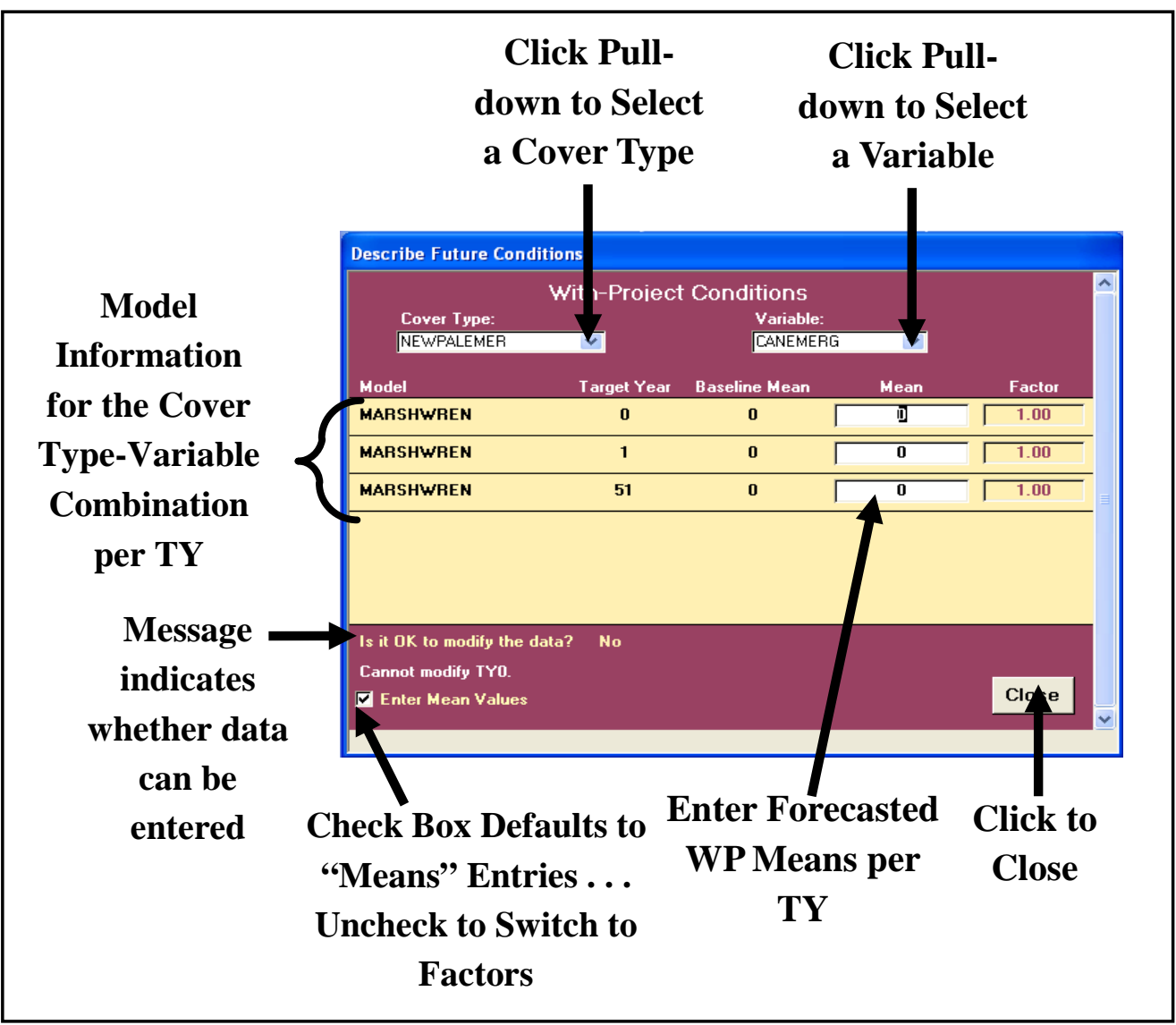

Figure 123. Describe Future Conditions entry window used to forecast with-project conditions for single formula models in EXHEP. 
Use the same protocol described above in the without-project section to enter variable trends for the cover types on the site under the with-project scenario (Figure 124).

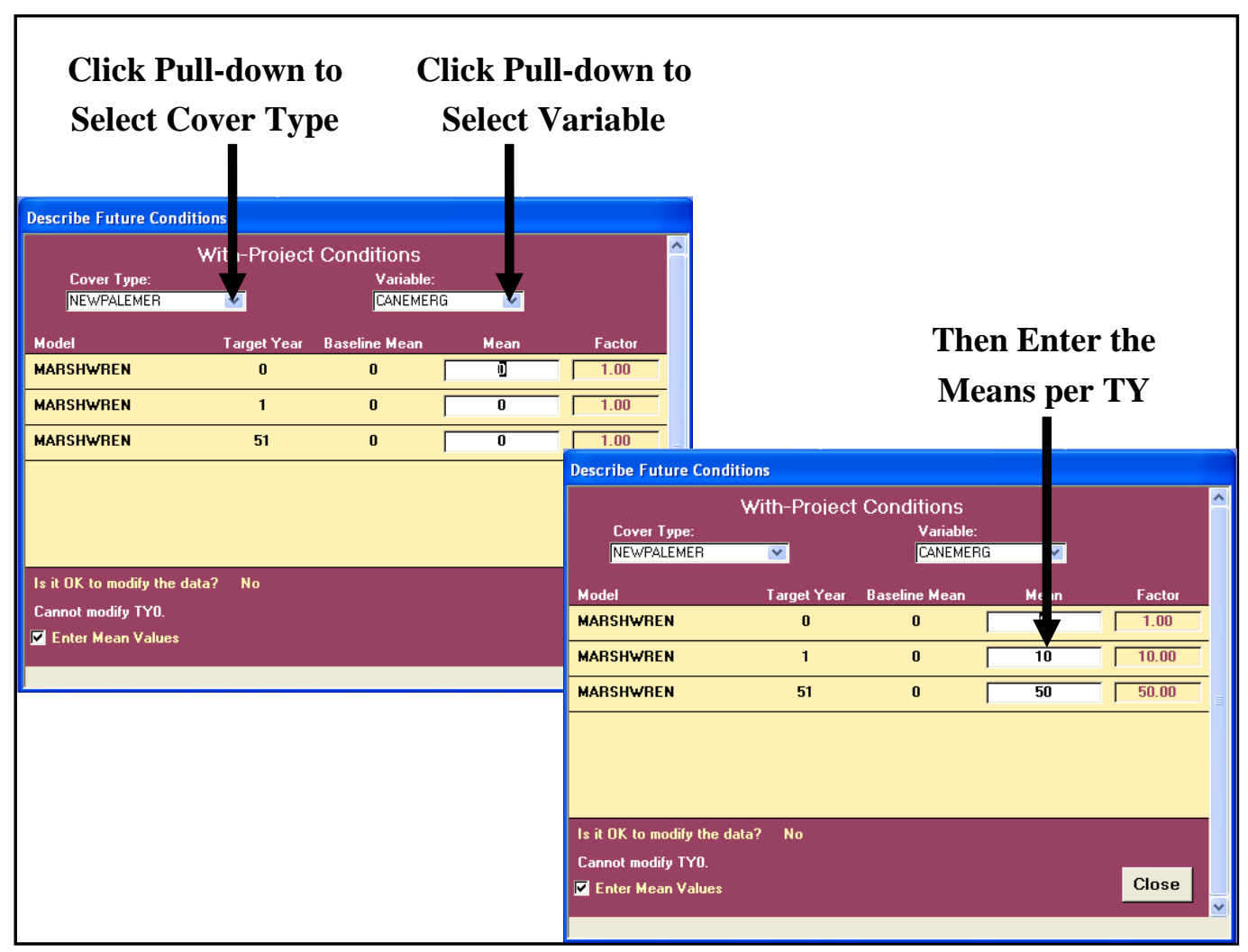

Figure 124. Enter the with-project conditions for the single formula models in EXHEP.

When finished, click on the Close button, and EXHEP asks about saving the data:

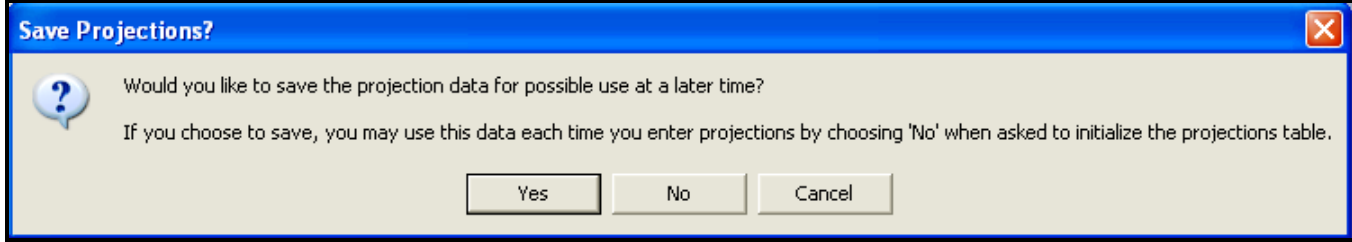

If an error was made, click the No button, and all the changes will be discarded. To correct the error immediately, click the Cancel button and EXHEP will return to the data entry interface. If the data were entered correctly, click on the Yes button, and

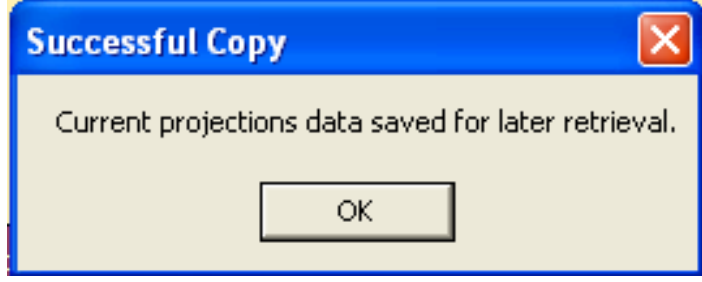


EXHEP affirms that the information was saved successfully. Click OK and EXHEP returns to the primary interface.

Additional actions for the with-project projection entries in multiple formula models

If multiple formula models are used in the analysis, the with-project variable projections must be completed using the second set of buttons on EXHEP's primary interface (Figure 125).

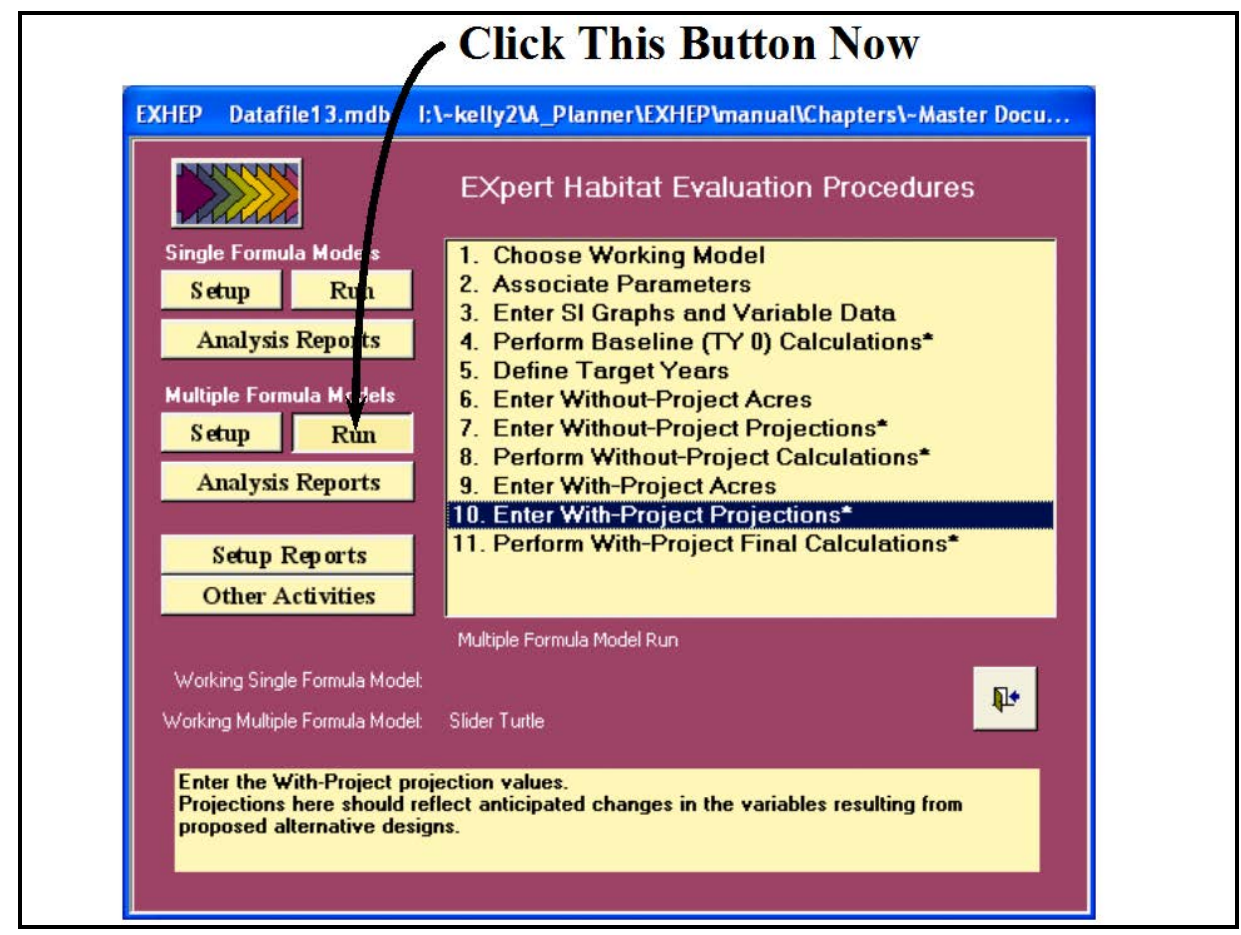

Figure 125. Click on the Run button to begin the with-project variables entry process for multiple formula models in EXHEP.

As it did before, a screen appears in the center of the primary interface with 11 options:

\section{Choose Working Model}

2. Associate Parameters

3. Enter SI Graphs and Variable Data

4. Perform Baseline (TY 0) Calculations*

5. Define Target Years

6. Enter Without-Project Acres

7. Enter Without-Project Projections*

8. Perform Without-Project Calculations* 


\section{Enter With-Project Acres \\ 10. Enter With-Project Projections* \\ 11. Perform With-Project Final Calculations*}

Check to assure that the "Working Model" is set to the correct model, and click on the tenth option (Enter With-project Projections*) to continue. In response, EXHEP asks the question, "Do you want to initialize the future factors first?"

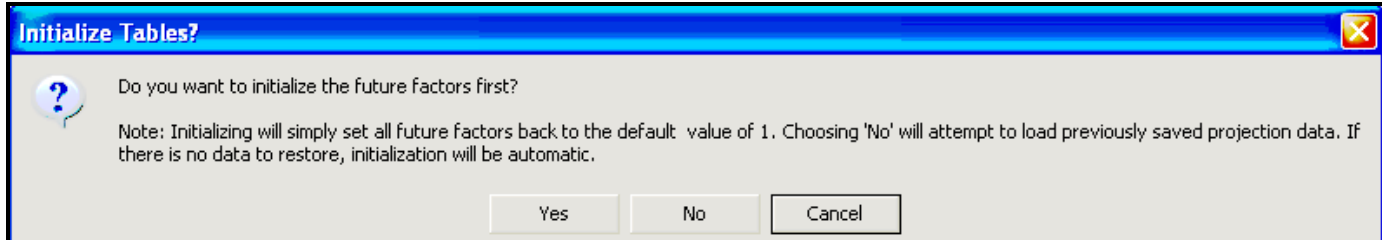

No $\quad$ Cancel

Click Yes to proceed - initialization here will not affect the data entered above in the single formula model

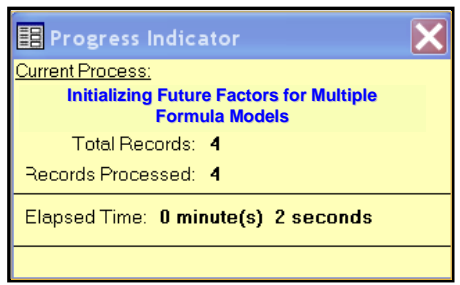

exercise. As

the initialization commences, a

Progress Indicator

window will open in the screen tracking the status of the process, providing a tentative estimate of Elapsed Time.

\section{USER'S NOTE:}

The initialization process is irreversible. If the Yes button is clicked in error, all the variables must be re-entered by hand.

\section{USER'S NOTE:}

The initialization process focuses on the "type" of model (single vs. multiple formula models), and initializes the database across all models within that "type." If the "Working Model" is changed, and this question is asked a second time, answer NO to proceed.

USER'S NOTE:

Say "Yes" to initialization if:

1. Variable projections have not been made for any model.

2. Variable projections have been made for single formula models, but multiple formula models are now the focus (i.e., each "type" must experience initialization once).

3. Baseline data have been modified after an initialization.

4. Target years have been modified after an initialization.

5. Model associations have been modified after an initialization.

6. Models, cover types, life requisites, and/or variables have been added/deleted/modified post-initialization (basically any change in Setup necessitates re-initialization).

Say "No" to initialization when:

1. Variable projections have been made for at least one model in the "type."

2. Modifying current projection trends - post-initialization.

3. Running models through the without- and/or with-project calculations in series.

4. Modifying acreages in the baseline, without- and/or with-project interfaces. 
When complete, EXHEP opens the following window (Figure 126).

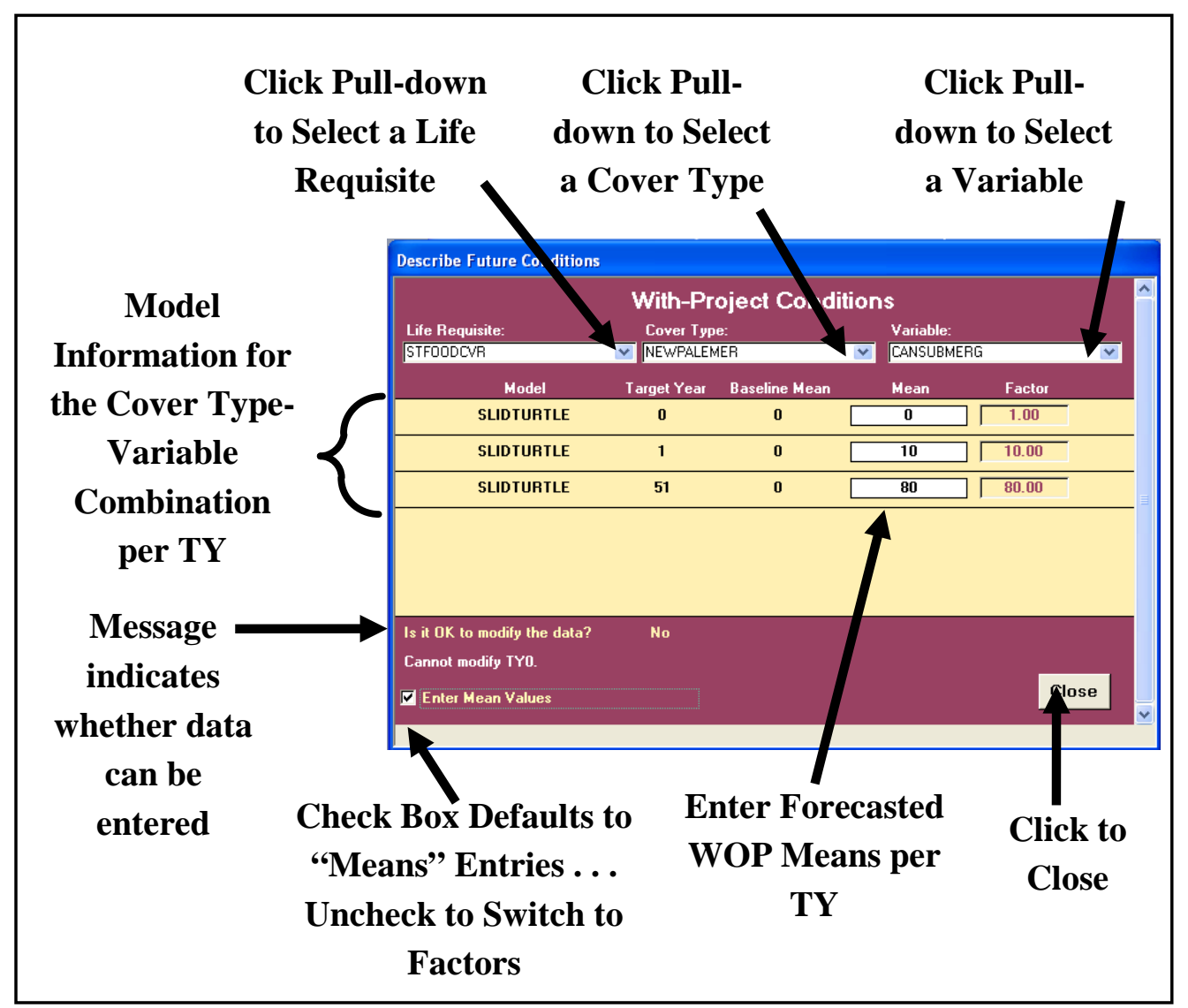

Figure 126. Describe Future Conditions entry window used to forecast with-project conditions for multiple formula models in EXHEP.

Use the same protocol described above in the without-project section to enter variable trends on the site under the with-project scenario. When finished, close the forms and return to the primary interface.

Checking the with-project variable entries with EXHEP reports

To review and check the results, click on the Analysis Reports button(s) on the primary interface (Figure 127). 


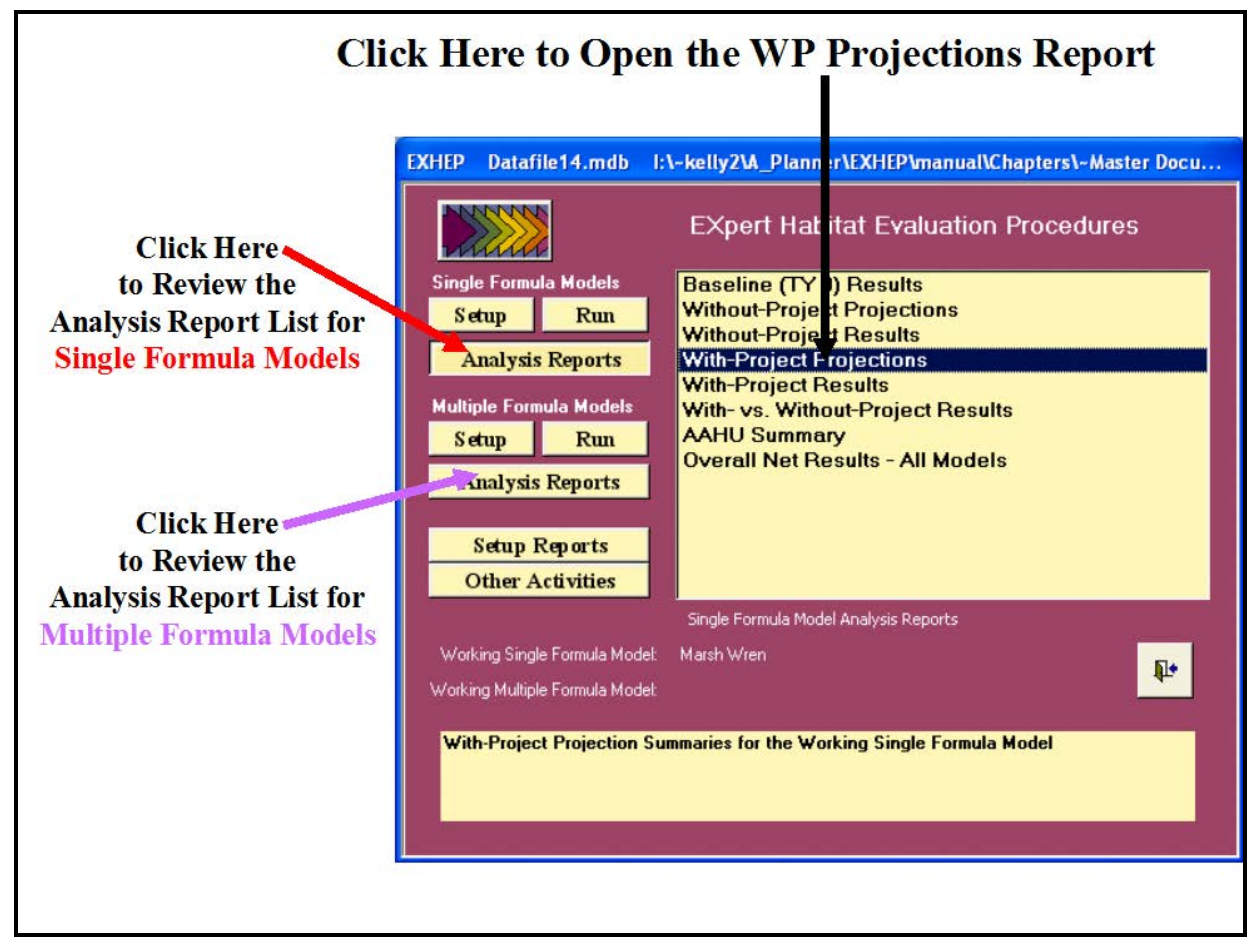

Figure 127. Click on the Analysis Reports buttons to review the with-project forecasted entries in EXHEP.

A list of available reports appears in the center of the primary interface. The fourth report listed, With-Project Projections, is ready for inspection. Double-click on this report to review the results (Figure 128).

Click on the Close button from the suite of buttons offered in the Report Control toolbar, and EXHEP returns to the primary interface. Now that the with-project forecasts have been entered into the system, proceed to the next step to learn how to assess the with-project conditions of the site in EXHEP.

\section{Perform the with-project calculations}

EXHEP will calculate the with-project results on a model-by-model basis for both single and multiple formula models. To proceed, click on the Run button under the Single Formula Models section of the primary interface (Figure 129). 


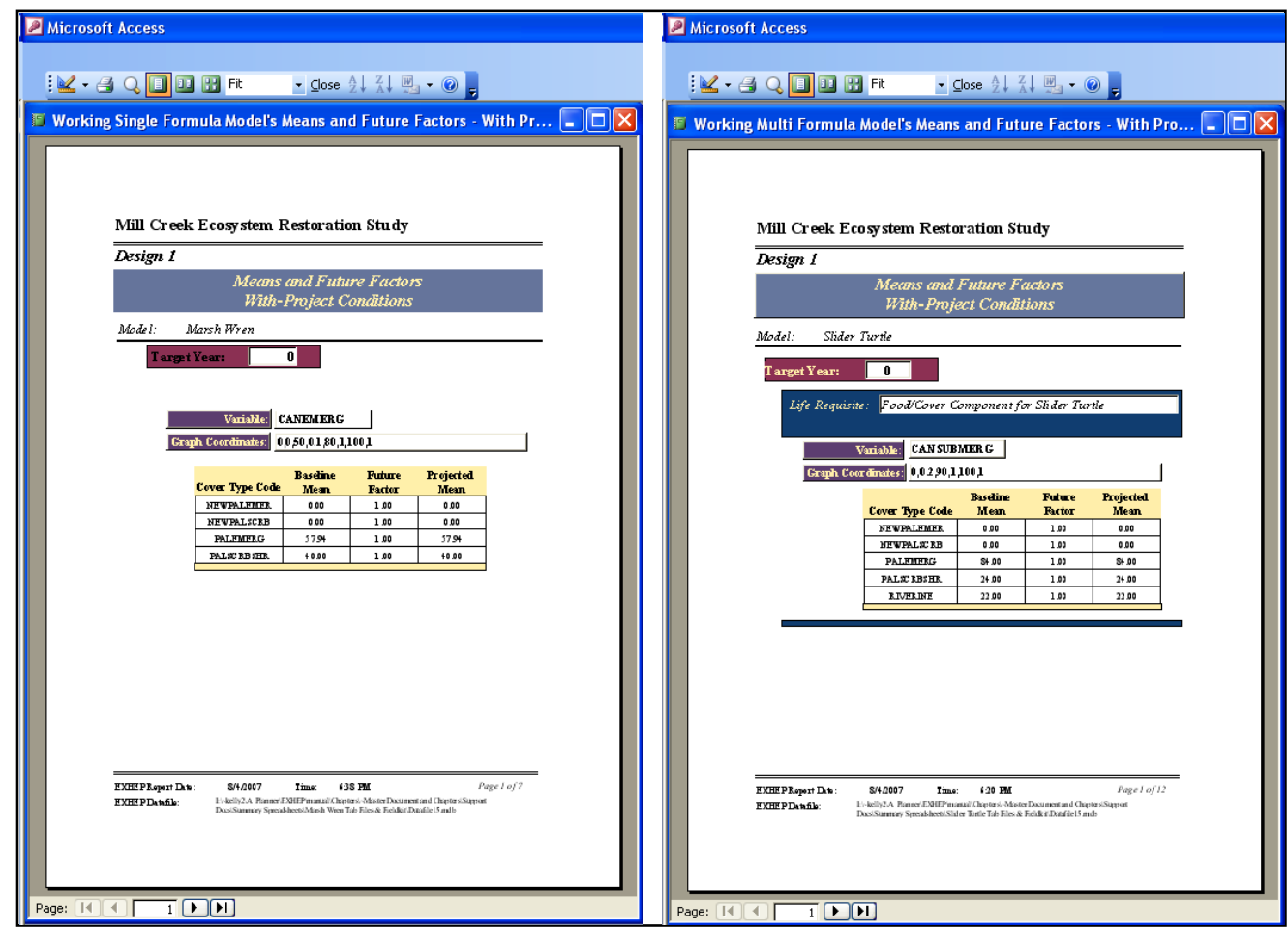

Figure 128. Example of With-Project Projections reports in EXHEP.

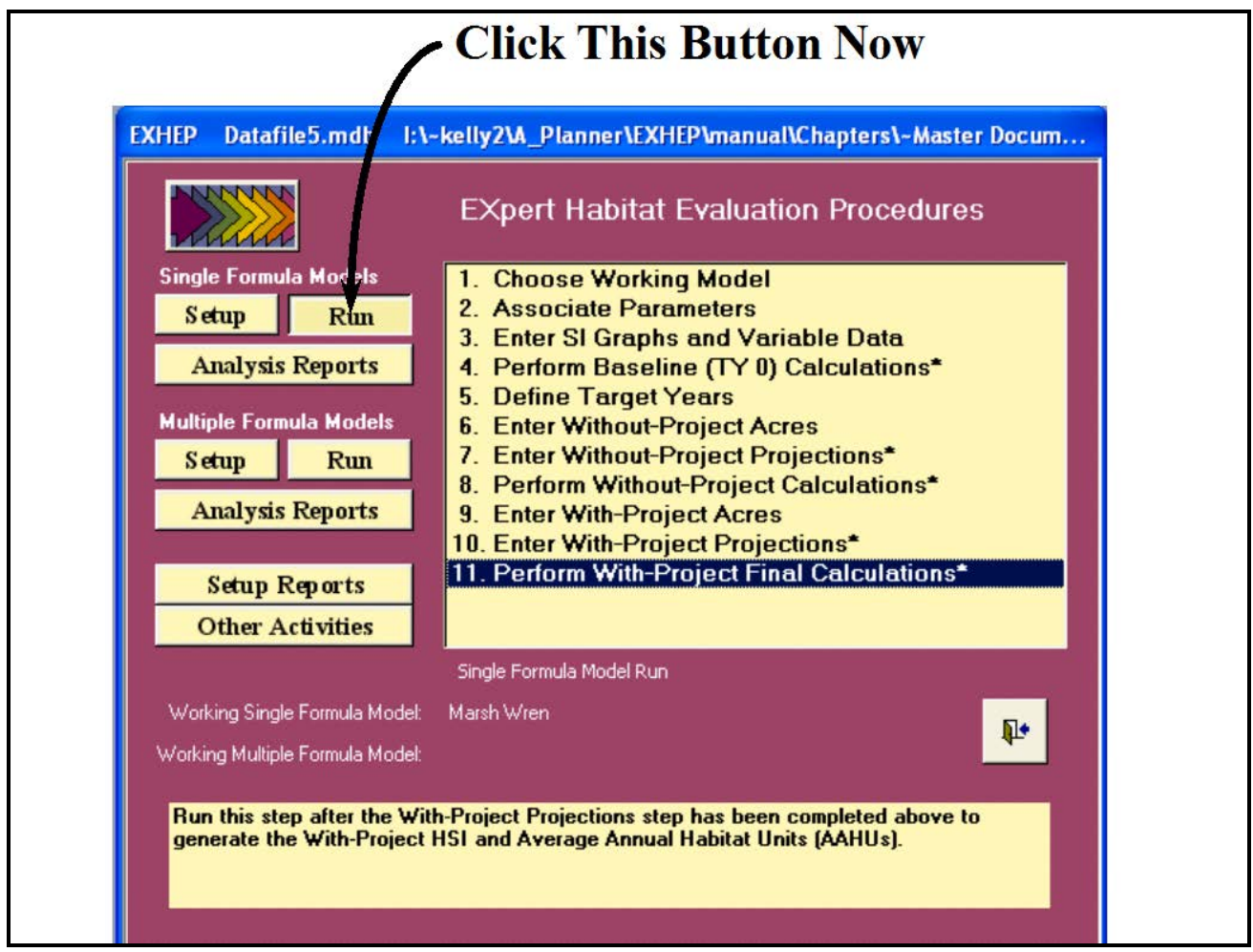

Figure 129. Click on the Run button to begin the with-project calculation process for single formula models in EXHEP. 
As it did before, a screen appears in the center of the primary interface with 11 options:

\section{Choose Working Model}

2. Associate Parameters

3. Enter SI Graphs and Variable Data

4. Perform Baseline (TY 0) Calculations*

5. Define Target Years

6. Enter Without-Project Acres

7. Enter Without-Project Projections*

8. Perform Without-Project Calculations*

9. Enter With-Project Acres

10. Enter With-Project Projections*

11. Perform With-Project Final Calculations*

Check to assure that the "Working Model" is set to the correct model, and click on the last option (Perform With-Project Calculations*) to continue. In response, EXHEP will begin the calculation process. As the calculations continue, a Progress Indicator

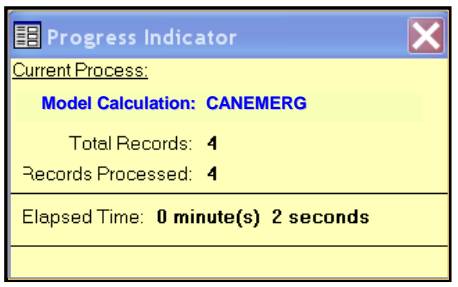
window will open in the screen. This progress window will track the status of the analysis (number of records to assess vs. number of records processed) and provide a tentative estimate of Elapsed Time. When completed, EXHEP returns a message that the calculations are concluded. This process must be completed for each model in the

\section{Final Calculation Status} The Working Model's Final With-Project Calculations completed successfully.

$$
\text { OK }
$$
analysis. 


\section{Exporting the AAHU results}

Before continuing to the next model, it is critical that the annualized outputs of the analysis are saved to an MS Word file for documentation purposes (these are overwritten each time the "Working Model" is changed). To begin, click on the Analysis Reports button(s) on the primary interface (Figure 130).

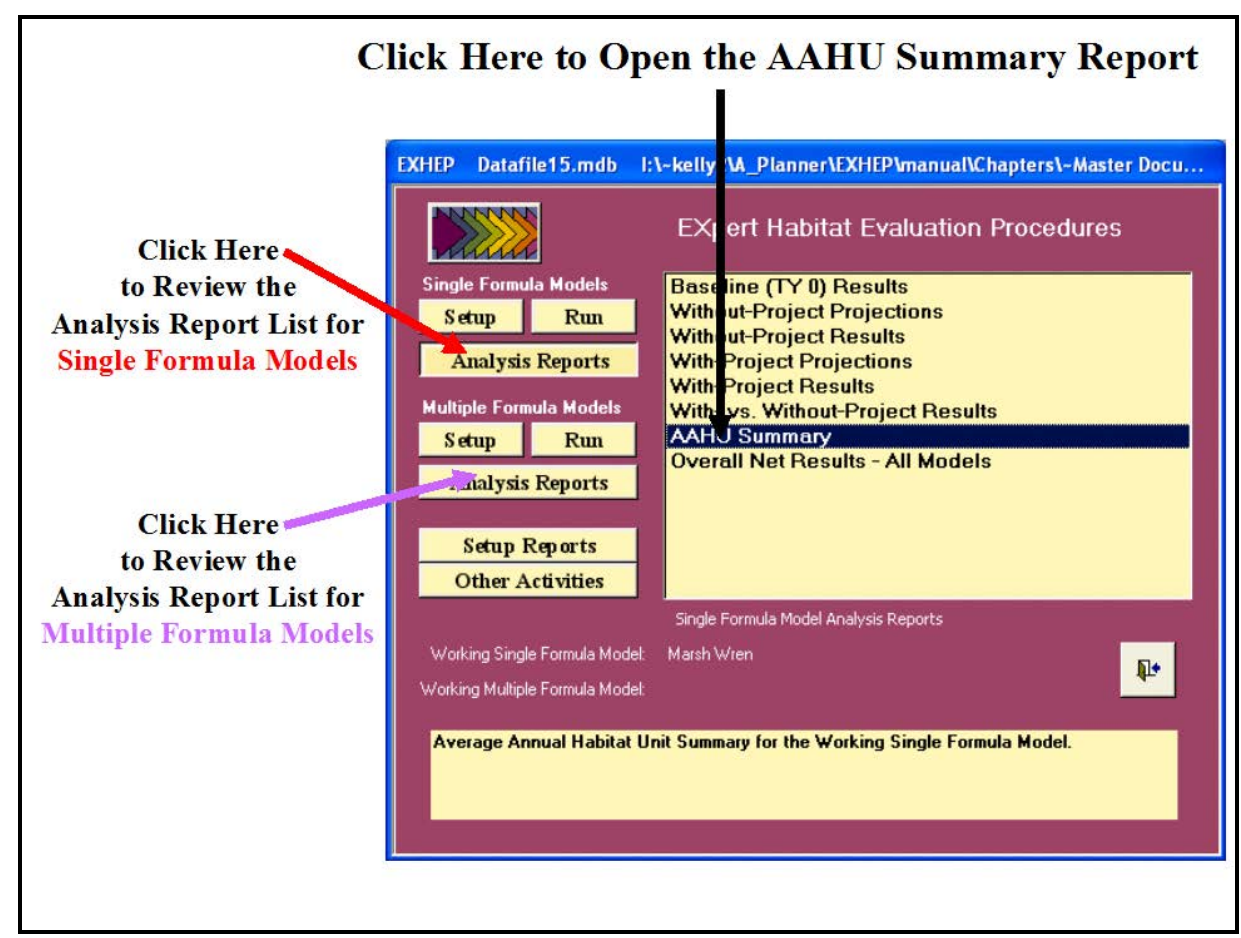

Figure 130. Click on the Analysis Reports buttons to find the AAHU Summary report in EXHEP.

A list of available reports appears in the center of the primary interface. The seventh report listed, AAHU Summary, is ready for inspection and export. Double-click on this report to review the results (Figure 131). 


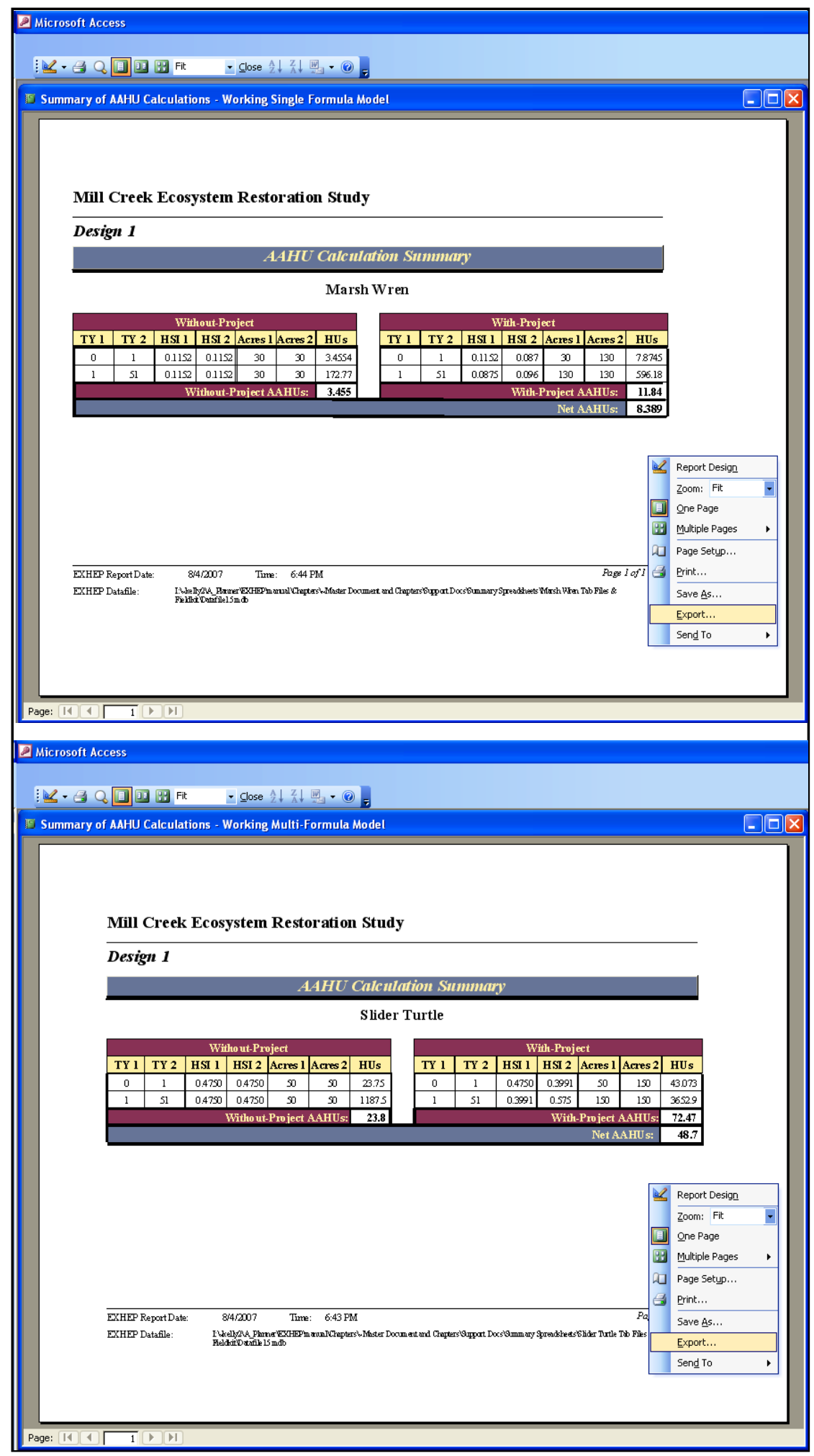

Figure 131. Example of $A A H U$ Summary reports in EXHEP. 
Right-click on the report, and select the Export option from the list of choices presented. An Export Report entry screen will open in response to this action (Figure 132).

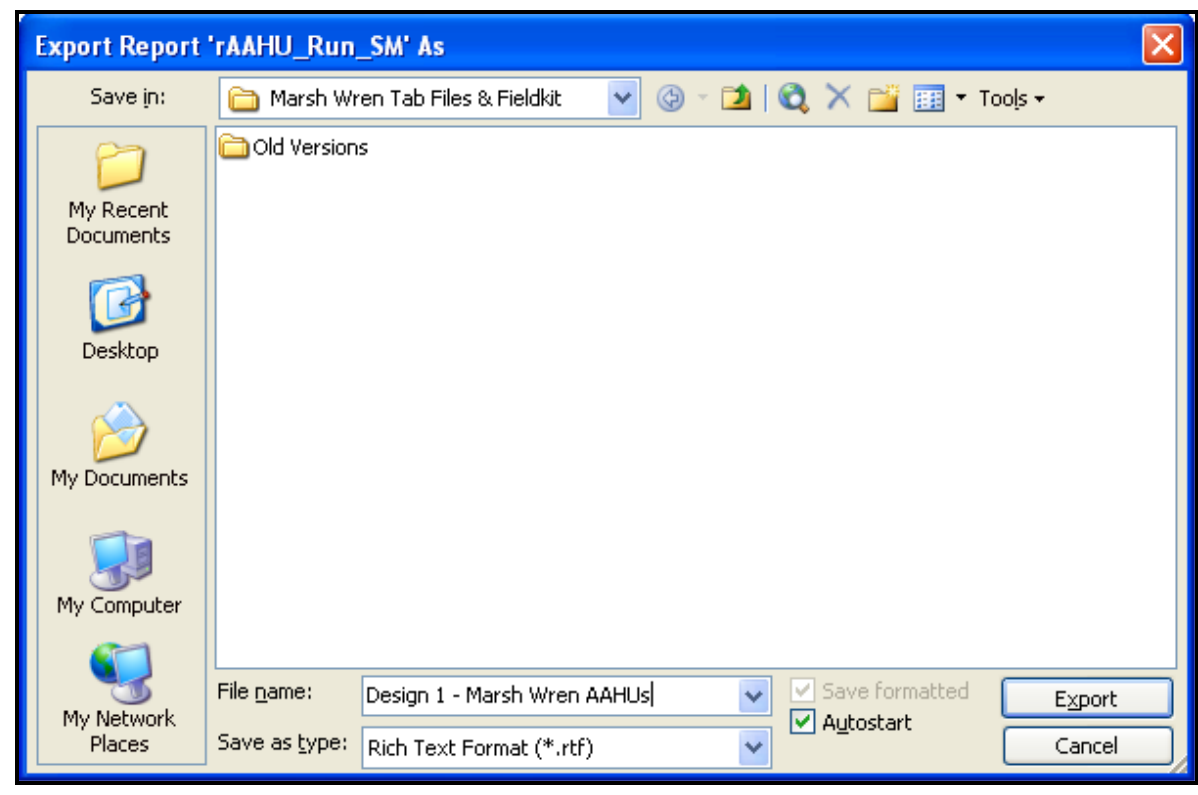

Figure 132. Export Report entry screen - exporting report information to MS Word.

Select the export location for the report in the Save in: window at the top of the entry screen. Provide a name for the report in the File name: window in the bottom of the entry screen. Use the Save as type: pulldown list to select the type of file to be exported [i.e., choose Rich Text Format (*.rtf) for the word-processing format]. To associate the software (i.e., MS Word for word processing documents), such that the medium opens and displays the information in the new environment upon exportation, click the Autostart checkbox immediately to the right of the Save as type: pull-down list. Press the Export button in the lower righthand corner of the screen. EXHEP will export the data displayed in the report to the new file, and if the Autostart option was clicked, MS Word will open and display the report in the new environment (Figure 133). 


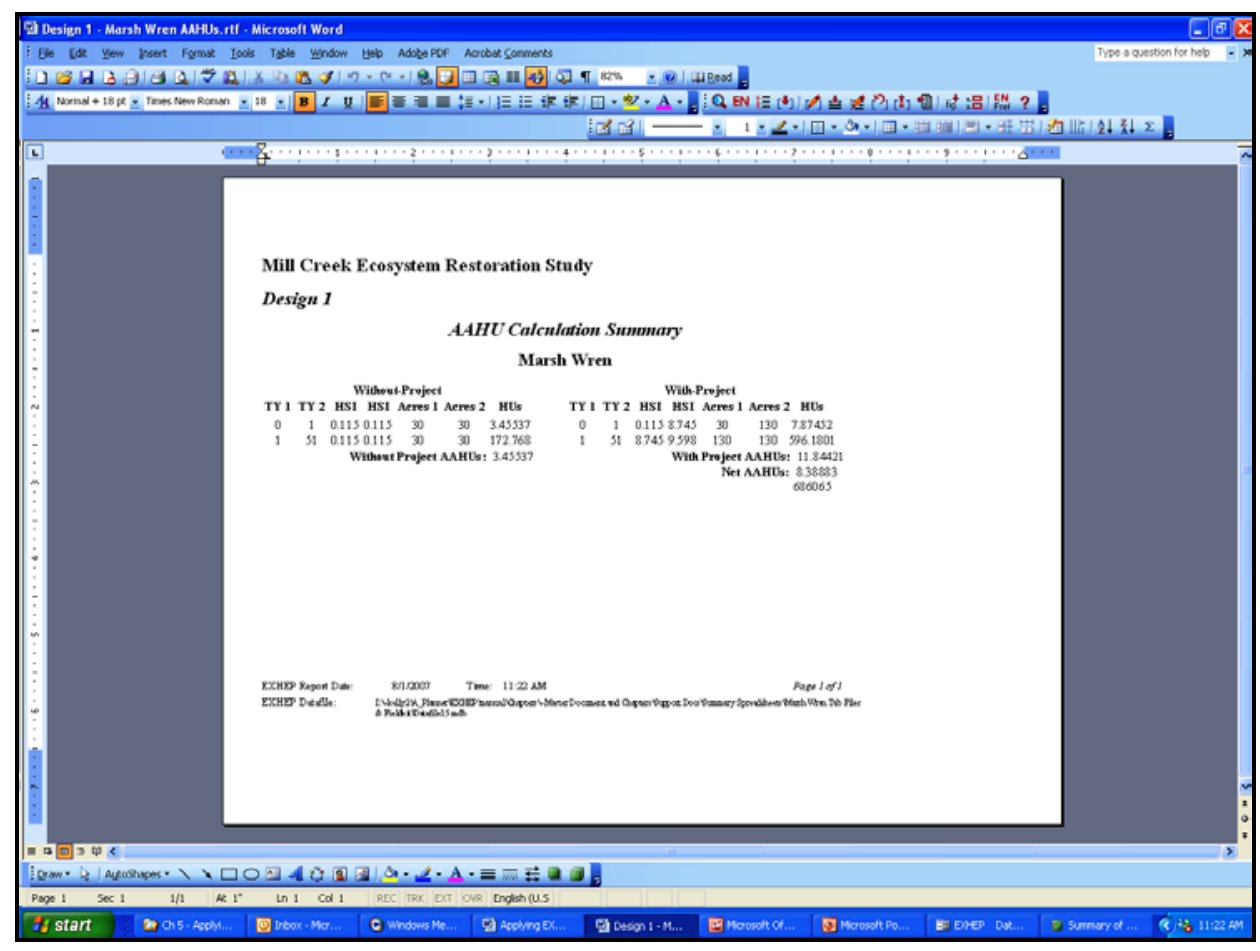

Figure 133. Exported AAHU summary in the MS Word environment.

Format and save the files as needed, close the MS Word application and

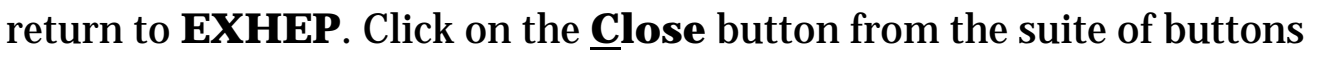
offered in the Print Preview toolbar - EXHEP returns to the primary interface. Now proceed to the next section.

Running more models through the assessment process

Each time a "Working Model" is selected, the options on the primary interface that have stars next to their titles must be re-run to focus the database. The "quick steps" are as follows:

1. Click on 1. Choose Working Model and select the next model for processing.

2. Click on 4. Perform Baseline (TY 0) Calculations*, let the system run, and click $\mathbf{O K}$ when it is finished.

3. Click on 7. Enter Without-project Projections*, click on the No button for initialization, click the Close button at the bottom of the interface, click Yes to save the data, and click $\mathbf{O K}$ when that save is affirmed.

USER'S NOTE:

Remember, when asked about initializing the data in option 7. Enter Without-Project Projections*, answer No or the data inside will be overwritten.

4. Click on 8. Perform Without-project Calculations*, let the system run, and click $\mathbf{O K}$ when it is finished. 
5. Click on 10. Enter With-project

Projections*, click on the No button for initialization, click the Close button at the bottom of the interface, click Yes to save the data, and click $\mathbf{O K}$ when that save is affirmed.
USER'S NOTE:

Remember, when asked about initializing the data in option 10. Enter With-Project Projections*, answer No or the data inside will be overwritten.

\section{Click on 11. Perform With-project}

Calculations*, let the system run, and click $\mathbf{O K}$ when it is finished.

7. Export the AAHU results to MS Word (i.e., click the Analysis Reports button; double-click the AAHU Summary report, right-mouse click on the report and select Export; name and save the file; close MSWord; and close the report).

Additional actions for the with-project calculations of multiple formula models

If multiple formula models are used in the analysis, calculate with-project results for the models using the second set of buttons on EXHEP's primary interface (Figure 134).

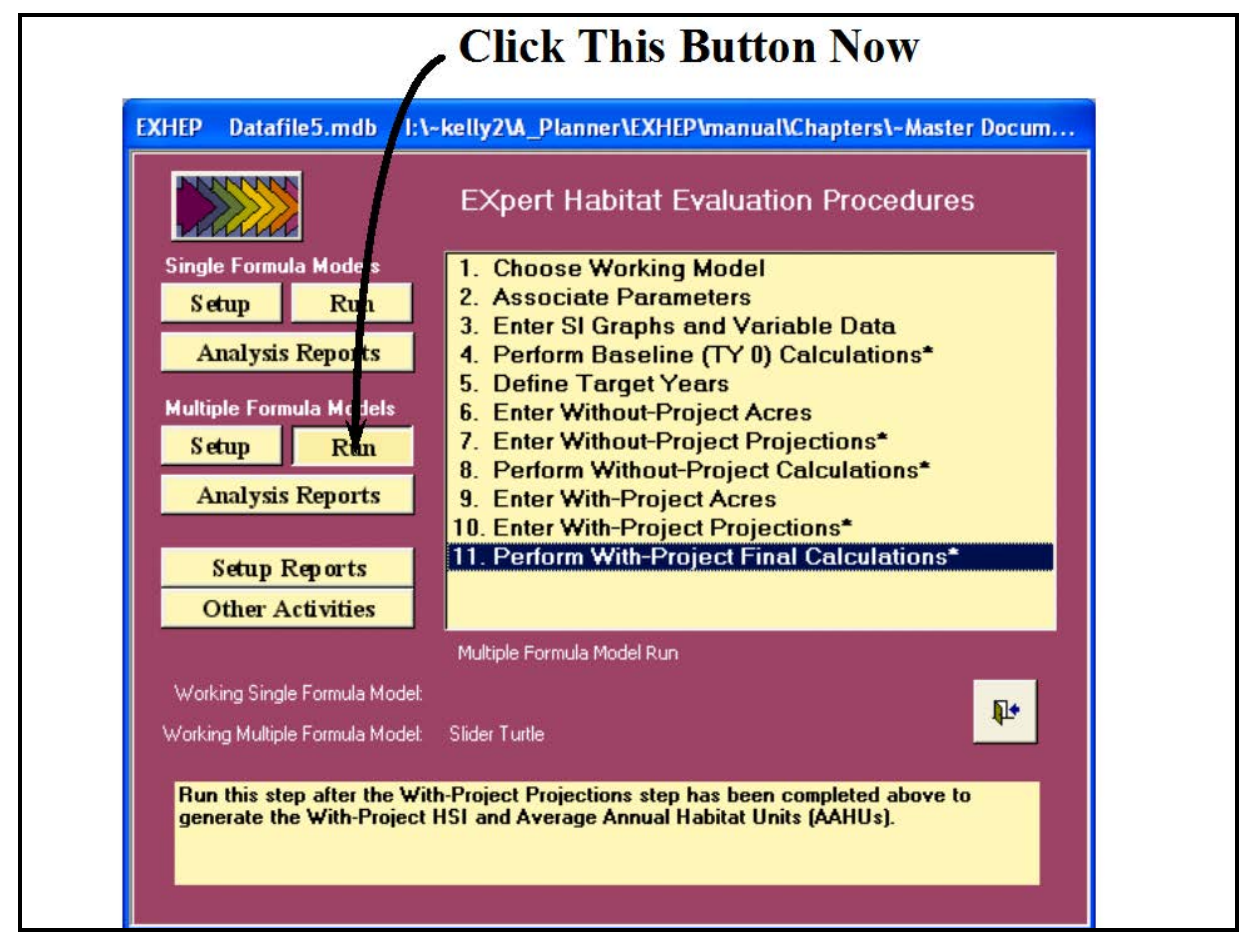

Figure 134. Click on the Run button to begin the with-project calculation process for multiple formula models in EXHEP.

As it did before, the screen appears in the center of the primary interface with 11 options: 
1. Choose Working Model

2. Associate Parameters

3. Enter SI Graphs and Variable Data

4. Perform Baseline (TY 0) Calculations*

5. Define Target Years

6. Enter Without-Project Acres

7. Enter Without-Project Projections*

8. Perform Without-Project Calculations*

9. Enter With-Project Acres

10. Enter With-Project Projections*

11. Perform With-Project Final Calculations*

Use the same protocol described above in the single formula models section to run the with-project analysis for each of the models in the analysis.

\section{Checking the with-project analysis with EXHEP reports}

To review and check the results, click on the Analysis Reports button(s) on the primary interface (Figure 135).

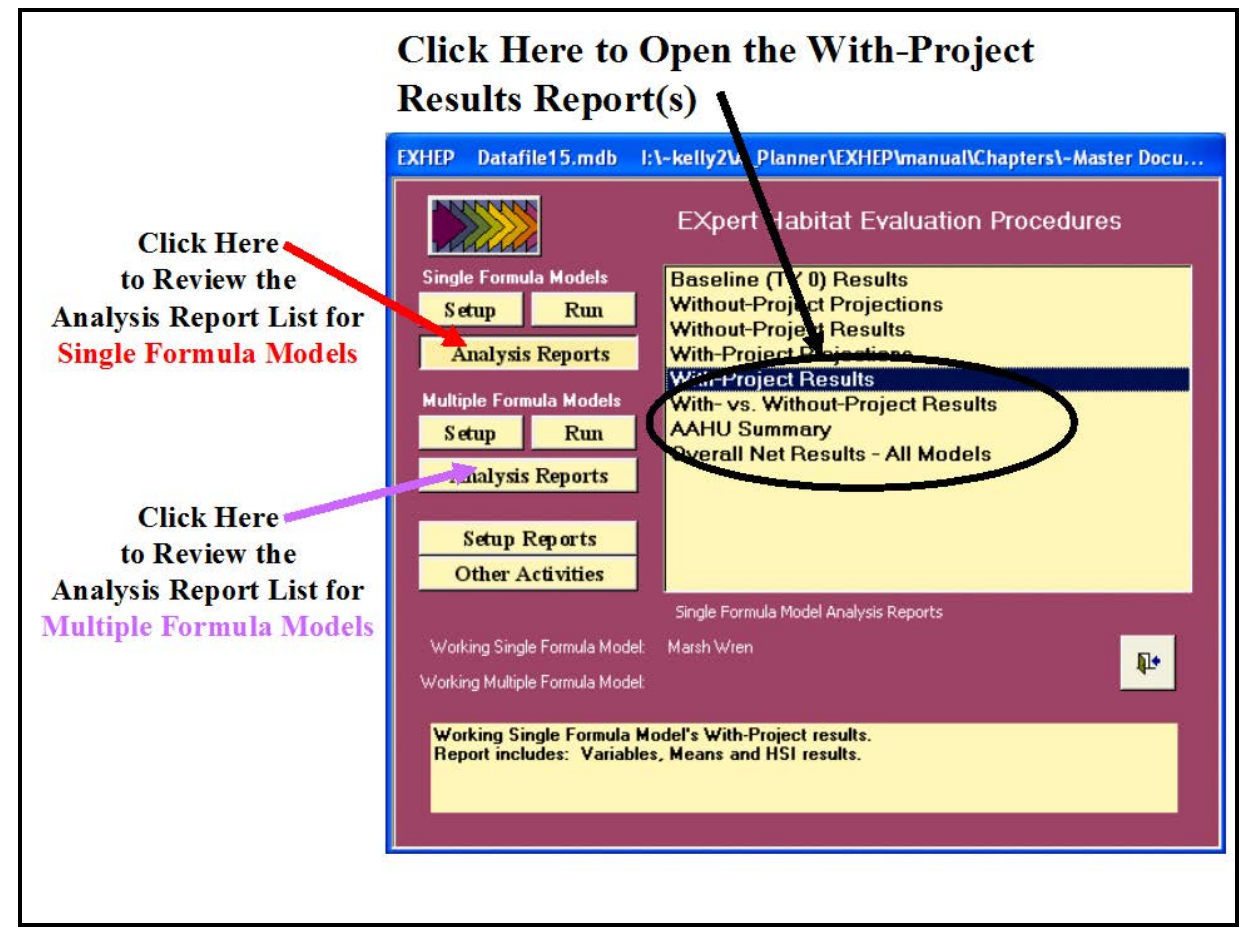

Figure 135. Click on the Analysis Reports buttons to review the with-project results in EXHEP. 
A list of available reports appears in the center of the primary interface. The fifth report listed, With-Project Results, is ready for inspection. Double-click on this report to review the results (Figure 136).

The site's overall average annual outputs (i.e., AAHUs) are reported just under each model's name. The HSI scores, associated acreages, and the resulting HU scores are then reported on a target year-by-target year basis below the AAHU values. The specifics used to generate the overall scores are reported in the tables below this summary. These details are sorted alphabetically by life requisite (where applicable), cover type, and variable. These reports are cumulative; thus, all models assessed in the EXHEP analyses are presented herein. Click on the Close button from the suite of buttons offered in the Report Control toolbar, and EXHEP returns to the primary interface.

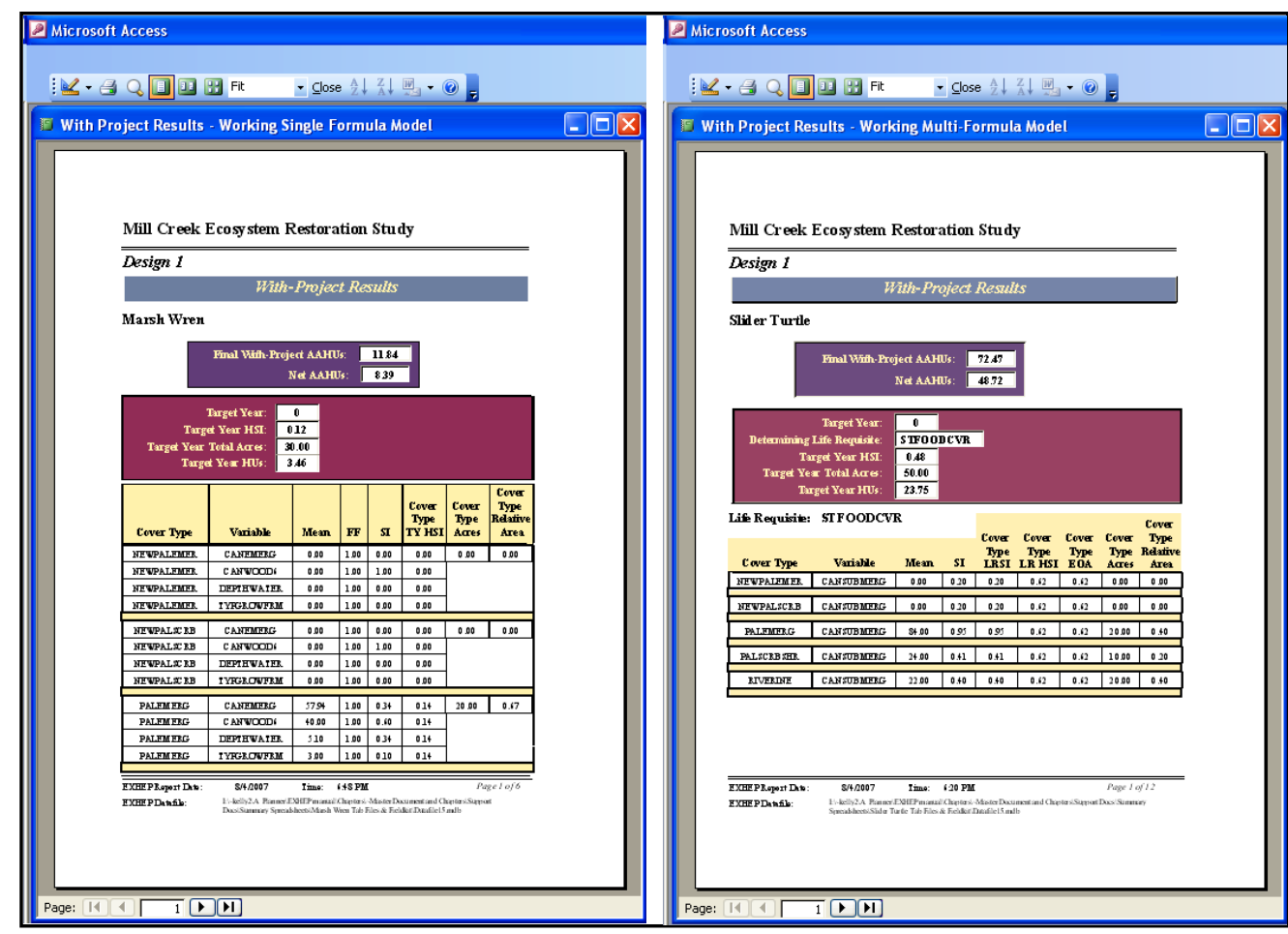

Figure 136. Examples of With-Project Results reports in EXHEP.

A side-by-side report is available to compare the without- and with-project conditions on the same page. Double-click on the With- vs. WithoutProject Results report(s) to review this information (Figure 137). 


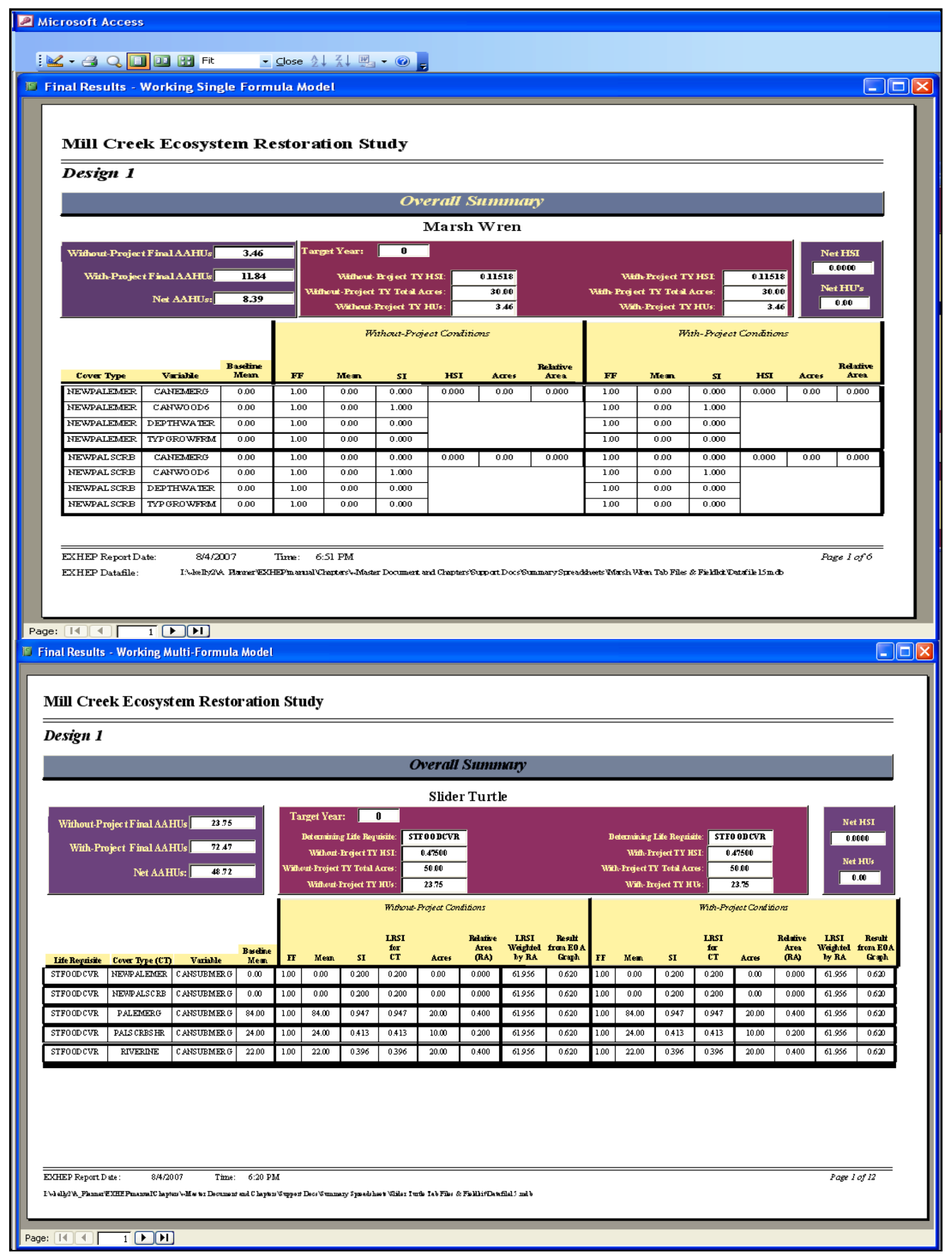

Figure 137. Examples of With- vs. Without-Project Results reports in EXHEP. 
Click on the Close button from the suite of buttons offered in the Report Control toolbar, and EXHEP returns to the primary interface.

One final report is available to compare outcomes across the entire suite of models assessed on the site. Double-click on the Overall Net Results All Models report to review this information (Figure 138).

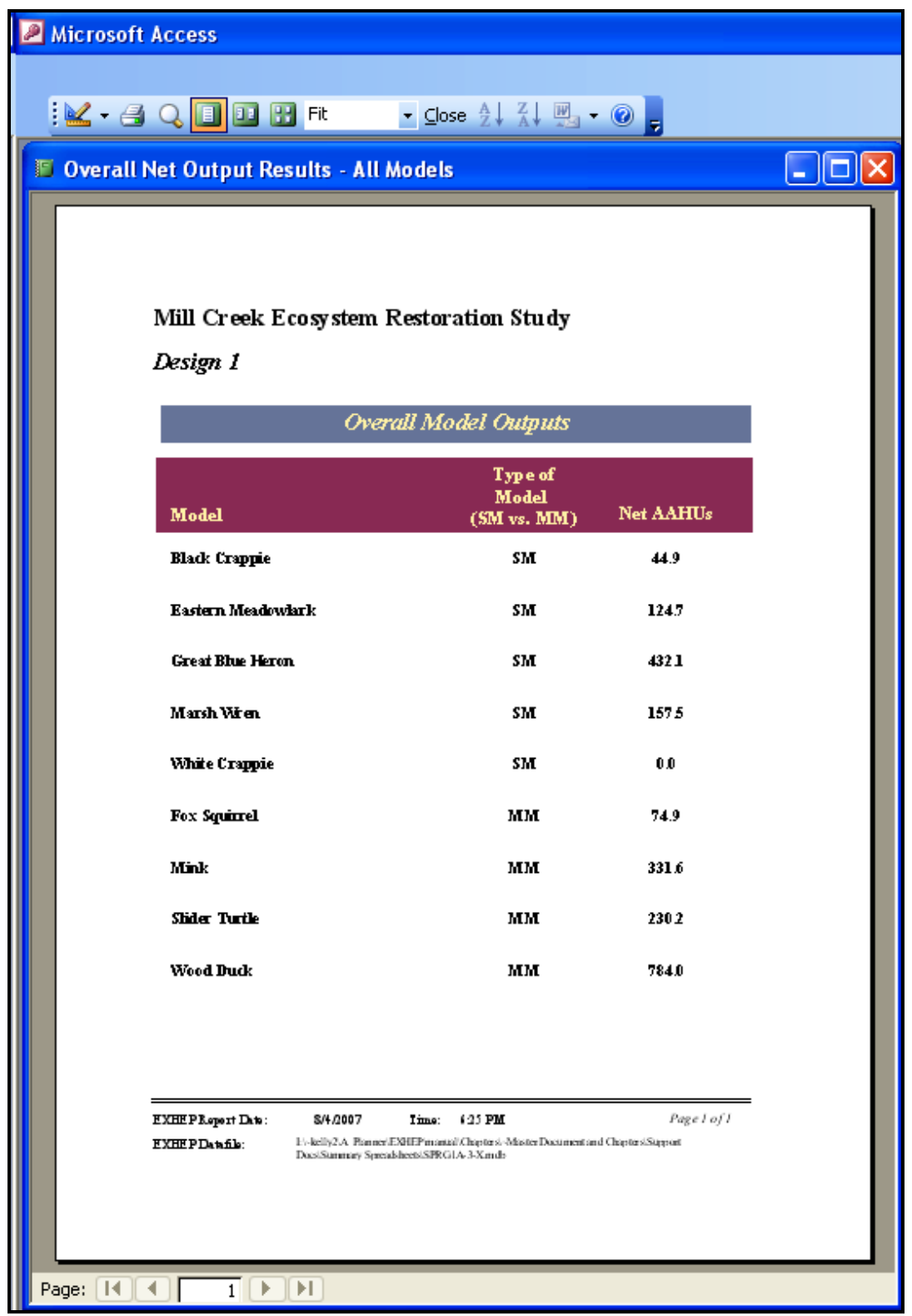

Figure 138. Examples of Overall Net Results - All Models report in EXHEP.

Click on the Close button from the suite of buttons offered in the Report Control toolbar, and EXHEP returns to the primary interface. 


\section{Step 7: Recycle the datafile and evaluate alternative designs}

Often multiple alternatives are formulated to address the goals and objectives in a single study. The HEAT modules were designed to assess multiple outputs per alternative, and can be used to generate outputs for multiple alternatives per project with a minimum amount of effort. It is not necessary to rebuild the models, cover type interdependencies, or life requisite interrelationships from a blank datafile. Instead, a "master" datafile can be constructed. ${ }^{1}$ This "master" datafile is then copied, renamed, and changed to capture the with-project trends (i.e., alternative variable and cover type projections are input) of an alternative design. In HEAT this process is referred to as "recycling," and can be performed as many times as necessary to assess the numerous alternatives formulated in a single study (Figure 139). ${ }^{2}$

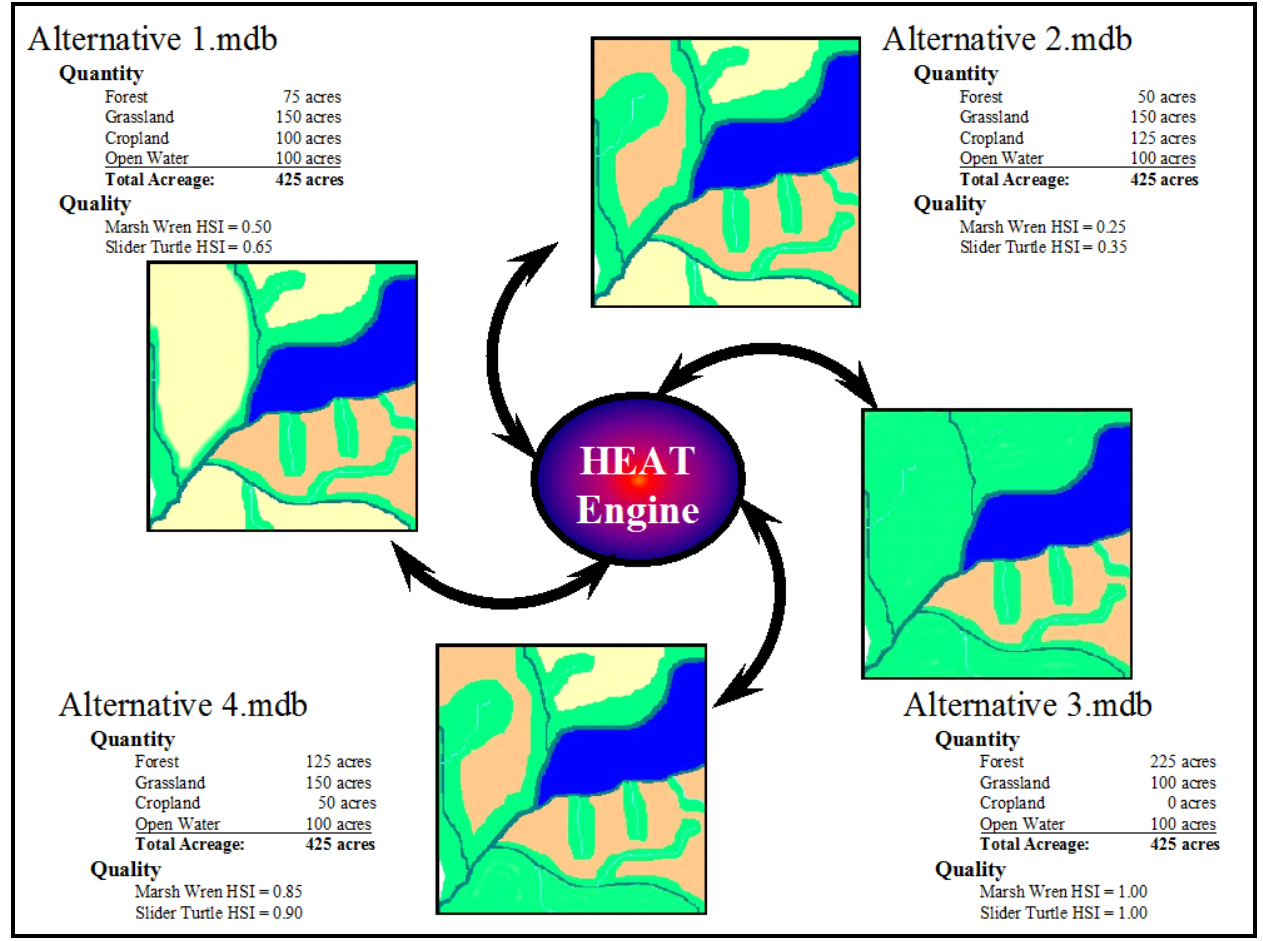

Figure 139. Recycling datafiles in HEAT to streamline the development of alternatives for a single project

\footnotetext{
${ }^{1}$ A "master" datafile here refers to an EXHEP file that has been run through the entire evaluation of a proposed design alternative [i.e., all models (both single and multiple formula "types") have been processed through the 11 steps on the primary interface].

2 To assist in the software application, a series of training exercises have been included in Appendix E refer to Exercise 9 for relevant training in master file creation in EXHEP.
} 
To begin, use the steps described in this user guide to construct and assess the first alternative in the study. Copy the "master" file and rename it based on the second alternative in Windows Explorer. Open this new file with HEATEXHEP and change the alternative name in the Methods and Assumptions option. Then change the with-project acreages and variable trends (i.e., 9. Enter With-Project

USER'S NOTE:

In environmental studies, it is common practice to hold the site size static and maintain a common timescale (i.e., target years) across alternative designs project-wide.

Acres, and 10. Enter With-Project Projections, * respectively) to capture the context of the new alternative. Re-run the calculations and report the outcomes in a cumulative fashion.

Alternatively, "master" datafiles can be used to assess multiple sites (i.e., as would be the case in an impact study where both the impact and mitigation sites must be evaluated). In this instance, use the steps described in this user guide to construct and assess the first alternative at the first site in the study. Copy the "master" file and rename it based on the second site in Windows Explorer. Open this new file with HEAT-EXHEP and change the alternative name in the Methods and Assumptions option. Now change all features relevant to the

USER'S NOTE:

In impact/mitigation studies, it is common practice to use the same timescale (i.e., target years) across locations and alternative designs.

change in location (i.e., baseline acreages data, without-project conditions, and with-project forecasts). Re-run the calculations and report the outcomes in a cumulative fashion. 


\section{Applying EXHGM}

As discussed in earlier chapters, there are 12 steps to complete when applying HGM in an ecosystem evaluation. Once a function or functions have been selected/ developed (Step 4), and the evaluation team has inventoried the site using the function's parameters (i.e., Step 5), it becomes necessary to generate outputs (i.e., Functional Capacity Units or FCUs). At this point, the HEAT software ${ }^{1}$ can be fully deployed. A series of steps have been devised to move through this process quickly and cleanly:

1. Gather the pertinent information.

2. Set up the models in EXHGM.

3. Associate the functions in the models.

4. Enter the baseline data and generate baseline results.

5. Enter the without-project conditions and calculate the effects.

6. Enter the with-project conditions and calculate the effects.

7. Recycle the datafileand evaluate alternative designs.

\section{Step 1: Gather the pertinent information}

Basic information regarding the study's goals, objectives, opportunities, and constraints is always useful to capture early on in the analysis process. EXHGM allows users to store this information iteratively, and provides access to this data at the touch of a button. Issues surrounding model selection, modification, and creation can also be addressed and documented in the software. Model architecture can have a profound effect on the analysis strategy, and should be recorded and incorporated into this data-gathering process as well.

\section{Data checklist for the EXHGM analyses}

It is also important to gather all information supporting the application of the models prior to setting up the software. Collect basic information (e.g., references, PWAAs, variables, sampling protocols, VSI curves, FCI formulas, etc.) early on. The software can be set up incrementally as this information becomes available, but analysis cannot commence without these

\footnotetext{
${ }_{1}$ An installation CD for the Habitat Evaluation and Assessment Tools (HEAT) program can be obtained by contacting Kelly Burks-Copes at 601-634-2290 (Kelly.A.Burks-Copes@usace.army.mil).
} 


\section{basics. A checklist is provided in Table 5 to help organize the necessary information and data. ${ }^{1}$}

Table 5. Pertinent information needed to complete an HGM analysis. ${ }^{1}$

\begin{tabular}{|c|c|}
\hline Items & Example \\
\hline \multicolumn{2}{|r|}{ Background Information } \\
\hline Project Name & Turkey Creek Project \\
\hline Alternative Name & Design 1 \\
\hline Methods & $\begin{array}{l}\text { Model References and Support Documentation } \\
\text { Model Modifications } \\
\text { List of Evaluation Team Members } \\
\text { Goals and Objectives } \\
\text { Data Management Strategies } \\
\text { Evaluation Strategies (including tradeoff approaches) } \\
\text { Field Sampling Team and Metadata (include locations, assumptions, dates, etc.) }\end{array}$ \\
\hline \multicolumn{2}{|r|}{ Model Specifics } \\
\hline $\begin{array}{l}\text { Model Name(s) and } \\
\text { Function(s) }\end{array}$ & $\begin{array}{l}\text { Wetland Subclass and functions performed by that subclass (both short-hand names or codes and } \\
\text { detailed descriptions). } \\
\text { Examples: } \\
\text { Wetland Subclass: Wet Pine Flats } \\
\text { Function: Maintain Characteristic Water Level Regime }\end{array}$ \\
\hline $\begin{array}{l}\text { Partial Wetland } \\
\text { Assessment Areas } \\
\text { (PWAA) }\end{array}$ & $\begin{array}{l}\text { Short-hand names or codes and detailed descriptions } \\
\text { Examples: } \\
\text { Bunchgrass-Pine Savanna } \\
\text { Cypress-Pine Savanna } \\
\text { Switchcane-Pine Savanna }\end{array}$ \\
\hline Variables & $\begin{array}{l}\text { Short-hand names or codes, detailed descriptions, sampling protocols, and data management } \\
\text { (statistical) activities. } \\
\text { Examples: } \\
\text { Density of pond cypress } \\
\text { Evapotranspiration potential } \\
\text { Herbaceous indicator score }\end{array}$ \\
\hline VSI Curves & $\begin{array}{l}X, Y \text { coordinates for all variables included in the model(s). } \\
\text { For example: } 0,1,1,1,3,1,10,0.7,15,0.7,25,0.7\end{array}$ \\
\hline FCl Formulas & 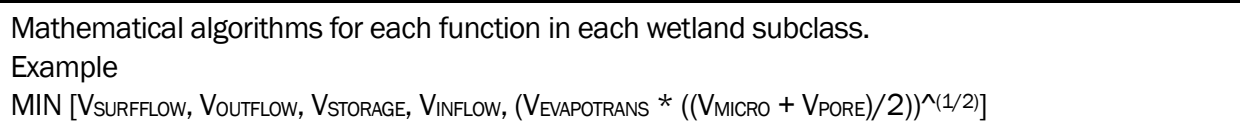 \\
\hline \multicolumn{2}{|r|}{ Evaluation Data } \\
\hline Target Years & $\begin{array}{l}\text { Baseline, end of construction, and life of the project (including additional years when needed). } \\
\text { Example: } \\
\begin{array}{rl}\frac{\text { TY }}{0} & \frac{\text { Calendar Year }}{2002} \\
1 & 2003 \\
51 & 2053\end{array}\end{array}$ \\
\hline
\end{tabular}

1 The wet pine flats FCl model has been provided on the installation CD (Wet Pine Flats Model). 
Table 5. (Concluded)

\begin{tabular}{|l|l||}
\hline \multicolumn{2}{|c||}{ Evaluation Data (Cont.) } \\
\hline \multirow{3}{*}{ Acres } & $\begin{array}{l}\text { Baseline acres per PWAA } \\
\text { Without-project acres per PWAA } \\
\text { With-project acres per PWAA for each alternative }\end{array}$ \\
\hline \multirow{3}{*}{ Variables } & $\begin{array}{l}\text { Baseline means/modes per PWAA } \\
\text { Without-project means/modes per PWAA } \\
\text { With-project means/modes per PWAA for each alternative }\end{array}$ \\
\hline
\end{tabular}

1 Data and material reported herein were taken from Rheinhardt et al. (2002).

\section{Step 2: Model setup}

To begin the setup process, open the HEAT software, select the EXHGM module, and load a blank datafile (Chapter 4). Name this file immediately (Other Activities button), and store it in a local folder of the user's preference (refer to Chapter 4 for details). When finished, click on the Setup button on the primary interface (upper left-hand corner under the EXHGM logo) (Figure 140). ${ }^{1}$

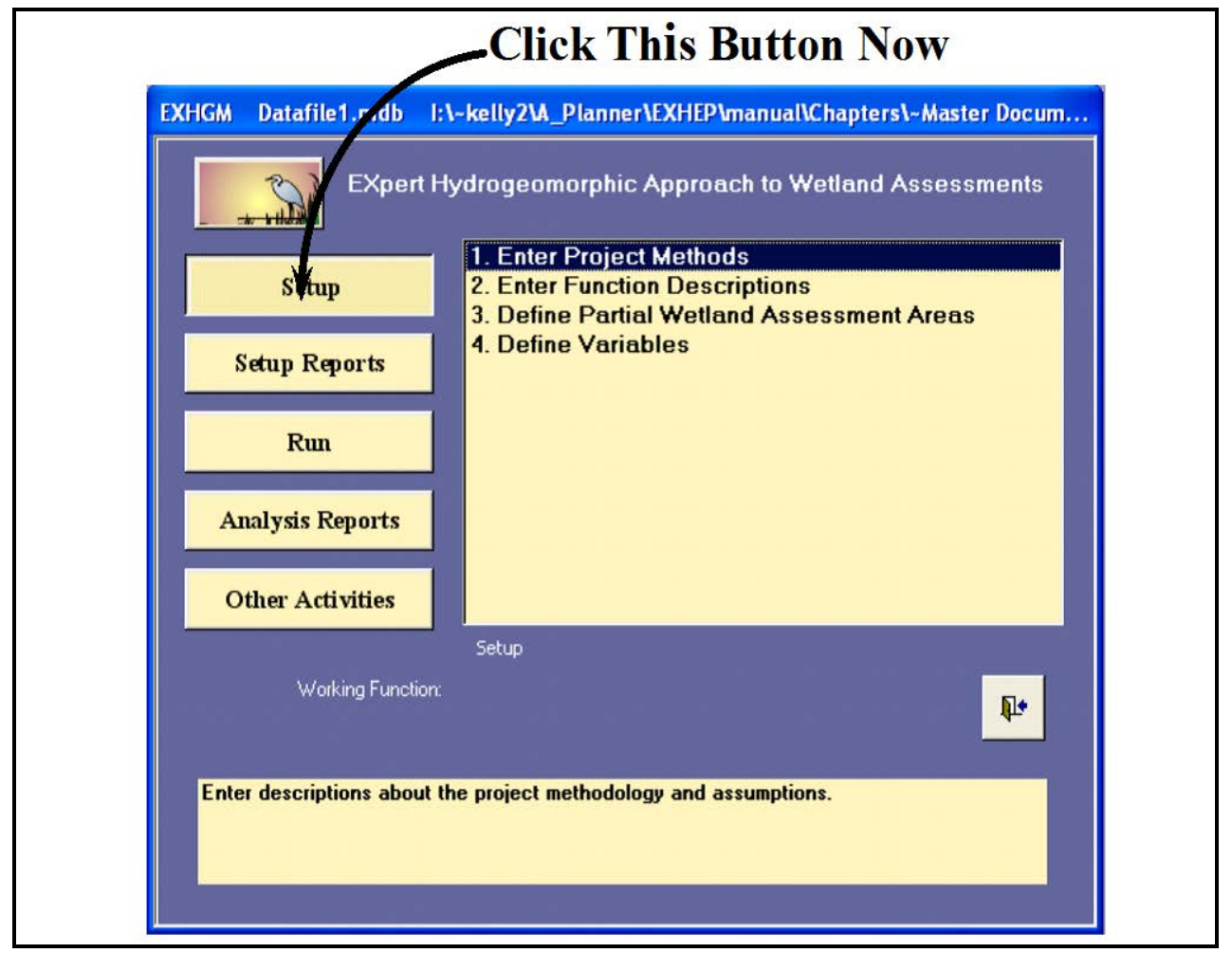

Figure 140. Click on the Setup button to begin the setup process in EXHGM.

\footnotetext{
${ }^{1}$ To assist in the software application, a series of training exercises have been included in Appendix E refer to Exercise 10 for relevant training in model setup in EXHGM.
} 
A screen appears in the center of the primary interface with four options:

\section{Enter Project Methods}

2. Enter Function Descriptions

3. Define Partial Wetland Assessment Areas

4. Define Variables

Moving through the process in a step-by-step fashion, systematically double-click each option from top to bottom to enter the methods, functions, partial wetland assessment areas ( or PWAAs), acres, and variables

USER'S NOTE:

If at any time new information is acquired that affects the analysis, return to these options and update the data. as described below.

\section{Enter, delete, and/or copy project methods}

Double-click on the first option in the primary interface (Enter Project Methods), to open the following window (Figure 141).

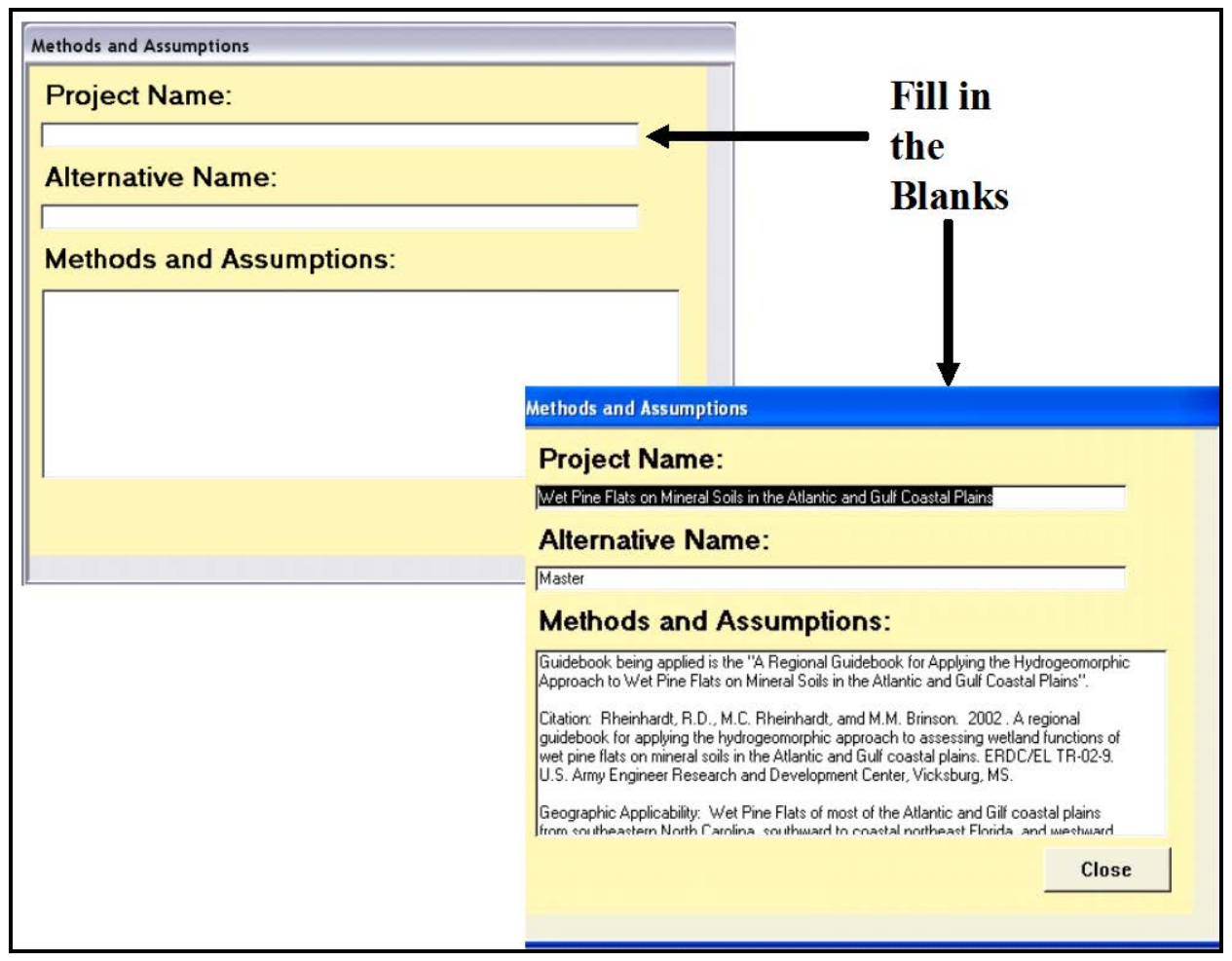

Figure 141. Methods and Assumptions entry screen in EXHGM. 
Fill in the blanks with all of the available information. Return to this screen as often as necessary to adequately document the analyses and applications. The key to success in HGM applications is documentation. At the absolute minimum, provide a Project Name (limit 255 characters) and an Alternative Name (limit 20 characters) in the spaces provided. The EXHGM module has no restrictions on the number of characters for the Methods and Assumptions section - there should be adequate space to fully document the study activities. Include any references for the function(s) used, and list the evaluation team participants with the associated affiliations. Consider including items such as PWAA codes and definitions, function modifications, dates of field collections, sources on historically gathered data (including GIS-based information), a list of the target years with their associated calendar years, sources for acreage estimates, descriptions of the future condition, forecasting strategies and decisions, information regarding who was responsible for the overall analysis and results, and points of contact for further information and support. Once this information has been entered, click on the Close button at the bottom of the screen and EXHGM returns to the primary interface.

If an entry must be entirely deleted from the datafile, click on the Other Activities button on EXHGM's primary interface, choose the Clear Database Information option, and proceed through the process as indicated in Chapter 4 of this guide. If details regarding the study's methods have been stored in an MS Word or MS Excel file, these details can be cut and pasted into this screen now. Highlight the information in the external file. Hold down the CTRL key and type $\mathbf{C}$ on the keyboard to copy the information.

USER'S NOTE:

Ctrl $+\mathbf{C}$ is the universal Microsoft shortcut key to copying text and numbers onto the clipboard and works well in these instances.

Ctrl $+\mathbf{V}$ is the universal Microsoft shortcut key to paste text and numbers from the clipboard.

Ctrl $+Z$ is the universal Microsoft shortcut key to undo an action.

F7 is the universal Microsoft shortcut key to check spelling.

EsC is an additional universal Microsoft shortcut key to undo an action.

These shortcut keys operate on a cell-bycell basis and cannot be used to copy entire spreadsheet rows or records in the data entry screens.

Return to EXHGM and click on the Methods and Assumptions section and click once. Hold down the CTRL key and type $\mathbf{V}$ on the keyboard to paste the information into this space. When finished with these activities, click on the Close button at the bottom of the screen and EXHGM returns to the primary interface. 


\section{Enter or delete the function descriptions}

Double-click on the second option in the primary interface (Enter

Function Descriptions), to open the following window (Figure 142).

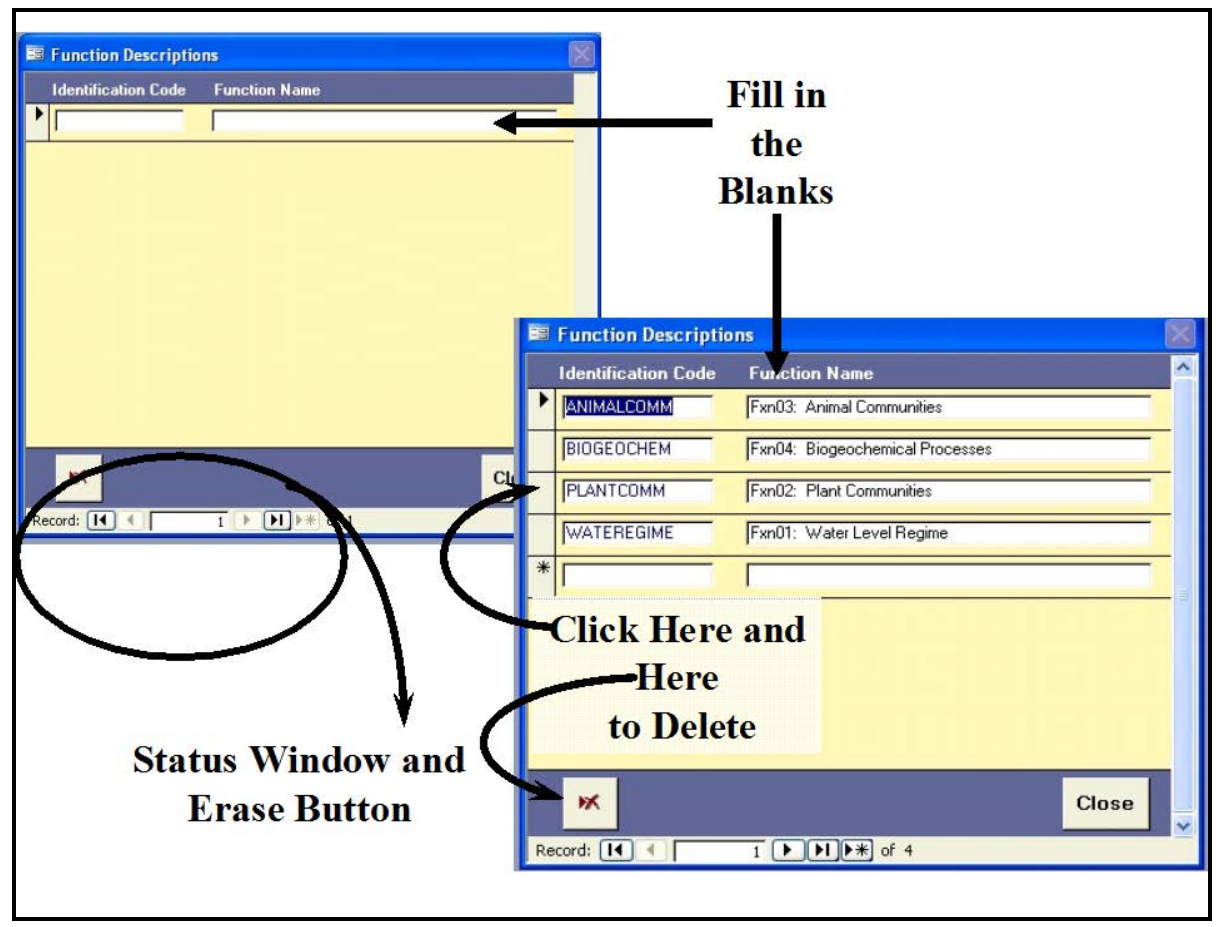

Figure 142. Function Descriptions entry screen in EXHGM.

Provide an Identification Code for the function (limit 10 characters) and a Function Name (limit 100 characters) for each functional index in the wetland model(s). A new row is

USER'S NOTE:

Each function's Identification Code must be unique and contain only alphanumeric characters. assigned to each function. EXHGM has set no limits on the number of functions that can be used for any analyses. A status window at the bottom of the form indicates the number of records (i.e., models) entered into the database. Restrictions on the data entry requirements of this form are offered at the bottom left-hand corner of the screen (Figure 143). 


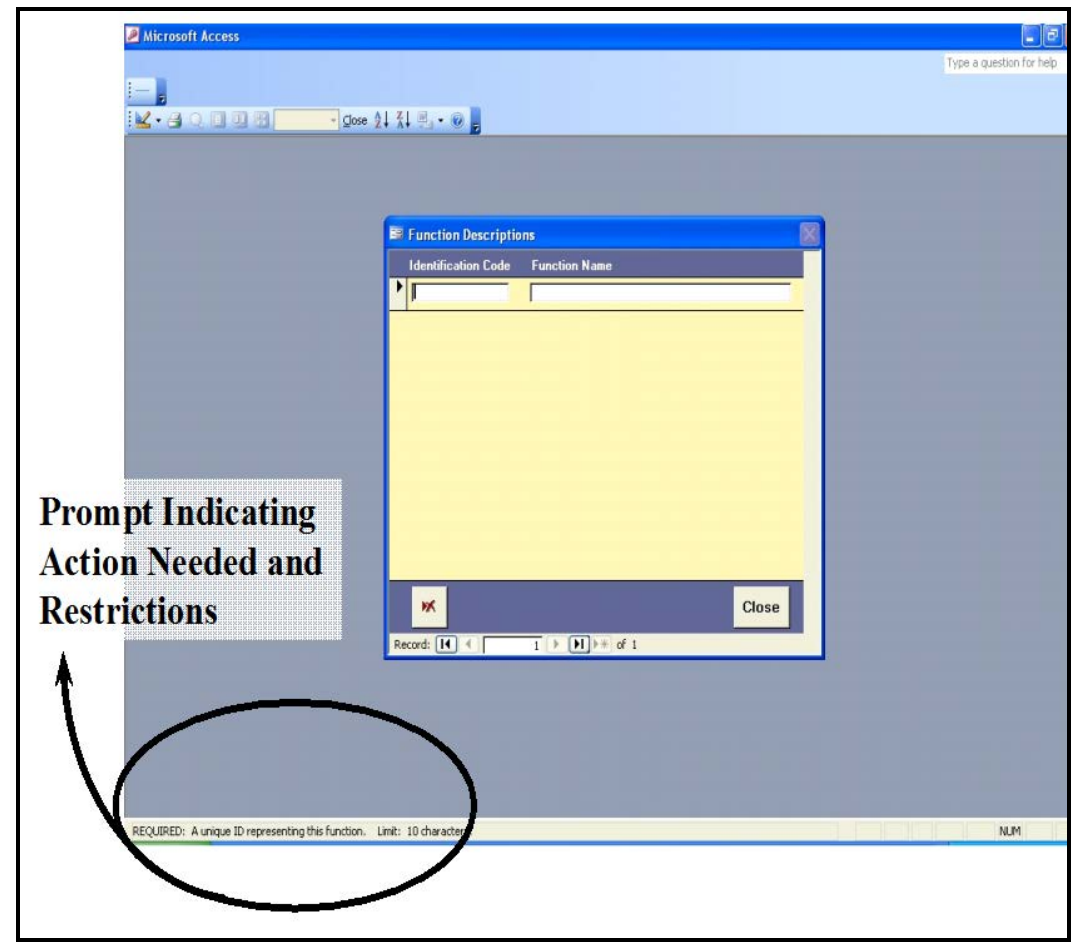

Figure 143. Common EXHGM data entry screen prompts.

This is a common feature of every EXHGM screen. If a function must be removed, click on the gray block immediately to the left of the function's Identification Code and click on the erase button in the lower left-hand corner of this form (see Figure 142 above). When finished with these activities, click on the Close button at the bottom of the screen and EXHGM returns to the primary interface.

\section{Define or delete PWAAs and baseline acres}

Double-click on the third option in the primary interface (Define Partial Wetland Assessment Areas), to open the following window (Figure 144). 


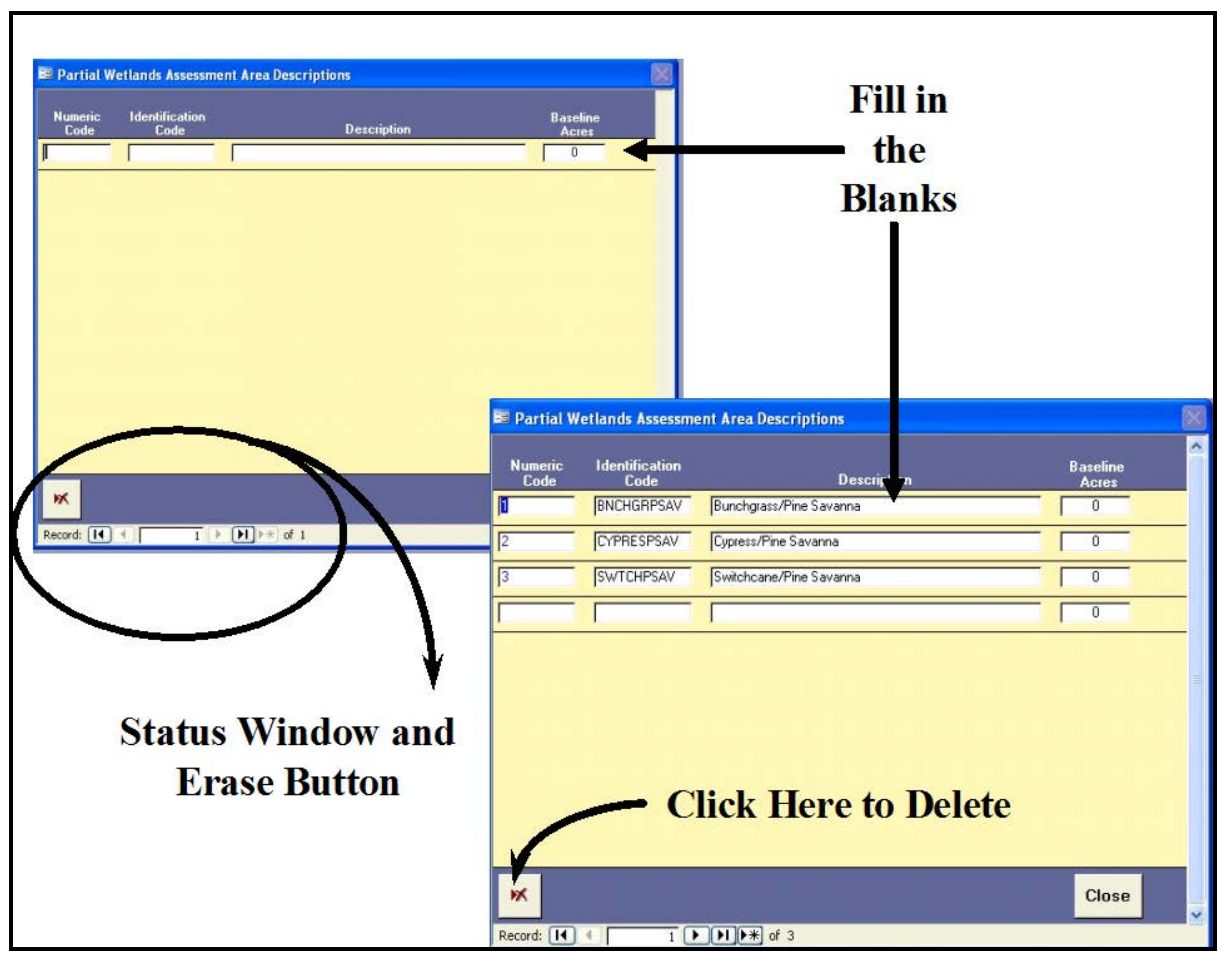

Figure 144. Partial Wetland Assessment Areas Descriptions entry screen in EXHGM.

Provide a Numeric Code ( 1 - $\infty)$, a shorthand Identification Code (limit 10 characters), a PWAA Description (limit 100 characters), and the number of Baseline Acres for each PWAA on the site. A new row is

USER'S NOTE:

Each PWAA's Numeric Code and Identification Code must be unique and contain only alphanumeric characters. assigned to each PWAA. There are no limits to the number of PWAAs EXHGM can process within the analysis. Again, a status window at the bottom of the form indicates the number of records (i.e., PWAAs) entered into the database, and restrictions on the data entry requirements of this form are offered at the bottom left-hand corner of the screen.

Account for every acre within the project boundary, even if the PWAA is not associated with a specific wetland model. In some instances, these unassociated acres are not considered wetlands today, but could be restored to functioning wetlands in the future, and thus the current acres should be tracked (i.e., AGCROP $=100$ acres of soybeans that could be replaced with forested wetlands under a proposed with-project scenario). Create "placeholder" PWAAs for future development options. Thus, for every existing PWAA that is associated with the
USER'S NOTE:

If the baseline acreages for the PWAAs are unavailable at this time, EXHGM assumes zero acres. Once the mapping is complete, return to this screen and refine the acreage estimates. 
function, create a "with-project" PWAA (e.g., Cypress/ Pine Savanna and Newly Developed Cypress/ Pine Savanna). If a PWAA must be removed, left-click once anywhere on the PWAA's row and click on the erase button in the lower left-hand corner of this form. When finished with these activities, click on the Close button at the bottom of the screen and EXHGM returns to the primary interface.

\section{Define or delete the variables}

Double-click on the fourth option in the primary interface (Define Variables), to open the following window (Figure 145).

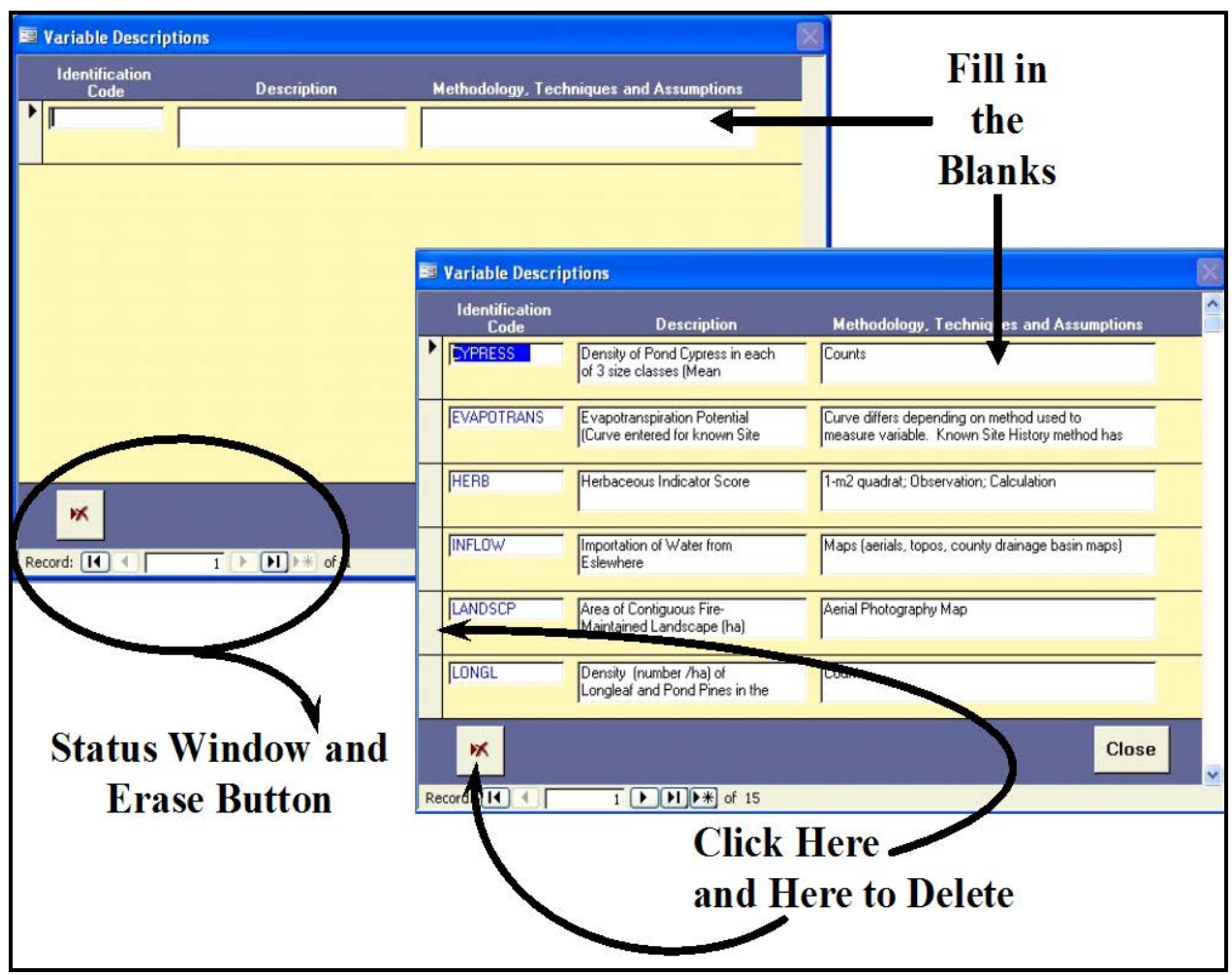

Figure 145. Variable Descriptions entry screen in EXHGM.

Provide an Identification Code (limit 10 characters), a variable Description (limit 100 characters), and a brief description of the Methodology, Techniques and Assumptions used to gather/ generate the analyses variable data (limit 255 characters - including periods and spaces). A new row is assigned to each variable. There are no limits to the number of variables EXHGM can
USER'S NOTE:

Each variable's Identification Code must be unique and contain only alphanumeric characters. The variable Description and Methodology, Techniques and Assumptions can contain symbols (i.e., \%, \&,\#). 
process in the analysis. When creating variable codes, begin with the action to be measured, and follow this with specifics. For example:

- Shrub canopy cover $=$ CANSHRUB

- Tree canopy cover $=$ CANTREE

- Water depth = DEPTHWATER

- Percent of the area covered by open water $=$ AREAVEGOPW

- Ratio of wet to total prairie acreage =AREAWETDRY

To shorten the codes (to meet the 10-character limit), try removing spaces, periods, conjunctions and vowels, and then use acronyms where possible (e.g., species $=$ SPP or vegetation =VEG). This approach allows EXHGM to cluster "like" variables together, and will help position variables in lists throughout the

USER'S NOTE:

The 255-character limit in the Methodology, Techniques and Assumptions cell is strictly enforced. To shorten the amount of information entered in this field, drop periods and use acronyms and symbols whenever possible. process. ${ }^{1}$ Remember, if variables share the same sampling protocol, use the $\mathbf{C t r l}+\mathbf{C}$ and $\mathbf{C t r l}+\mathbf{V}$ shortcut keys to copy the sampling methodology information from one variable to the next. This can only be used on a cellby-cell basis - an entire row cannot be copied using this technique. Once again, a status window at the bottom of the form indicates the number of records (i.e., PWAAs) entered into the database, and restrictions on the data entry requirements of this form are offered at the bottom left-hand corner of the screen. If a USER'S NOTE: Use Ctrl + Enter to insert hard returns into the Description and Methodology, Techniques and Assumptions fields of this form.

The Esc key on the keyboard allows users to jump out of cells if a mistake is made during the data entry process. variable must be removed, click on the gray block immediately to the left of the variable's Identification Code and click on the erase button in the lower left-hand corner of this form (see Figure 145 above). Initial function setup is now complete. Click on the Close button at the bottom of the screen. EXHGM returns to the primary interface. Proceed to the "Checking the setup process

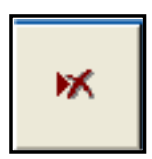
with EXHGM reports” section below.

\footnotetext{
${ }^{1}$ Appendix E provides a general naming convention protocol the authors have developed to simplify and standardize this process.
} 


\section{Checking the setup process with EXHGM reports}

EXHGM provides a series of summary reports as each step in the process above is completed. To review and check the setup information entered thus far, click on the Setup Reports button (Figure 146).

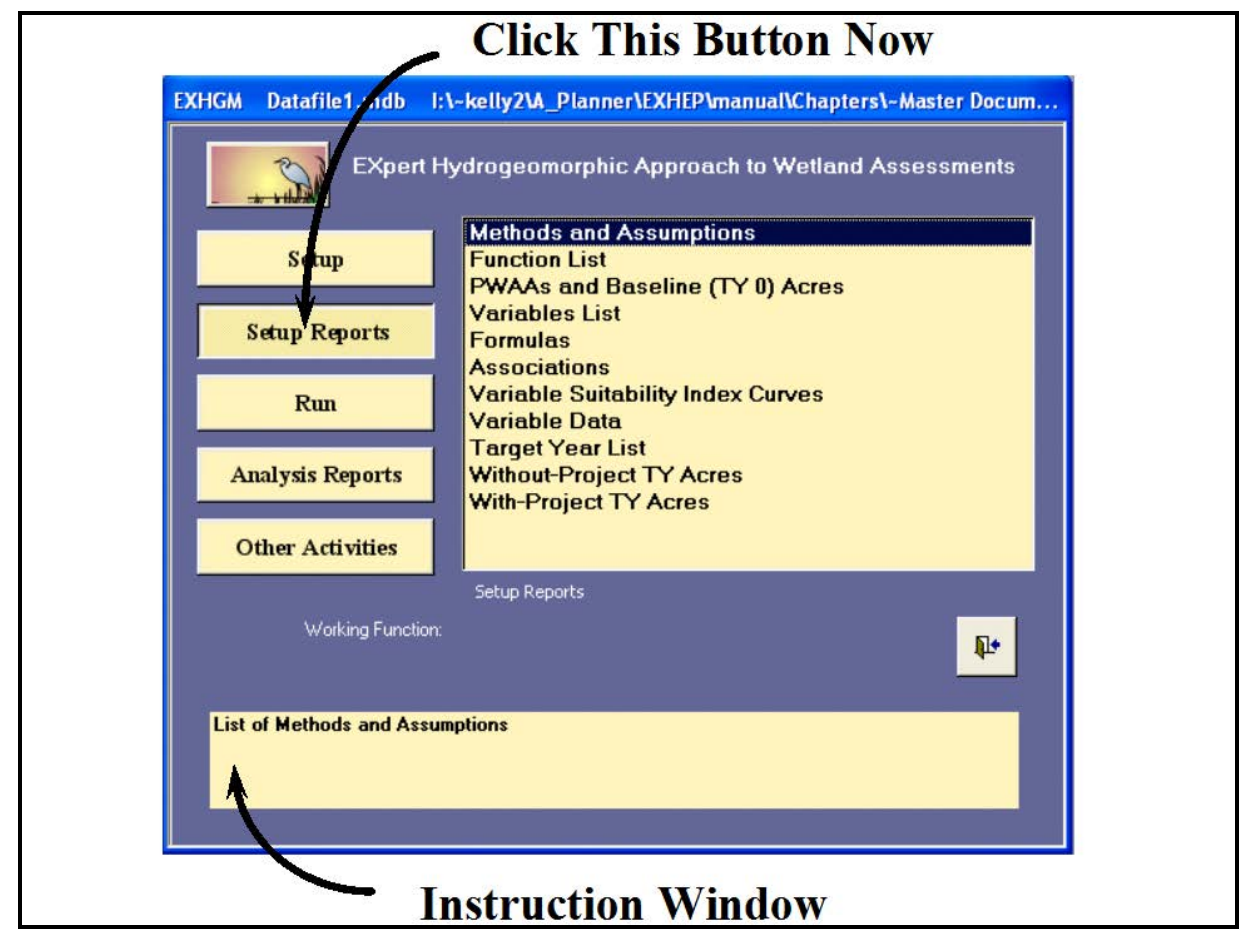

Figure 146. Click on the Setup Reports button to view the setup reports in EXHGM.

A list of available reports appears in the center of the primary interface. The first four reports should be populated at this point in the analysis:

- Methods and Assumptions

- Function List

- PWAAs and Baseline (TY 0) Acres

- Variables List

Single-click on any report and look to the Instruction Window to preview the contents of the report. Double-click on any report, and EXHGM opens a new window with the report inside (Figure 147).

\section{USER'S NOTE:}

A printer driver must be installed in order to view any of these reports. 


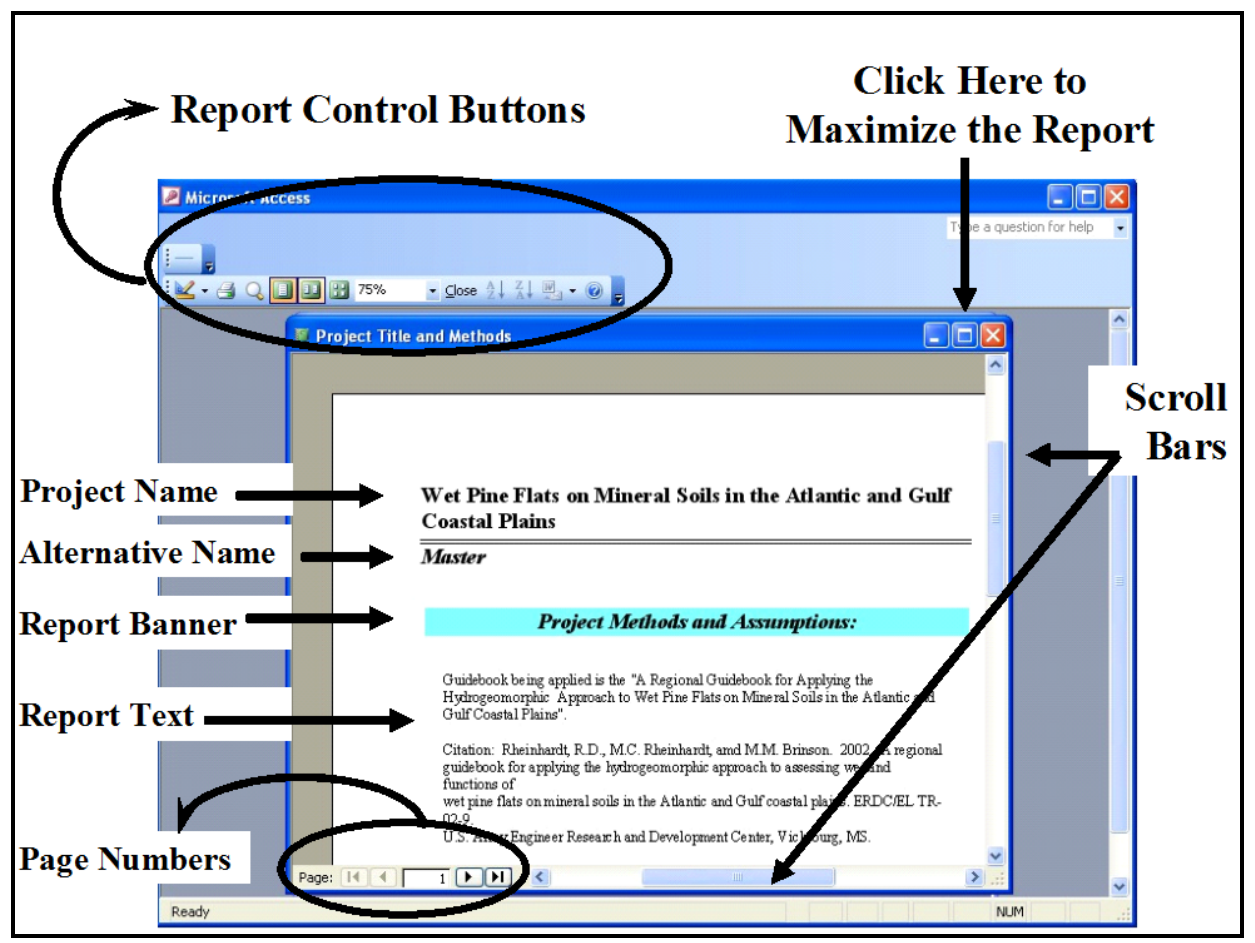

Figure 147. Report interface in EXHGM.

EXHGM reports are somewhat standardized to present the datafile's information in a clear and concise manner. The header of each report contains the project and alternative names separated by a heavy line. A banner below these names indicates the name of the report (in this case Project Methods and Assumptions). The report's contents are presented immediately below the banner. Standard MS Access scroll bars are provided on the bottom and right-hand edges of the report itself to aid in navigation. Page numbers are presented in the lower left-hand corner of the report to help move through the report as well. Standard Minimize/ Maximize/ Close buttons are located in the upper right-hand corner of the report. Outside of the report, on the toolbar in the upper left-hand corner of the screen, a series of report control buttons on the Print Preview toolbar can be found that allow users to review, print, close, and export the report as needed. 
Some default printer settings may cause EXHGM reports to span multiple pages if the margins are set too narrowly. To fix this problem, rightclick on the report, and select Page Setup from the list of options. Reset the margins, column widths, and page sizes

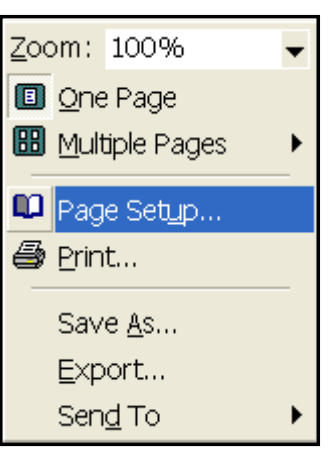
as necessary using the Margins, Page, and Columns tabs to customize the report for the printing environment.

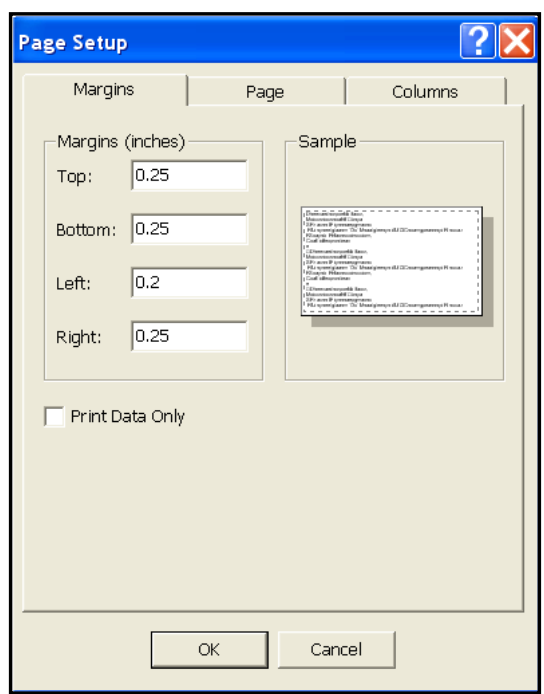

Click on the Maximize button and select Fit from the Zoom pull-down box on the toolbar. The footer of the report is revealed. Here, EXHGM provides two additional pieces of information that will assist in assessment logistics - the date and time the report was run, and the datafile's storage path (Figure 148).

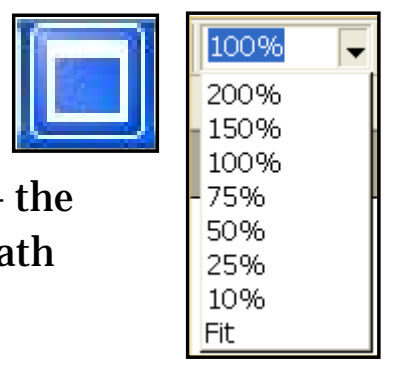

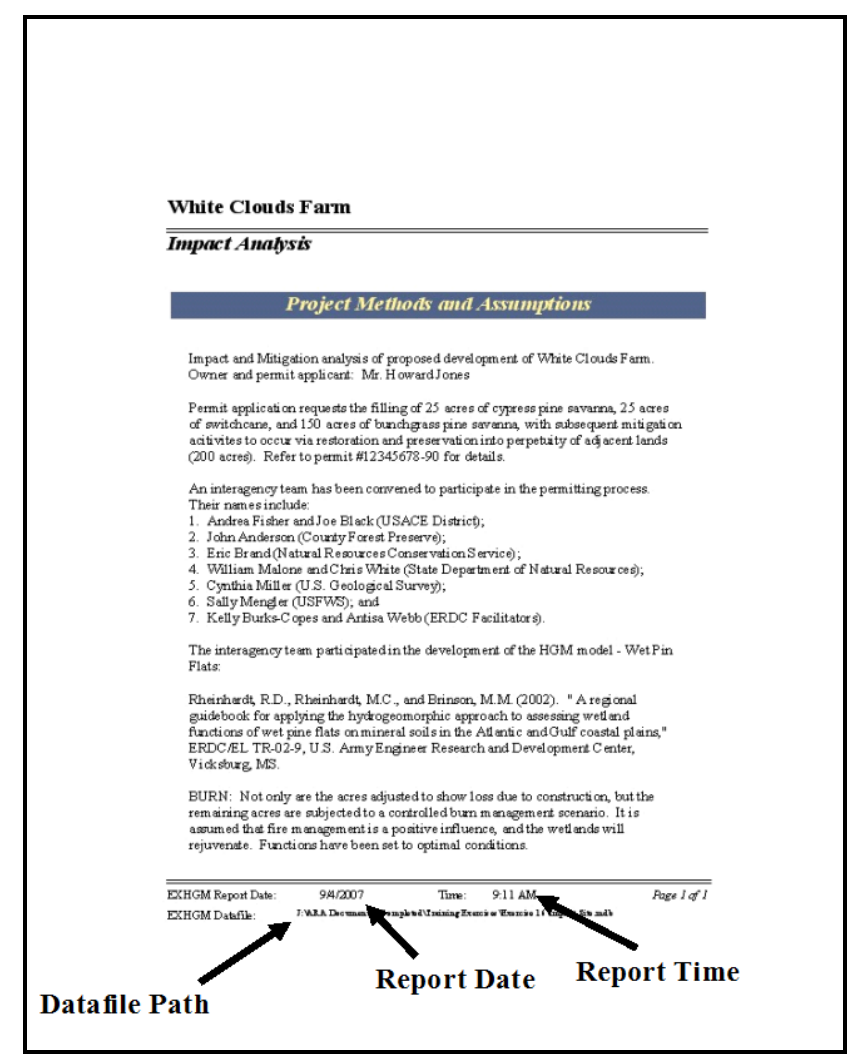

Figure 148. An expanded EXHGM report detailing additional fields. 
One convenient advantage of using the EXHGM software is its compatibility with other Microsoft software packages (namely MS Word and MS Excel). By simply clicking on the Officelinks pull-down box, and clicking

II.

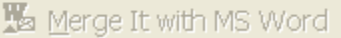
II Publish It with MS Word

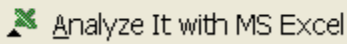
on either option, the report is exported out to the new medium, allowing customization to meet documentation needs.

If the Print Preview toolbar is not available in this screen, right-click on the report, and select the Export option from the list of choices presented. An Export entry screen will open in response to this action (Figure 149).

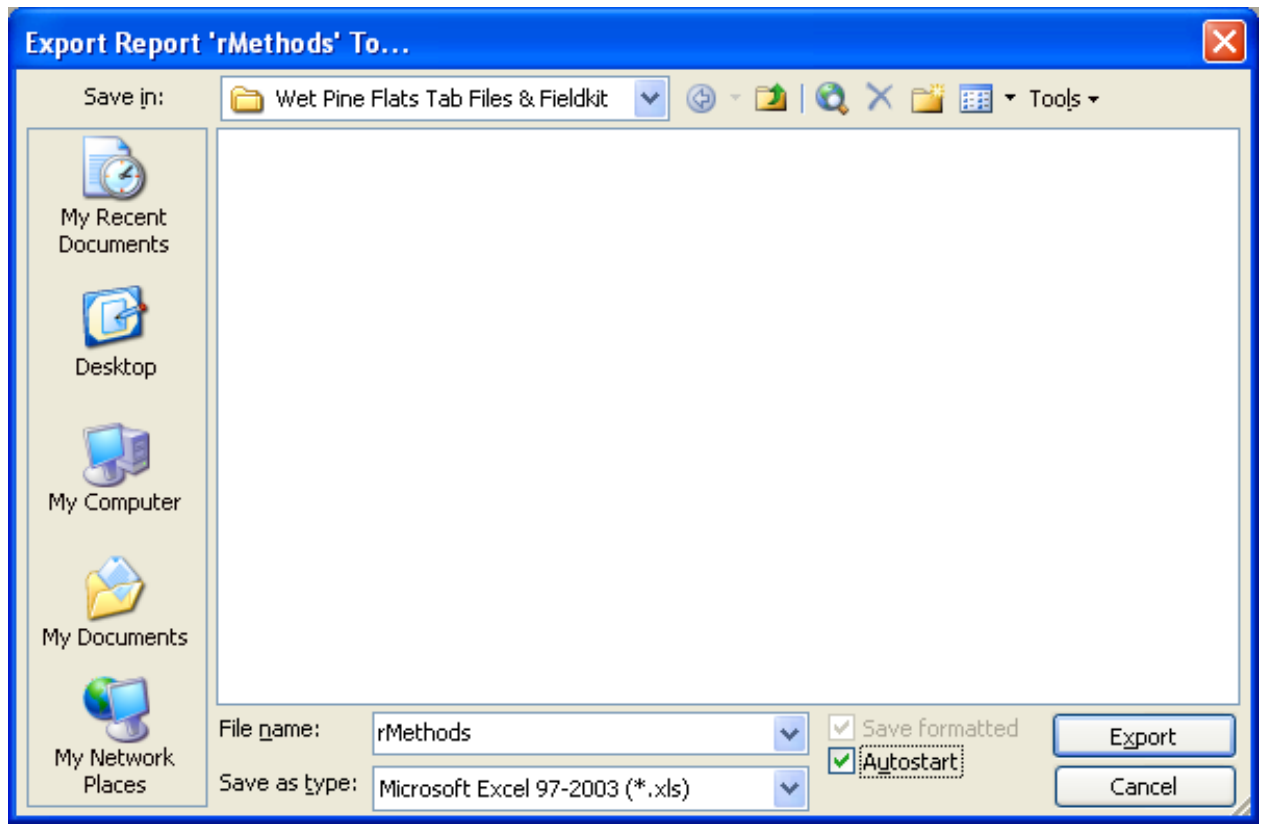

Figure 149. Export Report entry screen - exporting report information to MS Excel or MS Word.

Select the export location for the report in the Save in: window at the top of the entry screen. Provide a name for the report in the File name: window in the bottom of the entry screen. Use the Save as type: pulldown list to select the type of file to be exported [i.e., choose either MS Excel 97-2000 (*.xls) for a spreadsheet format or Rich Text Format (*.rtf) for a word-processing format]. To associate the software (i.e., MS Excel for spreadsheets or MS Word for word processing documents) , such that the medium opens and displays the information in the new environment upon exportation, click the Autostart checkbox immediately to the right of the Save as type: pull-down list. Press the Save button in the lower right-hand corner of the screen. EXHGM will export the data displayed in the report to the new file, and if the Autostart option was 
clicked, MS Excel/ MS Word will open and display the report in the new environment (Figure 150).

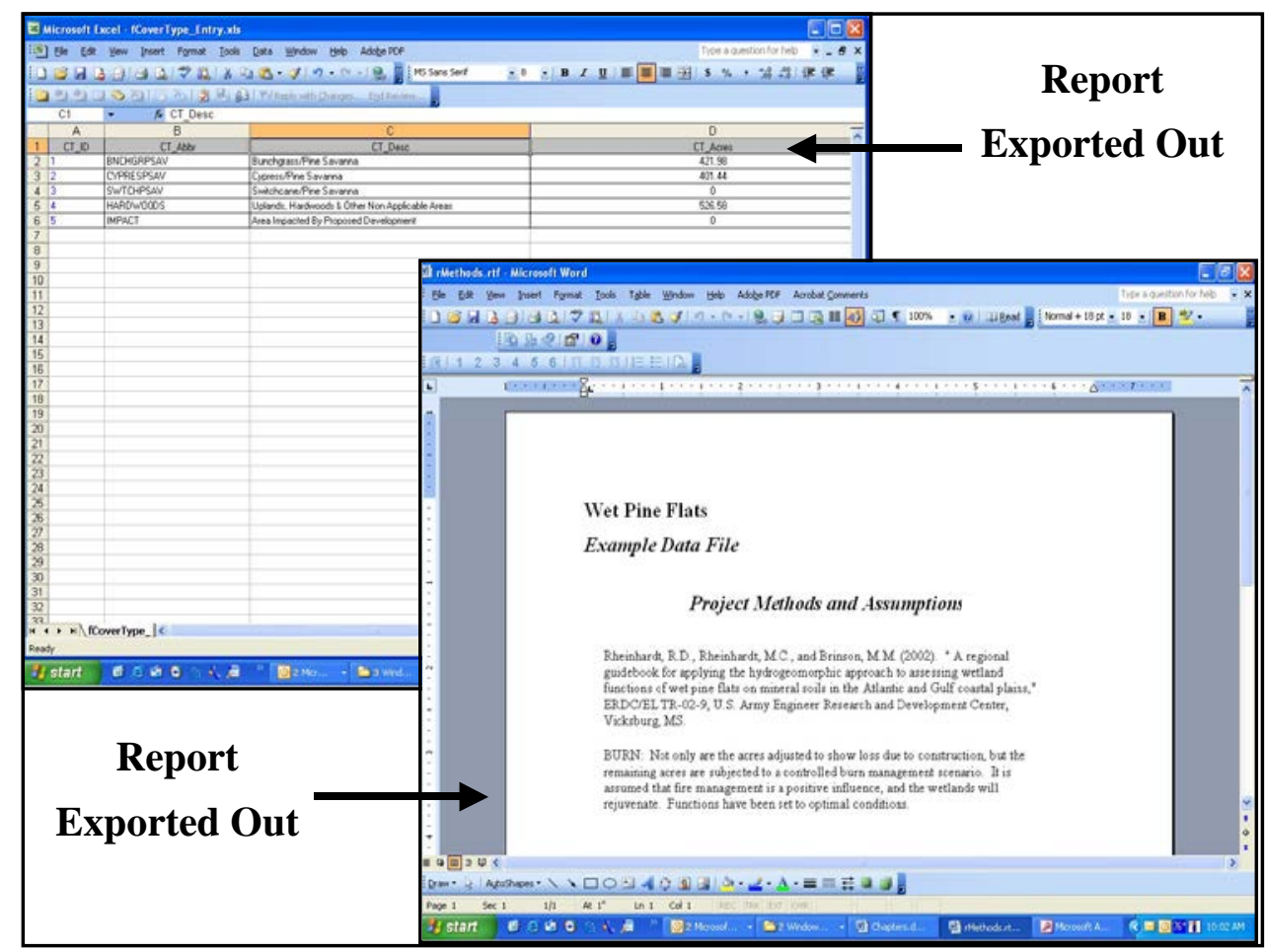

Figure 150. Exported reports in MS Excel and MS Word environments.

Format and save the files as needed, close the external applications (i.e., MS Excel or MS Word), and return to EXHGM. Click on the Close button from the suite of buttons offered in the Print Preview toolbar - EXHGM returns to the primary interface. Repeat the process as often as necessary to check the products developed thus far in the setup activities. Proceed to Step 3: Model Association to continue.

\section{Step 3: Function association}

As soon as the functional (FCI) models are set up in EXHGM, run the models through an assessment by clicking on the Run button on the primary interface (Figure 151). ${ }^{1}$

\footnotetext{
1 To assist in the software application, a series of training exercises have been included in Appendix E refer to Exercise 11 for relevant training in function association in EXHGM.
} 


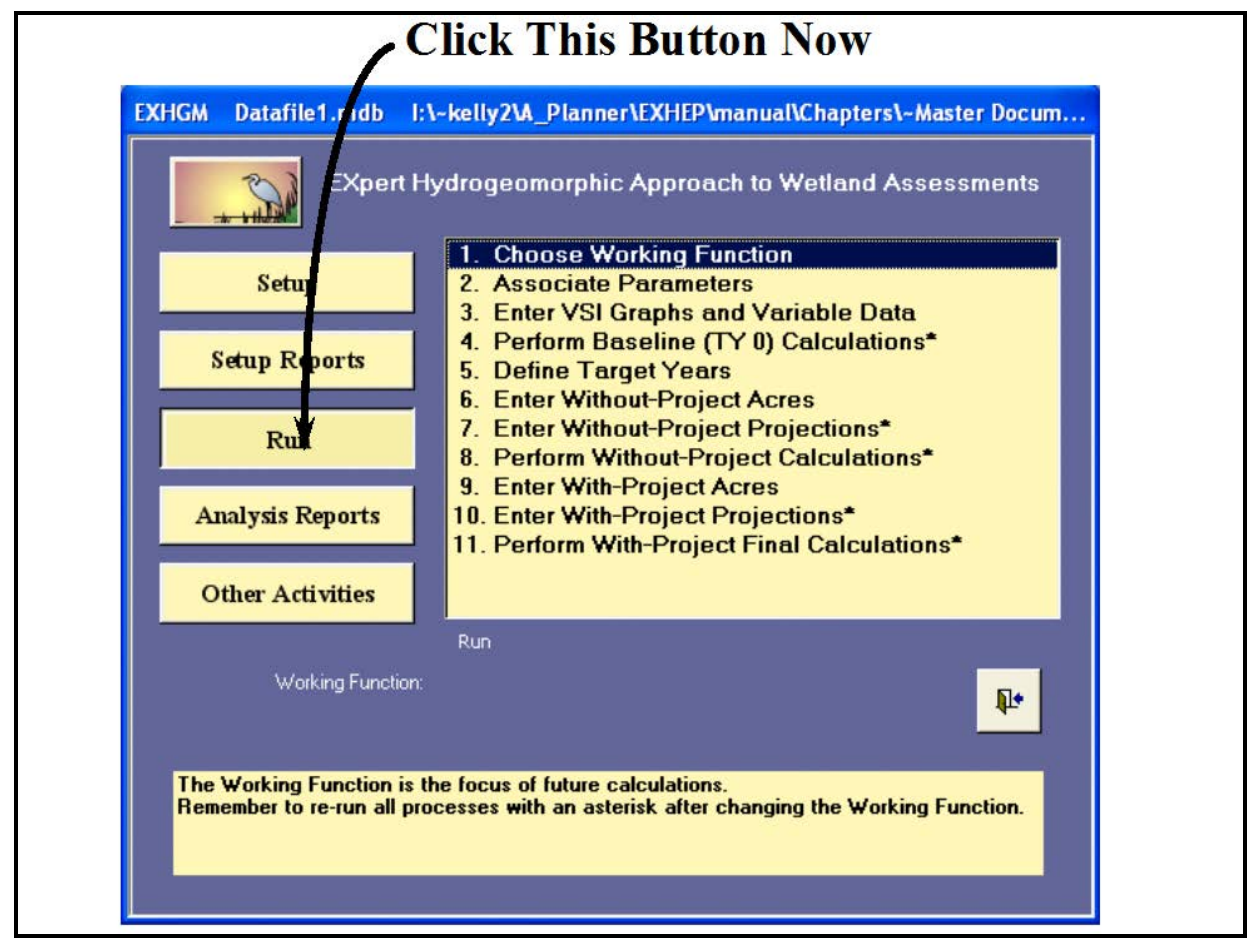

Figure 151. Click on the Run button to begin the association process in EXHGM.

A screen appears in the center of the primary interface with 11 options:

\section{Choose Working Function}

2. Associate Parameters

3. Enter VSI Graphs and Variable Data

4. Perform Baseline (TY 0) Calculations*

5. Define Target Years

6. Enter Without-Project Acres

7. Enter Without-Project Projections*

8. Perform Without-Project Calculations*

9. Enter With-Project Acres

10. Enter With-Project Projections*

11. Perform With-Project Final Calculations*

In systematic fashion, start at the top of the options list, click on each option, and provide the data or perform the action requested in each interface screen.

\section{USER'S NOTE:}

If at any time new information is acquired that affects the analysis, return to these options and update the data. 


\section{Choose the "Working Function"}

Initially, EXHGM was developed to associate and compute all functions simultaneously. Although this provided answers in a singlestep fashion, the rigorous processing cycles tied up computers for long periods of time. In addition, alteration of the information within any function made it necessary to re-process

USER'S NOTE:

Once a "Working Function" has been identified, EXHGM will focus solely on the data entry and evaluation of this model. If users wish to enter data for another model, a new "Working Function" must be identified. the entire database. This proved to be an inefficient mode of operation. EXHGM was re-designed to incorporate a means to directly process functions in a more focused approach. EXHGM's new evaluation strategy requires users to focus the EXHGM analysis engine on a single "Working Function" for function association and processing. Thus, to begin the run process, first identify a "Working Function." To do this, click on the first option (Choose Working Function) - the following window opens (Figure 152).

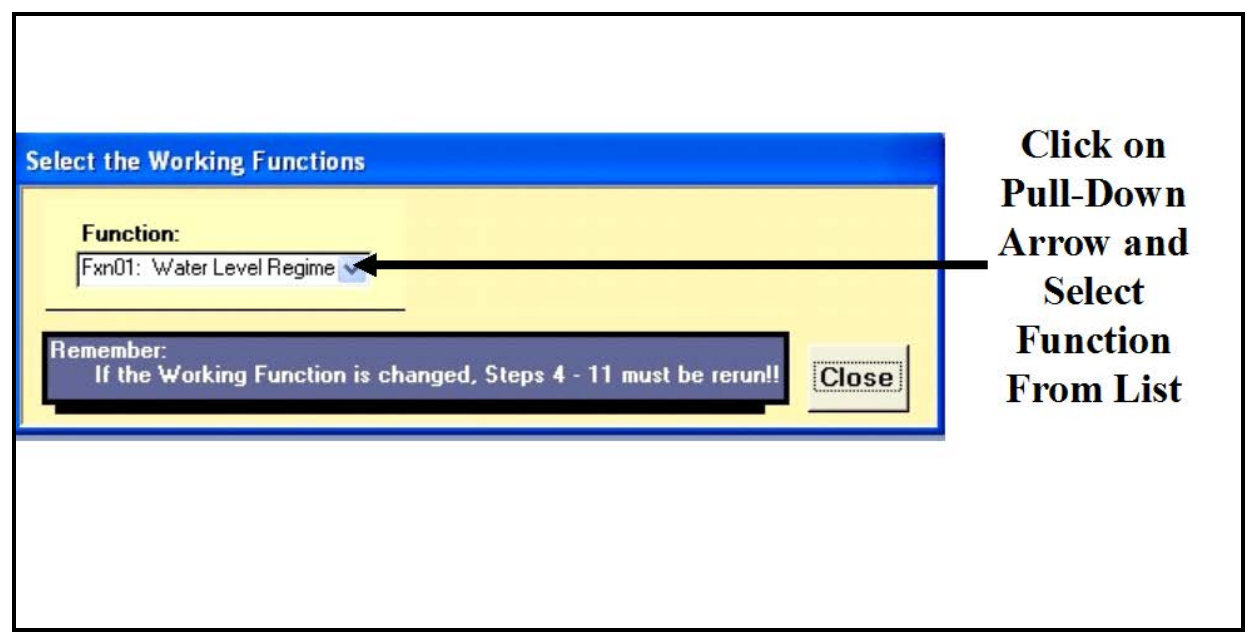

Figure 152. Select a function from the pull-down list.

Simply choose the function from the pull-down list on the right-hand box in this screen and click Close. EXHGM returns to the primary interface. At the bottom of the screen, the "Working Function" is now identified (Figure 153). Proceed to the next option. 


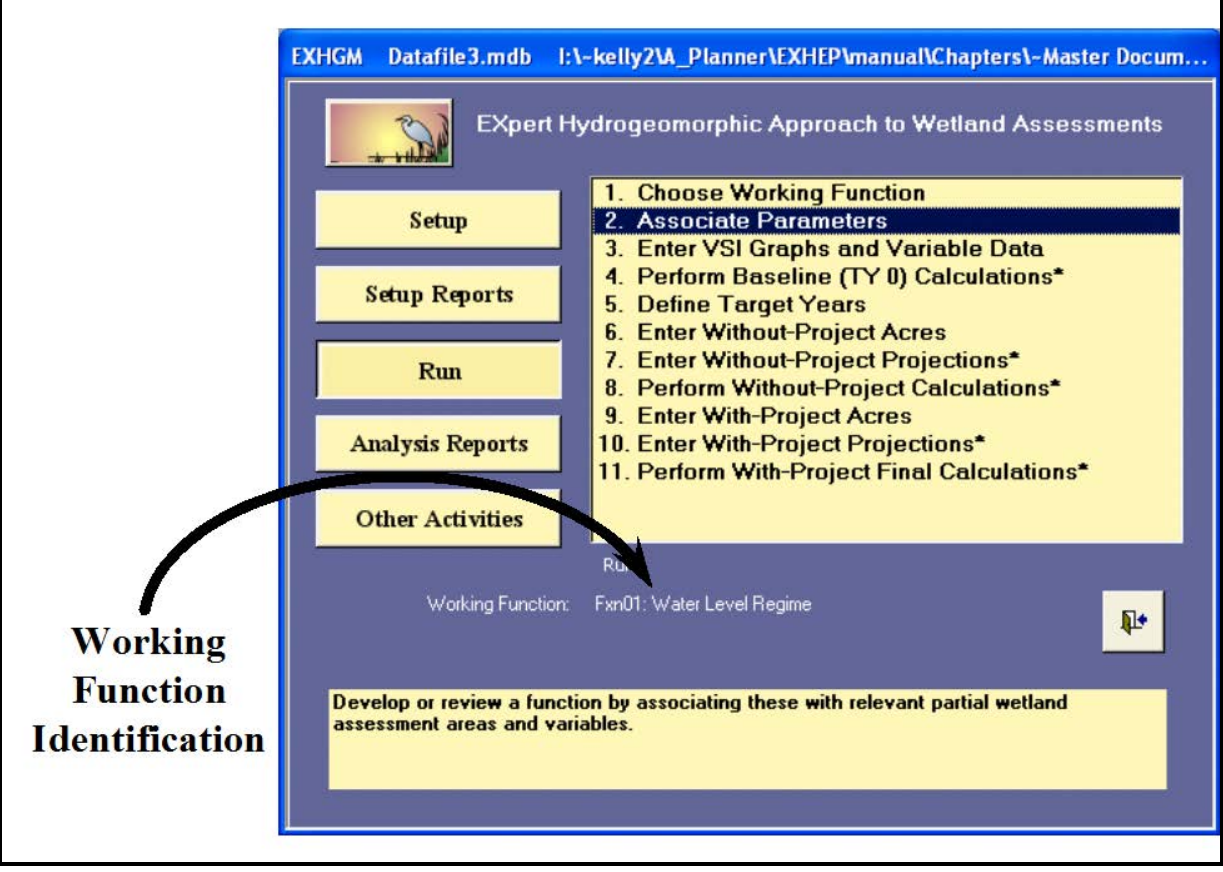

Figure 153. Identification of the Working Function in EXHGM's primary interface.

\section{Associate the model parameters}

In EXHGM, association is the process of combining model PWAAs and variables in a meaningful manner. In other words, association provides an interface with the EXHGM analysis engine and guides it in model definition and application. In this process, model definition is the translation of mathematical equations (e.g., FCI $=$ Variable $1 *$ Variable $2 *$ Variable 3 , etc.), often derived from the guidebook publications, into MS Accesscompatible language. Model application refers to the act of tying these models to specific wetland conditions (i.e., PWAAs). The association activity should be viewed as an opportunity to define and communicate the sensitivity of FCI models to the EXHGM program. Click on the second option (Associate Parameters) to open the following windows (Figure 154). 


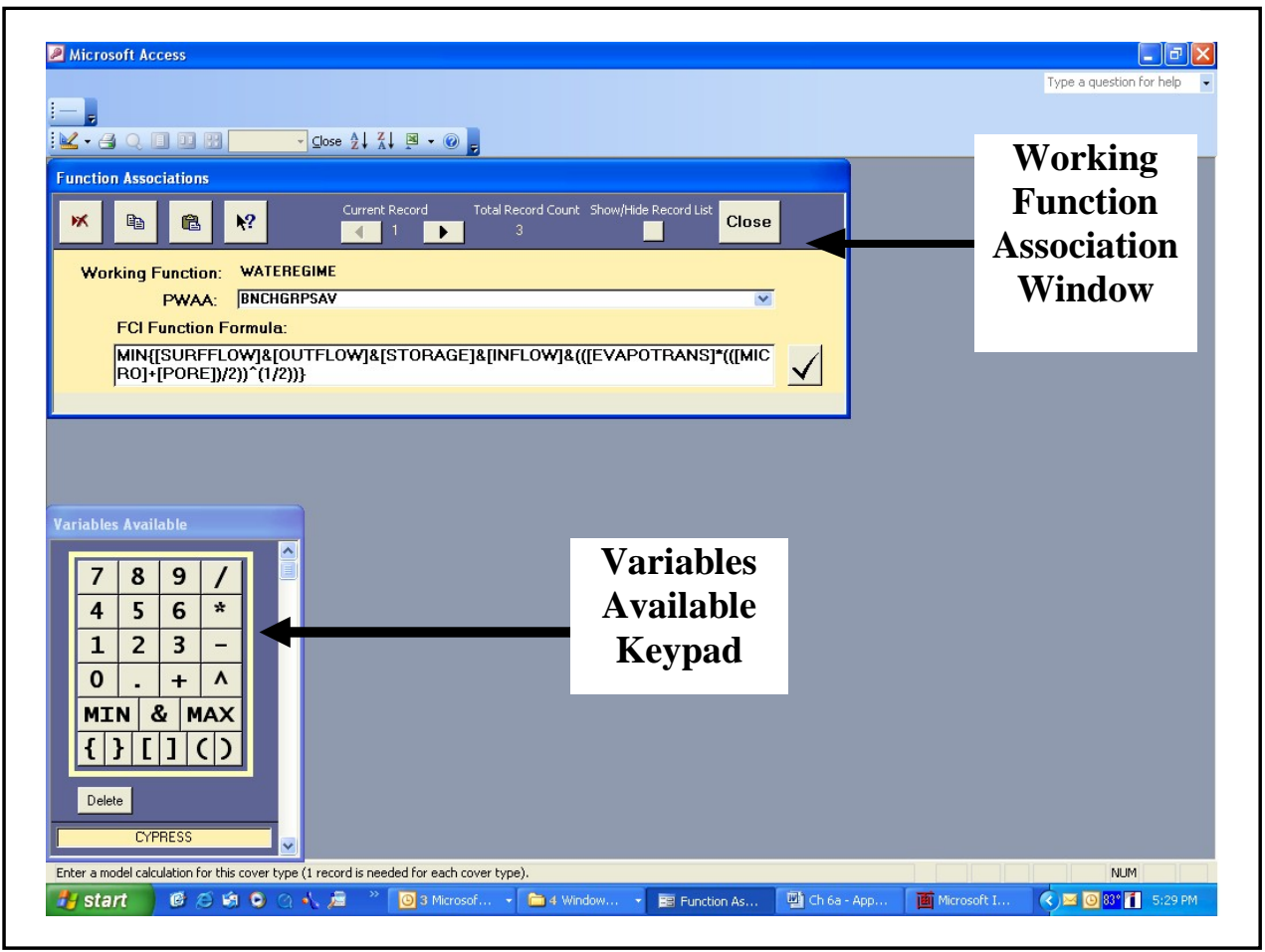

Figure 154. Function Associations window and Variables Available keypad used to associate models in EXHGM.

The Functions Associations window is the activity platform for the function association process. Indicate which PWAAs are evaluated in the function, and what formula will be used to determine the PWAA's functional capacity. The current Working Function is provided as the first item in the yellow box in the center of this form (Figure 155).

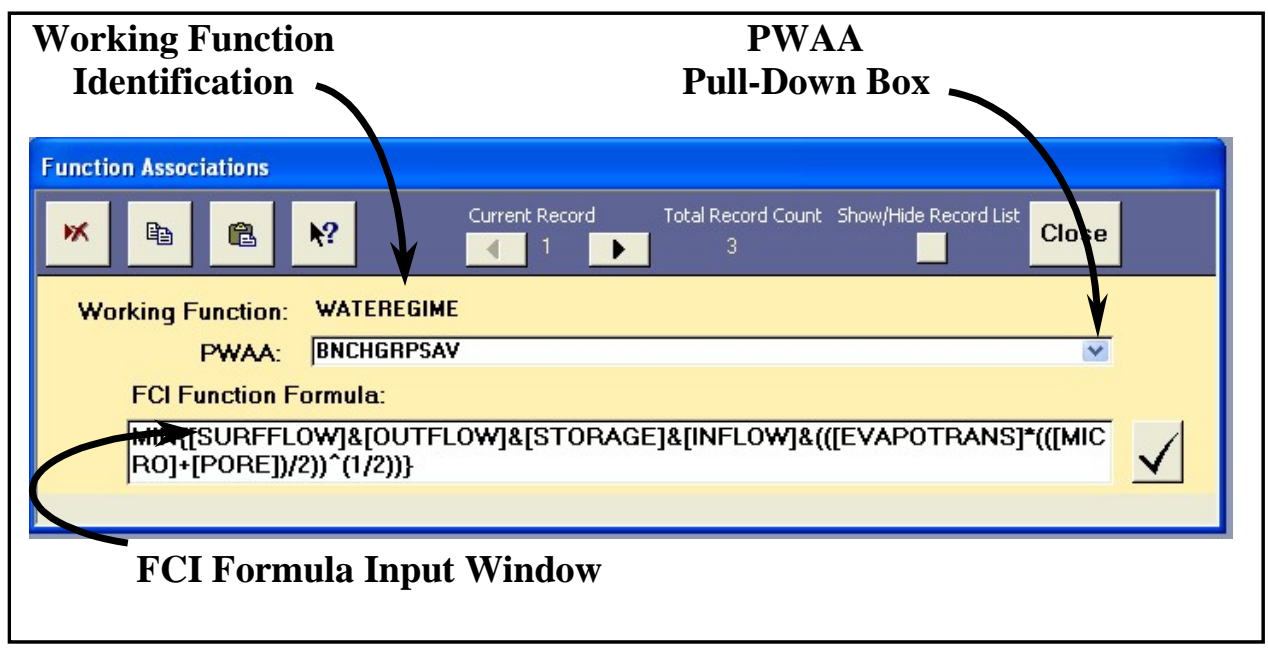

Figure 155. Function Associations entry screen. 
Immediately below the Working Function line, a pull-down box for PWAA identification and an FCI Model Formula input box are provided. Function association is a two-step process - select a PWAA first, and then enter an FCI formula. For details regarding these two steps read below.

Select a PWAA

To begin, click on the PWAA drop-down box, and choose the first PWAA that will be associated with the function (Figure 156).

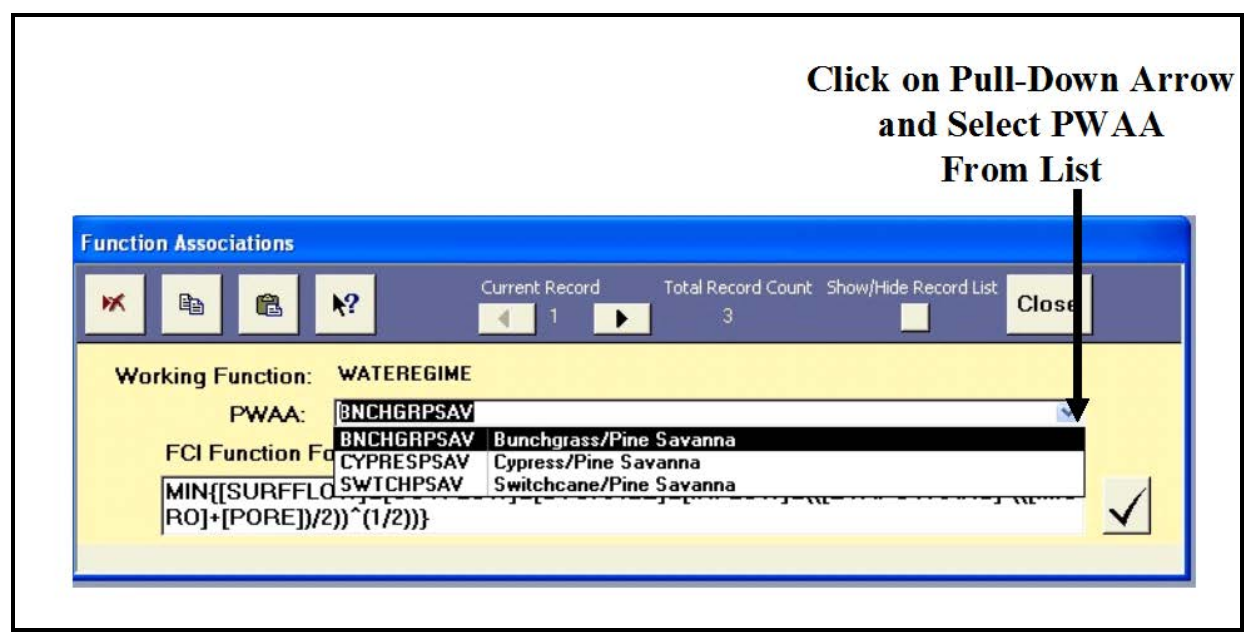

Figure 156. Select a partial wetland assessment area from the pull-down list.

\section{Enter an $\mathrm{FCl}$ formula}

Now left-click once in the FCI Function Formula input box in the lower portion of the screen. Define the combination of variables that together formulate the FCI function for the wetland ecosystem. In other words, in mathematical equations, provide the FCI function in a syntax that MS Access will understand. There are five basic syntax rules in EXHGM:

1. Every open parenthesis (or bracket) must have a corresponding closing parenthesis (or bracket).

2. Capitalize all letters in the variable codes.

3. Surround all variable codes with brackets (i.e., [VARIABLE]).

4. Use only the operators provided in the Function Equation/FCI Calculation Syntax window.

5. If the MIN/MAX function is used in a formula, use braces ( \{\} ) to surround the statement and separate the variables themselves with an ampersand ( $\boldsymbol{\&})$. For example: 


\section{MIN\{[VARIABLE A] \&[VARIABLE B] $\}$.}

\section{Formula entry assistance}

The equation entry task is undoubtedly the hardest step to complete in the EXHGM analysis process. Although this task can be tedious and somewhat technical, a series of equation development support tools have been included in the EXHGM package to ease the process. First, EXHGM offers a keypad and list of potential variables in an interactive floating window (Figure 157).

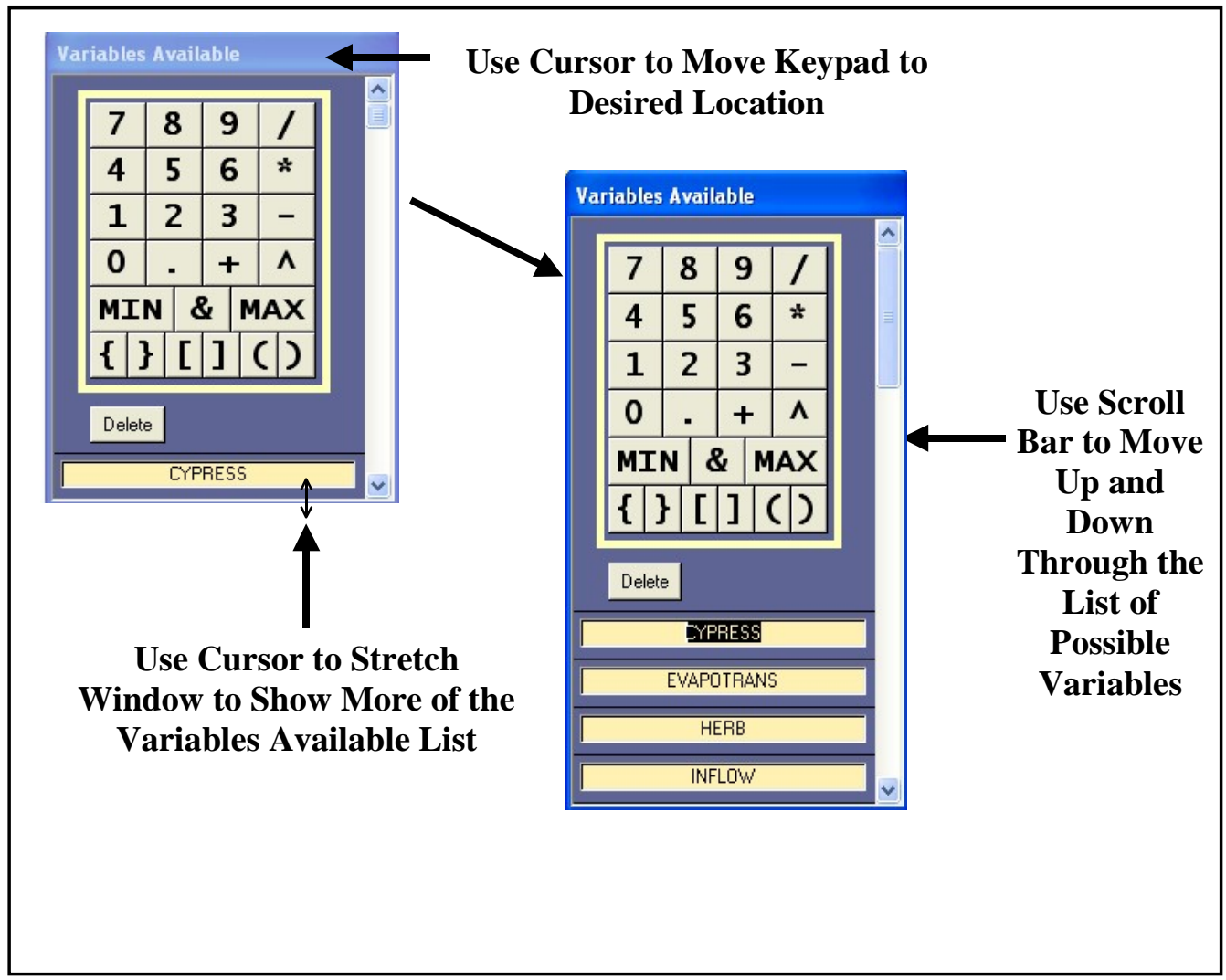

Figure 157. Customize the size and location of the floating Variables Available keypad.

By left-clicking on the banner at the top of the keypad and dragging it across the screen, the keypad can be moved anywhere on the desktop. Use the cursor to stretch the window - showing more of the available list of variables. To do this, float the cursor near the bottom of the window until it converts to a double-ended arrow. Left-click and drag the window down to the desired size. Use the scroll bar on the right-hand side of the keypad to scroll down through the variable list as well. All variables entered during the setup process are listed in alphabetical order in this list to 
simplify the search. If a variable is missing from this list, then it was not entered correctly in the variable setup process. To correct this problem return to Step 2 above and complete the variable setup process, then return here and find the variable in the list.

A help window has been developed to provide assistance during the equation entry process. This window is opened by clicking the Help button in the toolbar at the top of the Function Associations screen (Figure 158).

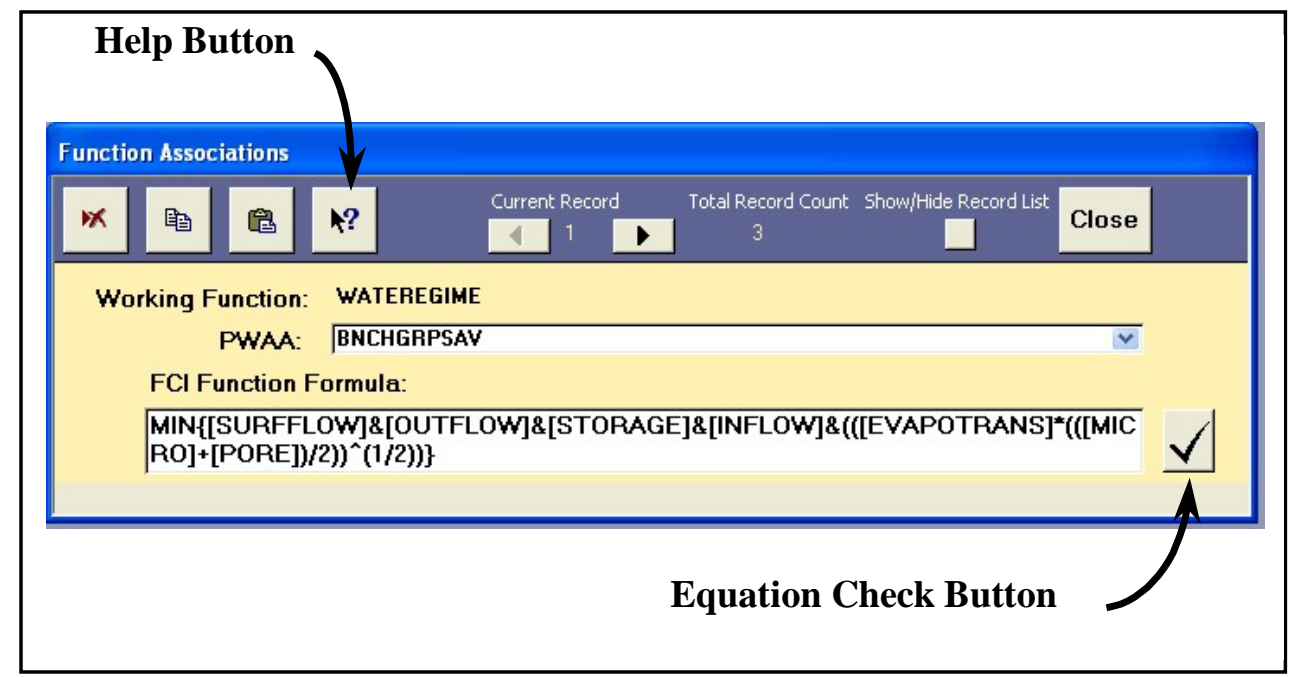

Figure 158. Help and Equation Check buttons provided to assist with $\mathrm{FCl}$ formula entry in EXHGM.

Once opened, a list of operands, basic equation development criteria, and several equation techniques (i.e., helpful examples) are displayed to further demonstrate proper equation syntax in EXHGM (Figure 159). 


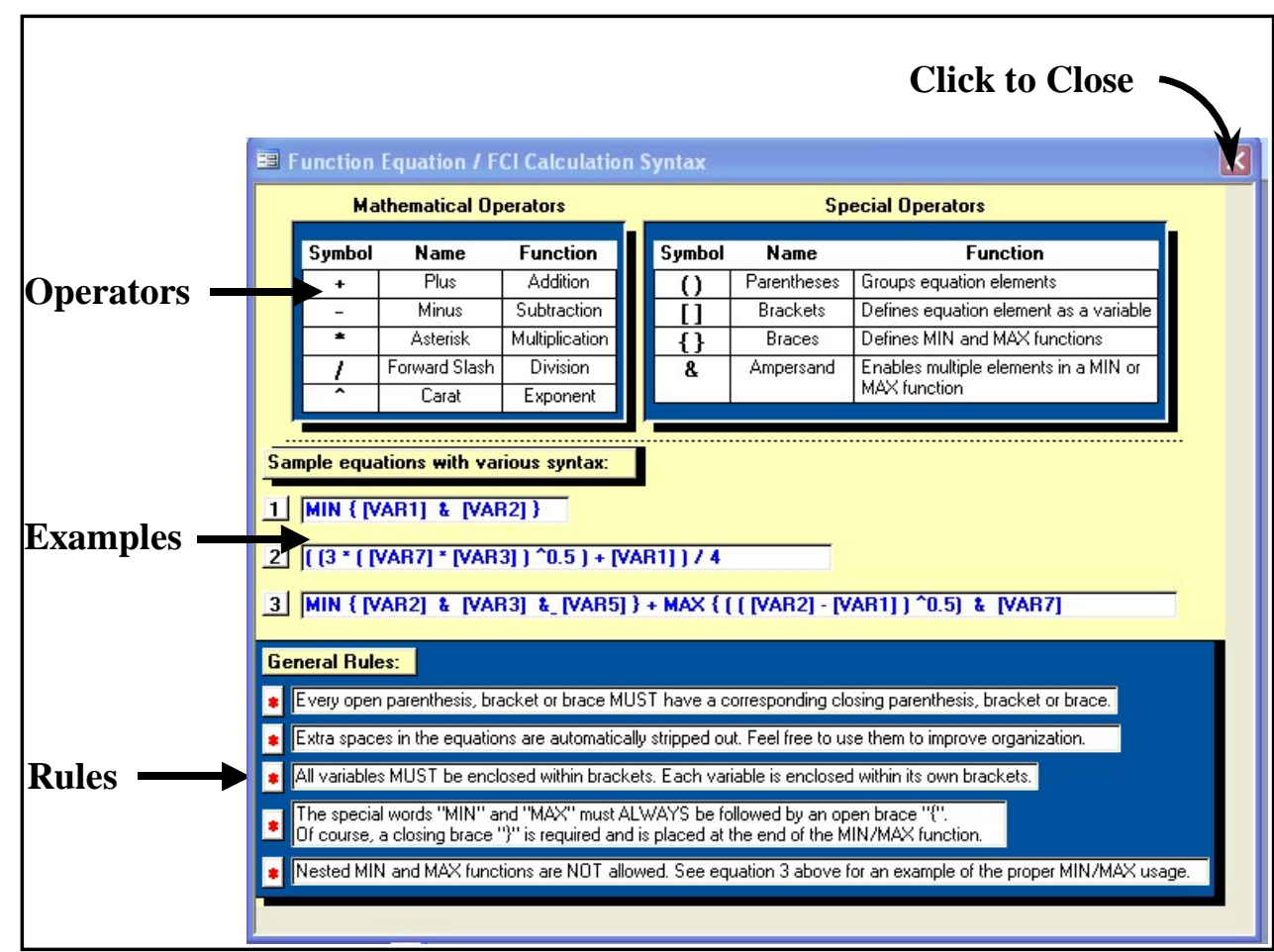

Figure 159. Function Equation/FCl Calculation Syntax window in EXHGM.

To close the Function Equation/FCI Calculation Syntax window, click on the " $\mathbf{X}$ " in the upper right-hand corner of the screen.

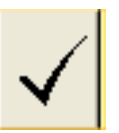

To further assist in the equation entry process, a testing button has been installed in the Function Associations screen to the immediate right of the FCI Function Formula input box, and

should help verify each equation entry as it is supplied. Once the formula has been entered, click on this button and receive either confirmation that the formula is mathematically sound, or helpful

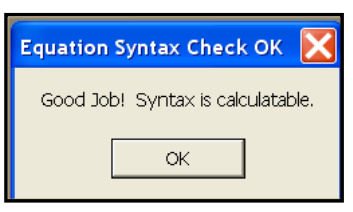
suggestions to modify the formula. The syntax verification process in EXHGM is a substitution process. EXHGM verifies the equation syntax by substituting the value of 1.0 for each variable and calculating the result. If the equation is written correctly, a message box

USER'S NOTE:

EXHGM can only review the accountability of the $\mathrm{FCl}$ formula entered - it cannot verify that the model is biologically correct - i.e., the software is unable to determine whether the limiting resources have been captured in the function. 
will verify that the entry is acceptable. If something is wrong with the equation's syntax, EXHGM will provide message windows with suggestions on how to correct the situation.

\section{Input the variable codes}

To begin the equation entry task, left-click once on the FCI Function Formula input box and put the cursor to the immediate right of the "1*" placeholder. Now move the cursor to the floating Variables Available keypad and scroll down through the list of variables. Single-click on the variable to be entered into the formula. EXHGM automatically adds the variable code (fully capitalized) to the FCI Function Formula input box, and places "[ ]" characters around the variable's code (Figure 160).

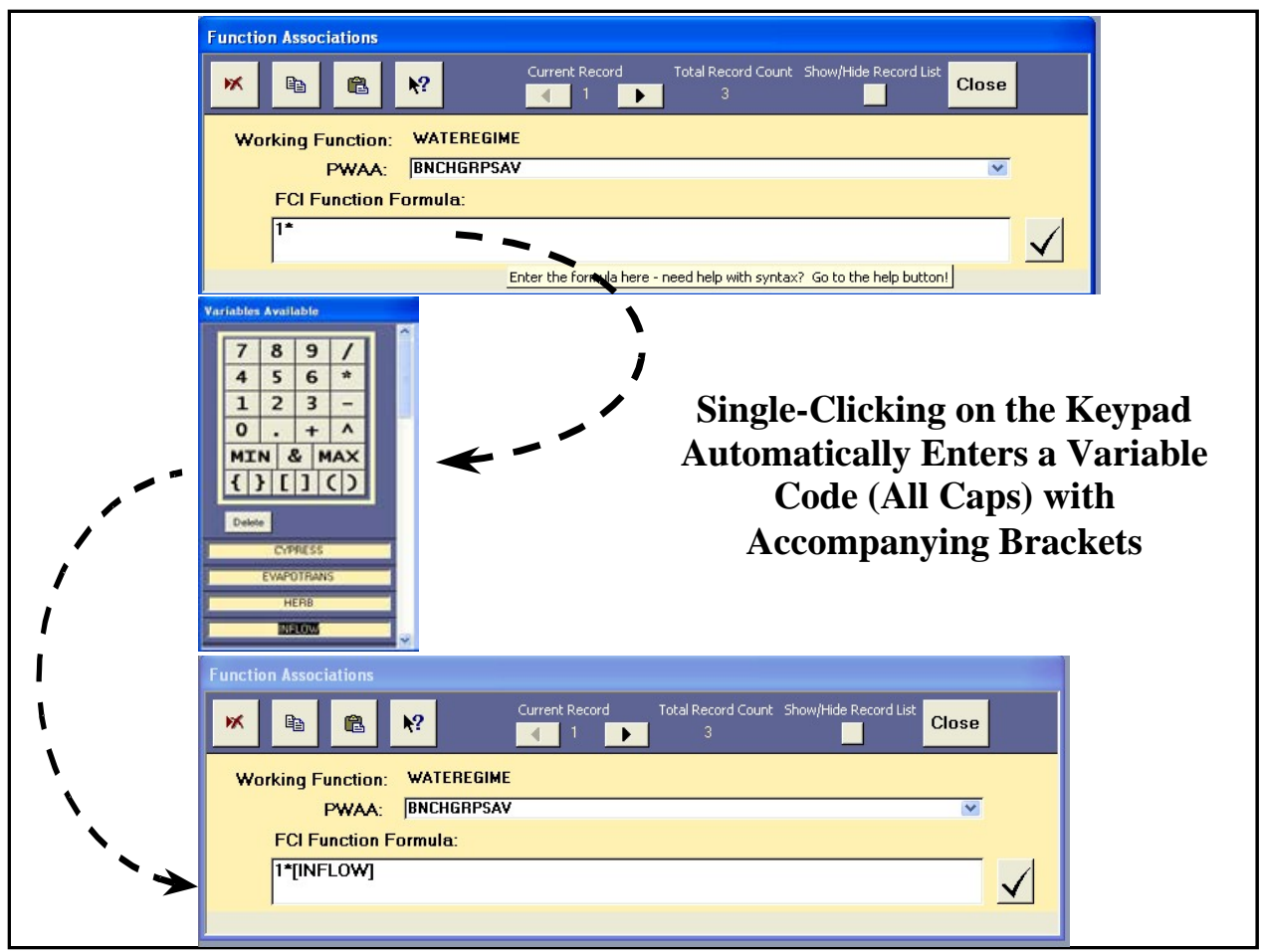

Figure 160. Use the keypad to correctly enter variable codes into the $\mathrm{FCl}$ Function Formula input box.

Repeat the process as often as necessary to populate the entry window with all the variables required to complete the FCI algorithm for this function (Figure 161). Alternatively, formulas may be manually typed into the space provided. Remember

USER'S NOTE:

Users who leave this screen and return to complete the formula entry process need to click on the FCl Function Formula before using the keypad. to fully capitalize the variable codes and place square brackets around each 
code. Spelling is critical - the database must be able to find the code in the list of available variables, so be sure to spell-check the entries.

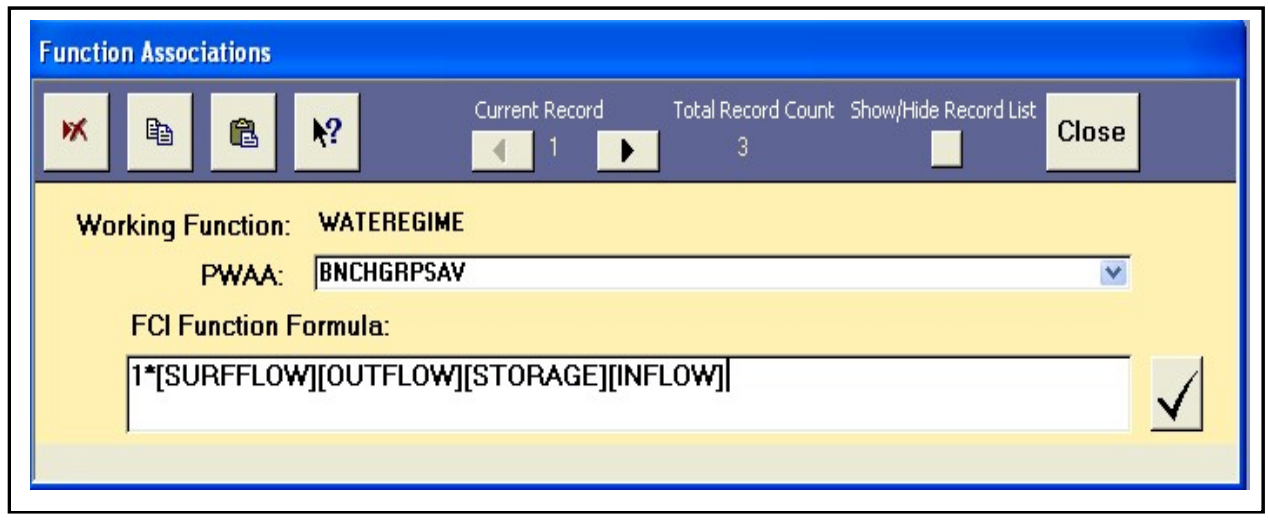

Figure 161. Begin by entering the variable codes (surrounded by brackets) into the $\mathrm{FCl}$ Function Formula input box.

Add the mathematical operators

Now enter the mathematical operators that combine the variables in a meaningful manner. There are five basic mathematical operators in EXHGM: 1) plus (+) for additions; 2) minus (-) for subtractions; 3) asterisks (*) for multiplications; 4) forward slashes (/) for divisions; and 5) carets ( $\wedge$ ) for exponential expressions. Four additional operators define special relationships inside the equation: 1) parentheses ( $(\mathbf{)})$ to group or cluster equation elements; 2) brackets ([ ]) to compartmentalize variable codes; 3) braces ( $\{\boldsymbol{\}})$ to distinguish minimum (or maximum) arguments; and 4) ampersands ( $\boldsymbol{\&}$ ) to separate the variables within the minimum (or maximum) arguments.

To proceed, systematically place the cursor between each of the variable codes in the FCI Function Formula entry box, and insert an operator using either the operator keys on the floating keypad, or the keys on the keyboard to type them in (Figure 162). 


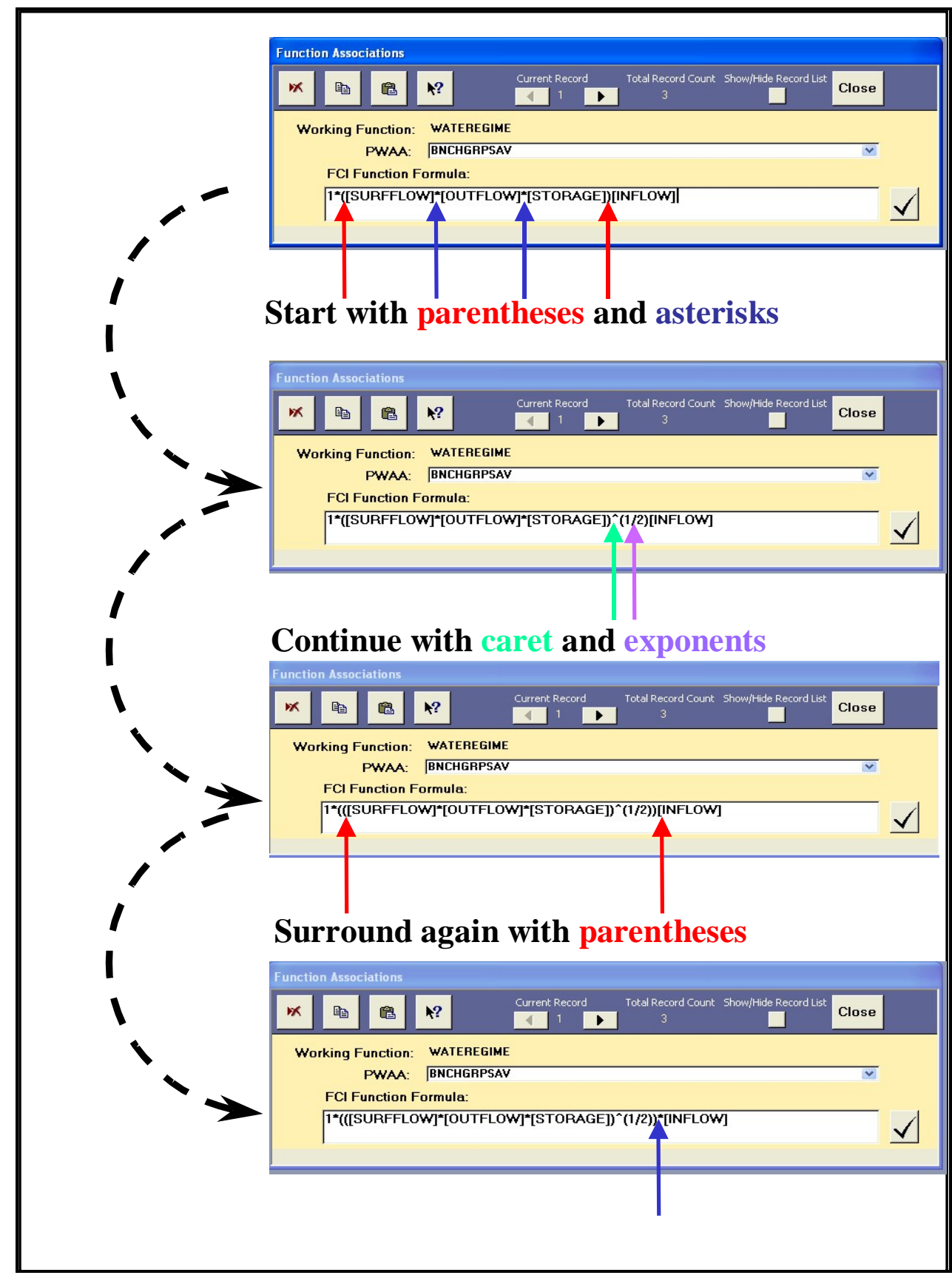

Figure 162. Example of the formula entry process in the Function Associations entry form.

At this point, it is okay to delete the "1*" placeholder. To do this, place the cursor between this first variable and the " $1 *$ " and backspace twice or put the cursor immediately before the "1" and hit the Delete button on the floating keypad

\section{USER'S NOTE:}

The Delete button on the floating Variables Available keypad can be used at any time to delete errors in the formula - note that this button removes items to the immediate right of the cursor. (Figure 163). 


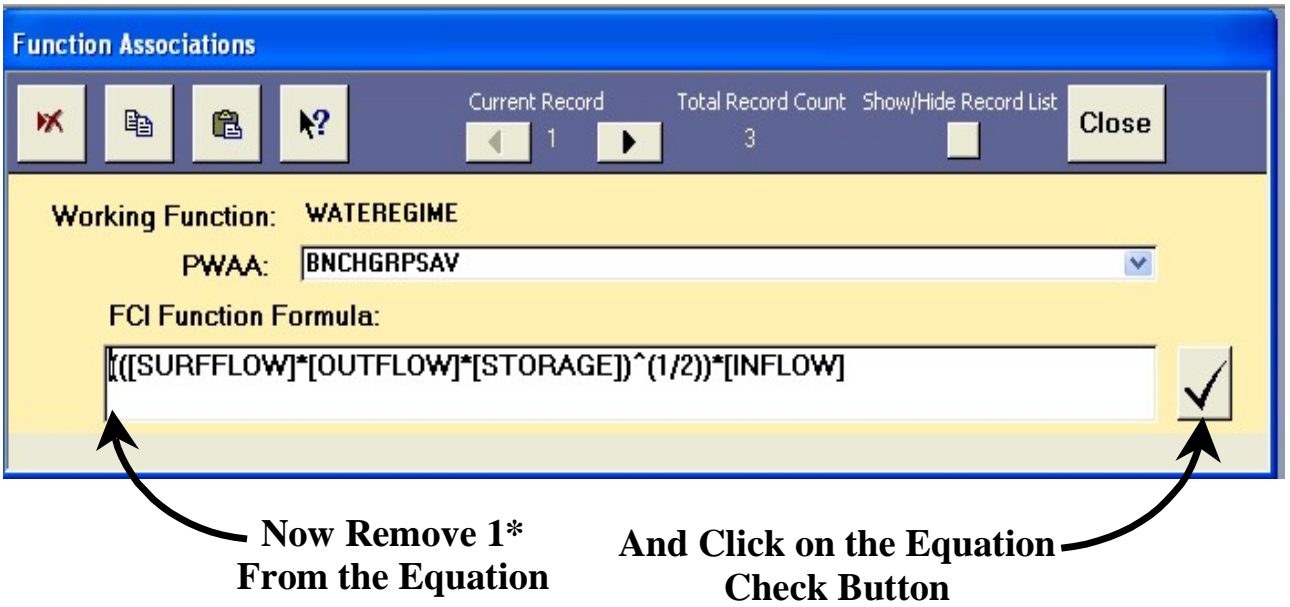

Figure 163. Completing the $\mathrm{FCl}$ formula entry process - removal of the 1* placeholder and formula checking.

Check the formula

To check the formula, click on the check button immediately to the right of the FCI Function Formula input box. If the function is mathematically calculable, a confirmation will be received. If there are problems

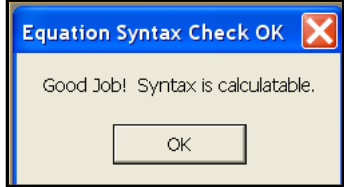
with the formula entry, the system will provide direction on the best possible solutions.

\section{Remove any phantom record}

If, as is the case in the example, the first PWAA in the list of PWAAs is not associated with the function, EXHGM assigns the first PWAA in the list a default association that must now be removed (i.e., it is considered a "phantom record"). To determine if a phantom record exists, look to the top of the Function Associations window and note the number of records showing. If only one association has been made thus far, and the record counter shows that there are two records instead, assume that a phantom record exists (Figure 164). 


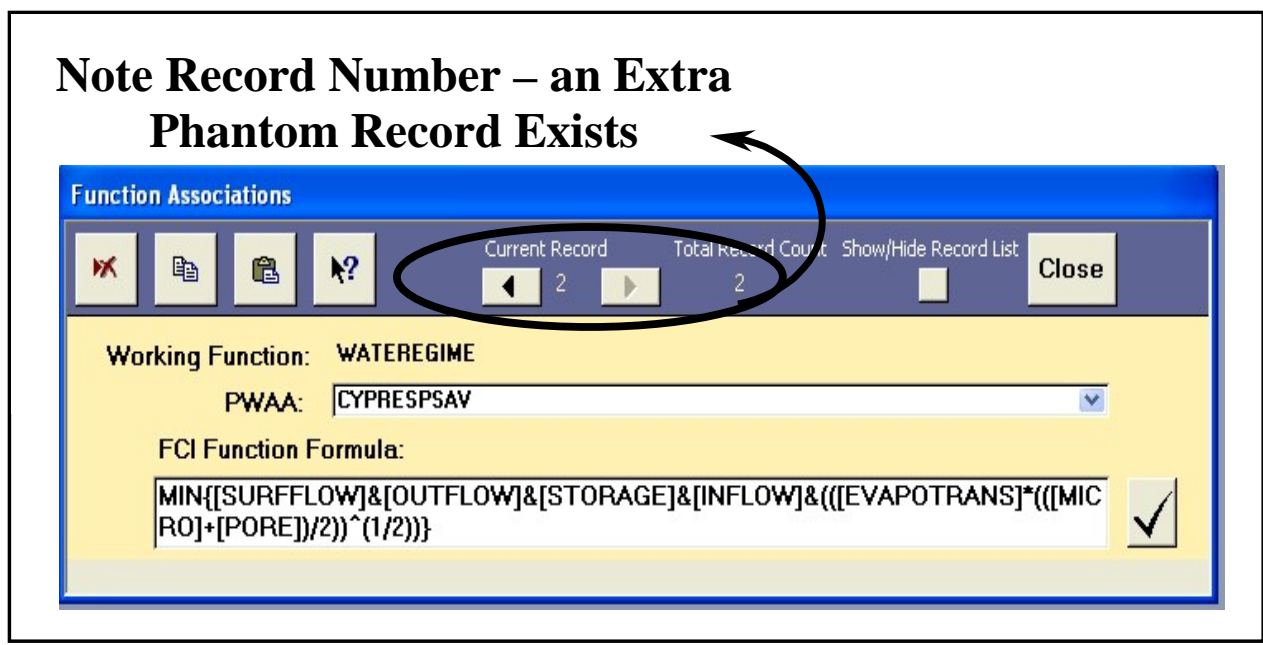

Figure 164. Extra records indicate phantom records that must be manually removed.

To remove this phantom record, complete the following steps: 1) click on the Back Record button, and 2) click on the Delete Record button.

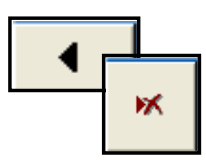

Duplicate the records for multiple PWAAs

As a general rule, each record in the Function Associations window represents a single application of the model. Functional models with one PWAA require one record of associative information. Functions with multiple PWAAs require an individual record for each PWAA associated with the wetland. In most instances, the same FCI formula applies across multiple PWAAs within a wetland function. EXHGM provides a copy feature in this window to expedite the formula entry process for these situations. To enter a multi-PWAA functional USER'S NOTE:

A new record must be created for every PWAA utilized in a function. To capture the potential for restorative or rehabilitative actions in the future condition, it is essential to associate both an existing and a newly developed PWAA for each function (e.g., two records would be associated for savanna - existing and newly restored savanna). model into EXHGM, follow the directions above to enter the first PWAA. Now click on the PWAA drop-down box, and choose the next PWAA to associate with the function (Figure 165). 


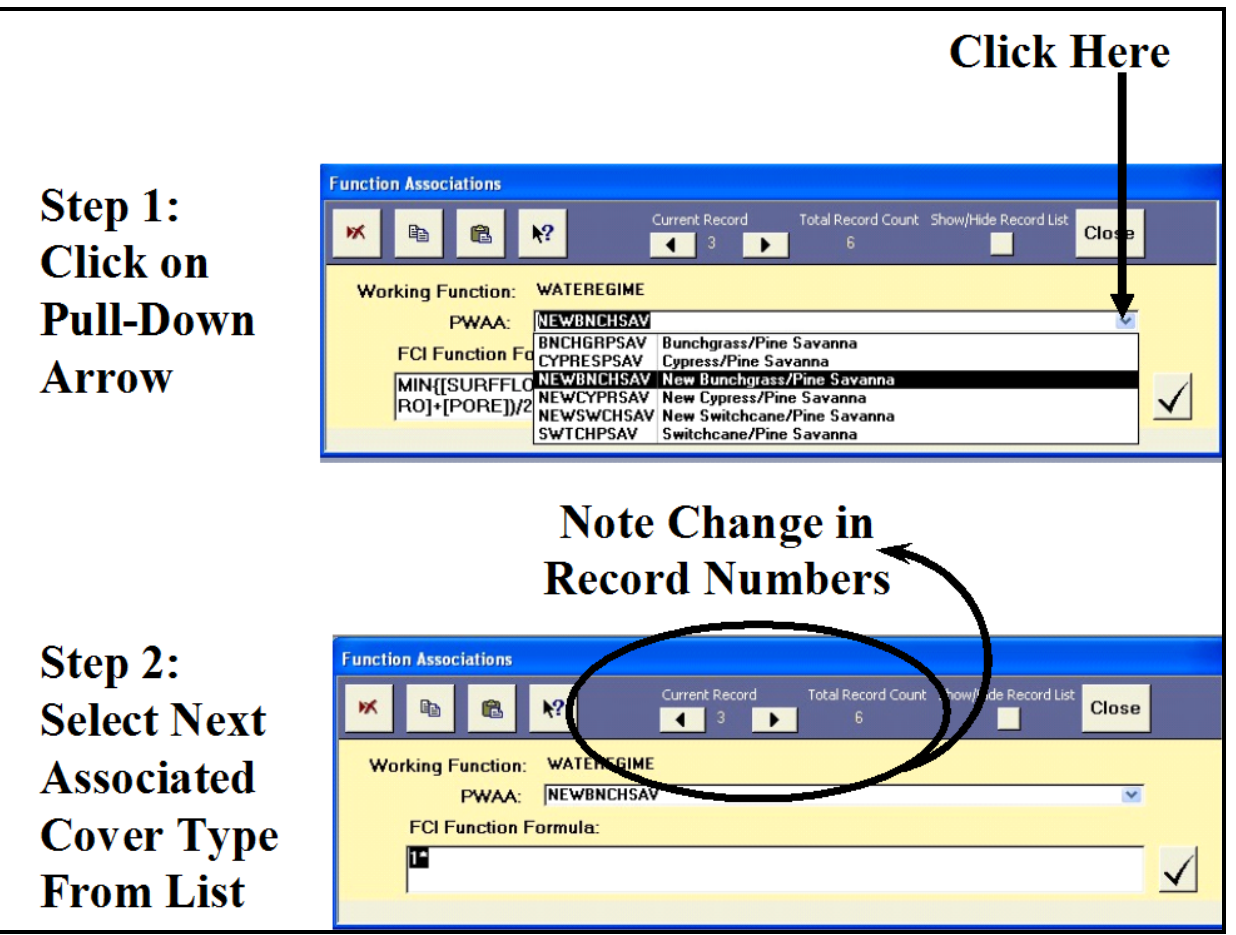

Figure 165. Select the next associated PWAA from the pull-down list.

EXHGM creates a new record and assigns the PWAA a formula of "1*." In stepwise fashion, complete the following quick steps:

1. Click on the Back Record button.

2. Click on the Copy button.

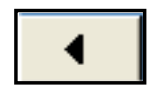

3. Click on the Forward Record button.

4. Click on the Paste button (Figure 166).
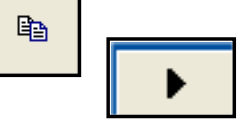


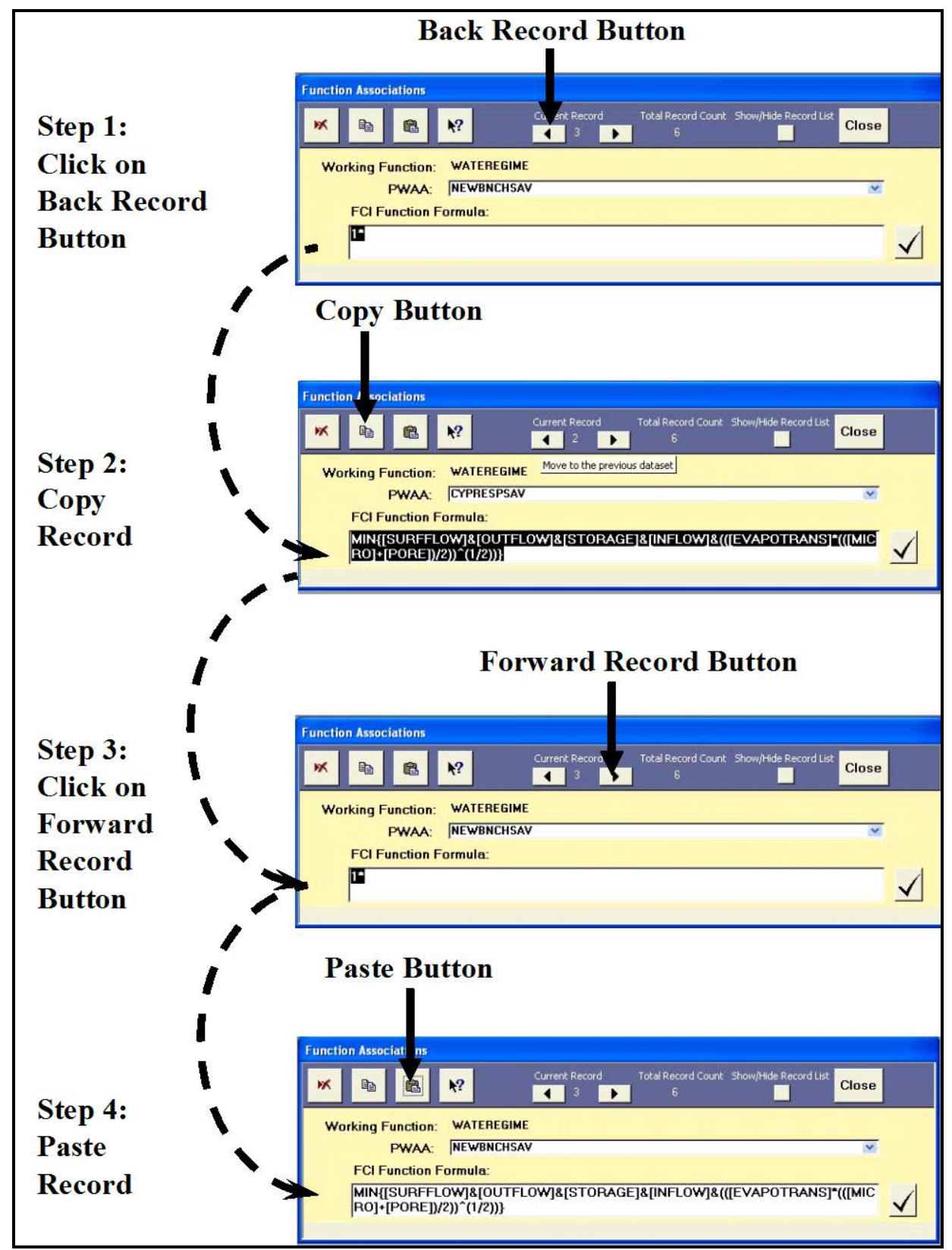

Figure 166. Make a new association by duplicating the previous data.

Repeat this process as many times as necessary to fully associate the various PWAAs (and FCI formulas) with the "Working Function." If at any time, a function's associated
USER'S NOTE:

There is one exception to this rule - if the targeted record is the only PWAA association for the function, EXHGM will not allow this deletion using this protocol. Instead, simply overwrite the $\mathrm{FCl}$ formula with " 1 *" and click on the Close button. This will remove the one and only association for the model. information must be removed, open the Function Associations window, left-click once on the FCI Functions Formula entry box, $\sqrt{ }$ and click on the Delete button. 
Reviewing the associations

To review the list of associated records entered thus far, click on the Show/ Hide Records List toggle button. In response, EXHGM opens the listing window (Figure 167).

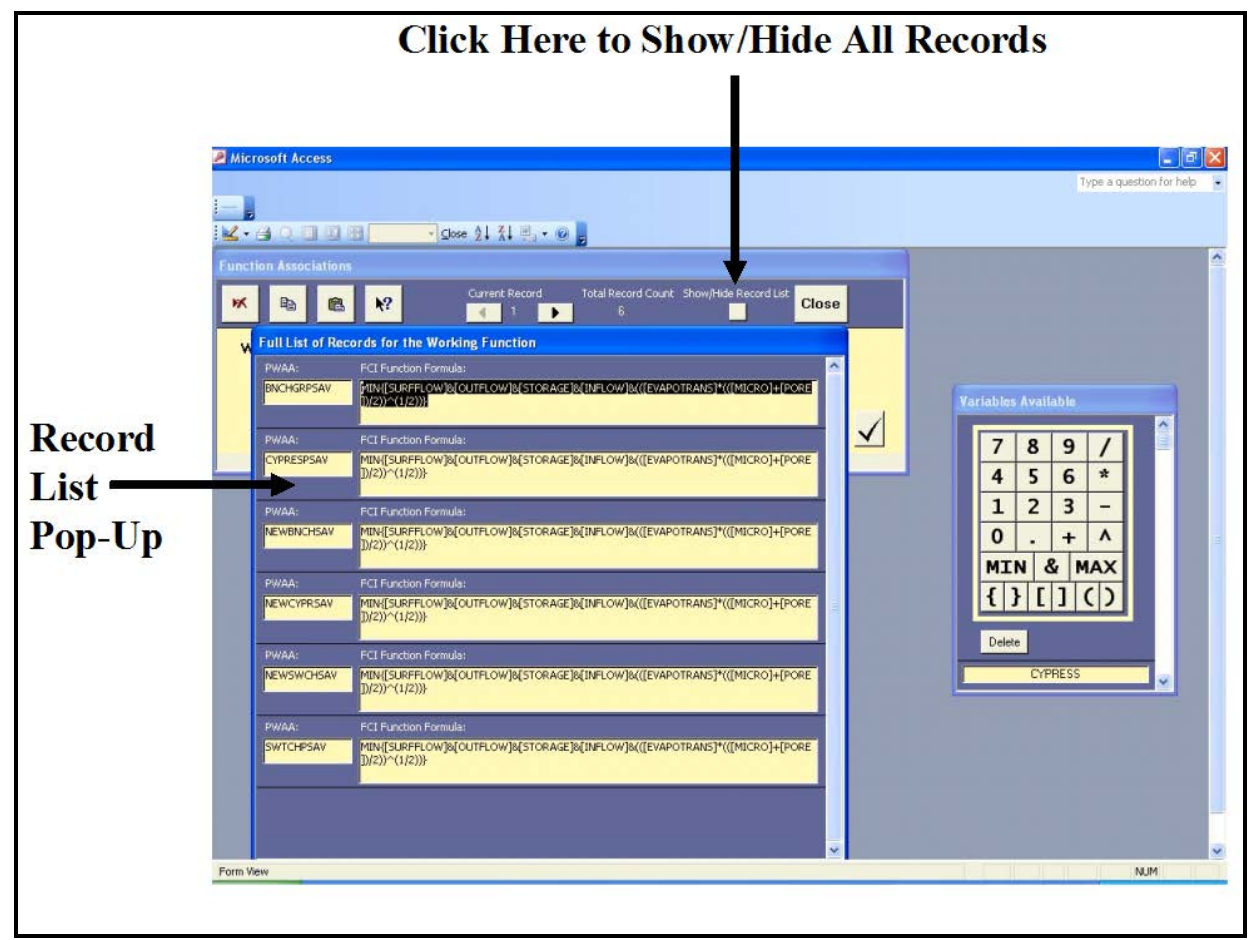

Figure 167. Show or hide all associated records simultaneously.

Scroll down this list with the scroll bar on the far right-hand side of this window. To close the window, click the toggle button a second time. When finished with the associations, click on the Close button in the upper right-hand corner of the toolbar and EXHGM returns to the primary interface. Repeat Step 3 (i.e., Function Association) for each "Working Function" in the analysis.

\section{Checking the association process with EXHGM reports}

EXHGM provides a series of summary reports as each step in the process above is completed. To review and check the associations made thus far, click on the Setup Reports button in the center of the primary interface (Figure 168). 


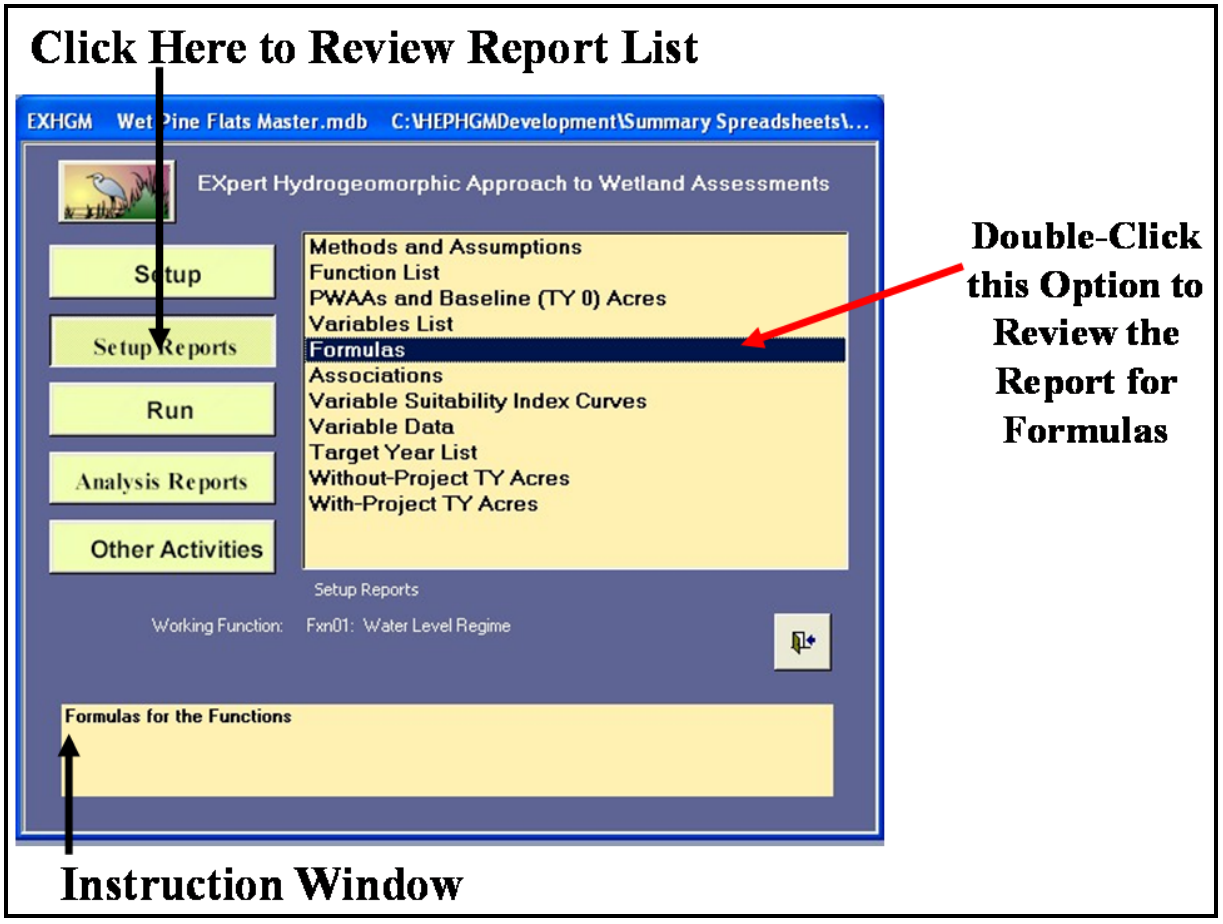

Figure 168. Click on the Setup Reports button to view the association reports in EXHGM.

A list of available reports appears in the center of the primary interface. Two additional reports should be populated by the time the function association process is complete:

\section{- Formulas}

\section{- Associations}

Single-click on either report and look to the Instruction Window to check the contents of the report. Double-click on any report, and EXHGM opens a new window with the report inside (Figure 169). 


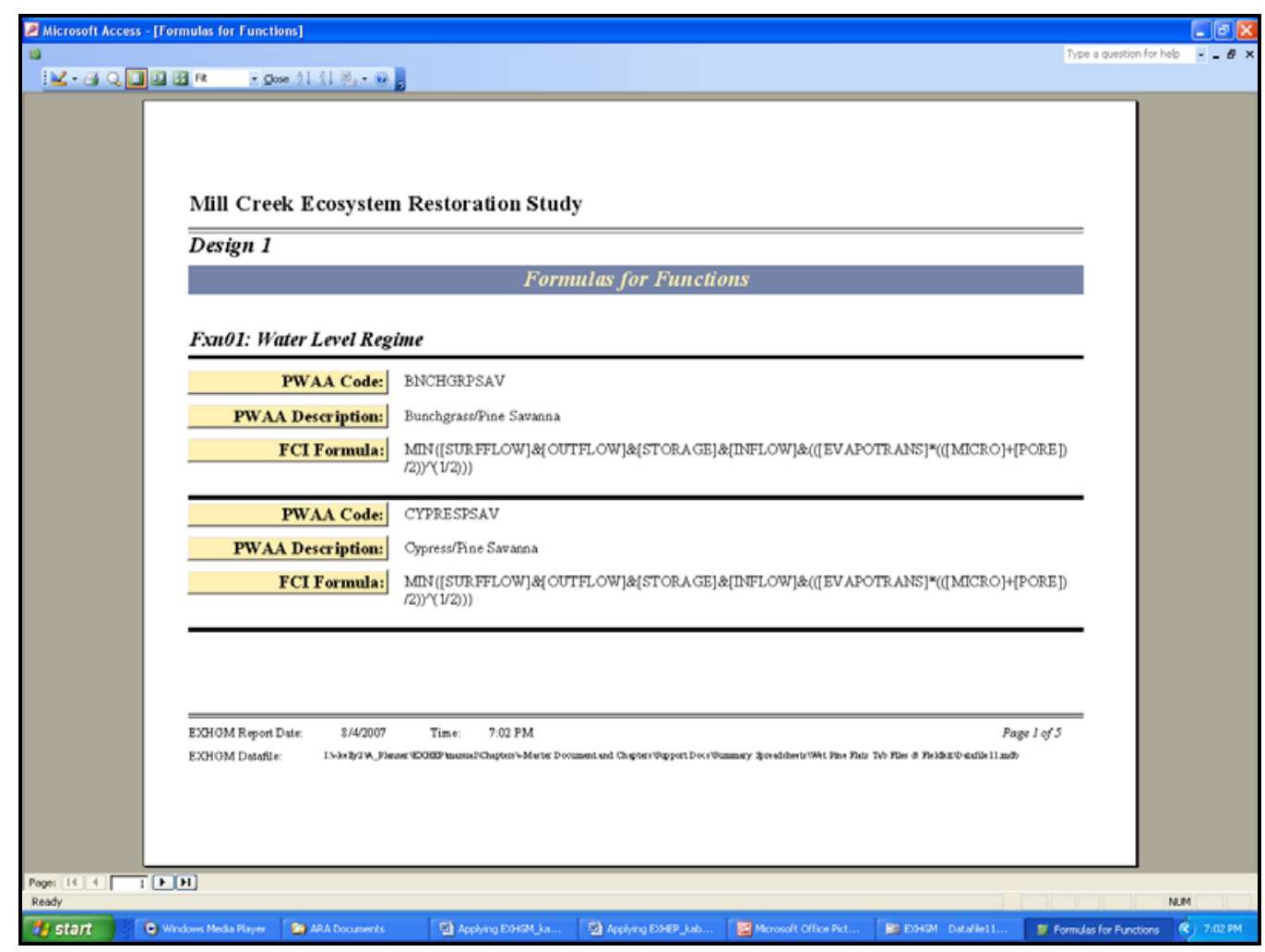

Figure 169. Example of EXHGM's formulas report for the functional models.

Click on the Close button from the suite of buttons offered in the Print Preview toolbar - EXHGM returns to the primary interface. Repeat the process as often as necessary to check the products developed thus far in the association activities. Proceed to Step 4: Enter Baseline Data and Generate Baseline Results to continue.

\section{Step 4: Enter baseline data and generate baseline results}

Once the FCI functions are set up and associated in EXHGM, the baseline data can be entered into the system. In some studies, several functional models are constructed with similar variables in an attempt to characterize the quality of the site in an efficient manner. EXHGM provides entry screens to import variable data, automating the data entry task. This capability reduces the amount of labor required to complete the analysis, while increasing the system's evaluation efficiency. Automated data input into EXHGM streamlines access to this data by importing it from a single 
source. This capability further enhances the system's efficiency and reduces the amount of time necessary to input the data. ${ }^{1}$

\section{VSI graph coordinate entry, deletion, and/or copying}

Prior to entering data into EXHGM, a normalized relationship (scale from 0 - 1) must be defined between each variable and the functional capacity of the wetland it represents. To begin the process, click on the Run button on the primary interface (Figure 170).

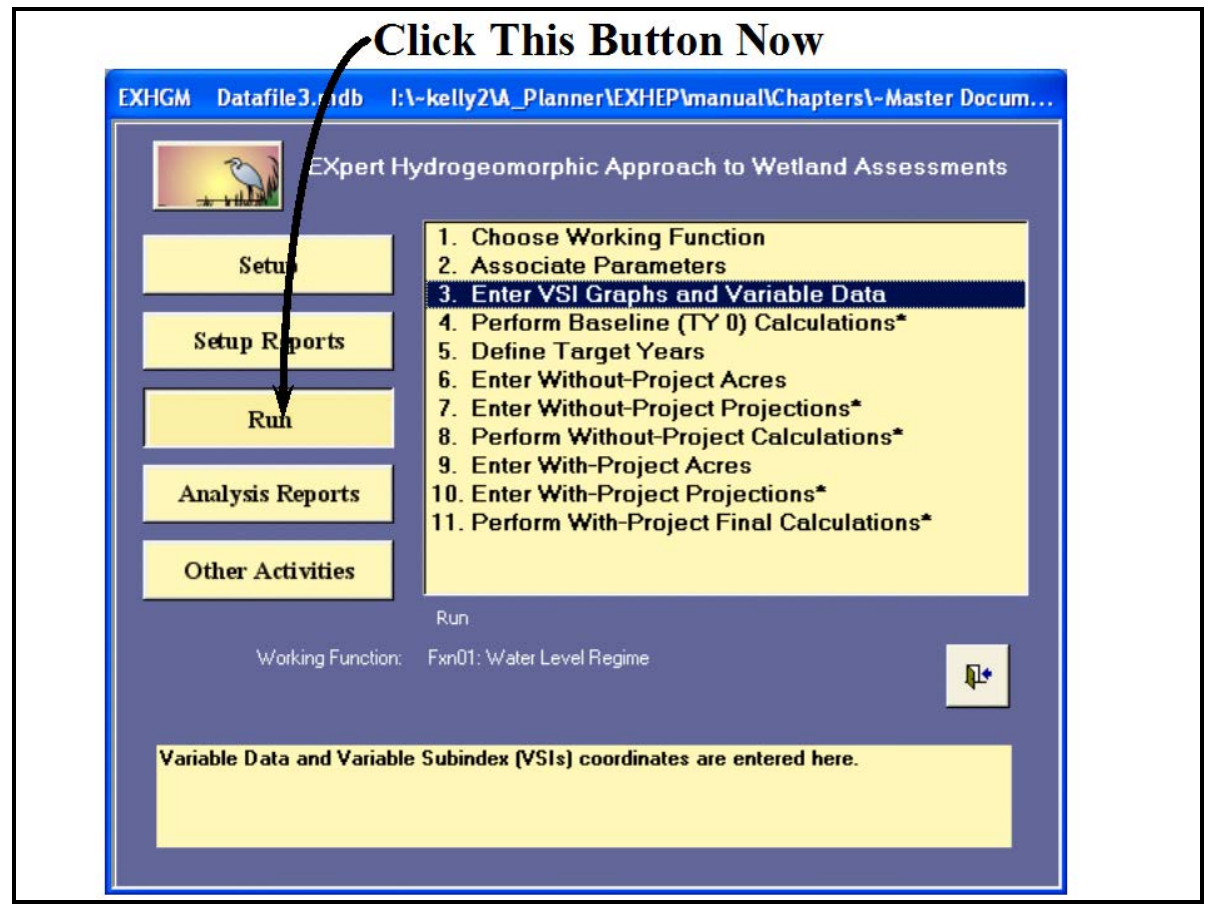

Figure 170. Click on the Run button to begin the baseline data entry process in EXHGM.

The screen appears in the center of the primary interface with 11 options:

\section{Choose Working Function}

2. Associate Parameters

3. Enter VSI Graphs and Variable Data

4. Perform Baseline (TY 0) Calculations*

5. Define Target Years

6. Enter Without-Project Acres

7. Enter Without-Project Projections*

8. Perform Without-Project Calculations*

\footnotetext{
1 To assist in the software application, a series of training exercises have been included in Appendix E refer to Exercises 12 and 13 for relevant training in baseline data entry in EXHGM.
} 


\section{Enter With-Project Acres \\ 10. Enter With-Project Projections* \\ 11. Perform With-Project Final Calculations*}

By now, the first two steps in this options list have been completed. However, the software may not be ready to accept coordinates for the models in the analysis - particularly if several models were defined earlier in the setup process. A systematic approach works best in this instance choose a "Working Function" (refer to Step 2: Function Association, Choose the Working Function above for assistance) to proceed. Now double-click on 3. Enter VSI Graphs and Variable Data to open the following windows (Figure 171).

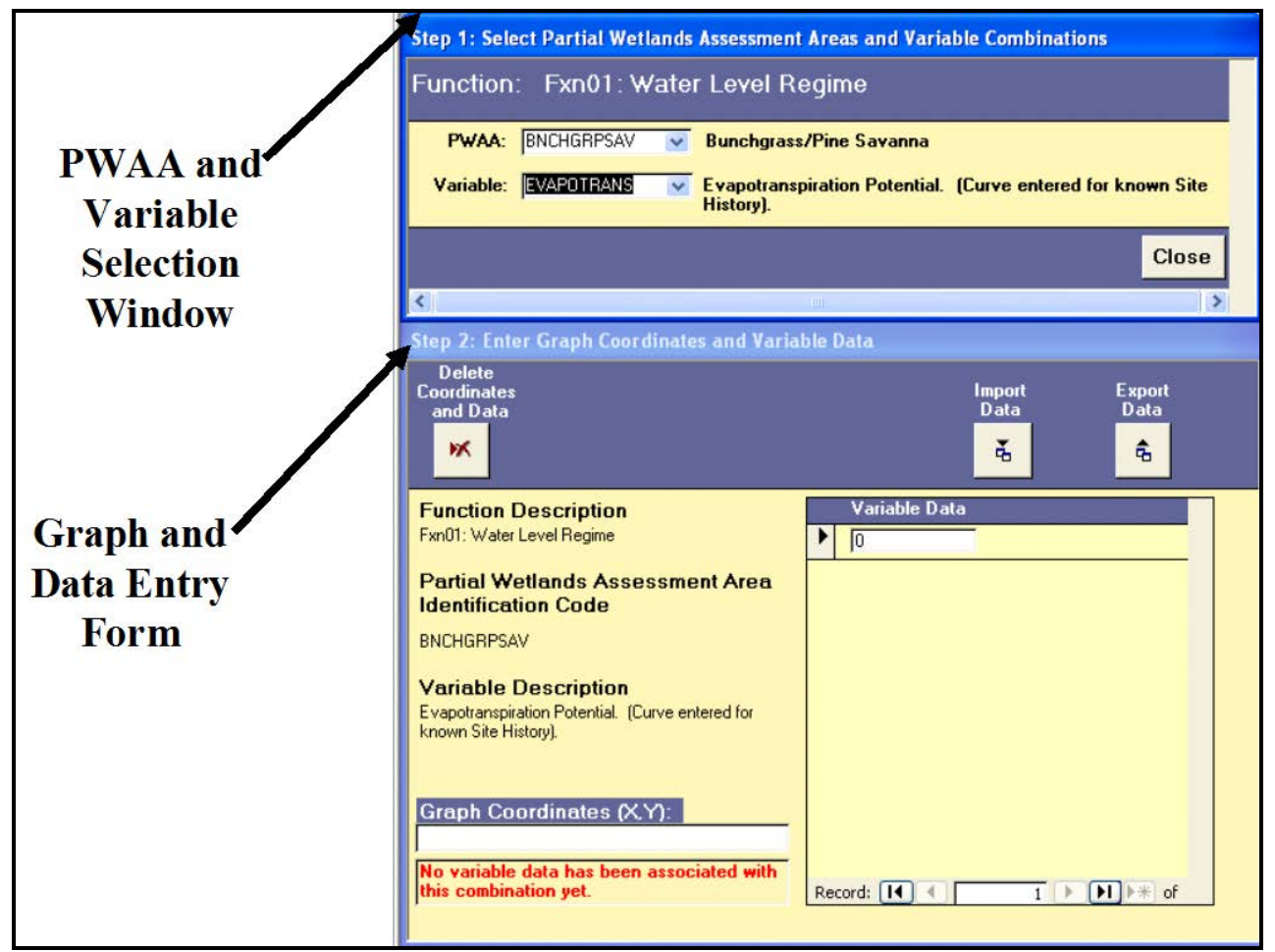

Figure 171. Baseline Data Entry windows in EXHGM.

The Step 1: Select Partial Wetland Assessment Areas and Variable Combinations window focuses the system on a PWAAvariable combination. The Step 2: Enter Graph Coordinates and Variable Data form is the interface that will accept the coordinates for the variable's VSI curve and the data points collected by the evaluation team. 
Step 1: Select a PWAA and a variable

To begin, select a PWAA and a variable from the pull-down menus in the upper window (Step 1: Select Partial Wetland Assessment Areas and Variable Combinations) (Figure 172).

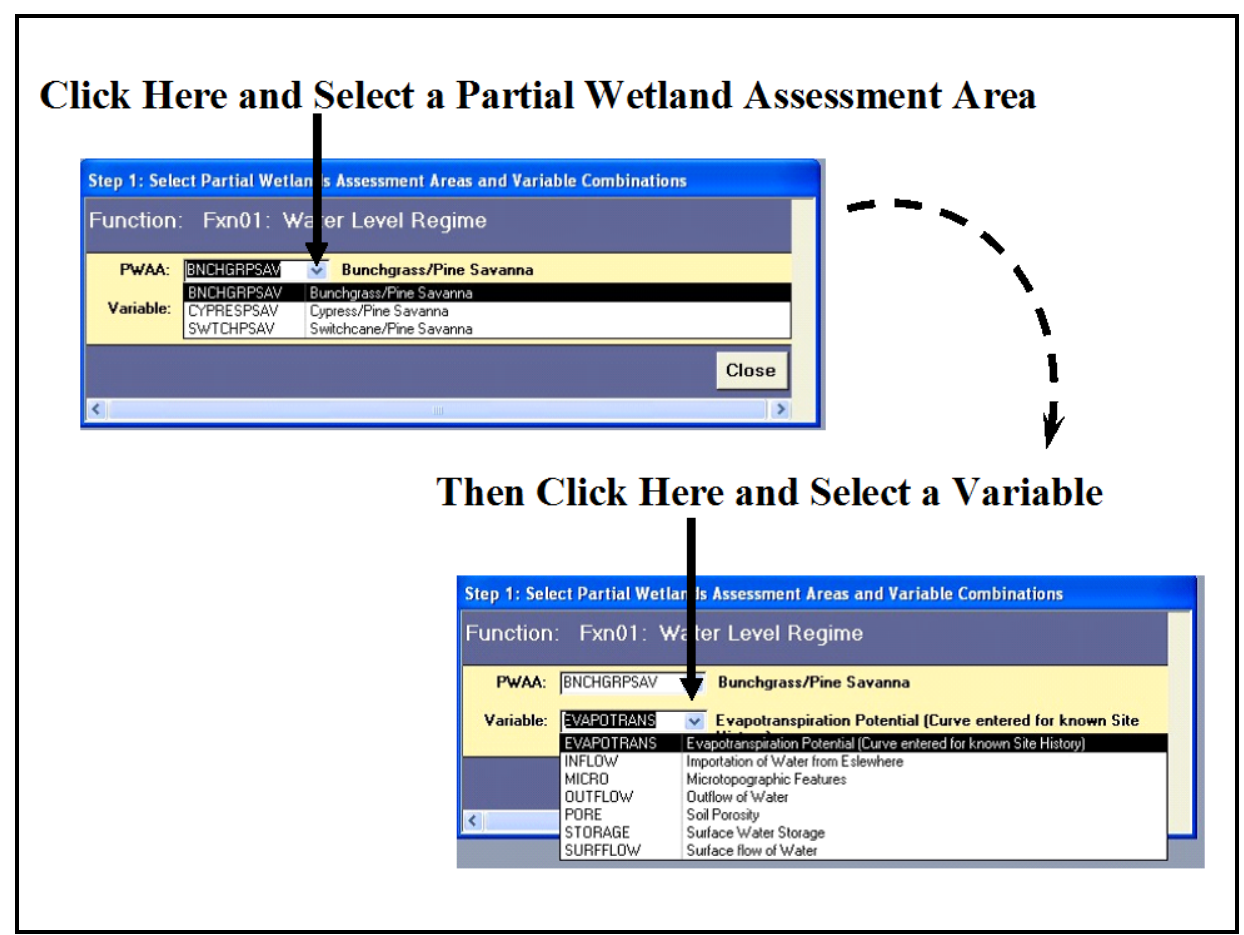

Figure 172. Begin data entry by selecting a partial wetland assessment area and a variable from the pull-down lists provided.

Step 2: Enter the VSI graph coordinates

Now move to the lower window (Step 2: Enter Graph Coordinates and Variable Data) and provide the VSI graph coordinates for the selected variable in the selected PWAA (Figure 173). 


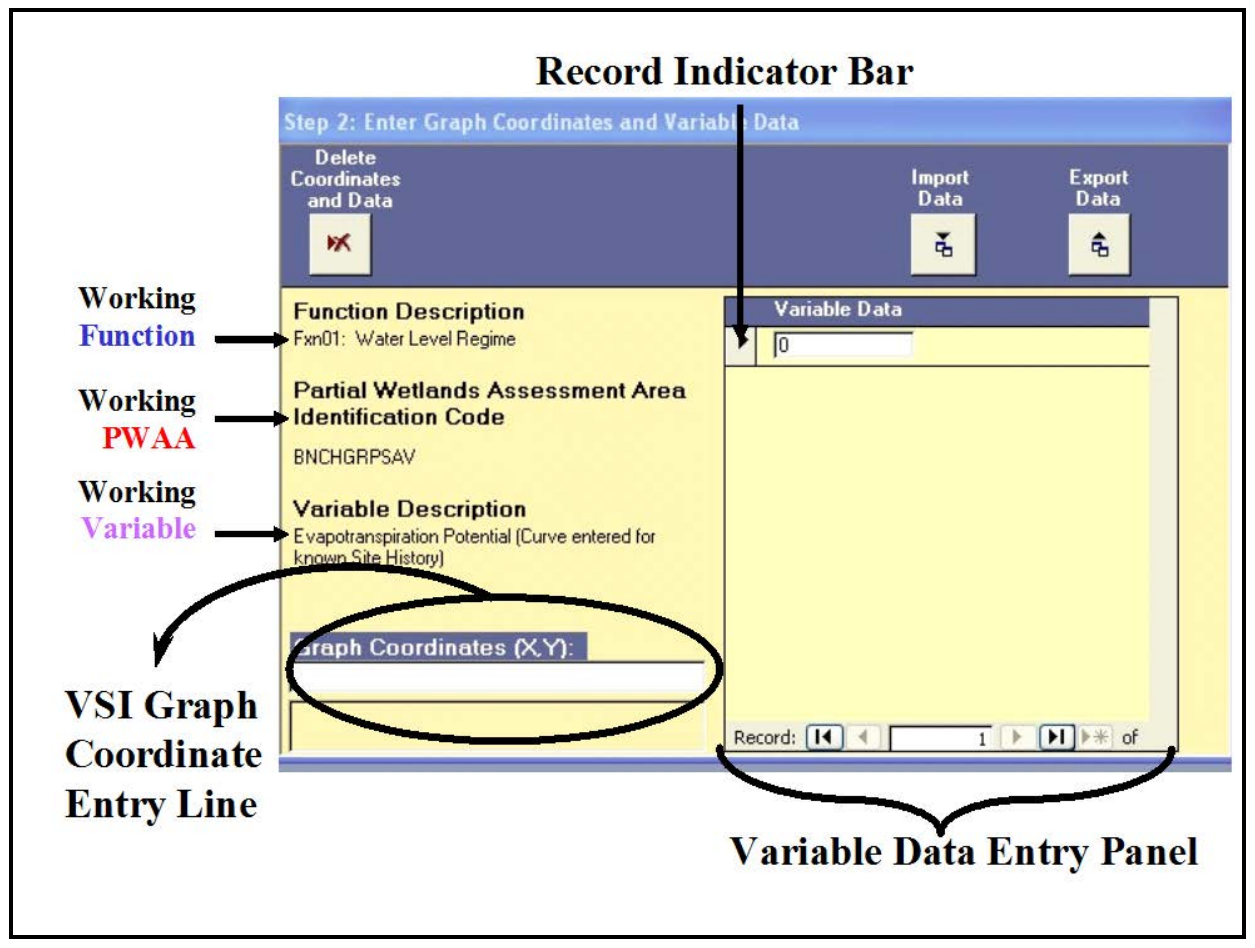

Figure 173. Move to the lower window and enter the VSI graph coordinates in the space provided.

To do this, enter sets of $X$ and $Y$ coordinates (separated by commas - $X_{1}, Y_{1}$, $\mathrm{X}_{2}, \mathrm{Y}_{2}, \mathrm{X}_{3}, \mathrm{Y}_{3}, \ldots \mathrm{X}_{\mathrm{n}}, \mathrm{Y}_{\mathrm{n}}$ ) into the lower left-hand space provided [i.e., Graph Coordinates $(\mathbf{X}, \mathbf{Y})$ and hit the Tab key on the keyboard (Figure 174).

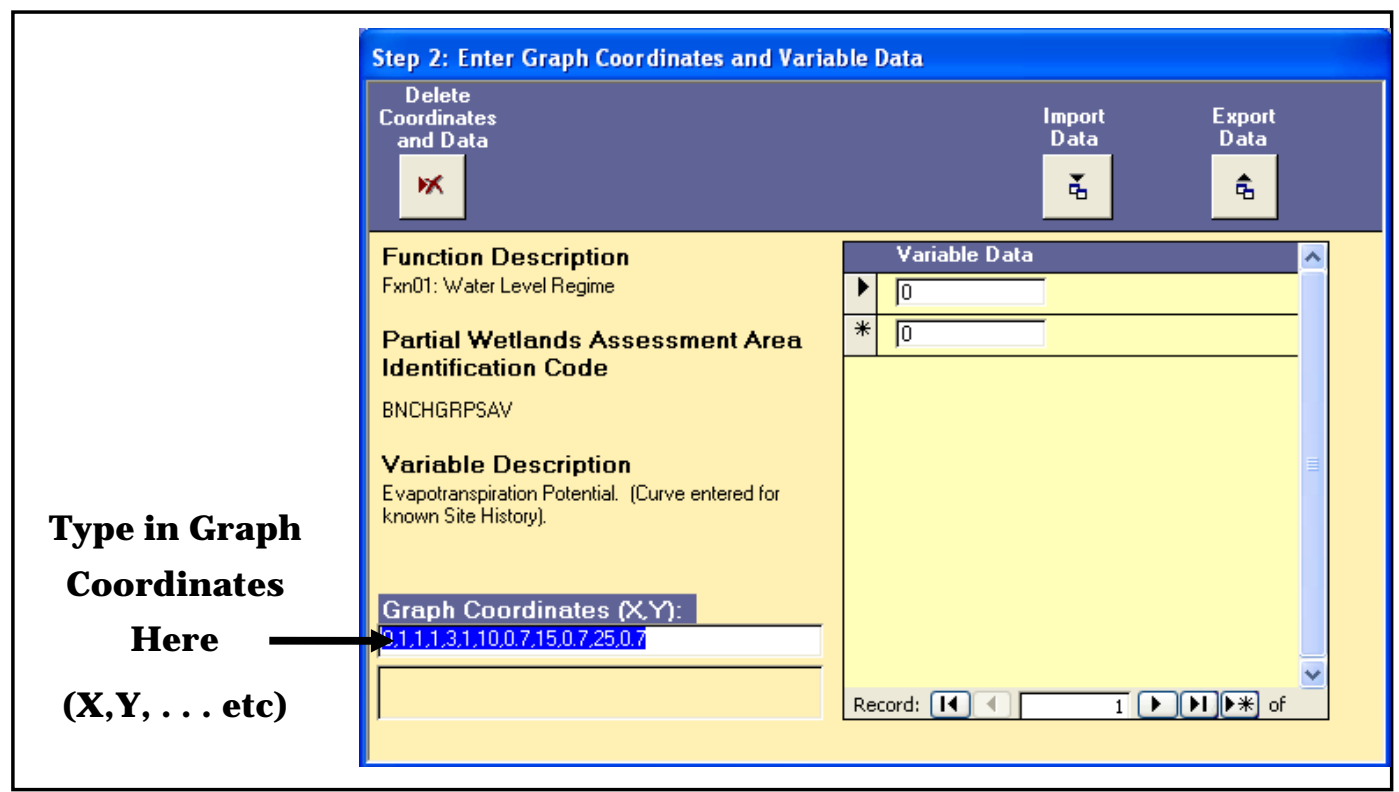

Figure 174. Example of a VSI coordinate entry. 
EXHGM ignores spaces in these entries, so use spaces within the entry to clarify and organize the $\mathrm{X}$ and $\mathrm{Y}$ values entered. As soon as

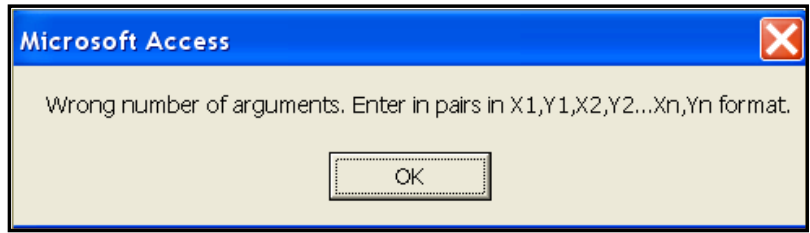
the coordinates are entered, hit Enter on the keyboard. EXHGM will check the numbers - if an entry is missing commas, or has too many commas, EXHGM will flash a warning and directions on the best way to proceed. Once the coordinates have been checked by the program, EXHGM will move the cursor to the first entry of the Variable Data entry panel on the right-hand side of the screen.

Continue the process of VSI coordinate entry for all PWWA/variable combinations in the function before proceeding to the data entry phase of the process. If the need should arise, the VSI graph coordinates can be deleted by highlighting the string of "X/Y" coordinates and hitting the Delete button on the keyboard. As is often the case, PWAAs may share the same VSI curve. In this instance, save time by copying the VSI graph coordinates to the clipboard and pasting them back into the next record. To do this, follow these quick steps:

1. Highlight the coordinates and press Ctrl C on the keyboard.

2. Move up to the upper form (Step 1: Select Partial Wetland Assessment Areas and Variable Combinations) and pull down the next PWAA. Do not change the variable selection.

3. Move down to the lower form (Step 2: Enter Graph Coordinates and Variable Data) and click once in the Graph Coordinates (X,Y) box.

4. Press Ctrl V on the keyboard and Tab to aocept the coordinates (Figure 175). 


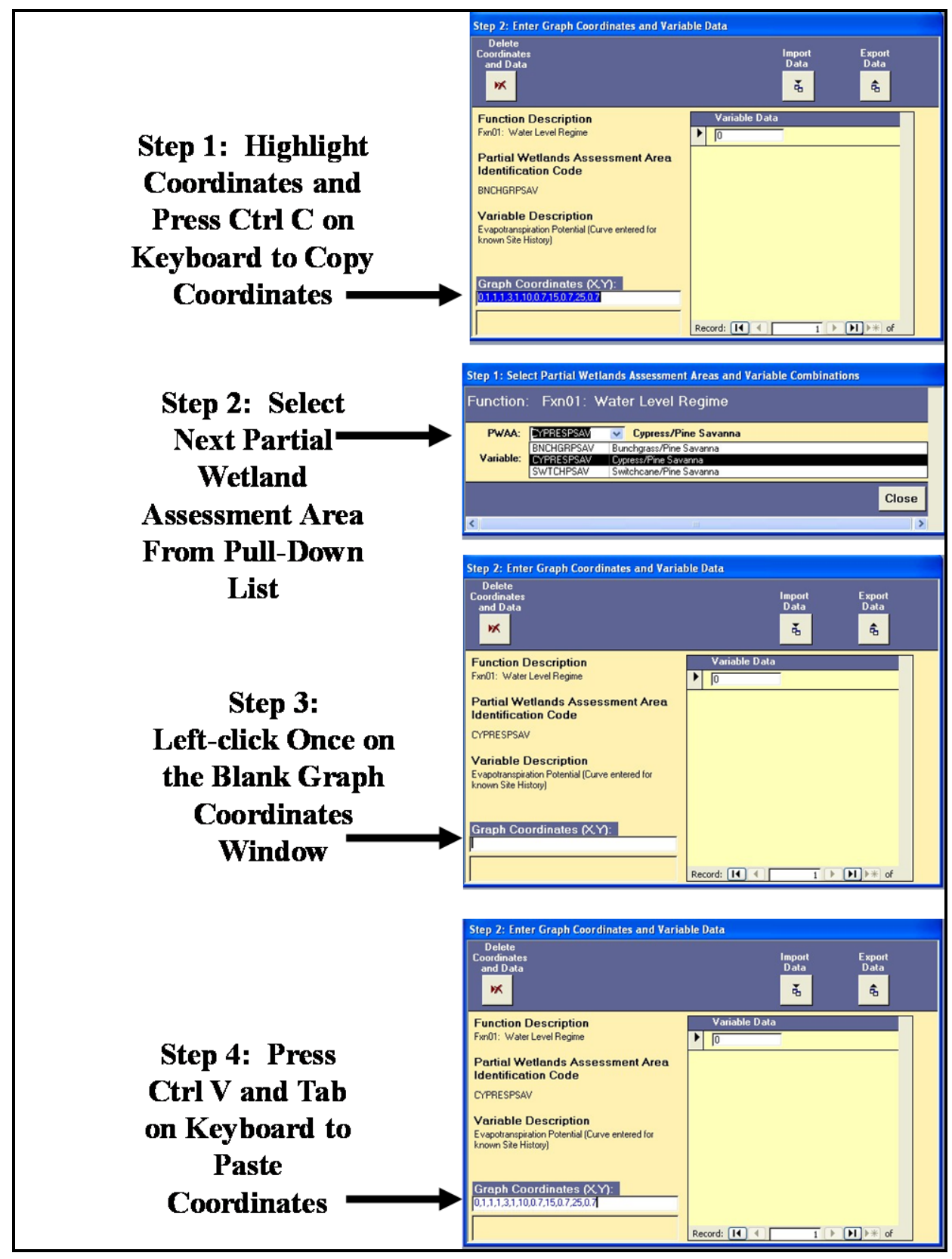

Figure 175. Copying VSI graph coordinates from one record to the next.

If there were no errors in the process, EXHGM will move the cursor up to the first entry of the Variable Data entry panel on the right-hand side of the screen. Repeat this process for every variable-PWAA combination for every function in the analysis. Return to the top of the PWAA

USER'S NOTE:

Although this process may seem tedious at first, the flexibility of the EXHGM software allows users to sensitize the model to conditions in their region. In other words, because the software asks for these graphical coordinates on a variable-by-variable basis for every PWAA, the system allows development of highly specialized relationships between the model's various components. 
and variable lists, and double-check each record to assure that the VSI graph coordinates have been entered for all records. Click Close to return to the primary interface. Choose the next "Working Function" and repeat the process as often as necessary to fully set up the datafile for all applicable models. ${ }^{1}$

\section{Checking the VSI graph coordinates with EXHGM reports}

To review and check the entries made thus far, click on the Setup Reports button (Figure 176).

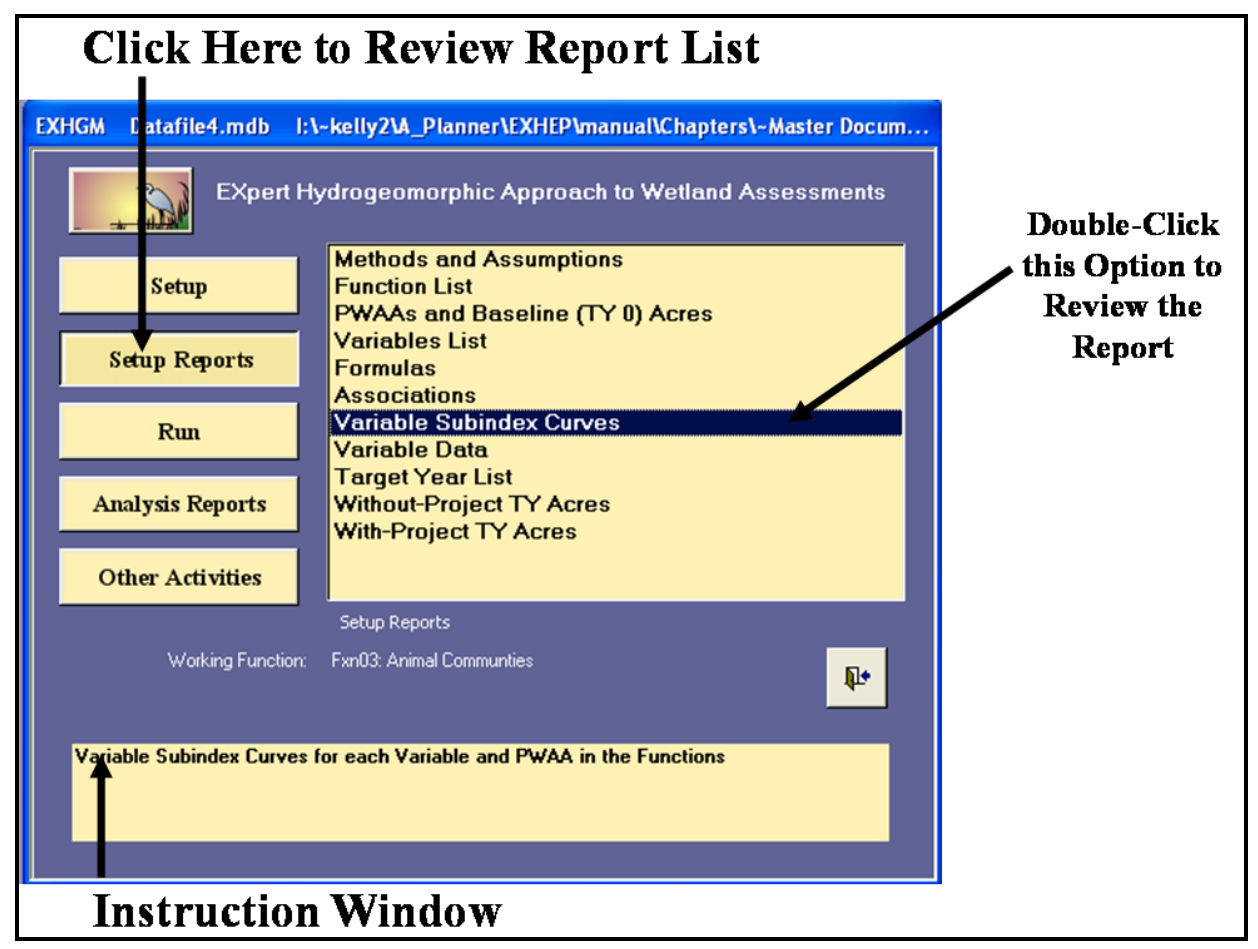

Figure 176. Click on the Setup Reports button to view the VSI graph coordinate reports in EXHGM.

A list of available reports appears in the center of the primary interface. The Variable Subindex Curves report is now ready for review. Single click on the report and look to the Instruction Window to preview the contents of the report. Double-click on the report, and EXHGM opens a new window with the report inside (Figure 177).

\footnotetext{
1 Once the suite of VSI coordinates have been entered into the file for the functions, the authors suggest saving the file as a "master." This is a particularly useful approach if the functions are to be used at multiple sites (with unique baseline conditions such as an impact site and a subsequent mitigation site or perhaps multiple restoration sites under the premise of a watershed study). By opening and saving the "master" as a new file, the setup information is retained, and the data entry process for the new site can begin immediately thereafter.
} 


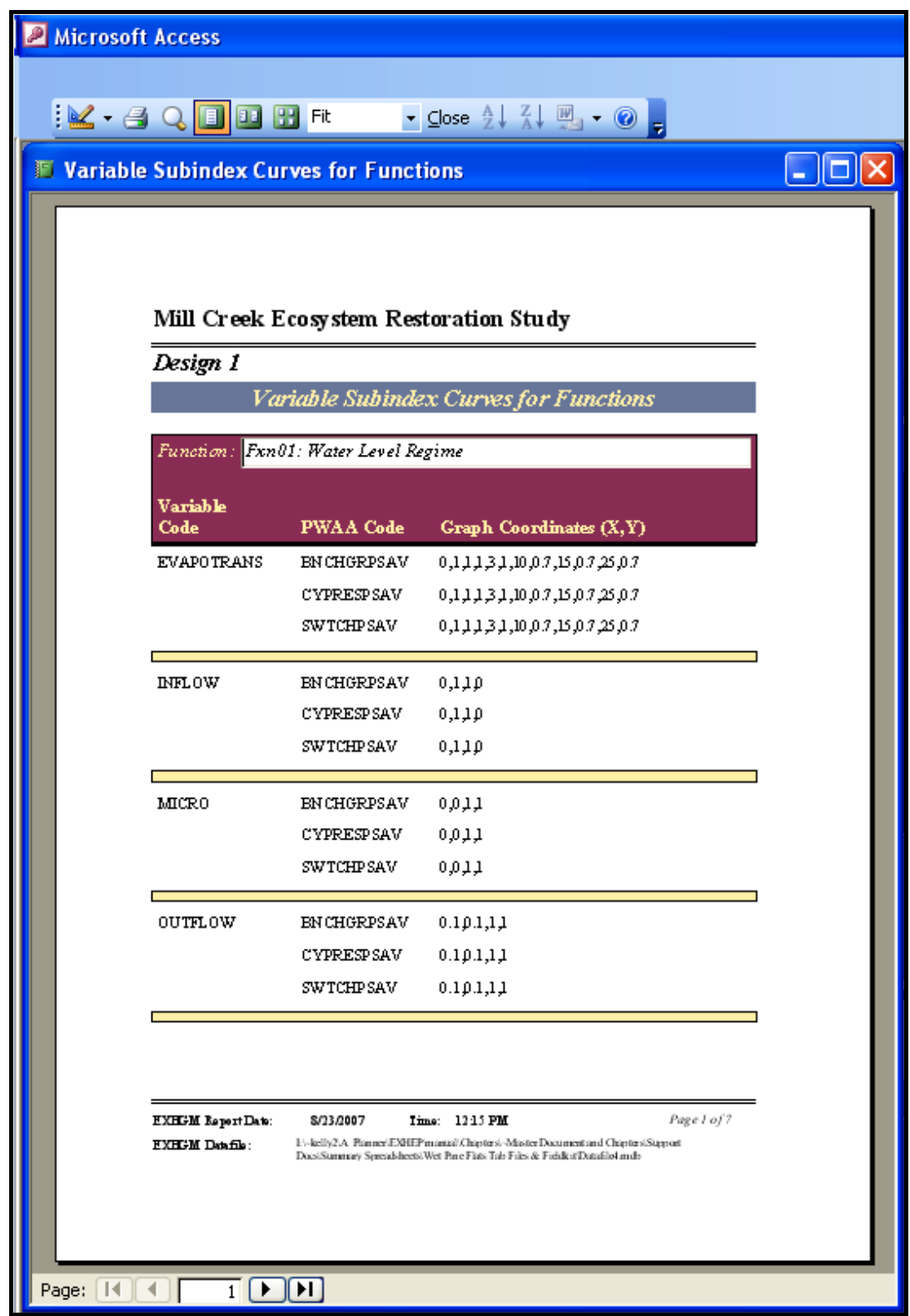

Figure 177. Example of Variable Subindex Curves for Functions Report in EXHGM.

Click on the Close button from the suite of buttons offered in the Report Control toolbar, and EXHGM returns to the primary interface. Now that the VSI graph coordinates entry process is complete, and the information has been confirmed, proceed to the next section to learn how to enter variable data into EXHGM.

\section{Baseline data entry}

It is often useful to organize the PWAAs and their associated variables in some fashion or manner before beginning the process of entering data. In 
most HGM studies, several data sets are generated for each variable (in each applicable PWAA). In its original form, this information is often unwieldy and prone to disorganization. One option is to store the data in a spreadsheet - EXHGM was designed to import data from such electronic formats (specifically Microsoft-compatible formats such as MS Excel). ${ }^{1}$

Now, begin the data entry process by clicking on the Run button on the primary interface (Figure 178).

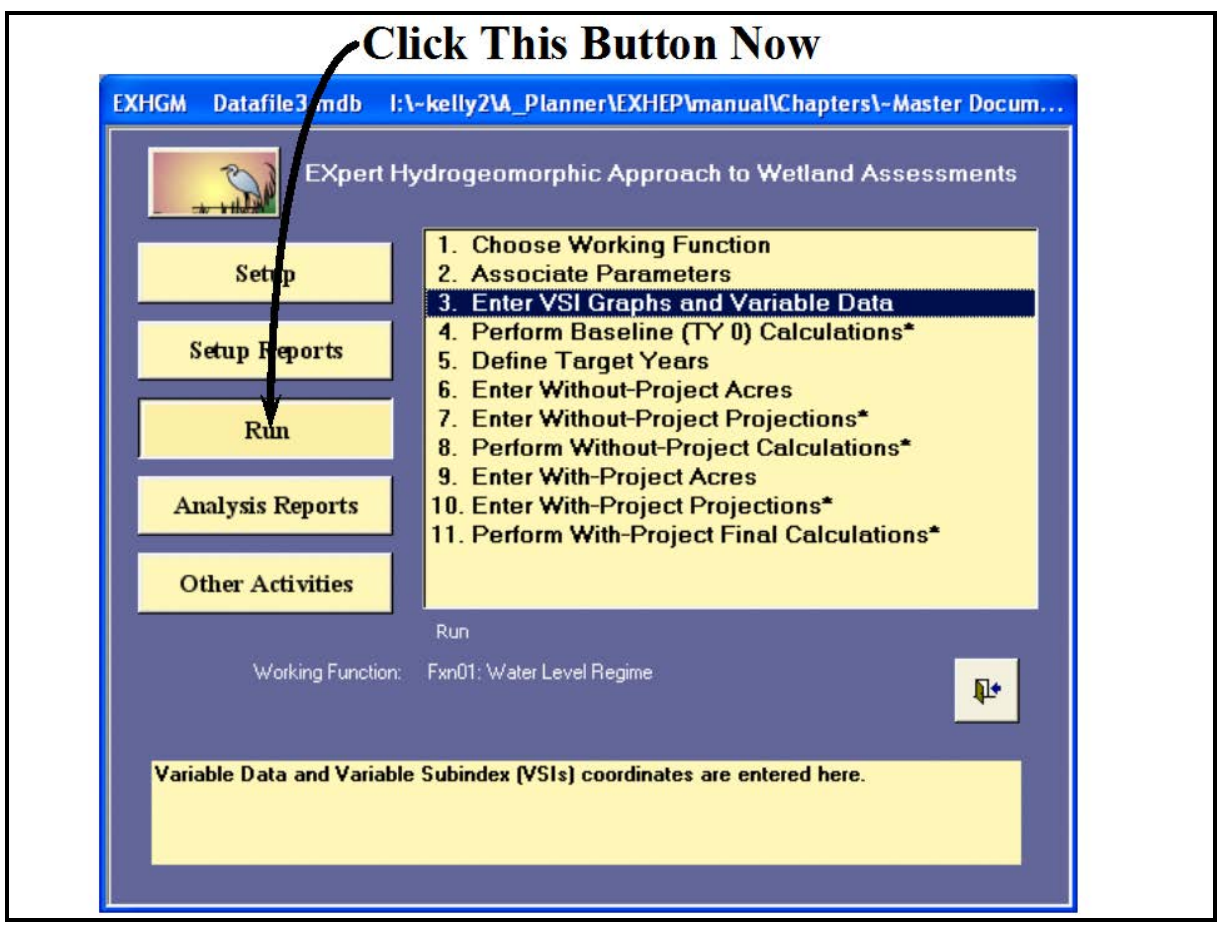

Figure 178. Click on the Run button to begin the baseline data entry process in EXHGM.

As it did before, a screen appears in the center of the primary interface with 11 options:

\section{Choose Working Function}

2. Associate Parameters

3. Enter VSI Graphs and Variable Data

4. Perform Baseline (TY 0) Calculations*

5. Define Target Years

\footnotetext{
${ }^{1}$ An example spreadsheet has been provided on the installation CD (Wet Pine Flats Field Kit).
} 


\section{Enter Without-Project Acres}

7. Enter Without-Project Projections*

8. Perform Without-Project Calculations*

9. Enter With-Project Acres

10. Enter With-Project Projections*

11. Perform With-Project Final Calculations*

Before continuing, assure that the correct "Working Function" has been selected (refer to Step 2: Model Association, Choose the Working Function above for assistance). Click on the third option (Enter VSI

Graphs and Variable Data) to continue. EXHGM will again open the Step 1: Select Partial Wetland Assessment Areas and Variable Combinations and Step 2: Enter Graph Coordinates and Variable Data forms. Select the first PWAA and variable combination and click on the " 0 " space of the Variable Data entry panel in the bottom window. Enter the field data (either manually or electronically) in the space provided. ${ }^{1}$

USER'S NOTE:

EXHGM will not accept negative data in the variable data entry screen. Refer to Chapter 7 of this User's Guide to review situations where this condition might arise, and helpful suggestions to address this issue.

\section{Manual data entry}

To enter data manually, type in the first value and press Enter on the keyboard (Figure 179).

\footnotetext{
1 In this first edition of the EXHGM software, it is assumed that each variable's index was developed in relation to the standard means of the data gathered. Thus all data are summed and averaged across the suite of data points collected, and the graph is plotted using direct linear configuration. If for any reason the data must be statistically analyzed in a different fashion (e.g., modes taken instead of means), perform this calculation outside the EXHGM software and provide the result(s) in the Variable Data entry panel.
} 


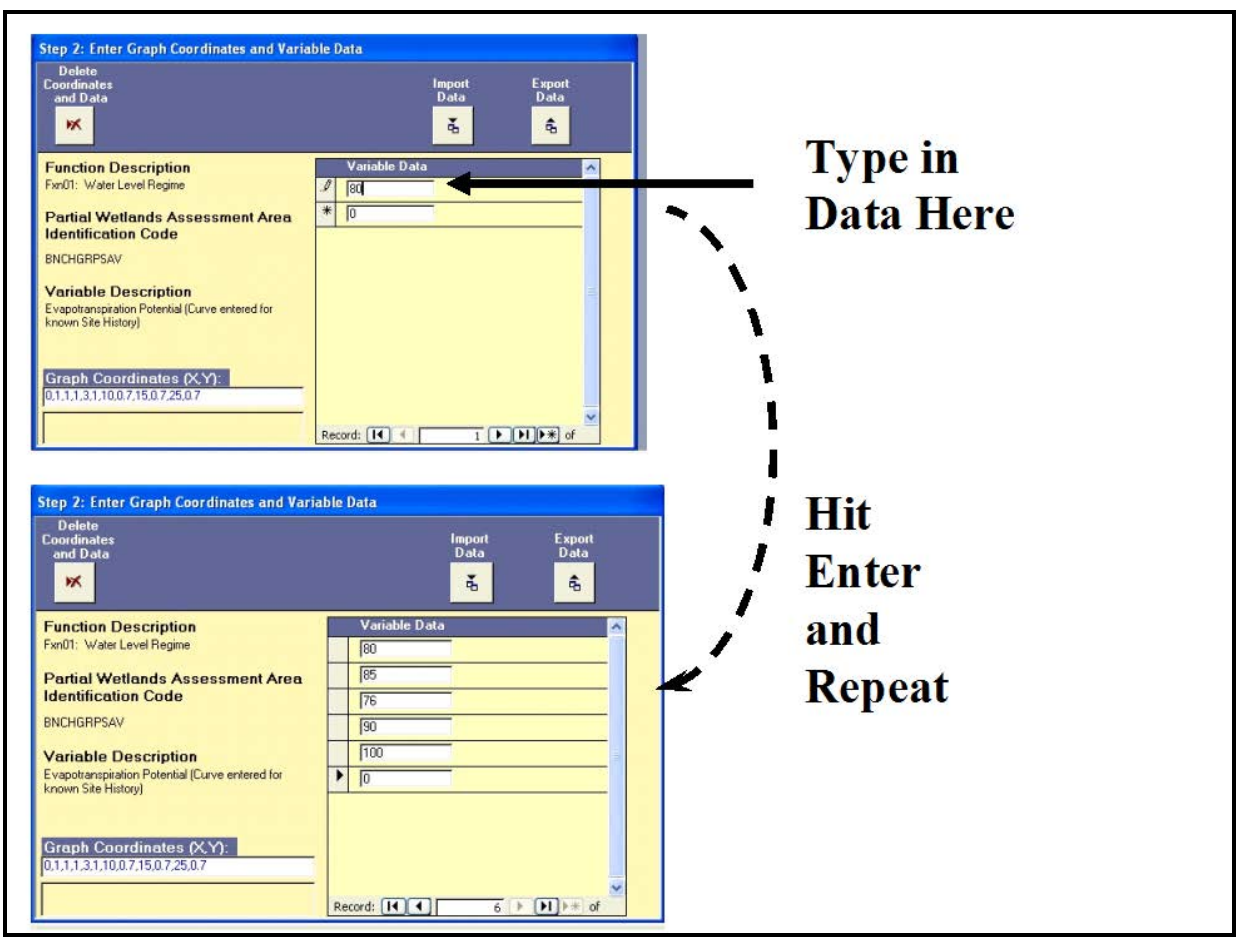

Figure 179. An example of the manual data entry process in EXHGM.

With this action, EXHGM will save the data point to the datafile and proceed to the next line. Repeat the process, entering all of the data for this variable and PWAA combination. Use the scroll bar on the right-hand side of this panel (or the arrow keys on the keyboard) to move up and down through the entries. Return to the upper form (Step 1: Select Partial Wetland Assessment Areas and Variable Combinations) and choose the next combination of variables and PWAA to continue the process. Repeat as often as necessary to fully populate the datafile.

\section{Electronic data entry}

If data are stored electronically in a spreadsheet, they can be imported into the datafile relatively easily. The data in the external spreadsheet must be organized in a variable per PWAA manner, and the data must be stored in columns. Now, import the data by following these steps:

1. Open the external spreadsheet.

2. Highlight a single column of data.

3. Press Ctrl C on the keyboard to copy the data to the clipboard.

4. Return to the EXHGM software (press ALT + Tab on the keyboard until the Microsoft Access icon appears in the viewer 
or click on the Microsoft Access icon on the taskbar at the bottom of the screen).

5. Click the Import Data button above the Variable Data entry panel (Figure 180).

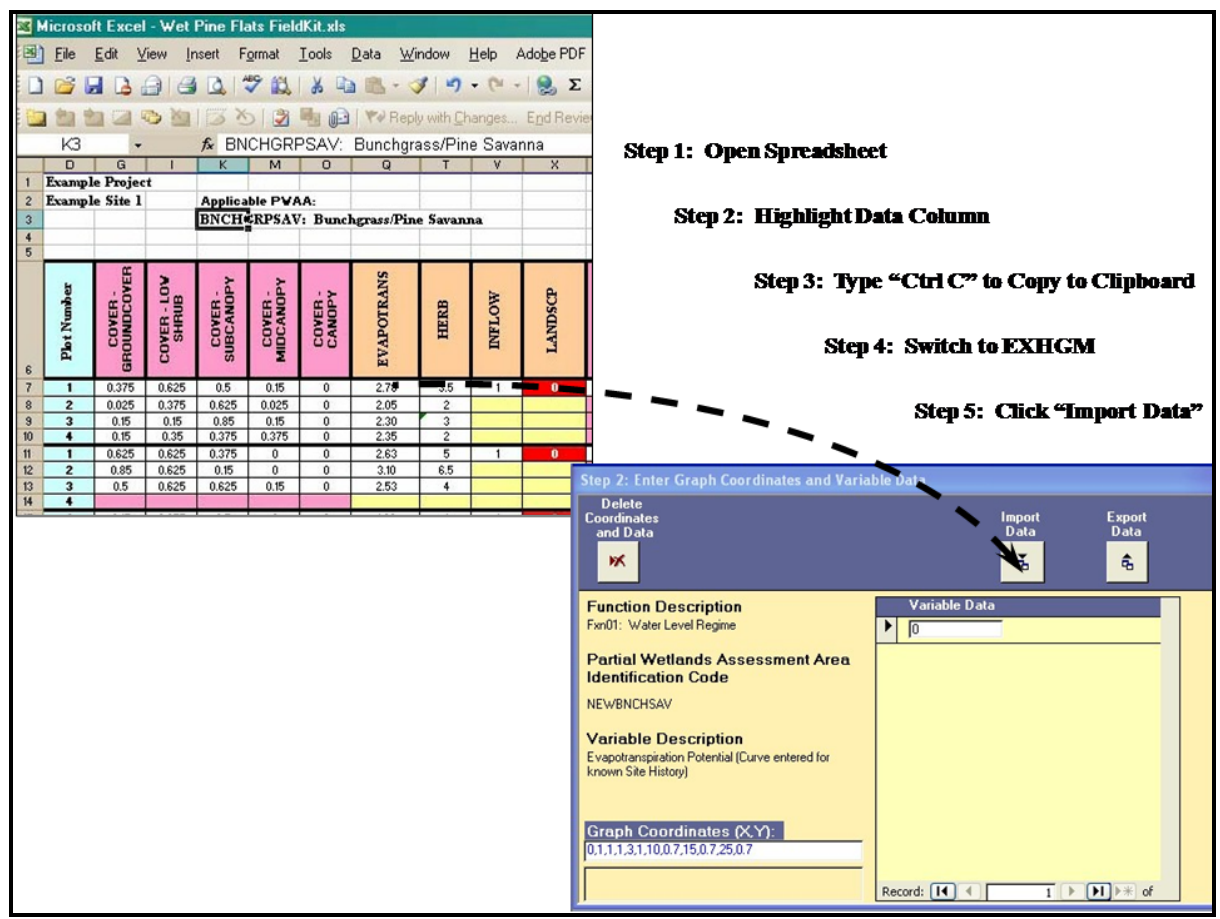

Figure 180. Importing data from an external spreadsheet into EXHGM.

6. Click on the Import Data button in the lower left-hand corner of the Field Data Import Process screen to continue.

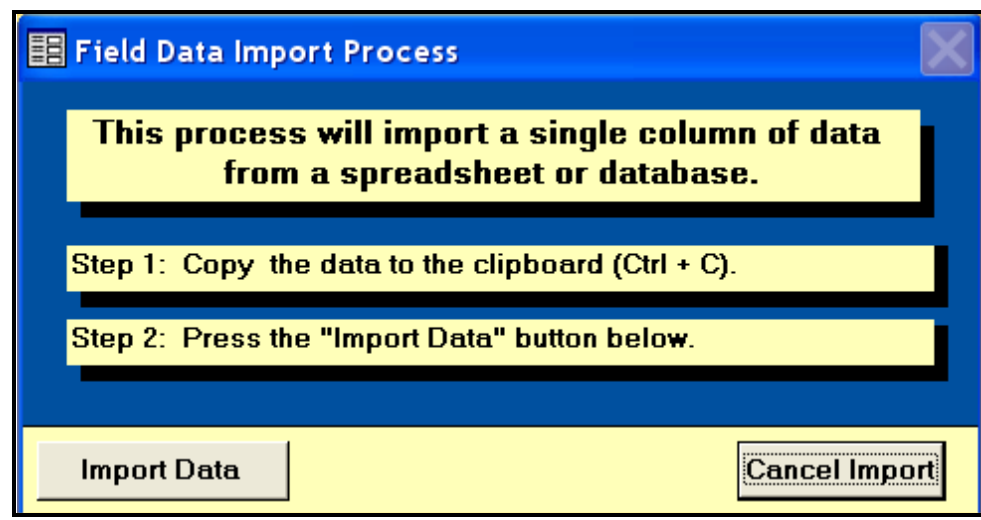


EXHGM pastes each cell from the copied dataset into the Variable Data entry panel. The Cancel Import button (lower right-hand corner) will halt the importation process and return to the Variable Data

Field Data Import Process $X$

Import successful!

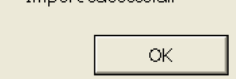
entry panel. When the importation process has finished, EXHGM provides a message indicating that the import action was completed success-

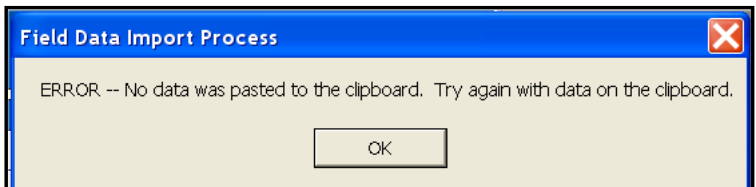
fully. Press OK to proceed. If the process failed, EXHGM will display an error message. To correct the error, empty the clipboard and try copying the data once again. Repeat the process, entering all of the data for the variable-PWAA combinations in the model.

\section{Exporting data to MS Excel}

Whether entering the data manually or electronically, every variable per PWAA in each function must be entered into the software systematically. Often, variables are shared across functions or even across PWAAs. Rather than re-entering the same data multiple times, a process has been devised to save this information in a compatible spreadsheet file for importation via the protocol described above. To do this, follow these quick steps:

1. Manually enter the data for the variable into the software following the directions above.

2. Save the data to an MS Excel file by clicking the Export Data button above the Variable Data entry panel (Figure 181).

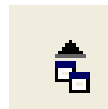

3. Select the location to save the datafile in via the Save in: window, provide a new name (only) for the datafile in the File name: window, and press Save $^{1}$ (Figure 182).

\footnotetext{
1 It is not necessary to provide an extension for the datafile in the File name: - MS Access pre-determines the extension and assigns an *.xls to the file name provided.
} 


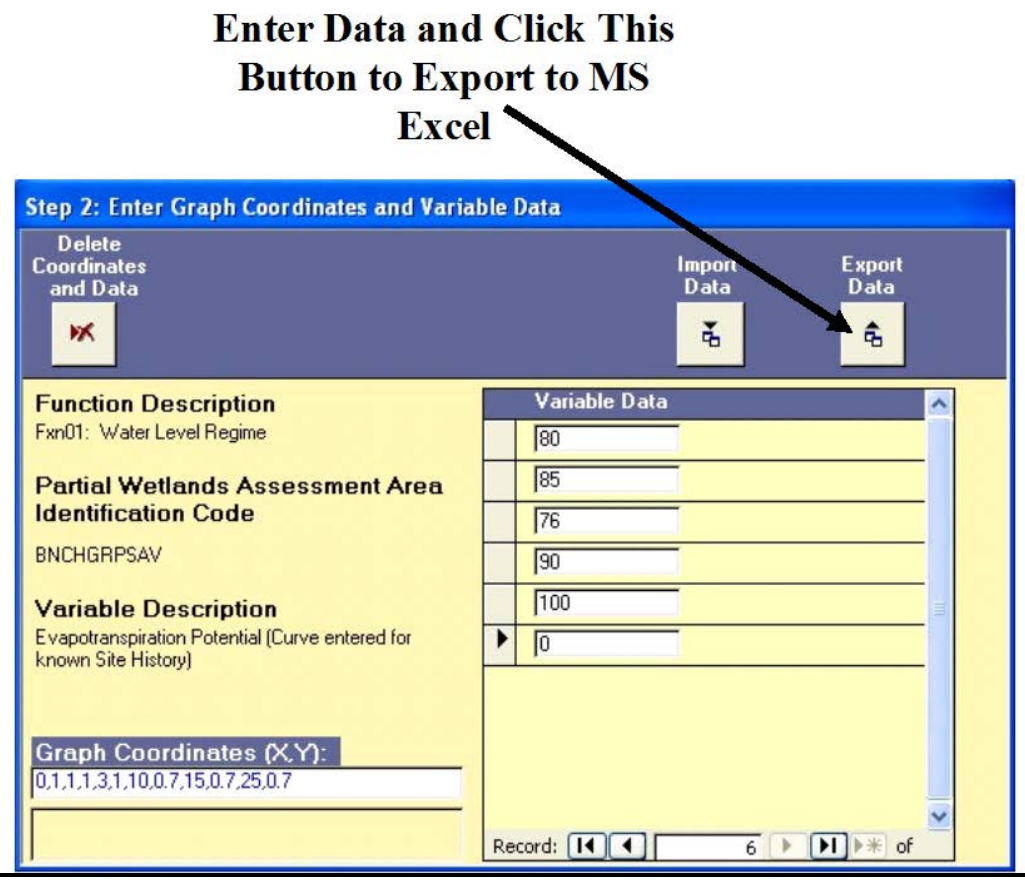

Figure 181. Exporting data from EXHGM to MS Excel - click the Export Data button.

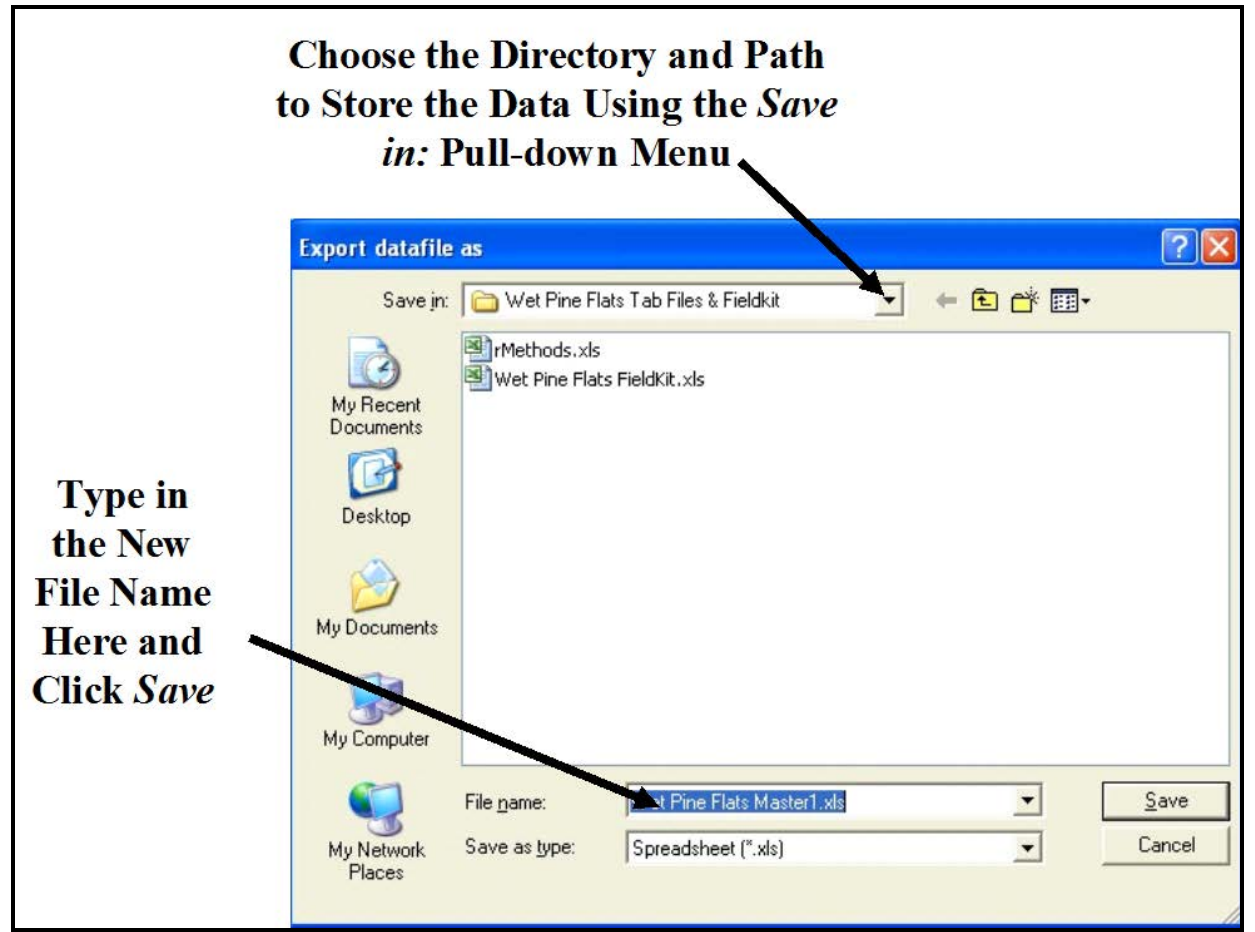

Figure 182. Exporting data from EXHGM to MS Excel - assign a directory and path for the new file. 
If EXHGM was able to export the data to MS Excel, a confirmation message is displayed. Click OK to close the message. EXHGM will return to the entry screens when finished. Select the next variable and PWAA combination, and Microsoft Access Docs\Summary Spreadsheets Marsh Wren Tab Files \& Fieldkitłpatafile51.xls repeat the process until OK all the field data have been entered, exported, and saved.

If the need should arise, delete an individual data point by clicking on the Record Indicator Bar to the immediate left of the data point and pressing Delete on the keyboard (Figure 183).

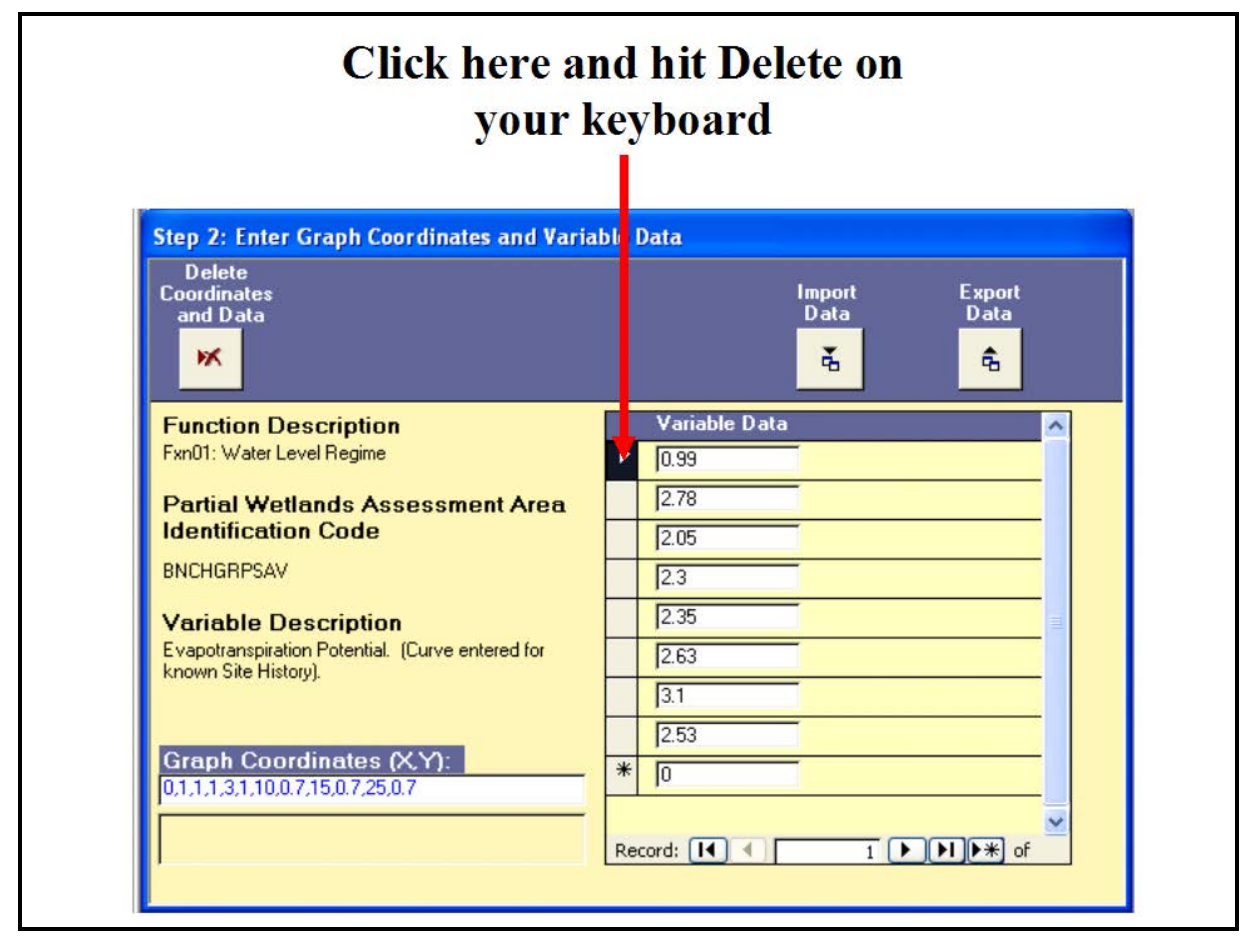

Figure 183. Deleting single data points in EXHGM.

To delete the entire data set, (if for example, a mistake was made and data were entered against the wrong variable or PWAA), press the Delete Coordinates and Data button in the upper left-hand corner of the Step 2: Enter Graph Coordinates and Variable Data screen. 
Before deleting the entire data set and VSI coordinates, EXHGM explains the implications of this action and provides an avenue to cancel the action

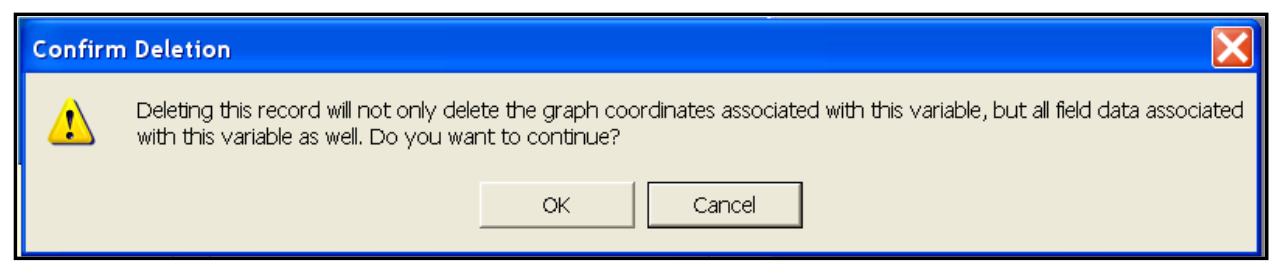

or proceed. Click on OK to proceed with the deletion or Cancel to stop the deletion process. Once deleted, the information cannot be recovered from the EXHGM datafile, and will need to be re-entered. When the data have been removed from the database, a status message appears. Click on OK to proceed with the data entry process.

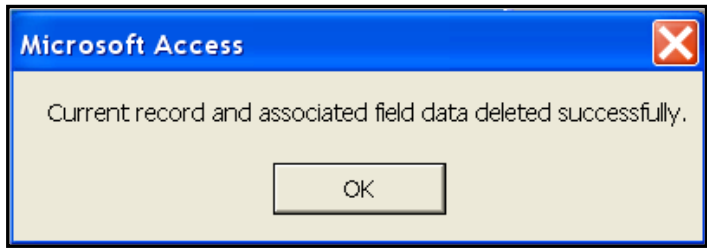

\section{Checking the baseline data entry with EXHGM reports}

To review and check the entries made thus far, click on Setup Reports on the primary interface (Figure 184).

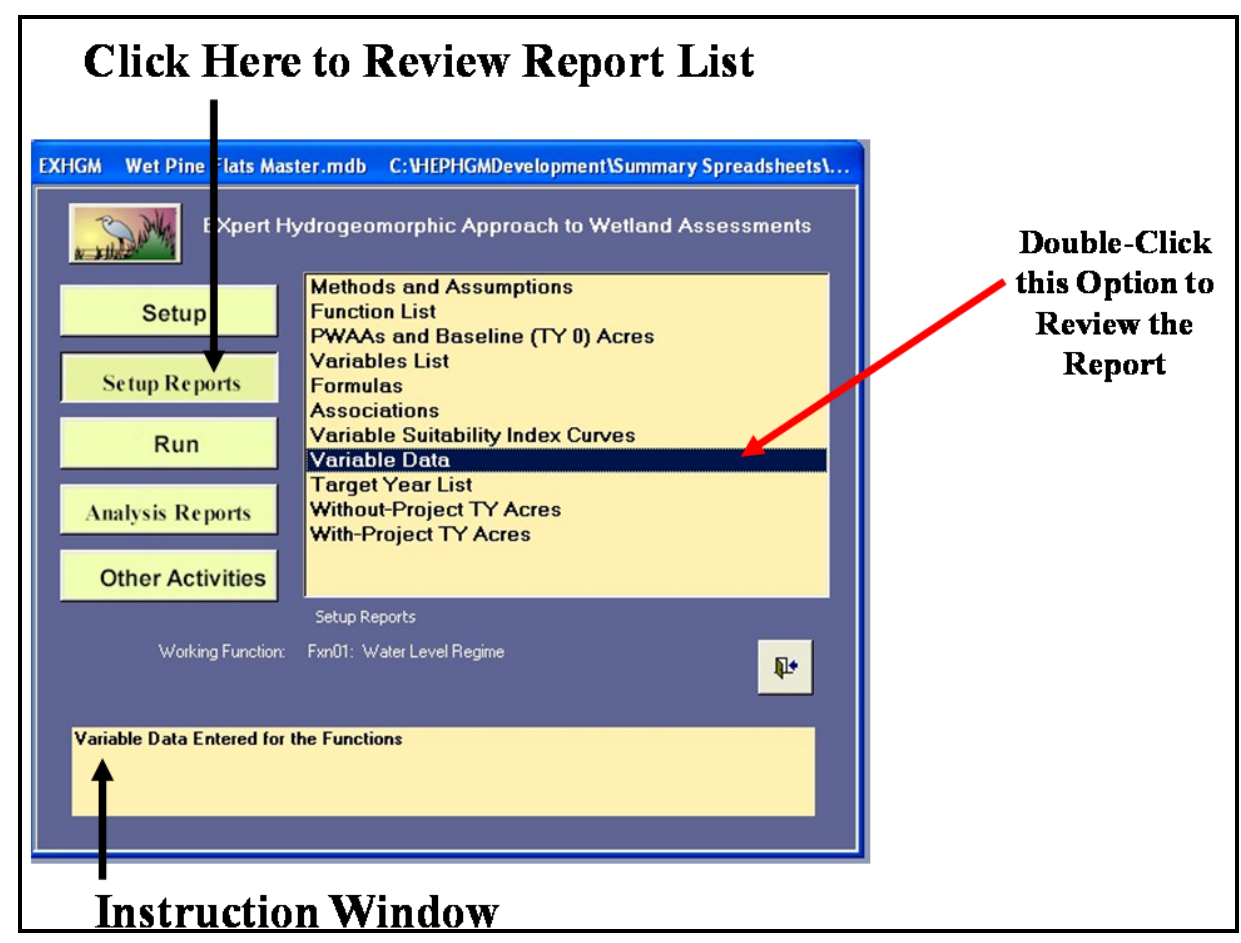

Figure 184. Click on the Setup Reports button to view the variable data reports in EXHGM. 
A list of available reports appears in the center of the primary interface. The Variable Data report is now ready for review. Single-click on the report and look to the Instruction Window to preview the contents of the report. Double-click on the report, and EXHGM opens a new window with the report inside (Figure 185).

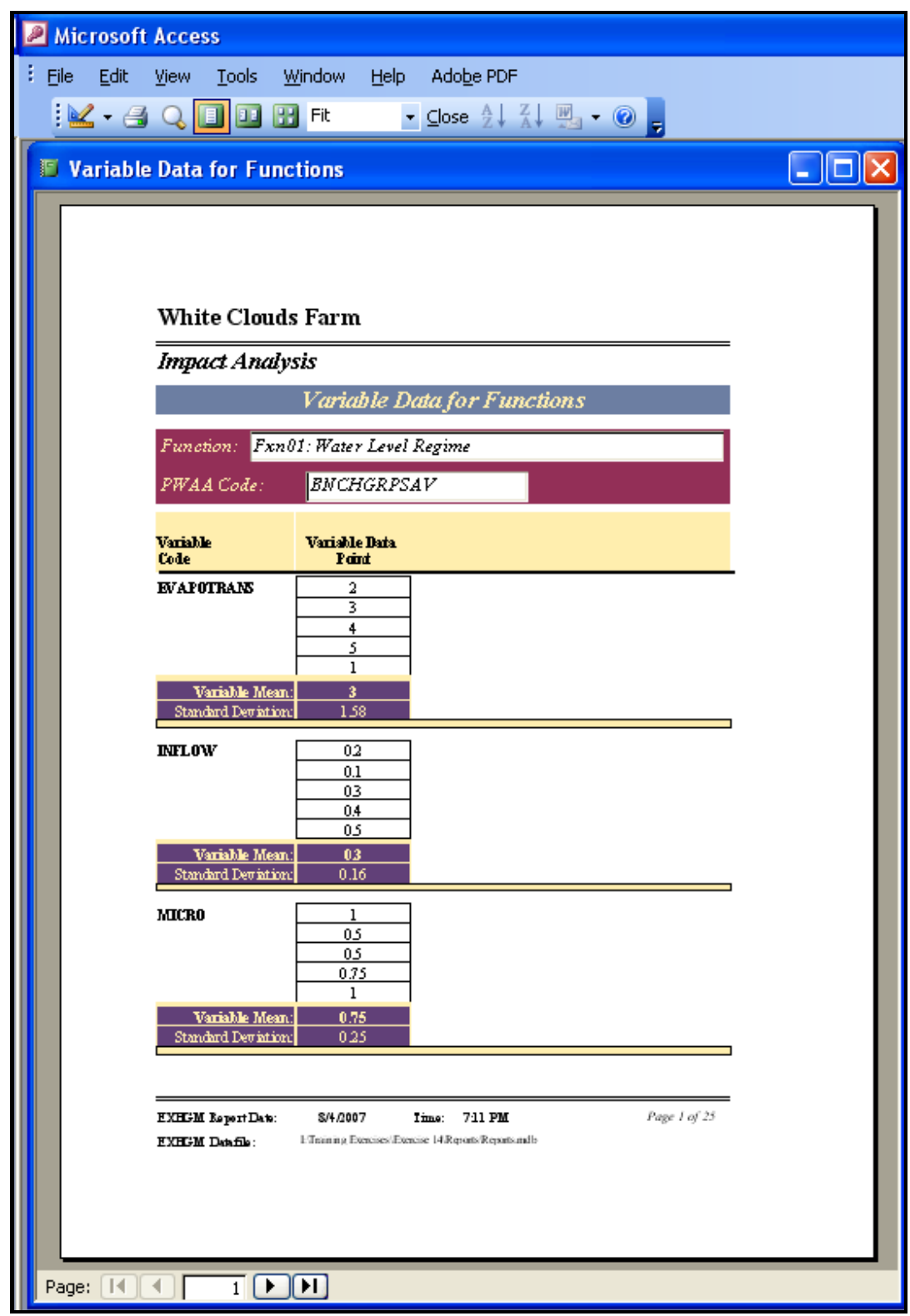

Figure 185. Example of Variable Data for Functions report in EXHGM.

Click on the Close button from the suite of buttons offered on the Report Control toolbar, and EXHGM returns to the primary interface. Now that the data entry process is complete, and the information has been 
confirmed, proceed to the next section to learn how to perform the baseline analyses in EXHGM.

\section{Perform baseline (TY 0) calculation}

EXHGM will calculate the values on a function-by-function basis. To proceed, click on the Run button on the primary interface (Figure 186).

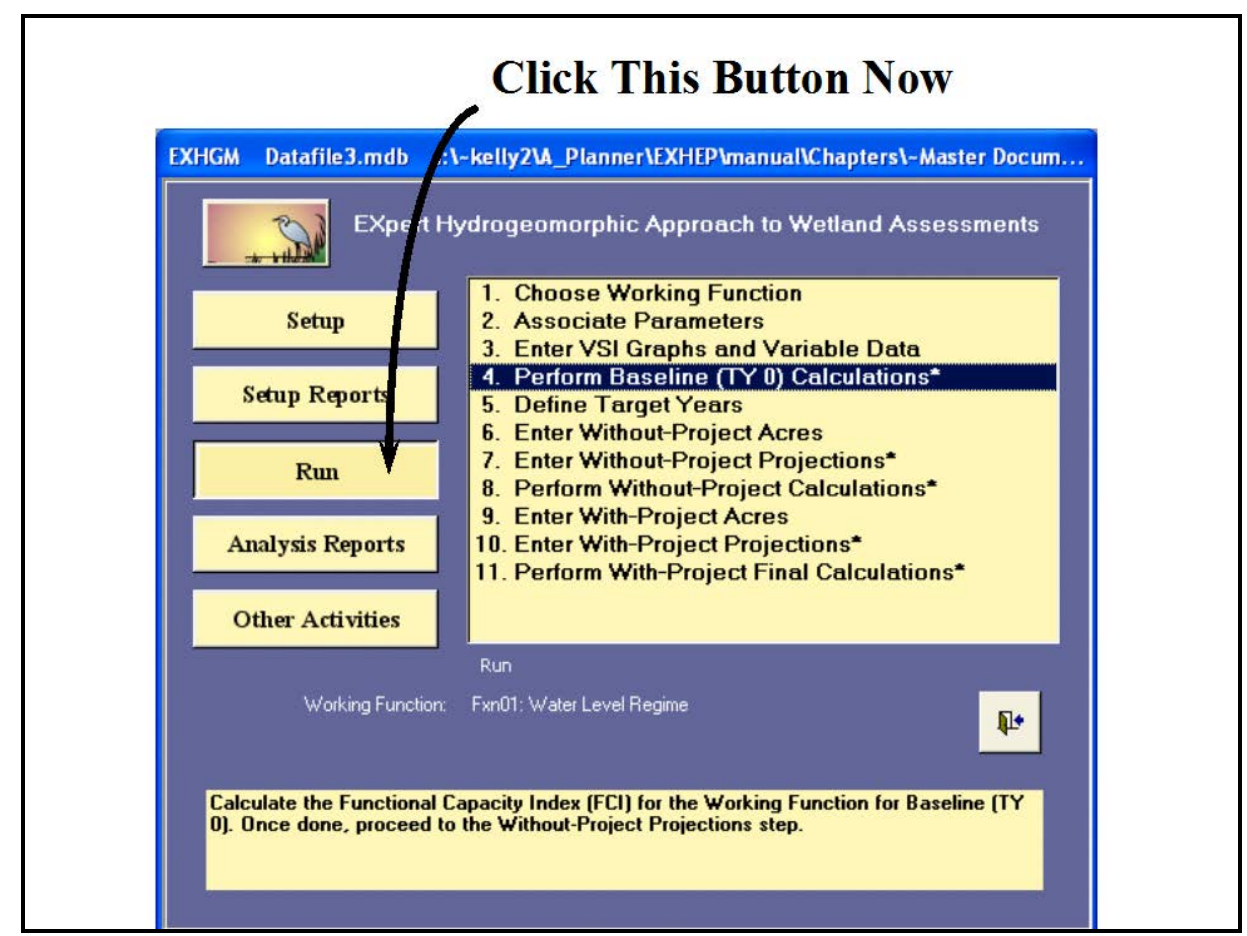

Figure 186. Click on the Run button to begin the baseline calculation process in EXHGM.

The screen appears in the center of the primary interface with 11 options:

\section{Choose Working Function}

2. Associate Parameters

3. Enter VSI Graphs and Variable Data

4. Perform Baseline (TY 0) Calculations*

5. Define Target Years

6. Enter Without-Project Acres

7. Enter Without-Project Projections*

8. Perform Without-Project Calculations*

9. Enter With-Project Acres

10. Enter With-Project Projections*

11. Perform With-Project Final Calculations* 
Check to assure that the "Working Function" is set to the correct function, and click on the fourth option [Perform Baseline (TY 0) Calculations*] to continue. In response, EXHGM will begin the calculation process. As the calculations continue, a Progress

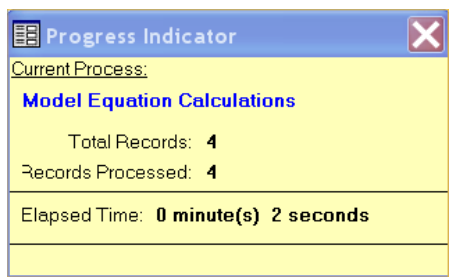

Indicator window will open in the upper right-hand corner of the screen. This progress window will track the status of the analysis (number of records to assess versus number of records processed) and provide a tentative estimate of Elapsed Time. When

\section{Baseline(TYO) Calculation Status} Baseline(Tro) Calculations completed successfully OK completed, EXHGM returns a message that the calculations are concluded.

\section{Checking the baseline analysis with EXHGM reports}

To review and check the results, click on the Analysis Reports button on the primary interface (Figure 187).

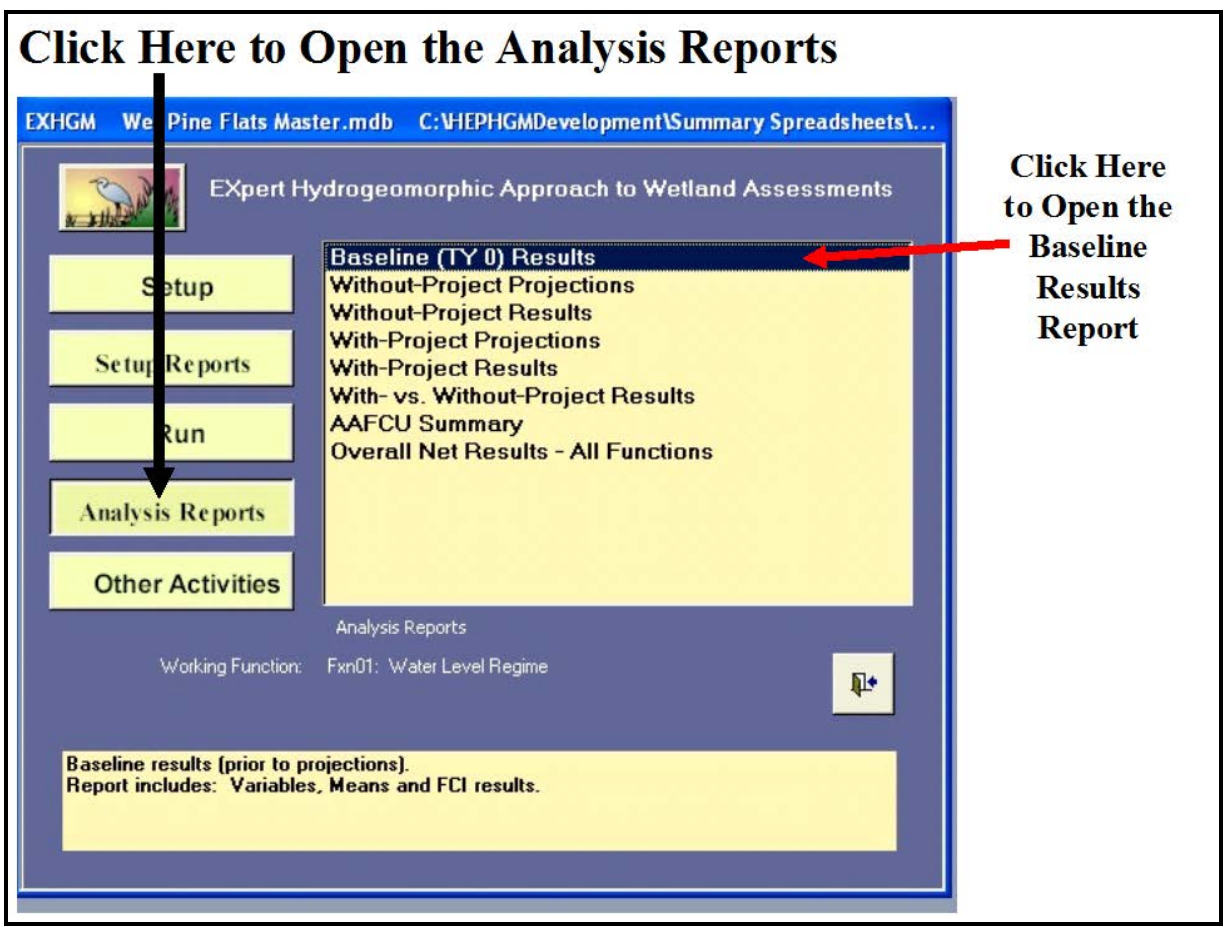

Figure 187. Click on the Analysis Reports button to review the baseline results in EXHGM. 
A list of available reports appears in the center of the primary interface. The first report listed, Baseline (TY0) Results, is ready for inspection. Double-click on this report to review the results (Figure 188).

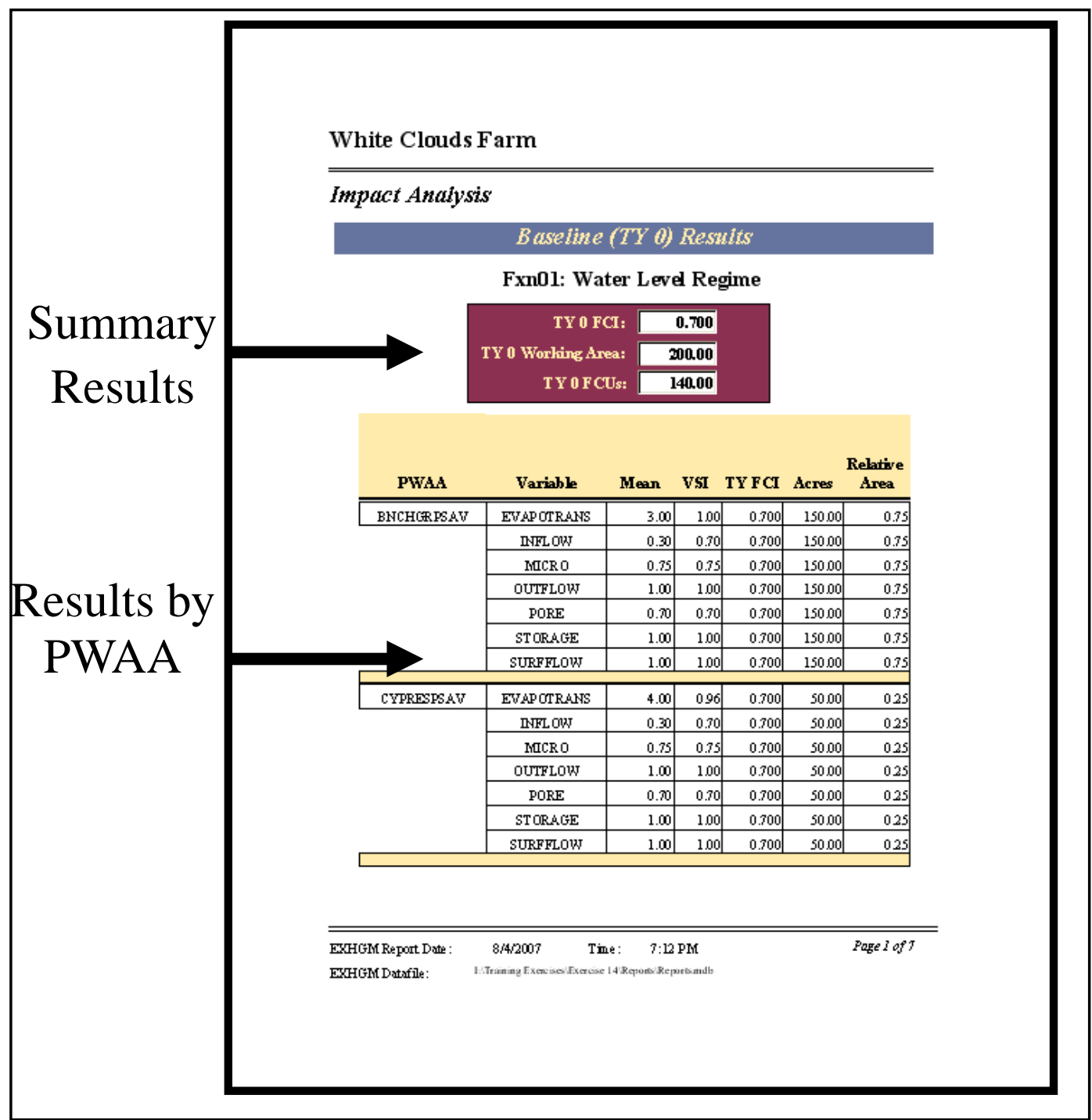

Figure 188. Example of Baseline ( $T Y O$ ) Results report in EXHGM.

The site's overall FCI score (i.e., TY 0 FCI), associated acres (i.e., TY 0 Working Area), and the resulting FCU score (i.e., TY 0 FCUs derived from the multiplication of the FCI score and the associated acres) are reported under the function name. The specifics used to generate the overall scores are reported in the tables below this summary. These details are sorted alphabetically by PWAA and variable. This report is cumulative; thus, all functions assessed in the EXHGM analyses are presented in this report. 
Click on the Close button from the suite of buttons offered in the Report Control toolbar, and EXHGM returns to the primary interface. Now that the baseline assessment is complete, proceed to the next step to learn how to assess the without-project conditions of the site in EXHGM.

\section{Step 5: Enter the without-project conditions and calculate the effects}

Often confused with the terms "Baseline Condition" or "Existing Condition," the Without-Project Condition (WOP) is the forecasted change in site conditions over the life of the project when no action is taken to counteract or change the current situation, and is also referred to as the "No Action Alternative" or the "No Action Plan" in traditional planning studies. In order to capture the WOP conditions in EXHGM, significant points in time (i.e., target years) must be defined to compartmentalize and focus the evaluation on critical temporal dimensions across the life of the project. These "target years" (TYs) then become the focus of the forecasting effort - predictions of site conditions are scaled to these snapshots in time. In HGM there can be no less than three TYs, and they are by definition:

- $\mathrm{TY}_{0}$ - baseline

- $\mathrm{TY}_{1}$ - first year of construction/ restoration

- $\mathrm{TY}_{\mathrm{X}}$ - last year of project life or evaluation period

Between $\mathrm{TY}_{1}$ and $\mathrm{TY}_{\mathrm{X}}$, it is not uncommon for the team to add additional TYs to capture changes in vegetative cover and structure or other parameters resulting from anticipated impacts or proposed designs. ${ }^{1}$

To quantify the projected change to the site if no action is taken, both without-project acreage and wetland quality must be documented and incorporated into the evaluation. "No Action" might imply that the site will not change over time - this is not necessarily the case. Often, vegetation matures and succeeds over time. Or artificial constructs (e.g., dams, levees, ditches, etc.) degrade the wetland over time. The timing, nature,

1 The authors wish to offer a precautionary note - the selection of too few TYs can result in a coarse analysis insensitive to key ecological/physical changes in the environment. The selection of too many TYs can lead to excessive data handling, often with no return on investment. Five TYs spread over the course of 50 years offers a reasonable expectation of return for the level of data analysis and management required. 
and magnitude of these changes must be quantified before the team can devise an alternative future for the site. ${ }^{1}$

\section{Without-project forecasting and data entry}

In EXHGM, the projected without-project changes to the site are undertaken first by defining target years and forecasting alterations of aerial extent given this timeframe. These changes in space and time are then coupled with changes in physical and biological conditions along this space-time continuum to characterize the quality and magnitude of change for the site under the without-project paradigm.

Enter, change, or delete the target years

To proceed, first click on the Run button on the primary interface (Figure 189).

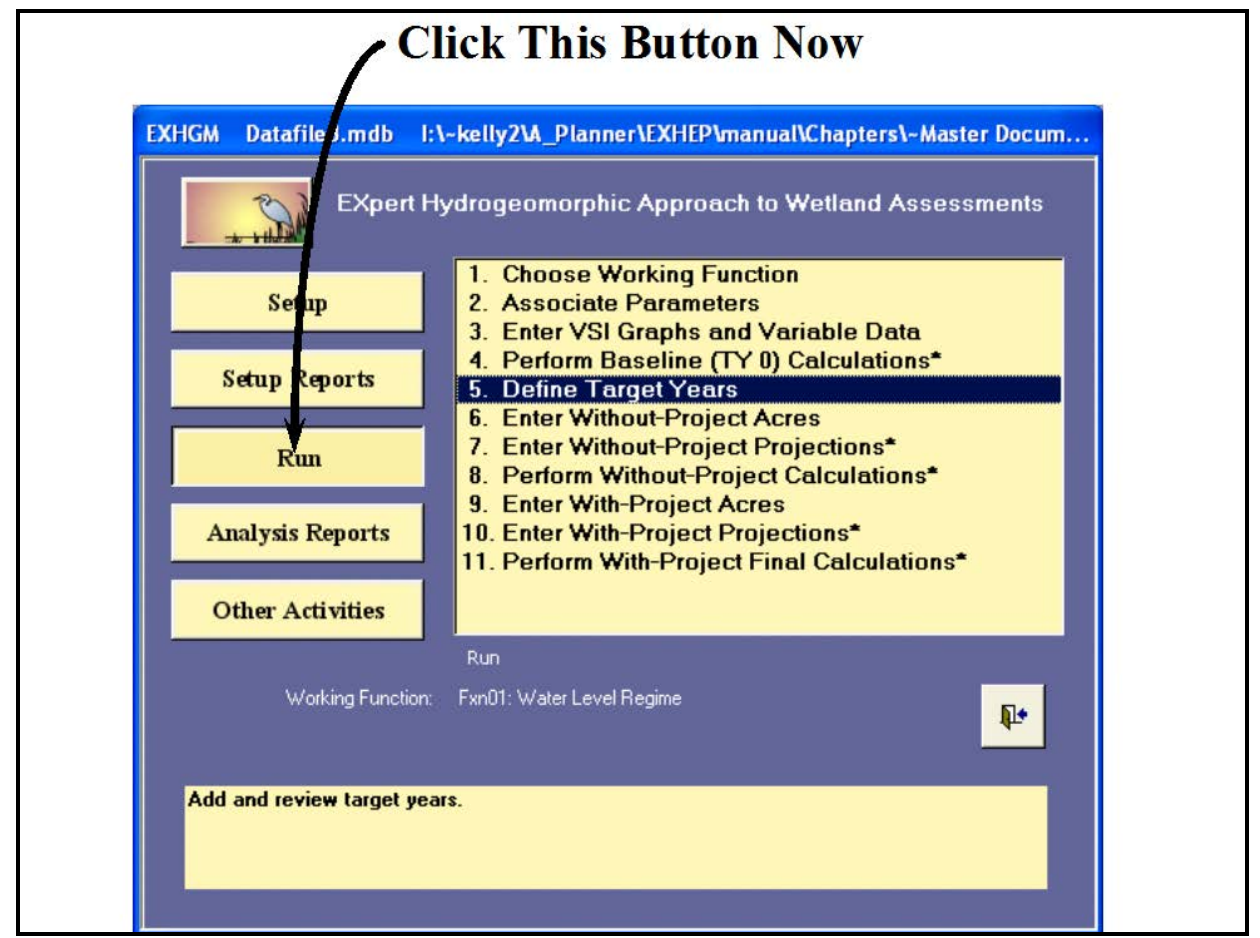

Figure 189. Click on the Run button to begin the target year entry process in EXHGM.

As it did before, a screen appears in the center of the primary interface with 11 options:

\footnotetext{
1 To assist in the software application, a series of training exercises have been included in Appendix E refer to Exercise 14 for relevant training in without-project data entry and analysis in EXHGM.
} 
1. Choose Working Function

2. Associate Parameters

3. Enter VSI Graphs and Variable Data

4. Perform Baseline (TY 0) Calculations*

5. Define Target Years

6. Enter Without-Project Acres

7. Enter Without-Project Projections*

8. Perform Without-Project Calculations*

9. Enter With-Project Acres

10. Enter With-Project Projections*

11. Perform With-Project Final Calculations*

Check to assure that the "Working Function" is set to the correct function, and click on the fifth option (Define Target Years) to continue. In response, EXHGM opens the following window (Figure 190).

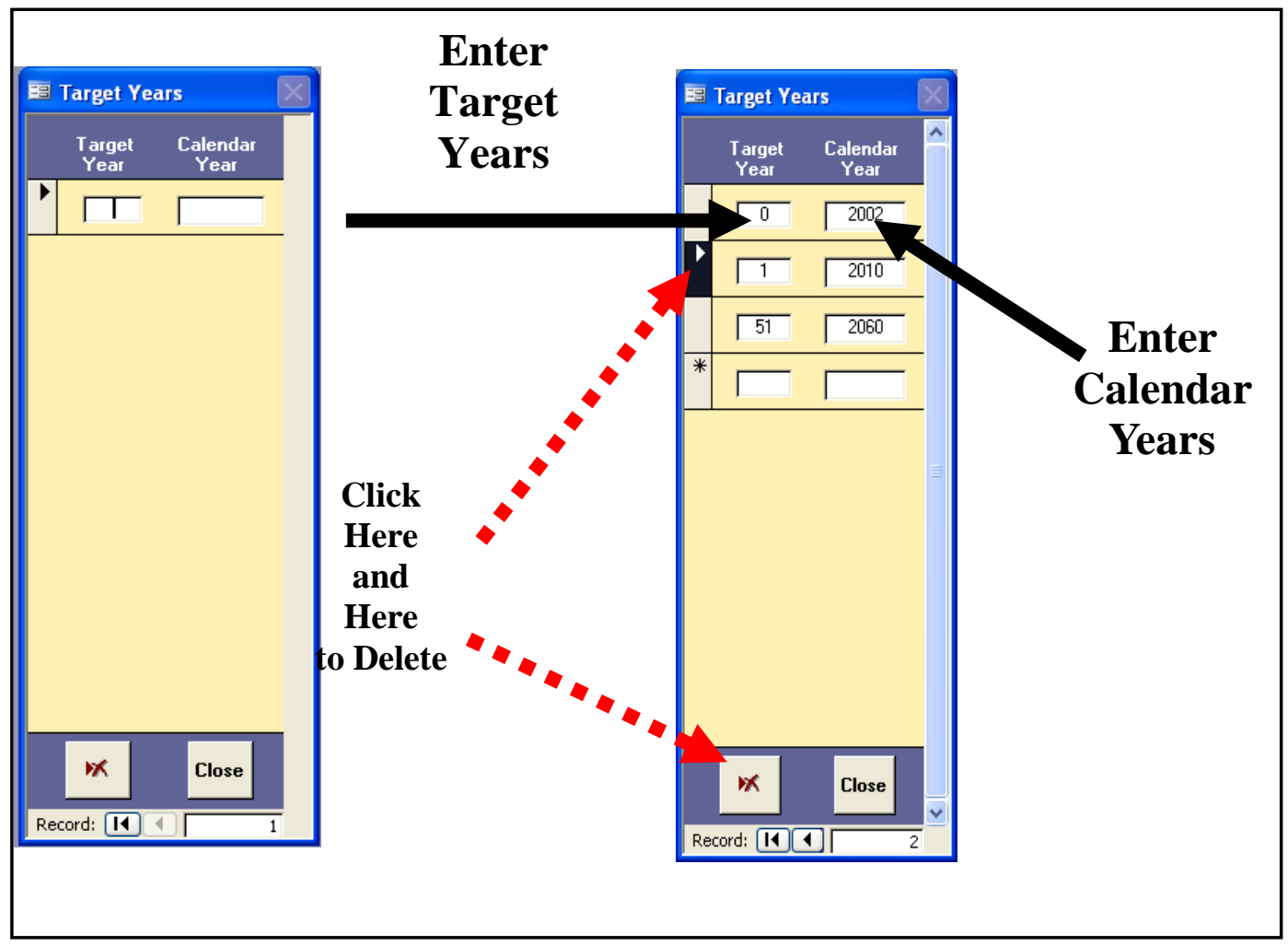

Figure 190. Define the target years in EXHGM.

The cursor is automatically placed in the first entry space in the left-hand column (Target Years). Type in the first target year (i.e., this should be "0") and hit the
USER'S NOTE:

The EXHGM software uses the TYs to calculate annualized outputs - not the calendar years. 
Tab button on the keyboard to move to the right-hand column. Now enter the corresponding calendar year in this column. Hit Enter on the keyboard to accept the entry. Repeat the process for each set of TYs and calendar years. ${ }^{1}$

If for some reason a TY must be modified, it must be completely erased from the database and re-entered. To do this, click once on the Record Indicator Bar (i.e., the square to the immediate left of the TY) and press the Delete button at the bottom of the form (left-hand corner). A warning message is displayed asking to continue or cancel the action.

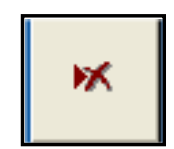

\section{Microsoft Office Access}

This will delete all data related to this target year from the database. Do you wish to continue?

$$
\text { OK }
$$
Cancel

The removal of a TY is a very serious undertaking in EXHGM - one click of the OK key removes every record associated with the TY database-wide. Click OK to continue, or Cancel to halt the process. Either way, the software returns to the Target Year Description entry screen, where the TY correction can be entered and/ or the data entry can be completed.

When finished, click on the Close button. EXHGM offers a reminder to enter future conditions into the system to support the TY identification,

You have added target years - be sure to forecast future conditions (variables and acres) for each Target Year.

so click the $\mathbf{O K}$ button and proceed to the next section (i.e., Enter and/ or modify without project acres) to focus on the without-project forecasting task. But first, it is important to note that the TYs assigned to the "Working Function" apply to all functions in the software. Therefore it is

\footnotetext{
1 As is evidenced in the examples above, a period of construction activity is often needed to implement the designs, and the sites are not ready for assessment for several calendar years (i.e., 8 years in the example). In HGM, it is as if those years do not count - they are condensed between $\mathrm{TY}_{0}$ and $\mathrm{TY}_{1}$. The period of analysis pertinent to HGM begins once construction ends, and continues until the end of the project life (i.e., oftentimes 50 years later). Thus it is not unusual to find TYs and calendar years "outof-sync" so to speak.
} 
only necessary to enter the TYs once - they will be carried into the analysis for the remaining functions.

Enter, change, or delete the without-project acres

Now click on the Run button on the primary interface (Figure 191).

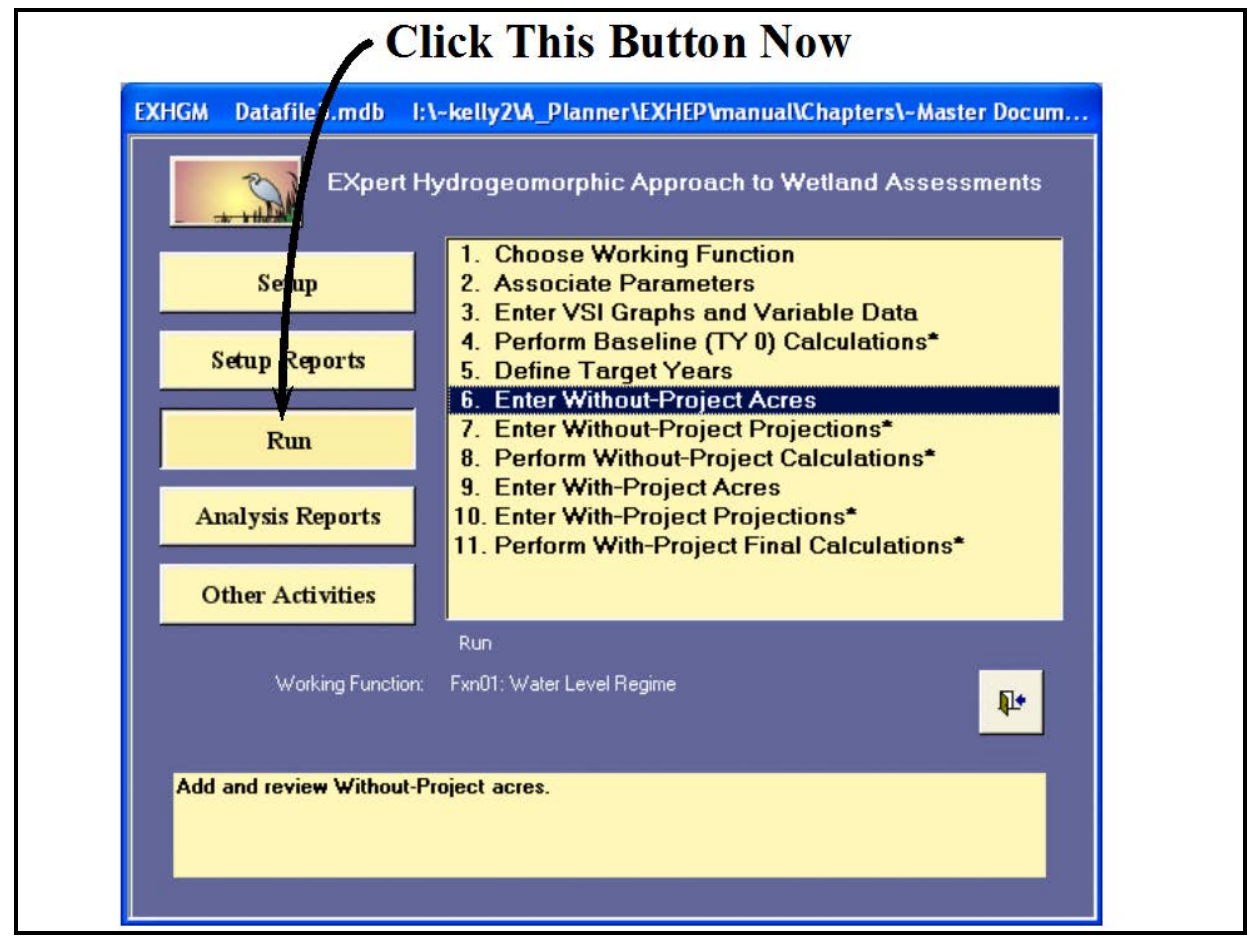

Figure 191. Click on the Run button to begin the without-project acres entry process in EXHGM.

As it did before, a screen appears in the center of the primary interface with 11 options:

\section{Choose Working Function}

2. Associate Parameters

3. Enter VSI Graphs and Variable Data

4. Perform Baseline (TY 0) Calculations*

5. Define Target Years

6. Enter Without-Project Acres

7. Enter Without-Project Projections*

8. Perform Without-Project Calculations*

9. Enter With-Project Acres

10. Enter With-Project Projections*

11. Perform With-Project Final Calculations* 
Check to assure that the "Working Function" is set to the correct function, and click on the sixth option (Enter Without-Project Acres) to continue. In response, EXHGM opens the following window (Figure 192).

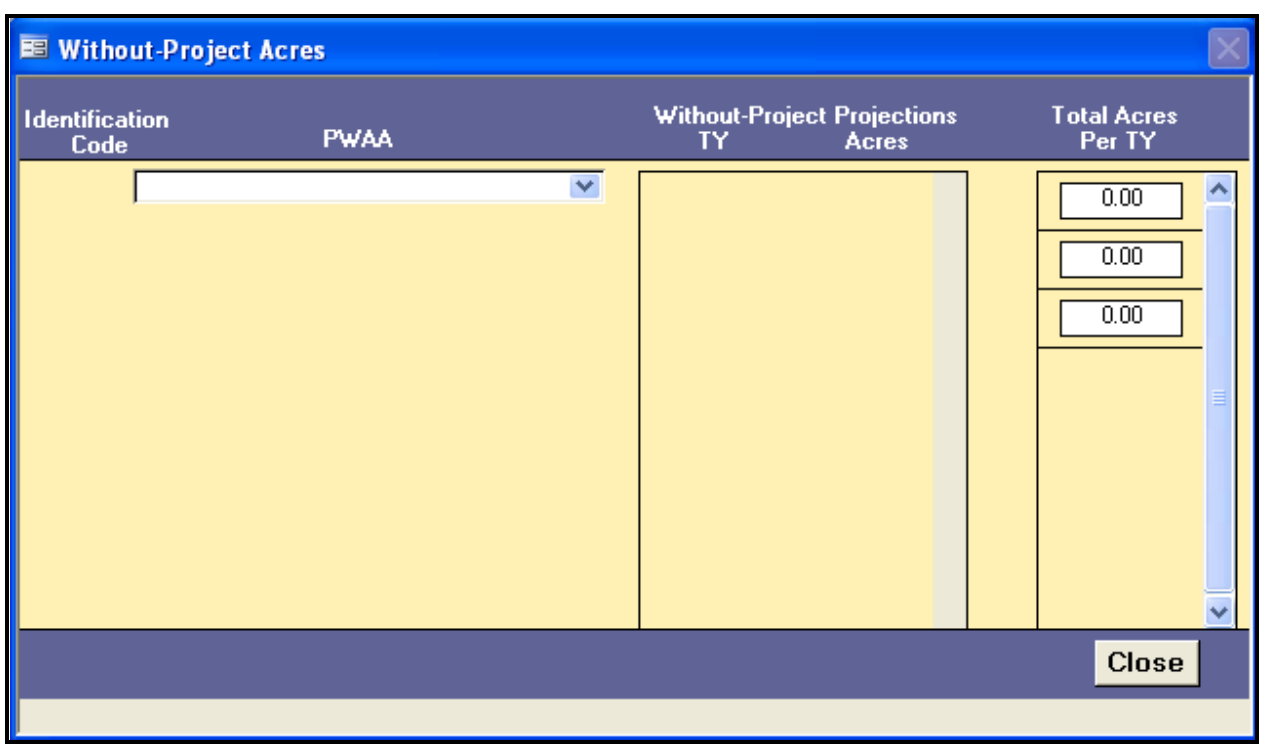

Figure 192. Without-Project Acres entry window in EXHGM.

Click once on the PWAA pull-down menu and select the first PWAA from the list of available wetland types. EXHGM queries the database for target years and baseline acres (based on the entries made in the steps above), and presents these in a tabular format in anticipation of projected future without-project conditions (Figure 193).

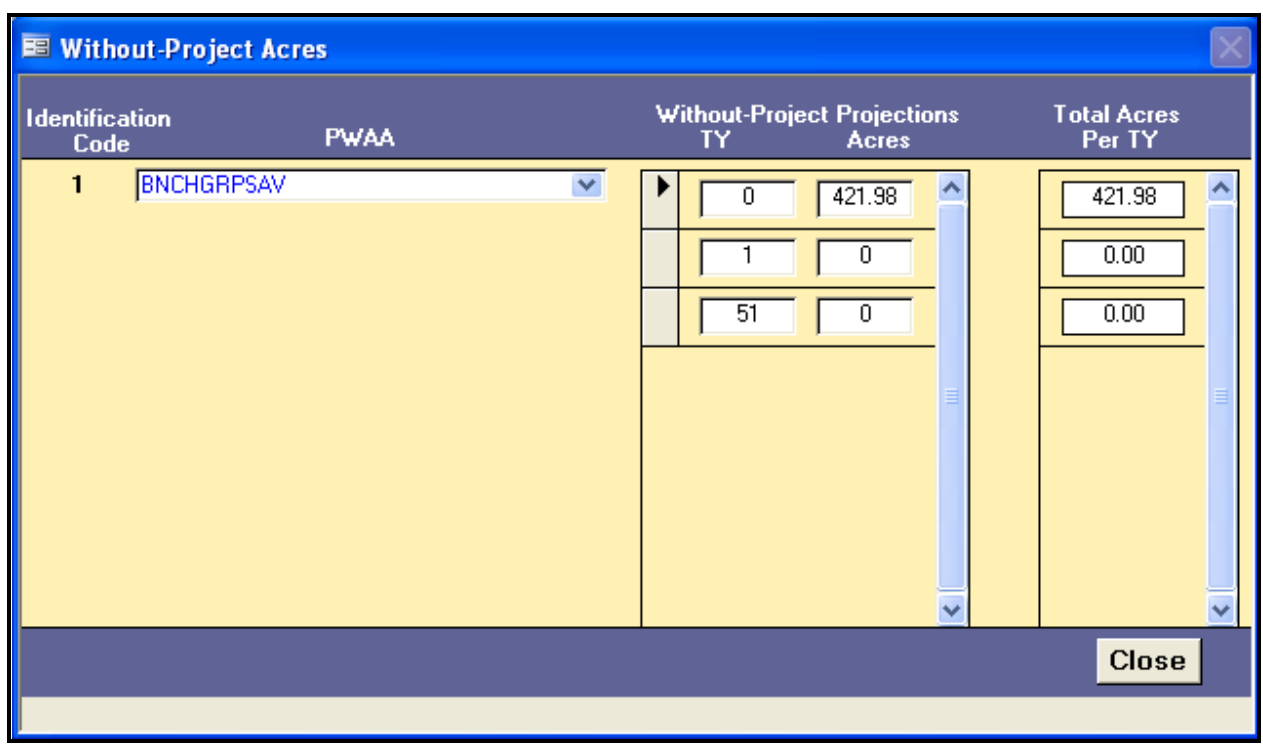

Figure 193. Select a PWAA from the pull-down menu, and EXHGM populates the entry form. 
Note that target years themselves cannot be modified in this interface. To make a target year change, return to Option 5. Define Target Years and follow the directions above to modify these values. It is also important to note that the system's query automatically populated the acreages in the rows of this table. Modifications to the baseline $\left(\mathbf{T Y}_{\mathbf{0}}\right)$ acreages cannot be made in this interface. Return to the setup interfaces to make these acreage changes (refer to Step 2: Model Setup section above for details). Zeroes are used as defaults in all outlying years for the acreages of this wetland.

Now forecast and enter the number of without-project (WOP) acres for this wetland in the cells to the right of the corresponding target years by highlighting and typing in the correct acres for that time frame (Figure 194).

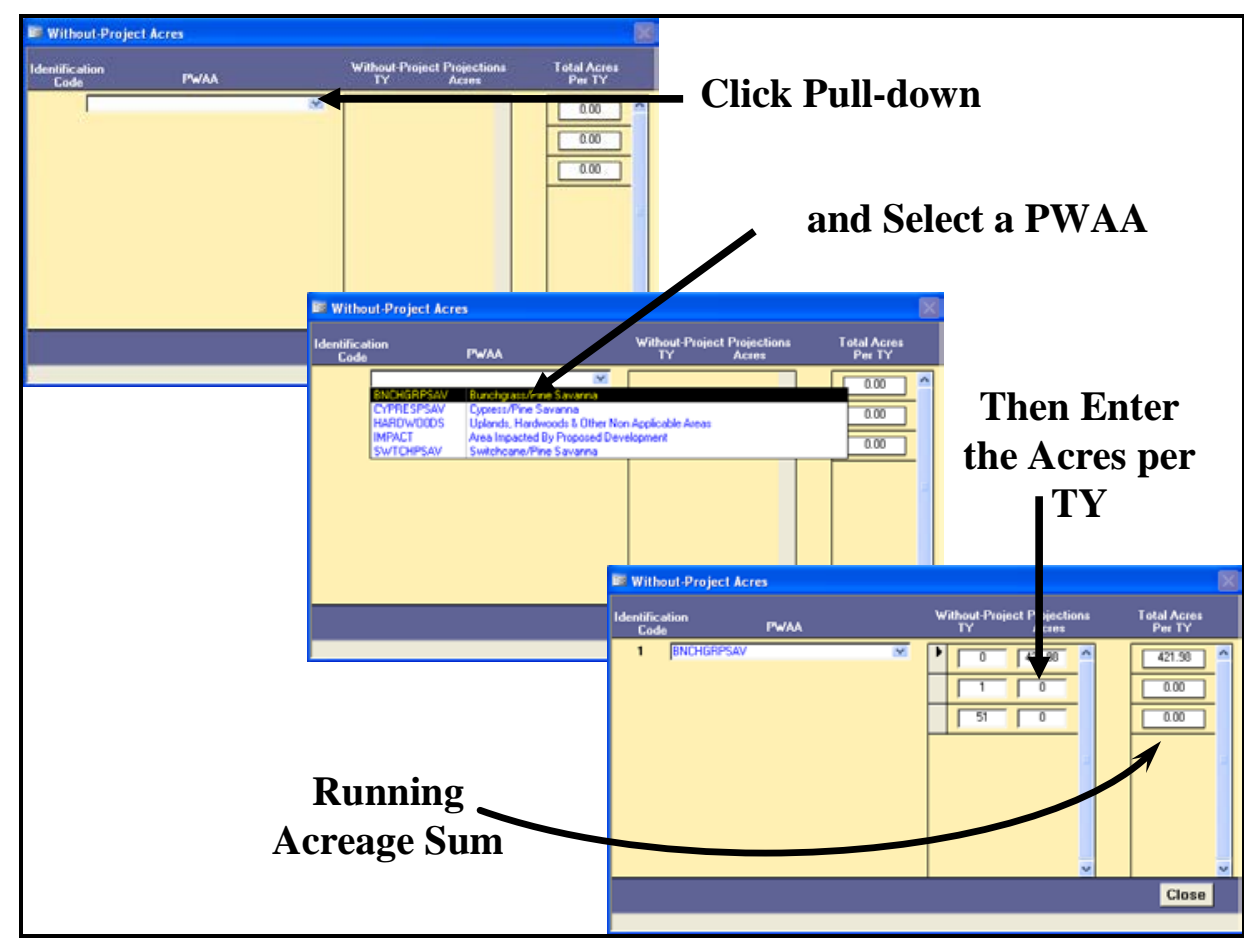

Figure 194. Enter the without-project acreage projections into the cells provided to the right of their corresponding target years.

Hit Enter once on the keyboard to accept the change, and hit Enter a second time to move down to the next row. Continue to the end of the column, at which point the final Enter hit on the keyboard will complete the process for this PWAA. Systematically "select" the remaining PWAAs one by one from the pull-down menu, forecast their acreages, and enter these values into the software. All PWAAs must be accounted for in this 
activity - even if they are not associated with the functions in the assessment.

A running sum of acres on the site is calculated by TY and presented in the third column running along the right-hand side of this form (Total Acres per TY). At the end of this exercise, the numbers in each row of this column should be the same (i.e., if there are 100 acres at baseline, there are 100 acres at the end of the study), or a problem has occurred in the entry process. Revisit and check the entries in each PWAA to find and correct any errors. If at any time acreages must be modified, simply return to this interface, highlight the acreage that must be changed, type in the new values, and hit Enter twice on the keyboard to

\section{USER'S NOTE:}

Inevitably, acreages and/or data are modified throughout the planning of a study. Moving in and amongst functions can lead to a break in the system's focus on a particular "Working Function." If the focus has changed, and the data have been changed (i.e., baseline acres, target tears, WOP acres, etc.) it is prudent to re-run the starred $(*)$ options in the primary interface [i.e., in particular 4. Perform Baseline (TY 0) Calculations* at this point in timel.

\section{USER'S NOTE:}

WOP acres assigned to the "Working

Function" apply to all models in the software. Therefore it is only necessary to enter the WOP acres once - they will be carried into the analysis for the remaining functions. accept the change. When finished, click on the Close button, and EXHGM returns to the primary interface. Now proceed to the next section to check the entries and focus on the without-project forecasting task.

Checking the without-project target years and without-project acres with

\section{EXHGM reports}

To review and check the target year and acreage entries made thus far, click on the Setup Reports button on the primary interface (Figure 195).

Two additional "setup" reports should be populated at this point:

\section{- Target Year List}

\section{- Without-Project TY Acres}

Single-click on either report and look to the Instruction Window to check the contents of the report. Double-click on either report, and EXHGM opens a new window with the report inside (Figure 196). 


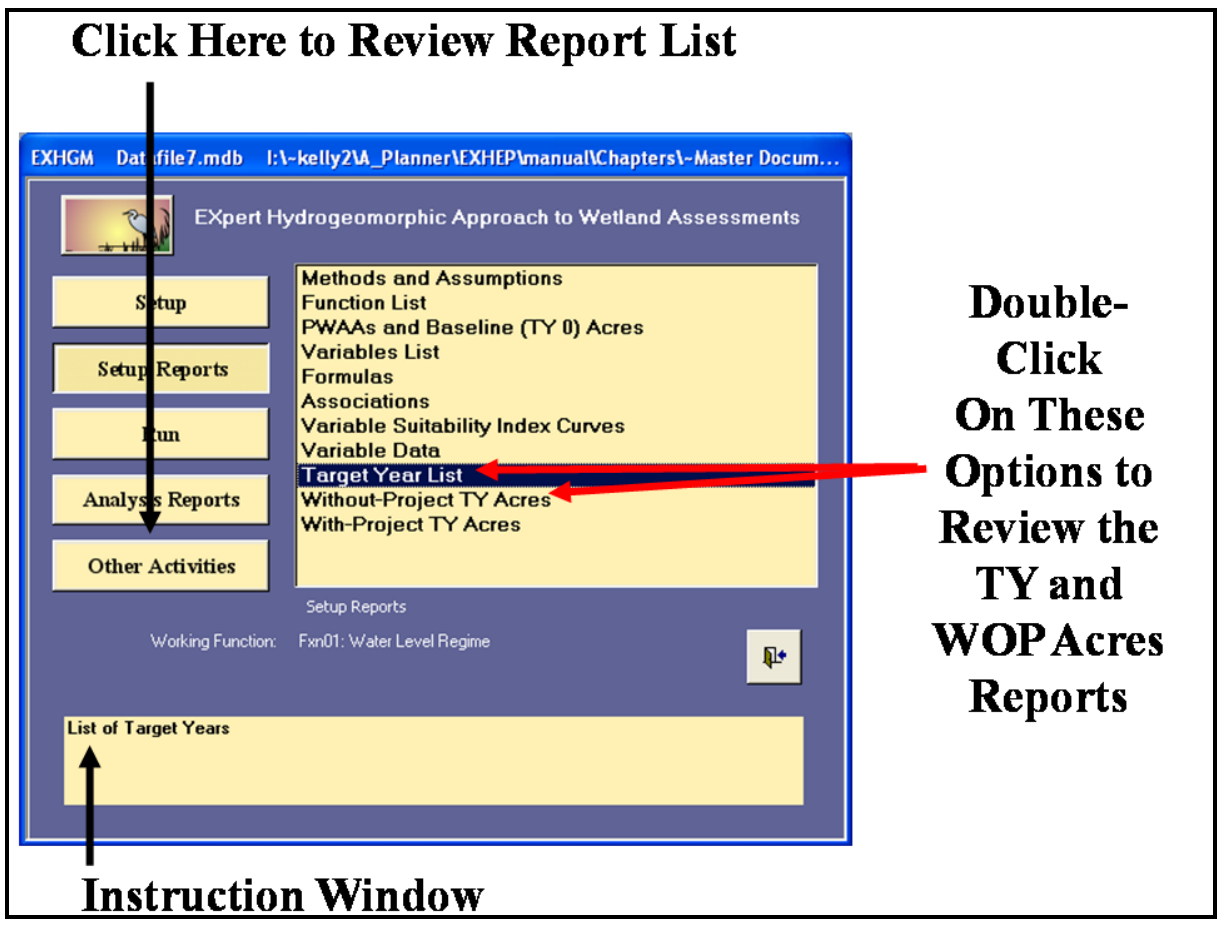

Figure 195. Click on the Setup Reports button to view the Target Year List report in EXHGM.

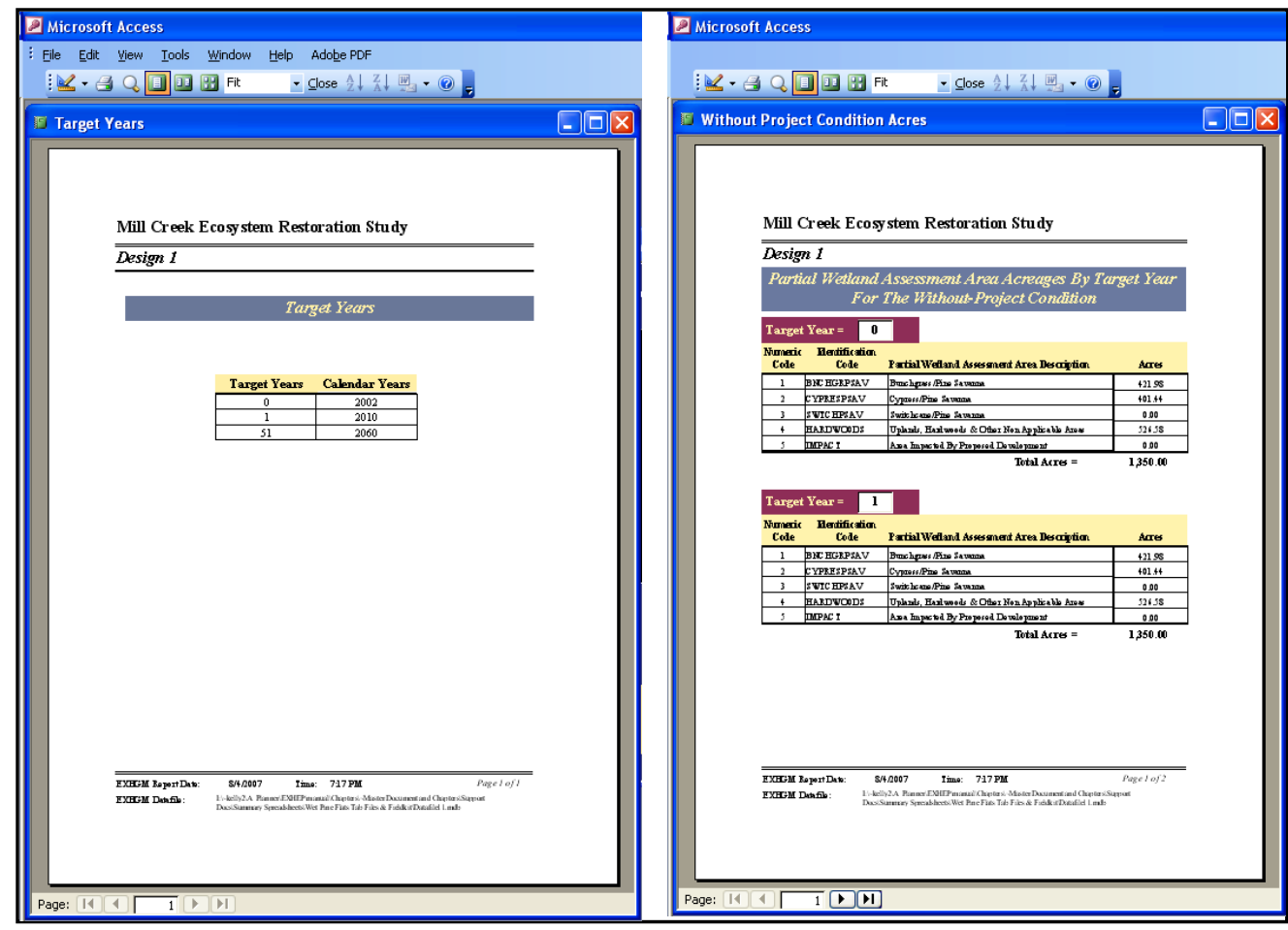

Figure 196. Example of Target Year List and Without-Project TY Acres reports in EXHGM.

Click on the Close button from the suite of buttons offered on the Report Control toolbar, and EXHGM returns to the primary interface. 
Enter the without-project variable projections

Now click on the Run button on the primary interface (Figure 197).

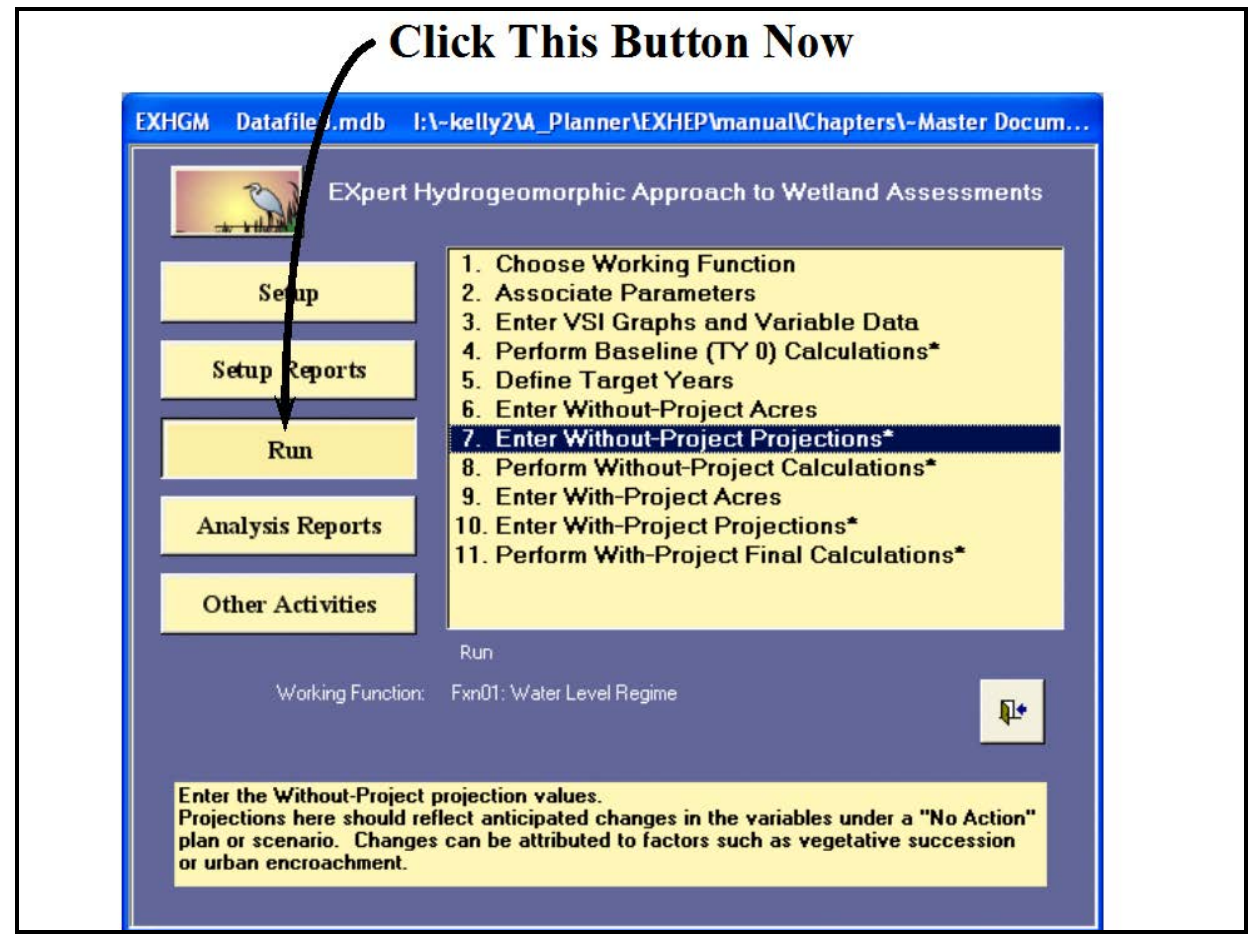

Figure 197. Click on the Run button to begin the without-project variables entry process in EXHGM.

As it did before, a screen appears in the center of the primary interface with 11 options:

1. Choose Working Function

2. Associate Parameters

3. Enter VSI Graphs and Variable Data

4. Perform Baseline (TY 0) Calculations*

5. Define Target Years

6. Enter Without-Project Acres

7. Enter Without-Project Projections*

8. Perform Without-Project Calculations*

9. Enter With-Project Acres

10. Enter With-Project Projections*

11. Perform With-Project Final Calculations*

Check to assure that the "Working Function" is set to the correct function, and click on the seventh option (Enter Without-Project Projections*) 
to continue. In response, EXHGM asks the question, "Do you want to initialize the future factors first?"

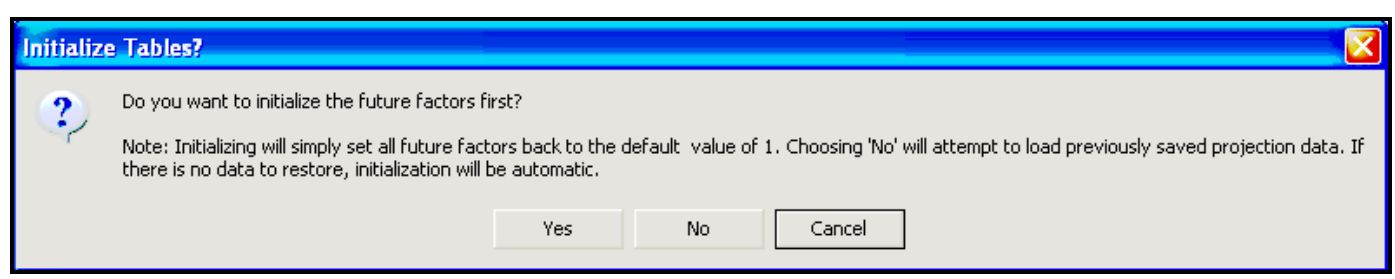

Initialization is EXHGM's attempt to save some time by automating the forecast conditions of the variables. In short, the program queries the database, finds the baseline means for each

USER'S NOTE:

The initialization process is irreversible. If the Yes button is clicked in error, all the variables must be re-entered by hand.

variable in each PWAA, and inputs this value into the projected forecast across all target years in the life of the project. For example, if the mean of a Variable X is 50 at baseline, EXHGM simply takes this value, copies it into every target year, and presents these values for inspection and modification. The key here is to only initialize the without-project

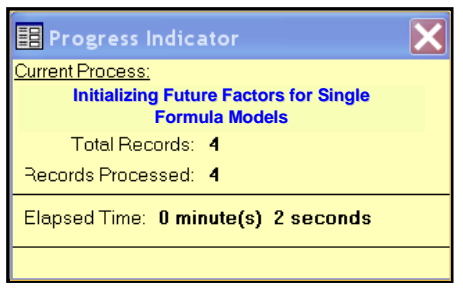

conditions once. Since this is the first time to enter this process, click Yes to proceed. As the initialization commences, a Progress Indicator window will open in the screen tracking the status of the process, providing a tentative estimate of Elapsed Time.

USER'S NOTE:

Say "Yes" to initialization if:

1. Variable projections have not been made for any function.

2. Baseline data have been modified after an initialization.

3. Target years have been modified after an initialization.

4. Function associations have been modified after an initialization.

5. Functions, PWAAs, and/or variables have been added/deleted/modified postinitialization (basically any change in Setup necessitates re-initialization).

Say "No" to initialization when:
1. Variable projections have been made for at least one function.
2. Modifying current projection trends - post-initialization.
3. Running functions through the without- and/or with-project calculations in series.
4. Modifying acreages in the baseline, without- and/or with-project interfaces. 
When complete, EXHGM opens the following window (Figure 198).

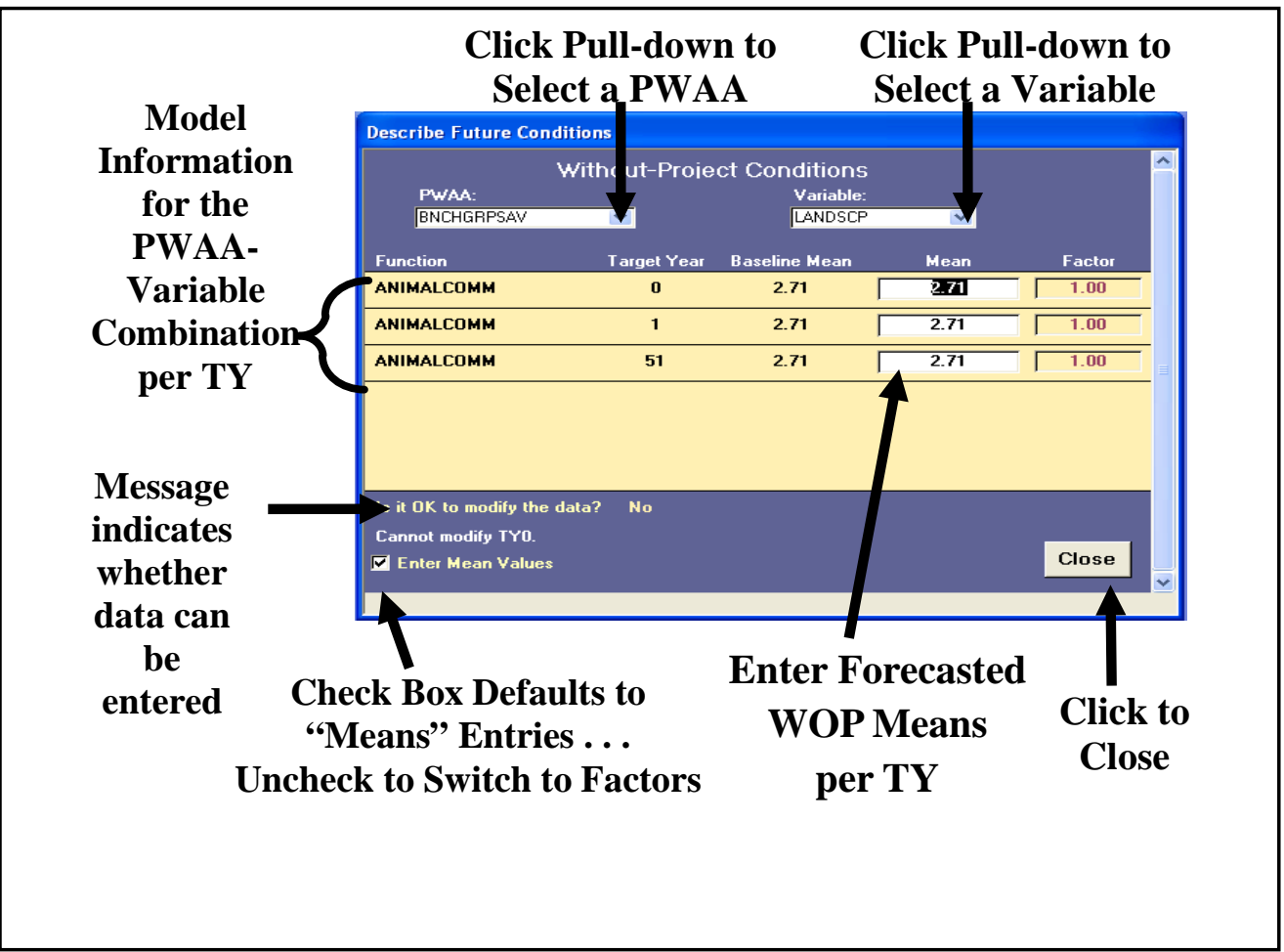

Figure 198. Describe Future Conditions entry window used to forecast without-project conditions in EXHGM.

Click on the PWAA pull-down menu and select the first PWAA from the list. Now click on the Variable pull-down menu and select the first variable from the list. EXHGM queries the database for target years and baseline data (based on the entries made in the steps above), and presents these in a tabular format. Note that outlying years are set to equal the baseline $\left(\mathrm{TY}_{0}\right)$ value.

Now make changes to the data where necessary to capture the withoutproject conditions of this variable in this PWAA over the course of the target years by highlighting the value and typing in the corrected version in the space provided. Press the Tab button on the keyboard to "accept" the change and move to the next row (Figure 199). 


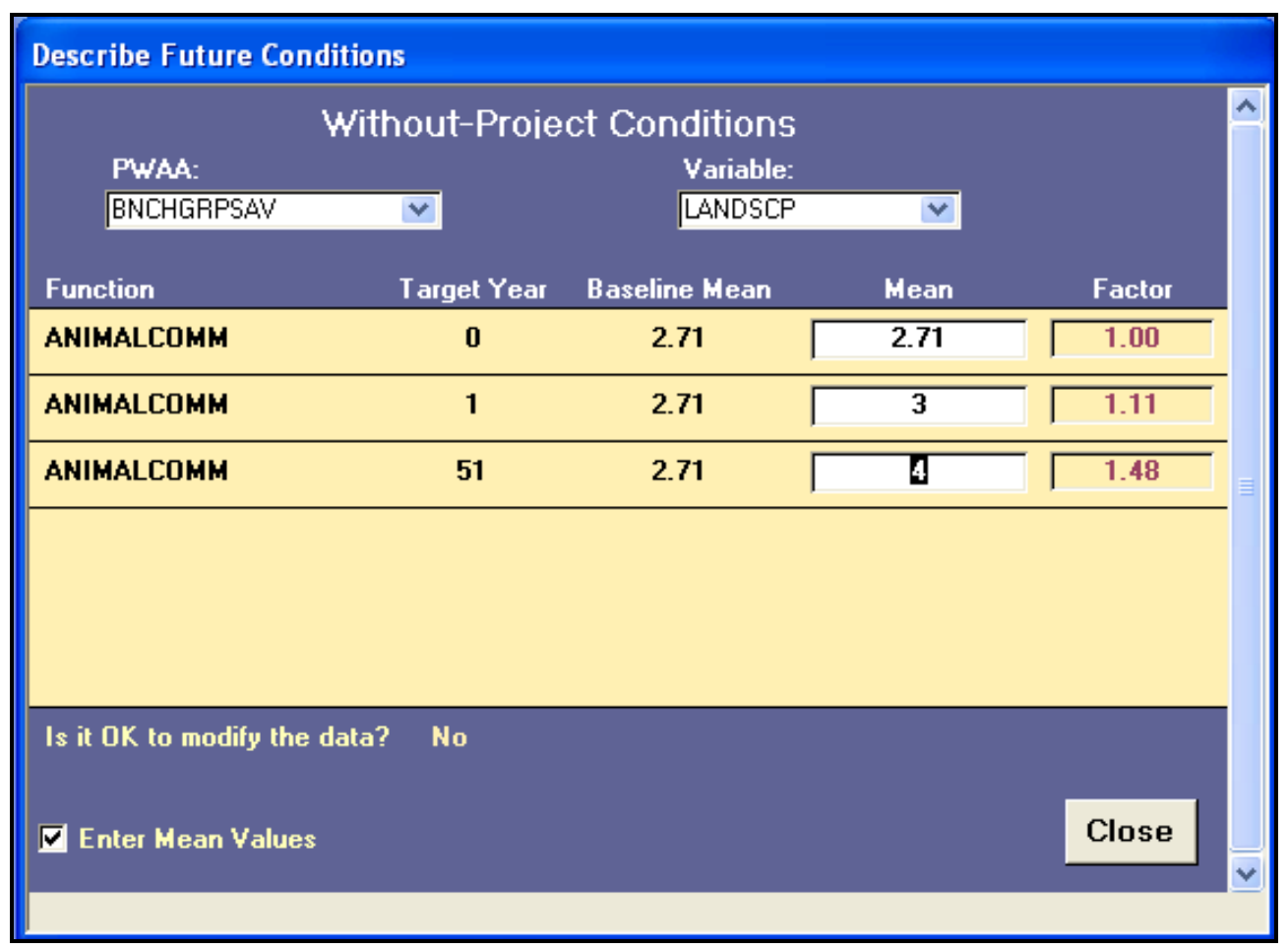

Figure 199. Type the new without-project values into the Mean column.

Note that the baseline means cannot be modified in this interface - a message in the lower left-hand corner of the screen indicates whether or not a field can be altered. To make changes to the baseline conditions, new data must be entered elsewhere (i.e., return to Option 3, Enter VSI Graphs and Variable Data and follow the directions above to modify these values). It is also important to note that the system's query filters the PWAAs and variables presented in this interface - only variables associated inside function formulas will be presented in this screen. Modifications to the associations cannot be made in this interface..Return to the function association interface to make these acreage changes (refer to Step 3: Function Association section above for details).

Functions that share similar variables will be presented simultaneously and sorted alphabetically by Function code in this interface. Changes to shared variables are automatically carried down into the subsequent functions, eliminating the need to enter the information into the system multiple times (Figure 200). 


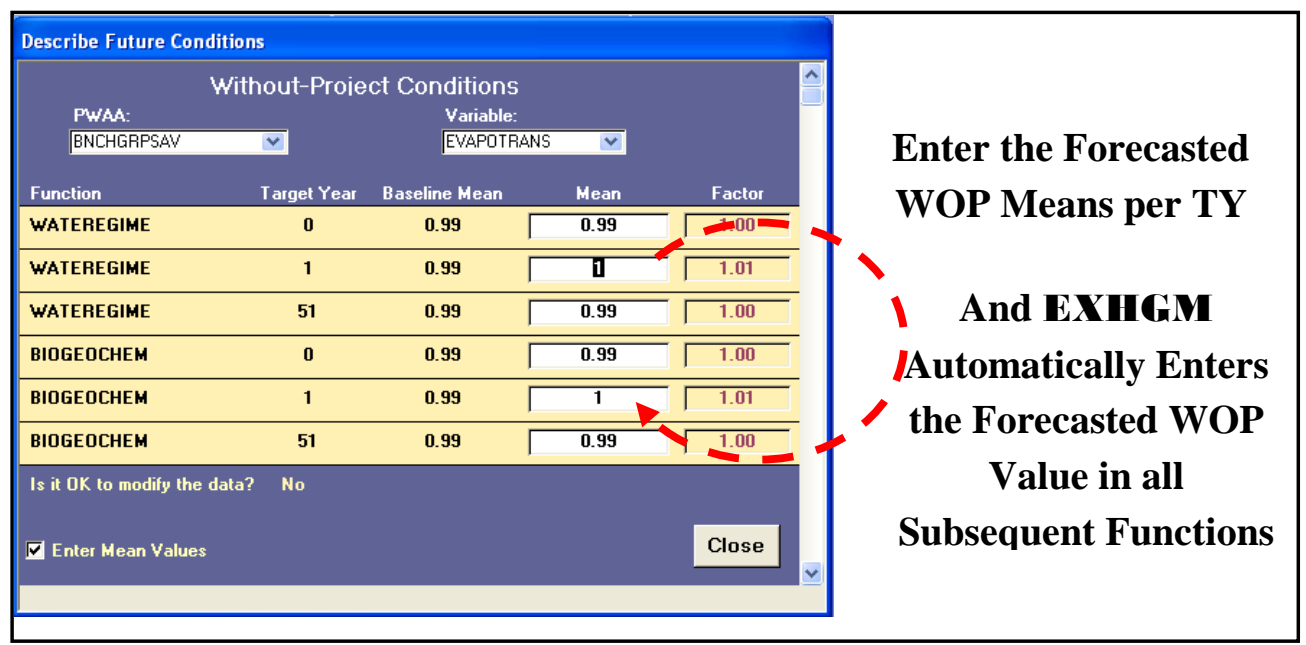

Figure 200. Automated data entry for functions that share variables.

Systematically pull down the PWAA and variable combinations and repeat the process as many times as necessary to fully document the withoutproject conditions at the site. When finished, click on the Close button, and EXHGM returns to the primary interface.

Although the system defaults are set to capture forecasted information documentation on a mean-by-mean basis, it is sometimes useful to forecast future conditions on the basis of percentages of the baseline mean. If this is a desired protocol, un-check the box to the immediate left of the Enter Mean Values label in the bottom left-hand corner of this form, and EXHGM will move the cursor to the Factor column (Figure 201).

Enter the without-project values for the variable in this PWAA by setting weights here (i.e., $50 \%=0.5 ; 125 \%=1.25$, etc.). EXHGM multiplies the factor against the baseline mean and generates the new mean for the TY. Systematically pull down the PWAA and variable combinations and repeat the process as many times as necessary to fully document the withoutproject conditions at the site. 


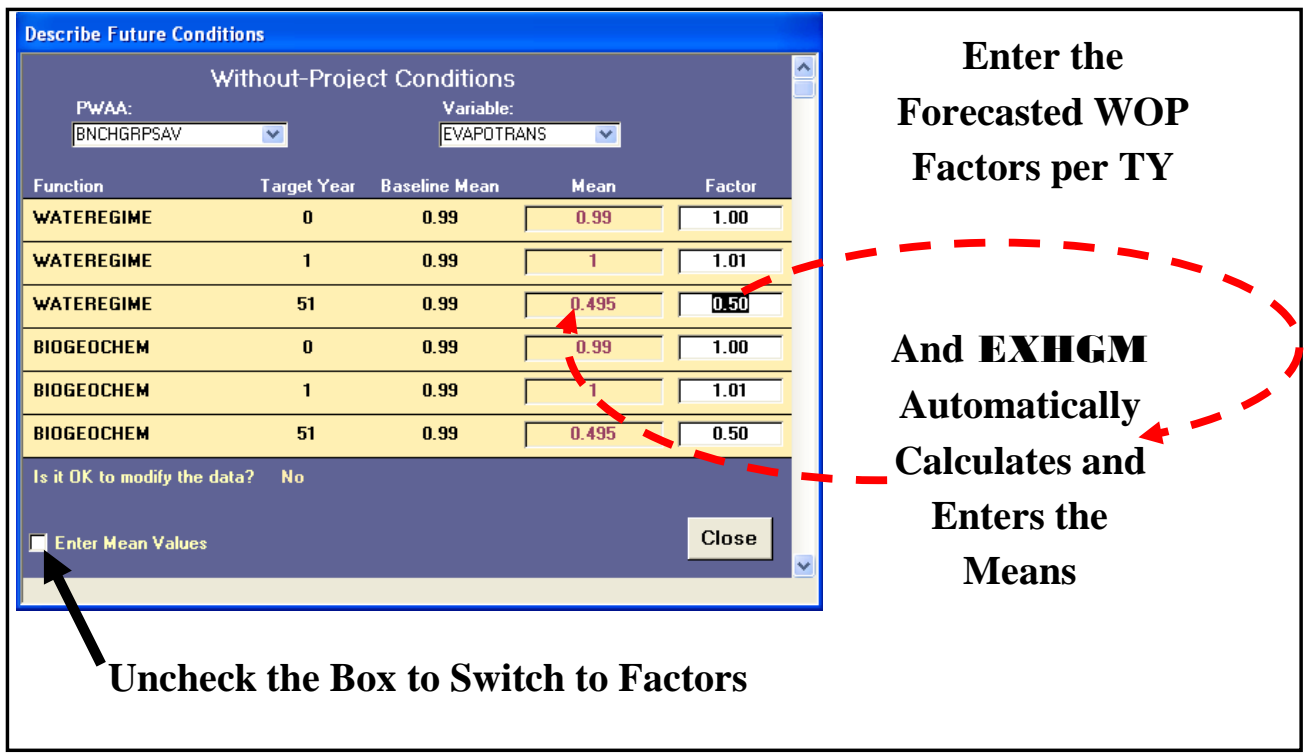

Figure 201. Use the Factor column to enter future projections in terms of percentages of baseline.

When the without-project data entry task is complete, click on the Close button, and EXHGM asks about saving the data:

? Would you like to save the projection data for possible use at a later time?

If you choose to save, you may use this data each time you enter projections by choosing 'No' when asked to initialize the projections table.

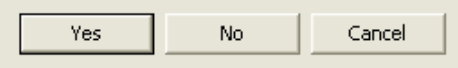

If an error was made, click the No button, and all the changes will be discarded. To correct the error immediately, click the Cancel button and EXHGM will return to the data entry interface. If the data were entered correctly, click on the Yes button, and EXHGM affirms that the information was saved successfully. Click

\section{Successful Copy}

Current projections data saved for later retrieval.

OK and EXHGM returns to the primary interface. 
Checking the without-project variable entries with EXHGM reports

To review and check the results, click on the Analysis Reports button on the primary interface (Figure 202).

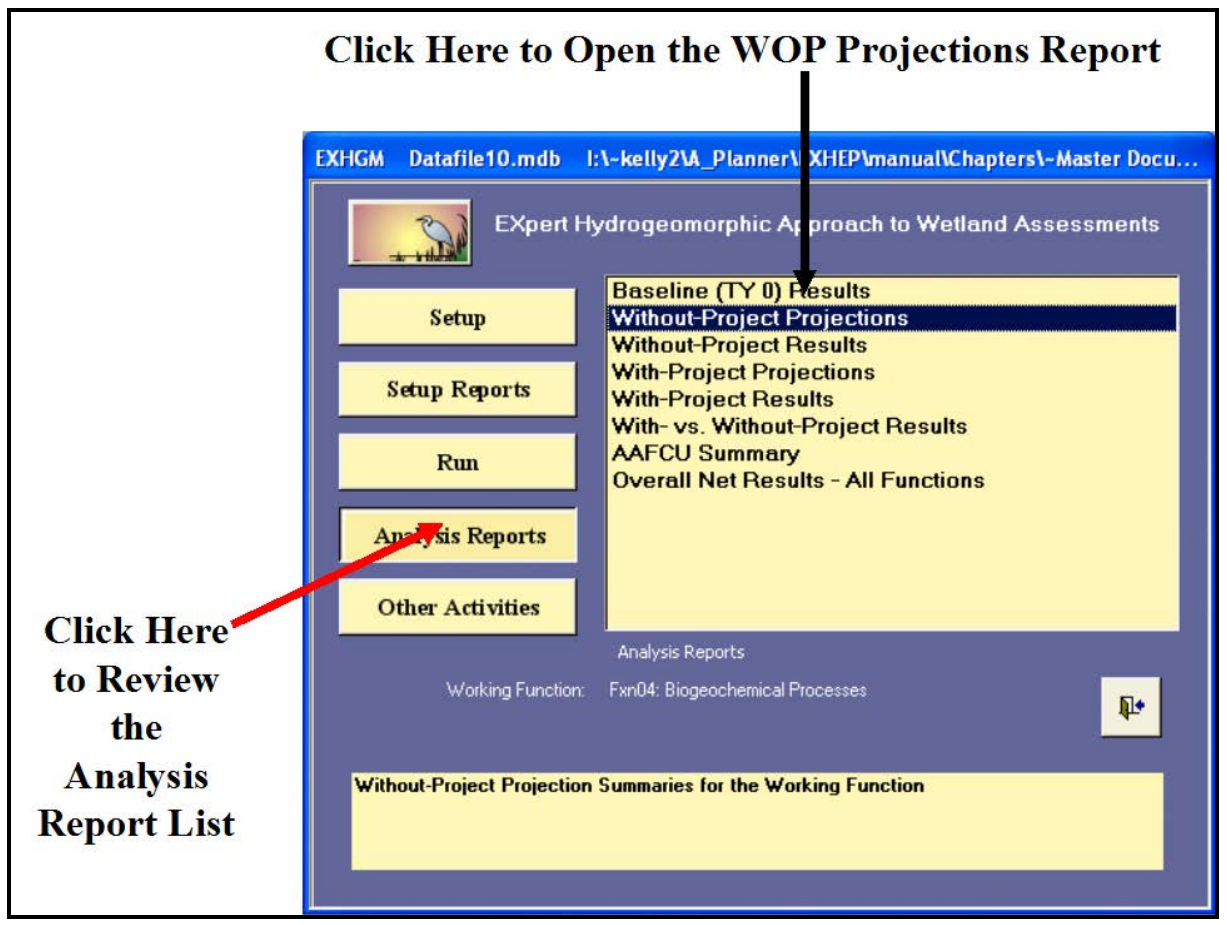

Figure 202. Click on the Analysis Reports button to review the without-project forecasted entries in EXHGM.

A list of available reports appears in the center of the primary interface. The second report listed, Without-Project Projections, is ready for inspection. Double-click on this report to review the results (Figure 203). 


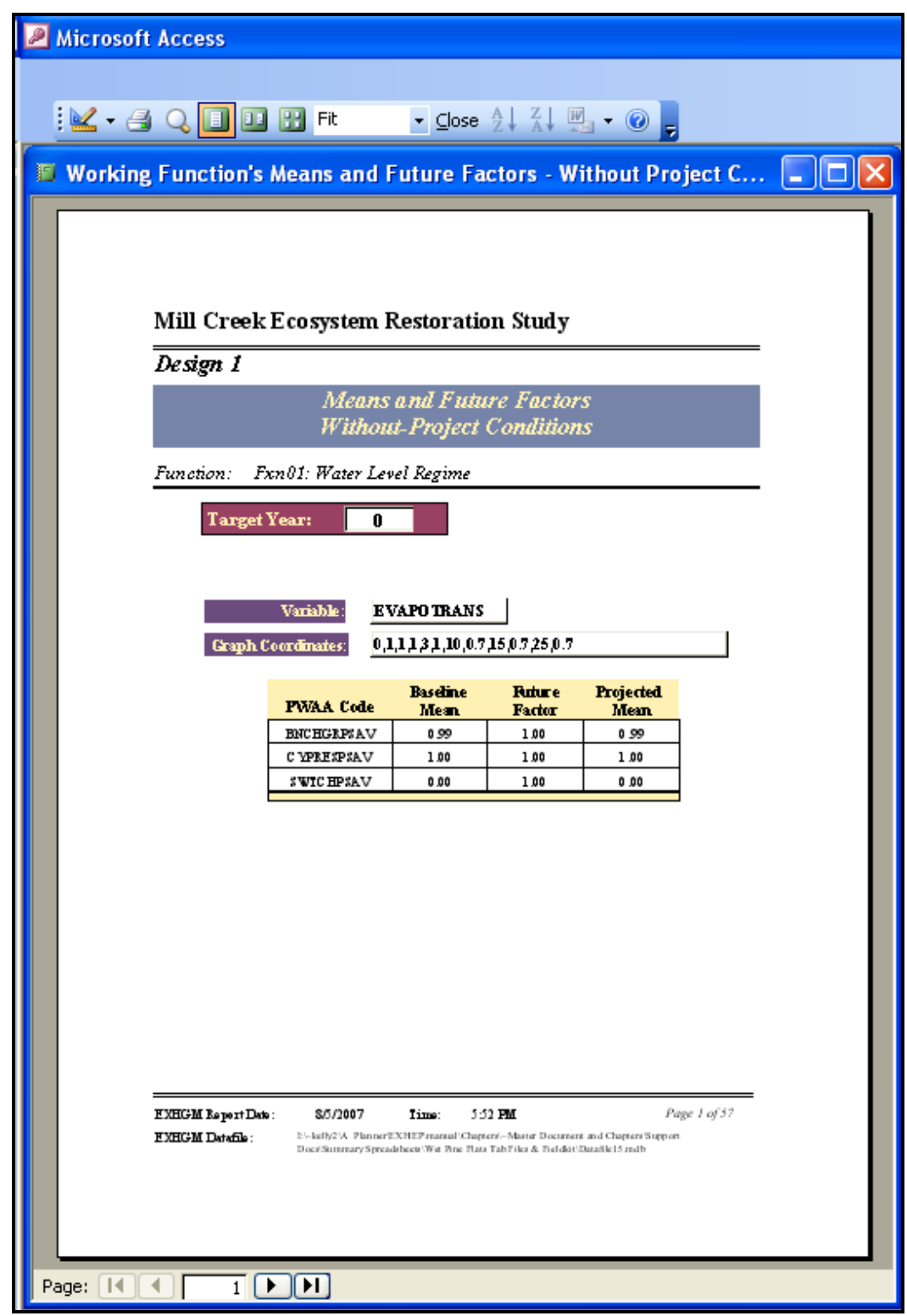

Figure 203. Example of Without-Project Projections report in EXHGM.

Click on the Close button from the suite of buttons offered in the Report Control toolbar, and EXHGM returns to the primary interface. Now that the without-project forecasts have been entered into the system, proceed to the next step to learn how to assess the without-project conditions of the site in EXHGM. 


\section{Perform the without-project calculations}

EXHGM will calculate the without-project results on a function-byfunction basis. To proceed, click on the Run button on the primary interface (Figure 204).

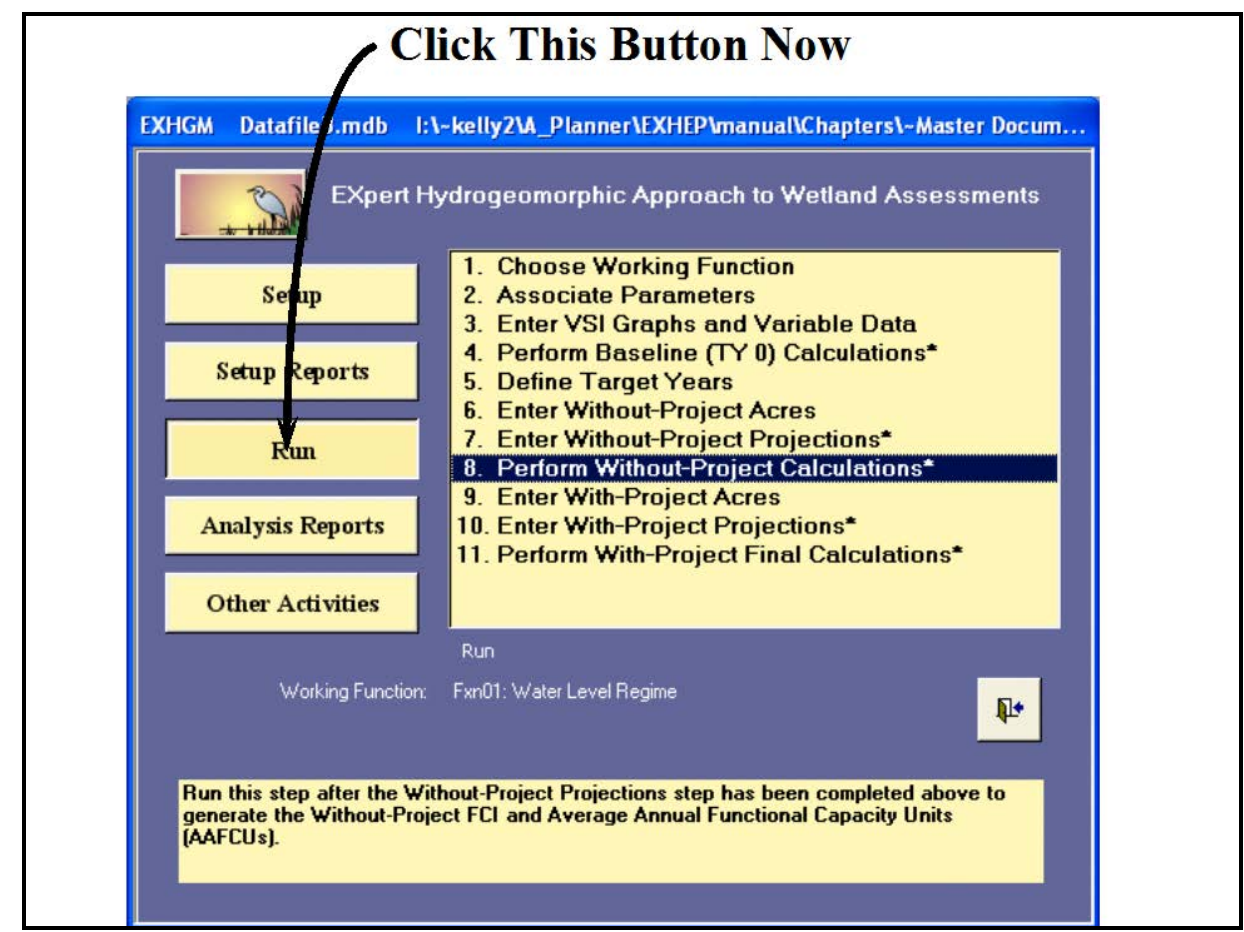

Figure 204. Click on the Run button to begin the without-project calculation process in EXHGM.

As it did before, a screen appears in the center of the primary interface with 11 options:

\section{Choose Working Function}

2. Associate Parameters

3. Enter VSI Graphs and Variable Data

4. Perform Baseline (TY 0) Calculations*

5. Define Target Years

6. Enter Without-Project Acres

7. Enter Without-Project Projections*

8. Perform Without-Project Calculations*

9. Enter With-Project Acres

10. Enter With-Project Projections*

11. Perform With-Project Final Calculations* 
Check to assure that the "Working Function" is set to the correct function, and click on the eighth option (Perform Without-Project Calculations*) to continue. In response, EXHGM will begin the calculation process. As

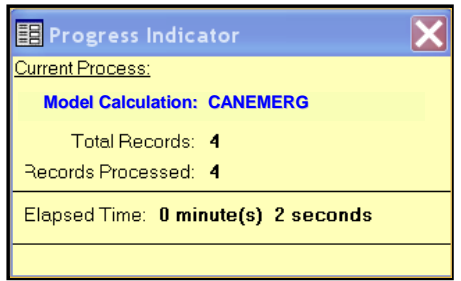
the calculations continue, a Progress

Indicator window will open in the Final Calculation Status screen. This progress window will track the status of the analysis (number of The Working Model's Final Without-Project Calculations completed successfully. records to assess vs. number of records processed) and provide a tentative estimate of Elapsed Time. When completed, EXHGM returns a message that the calculations are concluded. This process must be completed for each function in the analysis. Each time a "Working Function" is selected, the options on the primary interface that have stars next to their titles must be re-run to focus the database. The "quick steps" are as follows:

1. Click on 1. Choose Working Function and select the next function for processing.

2. Click on 4. Perform Baseline (TY 0) Calculations*, let the system run, and click $\mathbf{O K}$ when it is finished.

3. Click on 7. Enter WithoutProject Projections*, click on the No button for initialization, click the Close button at the bottom of the interface, click Yes to save the data, and click $\mathbf{O K}$ when that save is USER'S NOTE:

Remember, when asked about initializing the data in option 7. Enter Without-Project Projections*, answer No or the data inside will be overwritten. affirmed.

4. Click on 8. Perform Without-Project Calculations*, let the system run, and click $\mathbf{O K}$ when it is finished.

\section{Checking the without-project analysis with EXHGM reports}

To review and check the results, click on the Analysis Reports button on the primary interface (Figure 205). 


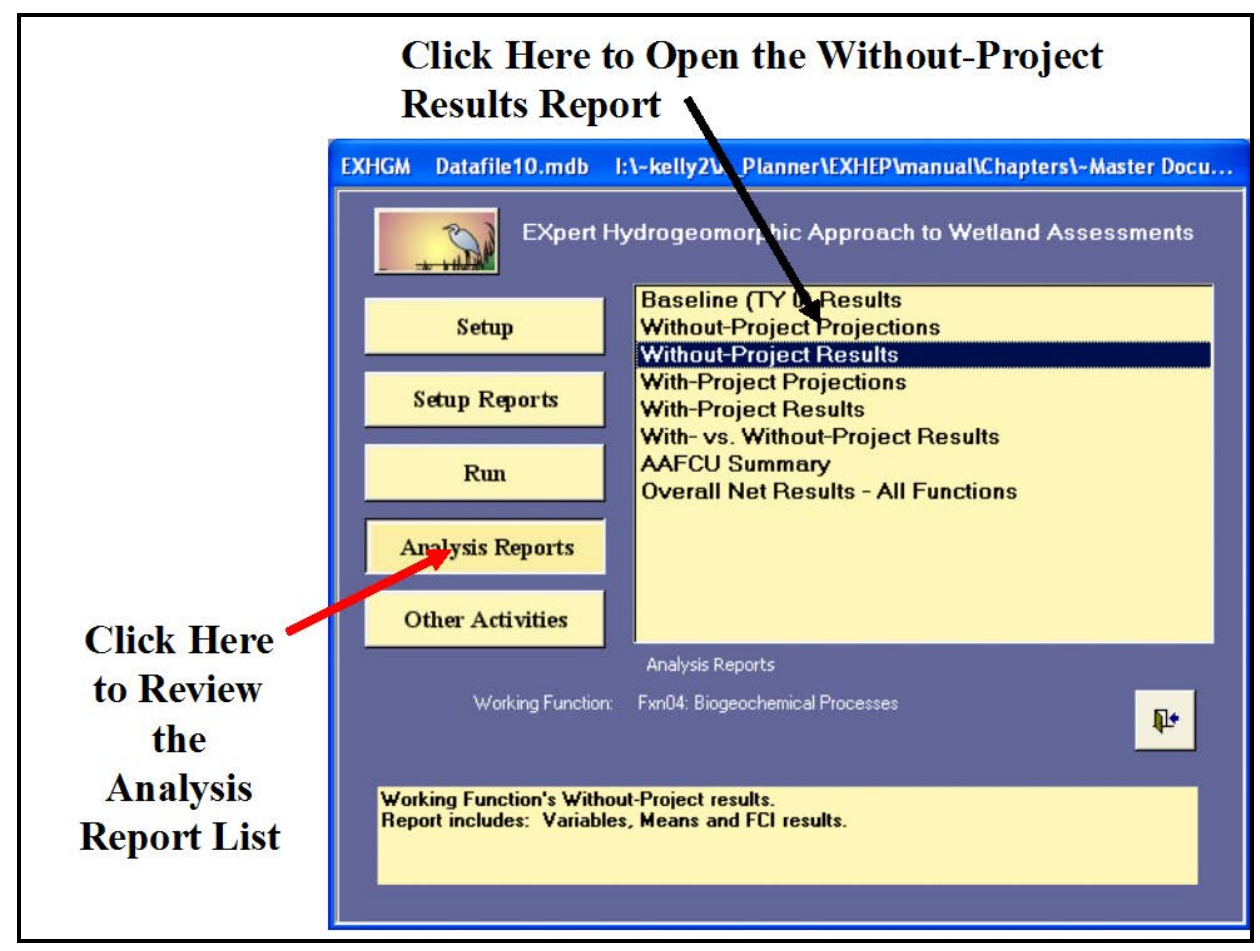

Figure 205. Click on the Analysis Reports button to review the without-project results in EXHGM.

A list of available reports appears in the center of the primary interface. The third report listed, Without-Project Results, is ready for inspection. Double-click on this report to review the results (Figure 206).

The site's overall average annual outputs (i.e., AAFCUs) are reported just under each function's name. The FCI scores, associated acreages, and the resulting FCU scores are then reported on a target year-by-target year basis below the AAFCU values. The specifics used to generate the overall scores are reported in the tables below this summary. These details are sorted alphabetically by PWAA and variable. These reports are cumulative; thus, all functions assessed in the EXHGM analyses are presented herein.

Click on the Close button from the suite of buttons offered in the Report Control toolbar, and EXHGM returns to the primary interface. Now that the without-project assessment is complete, proceed to the next step to learn how to assess the with-project conditions of the site in EXHGM. 


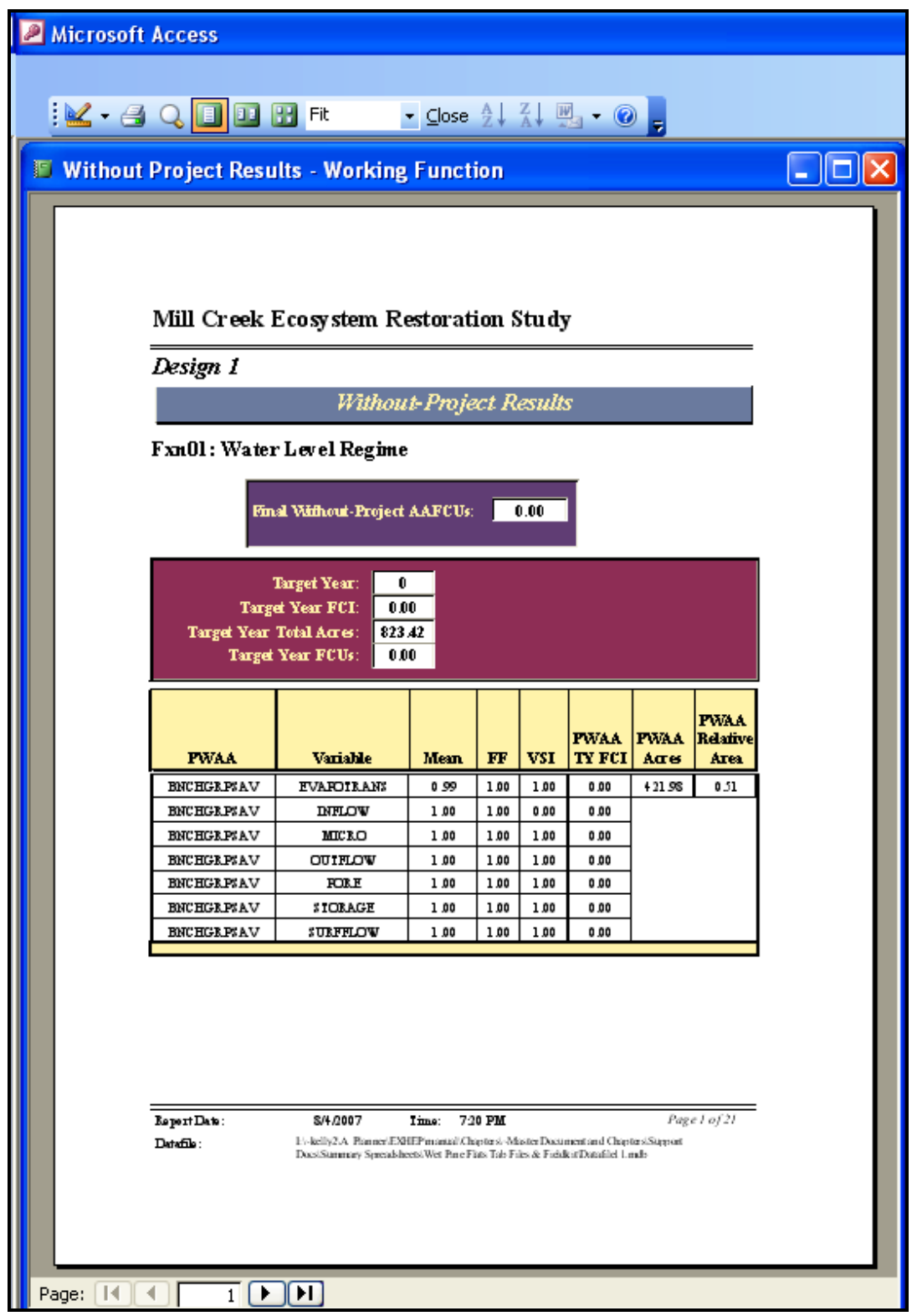

Figure 206. Examples of Without-Project Results report in EXHGM.

\section{Step 6: Enter the with-project conditions and calculate the effects}

Once the without-project conditions have been conceptualized, the evaluation team begins the iterative process of developing action plans (i.e., alternatives) to either counteract the degraded conditions (the goal of restoration or mitigation projects) or modify the existing site with activities that degrade the wetland (the focus of impact studies). To quantify the projected change to the site if one of these actions is taken, both withproject acreage and wetland quality must be documented and incorporated into the evaluation. The timing, nature, and magnitude of these changes must be quantified for each alternative proposed for the site. 
These values in turn must be entered into the software (one alternative at a time) and results are generated on an alternative-by-alternative basis. ${ }^{1}$

\section{With-project forecasting and data entry}

In EXHGM, the target years defined in the without-project condition are the same target years used in the with-project assessment, so it is not necessary to re-enter these milestones. Instead, the data entry efforts focus on the prediction of acreage and quality of that acreage over time with the proposed alternative in place.

USER'S NOTE:

The interfaces EXHGM uses to capture and organize this information will look very similar to those in the without-project section - only subtle differences in form titles indicate the with-project focus.

Enter and/or delete the with-project acres

To begin, click on the Run button on the primary interface (Figure 207).

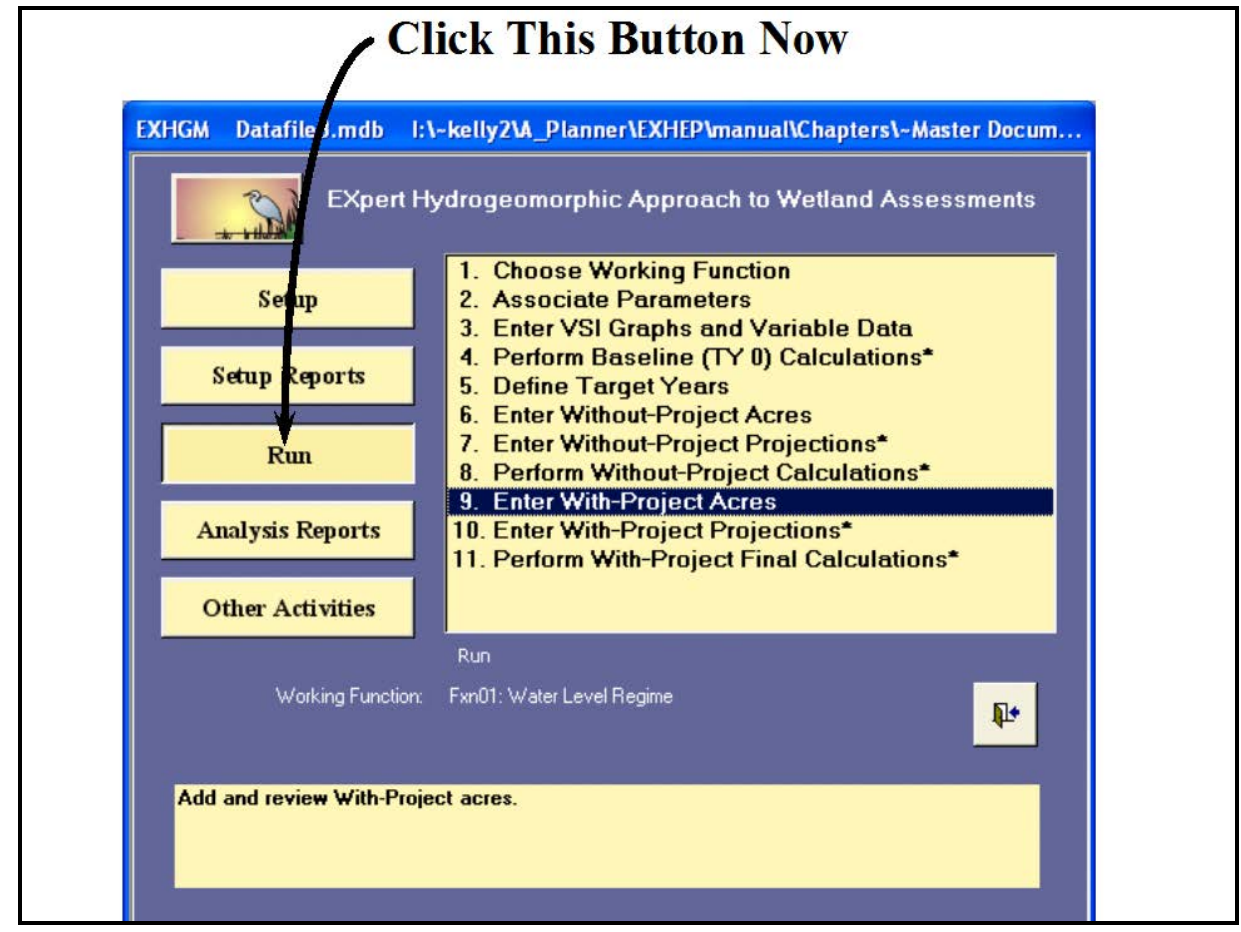

Figure 207. Click on the Run button to begin the with-project acres entry process in EXHGM.

\footnotetext{
1 To assist in the software application, a series of training exercises have been included in Appendix E refer to Exercise 15 for relevant training in with-project data entry and analysis in $\mathbf{E X H G M . ~}$
} 
As it did before, a screen appears in the center of the primary interface with 11 options:

1. Choose Working Function

2. Associate Parameters

3. Enter VSI Graphs and Variable Data

4. Perform Baseline (TY 0) Calculations*

5. Define Target Years

6. Enter Without-Project Acres

7. Enter Without-Project Projections*

8. Perform Without-Project Calculations*

9. Enter With-Project Acres

10. Enter With-Project Projections*

11. Perform With-Project Final Calculations*

Check to assure that the "Working Function" is set to the correct function, and click on the ninth option (Enter With-Project Acres) to continue. Use the same protocol described above in the without-project section to enter acres for the PWAAs on the site under the with-project scenario (Figure 208).

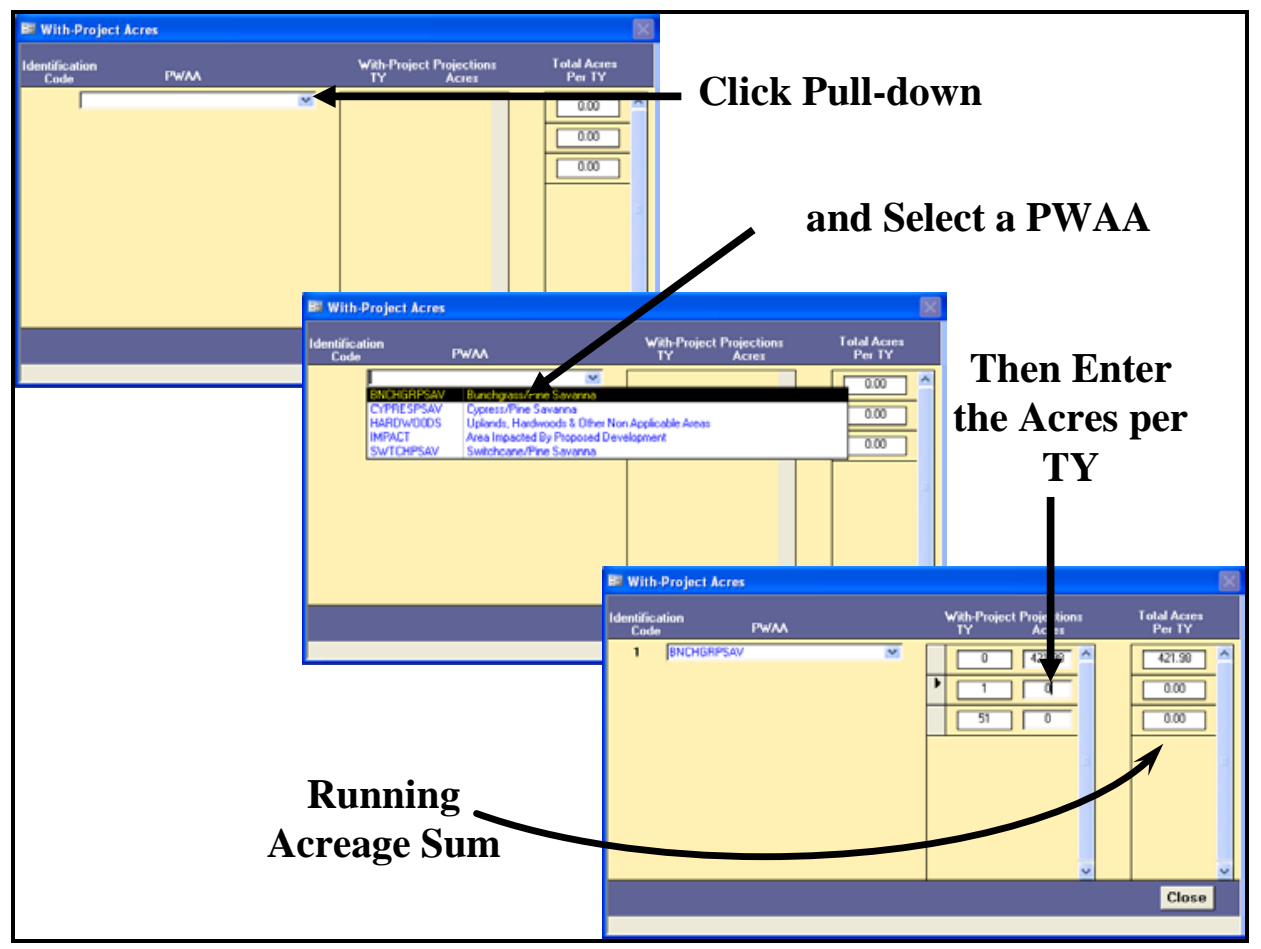

Figure 208. Enter the with-project acreage projections into the cells provided to the right of their corresponding target years. 
When finished, click on the Close button, and EXHGM returns to the primary interface. Now proceed to the next section to check the entries and focus on the with-project forecasting task.

Checking the with-project acres with EXHGM reports

To review and check the target year and acreage entries made thus far, click on the Setup Reports button on the primary interface (Figure 209).

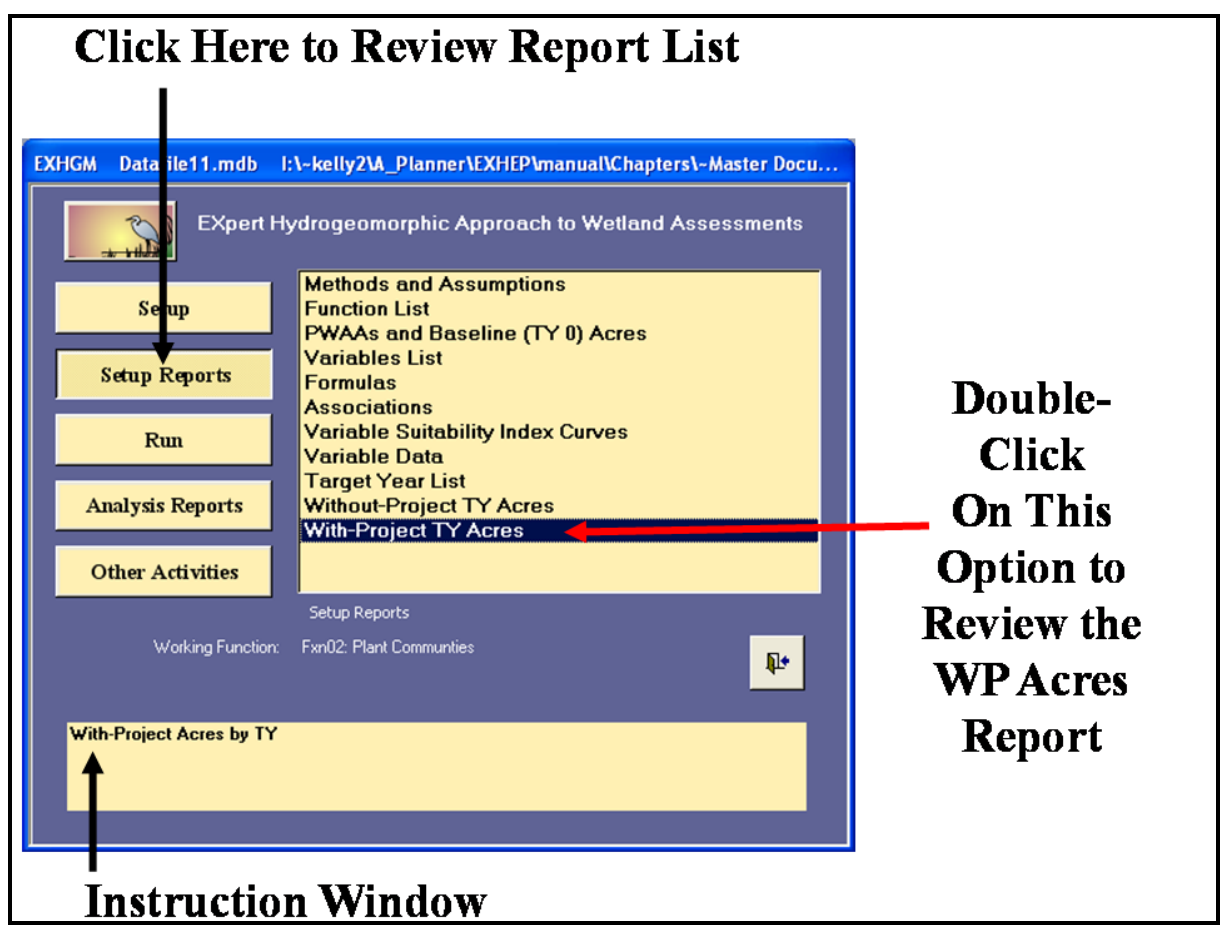

Figure 209. Click on the Setup Reports button to view the With-Project TY Acres report in EXHGM.

The With-Project TY Acres report should be populated at this point. Single-click on the report and look to the Instruction Window to check the contents of the report. Double-click on the report, and EXHGM opens a new window with the report inside (Figure 210).

Click on the Close button from the suite of buttons offered on the Report Control toolbar, and EXHGM returns to the primary interface.

Enter the with-project variable projections

Now click on the Run button on the primary interface (Figure 211). 


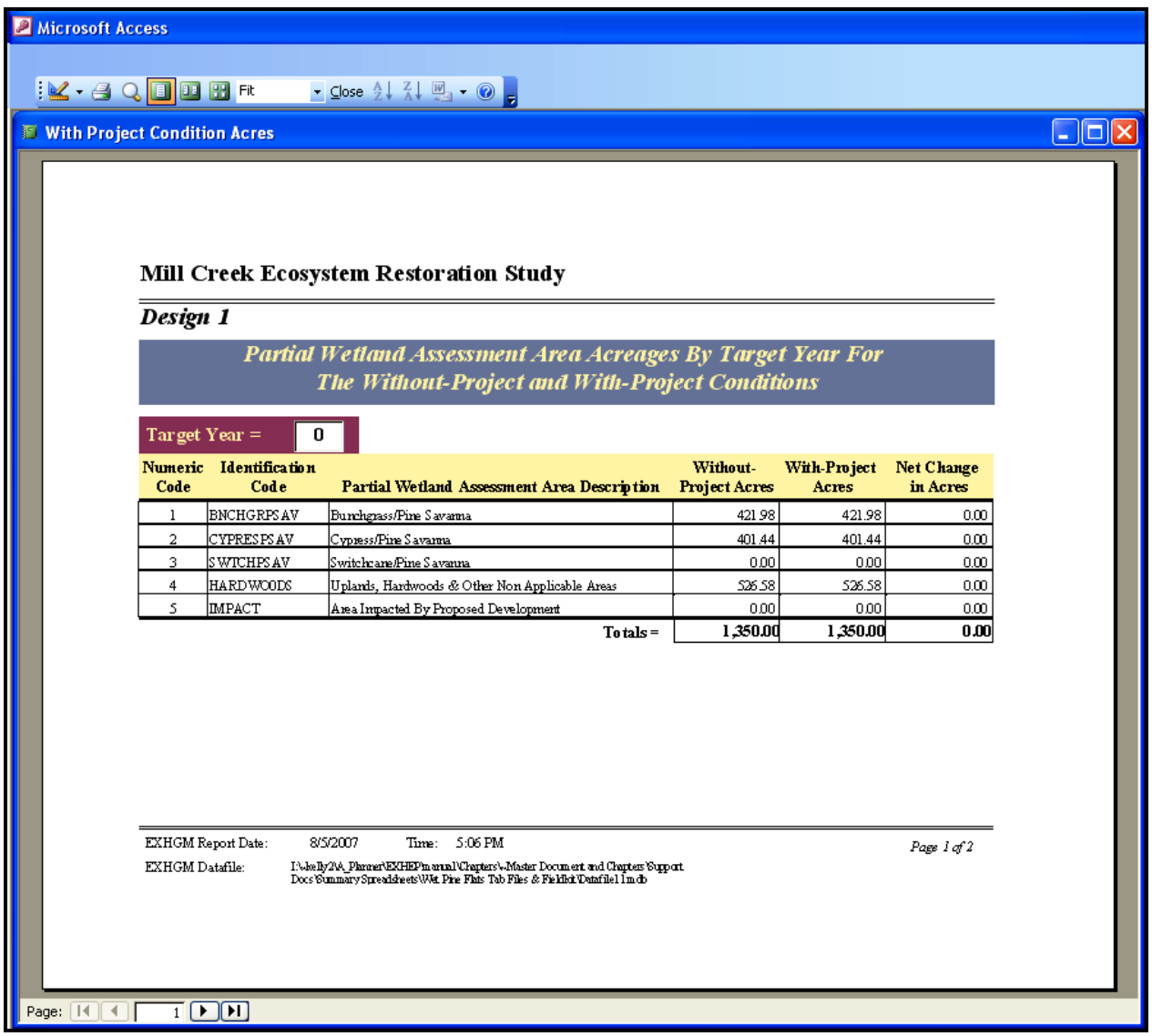

Figure 210. Example of With-Project TY Acres reports in EXHGM.

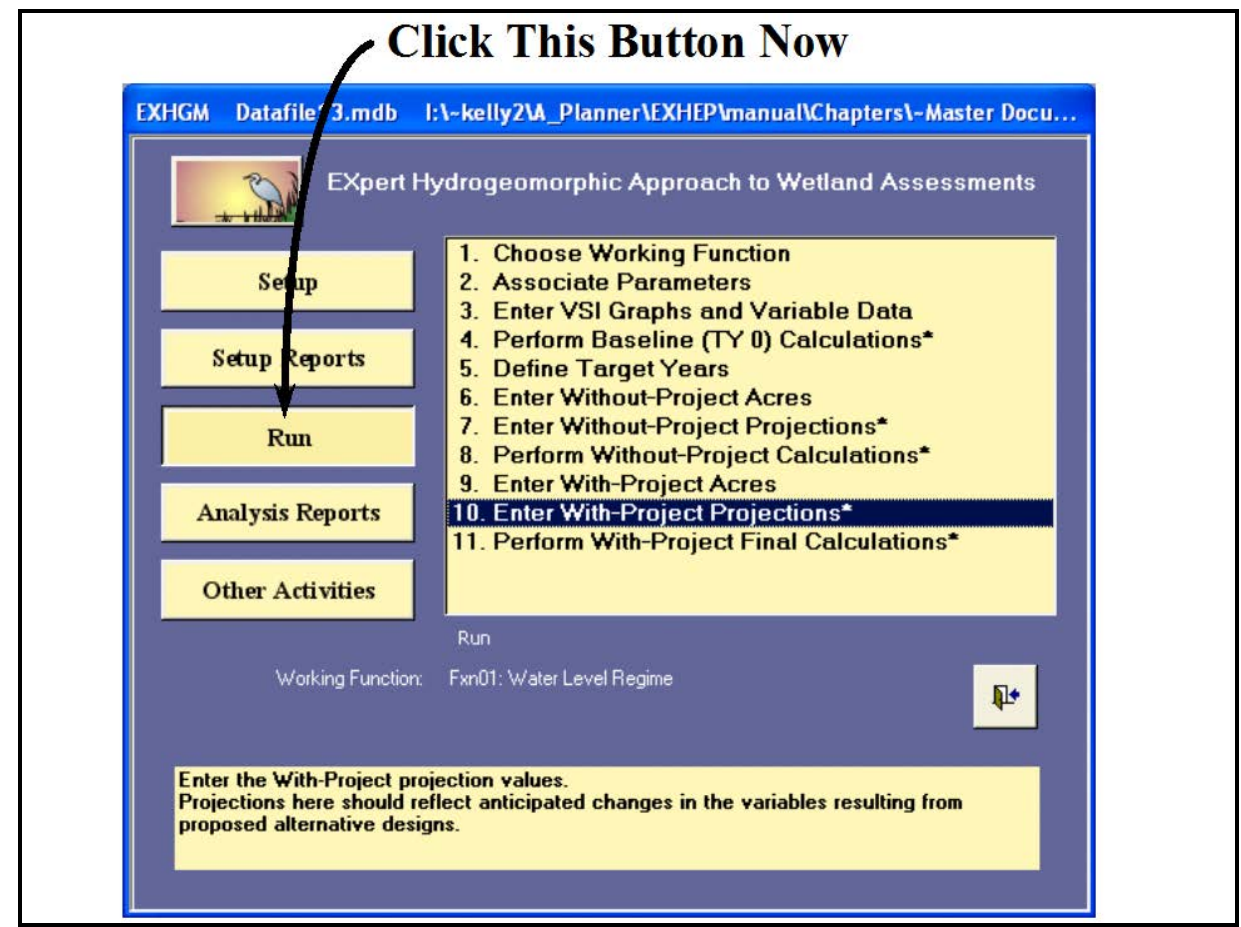

Figure 211. Click on the Run button to begin the with-project variables entry process in EXHGM. 
As it did before, a screen appears in the center of the primary interface with 11 options:

1. Choose Working Function

2. Associate Parameters

3. Enter VSI Graphs and Variable Data

4. Perform Baseline (TY 0) Calculations*

5. Define Target Years

6. Enter Without-Project Acres

7. Enter Without-Project Projections*

8. Perform Without-Project Calculations*

9. Enter With-Project Acres

10. Enter With-Project Projections*

11. Perform With-Project Final Calculations*

Check to assure that the "Working Function" is set to the correct function, and click on the tenth option (Enter With-Project Projections*) to continue. In response, EXHGM asks the question, "Do you want to initialize the future factors first?"

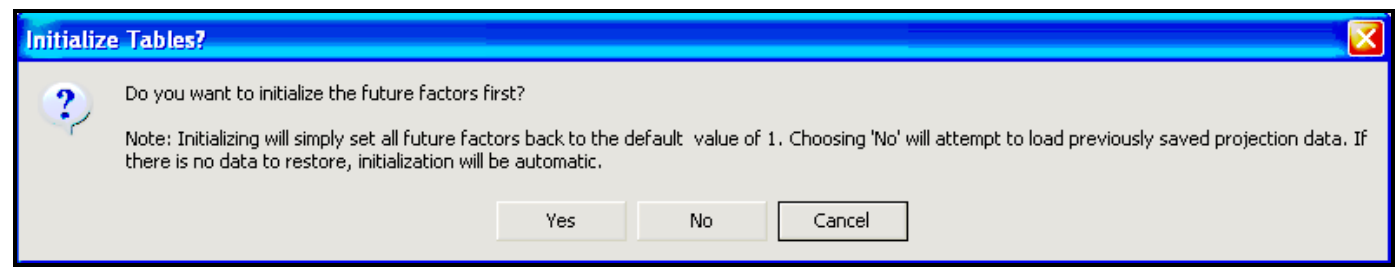

The key here again is to only initialize the with-project conditions once.

Since this is the first time to enter this process, click Yes to proceed. As the initialization commences, a Progress

Indicator window will open in the screen

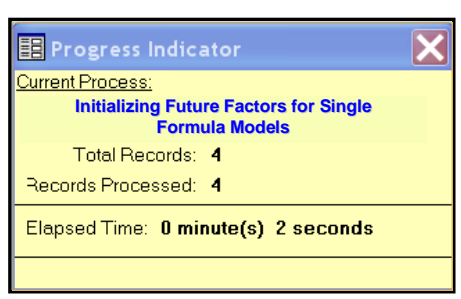

\section{USER'S NOTE:}

The initialization process is irreversible. If the Yes button is clicked in error, all the variables must be re-entered by hand. the process, providing a tentative estimate of Elapsed Time. 
USER'S NOTE:

Say "Yes" to initialization if:

1. Variable projections have not been made for any function.

2. Baseline data have been modified after an initialization.

3. Target years have been modified after an initialization.

4. Function associations have been modified after an initialization.

5. Functions, PWAAs, and/or variables have been added/deleted/modified postinitialization (basically any change in Setup necessitates re-initialization).

Say "No" to initialization when:

1. Variable projections have been made for at least one function.

2. Modifying current projection trends - post-initialization.

3. Running functions through the without- and/or with-project calculations in series.

4. Modifying acreages in the baseline, without- and/or with-project interfaces.

When complete, EXHGM opens the following window (Figure 212).

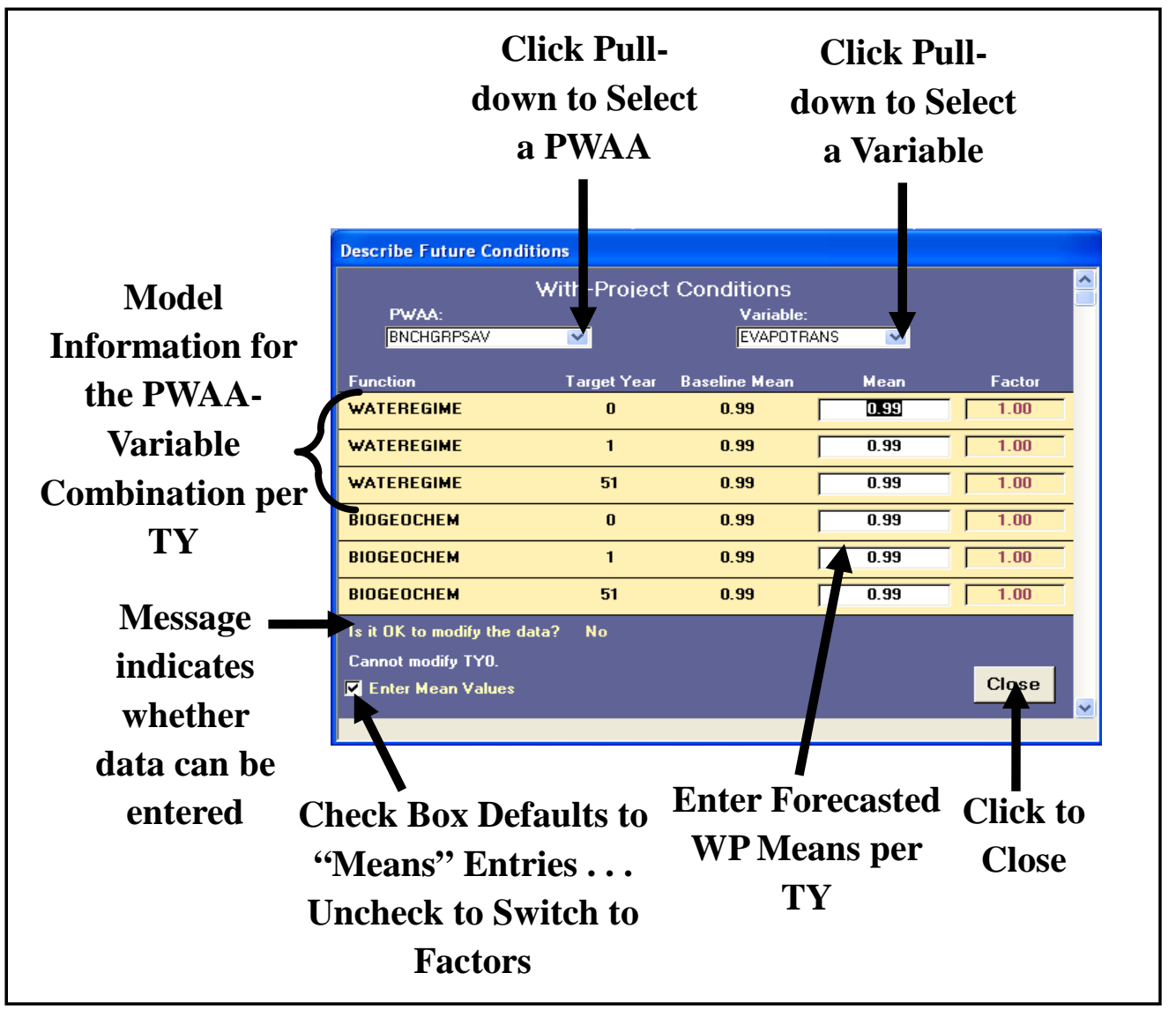

Figure 212. Describe Future Conditions entry window used to forecast with-project conditions in EXHGM. 
Use the same protocol described above in the without-project section to enter variable trends for the PWAAs on the site under the with-project scenario (Figure 213).

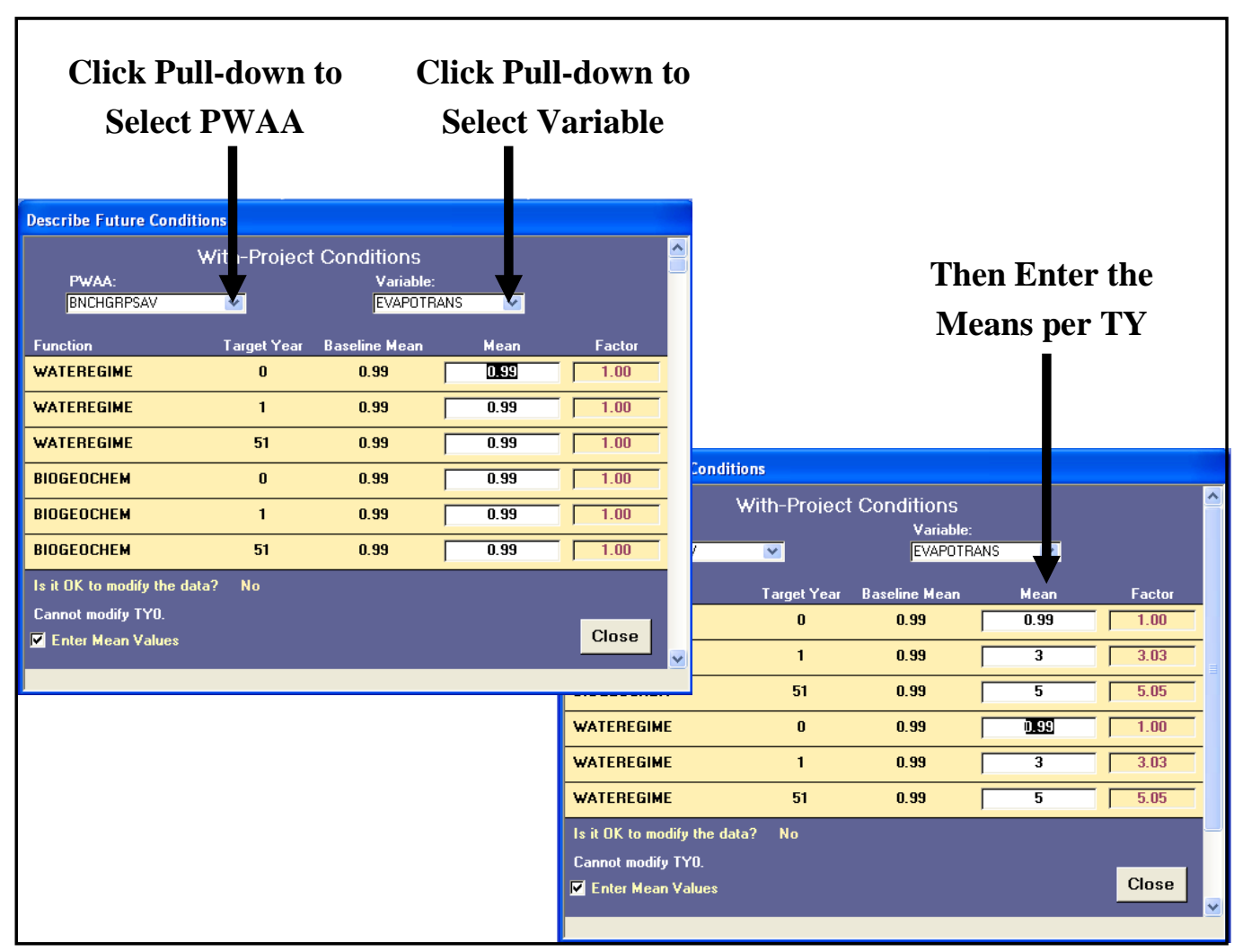

Figure 213. Enter the with-project conditions in EXHGM.

When finished, click on the Close button, and EXHGM asks about saving the data:

\section{Save Projections?}

? Would you like to save the projection data for possible use at a later time?

If you choose to save, you may use this data each time you enter projections by choosing 'No' when asked to initialize the projections table.

If an error was made, click the No button, and all the changes will be discarded. To correct the error immediately, click the Cancel button and EXHGM will return to the data entry interface. If the data were entered correctly, click on the Yes

\section{Successful Copy}

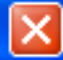

Current projections data saved for later retrieval.

$$
\text { OK }
$$


button, and EXHGM affirms that the information was saved successfully. Click OK and EXHGM returns to the primary interface.

Checking the with-project variable entries with EXHGM reports

To review and check the results, click on the Analysis Reports button(s) on the primary interface (Figure 214).

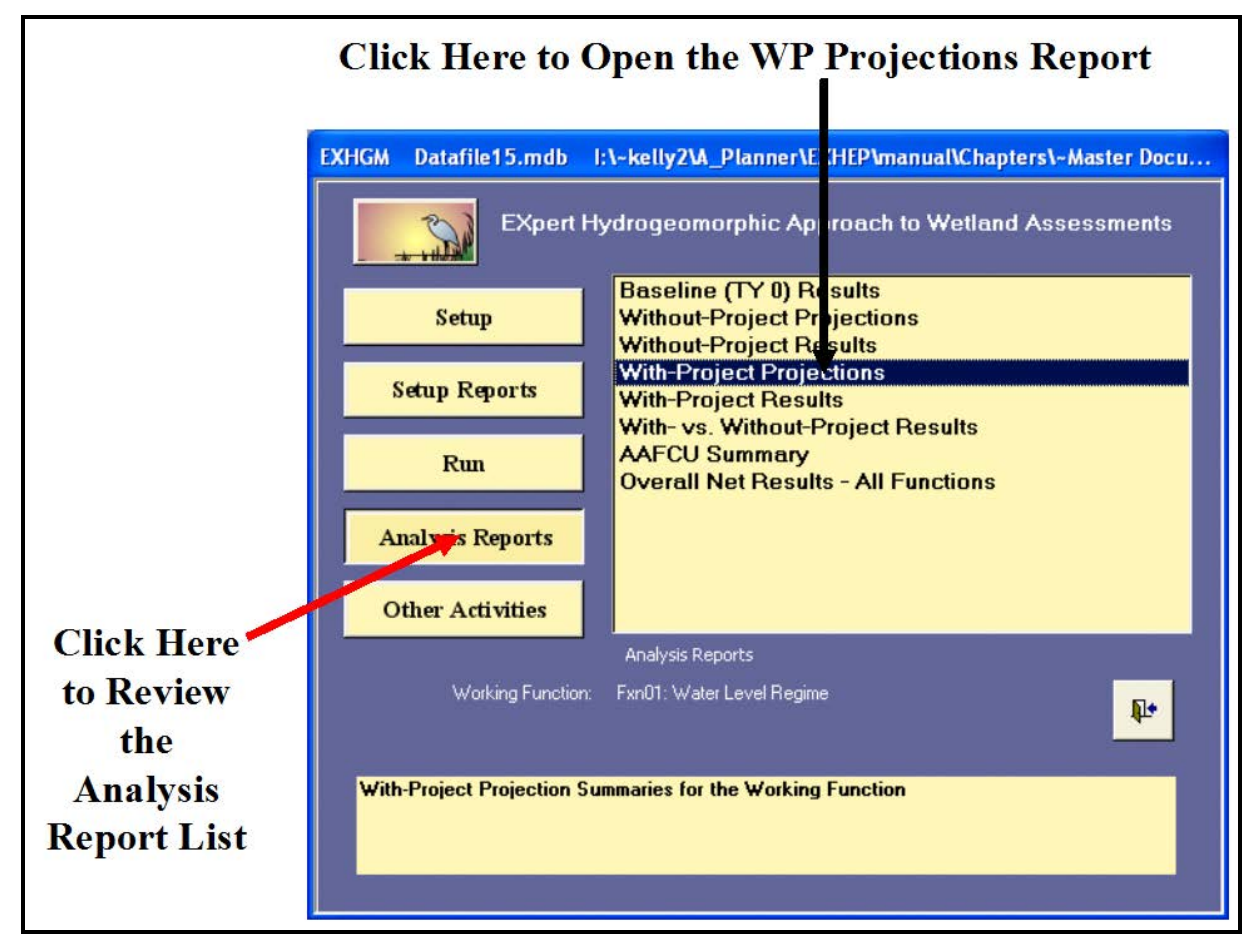

Figure 214. Click on the Analysis Reports button(s) to review the with-project forecasted entries in EXHGM.

A list of available reports appears in the center of the primary interface. The fourth report listed, With-Project Projections, is ready for inspection. Double-click on this report to review the results (Figure 215). 


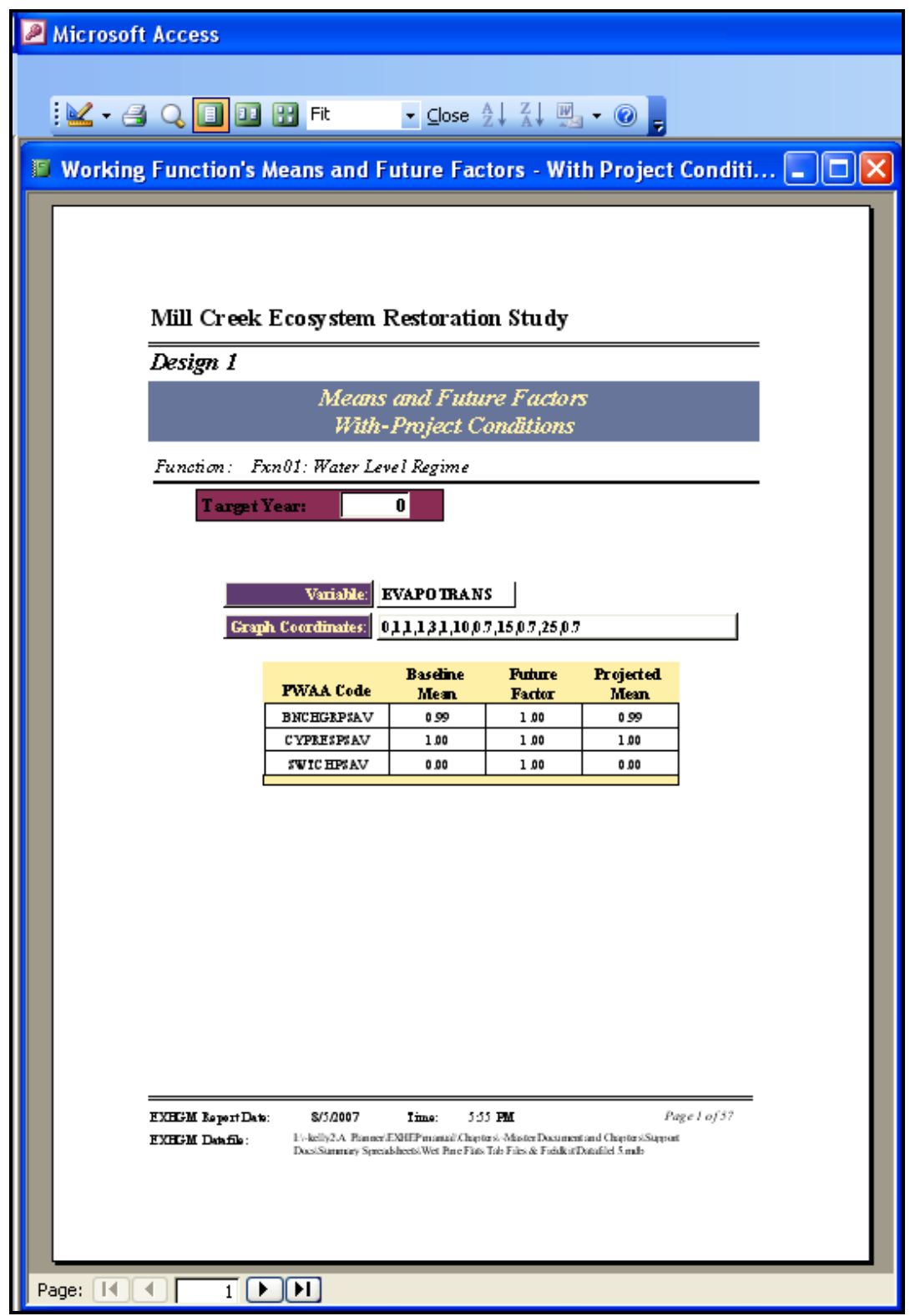

Figure 215. Example of With-Project Projections reports in EXHGM.

Click on the Close button from the suite of buttons offered in the Report Control toolbar, and EXHGM returns to the primary interface. Now that the with-project forecasts have been entered into the system, proceed to the next step to learn how to assess the with-project conditions of the site in EXHGM.

\section{Perform the with-project calculations}

EXHGM will calculate the with-project results on a function-by-function basis. To proceed, click on the Run button on the primary interface (Figure 216). 


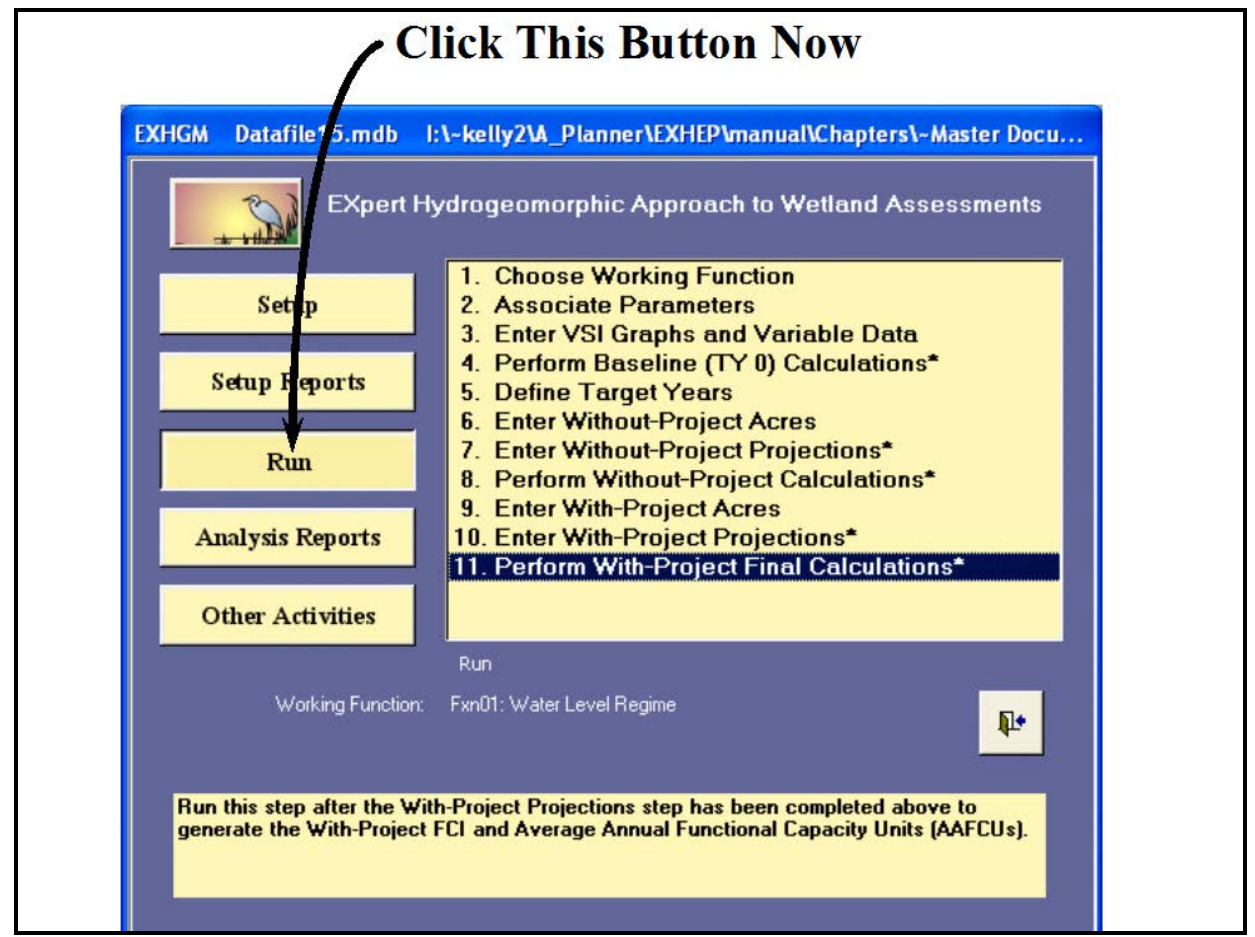

Figure 216. Click on the Run button to begin the with-project calculation process in EXHGM.

As it did before, a screen appears in the center of the primary interface with 11 options:

\section{Choose Working Function}

2. Associate Parameters

3. Enter VSI Graphs and Variable Data

4. Perform Baseline (TY 0) Calculations*

5. Define Target Years

6. Enter Without-Project Acres

7. Enter Without-Project Projections*

8. Perform Without-Project Calculations*

9. Enter With-Project Acres

10. Enter With-Project Projections*

11. Perform With-Project Final Calculations*

Check to assure that the "Working Function" is set to the correct function, and click on the last option (Perform With-Project Calculations*) to continue. In response, EXHGM will begin the calculation process. As the calculations continue, 
a Progress Indicator window will open in the screen. This progress window will track the status of the analysis (number of records to assess versus number of records processed) and provide a tentative estimate of Elapsed Time. When completed, EXHGM

returns a message that the calculations are concluded. This process must be completed for The Working Model's Final With-Project Calculations completed successfully. each function in the analysis.

\section{Exporting the AAFCU results}

Before continuing on to the next function, it is critical that the annualized outputs of the analysis are saved to an MS Word file for documentation purposes (these are overwritten each time the "Working Function" is changed). To begin, click on the Analysis Reports button(s) on the primary interface (Figure 217).

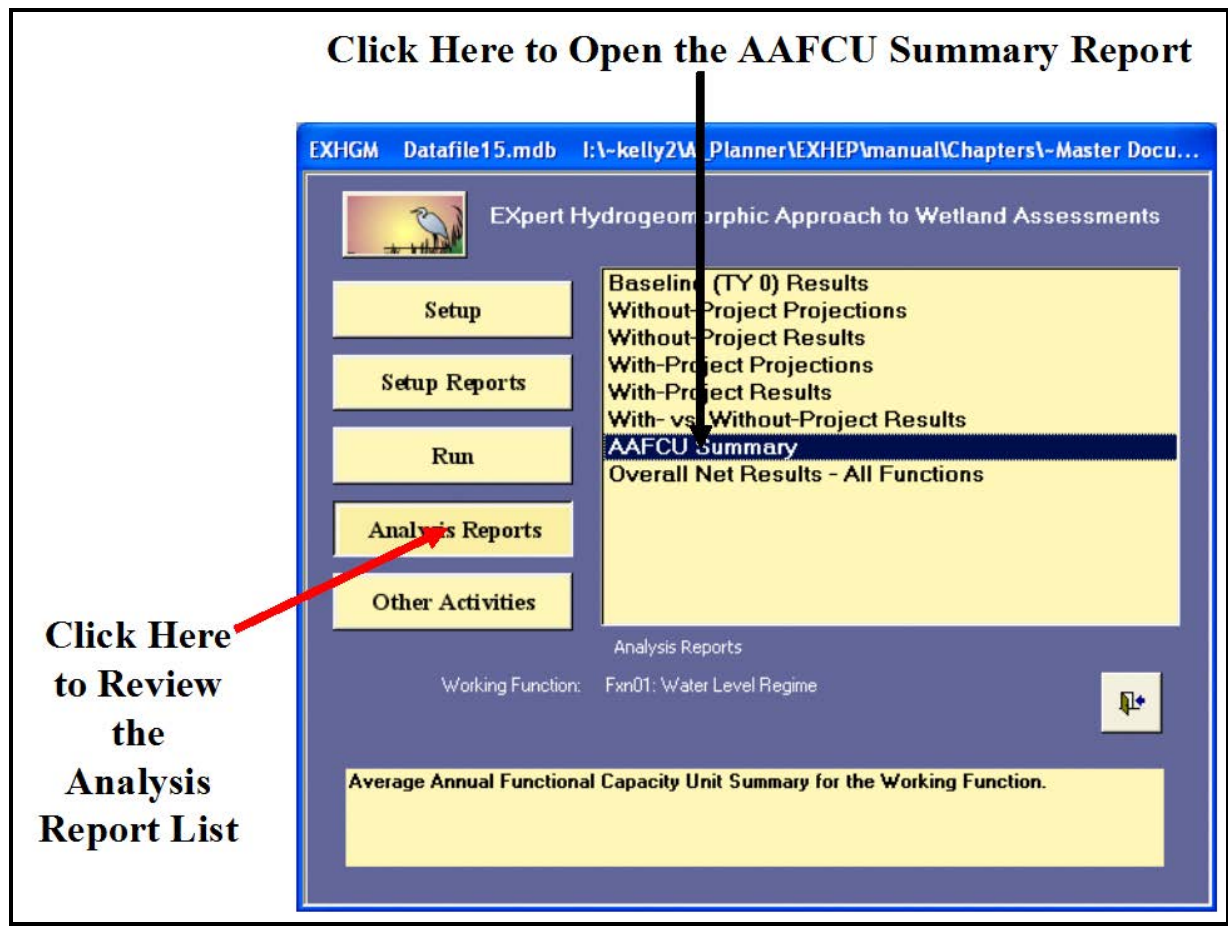

Figure 217. Click on the Analysis Reports buttons to find the AAFCU Summary report in EXHGM. 
A list of available reports appears in the center of the primary interface. The seventh report listed, AAFCU Summary, is ready for inspection and export. Double-click on this report to review the results (Figure 218).

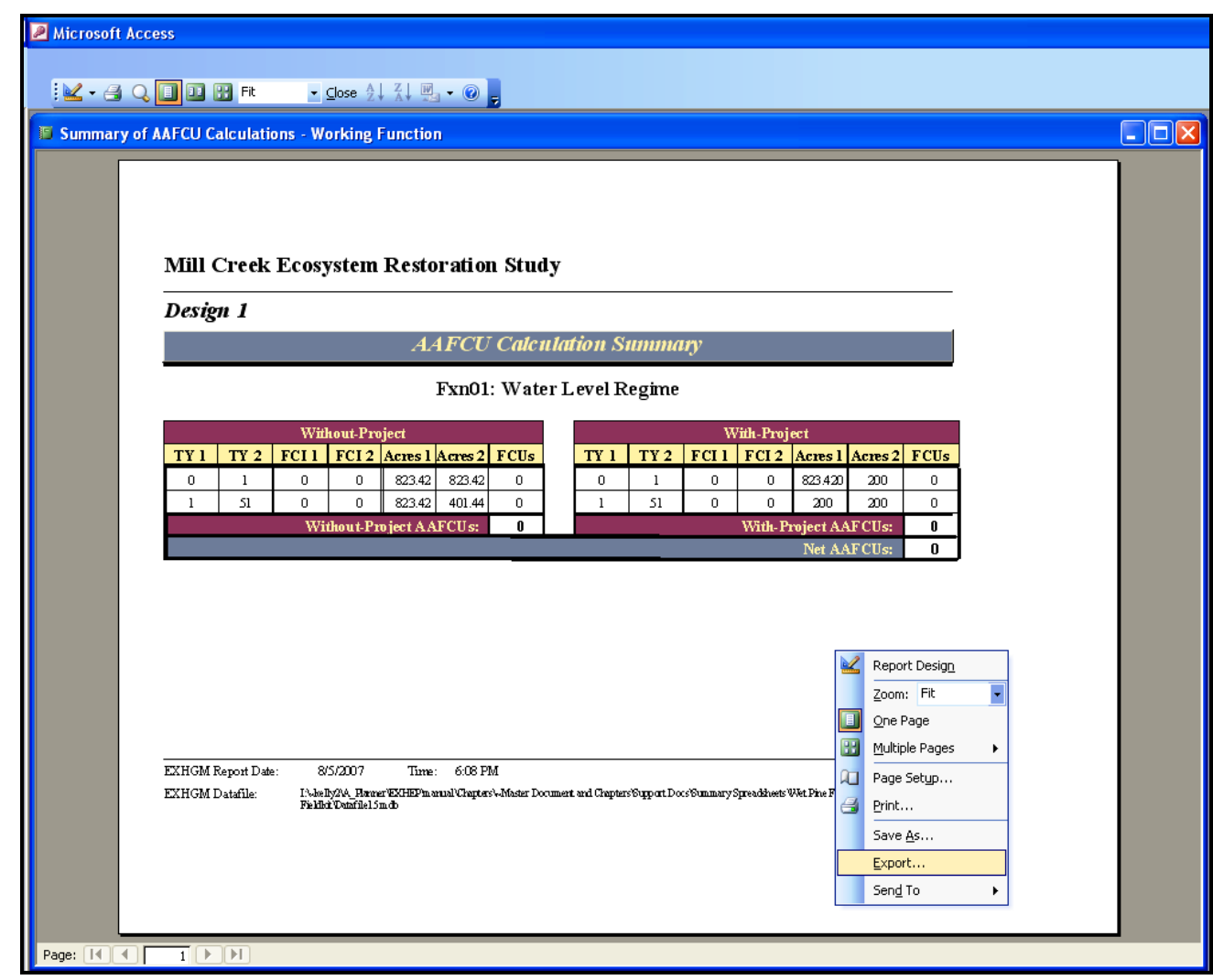

Figure 218. Example of $A A F C U$ Summary reports in EXHGM.

Right-click on the report, and select the Export option from the list of choices presented. An Export Report entry screen will open in response to this action (Figure 219). 


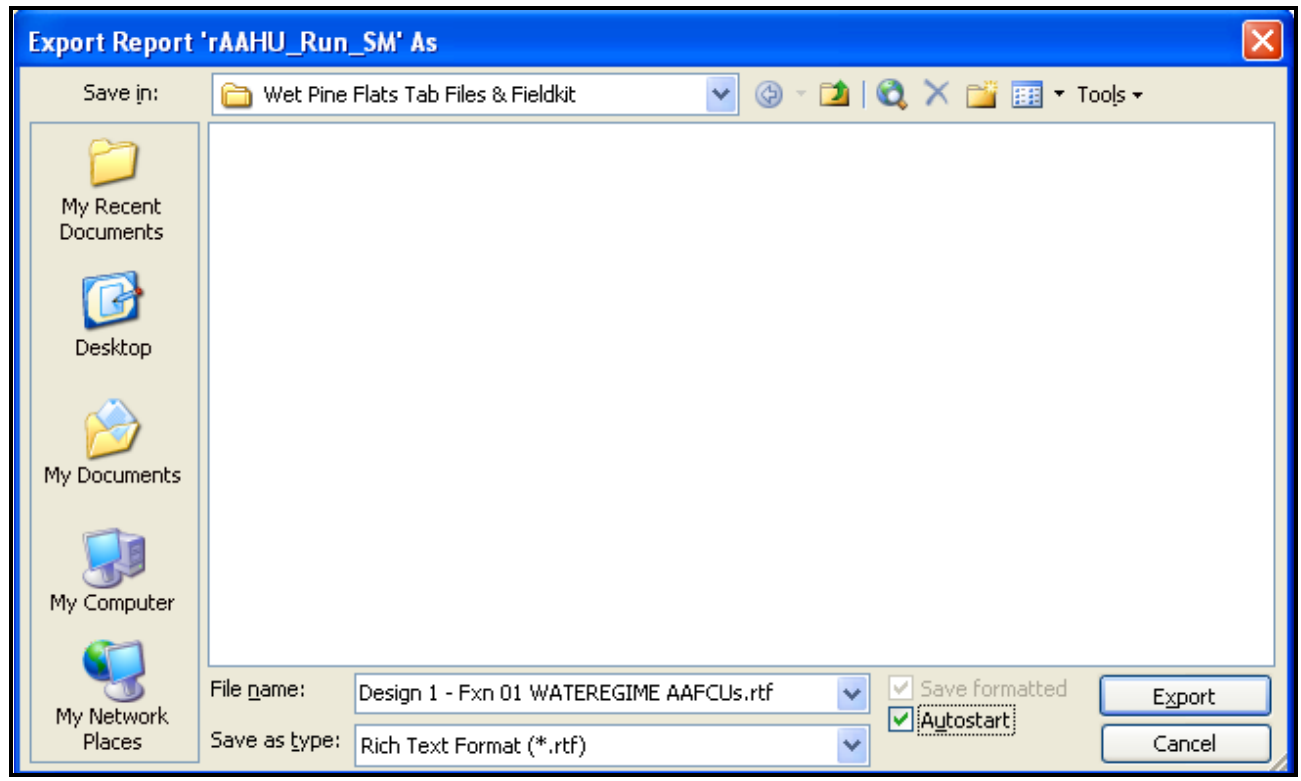

Figure 219. Export Report entry screen - exporting report information to MS Word.

Select the location to export the report to in the Save in: window at the top of the entry screen. Provide a name for the report in the File name: window at the bottom of the entry screen. Use the Save as type: pulldown list to select the type of file to be exported [i.e., choose Rich Text Format (*.rtf) for the word-processing format]. To associate the software (i.e., MS Word for word processing documents), such that the medium opens and displays the information in the new environment upon exportation, click the Autostart checkbox immediately to the right of the Save as type: pull-down list. Press the Export button in the lower righthand corner of the screen. EXHGM will export the data displayed in the report to the new file, and if the Autostart option was clicked, MS Word will open and display the report in the new environment (Figure 220).

Format and save the files as needed, close the MS Word application, and return to EXHGM. Click on the Close button from the suite of buttons offered in the Print Preview toolbar - EXHGM returns to the primary interface. Now proceed to the next section. 


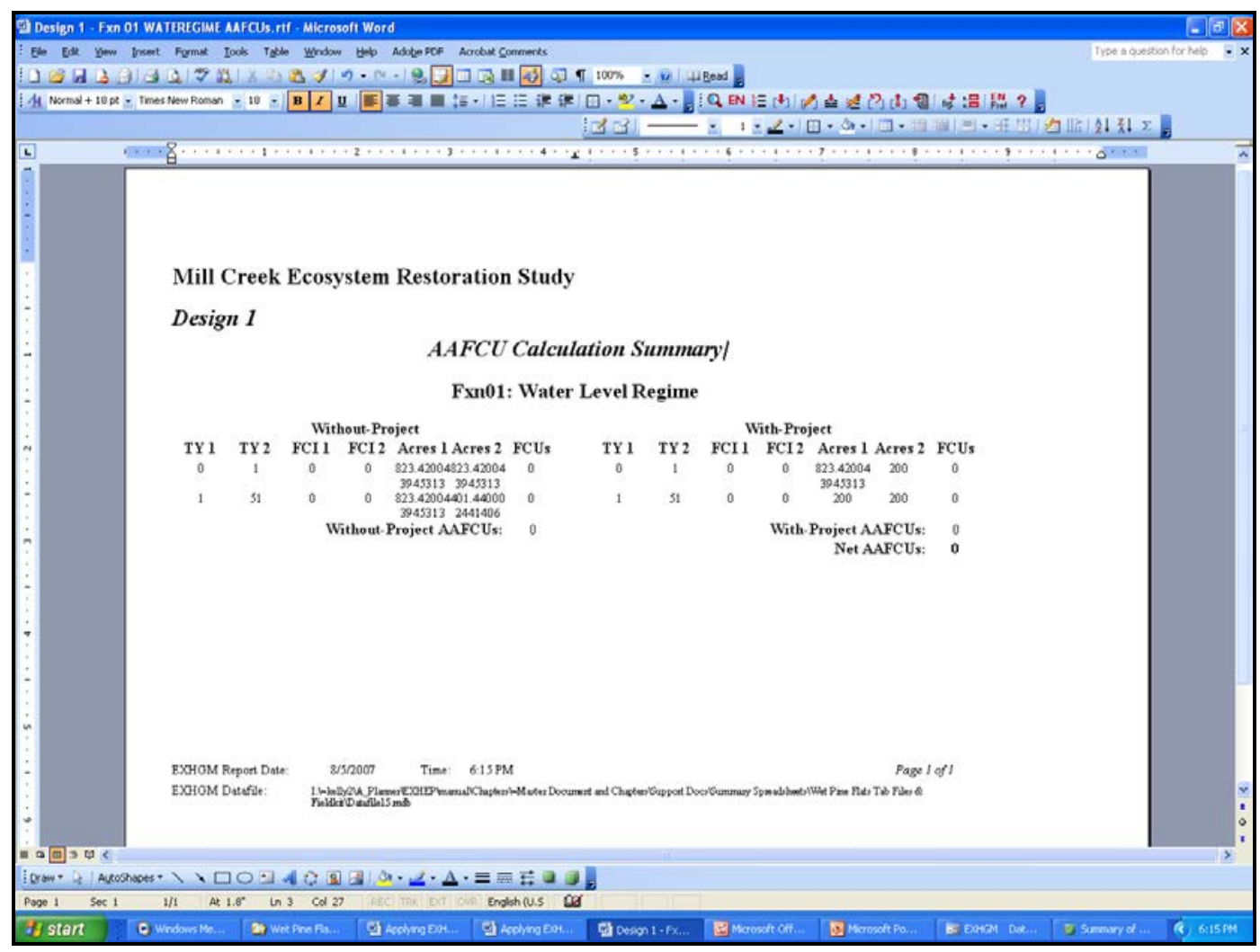

Figure 220. Exported AAFCU summary in the MS Word environment.

Running more functions through the assessment process

Each time a "Working Function" is selected, the options on the primary interface that have stars next to their titles must be re-run to focus the database. The "quick steps" are as follows:

1. Click on 1. Choose Working Function and select the next function for processing.

2. Click on 4. Perform Baseline (TY 0) Calculations*, let the system run, and click $\mathbf{O K}$ when it is finished.

3. Click on 7. Enter Without-Project Projections*, click on the No button for initialization, click the Close button at the bottom of the interface, click Yes to save the data, and click $\mathbf{O K}$ when that save is affirmed.

4. Click on 8. Perform Without-Project Calculations*, let the system run, and click OK when it is finished.

5. Click on 10. Enter With-Project Projections*, click on the No button for

USER'S NOTE:

Remember, when asked about initializing the data in option 7. Enter Without-Project Projections*, answer No or the data inside will be overwritten.

USER'S NOTE:

Remember, when asked about initializing the data in option 10. Enter With-Project Projections*, answer No or the data inside will be overwritten. 
initialization, click the Close button at the bottom of the interface, click Yes to save the data, and click $\mathbf{O K}$ when that save is affirmed.

6. Click on 11. Perform With-Project Calculations, ${ }^{*}$ let the system run, and click $\mathbf{O K}$ when it is finished.

7. Export the AAFCU results to MSWord (i.e., click the Analysis Reports button; double-click the AAFCU Summary report, right-click on the report and select Export; nameand save the file; close MS Word; and close the report).

\section{Checking the with-project analysis with EXHGM reports}

To review and check the results, click on the Analysis Reports button(s) on the primary interface (Figure 221).

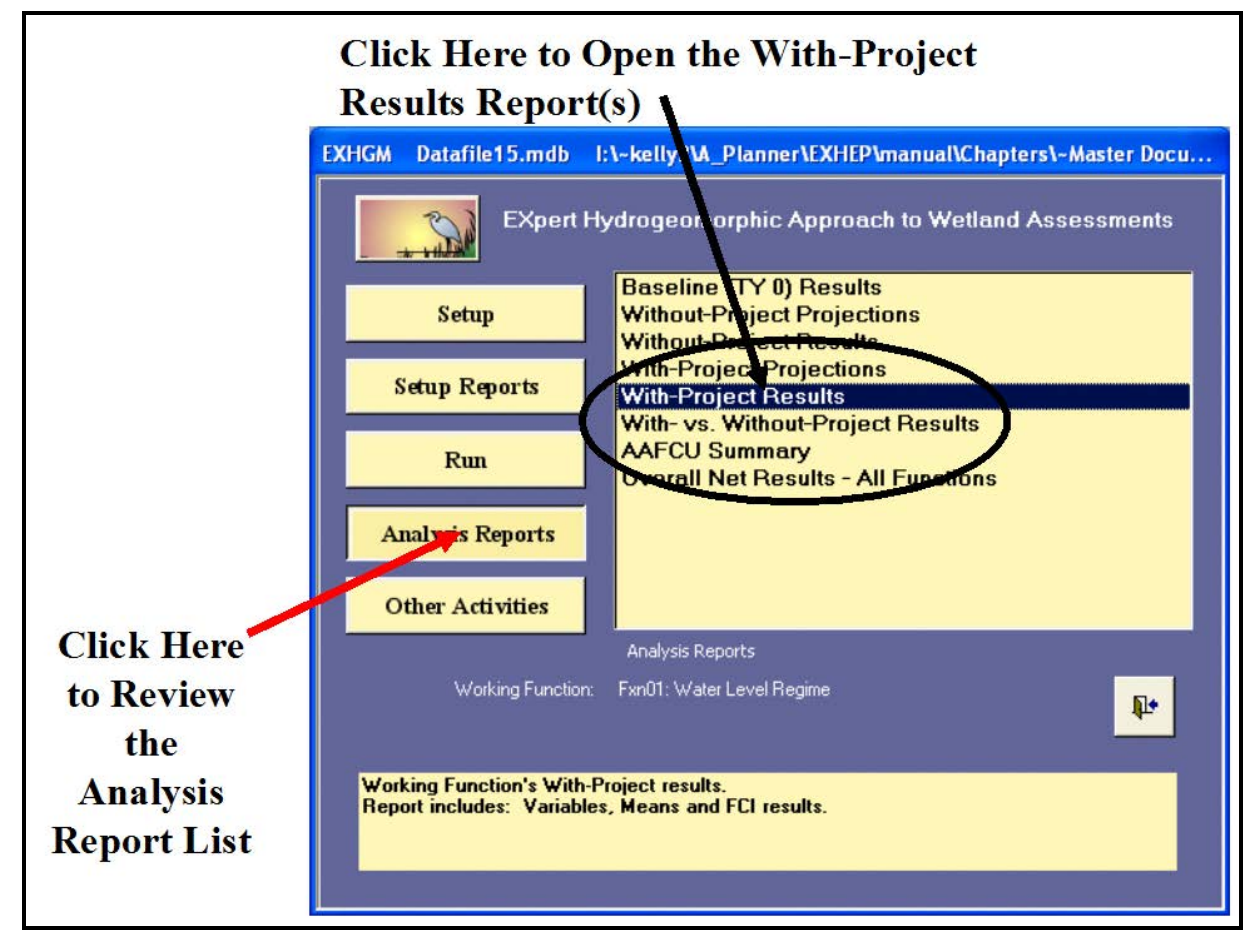

Figure 221. Click on the Analysis Reports button(s) to review the with-project results in EXHGM.

A list of available reports appears in the center of the primary interface. The fifth report listed, With-Project Results, is ready for inspection. Double-click on this report to review the results (Figure 222). 


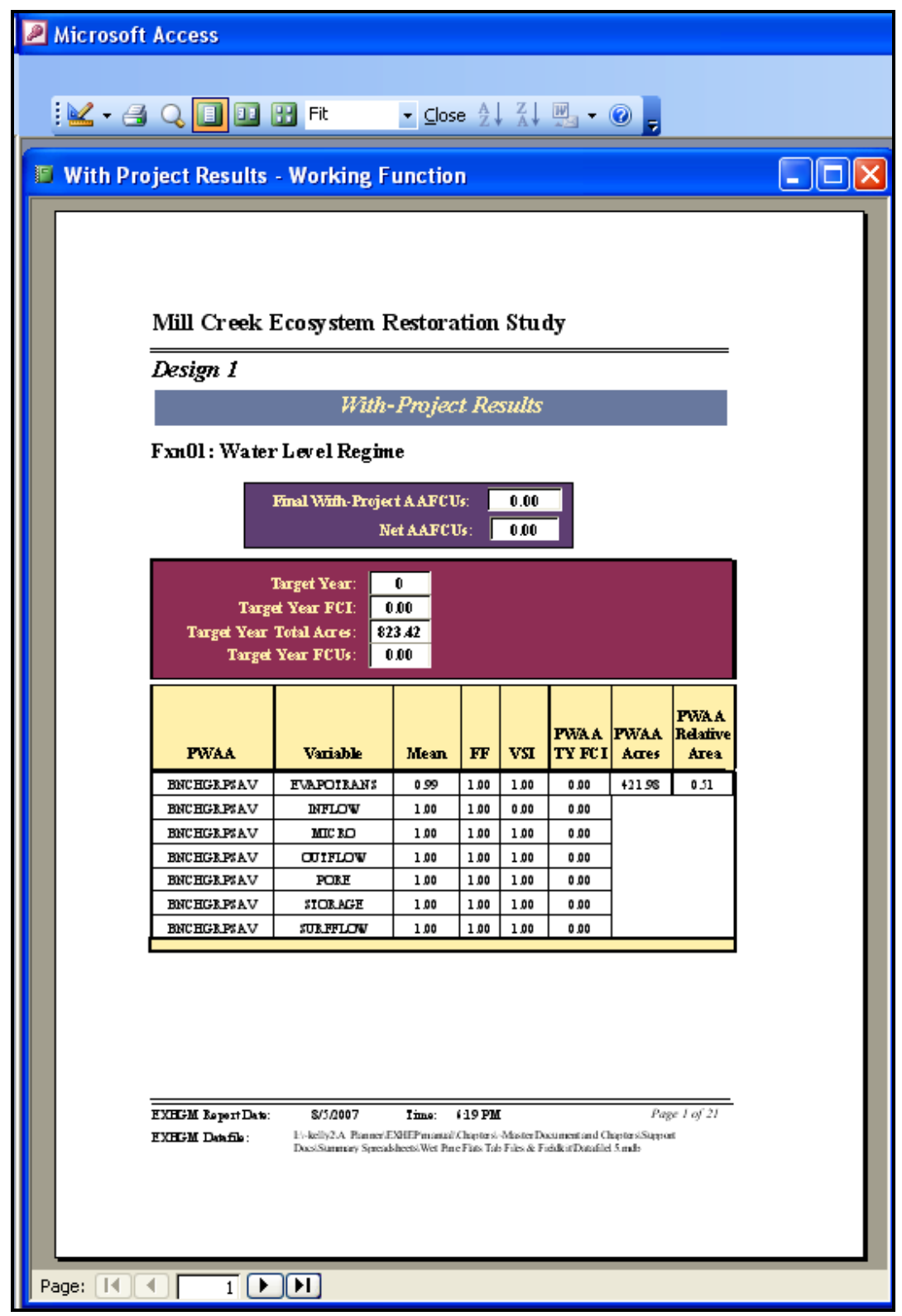

Figure 222. Examples of With-Project Results reports in EXHGM.

The site's overall average annual outputs (i.e., AAFCUs) are reported just under each function's name. The FCI scores, associated acreages, and the resulting FCU scores are then reported on a target year-by-target year basis below the AAFCU values. The specifics used to generate the overall scores are reported in the tables below this summary. These details are sorted alphabetically by PWAA and variable. These reports are cumulative; thus, all functions assessed in the EXHGM analyses are presented herein. Click on the Close button from the suite of buttons offered in the Report Control toolbar, and EXHGM returns to the primary interface. 
A side-by-side report is available to compare the without- and with-project conditions on the same page. Double-click on the With- vs. Without-

Project Results report(s) to review this information (Figure 223).

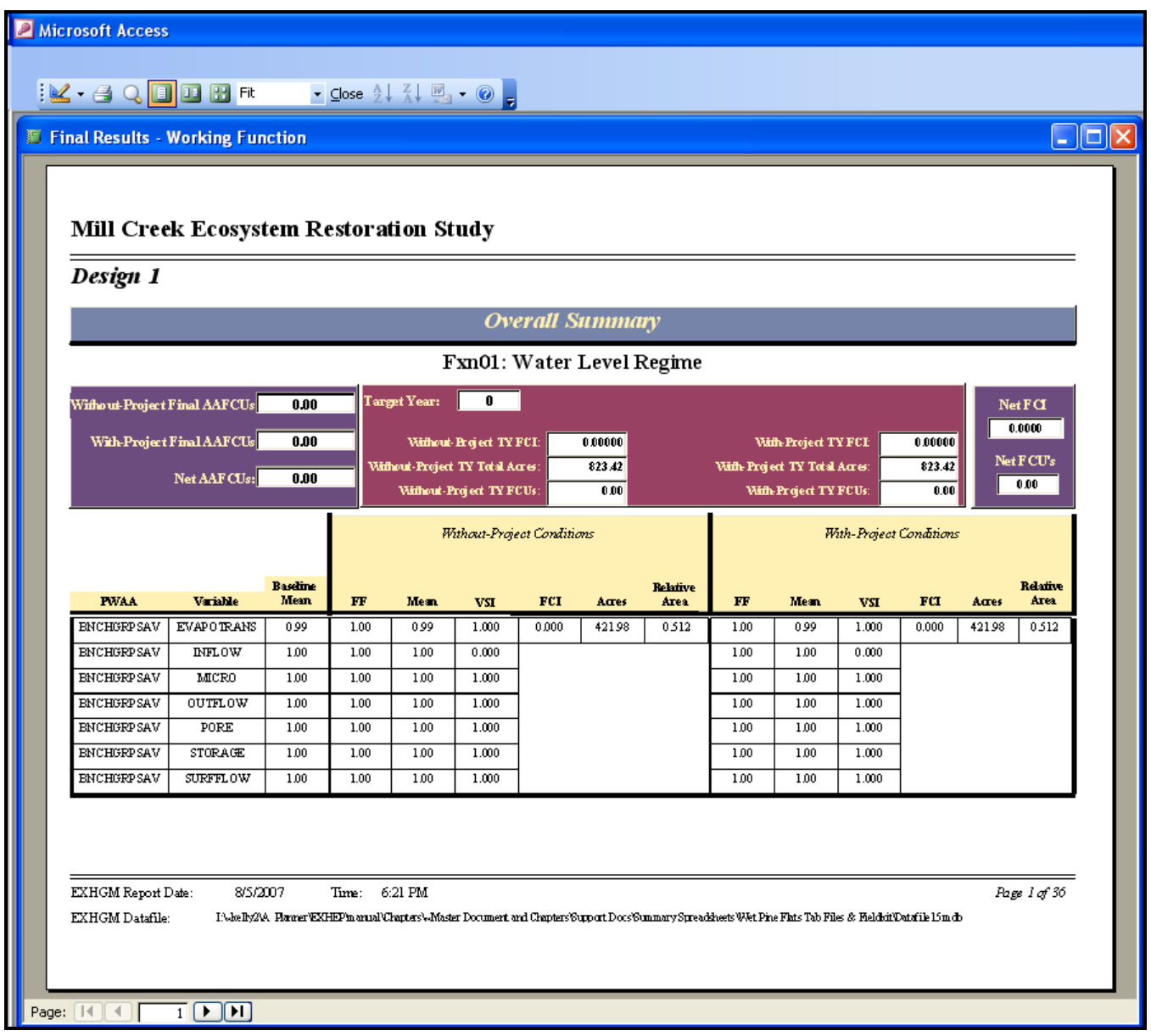

Figure 223. Examples of With- vs. Without-Project Results reports in EXHGM.

Click on the Close button from the suite of buttons offered in the Report Control toolbar, and EXHGM returns to the primary interface.

One final report is available to compare outcomes across the entire suite of functions assessed on the site. Double-click on the Overall Net Results

- All Functions report to review this information (Figure 224). 


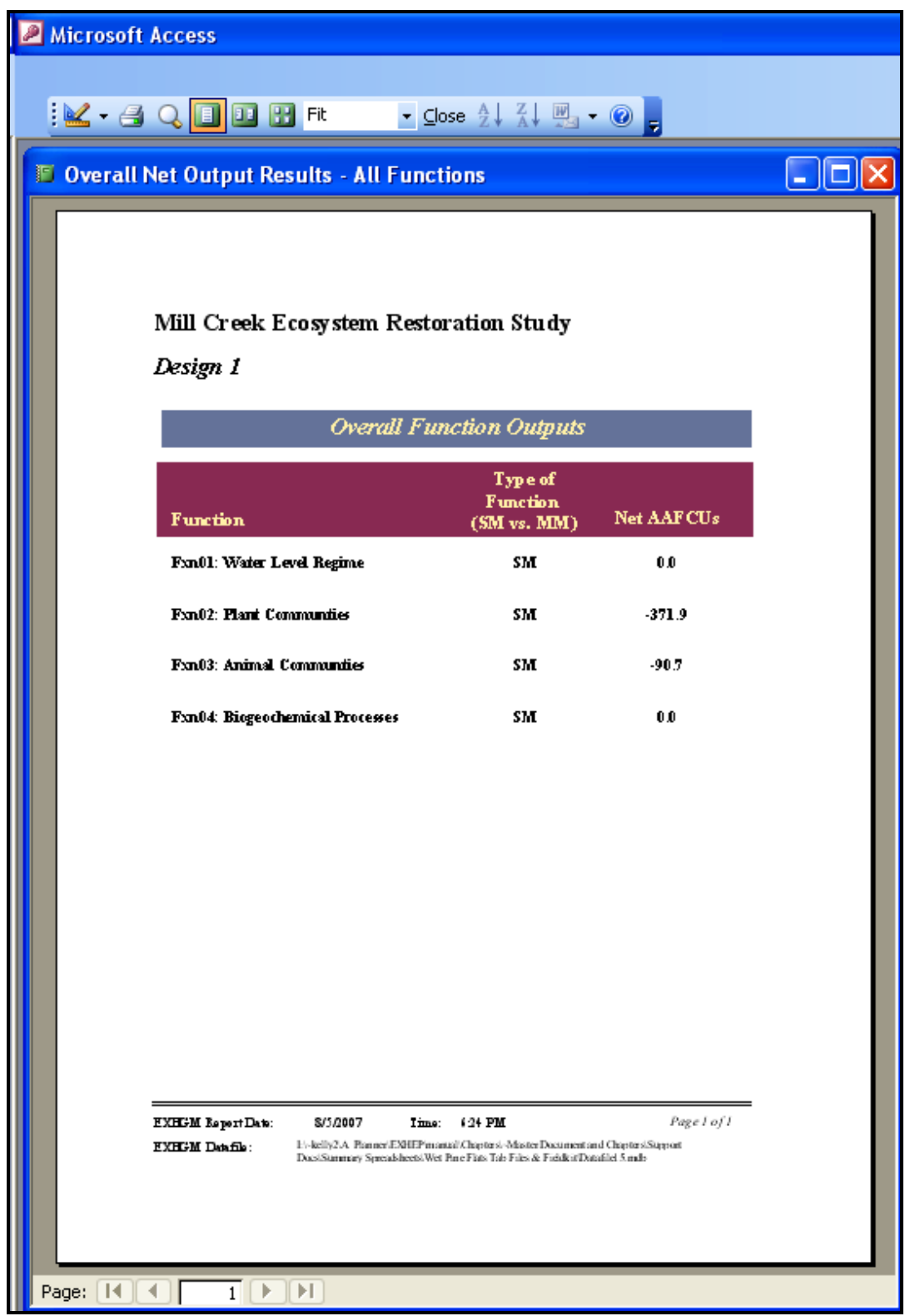

Figure 224. Examples of Overall Net Results - All Functions report in EXHGM.

Click on the Close button from the suite of buttons offered in the Report Control toolbar, and EXHGM returns to the primary interface. 


\section{Step 7: Recycle the datafile and evaluate alternative designs}

Often multiple alternatives are formulated to address the goals and objectives in a single study. The HEAT modules were designed to assess multiple outputs per alternative, and can be used to generate outputs for multiple alternatives per project with a minimum amount of effort. It is not necessary to rebuild the functions or PWAA interdependencies from a blank datafile. Instead, a "master" datafile can be constructed. ${ }^{1}$ This "master" datafile is then copied, re-named, and changed to capture the with-project trends (i.e., alternative variable and PWAA projections are input) of an alternative design. In HEAT this process is referred to as "recycling," and can be performed as many times as necessary to assess the numerous alternatives formulated in a single study (Figure 225). ${ }^{2}$

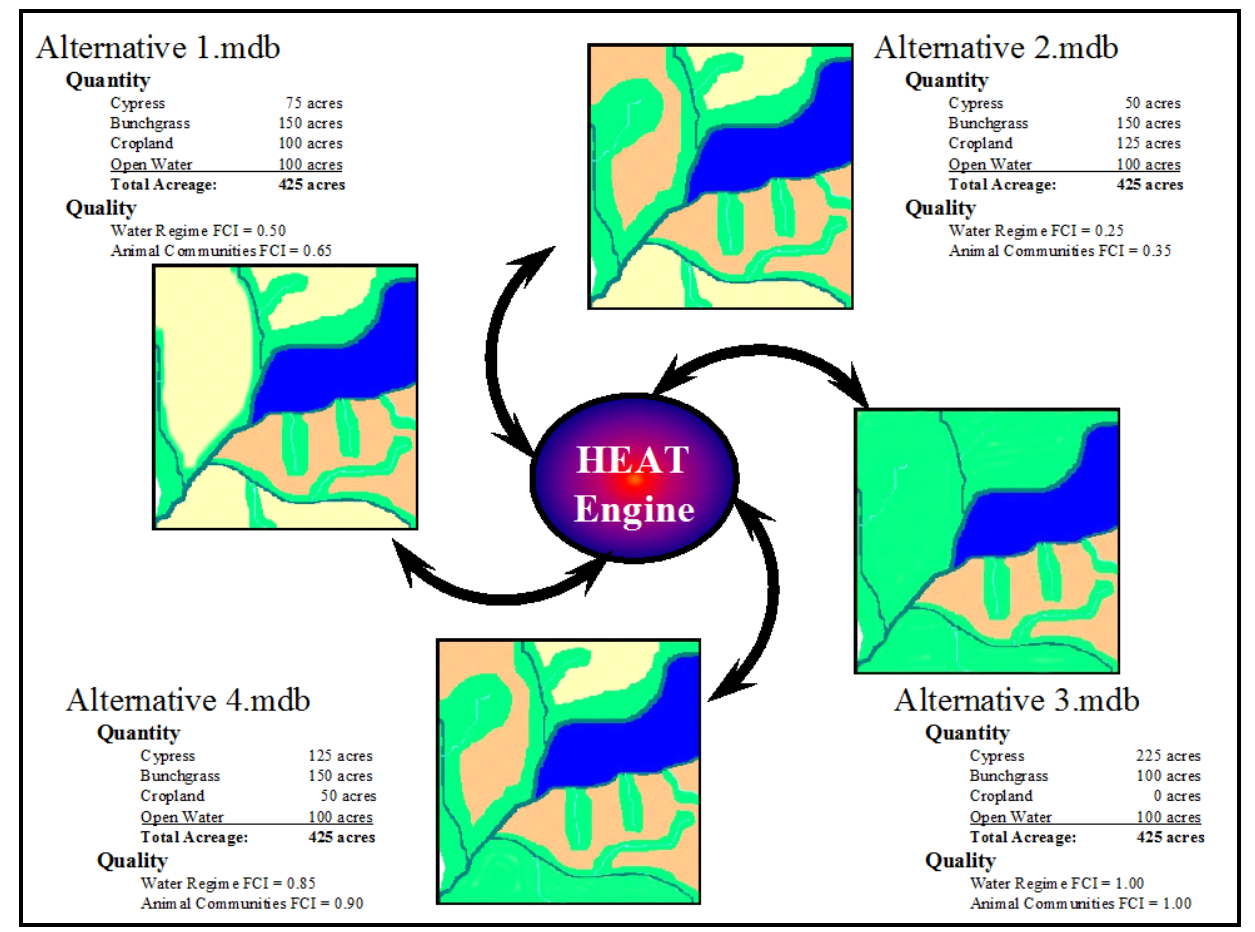

Figure 225. Recycling datafiles in HEAT to streamline the development of alternatives for a single project.

\footnotetext{
${ }^{1}$ A "master" datafile here refers to an EXHGM file that has been run through the entire evaluation of a proposed design alternative (i.e., all functions have been processed through the 11 steps on the primary interface).

2 To assist in the software application, a series of training exercises have been included in Appendix E refer to Exercise 16 for relevant training in master file creation in EXHEP.
} 
To begin, use the steps described in this user guide to construct and assess the first alternative in the study. Copy the "master" file and rename it based on the second alternative in Windows Explorer. Open this new file with HEATEXHGM and change the alternative name in the Methods and Assumptions option. Then change the with-project acreages and variable trends (i.e., 9. Enter With-Project

\section{USER'S NOTE:}

In environmental studies, it is common practice to hold the site size static and maintain a common timescale (i.e., target years) across alternative designs project-wide.

Acres, and 10. Enter With-Project Projections,* respectively) to capture the context of the new alternative. Re-run the calculations and report the outcomes in a cumulative fashion. 


\section{Troubleshooting}

In a perfect world, the earlier chapters should provide a straightforward, comprehensive guide to navigate users through the complexities of ecosystem evaluation with HEAT, but in the real world, an iterative planning process leads to multiple re-evaluation scenarios. As a direct result, users will likely be forced to return again and again to the software ${ }^{1}$ to modify the assessment conditions to better capture the fluid planning environment. As such, two primary issues will likely arise: 1) users will need to alter information "on-the-fly" to capture changes in the planning outlook; and 2) as these changes are made, illegal operations (i.e., user errors) will inevitably cause the system to fail to process commands and the analysis will stall. Over the course of the last 15 years, the software has been tested on many USACE feasibility studies and at numerous software training workshops. In this process, a few lessons or "tricks of the trade" have been learned. The "top ten" lessons learned in applying the software to an ecosystem assessment are listed below.

\section{Lesson 1: Get Organized Early (Outside the Software)}

Before opening the software, the ERDC team usually works out the details of the models in simple MS Excel spreadsheets. One might ask, if I follow this strategy, at what point should I transition into HEAT? Well, in all likelihood, one will have to show baseline results to the evaluation team in order to garner review and feedback of each model's performance. So after going to the field, but before scheduling a baseline results meeting, the model(s) should be transitioned into HEAT. These files are "zero draft" files and should contain nothing to be released to anyone outside the evaluation team; they are "first blush" results of endeavors to characterize the existing conditions for feedback and review. In all likelihood, these files will be thrown out or re-worked to better capture the evaluation team's perceptions of the baseline condition. It may even be appropriate to put off running HEAT until after the without- and with-project trend meetings because the models were so fluid and likely to change, that the effort to enter and modify them in the software may be too time-consuming and the effort might be counter-productive. Remember, future trends do not

\footnotetext{
${ }^{1}$ An installation CD for the Habitat Evaluation and Assessment Tools (HEAT) program can be obtained by contacting Kelly Burks-Copes at 601-634-2290 (Kelly.A.Burks-Copes@usace.army.mil).
} 
depend upon the baseline model results, but rather on the means of the baseline variables, so putting off the model setup in the software is not going to jeopardize the assessment process. The fieldkits provided in the training exercises (Appendix E) represent examples of how a team gets organized outside the software. These fieldkits can be adapted to users' unique situations. The authors advise moving into HEAT only when the models have settled into a stable configuration (i.e., they have been fully reviewed and tested on the reference data - they now characterize the baseline watershed conditions of import and are ready to be put into the software).

\section{Lesson 2: Data Format Incompatibility - Negative Numbers and Modes}

Although the issue of negative data was addressed in Chapters 5 and 6 , users are again reminded that the system cannot handle negative data. To address the problem, normalize the graph coordinates and the data. For example, assume that the operation of a reservoir is critical to the suitability of a particular fish species. The fish are well adapted to conditions +/ - $10 \mathrm{~m}$ within regular operating depths, but below or above these thresholds, the suitability declines (Figure 226).

To normalize the coordinates, add the absolute value of the minimum data point such that the most negative number becomes zero and all other numbers become positive. For example, if the data are -30, -20, -10, 0, $\mathbf{1 0}, \mathbf{2 0}, \mathbf{3 0}$, then the minimum value is $\mathbf{- 3 0}$. Increase all the coordinates by 30 and the new curve breakpoints become: $0,10,20,30,40,50,60$. The data applied to this curve must also be normalized (i.e., add $\mathbf{3 0}$ to each data point) prior to entry into the software.

In this first edition of the HEAT software, it is assumed that each variable's index was developed in relation to the standard means of the data gathered. Thus all data are summed and averaged across the suite of data points collected, and the graph is plotted using direct linear configuration. If for any reason the data must be statistically analyzed in a different fashion (e.g., modes taken instead of means), this calculation must be performed outside the HEAT software, and the result (i.e., the mode) must be entered into software in the space provided. The authors advise that all statistical analyses be performed outside the software (probably necessary anyway to calibrate the index curves), and only the means or modes should be entered into the software. In that way, other statistics important to the model calibration (covariance for example) can be 
calculated, and it is easier to change a single data point (i.e., which is likely to happen as assumptions and iterative planning affect the data analyses) than a series of data points.

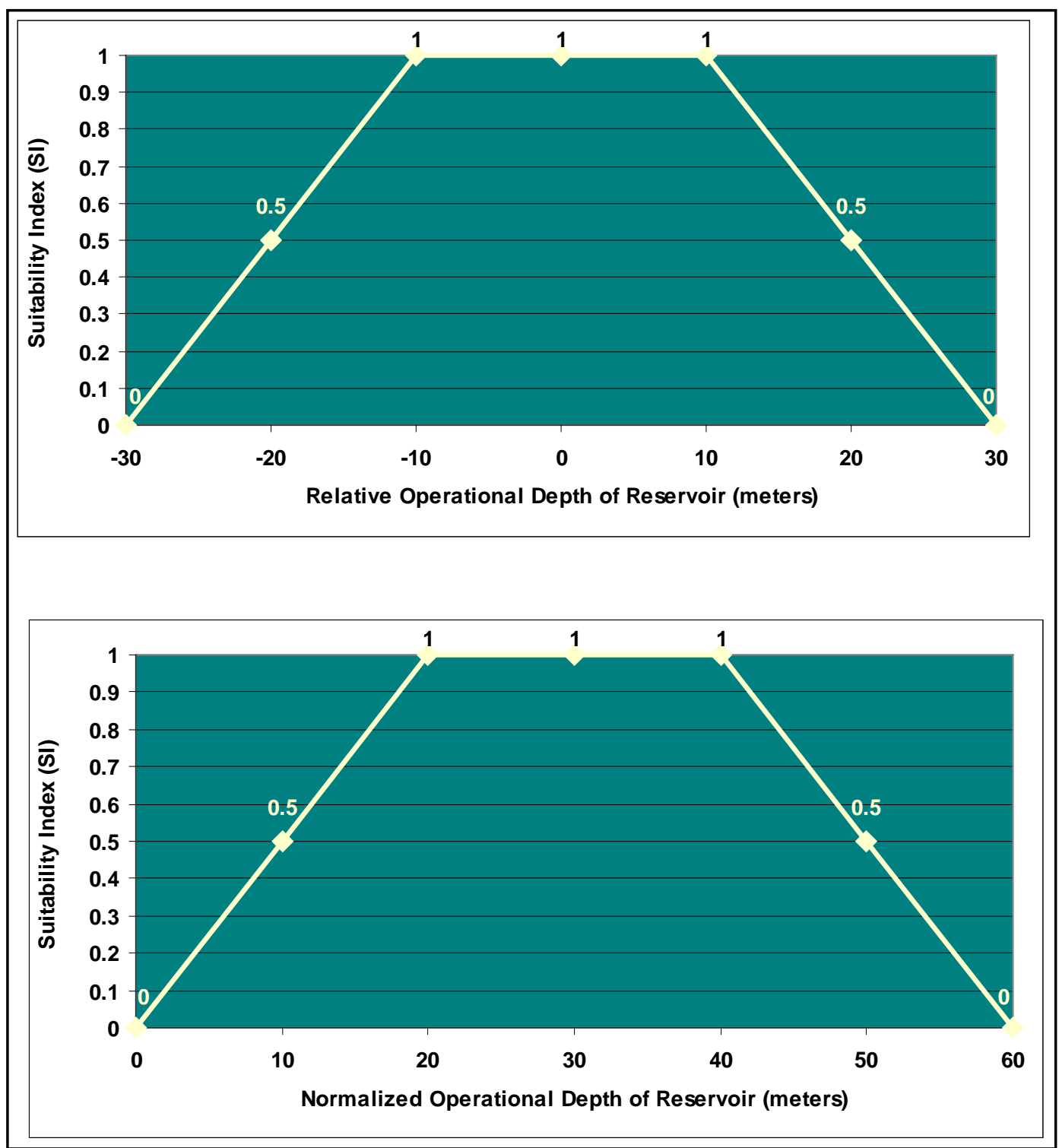

Figure 226. Normalizing negative data for use in the software. 


\section{Lesson 3: Install and Run the Software Locally}

One of the more unusual problems encountered over the past 15 years has been the evolution of networked offices and the storage of software and files on a network rather than on a local machine. Network configurations directly conflict with the functionality of the HEAT software modules. The HEAT software was designed to run on a local machine - both the engine and all alternative data files must be stored on a user's hard drive and cannot be run across a network. In one unusual case, problems were encountered with network printer configurations- in that instance, the software shut down when asked to perform calculations. The problem was solved by switching to a local printer; after that, the system ran fine. If this solution does not work (or if users have access only to network printers), try changing the default printer to a different network printer.

\section{Lesson 4: Make Backups and Organize Datafiles in a Few Key Subdirectories}

This lesson cannot be stressed enough. At some point in the analysis, users will have to revert to an old version of the datafile. It seems a little obvious to suggest making backups and getting organized, but it is surprising how often a little amount of maintenance and forward thinking can improve efficiency. It takes only a few seconds to make a backup - it could take hours to rebuild a database. Also, users may need to revert to the backup if the model modification process is unsuccessful, and error messages arise.

The Other Activities options are extremely powerful, and therefore extremely sensitive to user actions. Often, the system halts if the datafiles are stored too deep into hierarchical subdirectories - too much organization is not effective either. The authors suggest storing the datafiles loosely - only a few layers deep into the subdirectories (a good general rule of thumb is no deeper than three subdirectories). If an error is thrown, close the software, reduce the number of subdirectory levels, and re-open the datafile (with the HEAT engine) from the new subdirectory. 


\section{Lesson 5: Use the HEAT Engine - Do Not Open the Datafile with MS Access}

The most common error found to date is quite simple - users continue to attempt to open a datafile from MS Explorer or with MS Access rather than using the HEAT analysis engine and the datafile pops open without the interfaces to run the software (Figure 227).

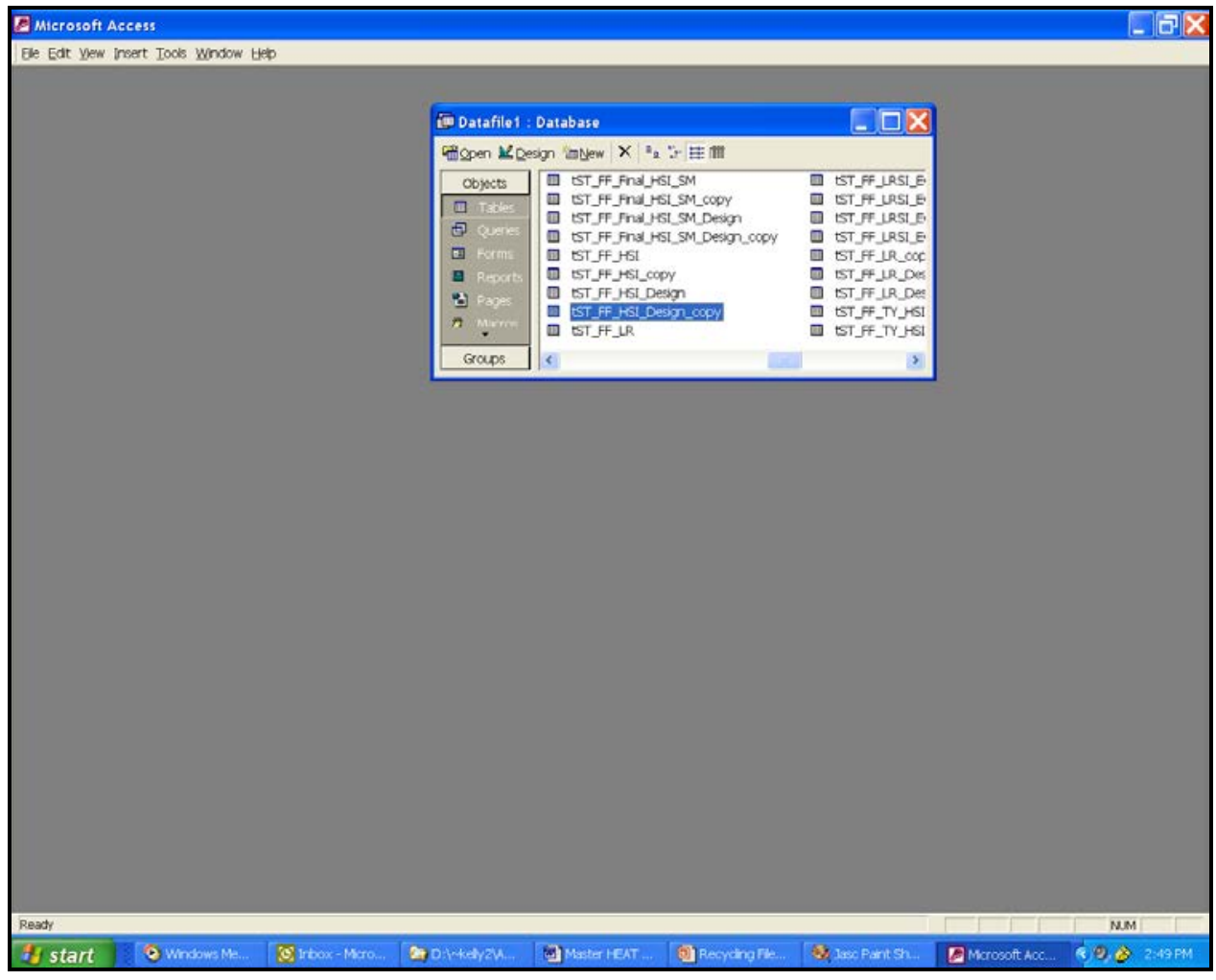

Figure 227. Opening a datafile incorrectly.

If this happens, close MS Access, and start the HEAT engine correctly:

1. Click on the Start button.

2. Move the cursor up to the All Programs option.

3. Scroll the menu to HEAT-Habitat Evaluation and Assessment Tools.

4. Hold down the mouse button, move across to HEAT and click.

5. Identify the datafile name and location. 


\section{Lesson 6: Error Messages and Installation Problems}

If reports open with blacked-out title bars, or an error message is "thrown," close and re-start the software. If the error occurs again, a re-boot might solve the problem. The authors welcome contact from users when these simple solutions do not correct the problem. Before calling, try checking the following issues:

1. Make a screen capture of the error (or write it down) and be prepared to email it (along with a zipped copy of your datafile).

2. Make sure the HEAT software (USACE_HEAT.mdb) and all the associated datafiles (including Heproll.mdb in the root directory) are housed on a local drive (not shared on a network drive) and their attributes are set as "read/ write," NOT "read only."

3. Users will probably be asked what version of MSWindows and Office they are running, and users should ensure that they have installed the latest updates to these products.

4. If users are having problems with installation, in all likelihood they do not have administrative privileges. Before contacting the authors, make sure these privileges are in place, or that support staff is on hand to allow operation under the administrator environment.

5. If running under the Vista operating system, run this installation by browsing to the setup.exe file on the $\mathrm{CD}$, right-clicking on the setup.exe file, and selecting "Run as Administrator."

6. The security settings of USACE's IWR-Plan software (versions prior to IWR Planning Suite 2007) may restrict aocess to any MS Access software package - including HEAT. To solve this problem, contact the authors of this report to troubleshoot this problem.

\section{Lesson 7: Printing and Export Issues}

Printing is the key to conveying the results of the analysis to an evaluation team and customers. Several things can go wrong during the printing process, as listed below, along with simple solutions: 
1. If users open a report and it does not populate (i.e., the basic template is shown, but no numbers are filled into the cells), in all likelihood the Project Name and Alternative Name in the Methods and Assumptions section have not been
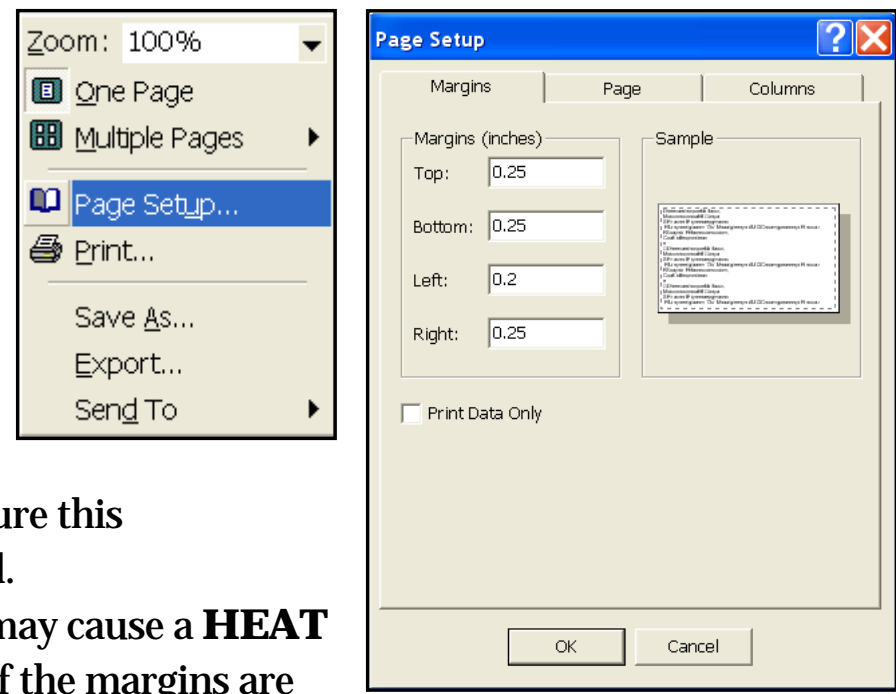

filled out. Go back and make sure this information has been provided.

2. Some default printer settings may cause a HEAT report to span multiple pages if the margins are too narrow. To fix this problem, right-click on the report, and select Page Setup from the list of options. Reset the margins, column widths, and page sizes as necessary using the Margins, Page, and Columns tabs to customize the report for the printing environment.

3. When using Officelinks to export the reports to MS Excel, some of the information (in particular the summary information) is not exported out of MS Access. As this is a Microsoft issue, and not a HEAT issue, the only suggestion is to export the information out to MSWord first, then block and copy the information into MS Excel for formatting.

\section{Lesson 8: Delete a Model by Working Backwards}

The first thing to realize about the HEAT software is that the "backbone" of the program is a relational database. Any change made in one interface is likely to have a domino effect database-wide. To that end, the "trick" to deleting a model is to work backwards.

1. Open the graphs and baseline data information first, and delete ${ }^{1}$ the data and graphs coordinates (Run button).

2. Open the associations next, and delete the associated records for the model (Run button).

3. Open the variables and delete them (if they are not used by another model) (Setup button).

4. Open the cover types and delete them (if they are not used by another model) (Setup button).

5. Open the life requisites (if applicable) and delete them (Setup button).

\footnotetext{
1 Look to Chapters 5 and 6 for details regarding the deletion of components.
} 
6. And finally, open the models and delete them (Setup button).

Deleting each component of the model is dictated by the interfaces in which they are presented - be sure to take advantage of the Delete keys when they are available. To completely remove the model from the analysis, the without- and with-project information must be re-initialized and re-run. This of course means that the future projections must be reentered into the projection screens.

\section{Lesson 9: Change a Model by Working Backwards}

Again, remember that changing the information in one interface has farreaching effects database-wide. The simple act of changing a cover type name or variable code will cause the system to disassociate the information from the model. The "trick" again is to work backwards.

1. Remove all reference to the parameter prior to changing the name or code of a variable, cover type, life requisite, or model (i.e., look to Lesson 1 above).

2. Make the change. If the change was in graph coordinates for baseline data, move to the next step. If the change affects the model formulas, re-associate the information first, and then move to the next step.

3. Re-run the analysis (which includes the dreaded re-initialization and re-entry of the data).

\section{Lesson 10: Not Everything Works the Same Way in the Software}

The software was designed to be somewhat flexible - certain model components can be altered without removing them entirely from the database. Here's the list of components that can be altered quickly:

1. Acres (baseline, without- and with-project) can be changed without having to remove the cover types or re-initializing the system. Simply make the changes and re-run the numbers.

2. Without-project and with-project variable trends can be changed without having to remove model components (i.e., variables, associations, curves, etc.) and the system does not need to be re-initialized. Again, make the changes and re-run the numbers.

3. Methods and alternative names can be changed without having to reinitialize. 
Below is a short list of components that require a little more effort to modify:

1. Changes to baseline information (model names, associations, cover types, variables, graph coordinates, baseline data etc.) require re-initialization and re-entry of without- and with-project projections.

2. Changes to target years are significant - the system completely removes any association with the target year of concern. Again, do not simply type in the new information and expect the system to track the change. Delete the target year, and re-enter the newvalues. Re-initialization and re-entry of projection trends are required to capture these changes.

At this point, it is probably appropriate to re-visit the "rules" for initialization:

USER'S NOTE:

Say "Yes" to initialization if:

1. Variable projections have not been made for any model.

2. Variable projections have been made for single formula models, but multiple formula models are now the focus (i.e., each "type" must experience initialization once).

3. Baseline data have been modified after an initialization.

4. Target years have been modified after an initialization.

5. Model associations have been modified after an initialization.

6. Models (functions), cover types (PWAAs), life requisites and/or variables have been added/deleted/modified post-initialization (basically any change in Setup necessitates re-initialization).

Say "No" to initialization when:

1. Variable projections have been made for at least one model in the "type."

2. Modifying current projection trends - post-initialization.

3. Running models (functions) through the without- and/or with-project calculations in series.

4. Modifying acreages in the baseline, without- and/or with-project interfaces.

This summarizes the top 10 lessons learned in deploying and applying the HEAT software. The authors hope these tools serve users well, and users are invited to contact the authors directly if these solutions do not address their problems. Comments and critiques of these tools are welcomed and discussion with regards to upgrades or changes to the system are invited. St. Louis, Los Angeles, Galveston, Mobile, Chicago, Tulsa, Omaha, Albuquerque, and Kansas City USACE Districts have all received training 
in beta versions ${ }^{1}$ of the software and are currently or have recently used the software to assess feasibility studies in their areas. The basic software testing premise has been to apply the software modules to real-world problems, and the authors are forever grateful to these Districts for taking the risk on a new tool, and for their ongoing support in this venture. Users are encouraged to contact the authors to discuss ecosystem assessments, and the authors can offer guidance and suggestions with regards to all aspects of the planning process.

\footnotetext{
${ }^{1}$ Release notes have been included with the installation CD. All users with beta versions of the software
} should uninstall and reinstall the HEAT software using Control Panel-Add or Remove Programs. 


\section{References}

Adamus, P. R., Eco-Analysis, Inc., E. Clairain, R. D. Smith, and R. E. Young. 1987. Wetland evaluation technique (WET), Volume II. Technical Report Y-87. Vicksburg, MS: U.S. Army Engineer Waterways Experiment Station.

Angermeier, P. L., and J . R. Karr. 1994. Biological integrity versus biological diversity as policy directives: Protecting biotic resources. Bioscience 44:690-697.

Barry, D., R. A. Fischer, K. W. Hoffman, T. Barry, E. G. Zimmerman, and K. L. Dickson. 2006. Assessment of habitat values for indicator species and avian communities in a riparian forest. Southeastern Naturalist 5:295- 310.

Bormann, F. H., and G. E. Likens. 1969. The watershed-ecosystem concept and studies of nutrient cycling. In The ecosystem concept in natural resources management, ed. G. M. VanDyne, 49- 76. New York: Academic Press.

Brinson, M. M. 1993. A hydrogeomorphic classification for wetlands. Technical Report WRP-DE-4. Vicksburg, MS: U.S. Army Engineer Waterways Experiment Station.

. 1995. The HGM approach explained. National Wetlands Newsletter, November-December: 7- 13.

. 1996. Assessing wetland functions using HGM. National Wetlands Newsletter, January-February: 10- 16.

Brooks, R. P. 1997. Improving habitat suitability index models. Wildlife Society Bulletin 25:163- 167.

Brown, S. K., K. R. Buja, S. H. J ury, M. E. Monaco, and A. Banner. 2000. Habitat suitability index models for eight fish and invertebrate species in Casco and Sheepscot Bays, Maine. North American J ournal of Fisheries Management 20:408- 435.

Burgman, M. A., D. R. Breininger, B. W. Duncan, and S. Ferson. 2001. Setting reliability bounds on habitat suitability indices. Ecological Applications 11:70- 78.

Cole, C. A., and J . G. Kooser. 2002. HGM: Hidden, gone, missing? National Wetland Newsletter 24(2):15- 18.

Conservation Foundation. 1988. Protecting America's wetlands: An action agenda. Final report of the National Wetlands Policy Forum. Washington, DC: The Conservation Foundation.

Cooperrider, A. Y. 1986. Habitat evaluation systems. In Inventory and monitoring of wildlife habitat, ed. A. Y. Cooperrider, R. J . Boyd, and H. R. Stuart, 757- 776. Denver, CO: U.S. Department of Interior and Bureau of Land Management Service Center.

Dale, V. H., and S. C. Beyeler. 2001. Challenges in the development and use of ecological indicators. Ecol. Indicators 1:3- 10. 
Felix, A. B., H. Campa, K. F. Millenbah, S. R. Winterstein, and W. E. Moritz. 2004. Development of landscape-scale habitat-potential models for forest wildlife planning and management. Wildlife Society Bulletin 32:795- 806.

Gillenwater, D., T. Granata, and U. Zika. 2006. GIS-based modeling of spawning habitat suitability for walleye in the Sandusky River, Ohio, and implications for dam removal and river restoration. Ecological Engineering 28:311- 323.

Groves, C., L. Valutis, D. Vosick, B. Neely, K. Wheaton, J. Touval, and B. Runnels. 2000. Designing a geography of hope: A practitioner's handbook to ecoregional conservation planning, Volumes 1 and 2, 2nd ed. Conserveonline.

Groves, C. R. 2003. Drafting a conservation blueprint: A practitioner's guide to planning for biodiversity. Washington, DC: Island Press.

Groves, C. R., D. B. J ensen, L. L. Valutis, K. H. Redford, M. L. Shaffer, J . M. Scott, J . F. Baumgartner, J. V. Higgins, M. W. Beck, and M. G. Anderson. 2002. Planning for biodiversity conservation: Putting conservation science into practice. BioScience 52:499-512.

Gutzwiller, K. J ., and S. H. Anderson. 1987. Habitat suitability index models: Marsh wren (Cistothorus palustris). Biological Report 82(10.139). Washington, DC: U.S. Department of the Interior, Fish and Wildlife Service.

Hays, R. L. 1987. A users manual for micro-HSI, habitat suitability index modeling software for microcomputers, Version 2. Ft. Collins, CO: U.S. Department of the Interior, Fish and Wildlife Service, National Ecology Center.

Higgins, J ., M. Lammert, and M. Bryer, ed. 1999. Designing a geography of Hope. Update \#6: Including aquatic targets in ecoregional portfolios: Guidance for ecoregional planning teams. Arlington, VA: The Nature Conservancy, obtained from: http://www.geographyofhope.org/frm.htm (Accessed on 26 J une 2007).

Hirzel, A. H., G. Le Lay, V. Helfer, C. Randin, and A. Guisan. 2006. Evaluating the ability of habitat suitability models to predict species presences. Ecological Modelling 199:142- 152.

Hruby, T. 1997. Continuing the discussion: Scientific and technical issues regarding the hydrogeomorphic approach to function assessment of wetlands. Society of Wetland Scientists Bulletin 14(3):23-24.

. 2001. Testing the basic assumption of the hydrogeomorphic approach to assessing wetland functions. Environmental Management 27(5):749- 761.

Inglis, G. J ., H. Hurren, J . Oldman, and R. Haskew. 2006. Using habitat suitability index and particle dispersion models for early detection of marine invaders. Ecological Applications 16:1377- 1390.

Kapustka, L. A. 2003. Rationale for use of wildlife habitat characterization to improve relevance of ecological risk assessments. Human and Ecological Risk Assessment 9:1425-1430.

. 2005. Assessing ecological risks at the landscape scale: Opportunities and technical limitations. Ecology and Society 10:Article 11. 
Kusler, J . A., and W. Niering. 1988. Wetland assessment: Have we lost our way? National Wetlands Newsletter 20:8-14.

Morreale, S. J., and J. W. Gibbons. 1986. Habitat suitability index models: Slider turtle. Biological Report 82(10.125). Washington, DC: U.S. Department of the Interior, Fish and Wildlife Service.

National Biological Service. 1995. Habitat evaluation procedures workbook (revised). Fort Collins, CO: Mid Continent Ecological Science Center.

National Research Council. 2001. Compensating for wetland losses under the Clean Water Act. Washington, DC: National Academy Press.

Noss, R. F., and A. Cooperrider. 1994. Saving nature's legacy: Protecting and restoring biodiversity. Washington, DC: Defenders of Wildlife and Island Press.

Ortigosa, G. R., G. A. De Leo, and M. Gatto. 2000. VVF: Integrating modelling and GIS in a software tool for habitat suitability assessment. Environmental Modelling \& Software 15:1- 12.

Poiani, K. A., B. D. Richter, M. G. Anderson, and H. E. Richter. 2000. Biodiversity conservation at multiple scales: Functional sites, landscapes, and networks. BioScience 50:133- 146.

Radeloff, V. C., A. M. Pidgeon, and P. Hostert. 1999. Habitat and population modelling of roe deer using an interactive geographic information system. Ecological Modelling 114:287- 304.

Ray, N., and M. A. Burgman. 2006. Subjective uncertainties in habitat suitability maps. Ecological Modelling 195:172- 186.

Rheinhardt, R. D., M. C. Rheinhardt, M. M. Brinson, and K. E. Faser, Jr. 1999. Application of reference data for assessing and restoring headwater ecosystems. Restoration Ecology 7(3):241- 251.

Rheinhardt, R. D., M. C. Rheinhardt, and M. M. Brinson. 2002. A regional guidebook for applying the hydrogeomorphic approach to assessing wetland functions of wet pine flats on mineral soils in the Atlantic and Gulf Coastal Plains. ERDC/ EL TR-02-9. Vicksburg, MS: U.S. Army Engineer Research and Development Center.

Robinson, R. R., W. Hansen, and K. Orth in collaboration with S. Franco. 1995. Evaluation of environmental investments procedures manual: Cost effectiveness and incremental cost analyses. IWR Report 95-R-1. Alexandria, VA: U.S. Army Corps of Engineers, Evaluation of Environmental Investments Research Program, Instate for Water Resources, and Vicksburg, MS: U.S. Army Engineer Waterways Experiment Station.

Schluter, M., N. Ruger, A. G. Savitsky, N. M. Novikova, M. Matthies, and H. Lieth. 2006. TUGAI: An integrated simulation tool for ecological assessment of alternative water management strategies in a degraded river delta. Environmental Management 38:638- 653. 
Shifley, S. R., F. R. Thompson, W. D. Dijak, M. A. Larson, and J. J . Millspaugh. 2006. Simulated effects of forest management alternatives on landscape structure and habitat suitability in the Midwestern United States. Forest Ecology and Management 229:361- 377.

Smith, R. D., A. Ammann, C. Bartoldus, and M. M. Brinson. 1995. An approach for assessing wetland functions using hydrogeomorphic classification, reference wetlands, and functional indices. Technical Report WRP-DE-10.Vicksburg, MS: U.S. Army Engineer Waterways Experiment Station.

Smith, R. D., and C. C. Bartoldus. 1995. A snap-shot of the Corps' new wetland assessment procedure. Environmental Concern Wetland J ournal 6:3-5.

Society for Ecological Restoration International (SERI). 2004. The Society of Ecological Restoration International Primer on Ecological Restoration, Version 2. (http://www.ser.org/content/ecological_restoration_primer.asp) (September 2008).

Store, R., and J . J okimaki. 2003. A GIS-based multi-scale approach to habitat suitability modeling. Ecological Modelling 169:1- 15.

Store, R., and J . Kangas. 2001. Integrating spatial multi-criteria evaluation and expert knowledge for GIS-based habitat suitability modeling. Landscape and Urban Planning 55:79- 93.

Thiesing, M. A. 2001. An evaluation of wetland assessment techniques and their applications to decision making. In Wetland inventory, assessment and monitoring: Practical techniques and identification of major issues. Proceedings of Workshop 4, 2nd International Conference on Wetlands and Development Dakar, Senegal, Nov. 8- 14, 1998, ed. C. M. Finlayson, N. C. Davidson, and N. J . Stevenson. Dakar, Senegal.

U.S. Army Corps of Engineers (USACE). 1980. A habitat evaluation system for water resources planning. Vicksburg, MS: Lower Mississippi Valley Division. . 1996. National action plan to develop the hydrogeomorphic approach for assessing wetland functions. Federal Register 61(160):42593- 42603. . 2000. Planning guidance notebook. Engineer Regulation 1105-2-100. Washington, DC.

U.S. Fish and Wildlife Service (USFWS). 1980a. Habitat as a basis for environmental assessment. Ecological Service Manual 101. Washington, DC.

. 1980b. Habitat evaluation procedures (HEP). Ecological Service Manual 102. Washington, DC.

. 1980c. Standards for the development of habitat suitability index models. Ecological Service Manual 103. Washington, DC.

. 1986. Habitat management evaluation model (HMEM). Version 1. Fort Collins, CO: National Ecology Center. 
Van der Lee, G. E. M., D. T. Van der Molen, H. F. P. Van den Boogaard, and H. Van der Klis. 2006. Uncertainty analysis of a spatial habitat suitability model and implications for ecological management of water bodies. Landscape Ecology 21:1019- 1032.

Van Lonkhuyzen, R. A., K. E. Lagory, and J . A. Kuiper. 2004. Modeling the suitability of potential wetland mitigation sites with a geographic information system. Environmental Management 33:368- 375.

VanHorne, B., and J . A. Wiens. 1991. Forest bird habitat suitability models and the development of general habitat models. Research Report No. 8. Washington, DC: Department of the Interior, U.S. Fish and Wildlife Service.

Vospernik, S., M. Bokalob, F. Reimoserc, and H. Sterbaa. 2007. Evaluation of a vegetation simulator for roe deer habitat predictions. Ecological Modelling 202:265- 280 .

White, D., and S. Fennessy. 2005. Modeling the suitability of wetland restoration potential at the watershed scale. Ecological Engineering 24:359- 377.

Williams, G. L. 1988. An assessment of HEP (Habitat Evaluation Procedures) applications to Bureau of Reclamation projects. Wildlife Society Bulletin 16:437447.

Yoe, C. E., and K. D. Orth. 1996. Planning manual. IWR Report 96-R-21. Alexandria, VA: U.S. Army Engineer Institute for Water Resources. 


\section{Appendix A: Methods}

\section{Background}

The survival of a species or community is often determined by the presence or absence of limiting habitat features (e.g., life requisites) that are defined by their primary life-giving component, be it food, water, cover, and/ or reproduction. Habitats that optimize life requisites afford a higher level of productivity, and restoring habitats to these optimal conditions is the challenge of many local, state, and federal agencies. It then becomes the responsibility of agency biologists to quantify the success of their plans and projects based on established scientific methodology. In general, restoration can be measured on the basis of quantity (area of land) and/ or quality (rating of the value of the habitat). It is important then, that any technique used to quantify benefits be repeatable, efficient and effective, as results are often questioned by outside interests, and most agencies cannot afford to spend copious amounts of time justifying designs. Many such rapid assessment techniques are readily available in off-the-shelf formats. The basic premises of these methods are presented in a condensed, tabular approach (Table A1-A7).

Of the more than 60 available techniques used across the nation to assess environmental conditions, 16 are summarized herein (Tables A1-A7). Detailed descriptions, output levels, and software/ training information are provided in tabular format in an attempt to provide the reader with a basis for selection of an appropriate technique for future activities. The formal citations of each method immediately follow these tables.

The intent of this appendix is to summarize some of the methods and procedures currently being used in the field of habitat evaluation without implying which is the best solution for the reader. Each method has inherent strengths and weaknesses - often a result of the specific purposes for which they were designed. More information may be required prior to the selection of a particular method for ongoing efforts, and the authors strongly suggest a team fully review the method's full documentation prior to application. 
Table A1. Habitat assessment techniques that can be used to assess ecosystem restoration: AHAG and AREM.

\begin{tabular}{|c|c|c|c|c|}
\hline Method & Acronym & Output & Description & Software \\
\hline $\begin{array}{l}\text { Aquatic Habitat } \\
\text { Appraisal Guide } \\
\text { (Mathias et al. } \\
\text { 1996) }\end{array}$ & AHAG & $\begin{array}{l}\text { Index between } 0 \text { (high quality) } \\
\text { and } 1 \text { (low quality). Quality is } \\
\text { multiplied by Area resulting in } \\
\text { Habitat Units (HUs). When future } \\
\text { projections are used, results are } \\
\text { Average Annual Habitat Units } \\
\text { (AAHUs). }\end{array}$ & $\begin{array}{l}\text { Used to assess changes in the habitat } \\
\text { components of aquatic resources (pri- } \\
\text { marily fish species), given existing and } \\
\text { projected conditions. AHAG assesses both } \\
\text { fishery impacts and benefits resulting } \\
\text { from environmental activities of project } \\
\text { design. Based on the Habitat Evaluation } \\
\text { Procedure (HEP) concept, AHAG numer- } \\
\text { ically rates habitat quality using Habitat } \\
\text { Suitability Indices (HSIs). Scores can be } \\
\text { linked to HEP for calculating Habitat Units } \\
\text { (HUs). The software contains a default } \\
\text { matrix of HSI scores for } 8 \text { evaluation } \\
\text { species. The default matrix uses five types } \\
\text { of variables to evaluate fish habitat. }\end{array}$ & $\begin{array}{l}\text { MS-DOS-based software is available } \\
\text { from the authors. Written in: C } \\
\text { Hardware Requirements: PC with } \\
\text { 386+ Processor Op. Sys. } \\
\text { Requirements: MS DOS 3.1+ } \\
\text { Distribution: } 3.5 \text { hd disks }\end{array}$ \\
\hline $\begin{array}{l}\text { Avian Richness } \\
\text { Evaluation Method } \\
\text { (Adamus 1993) }\end{array}$ & AREM & $\begin{array}{l}\text { Habitat Index ( } 0 \text { - infinity) for } \\
\text { habitat suitability. Avian richness } \\
\text { Synthesis Score (0- infinity), } \\
\text { where } 0 \text { = low biodiversity. } \\
\text { Sensitivity analysis (probabilities) } \\
\text { can be included in the analysis. } \\
\text { Species Habitat Score }(0-1.0 \text { ) for } \\
\text { species composition. The } \\
\text { Synthesis Scores can be } \\
\text { weighted (on a scale of } 1 \text { - } 10 \text { ) to } \\
\text { reflect conservation priorities for } \\
\text { individual species. }\end{array}$ & $\begin{array}{l}\text { AREM estimates bird species composition } \\
\text { and richness for forecasting site bio- } \\
\text { diversity (number of species present), } \\
\text { determining species identification, and } \\
\text { evaluating relative habitat suitability for } \\
\text { each species. }\end{array}$ & $\begin{array}{l}\text { Software is available from author. } \\
\text { Written in: Undocumented } \\
\text { Language Hardware Requirements: } \\
\text { PC with 386+ Processor Op. Sys. } \\
\text { Requirements: MS DOS 3.1+ } \\
\text { Distribution: } 3.5 \text { hd disks }\end{array}$ \\
\hline
\end{tabular}


Table A2. Habitat assessment techniques that can be used to assess ecosystem restoration: BEST and CT-METHOD.

\begin{tabular}{|c|c|c|c|c|}
\hline Method & Acronym & Output & Description & Software \\
\hline $\begin{array}{l}\text { Biological } \\
\text { Evaluation } \\
\text { Standardized } \\
\text { Technique } \\
\text { (Robinson 1983) }\end{array}$ & BEST & $\begin{array}{l}\text { Numerical value }(0 \text { - infinity) that } \\
\text { reflects the "ecological value" of } \\
\text { a habitat based on three } \\
\text { biological components: area, } \\
\text { species identification, and } \\
\text { ecological criteria (where } 0=\text { low } \\
\text { "value"). }\end{array}$ & $\begin{array}{l}\text { A relatively non-subjective, ecologically } \\
\text { based technique for quantifying habitat } \\
\text { value in relation to various fish species. } \\
\text { This technique implies that the value of a } \\
\text { species' habitat can be determined by } \\
\text { evaluating living space, nursery area, food } \\
\text { resources, spawning area, and/or site } \\
\text { productivity. A matrix is used to integrate } \\
\text { these components in a meaningful } \\
\text { manner. }\end{array}$ & $\begin{array}{l}\text { Software is available from author } \\
\text { Written in: ProDAS Hardware } \\
\text { Requirements: PC with 386+ } \\
\text { Processor Op. Sys. } \\
\text { Requirements: MS DOS 5.0+ } \\
\text { Distribution: Unknown }\end{array}$ \\
\hline $\begin{array}{l}\text { Connecticut Method } \\
\text { (Ammann et al. } \\
\text { 1986) }\end{array}$ & CT Method & $\begin{array}{l}\text { Functional Value Index (FVI) } \\
(0-1) \text { that can be multiplied by } \\
\text { acreage to generate a total } \\
\text { wetland score (Wetland Value } \\
\text { Unit WVU) for each function } \\
(0=\text { low functionality). }\end{array}$ & $\begin{array}{l}\text { A scoring technique that ranks groups of } \\
\text { wetlands based on functionality. Several } \\
\text { scores are generated for each series of } \\
\text { wetlands by ranking ecological integrity, } \\
\text { wildlife habitat, fish habitat, educational } \\
\text { potential, visual quality, water-based } \\
\text { recreation, flood control potential, } \\
\text { groundwater use potential, sediment } \\
\text { trapping, nutrient attenuation, shoreline } \\
\text { anchoring, urban quality of life, historical } \\
\text { site potential, and noteworthiness. }\end{array}$ & Software is not available. \\
\hline
\end{tabular}


Table A3. Habitat assessment techniques that can be used to assess ecosystem restoration: HEP and HGM.

\begin{tabular}{|c|c|c|c|c|}
\hline Method & Acronym & Output & Description & Software \\
\hline $\begin{array}{l}\text { Habitat Evaluation } \\
\text { Procedures } \\
\text { (U.S. Fish and } \\
\text { Wildlife Service } \\
\text { (USFWS) 1980) }\end{array}$ & HEP & $\begin{array}{l}\text { Habitat Suitability Index (HSI) } \\
(0-1) \text { is used to measure the } \\
\text { limiting resources of a site }(0 \\
=\text { low quality habitat, } 1.0=\text { high } \\
\text { quality habitat). This index is } \\
\text { multiplied against the site's } \\
\text { available area to generate } \\
\text { Habitat Units (HUs), which can be } \\
\text { averaged over a series of target } \\
\text { years to generate Average Annual } \\
\text { Habitat Units (AAHUs). }\end{array}$ & $\begin{array}{l}\text { HEP is a biological accounting system that } \\
\text { quantifies the suitability of a habitat for a } \\
\text { species or community based on the com- } \\
\text { bination of measurable limiting factors } \\
\text { (e.g., distance to cover, height of grass, } \\
\text { percent canopy cover, etc.) in a mathe- } \\
\text { matical equation. In HEP, a user can make } \\
\text { projections on these parameters to } \\
\text { simulate/anticipate changes in future out- } \\
\text { years. HEP combines the quality of these } \\
\text { factors with the quantity of available } \\
\text { habitat to generate the Habitat Units (HUs) } \\
\text { and Average Annual Habitat Units for a set } \\
\text { of target years. More than } 250 \text { HEP } \\
\text { models have been developed and } \\
\text { published by the USFWS. In HEP, a user } \\
\text { can easily adjust/modify these models } \\
\text { (the equations and variables) to better } \\
\text { reflect a new region's conditions. }\end{array}$ & $\begin{array}{l}\text { MS-DOS-based software is available } \\
\text { from the authors. } \\
\text { Written in: Fortran Hardware } \\
\text { Requirements: PC with } 386+ \\
\text { Processor Op. Sys. } \\
\text { Requirements: MS-DOS 2.0+ } \\
\text { Distribution: Distributed by USFWS } \\
\text { on } 3.5 \text { hd disk. } \\
\text { MS Windows-based software is } \\
\text { available from Ms. Kelly Burks- } \\
\text { Copes, USAE-ERDC, Kelly.A.Burks- } \\
\text { Copes@usace.army.mil. } \\
\text { Written in: MS Access }{ }^{\odot} \text { '97 } \\
\text { Requirements: PC with } 486+ \\
\text { Op. Sys. Requirements: Windows } \\
\text { '98 or Windows NT 4.0+ } \\
\text { Distribution: Distributed by USAE - } \\
\text { ERDC on CD. }\end{array}$ \\
\hline $\begin{array}{l}\text { Hydrogeomorphic } \\
\text { Classification of } \\
\text { Wetlands } \\
\text { (Brinson 1993) }\end{array}$ & HGM & $\begin{array}{l}\text { A Functional Capacity Index (FCI) } \\
(0-1) \text { is used to measure the } \\
\text { wetland function ( } 0=\text { low func- } \\
\text { tionality, } 1 \text { = high). This index is } \\
\text { multiplied against the site's } \\
\text { available area to generate } \\
\text { Functional Capacity Units (FCU), } \\
\text { which can be averaged over a } \\
\text { series of target years to produce } \\
\text { Average Annual Functional } \\
\text { Capacity Units (AAFCU). }\end{array}$ & $\begin{array}{l}\text { HGM is a wetland assessment tool based } \\
\text { on three basic wetland hydrogeomorphic } \\
\text { properties: 1) setting (landscape posi- } \\
\text { tion); 2) water source and transport; and } \\
\text { 3) hydrodynamics. These components } \\
\text { form a picture of the wetland from a } \\
\text { functional standpoint. Strengths of the } \\
\text { classification include clarification of the } \\
\text { relationship between hydrology, geo- } \\
\text { morphology, wetland function, and open } \\
\text { structure. }\end{array}$ & $\begin{array}{l}\text { MS Windows-based software is } \\
\text { available from USAE-ERDC, } \\
\text { Environmental Lab (601) 634-4862. } \\
\text { Written in: MS Access } 97 \\
\text { Hardware Requirements: PC with } \\
486+ \\
\text { Op. Sys. Requirements: Windows } 95 \\
\text { or Windows NT 4.0+ } \\
\text { Distribution: by USAE-ERDC on 3.5" } \\
\text { hd disk }\end{array}$ \\
\hline
\end{tabular}


Table A4. Habitat assessment techniques that can be used to assess ecosystem restoration: H-M(N)M, IBI, and PAMHEP.

\begin{tabular}{|c|c|c|c|c|}
\hline Method & Acronym & Output & Description & Software \\
\hline $\begin{array}{l}\text { Hollands-Magee } \\
\text { (Normandeau) } \\
\text { Method } \\
\text { (Hollands and } \\
\text { Magee 1985) }\end{array}$ & $\mathrm{H}-\mathrm{M}(\mathrm{N}) \mathrm{M}$ & $\begin{array}{l}\text { Function score (scale varies) of a } \\
\text { wetland site relative to the } \\
\text { functional model mean score } \\
\text { and/or scores for other wetlands } \\
\text { in a defined area and weighted } \\
\text { based on } 10 \text { evaluation func- } \\
\text { tions, thus a wetland score scale } \\
\text { is } 0-10, \text { with } 0 \text { indicating the } \\
\text { worst possible conditions. }\end{array}$ & $\begin{array}{l}\text { This technique addresses } 11 \text { common } \\
\text { wetland functions (biology, hydrology, } \\
\text { groundwater, storm/floodwater storage, } \\
\text { shoreline protection, water quality main- } \\
\text { tenance, cultural, economic, recreational, } \\
\text { aesthetics, and educational). The scoring } \\
\text { technique reflects the functional "unique- } \\
\text { ness" of the site. It is similar to the } \\
\text { Ontario, Connecticut, and New Hampshire } \\
\text { methods and was revised as the Rapid } \\
\text { Assessment Procedure. }\end{array}$ & Software is not available. \\
\hline $\begin{array}{l}\text { Index of Biotic } \\
\text { Integrity } \\
\text { (Lyons 1992) }\end{array}$ & $|\mathrm{B}|$ & $\begin{array}{l}\text { Index is calculated for a stream } \\
\text { site by comparing the observed } \\
\text { values of each fish attribute with } \\
\text { values expected in comparable } \\
\text { streams of high environmental } \\
\text { quality. Index from } 0 \text { to } 100 \text {. It } \\
\text { indicates the ability of a habitat } \\
\text { to support and maintain a } \\
\text { balanced, integrated biological } \\
\text { system. }\end{array}$ & $\begin{array}{l}\text { To assess the biological integrity of a } \\
\text { habitat using samples of living organisms } \\
\text { and to evaluate the consequences of } \\
\text { human actions on biological systems. Bio- } \\
\text { assessment and monitoring approach } \\
\text { allows attributes of fish communities (data } \\
\text { on biological populations) to be used to } \\
\text { assess biotic integrity and environmental } \\
\text { quality of streams and rivers (Karr 1981, } \\
\text { Karr et al. 1986). }\end{array}$ & Software is not available. \\
\hline $\begin{array}{l}\text { Pennsylvania } \\
\text { Modified HEP } \\
\text { (Palmer et al. 1985) }\end{array}$ & PAMHEP & $\begin{array}{l}\text { Habitat Suitability (HIS) of each } \\
\text { cover type and the entire project } \\
\text { area for each evaluation species. } \\
\text { Habitat Units (HU): HSI multi- } \\
\text { plied by area. }\end{array}$ & $\begin{array}{l}\text { PAMHEP is a modified version of HEP. } \\
\text { Used to assess baseline fish and wildlife } \\
\text { habitat conditions and to determine the } \\
\text { direct impacts of a project. The software } \\
\text { (GDI/HEP) links outputs to GIS databases. }\end{array}$ & $\begin{array}{l}\text { Software (GDI/HEP) is available from } \\
\text { Geo Decisions, Inc., } 301 \text { N. Science } \\
\text { Park Rd., State College, PA. 16803, } \\
\text { (814) 234-8625 or } \\
\text { http://www.geodecisions.com } \\
\text { Hardware Requirements: } \\
\text { PC with 512k memory } \\
\text { Op. Sys: MS-DOS 2.0+ based }\end{array}$ \\
\hline
\end{tabular}


Table A5. Habitat assessment techniques that can be used to assess ecosystem restoration: RCHARC, SAW, and WET.

\begin{tabular}{|c|c|c|c|c|}
\hline Method & Acronym & Output & Description & Software \\
\hline $\begin{array}{l}\text { Riverine Commun- } \\
\text { ity Habitat Assess- } \\
\text { ment and Restor- } \\
\text { ation Concept } \\
\text { (Nestler et al. } \\
\text { 1993) }\end{array}$ & RCHARC & $\begin{array}{l}\text { Values between } 0 \text { and } 1 \\
\text { that reflect the degree of } \\
\text { similarity between the } \\
\text { reference and each project } \\
\text { flow. }\end{array}$ & $\begin{array}{l}\text { RCHARC contrasts depth and velocity distributions } \\
\text { at specific time intervals from a reference stream } \\
\text { against distributions from different operational or } \\
\text { channel alternatives in a target stream. For each } \\
\text { flow there is an associated distribution of depth or } \\
\text { velocity. Occurrence of many fish species can be } \\
\text { ordinated along these distributions. The higher the } \\
\text { depth and velocity distributions for an alternative } \\
\text { resemble the reference stream, the higher the } \\
\text { ranking. }\end{array}$ & $\begin{array}{l}\text { Software is available from Dr. John } \\
\text { Nestler, USAE-ERDC, } \\
\text { John.M.Nestler@usace.army.mil. }\end{array}$ \\
\hline $\begin{array}{l}\text { Synoptic Approach } \\
\text { for Wetlands } \\
\text { Cumulative Effects } \\
\text { Analysis } \\
\text { (Abbruzzese et al. } \\
1990)\end{array}$ & SAW & $\begin{array}{l}\text { Index measures function, } \\
\text { value, functional loss, } \\
\text { and/or replacement } \\
\text { potential of landscape } \\
\text { subunit within a defined } \\
\text { geographical area. }\end{array}$ & $\begin{array}{l}\text { Method categorizes landscape units instead of } \\
\text { ranking individual wetlands. Four general } \\
\text { indicators can be assessed: function (habitat, } \\
\text { water quality, and hydrology), value, functional } \\
\text { loss, and replacement potential. Most rapid } \\
\text { technique for ranking landscapes and can help } \\
\text { focus later site-specific analyses using other } \\
\text { techniques. }\end{array}$ & Software is not available. \\
\hline $\begin{array}{l}\text { Wetland Evaluation } \\
\text { Techniques } \\
\text { (Adamus et al. } \\
\text { 1987) }\end{array}$ & WET & $\begin{array}{l}\text { Probability rating measures } \\
\text { the potential of a wetland } \\
\text { to perform a function } \\
\text { (actually an estimate of } \\
\text { probability) with respect to } \\
\text { social significance, effec- } \\
\text { tiveness, and/or } \\
\text { opportunity. }\end{array}$ & $\begin{array}{l}\text { WET provides wetland assessment of recognized } \\
\text { wetland functions and values. Ten functions are } \\
\text { addressed: groundwater recharge; discharge; } \\
\text { flood flows; sediment stabilization; sediment } \\
\text { retention; nutrient removal; production export; } \\
\text { wildlife diversity/abundance; recreation; and } \\
\text { uniqueness. WET evaluates habitat suitability for } \\
14 \text { waterfowl species, } 4 \text { freshwater fish species, } \\
120 \text { wetland bird species, and } 133 \text { saltwater fish } \\
\text { and invertebrate species. Wetlands are placed into } \\
\text { one of three broad categories representing the } \\
\text { qualitative probability of functions occurring in a } \\
\text { wetland rather than the value of the functions. }\end{array}$ & $\begin{array}{l}\text { Software available from Dr. Dan } \\
\text { Smith, USAE-ERDC, } \\
\text { Ronald.D.Smith@usace.army.mil. }\end{array}$ \\
\hline
\end{tabular}


Table A6. Habitat assessment techniques that can be used to assess ecosystem restoration: WRAP.

\begin{tabular}{|l|l|l|l|l|}
\hline Method & Acronym & Output & Description \\
\hline $\begin{array}{l}\text { Wetland Rapid } \\
\text { Assessment } \\
\text { Procedure } \\
\text { (Miller and } \\
\text { Gunsalus 1997) }\end{array}$ & WRAP & $\begin{array}{l}\text { Measures the functionality } \\
\text { provided by a wetland. }\end{array}$ & $\begin{array}{l}\text { WRAP evaluates freshwater wetlands that } \\
\text { have been created, enhanced, preserved, or } \\
\text { restored. Six variables are measured: wildlife } \\
\text { utilization; overstory/shrub canopy; wetland } \\
\text { vegetative ground cover; adjacent upland/ } \\
\text { wetland buffer; hydrology; and water quality. } \\
\text { M-WRAP is a modified version designed for } \\
\text { reviewing mitigation banks and to aid in } \\
\text { determining credits. E-WRAP is a draft } \\
\text { (modified) version designed for use in } \\
\text { assessing estuarine systems. }\end{array}$ \\
\hline
\end{tabular}


Table A7. Habitat assessment techniques that can be used to assess ecosystem restoration: WVA, WHAG, and WHAP.

\begin{tabular}{|c|c|c|c|c|}
\hline Method & Acronym & Output & Description & Software \\
\hline $\begin{array}{l}\text { Wetland Value } \\
\text { Assessment } \\
\text { Methodology } \\
\text { (Mitchell 1993) }\end{array}$ & WVA & $\begin{array}{l}\text { Models use an index on a } 0 \text { to } 1 \\
\text { scale. Results are measured in } \\
\text { Average Annual Habitat Units } \\
\text { (AAHUs) }\end{array}$ & $\begin{array}{l}\text { WVA, a quantitative habitat-based assessment } \\
\text { method, developed for use in prioritizing } \\
\text { project proposals submitted for funding under } \\
\text { the Coastal Wetlands Planning, Protection, } \\
\text { and Restoration Act (CWPPRA). WVA quantifies } \\
\text { change in fish and wildlife habitat quality and } \\
\text { acreage that is projected as a result of a } \\
\text { proposed wetland enhancement project. } \\
\text { Results can be combined with economic data } \\
\text { to provide a measure of the effectiveness. }\end{array}$ & $\begin{array}{l}\text { May use HSI and HEP software if } \\
\text { models are input into the system. }\end{array}$ \\
\hline $\begin{array}{l}\text { Wildlife Habitat } \\
\text { Appraisal Guide } \\
\text { (Missouri Depart- } \\
\text { ment of Conserva- } \\
\text { tion and U.S. Soil } \\
\text { Conservation } \\
\text { Service 1990) }\end{array}$ & WHAG & $\begin{array}{l}\text { Index between } 0 \text { and } 1 \text { is multi- } \\
\text { plied by the area resulting in } \\
\text { Habitat Units (HUs). Final } \\
\text { Results are measured in } \\
\text { Average Annual Habitat Units } \\
\text { (AAHUs). }\end{array}$ & $\begin{array}{l}\text { Wildlife species are grouped in guilds, then } \\
\text { guilds for a habitat type are put in a Charac- } \\
\text { teristic Matrix. Within each matrix charac- } \\
\text { teristic variables are used to rate the habitat } \\
\text { quality. The } 1983 \text { revised guide describes } \\
21 \text { upland and } 7 \text { wetland species models. } \\
\text { Models are planning and management } \\
\text { oriented. }\end{array}$ & Software is available from author. \\
\hline $\begin{array}{l}\text { Wildlife Habitat } \\
\text { Appraisal } \\
\text { Procedures } \\
\text { (Frye 1995) }\end{array}$ & WHAP & $\begin{array}{l}\text { Habitat Quality measured in } \\
\text { Habitat Units (HU) derived from } \\
\text { multiplying average Habitat } \\
\text { Quality score (0-1) for a cover } \\
\text { type by the size of the areas. } \\
\text { Average Annual Habitat Units } \\
\text { (AAHU) determined by the total } \\
\text { number of HUs divided by the } \\
\text { life of the project. }\end{array}$ & $\begin{array}{l}\text { Purpose is to provide a qualitative assessment } \\
\text { of wildlife habitat. Points for component } \\
\text { criteria are assigned and calculated to } \\
\text { produce a score for } 3 \text { sections: Biological } \\
\text { Habitat, Significance to protected fauna and } \\
\text { flora, and Acquisition and Administration. }\end{array}$ & Software is not available. \\
\hline
\end{tabular}




\section{Method Citations}

Abbruzzese, B., S. G. Leibowitz, and R. Sumner. 1990. Application of the synoptic approach to wetland designation: A case study in Washington. Technical Report EPA/600/3-90/ 072. Corvallis, OR: U.S. Environmental Protection Agency Environmental Research Lab.

Adamus, P. R. 1993. User's manual: Avian Richness Evaluation Method (AREM) for lowland wetlands of the Colorado plateau. Technical Report EPA/600/R93/240. Corvallis, OR: U.S. Environmental Protection Agency Environmental Research Laboratory.

Adamus, P. R., E. J . Clairain, R. D. Smith, and R. E. Young. 1987. Wetland Evaluation Technique (WET), Vol II: Methodology. Technical Report Y-87. Vicksburg, MS: U.S. Army Engineer Waterways Experiment Station.

Ammann, A. P., R. W. Franzen, and J . L. J ohnson. 1986. Method for the evaluation of inland wetlands in Connecticut. Bulletin No. 9. Hartford, CT: Connecticut Department Environmental Protection and USDA Soil Conservation Service.

Brinson, M. M. 1993. A hydrogeomorphic classification for wetlands. Technical Report WRP-DE-4. Vicksburg, MS: U.S. Army Engineer Waterways Experiment Station.

Frye, R. 1995. Wildlife Habitat Appraisal Procedures (WHAP). Austin, TX: Texas Parks and Wildlife Department.

Hollands, G. G., and D. W. Magee. 1985. A method for assessing the functions of wetlands. Proceedings of the National Wetland Assessment Symposium. Association of Wetland Managers 108- 118.

Karr, J . R. 1981. Assessment of biotic integrity using fish communities. Fisheries 6(6):2127.

Karr, J. R., K. D. Fausch, P. L. Angermeier, P. R. Yant, and I. J . Schlosser. 1986. Assessing biological integrity in running waters: A method and its rationale. Special Publication 5. Champaign, IL: Illinois Natural History Survey.

Mathias, D., T. B. Hardy, K. J . Killgore, and J . W. J ordan. 1996. Aquatic habitat appraisal guide. Instruction Report EL-96-1. Vicksburg, MS: U.S. Army Engineer Waterways Experiment Station.

Miller, R. E., and B. E. Gunsalus. 1997. Wetland Rapid Assessment Procedure (WRAP). Technical Publication (REG-001). West Palm Beach, FL: South Florida Water Management District, Natural Resource Management Division.

Missouri Department of Conservation and U.S. Soil Conservation Service. 1990. Wildlife habitat appraisal guide. J efferson City, MO.

Mitchell, L. 1993 (Draft). Wetland Value Assessment Methodology (WVA). Lafayette, LA: U.S. Fish and Wildlife Service. 
Nestler, J . M., L. T. Schneider, and D. C. Latka. 1993. Physical habitat analysis of Missouri River main stem reservoir tailwaters using the Riverine Community Assessment and Restoration Concept (RCHARC). Technical Report EL-93-22. Vicksburg, MS: U.S. Army Engineer Waterways Experiment Station.

Palmer, J . H., M. T. Chezik, R. D. Heaslip, G. A. Rogalsky, D. J . Putnam, R. W. McCoy, and J . A. Arway. 1985. Pennsylvania modified 1980 habitat evaluation procedure instruction manual. State College, PA: U.S. Fish and Wildlife Service.

Robinson, D. 1983. Biological Evaluation Standardized Technique(BEST). Lafayette, CA: MEC Analytical Systems, Tetra Tech, Inc.

U.S. Fish and Wildlife Service (USFWS). 1980. Habitat Evaluation Procedures (HEP) Manual. Ecological Service Manual 102. Washington, DC: U.S. Fish and Wildlife Service. 


\section{Appendix B: Glossary}

Activity

Alternative (aka Alternative Plan, Plan, or Solution)

Assessment Model
In planning studies, an activity is the smallest component of a management measure that is typically a nonstructural, ongoing (continuing or periodic) action (Robinson et al. 1995).

An alternative can be composed of numerous management measures that in turn are comprised of multiple features or activities. Alternatives are mutually exclusive, but management measures may or may not be combinable with other management measures or alternatives (Robinson et al. 1995).

Some examples of alternatives include:

Alternative 1: Plant food plots, increase wetland acreage by 10 percent, install 10 goose nest boxes, and build a fence around the entire site.

Alternative 2: Build a dam, inundate 10 acres of riparian corridor, build 50 miles of supporting levee, and remove all wetlands in the levee zone.

Alternative 3: Reduce the grazing activities on the site by 50 percent, replant grasslands (10 acres), install a passive irrigation system, build 10 escape cover stands, use 5 miles of willow fascines along the stream bank for stabilization purposes.

A simple mathematical tool that defines the relationship between ecosystem/landscape scale variables and either functional capacity of a wetland or suitability of habitat for species and communities. Habitat Suitability Indices and Functional Capacity Indices are examples of assessment models that the HEAT software can be used to assess impacts/benefits of alternatives. 
Assessment

Team

(A-Team)
In HGM applications, the Assessment Team (e.g., ATeam) is an interdisciplinary group of regional and local scientists responsible for classification of wetlands within a region, identification of reference wetlands, construction of assessment models, definition of reference standards, and calibration of assessment models (Smith et al. 1995).

Average Annual In HGM applications, a quantitative result of annuFunctional Capacity Unit (AAFCU) alizing Functional Capacity Unit (FCU) gains or losses across all years in the period of analysis.

AAFCUs $=$ Cumulative FCUs $\div$ Number of years in the life of the project, where:

Cumulative FCUs =

$\sum\left(\mathrm{T}_{2}-\mathrm{T}_{1}\right)\left[\left\{\left(\left(\mathrm{A}_{1} \mathrm{~F}_{1}+\mathrm{A}_{2} \mathrm{~F}_{2}\right) / 3\right)\right\}+\left\{\left(\left(\mathrm{A}_{2} \mathrm{~F}_{1}+\mathrm{A}_{1} \mathrm{~F}_{2}\right) / 6\right)\right\}\right]$

and where:

$\mathrm{T}_{1}=$ First Target Year time interval

$\mathrm{T}_{2}=$ Second Target Year time interval

$\mathrm{A}_{1}=$ Area of available wetland assessment area at beginning of $\mathrm{T}_{1}$

$\mathrm{A}_{2}=$ Area of available wetland assessment area at end of $\mathrm{T}_{2}$

$\mathrm{F}_{1}=\mathrm{FCI}$ at beginning of $\mathrm{T}_{1}$

$\mathrm{F}_{2}=\mathrm{FCI}$ at end of $\mathrm{T}_{2}$ 
Average Annual In HEP applications, a quantitative result of annualHabitat Unit

(AAHU) izing Habitat Unit (HU) gains or losses across all years in the period of analysis.

AAHUs $=$ Cumulative HUs $\div$ Number of years in the life of the project, where

Cumulative HUs $=$

Sum $\left(\mathrm{T}_{2}-\mathrm{T}_{1}\right)\left[\left(\left(\mathrm{A}_{1} \mathrm{H}_{1}+\mathrm{A}_{2} \mathrm{H}_{2}\right) / 3\right)+\left(\left(\mathrm{A}_{2} \mathrm{H}_{1}+\mathrm{A}_{1} \mathrm{H}_{2}\right) /\right.\right.$

6)]

and where:

$\mathrm{T}_{1}=$ First Target Year time interval

$\mathrm{T}_{2}=$ Second Target Year time interval

$\mathrm{A}_{1}=$ Area of available habitat at beginning of $\mathrm{T}_{1}$

$\mathrm{A}_{2}=$ Area of available habitat at end of $\mathrm{T}_{2}$

$\mathrm{H}_{1}=\mathrm{HSI}$ at beginning of $\mathrm{T}_{1}$

$\mathrm{H}_{2}=\mathrm{HSI}$ at end of $\mathrm{T}_{2}$

\section{Baseline \\ Condition \\ (aka Existing \\ Conditions)}

\section{Blue Book}

In habitat/functional assessments, baseline is the point in time before proposed changes, and is synonymous with Target Year $(\mathrm{TY}=0)$.

In the past, the U. S. Fish and Wildlife Service was responsible for publishing documents identifying and describing HSI models for numerous species across the nation. Referred to as "Blue Books" in the field, due primarily to the light blue tint of their covers, these references fully illustrate and define habitat relationships and limiting factor criteria for individual species nationwide. Blue Books provide: HSI Models, life history characteristics, SI curves, methods of variable collection, and referential material that can be used in the application of the HSI model in the field. For copies of Blue Books, or a list of available Blue Books, contact the local USFWS office. 
Calibration

Cover Type

(CT)

\section{Ecological Integrity}

The use of known (reference) data on the observed relationship between a dependent variable and an independent variable to estimate other values of the independent variable from new observations of the dependent variable.

In HEP applications, cover types are homogenous zones of similar vegetative species, geographic similarities, and physical conditions that make the area unique. In general, cover types are defined on the basis of species recognition and dependence.

Angermeir and Karr (1994) define this as "system wholeness, including the presence of appropriate species, populations, and communities and the occurrence of ecological process at appropriate rates and scales as well as the environmental conditions that support these taxa" as cited in Dale and Beyeler (2001) on page 3.

An ecosystem is a biotic community, together with its physical environment, considered as an integrated unit. Implied within this definition is the concept of a structural and functional whole, unified through life processes. Ecosystems are hierarchical, and can be viewed as nested sets of open systems in which physical, chemical, and biological processes form interactive subsystems. Some ecosystems are microscopic, and the largest comprises the biosphere. Ecosystem restoration can be directed at differentsized ecosystems within the nested set, and many encompass multi-states, more localized watersheds, or a smaller complex of aquatic habitat. 
Ecosystem Functions

Ecosystem Integrity
Ecosystem functions are the dynamic attributes of ecosystems, including interactions among organisms and interactions between organisms and their environment (Society for Ecological Restoration International (SERI) 2004). Some restoration ecologists limit the use of the term "ecosystem functions" to those dynamic attributes which most directly affect metabolism, principally the sequestering and transformation of energy, nutrients, and moisture. Examples are carbon fixation by photosynthesis, trophic interactions, decomposition, and mineral nutrient cycling. When ecosystem functions are strictly defined in this manner, other dynamic attributes are distinguished as "ecosystem processes" such as substrate stabilization, microclimatic control, differentiation of habitat for specialized species, pollination, and seed dispersal. Functioning at larger spatial scales is generally conceived in more general terms, such as the long-term retention of nutrients and moisture and overall ecosystem sustainability.

The state or condition of an ecosystem that displays the biodiversity characteristic of the reference, such as species composition and community structure, and is fully capable of sustaining normal ecosystem functioning (SERI 2004). These characteristics are often defined in terms such as health, biodiversity, stability, sustainability, naturalness, wildness, and beauty. 
Equivalent Optimal Area (EOA)

Evaluation

Team

(E-Team)

\section{Existing Condition}

Feature
The concept of equivalent optimal area (EOA) is used in HEP applications where the composition of the landscape, in relation to providing life requisite habitat, is an important consideration. An EOA is used to weight the value of the LRSI score to compensate for this inter-relationship. For example, for optimal wood duck habitat conditions, at least 20 percent of an area should be composed of cover types providing broodcover habitat (a life requisite). If an area has less than 20 percent in this habitat, the suitability is adjusted downward.

In planning studies, the Evaluation Team (e.g., E-Team) is an interdisciplinary group of regional and local scientists responsible for determining significant resources, identifying reference sites, constructing assessment models, defining reference standards, and calibrating assessment models. In some instances the E-Team is also referred to as the Ecosystem Assessment Team.

Also referred to as the "Baseline Condition," the existing condition is the point in time before proposed changes, and is designated as Target Year TY $=0$ in HEP and HGM applications.

In planning studies, a feature is the smallest component of a management measure that is typically a structural element requiring construction (Robinson et al. 1995). 
Field Data

Functional Assessment

Functional Capacity

Functional Capacity Index Model (FCI)
In HEP and HGM applications, this information is collected on various parameters (i.e., variables) in the field, and from aerial photos, following defined, welldocumented methodology. An example is the measurement of percent herbaceous cover, over 10 quadrants, within a riparian forest cover type. The values recorded are each considered "field data." Means of variables are applied to derive suitability indices and/or functional capacity indices.

In HGM applications, a functional assessment is the process by which the capacity of a wetland to perform a function is measured. This approach measures capacity using an assessment model to determine an FCI (Smith et al. 1995).

The magnitude to which an area of wetland performs a function. Functional capacity is dictated by attributes of the wetland ecosystem and the surrounding landscape and interaction between the two (Smith et al. 1995).

In HGM applications, the FCI model is an index of the capacity of a wetland to perform a function relative to other wetlands from a regional wetland subclass in a reference domain (Smith et al. 1995). The ideal goal of a Functional Capacity Index (FCI) model is to quantify and produce an index that reflects functional capacity at the site. The results of an FCI analysis can be quantified on the basis of a standard 0-1.0 scale, where 0.00 represents low functional capacity for the wetland, and 1.0 represents high functional capacity for the wetland. An FCI model can be defined in words, or mathematical equations, that clearly describe the rules and assumptions necessary to combine functional capacity indices in a meaningful manner for the wetland. For example: 
$\mathrm{FCI}=\left(\mathrm{VSI} \mathrm{V}_{1} * \mathrm{VSI} \mathrm{V}_{2}\right) / 4$,

where:

VSI V $\mathrm{V}_{1}$ is the Variable Subindex (VSI) for variable 1;

VSI $V_{2}$ is the VSI for variable 2

Functional

Capacity Unit (FCU)

Future Factor (FF)

Goal
In HGM applications, a quantitative environmental assessment value considered the biological currency in HGM (Smith et al. 1995). Functional Capacity Units (FCUs) are calculated by multiplying the area of available wetland (quantity) by the quality of the wetland based on functionality. Quality is determined by measuring limiting factors describing wetland function, and is represented by values derived from Functional Capacity Indices (FCIs):

FCU $=$ AREA (acres) X FCI

Changes in FCUs represent potential impacts or improvements of proposed actions.

In the HEAT software modules, a Future Factor (FF) is a unit of quality change that is used to define the anticipated changes in mean field data, by target year, on a variable-per-cover type basis, rather than on a species-by-species basis. FF values are multiplicative factors (e.g., 1, 1.5, 0.5, etc.) directly multiplied against the mean baseline condition, which allows evaluation teams the opportunity to forecast changes over time on the site or project. For example, a team might envision a 50-percent increase in height of grass in the grassland cover types between TY0 and TY1 - this expected future trend is considered a Future Factor in the HEAT modules.

In planning studies, a goal is defined as the end or final purpose. Goals provide the reason for a study rather than a reason to formulate alternative plans (Yoe and Orth 1996). 
Guild

Habitat Assessment

\section{Habitat \\ Suitability Index Model (HSI)}

A group of functionally similar species with comparable habitat requirements whose members interact strongly with one another, but weakly with the remainder of the community. Often a species HSI model is selected to represent changes (impacts) to a guild.

In HEP applications, a habitat assessment is the process by which the suitability of a site to provide habitat for a community or species is measured. This approach measures habitat suitability using an assessment model to determine an HSI.

In HEP applications, a Habitat Suitability Index (HSI) model is a quantitative estimate of habitat conditions for an evaluation species or community. The ideal goal of an HSI model is to quantify and produce an index that reflects carrying capacity at the site. The results of an HSI analysis can be quantified on the basis of a standard 0-1.0 scale, where 0.00 represents low quality habitat for the species/ community and 1.0 represents high quality habitat for the species/ community. An HSI model can be defined in words, or mathematical equations that clearly describe the rules and assumptions necessary to combine suitability indices in a meaningful manner for the species.

For example:

$\mathrm{HSI}=\left(\mathrm{SI} \mathrm{V}_{1} * \mathrm{SI} \mathrm{V}_{2}\right) / 4$

where:

SI $V_{1}$ is the SI for variable 1 ;

SI $V_{2}$ is the SI for variable 2. 
Habitat Unit (HU)

Hydrogeomorphic Wetland Class

Life Requisite Suitability Index (LRSI)
In HEP applications, a quantitative environmental assessment value, considered the biological currency in HEP. Habitat Units (HUs) are calculated by multiplying the area of available habitat (quantity) by the quality of the habitat for each species or community. Quality is determined by measuring limiting factors for the species (or community), and is represented by values derived from Habitat Suitability Indices (HSIs).

$\mathrm{HU}=\mathrm{AREA}$ (acres) X HSI.

Changes in HUs represent potential impacts or improvements of proposed actions.

The highest level in the hydrogeomorphic wetland classification (Smith et al. 1995).

In HEP applications, a Life Requisite Suitability Index (LRSI) is a mathematical equation that reflects a species' or community's sensitivity to a change in a limiting life requisite component within the habitat type. In HEP, LRSIs are depicted using scatter plots and bar charts (i.e., life requisite suitability curves). The LRSI value ( $Y$ axis) ranges on a scale from 0.0 to 1.0 , where an LRSI $=0.0$ means the factor is extremely limiting and an LRSI $=1.0$ means the factor is in abundance (not limiting) in most instances.

Limiting Factor In HEP and HGM applications, a variable whose presence/ absence directly restrains the existence of a species or community in a habitat. A deficiency of the limiting factor can reduce the quality of the habitat for the species or community, while an abundance of the limiting factor can indicate an optimum quality of habitat for the same species or community. 
Management Measure

Measure

Multiple

Formula Model (MM) (aka Life Requisite Model)
In planning studies, management measures are the components of a plan that may or may not be separable actions that can be taken to affect environmental variables and produce environmental outputs. A management measure is typically made up of one or more features or activities at a particular site (Robinson et al. 1995).

In HEP and HGM applications, this is the act of physically sampling variables such as height, distance, percent, etc., and the methodology followed to gather variable information (i.e., see "Sampling Method" below).

In HEP applications, there are two types of HSI models, the Single Formula Model (SM) (refer to the definition below) and the Multiple Formula Model (MM). In this case a multiple formula model is, as one would expect, a model that uses more than one formula to assess the suitability of the habitat for a species or a community. If a species/ community is limited by the existence of more than one life requisite (food, cover, water, etc.), and the quality of the site is dependent on a minimal level of each life requisite, then the model is considered an MM model. In order to calculate the HSI for any MM, one must derive the value of a Life Requisite Suitability Index (LRSI) (see definition below) for each life requisite in the model a process requiring the calculation of multiple LRSI formulas. This Multiple Formula processing has led to the name "Multiple Formula Model" in HEP.

No Action Plan Also referred to as the without-project condition, the (aka No Action Alternative or No Action Plan describes the project area's future if no Federal action is taken to solve the problem(s) at Without-Project hand. Every alternative is compared to the same Condition) without-project condition (Yoe and Orth 1996). 
Objective

Partial Wetland Assessment Area (PWAA)
An objective is a statement of the intended purposes of the planning process; it is a statement of what an alternative plan should try to achieve. More specific than goals, a set of objectives will effectively constitute the mission statement of the Federal/ non-Federal planning partnership. A planning objective is developed to capture the desired changes between the without- and with-project conditions that when developed correctly identify effect, subject, location, timing, and duration (Yoe and Orth 1996).
Plan

(aka Alternative, Alternative Plan, or Solution)

Project Area

\section{Reference Domain}

\section{Reference Sites}

A set of one or more management measures vegetative species, geographic similarities and physical conditions that make the area unique. In general, PWAAs are defined on the basis of species recognition and dependence, soils types and topography (Smith et al. 1995).

functioning together to address one or more planning objectives (Yoe and Orth 1996). Plans are evaluated at the site level with both HEP, HGM or other assessment techniques and CEA/ICA in restoration studies (Robinson et al. 1995).

The area that encompasses all activities related to an ongoing or proposed project.

The geographic area from which reference wetlands are selected. A reference domain may, or may not, include the entire geographic area in which a regional wetland subclass occurs (Smith et al. 1995).

In HEP applications, these sites encompass the variability of all conditions within the region. Reference sites are used to establish the range of conditions for construction and calibration of HSIs and establish reference standards. 
Reference Standard Sites

Reference Standard Wetlands

Reference Wetlands

Regional Wetland Subclass

Relative Area (RA)
In HEP applications, these sites represent the highest level of habitat suitability or function found within the region for a given species or community.

In HGM applications, reference standard wetlands are conditions exhibited by a group of reference wetlands that correspond to the highest level of functional capacity (highest, sustainable level of functioning) across the suite of functions performed by the regional wetland subclass. The highest level of functional capacity is assigned an index value of 1.0 by definition (Smith et al. 1995).

In HGM applications, wetland sites that encompass the variability of a regional wetland subclass in a reference domain. Reference wetlands are used to establish the range of conditions for construction and calibration of functional indices and establish reference standards (Smith et al. 1995).

In HGM applications, wetlands within a region that are similar based on hydrogeomorphic classification factors. More than one regional wetland subclass may be identified within each hydrogeomorphic wetland class, depending on the diversity of wetlands in a region and the assessment objectives (Smith et al. 1995).

In HGM, the relative area is a mathematical process used to "weight" the various applicable cover types on the basis of quantity. To derive the relative area of a model's PWAA, the following equation can be utilized:

Relative Area $=\underline{\text { Partial Wetland Assessment Area }}$ Total Area 
where:

Partial Wetland Assessment Area = only those acres assigned to the cover type of interest

Total Area = the sum of the acres associated with the Model.

Sampling Method

Scale
In HEP and HGM applications, this is the mode/ protocol followed to collect and gather field data. It is important to document the relevant criteria limiting the collection methodology. For example, the time of data collection, the type of techniques used, and the details of gathering this data should be documented as much as possible. An example of a sampling method would be:

Between March and April, run five random 50-m transects through the relevant cover types. Every 10$\mathrm{m}$ along the transect, place a $10-\mathrm{m}^{2}$ quadrant on the right side of the transect tape and record the percent herbaceous cover within the quadrant. Average the results per transect.

(1) In some geographical methodologies, scale is the defined size of the image in terms of miles per inch, feet per inch, or pixels per acre; (2) scale can also refer to different "sizes" of plans (Yoe and Orth 1996); or (3) variations of a management measure in cost analyses. Scales are mutually exclusive, and therefore a plan or alternative may only contain one scale of a given management measure (Robinson et al. 1995). 
Single Formula Model

(SM)

Site

Solution

(aka Alternative, more planning objectives (Yoe and Orth 1996).

\section{Alternative Plan,} or Plan)

Spreadsheet
In habitat/functional assessments, two potential types of models are selected to assess change at a site - the Single Formula Model and the Multiple Formula Model (refer to the definition above). In this instance, an HSI model (or an FCI model in HGM) is based on the existence of a single life requisite requirement (or single wetland function requirement in HGM), and a single formula is used to depict the relationship between quality and carrying capacity (or functional capacity in HGM) for the site.

The location upon which the project manager will take action, evaluate alternatives, and focus cost analysis (Robinson et al. 1995).
A solution is a way to achieve all or part of one or 
Target Year (TY)

\section{Trade-offs (TOs)}

Validation
In HEP and HGM applications, TY is a unit of time measurement that allows the project manager to anticipate and direct significant changes (in area or quality) within the project (or site). As a rule, the baseline TY is always TY $=0$, where the baseline year is defined as a point in time before proposed changes would be implemented. As a second rule, there must always be a $T Y=1$, and a $T Y=X_{2}$. TY 1 is the first year land- and water-use conditions are expected to deviate from baseline conditions. $\mathrm{TY}_{\mathrm{X} 2}$ designates the ending target year. A new target year must be assigned for each year the project manager intends to develop or evaluate change within the site or project. The habitat conditions (quality and quantity) described for each TY are the expected conditions at the end of that year. It is important to maintain the same target years in both the environmental and economic analyses.

Trade-offs are used to adjust the model outputs by considering human values. There are no right or proper answers, only acceptable ones. If trade-offs are used, outputs are no longer directly related to optimum habitat or wetland function (Robinson et al. 1995).

Establishing by objective, yet independent, evidence that the model specifications conform to needs and intended use(s). The validation process questions whether the model is an accurate representation of the system based on independent data not used to develop the model in the first place. Validation can encompass all of the information that can be verified, as well as all of the things that cannot - i.e., all of the information that the model designers might never have anticipated you might want or expect the product to do. 
For purposes of this effort, validation refers to independent data collections (bird surveys, water quality surveys, etc.) that can be compared to the model outcomes to determine whether the model is capturing the essence of the ecosystem's functionality.

Variable

Variable Subindex (VSI)
A measurable parameter that can be quantitatively described, with some degree of repeatability, using standard field sampling and mapping techniques. Often, the variable is a limiting factor for a wetland's functional capacity used in the development of VSI curves and measured in the field (or from aerial photos) by personnel, to fulfill the requirements of field data collection in a HEP or HGM application. Some examples of variables include: height of grass, percent canopy cover, distance to water, number of snags, and average annual water temperature.

In HGM applications, this index is a mathematical equation that reflects a wetland function's sensitivity to a change in a limiting factor (i.e., variable) within the PWAA that is compared to the reference standards of a regional wetland subclass in a reference domain (Smith et al. 1995). In HGM, VSIs are depicted using scatter plots and bar charts (i.e., functional capacity curves). The VSI value (Y-axis) ranges on a scale from 0.0 to 1.0 , where a VSI $=0.0$ represents a variable that is extremely limiting and a VSI $=1.0$ represents a variable in abundance (not limiting) for the wetland. 
Verification

Wetland Assessment Area (WAA)

Wetland Functions
Model verification refers to a process by which the development team confirms by examination and/ or provision of objective evidence that specified requirements of the model have been fulfilled with the intention of assuring that the model performs ( $0 \mathrm{r}$ behaves) as it was intended.

Sites deemed to be highly functional wetlands according to experts, should produce high HSI scores. Sites deemed dysfunctional (by the experts) should produce low HSI scores.

The wetland area to which results of an HGM assessment are applied (Smith et al. 1995).

The normal activities or actions that occur in wetland ecosystems. Wetland functions result directly from the characteristics of a wetland ecosystem, the surrounding landscape and the interaction between the two (Smith et al. 1995).

Without-Project Often confused with the terms "Baseline Condition" Condition (WOP) (aka No Action Plan or No Action Alternative) and "Existing Condition," the Without-Project Condition is the expected condition of the site without implementation of an alternative over the life of the project, and is also referred to as the "No Action Plan" in traditional planning studies (Yoe and Orth 1996; U.S. Army Corps of Engineers (USACE) 2000).

With-Project Condition (WP)

In planning studies, this term is used to characterize the condition of the site after an alternative is implemented (Yoe and Orth 1996; USACE 2000). 


\section{Appendix C: Notation}

$\begin{array}{ll}\text { AAFCU } & \text { Average Annual Functional Capacity Unit } \\ \text { AAHU } & \text { Average Annual Habitat Unit } \\ \text { A-Team } & \text { Assessment Team in HGM Applications } \\ \text { CT } & \text { Cover Type } \\ \text { EMRRP } & \text { USACE's Ecosystem Management and Restoration } \\ & \text { Research Program } \\ \text { EOA } & \text { Equivalent Optimal Area } \\ \text { ERDC-EL } & \text { U.S. Army Engineer Research and Development } \\ \text { E-Team } & \text { Center, Environmental Laboratory } \\ \text { EXHEP } & \text { Evaluation Team } \\ \text { EXHGM } & \text { EXpert Habitat Evaluation Procedures Module } \\ \text { FCI } & \text { Assessments Module } \\ \text { FCU } & \text { Functional Capacity Index } \\ \text { FF } & \text { Functional Capacity Unit } \\ \text { GIS } & \text { Future Factor } \\ \text { HEAT } & \text { Geographic Information Systems } \\ \text { HEP } & \text { Habitat Evaluation and Assessment Tools } \\ \text { HGM } & \text { Habitat Evaluation Procedures } \\ \text { HSI } & \text { Hydrogeomorphic Assessment of Wetlands } \\ \text { HU } & \text { Habitat Suitability Index } \\ \text { LRSI } & \text { Habitat Unit } \\ \text { NEPA } & \text { Life Requisite Suitability Index } \\ \text { NRCS } & \text { National Environmental Policy Act } \\ \text { PWAA } & \text { Natural Resources Conservation Service } \\ \text { RA } & \text { Partial Wetland Assessment Area } \\ & \text { Relative Area } \\ & \end{array}$




$\begin{array}{ll}\text { SI } & \text { Suitability Index } \\ \text { SM } & \text { Single Formula Model } \\ \text { SWWRP } & \text { System-Wide Water Resources Program } \\ \text { TO } & \text { Trade-Off } \\ \text { TY } & \text { Target Year } \\ \text { USACE } & \text { U.S. Army Corps of Engineers } \\ \text { USEPA } & \text { U.S. Environmental Protection Agency } \\ \text { USFWS } & \text { U.S. Fish and Wildlife Service } \\ \text { VGA } & \text { Video Graphics Adapter } \\ \text { VSI } & \text { Variable Subindex } \\ \text { WAA } & \text { Wetland Assessment Area } \\ \text { WOP } & \text { Without-Project Conditions } \\ \text { WP } & \text { With-Project Conditions } \\ \text { WRAP } & \text { Wetlands Regulatory Assistance Program, USACE }\end{array}$




\section{Appendix D: Suggested Variable Naming Conventions}

In the HEAT software, index model components, (i.e., models, functions, life requisites, cover types, PWAAs, and variables) are assigned Identification Codes in the setup process that are then carried forward into the association, analysis, and report interfaces of the program. These codes are limited to just 10 characters, and in most instances, the software sorts pull-down menus and report tables alphabetically based on these codes. It is therefore in the user's best interest to develop codes for these components in a thoughtful manner - an intuitive strategy that keeps the user organized, and facilitates efficient use of the software (i.e., a well-thoughtout naming convention will save time and convey the results effectively). Over the course of using the software for the last 15 years, the authors have developed a component-naming protocol that seems to work well. Here are some suggestions.

Remember, the constraints in naming the parameters are:

1. They must be 10 characters or less.

2. No spaces are allowed (but the underline character can be used to forcea space).

3. Numbers areallowed.

4. Special characters (i.e.,,,$-+ /$,etc.) are not allowed.

The strategy is fairly straightforward, but changes slightly across components. The goal is to give a code to the component that is instantly recognizable. Strategies are described below along with numerous examples that have worked in the authors' applications. Users can make use of this information as they choose - there are no requirements to adopt these strategies in the software. In other words, users can choose to develop their own naming conventions and ignore these suggestions. The key to success is simplicity - make the names and codes work for you. 


\section{Suggestion \#1: Coding HSI Models}

The key to naming HSI models is to focus on the community or species and make the code as intuitive as possible. To begin, write out the full name of the species or community. Strip away everything but the most descriptive nouns. Remove the spaces between these words. Then shorten the nouns (by removing vowels and duplicated letters).

1st Example:

- Model Name:

Slider Turtle

- Keep Descriptive Nouns:

SLIDER TURTLE

- Remove Spaces:

SLIDERTURTLE

- Shorten the Nouns:

SLIDTURTLE (10 characters)

$2^{\text {nd }}$ Example:

- Model Name:

Forest Floodplain Community

- Keep Descriptive Nouns:

FOREST FLOODPLAIN

- Remove Spaces:

FORESTFLOODPLAIN

- Shorten the Nouns:

FORFLOOD (8 characters)

3rd Example:

- Model Name:

Gravel Bar Mussel Community

- Keep Descriptive Nouns:

GRAVEL BAR MUSSEL

- Remove Spaces:

GRAVELBARMUSSEL

- Shorten the Nouns:

GRVBMUSSEL (10 characters)

\section{Suggestion \#2: Naming FCI Functions}

The same approach described above in Suggestion \#1 can be applied to develop codes for functions. However, HGM developers often number the functions in their models, and then refer to these indices by number rather than by function name. It is therefore useful to include the function number in the naming convention. Because the software couples the Function Name and Function Identification Codes in all pull-down menus, it is only necessary to insert numbers into the Function Name cells provided. These numbers should be inserted into the beginning of each Function Name, so that the software sorts by function number when presenting information. 
Simply write out the full name of the function and insert the function number into the description. One word of caution - be sure to insert a zero in front of single digits, or the system will sort double digits inside single digits - 1, 10, 11, 2, 3, 4, etc. Up to 100 characters are allowed (but spaces count), and special characters can be used (i.e., colons).

1st Example:

- Function Name:

- New Function Name:

$2^{\text {nd }}$ Example:

- Function Name:

- New Function Name:

3rd Example:

- Function Name:

- New Function Name:
Maintain Characteristic Water Level Regime (Function \#1)

Fxn 01: Maintain Characteristic Water Level Regime (51 characters)

\author{
Maintain Characteristic Animal \\ Communities (Function \#15) \\ Fxn 15: Maintain Characteristic \\ Animal Communities
}

Maintain Characteristic Biogeochemical Processes (Function \#7)

Fxn 07: Maintain Characteristic Biogeochemical Processes

\section{Suggestion \#3: Creating Cover Type (or PWAAs) Codes}

The key to developing cover type (or PWAA) codes is to leave enough room in the codes to "capture" the future conditions of the site. For every forest today, there could be a potential newly developed forest tomorrow (i.e., a plot of land that is restored through some action to forest under the withproject alternative). Therefore it is important to name and pair these before and after scenarios in an easily recognizable code set. 
1st Example:

- Cover Type Name:

- Code:

- Cover Type Name:

- Code:

2nd Example:

- Cover Type Name:

- Code:

- Cover Type Name:

- Code:

3rd Example:

- PWAA Name:

- Code:

- PWAA Name:

- Code:
Floodplain Forest

FOREST (6 characters)

Newly Restored Floodplain Forest

NEWFOREST ( 9 characters)

Salt Marsh

SALTMARSH (9 characters)

Newly Restored Salt Marsh

NEWSALTMSH (10 characters)

\section{Suggestion \#4: Creating Life Requisites Codes}

HSI models often use similar naming conventions across models to describe life requisites (i.e., Cover, Reproduction, Food, etc.). It is therefore suggested that the name of the species or community be included in the codes for life requisites. The authors suggest writing out the life requisite in its entirety. Strip away everything but the key nouns and verbs. Insert the name of the species or community into the front of this description. Remove the spaces between these words. Shorten the species/ community name to fewer than three characters (remove vowels and most of the consonants). Then shorten the remaining nouns (by removing vowels and duplicated letters). 
1st Example:

- Life Requisite Name: $\quad$ Food/ Cover Component (Slider Turtle)

- Insert Species/Community: SLIDER TURTLE FOOD COVER COMPONENT

- Keep Nouns and Verbs: SLIDER TURTLE FOOD COVER

- Remove Spaces:

SLIDERTURTLEFOODCOVER

- Shorten Species/Community: SLTFOODCOVER

- Shorten the Nouns:

SLTFOODCOV (10 characters)

$2^{\text {nd }}$ Example:

- Life Requisite Name: Water Component (Slider Turtle)

- Insert Species/Community: SLIDER TURTLE WATER

COMPONENT

- Keep Nouns and Verbs: SLIDER TURTLE WATER

- Remove Spaces: SLIDERTURTLEFWATER

- Shorten Species/Community: SLTWATER

- Shorten the Nouns: SLTWATER (10 characters)

3rd Example:

- Life Requisite Name: Temperature Component (Slider Turtle)

- Insert Species/Community: SLIDER TURTLE TEMPERATURE

COMPONENT

- Keep Nouns and Verbs: SLIDER TURTLE TEMPERATURE

- Remove Spaces: SLIDERTURTLETEMPERATURE

- Shorten Species/Community: SLTTEMPERATURE

- Shorten the Nouns: $\quad$ SLTTEMP (10 characters)

\section{Suggestion \#5: Generating Variable Codes by Clustering Actions}

Write out the component in its entirety. Strip away everything but the nouns and verbs. Remove the spaces between these words. Reverse the actions (i.e., put the nouns last and the activity or verbs first), and count the number of characters remaining. Shorten the actions to fewer than five characters (by removing vowels and duplicated letters). Then shorten the nouns (by removing vowels and duplicated letters). 
1st Example:

- Variable Name:

Percent Tree Canopy Cover

- Keep Nouns and Verbs:

TREE CANOPY COVER

- Remove Spaces:

TREECANOPYCOVER

- Reverse the Actions:

CANOPY COVERTREE

- Shorten the Actions:

CANTREE ( 7 characters)

$2^{\text {nd }}$ Example:

- Variable Name:

Percent Shrub Canopy Cover

- Keep Nouns and Verbs:

SHRUB CANOPY COVER

- Remove Spaces:

SHRUBCANOPYCOVER

- Reverse the Actions:

CANOPY COVERSHRUB

- Shorten the Actions:

CANSHRUB (8 characters)

$3^{\text {rd }}$ Example:

- Variable Name:

Percent Herbaceous Canopy Cover

- Keep Nouns and Verbs:

HERBACEOUS CANOPY COVER

- Remove Spaces:

HERBACEOUSCANOPYCOVER

- Reverse the Actions:

- Shorten the Actions:

CANOPY COVERHERBACEOUS

CANHERBACEOUS

- Shorten the Nouns:

CANHERB ( 7 characters)

Common "actions" in index models include: height (e.g., of vegetation), depth (e.g., of water or soils), density (e.g., of stems or perches), presence/ absence, structure (e.g., vegetative layering), types (e.g., categories of vegetation), and distance (e.g., to or from an item of interest).

\section{Suggestion \#6: Generating Variable Codes by Clustering Elements}

Components can also be clustered based on elements (i.e., water, soils, vegetation, landscape, etc.). In this case, the component is written out in its entirety. Strip away everything but the nouns and verbs. Remove the spaces between these words. Start with the elements (i.e., insert the elements first and the measurements last), and count the number of characters remaining. Shorten the elements to fewer than five characters (by removing vowels and duplicated letters). Then shorten the measurements (by removing vowels and duplicated letters). 
1st Example:

- Variable Name:

Canopy Structure (Number of Layers)

- Keep Nouns and Verbs:

CANOPY STRUCTURE

- Remove Spaces:

CANOPYSTRUCTURE

- Elements First:

VEGETATIONCANOPYSTRUCTURE

- Shorten the Elements:

VEGCANOPYSTRUCTURE

- Shorten the Measurements:

VEGSTRUCT (9 characters)

$2^{\text {nd }}$ Example:

- Variable Name:

Depth of Organic Matter

- Keep Nouns and Verbs:

DEPTH ORGANIC MATTER

- Remove Spaces:

DEPTHORGANICMATTER

- Elements First:

SOILDEPTHORGANICMATTER

- Shorten the Elements: SOILDEPTHORGANICMATTER

- Shorten the Measurements:

SOILORGDEP (10 characters)

$3^{\text {rd }}$ Example:

- Variable Name:

Depth to Ground Water

- Keep Nouns and Verbs:

DEPTH GROUND WATER

- Remove Spaces:

DEPTHGROUNDWATER

- Elements First:

WATERGROUNDDEPTH

- Shorten the Elements:

WATERGROUNDEPTH

- Shorten the Measurements: WATERDEPTH (10 characters)

Using this approach, all of the variables that focus on soils (or water or vegetation) will be clustered.

\section{Suggestion \# 7: Generating Variable Codes by Clustering Sources}

Variables can also be named based on the medium or sources from which they are derived (i.e., field measurements, historical data, or even GIS). In this case, write out the component in its entirety. Strip away everything but the nouns and verbs. Remove the spaces between these words. Start with the source (i.e., insert the elements first and the measurements last), and count the number of characters remaining. Shorten the elements to fewer than five characters (by removing vowels and duplicated letters). Then shorten the measurements (by removing vowels and duplicated letters). 
1st Example:

- Variable Name:

Patch Size (GIS)

- Keep Nouns and Verbs:

PATCH SIZE

- Remove Spaces:

PATCHSIZE

- Source First:

GISPATCHSIZE

- Shorten the Source:

GISPATCHSIZE

- Shorten the Measurements: GISPATCHSZ (10 characters)

$2^{\text {nd }}$ Example:

- Variable Name:

Water Regime (FLOW2D Water Model)

- Keep Nouns and Verbs:

WATER REGIME

- Remove Spaces:

WATERREGIME

- Source First:

FLOW2DWATERREGIME

- Shorten the Source:

F2DWATERREGIME

- Shorten the Measurements: F2DREGIME (9 characters)

3rd Example:

- Variable Name:

Number of Perches (Field

Measurement)

- Keep Nouns and Verbs:

NUMBER PERCHES

- Remove Spaces:

NUMBERPERCHES

- Elements First:

FIELDNUMBERPERCHES

- Shorten the Elements:

FIELDNUMBERPERCHES

- Shorten the Measurements: FIELDPERCH (10 characters) 


\section{Appendix E: Training Exercises}

A series of interactive training exercises have been provided here to assist users in learning and practicing the skills necessary to effectively evaluate alternative designs using the HEAT software. Each exercise is accompanied by start-up datafiles that can be accessed from the training folder under the install directory and are indicated with blue text in this guide. Output files (i.e., MS Word documents exported from the HEAT reports) are also supplied for use and reference (identified by yellow highlighted text) and can be accessed from the installation CD. ${ }^{1}$

When the HEAT software package is installed, a series of tutorials are also installed on the hard drive. The following exercises direct users to open various datafiles within the tutorials. For convenience, all files have been stored in the following directory:

\section{- C: \Program Files $\backslash$ HEAT-Habitat Evaluation and Assessment Tools $\backslash$ Datafiles $\backslash$ appendix e}

Navigate to this subdirectory and locate the folder based on the exercise being undertaken to find the necessary files.

\section{Exercise 1: Project Information and Model Setup in EXHEP}

The U.S. Army Corps of Engineers has undertaken a feasibility study to develop a cost-effective restoration plan for a large parcel along Mill Creek in southern Texas. Without action, the wetlands will in all likelihood be drained and converted to agricultural croplands. The primary goal of the study is to purchase and restore the agricultural croplands on the site to a fully functioning wetland habitat while protecting and restoring the environmental integrity and beneficial uses of the current wetland habitats already present. The study's objectives include:

1. Protect and enhance water quality and habitat.

2. Preserve and restore natural resources.

\footnotetext{
${ }_{1}$ An installation CD for the Habitat Evaluation and Assessment Tools (HEAT) program can be obtained by contacting Kelly Burks-Copes at 601-634-2290 (Kelly.A.Burks-Copes@usace.army.mil).
} 
3. Preserve cultural resources.

4. Increase open space, recreational opportunities, and greenways.

Specific performance measures include:

1. Restoration of Marsh Wren habitat to sustainable levels (i.e., $100+$ acres with at least a 0.50 HSI score).

2. Restoration of Slider Turtle habitat to sustainable levels (i.e., 100+acres with at least a 0.50 HSI score).

An interagency team has been convened to participate in the study. Names and affiliations of the team members are as follows:

- Andrea Fisher and J oe Black (USACE District)

- J ohn Anderson (Harris County Forest Preserve)

- Eric Brand (Natural Resources Conservation Service)

- William Malone and Chris White (Texas Parks and Wildlife Department)

- Cynthia Miller (Texas State Geological Survey)

- Sally Mengler (USFWS)

- Ingrid East (Mill Creek Ecosystem Partnership)

- Kelly Burks-Copes and Antisa Webb (ERDC Facilitators)

The team has identified several restoration alternatives. However, they need to determine how much each alternative will cost and whether the alternatives are biologically productive. The project area covers a total of 150 acres. Below is a table containing a breakdown of acres by cover type (Table E1).

Table E1. Baseline cover types and acreages for the study.

\begin{tabular}{|c|c|c|c|}
\hline No. & Code & Cover Type Descriptions & $\begin{array}{l}\text { Baseline } \\
\text { Acres } \\
\text { (TYo) }\end{array}$ \\
\hline 1 & EMERGENT & Emergent Wetlands & 50 \\
\hline 2 & NEWEMERG & Newly Developed Emergent Wetlands & 0 \\
\hline 3 & SCRUBSHRUB & Scrub-Shrub Wetlands & 30 \\
\hline 4 & NEWSCRUB & Newly Developed Scrub-Shrub Wetlands & 0 \\
\hline \multirow[t]{2}{*}{5} & AGCROP & Agricultural Croplands & 70 \\
\hline & & TOTAL: & 150 \\
\hline
\end{tabular}


The team selected two HSI models, the Marsh Wren and the Slider Turtle, to assess the restoration alternatives. Details regarding each model's associated cover types and variables (i.e., model components, sampling protocols, data management, etc.) have been placed on the user's hard drive during installation of the software (refer to the Marsh Wren Field Kit; Slider Turtle Field Kit workbooks). For purposes of this exercise, the associations in these files have been simplified (i.e., not all associations in the model documentation have been presented in the files). Nonetheless, use this information to set up the models in the following exercises.

1. To begin, open the Blank datafile.mdb with EXHEP (go to C: \Program Files\HEAT-Habitat Evaluation and Assessment Tools \Datafiles\appendix el Exercise 1), name it something unique (say Exercise 1.mdb), and save it to the Exercise 1 folder under the Training Exercise subdirectory under Environmental Programs on the hard drive. Now, document the basic premises behind the study in the methods interface (click the Setup button under the Single Formula Models section and double-click the 1. Enter Project Methods option). The following information might be important to enter into the interface:

- Model references and support documentation.

- Model modifications (if any).

- List of evaluation team members.

- Goals and objectives.

- Data management strategies.

- Evaluation strategies (including tradeoff approaches).

- Field sampling team and metadata (include locations, assumptions, dates, etc.).

At the absolute minimum, a Project Name (i.e., Mill Creek Ecosystem Restoration Study) and Alternative Name (i.e., Design 1) must be entered in the spaces provided.

2. Now set up the marsh wren model by assigning names and describe the model in the model description interface (double-click the 2. Enter Model Descriptions option). Hint - look in the MS Excel "field kits" for the Methods worksheet to see details.

3. Define the cover types in the study area in the cover type interface (doubleclick the 3. Define Cover Types and Baseline Acres option). 
Hint - look in the MS Excel "fieldkits" for the "Cover Types" worksheet to see details.

4. Now enter the variables (double-click the 4. Define Variables option). Hint - look in the MS Excel "fieldkits" for the "Variables" worksheet to see details.

5. Repeat the process for the second model (Slider Turtle). Don't forget the extra step (i.e., entering the life requisites). Click on the Setup button under the Multiple Formula Models section and systematically go through thelast four options:

- Enter Model Descriptions

- Enter Life Requisite Descriptions

- Define Cover Types and Baseline Acres

- Define Variables

Note: It is not mandatory for the first option (1. Enter Project Methods) to be completed - EXHEP assumes the same overriding approach entered earlier in the marsh wren exercise is still applicable here. Hint - look in the MS Excel "fieldkits" for the ". . Life Requisites" tab for details on Step 3.

6. Use the reports in EXHEP to check work and document analysis (Setup Reports button). The following reports should be created as a result:

- Methods and Assumptions.

- Model List - Single Formula Models.

- Model List - Multiple Formula Models.

- Life Requisite List.

- Cover Types and Baseline (TY 0) Acres.

\section{- Variables List.}

The files on the disk are not formatted as they might appear in EXHEP (the actual EXHEP reports were exported to MS Word for purposes of distribution), but the information contained within is accurate. 


\section{Exercise 2: Single Formula Model Association and HSI Formula Writing in EXHEP}

For users who need help writing formulas in EXHEP, three helpful tools are provided in the software to make the process easier. The first tool is called a "Cheat Sheet." Just click on the Model Association screen (under Run, 2. Associate Parameters) to view this screen:

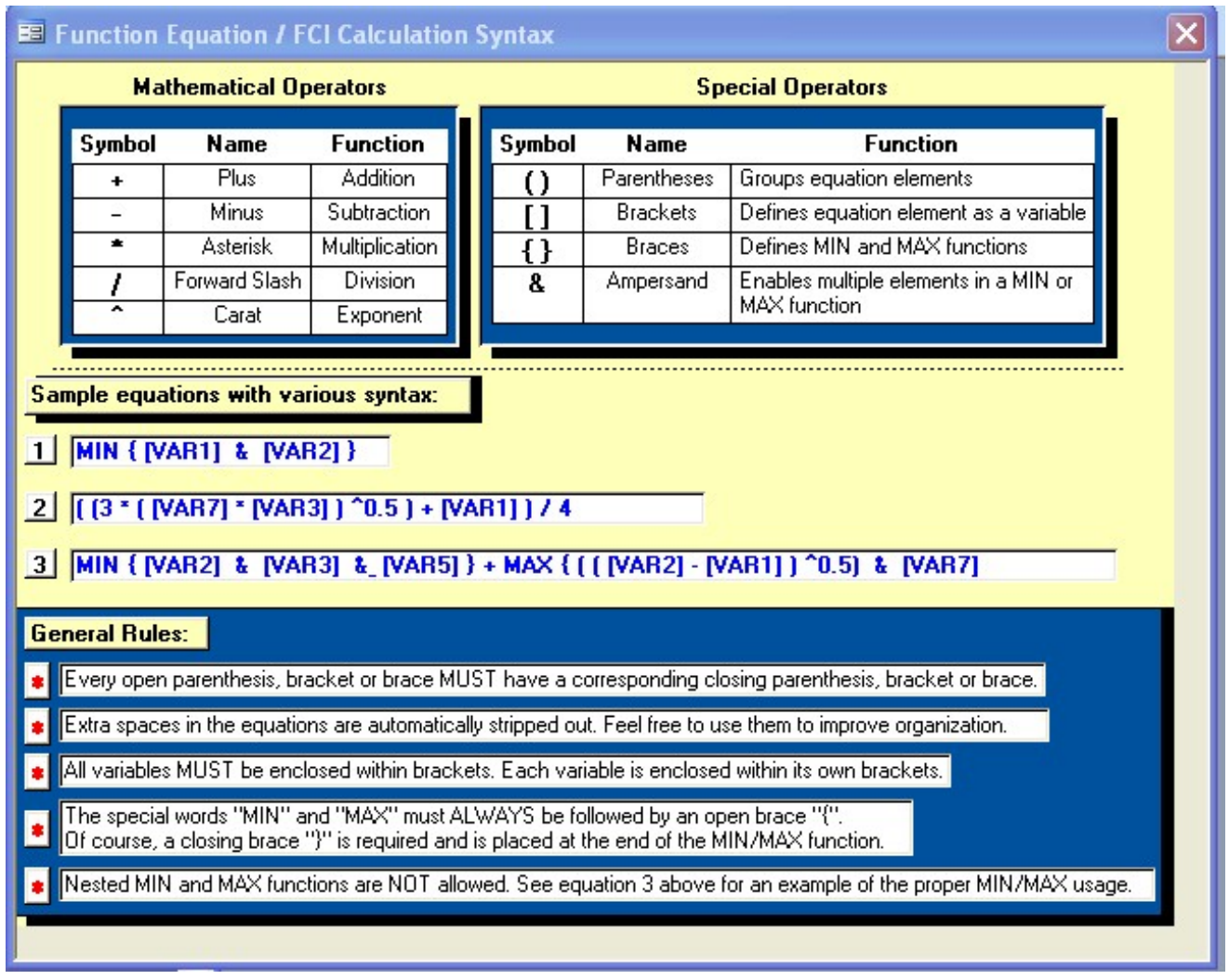

The HSI formulas for the Marsh Wren HSI model can be written using these syntax rules. 
Marsh Wren (single formula model)

$$
\text { Index } x_{\text {MARSHWREN }}=\left[\left(v_{1} \times v_{2} \times v_{3}\right)^{1 / 3}\right] \times v_{4}
$$

Where:

$$
\begin{aligned}
& \mathrm{V}_{1}=\text { Dominant Vegetative Type (TYPGROWFRM) } \\
& \mathrm{V}_{2}=\text { Emergent Canopy Cover (CANEMERG) } \\
& \mathrm{V}_{3}=\text { Water Depth (DEPTHWATER) } \\
& \mathrm{V}_{4}=\text { Woody Canopy Cover (CANWOOD6) }
\end{aligned}
$$

Follow the syntax rules to enter the formula into the software (correct formula can be found on the next page).

The Marsh Wren's HSI formula = 
Correct answer:

$(([T Y P G R O W F R M] *[$ CANEMERG $] *[$ DEPTHWATER $]) \wedge(1 / 3)) *[$ CANWOOD6]

The formula can now be entered into the software.

1. To begin, open the training datafile entitled Exercise 2.mdb and click on the Run button under the Single Formula Models section of the primary interface.

2. Double-click on the first option (1. Choose Working Model), select the Marsh Wren option from the pull-down list, and click Close.

3. Now double-click on the second option (2. Associate Parameters).

4. Click on the Cover Type drop-down box, and choose EMERGENT from the pull-down list.

5. Click once on the HSI Model Formula input box and assure that the cursor is to the immediate right of the " $\mathbf{1}$ " placeholder.

6. Now move the cursor to the floating Variables Available keypad and scroll down through the list of variables. Single-click on the variables TYPGROWFRM, CANEMERG, DEPTHWATER, and finally CANWOOD6.

7. Systematically place the cursor between each of the variable codes in the HSI Model Formula input box, and insert an operator (refer to the manual for these) using either the operator keys on the floating keypad, or type them in using the keys on the keyboard (look to the box above to check input).

8. Put the cursor between this first variable and the "1*" and backspace twice or put the cursor immediately before the "1*" and hit the Delete button on the floating keypad.

9. Click on the Equation Check button immediately to the right of the HSI Model Formula input box to check work.

10. Remove any phantom records if they exist.

Now duplicate the formula for all the cover types associated with the model.

1. To begin, click on the cover type drop-down box, and choose NEWEMERG.

2. Click on the Back Record button, click on the Copy button, click on the Forward Record button, and click on the Paste button. 
3. Click on the cover type drop-down box, and choose NEWSCRUB and click on the Paste button once again.

4. Click on the cover type drop-down box, and choose SCRUBSHRUB and click on the Paste button one last time.

5. Remove any phantom records if they exist.

By now you should have four records - one for each cover type in the model (Table E2).

Table E2. Record list for Exercise 2 after formula entry.

\begin{tabular}{|c|c|c|c|}
\hline $\begin{array}{l}\text { Record } \\
\text { Count }\end{array}$ & Model & Cover Type & HSI Formula \\
\hline 1 & MARSHWREN & EMERGENT & $\left(([\text { TYPGROWFRM }] *[\text { CANEMERG }] *[\text { DEPTHWATER }])^{\wedge}(1 / 3)\right)^{*}[$ CANWOOD 6$]$ \\
\hline 2 & MARSHWREN & NEWEMERG & $\left(([T Y P G R O W F R M] *[\text { CANEMERG }] *[\text { DEPTHWATER }])^{\wedge}(1 / 3)\right)^{*}[$ CANWOOD 6$]$ \\
\hline 3 & MARSHWREN & NEWSCRUB & $\left(\left([T Y P G R O W F R M] *[\text { CANEMERG }]^{*}[\text { DEPTHWATER }]\right)^{\wedge}(1 / 3)\right)^{*}[$ CANWOOD 6$]$ \\
\hline 4 & MARSHWREN & SCRUBSHRUB & $\left(([T Y P G R O W F R M] *[\text { CANEMERG }] *[\text { DEPTHWATER }])^{\wedge}(1 / 3)\right)^{*}[$ CANWOOD 6$]$ \\
\hline
\end{tabular}

Use the reports in EXHEP to check work and document analysis (Setup Reports button). The following reports should be created as a result:

- Formulas - Single Formula Models

- Associations - Single Formula Models 


\section{Exercise 3: Multiple Formula Model Association and LRSI/HSI Formula Writing in EXHEP}

For users who need help writing formulas in EXHEP, three helpful tools are provided in the software to make the process easier. The first tool is called a "Cheat Sheet." Just click on the Model Association screen (under Run, 2. Associate Parameters) to view this screen:

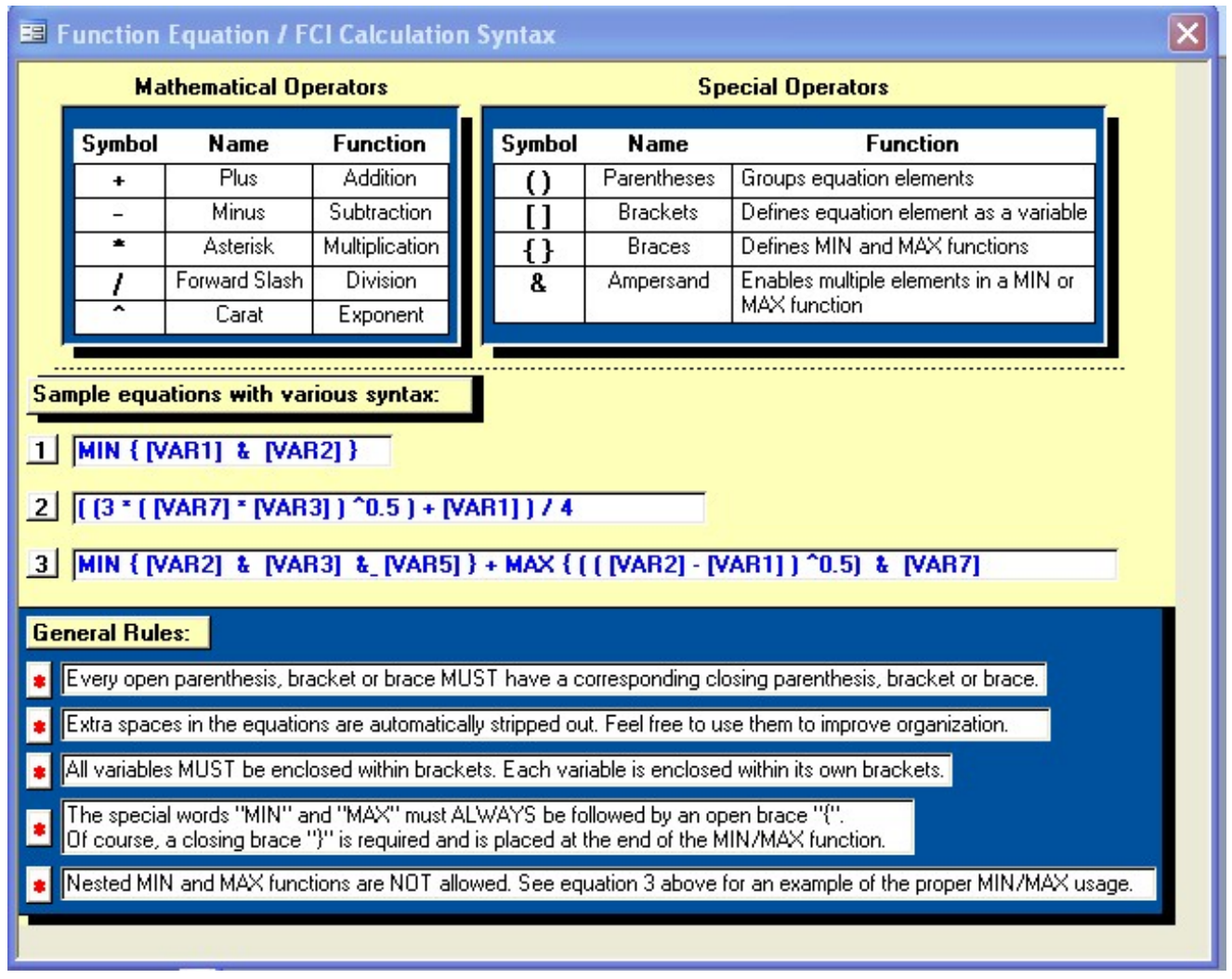

The HSI and LRSI formulas for the Slider Turtle HSI model can be written using these syntax rules. 


\section{Slider Turtle (multiple formula model) \\ IndeXSLIDTURTLE $=$ Minimum of LRSISTFOODCVR or LRSISTTEMP or LRSISTWATER}

Where:
LRSISTFOODCVR $=$
V CANSUBMERG
LRSISTTEMP $_{\text {S }}=\mathrm{V}_{\text {TEMPWATER }}$
LRSISTWATER $_{\text {S }}$ Minimum of V VELOCITY Or V VEPTHWATER Or V $\mathrm{V}_{\text {REGIMEWATR }}$

Follow the syntax rules to enter the formula into the software (correct formula can be found on the next page).

The Slider Turtle HSI formula =

The Slider Turtle's STFOODCVR LRSI formula =

The Slider Turtle's STTEMP LRSI formula =

The Slider Turtle's STWATER LRSI formula = 
Correct answers:

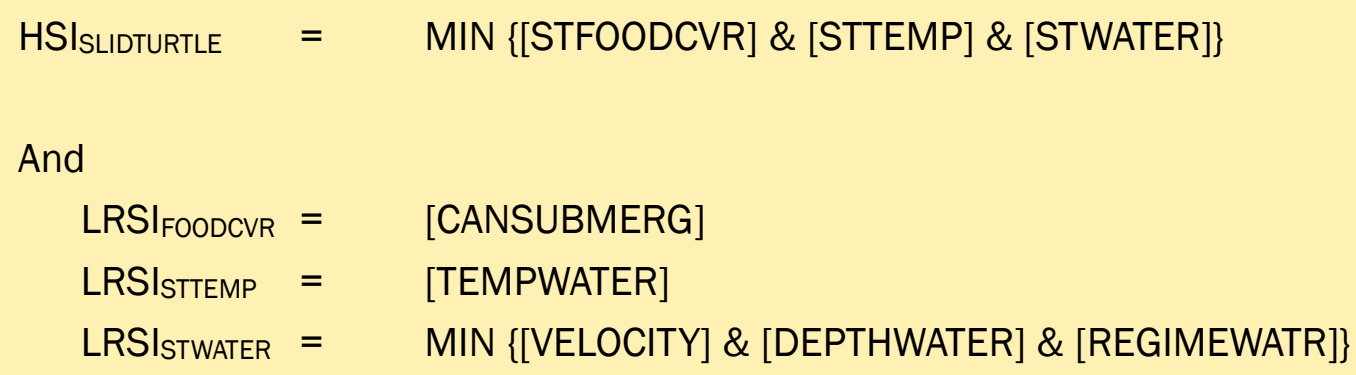

The formula can now be entered into the software.

1. To begin, open the training datafile entitled Exercise 3.mdb and click on the Run button under the Multiple Formula Models section of the primary interface.

2. Double-click on the first option (1. Choose Working Model), select the Slider Turtle option from the pull-down list, and click Close.

3. Now double-click on the second option (2. Associate Parameters).

Chapter 5 (Applying EXHEP) provides some good illustrations of the process about to be undertaken, so users might want to turn to the section on associating models now. Also, there are many approaches to associating models in the software - Chapter 5 focuses on completing one record at a time. Users comfortable with that approach are encouraged to use it! A different approach will be presented here - one that focuses on populating the specific fields in the records rather than Chapter 5's approach. First, placeholders will be created for all the LRSI-cover type combinations in the model.

1. Click on the Life Requisite drop-down box, and choose STFOODCVR; click on the Cover Type drop-down box and choose EMERGENT from the pull-down list.

2. There are many ways to approach the duplication of these associations. One approach is to systematically pull down the other three cover types associated with the model for this life requisite (i.e., NEWEMERG,

NEWSCRUB, and SCRUBSHRUB), then choose each of the remaining life requisites (STTEMP and STWATER) and repeat the process.

3. Remove any phantom reconds if they exist. 
By now there should be 12 records - one for each LRSI-cover type combination. It is time to put the individual LRSI formulas into each of these records. There are four records per LRSI (four cover types $\times$ three LRSIs) (Table E3).

Table E3. Record list for Exercise 3 prior to formula entry.

\begin{tabular}{|c|c|c|c|c||}
\hline Record Count & Life Requisite & Cover Type & HSI Formula & LRSI Formula \\
\hline 1 & {$[$ STFOODCVR] } & EMERGENT & $1^{*}$ & $1^{*}$ \\
\hline 2 & {$[$ STFOODCVR] } & NEWEMERG & $1^{*}$ & $1^{*}$ \\
\hline 3 & {$[$ STFOODCVR] } & NEWSCRUB & $1^{*}$ & $1^{*}$ \\
\hline 4 & {$[$ STFOODCVR] } & SCRUBSHRUB & $1^{*}$ & $1^{*}$ \\
\hline 5 & {$[$ STTEMP] } & EMERGENT & $1^{*}$ & $1^{*}$ \\
\hline 6 & {$[$ STTEMP] } & NEWEMERG & $1^{*}$ & $1^{*}$ \\
\hline 7 & {$[$ STTEMP] } & NEWSCRUB & $1^{*}$ & $1^{*}$ \\
\hline 8 & {$[$ STTEMP] } & SCRUBSHRUB & $1^{*}$ & $1^{*}$ \\
\hline 9 & {$[$ STWATER] } & EMERGENT & $1^{*}$ & $1^{*}$ \\
\hline 10 & {$[$ STWATER] } & NEWEMERG & $1^{*}$ & $1^{*}$ \\
\hline 11 & {$[$ STWATER] } & NEWSCRUB & $1^{*}$ & $1^{*}$ \\
\hline 12 & {$[$ STWATER] } & SCRUBSHRUB & $1^{*}$ & \\
\hline
\end{tabular}

Now it is time to enter the HSI formula into each of these records. The same HSI formula must be used for each LRSI, so the process is fairly simple.

1. Go back to the first record (use the forward/ back arrows), left-click once on the HSI Model Formula input box, and assure that the cursor is to the immediate right of the " $\mathbf{1}$ " placeholder.

2. Now move the cursor to the floating LR Codes Available for HSI Formulas keypad and scroll down through the list of life requisites.

3. Single-click on the life requisites STFOODCVR, STTEMP, and finally STWATER.

4. Systematically place the cursor between each of the variable codes in the HSI Model Formula input box, and insert an operator (refer to the manual for these) using either the operator keys on the floating keypad, or type them in using the keyboard. The formula should look like this:

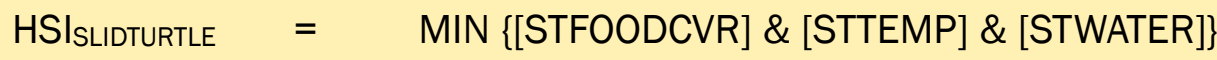


5. Place the cursor between this first variable and the "1*" and backspace twice or put the cursor immediately before the "1*" and hit the Delete button on the floating keypad.

6. Click on the Equation Check button immediately to the right of the HSI Model Formula input box to check your work.

Now it's time to copy the HSI formula to the other 11 records.

1. To begin, block the formula, hold down the CTRL button on the keyboard, and type $\mathbf{C}$ (copying the formula to the clipboard).

2. Use the forward arrow to move through each record and paste the formula into the HSI Model Formula input box by left-clicking once on the space, holding down $\mathbf{C T R L}$ on the keyboard and typing $\mathbf{V}$.

3. Repeat the process 11 times - there should be 12 records total (Table E4).

Table E4. Record list for Exercise 3 after HSI formula entry.

\begin{tabular}{|c|c|c|c|c|}
\hline $\begin{array}{l}\text { Record } \\
\text { Count }\end{array}$ & Life Requisite & Cover Type & HSI Formula & LRSI Formula \\
\hline 1 & [STFOODCVR] & EMERGENT & MIN $\{[$ STFOODCVR] \& [STTEMP] \& [STWATER] $\}$ & $1 *$ \\
\hline 2 & [STFOODCVR] & NEWEMERG & MIN $\{[$ STFOODCVR] \& [STTEMP] \& [STWATER] $\}$ & $1 *$ \\
\hline 3 & [STFOODCVR] & NEWSCRUB & MIN $\{[$ STFOODCVR] \& [STTEMP] \& [STWATER] $\}$ & $1 *$ \\
\hline 4 & [STFOODCVR] & SCRUBSHRUB & MIN $\{[$ STFOODCVR] \& [STTEMP] \& [STWATER]\} & $1 *$ \\
\hline 5 & [STTEMP] & EMERGENT & MIN $\{[S T F O O D C V R] \&[S T T E M P] \&$ [STWATER] $\}$ & $1 *$ \\
\hline 6 & [STTEMP] & NEWEMERG & MIN $\{[$ STFOODCVR] \& [STTEMP] \& [STWATER] $\}$ & $1 *$ \\
\hline 7 & [STTEMP] & NEWSCRUB & MIN $\{[$ STFOODCVR] \& [STTEMP] \& [STWATER] $\}$ & $1 *$ \\
\hline 8 & [STTEMP] & SCRUBSHRUB & MIN $\{[$ STFOODCVR] \& [STTEMP] \& [STWATER]\} & $1 *$ \\
\hline 9 & [STWATER] & EMERGENT & MIN $\{[$ STFOODCVR] \& [STTEMP] \& [STWATER]\} & $1 *$ \\
\hline 10 & [STWATER] & NEWEMERG & MIN $\{[S T F O O D C V R] \&[S T T E M P] \&$ [STWATER]\} & $1 *$ \\
\hline 11 & [STWATER] & NEWSCRUB & MIN $\{[S T F O O D C V R] ~ \&[S T T E M P] \&$ [STWATER]\} & $1 *$ \\
\hline 12 & [STWATER] & SCRUBSHRUB & MIN $\{[$ STFOODCVR] \& [STTEMP] \& [STWATER]\} & $1 *$ \\
\hline
\end{tabular}

Now it's time to enter each combination's unique LRSI.

1. To begin, use the back/ forward arrows to return to the first record ( $\mathrm{LR}=$ STFOODCVR, and cover type = EMERGENT)

2. Left-click once on the LRSI Entry Space. Now move the cursor to the floating Variables Available keypad and scroll down through thelist of variables. 
3. Single-click on the variable CANSUBMERG. This is the only variable needed to complete the LRSI formula for this life requisite. The LRSI formula is:
$\mathrm{LRSI}_{\text {FOODCVR }}=$
[CANSUBMERG]

4. Place the cursor between this variable and the "1*" and backspace twice or put the cursor immediately before the "1*" and hit the Delete button on the floating keypad.

5. Click on the Copy button, click on the Forward Record button, and click on the Paste button in the NEWEMERG record.

6. Click on the Forward Record button, and click on the Paste button in the NEWSCRUB recond.

7. Click on the Forward Record button, and click on the Paste button in the SCRUBSHRUB recond.

You should now have four records completely filled out (Table E5).

Table E5. Record list for Exercise 3 after first LRSI formula entries.

\begin{tabular}{|c|c|c|c|c|}
\hline $\begin{array}{l}\text { Record } \\
\text { Count }\end{array}$ & Life Requisite & Cover Type & HSI Formula & LRSI Formula \\
\hline 1 & [STFOODCVR] & EMERGENT & MIN $\{[S T F O O D C V R] \&$ [STTEMP] \& [STWATER] $\}$ & [CANSUBMERG] \\
\hline 2 & [STFOODCVR] & NEWEMERG & MIN $\{[S T F O O D C V R] \&[S T T E M P] \&[S T W A T E R]\}$ & [CANSUBMERG] \\
\hline 3 & [STFOODCVR] & NEWSCRUB & MIN $\{[S T F O O D C V R] \&[S T T E M P] \&[S T W A T E R]\}$ & [CANSUBMERG] \\
\hline 4 & [STFOODCVR] & SCRUBSHRUB & MIN $\{[$ STFOODCVR] \& [STTEMP] \& [STWATER] $\}$ & [CANSUBMERG] \\
\hline 5 & [STTEMP] & EMERGENT & MIN $\{[$ STFOODCVR] \& [STTEMP] \& [STWATER]\} & $1 *$ \\
\hline 6 & [STTEMP] & NEWEMERG & 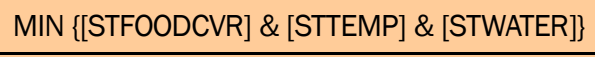 & $1 *$ \\
\hline 7 & [STTEMP] & NEWSCRUB & MIN $\{[S T F O O D C V R] \&[S T T E M P] \&[S T W A T E R]\}$ & $1 *$ \\
\hline 8 & [STTEMP] & SCRUBSHRUB & 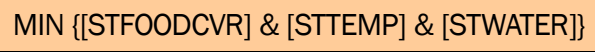 & $1 *$ \\
\hline 9 & [STWATER] & EMERGENT & MIN $\{[$ STFOODCVR] \& [STTEMP] \& [STWATER] $\}$ & $1 *$ \\
\hline 10 & [STWATER] & NEWEMERG & MIN $\{[$ STFOODCVR] \& [STTEMP] \& [STWATER] $\}$ & $1 *$ \\
\hline 11 & [STWATER] & NEWSCRUB & MIN $\{[S T F O O D C V R] ~ \&[S T T E M P] \&$ [STWATER] $\}$ & $1 *$ \\
\hline 12 & [STWATER] & SCRUBSHRUB & MIN $\{[$ STFOODCVR] \& [STTEMP] \& [STWATER] $\}$ & $1 *$ \\
\hline
\end{tabular}

Repeat the process for the LR $=\mathbf{S T T E M P}$, but this time the LRSI formula is:
LRSISTTEMP
[TEMPWATER] 
You should now have four more records completely filled out (Table E6).

Table E6. Record list for Exercise 3 after second LRSI formula entries.

\begin{tabular}{|c|c|c|c|c|}
\hline $\begin{array}{l}\text { Record } \\
\text { Count }\end{array}$ & Life Requisite & Cover Type & HSI Formula & LRSI Formula \\
\hline 1 & [STFOODCVR] & EMERGENT & MIN $\{[$ STFOODCVR] \& [STTEMP] \& [STWATER]\} & [CANSUBMERG] \\
\hline 2 & [STFOODCVR] & NEWEMERG & MIN $\{[S T F O O D C V R] \&[S T T E M P] \&[S T W A T E R]\}$ & [CANSUBMERG] \\
\hline 3 & [STFOODCVR] & NEWSCRUB & MIN $\{[$ STFOODCVR] \& [STTEMP] \& [STWATER] $\}$ & [CANSUBMERG] \\
\hline 4 & [STFOODCVR] & SCRUBSHRUB & MIN $\{[S T F O O D C V R] \&$ [STTEMP] \& [STWATER]\} & [CANSUBMERG] \\
\hline 5 & [STTEMP] & EMERGENT & MIN $\{[S T F O O D C V R] \&[S T T E M P] \&[S T W A T E R]\}$ & [TEMPWATER] \\
\hline 6 & [STTEMP] & NEWEMERG & MIN $\{[S T F O O D C V R] \&[S T T E M P] \&[S T W A T E R]\}$ & [TEMPWATER] \\
\hline 7 & [STTEMP] & NEWSCRUB & MIN $\{[$ STFOODCVR] \& [STTEMP] \& [STWATER] $\}$ & [TEMPWATER] \\
\hline 8 & [STTEMP] & SCRUBSHRUB & MIN $\{[S T F O O D C V R] \&[S T T E M P] \&$ [STWATER]\} & [TEMPWATER] \\
\hline 9 & [STWATER] & EMERGENT & MIN $\{[$ STFOODCVR] \& [STTEMP] \& [STWATER]\} & $1 *$ \\
\hline 10 & [STWATER] & NEWEMERG & MIN $\{[$ STFOODCVR] \& [STTEMP] \& [STWATER] $\}$ & $1 *$ \\
\hline 11 & [STWATER] & NEWSCRUB & MIN $\{[$ STFOODCVR] \& [STTEMP] \& [STWATER] $\}$ & $1 *$ \\
\hline 12 & [STWATER] & SCRUBSHRUB & MIN $\{[$ STFOODCVR] \& [STTEMP] \& [STWATER] $\}$ & $1 *$ \\
\hline
\end{tabular}

Repeat the process for the LR =STWATER, but this time the LRSI formula is a little trickier. Mathematical operators and the "Minimum" function on the keypad must be used to generate the following formula: MIN $\{[$ VELOCITY] \& [DEPTHWATER] \& [REGIMEWATR]\}

Remember, start with the variables, add the operators, and finish with the parentheses and braces. When finished, all 12 records should be completely filled out (refer to Table E7 to check work). 
Table E7. Record list for Exercise 3 after the third set of LRSI formula entries.

\begin{tabular}{|c|c|c|c|c|}
\hline $\begin{array}{l}\text { Record } \\
\text { Count }\end{array}$ & $\begin{array}{c}\text { Life } \\
\text { Requisite }\end{array}$ & Cover Type & HSI Formula & LRSI Formula \\
\hline 1 & [STFOODCVR] & EMERGENT & MIN\{[STFOODCVR]\&[STTEMP]\&[STWATER]\} & [CANSUBMERG] \\
\hline 2 & [STFOODCVR] & NEWEMERG, & MIN\{[STFOODCVR]\&[STTEMP]\&[STWATER]\} & [CANSUBMERG] \\
\hline 3 & [STFOODCVR] & NEWSCRUB, & MIN\{[STFOODCVR]\&[STTEMP]\&[STWATER]\} & [CANSUBMERG] \\
\hline 4 & [STFOODCVR] & SCRUBSHRUB & MIN\{[STFOODCVR]\&[STTEMP]\&[STWATER]\} & [CANSUBMERG] \\
\hline 5 & [STTEMP] & EMERGENT & MIN\{[STFOODCVR]\&[STTEMP]\&[STWATER]\} & [TEMPWATER] \\
\hline 6 & [STTEMP] & NEWEMERG, & MIN\{[STFOODCVR]\&[STTEMP]\&[STWATER]\} & [TEMPWATER] \\
\hline 7 & [STTEMP] & NEWSCRUB, & MIN\{[STFOODCVR]\&[STTEMP]\&[STWATER]\} & [TEMPWATER] \\
\hline 8 & [STTEMP] & SCRUBSHRUB & MIN\{[STFOODCVR]\&[STTEMP]\&[STWATER]\} & [TEMPWATER] \\
\hline 9 & [STWATER] & EMERGENT & MIN\{[STFOODCVR]\&[STTEMP]\&[STWATER]\} & $\begin{array}{l}\text { MIN }\{[V E L O C I T Y] \& \\
{[\text { DEPTHWATER]\& }} \\
{[\text { REGIMEWATR] }\}}\end{array}$ \\
\hline 10 & [STWATER] & NEWEMERG, & MIN $\{[S T F O O D C V R] \&[S T T E M P] \&[S T W A T E R]\}$ & $\begin{array}{l}\text { MIN\{[VELOCITY]\& } \\
\text { [DEPTHWATER]\& } \\
\text { [REGIMEWATR]\} }\end{array}$ \\
\hline 11 & [STWATER] & NEWSCRUB, & MIN $\{[S T F O O D C V R] \&[S T T E M P] \&[S T W A T E R]\}$ & $\begin{array}{l}\text { MIN\{[VELOCITY]\& } \\
{[\text { DEPTHWATER]\& }} \\
{[\text { REGIMEWATR]\} }}\end{array}$ \\
\hline 12 & [STWATER] & SCRUBSHRUB & MIN\{[STFOODCVR]\&[STTEMP]\&[STWATER]\} & $\begin{array}{r}\text { MIN }\{[V E L O C I T Y] \& \\
{[D E P T H W A T E R] \&} \\
{[\text { REGIMEWATR]\} }}\end{array}$ \\
\hline
\end{tabular}

Because there is no EOA limitation on this model, the EOA graph coordinates can be left in the default position (i.e., $\mathbf{0 , 0}, \mathbf{1 0 0}, \mathbf{1}$ ) and work is basically completed. Use the reports in EXHEP to check work and document analysis (Setup Reports button). The following reports should be created as a result:

- Formulas - Multiple Formula Models

- Associations - Multiple Formula Models 


\section{Exercise 4: Entering Model Curves into EXHEP}

\section{Part 1: Single Formula Models}

For users who need help reading and converting SI curves into X,Y coordinates in EXHEP, a series of graphs taken from the Marsh Wren HSI documentation are provided. The Marsh Wren happens to be a single formula model, so these four curves must be converted into X,Y coordinates, and exported into the software to complete the "setup" process in EXHEP. First, write out the $X, Y$ coordinates in tabular fashion, then convert these to EXHEP syntax (i.e., $\mathrm{X}_{1}, \mathrm{Y}_{1}, \mathrm{X}_{2}, \mathrm{Y}_{2}, \mathrm{X}_{3}, \mathrm{Y}_{3}, \ldots \mathrm{X}_{\mathrm{n}}, \mathrm{Y}_{\mathrm{n}}$ ). (Note: answers are included on the pages that follow):

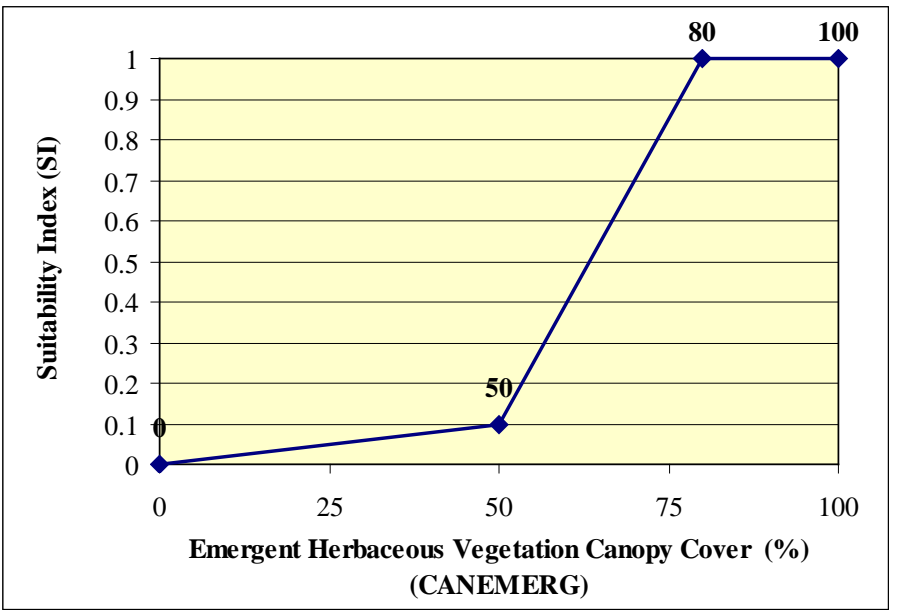

$\mathrm{X}, \mathrm{Y}$ coordinates in tabular fashion:

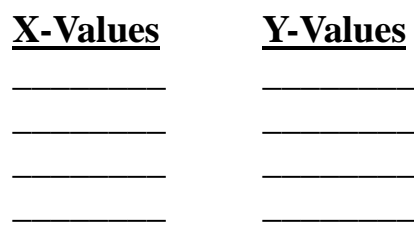

$\mathrm{X}, \mathrm{Y}$ coordinates in EXHEP syntax:

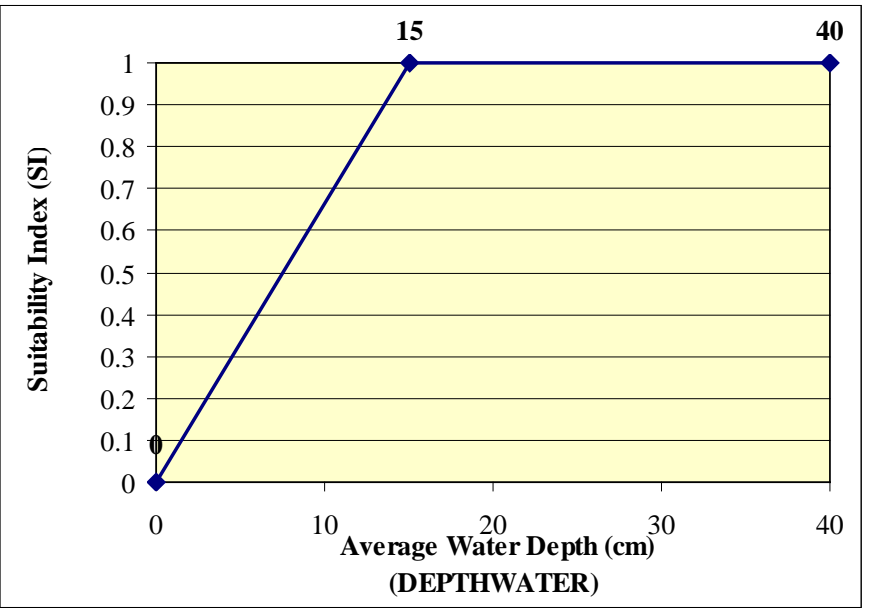

$\mathrm{X}, \mathrm{Y}$ coordinates in tabular fashion:

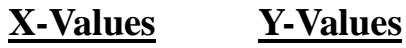

$\mathrm{X}, \mathrm{Y}$ coordinates in EXHEP syntax: 


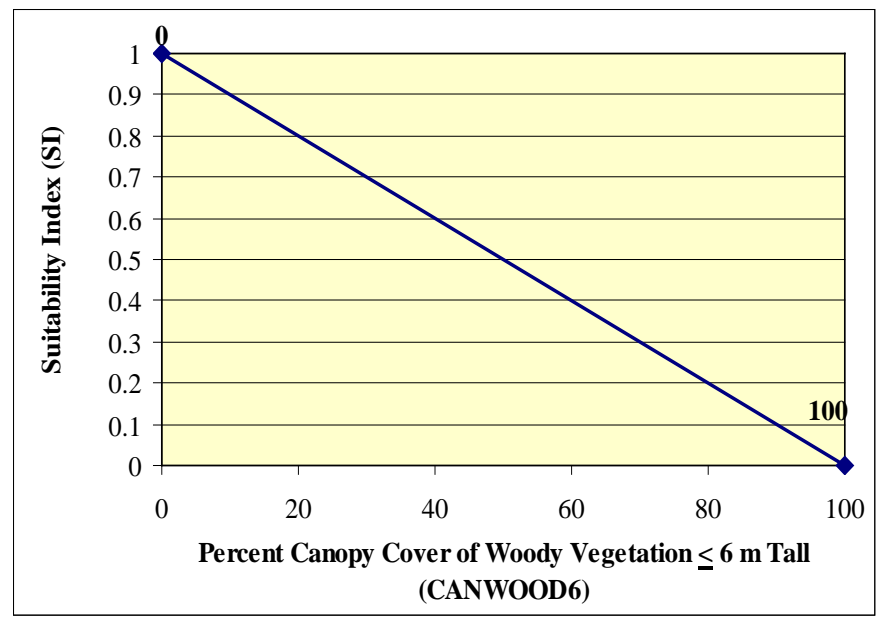

$\mathrm{X}, \mathrm{Y}$ coordinates in tabular fashion:

$\underline{\text { X-Values }} \quad \underline{\text { Y-Values }}$

$\mathrm{X}, \mathrm{Y}$ coordinates in EXHEP syntax:

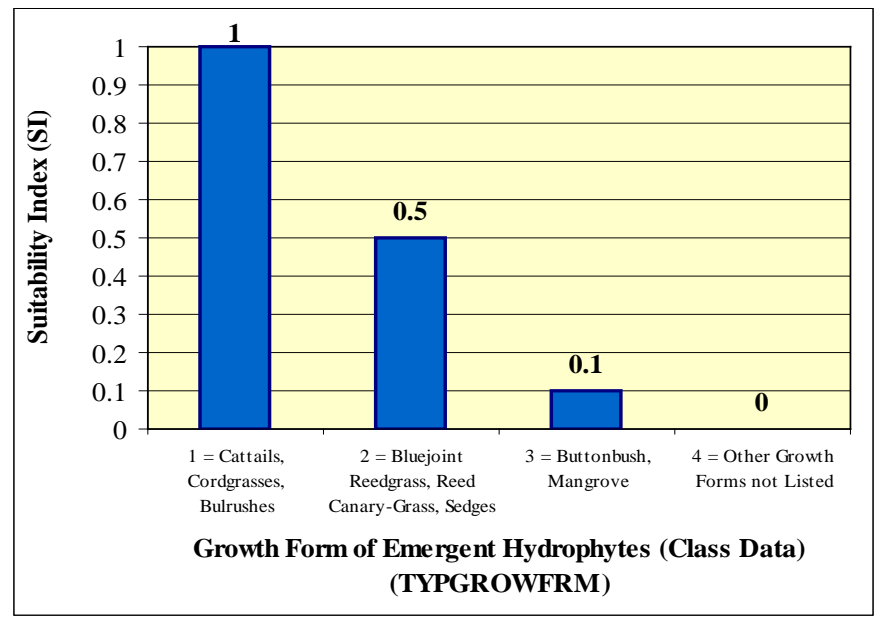

$\mathrm{X}, \mathrm{Y}$ coordinates in tabular fashion:

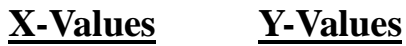

$\mathrm{X}, \mathrm{Y}$ coordinates in EXHEP syntax: 
Here are the answers:
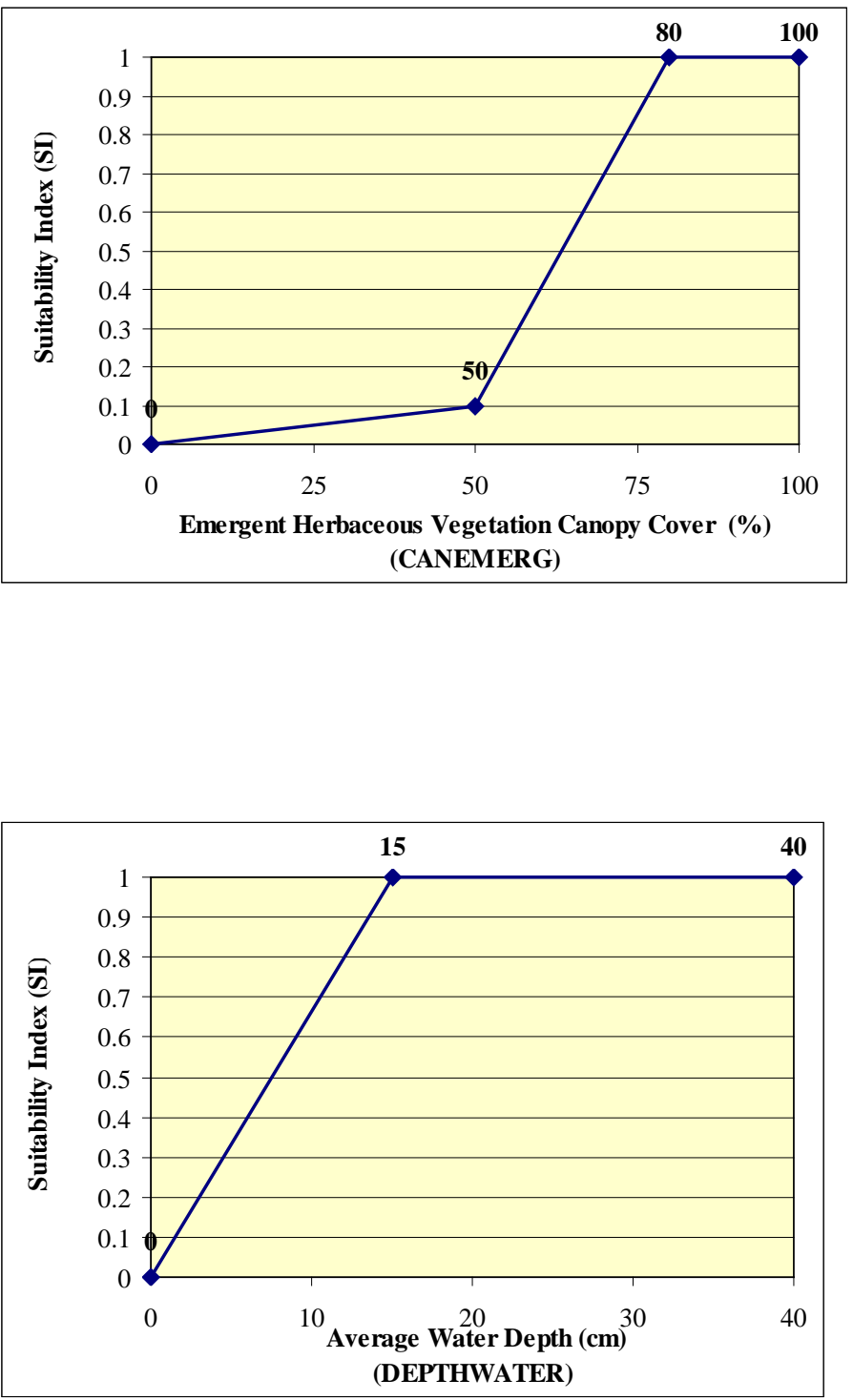

$\mathrm{X}, \mathrm{Y}$ coordinates in tabular fashion:

$\underline{\text { X-Values }} \quad \underline{\text { Y-Values }}$

0

50

80

100

0.1

1

1

$\mathrm{X}, \mathrm{Y}$ coordinates in

EXHEP syntax:

$\underline{0,0,50,0.1,80,1,100,1}$

$\mathrm{X}, \mathrm{Y}$ coordinates in tabular fashion:

$\underline{\mathrm{X} \text {-Values }} \quad \underline{\text { Y-Values }}$ 0 15

40

0
1
1

$\mathrm{X}, \mathrm{Y}$ coordinates in EXHEP syntax:

$0,0,15,1,40,1$ 

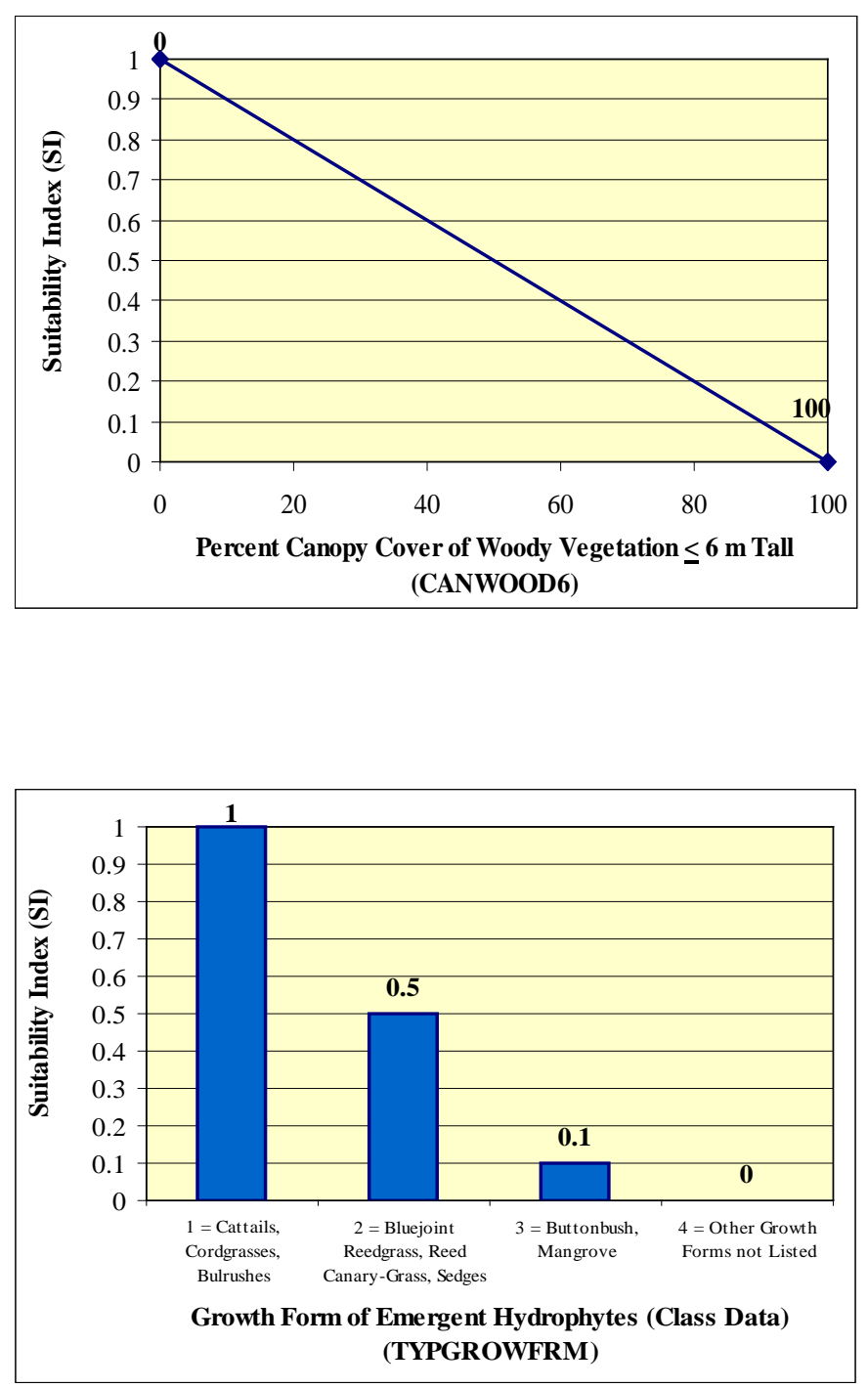

$\mathrm{X}, \mathrm{Y}$ coordinates in tabular fashion:

$\begin{array}{cc}\frac{\text { X-Values }}{0} & \text { Y-Values } \\ 100 & 0\end{array}$

$\mathrm{X}, \mathrm{Y}$ coordinates in EXHEP syntax:

$\mathbf{0 , 1 , 1 0 0 , 0}$
$\mathrm{X}, \mathrm{Y}$ coordinates in tabular fashion:

\begin{tabular}{ccc} 
X-Values & & Y-Values \\
\cline { 1 - 1 } 1 & & 0.5 \\
2 & & 0.1 \\
3 & & 0 \\
4 & &
\end{tabular}

$\mathrm{X}, \mathrm{Y}$ coordinates in EXHEP syntax:

$\underline{1,1,2,0.5,3,0.1,4,0}$

The coordinates can now be entered into the software.

8. To begin, open the training datafile entitled Exercise 4.mdb and click on the Run button under the Single Formula Models section of the primary interface.

9. Double-click on the first option (1. Choose Working Model), select the Marsh Wren option from the pull-down list, and click Close.

10. Now double-click on the third option (3. Enter SI Graphs and Variable Data).

11. Double-click on the Cover Type drop-down box, and choose EMERGENT from the pull-down list. 
12. Click on the Variable drop-down box, and choose CANEMERG from the pull-down list.

13. Click onœ on the Graph Coordinates $(\mathbf{X}, \mathbf{Y})$ entry box, type in the

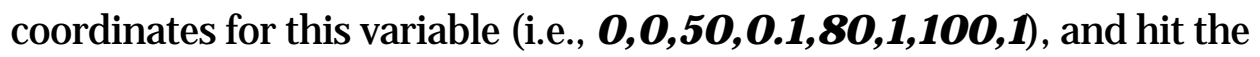
Tab key on the keyboard. If all is well, the cursor moves to the Variable Data entry window. Omitting a comma will generate an error message.

Copy this information to the remaining cover types in the model (i.e., NEWEMERG, NEWSCRUB, and SCRUBSHRUB).

1. Highlight the coordinates and press Ctrl C on the keyboard.

2. Now move to the upper form (Step 1: Select Cover Types and Variable Combinations) and pull down NEWEMERG from the Cover Type pull-down box. Leave the variable selection as it is.

3. Move down to the lower form (Step 2: Enter Graph Coordinates and Variable Data) and left-click once in the Graph Coordinates $(\mathbf{X}, \mathbf{Y})$ box.

4. Press Ctrl V on the keyboard and Tab to aocept the coordinates.

Now repeat the process for the remaining variables.

1. Click on the Cover Type drop-down box, and choose EMERGENT from the pull-down list.

2. Click on the Variable drop-down box, and choose CANWOOD6 from the pull-down list.

3. Click once on the Graph Coordinates $(\mathbf{X}, \mathbf{Y})$ entry box, type in the coordinates for this variable (i.e., $\mathbf{0 , 1 , 1 0 0 , 0}$ ), and hit the Tab key on the keyboard. If all is well, the cursor moves to the Variable Data entry window. Omitting a comma will generate an error message.

4. Copy the coordinates to the remaining three cover types, and repeat the entire process two more times for the DEPTHWATER and TYPGROWFRM variables.

5. Click Close to finish the session. 
By now you should have 16 records - (four variables $\times$ four cover types) (Table E8).

Table E8. Record list for Exercise 4 after X,Y coordinate entry into single formula model.

\begin{tabular}{|c|c|c|c|c||}
\hline $\begin{array}{c}\text { Record } \\
\text { Count }\end{array}$ & Model & Cover Type & Variable & X,Y Coordinates \\
\hline 1 & MARSHWREN & EMERGENT & CANEMERG & $0,0,50,0.1,80,1,100,1$ \\
\hline 2 & MARSHWREN & NEWEMERG & CANEMERG & $0,0,50,0.1,80,1,100,1$ \\
\hline 3 & MARSHWREN & NEWSCRUB & CANEMERG & $0,0,50,0.1,80,1,100,1$ \\
\hline 4 & MARSHWREN & SCRUBSHRUB & CANEMERG & $0,0,50,0.1,80,1,100,1$ \\
\hline 5 & MARSHWREN & EMERGENT & CANWOOD6 & $0,1,100,0$ \\
\hline 6 & MARSHWREN & NEWEMERG & CANWOOD6 & $0,1,100,0$ \\
\hline 7 & MARSHWREN & NEWSCRUB & CANWOOD6 & $0,1,100,0$ \\
\hline 8 & MARSHWREN & SCRUBSHRUB & CANWOOD6 & $0,1,100,0$ \\
\hline 9 & MARSHWREN & EMERGENT & DEPTHWATER & $0,0,15,1,40,1$ \\
\hline 10 & MARSHWREN & NEWEMERG & DEPTHWATER & $0,0,15,1,40,1$ \\
\hline 11 & MARSHWREN & NEWSCRUB & DEPTHWATER & $0,0,15,1,40,1$ \\
\hline 12 & MARSHWREN & SCRUBSHRUB & DEPTHWATER & $0,0,15,1,40,1$ \\
\hline 13 & MARSHWREN & EMERGENT & TYPGROWFRM & $1,1,2,0.5,3,0.1,4,0$ \\
\hline 14 & MARSHWREN & NEWEMERG & TYPGROWFRM & $1,1,2,0.5,3,0.1,4,0$ \\
\hline 15 & MARSHWREN & NEWSCRUB & TYPGROWFRM & $1,1,2,0.5,3,0.1,4,0$ \\
\hline 16 & MARSHWREN & SCRUBSHRUB & TYPEGROWFRM & $1,1,2,0.5,3,0.1,4,0$ \\
\hline
\end{tabular}

Use the reports in EXHEP to check work and document analysis (Setup Reports button). The following report should be created as a result:

\section{- Suitability Index Curves - Single Formula Models}

\section{Part 2: Multiple Formula Models}

A series of graphs taken from the Slider Turtle HSI documentation are presented below. The Slider Turtle happens to be a multiple formula model, so these curves must be converted into X,Y coordinates and exported into the software to complete the "setup" process in EXHEP. First, write out the $\mathrm{X}, \mathrm{Y}$ coordinates in tabular fashion, then convert these to EXHEP syntax (i.e., $\mathrm{X}_{1}, \mathrm{Y}_{1}, \mathrm{X}_{2}, \mathrm{Y}_{2}, \mathrm{X}_{3}, \mathrm{Y}_{3}, \ldots \mathrm{X}_{\mathrm{n}}, \mathrm{Y}_{\mathrm{n}}$ ). (Note: answers are included on the pages that follow): 


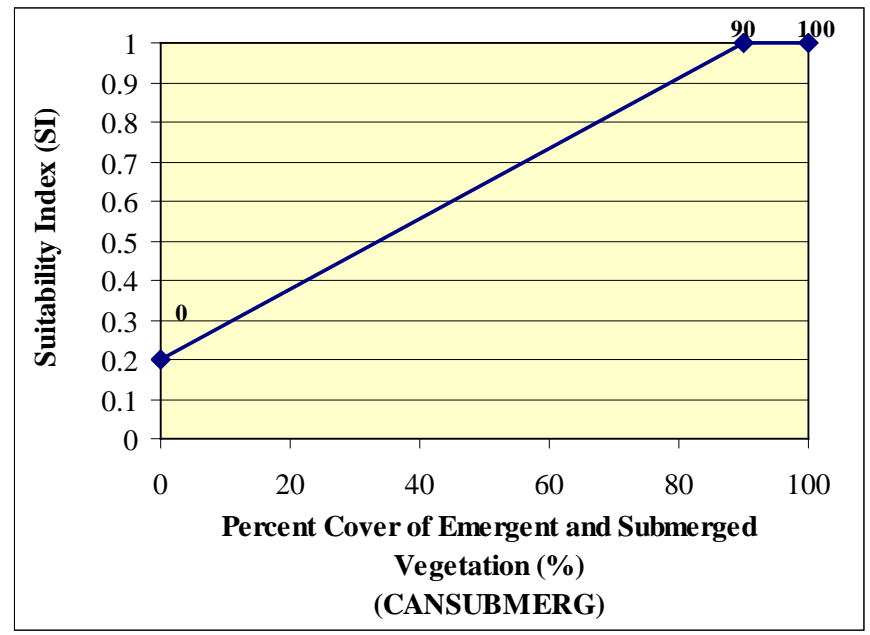

$\mathrm{X}, \mathrm{Y}$ coordinates in tabular fashion:

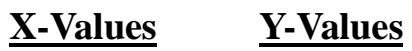

$\mathrm{X}, \mathrm{Y}$ coordinates in EXHEP syntax:

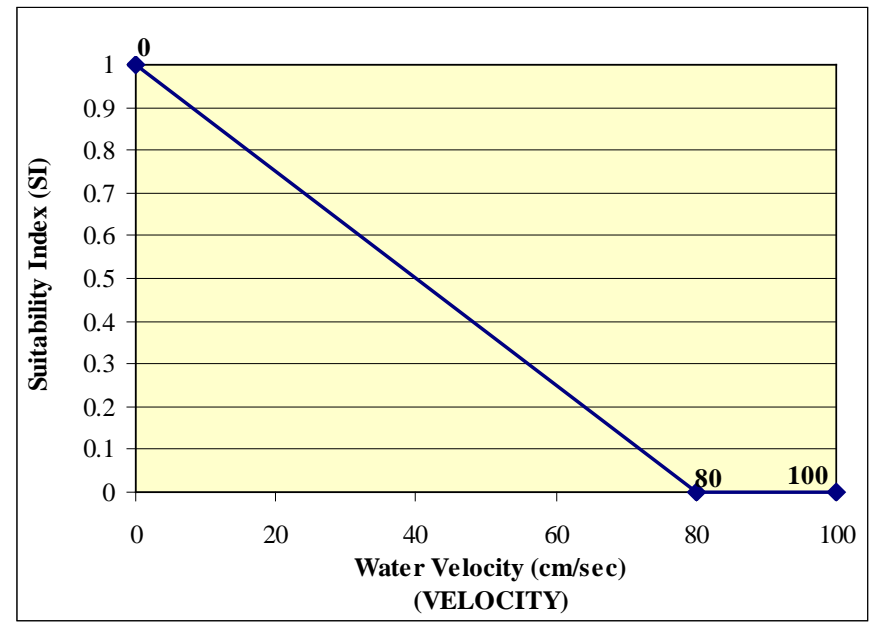

$\mathrm{X}, \mathrm{Y}$ coordinates in tabular fashion:

$\underline{\text { X-Values }} \underline{\text { Y-Values }}$

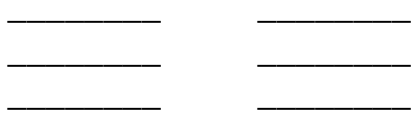

$\mathrm{X}, \mathrm{Y}$ coordinates in EXHEP syntax: 


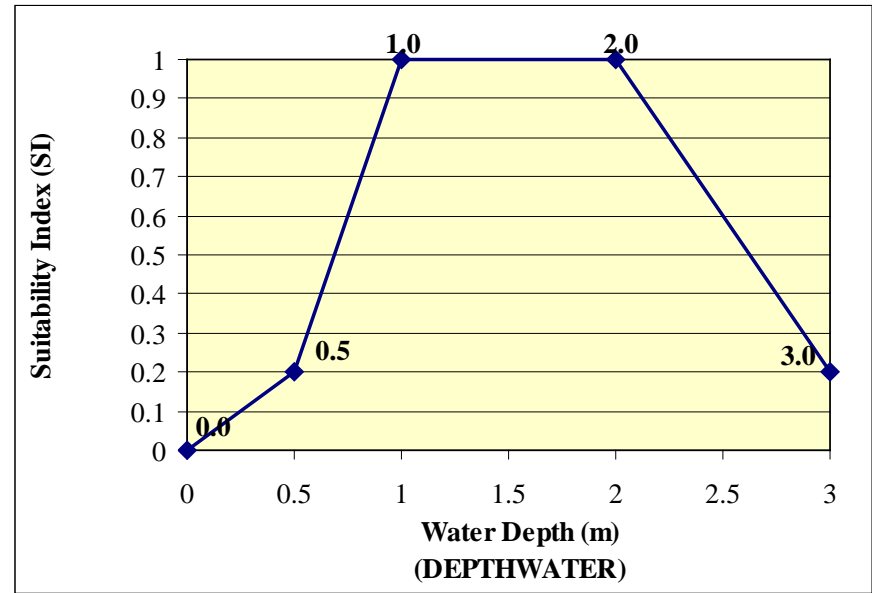

$\mathrm{X}, \mathrm{Y}$ coordinates in tabular fashion:

$\underline{\text { X-Values }} \quad \underline{\text { Y-Values }}$

$\mathrm{X}, \mathrm{Y}$ coordinates in EXHEP syntax:

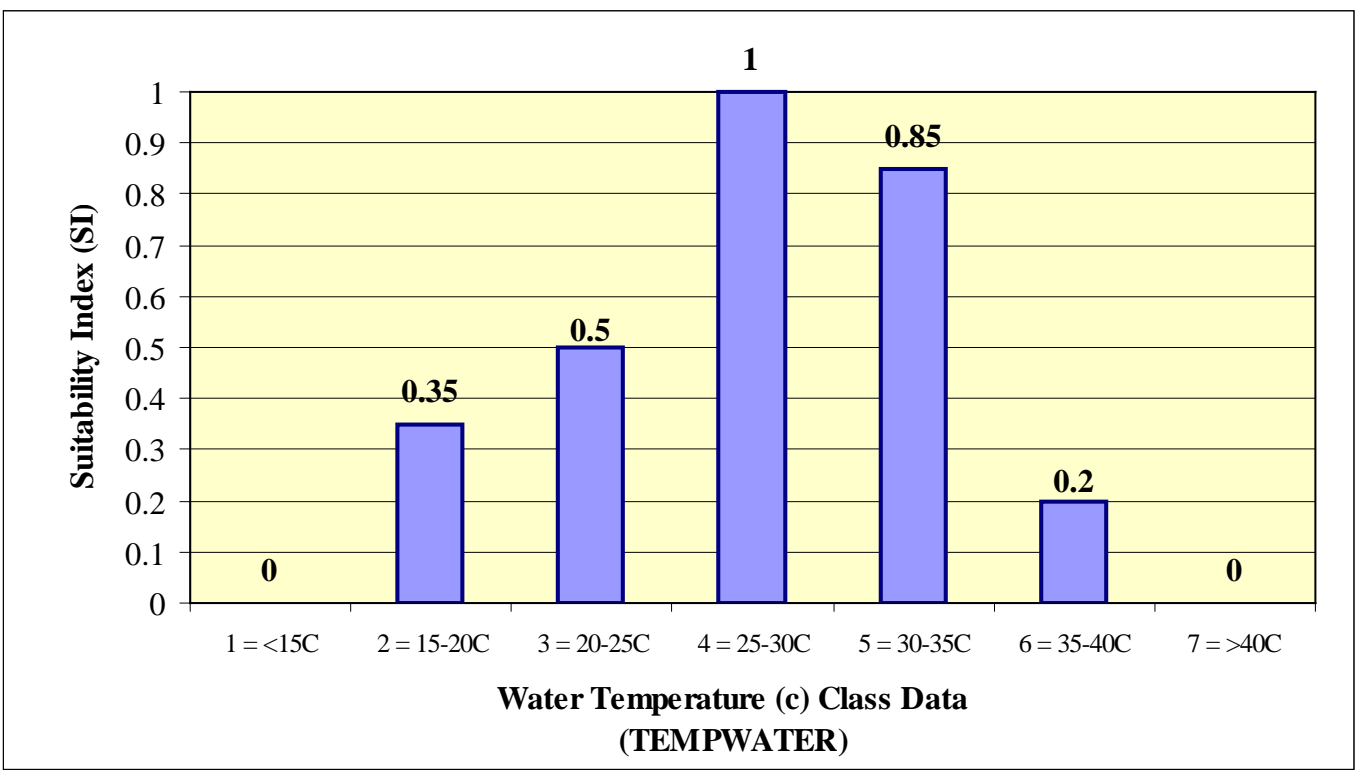

$\mathrm{X}, \mathrm{Y}$ coordinates in tabular fashion:

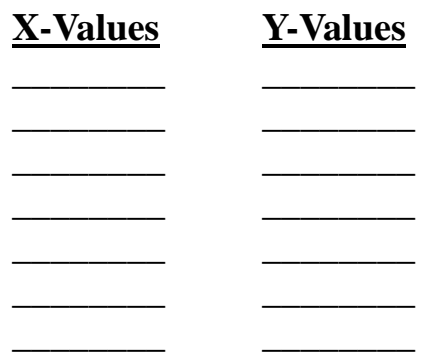

$\mathrm{X}, \mathrm{Y}$ coordinates in EXHEP syntax: 


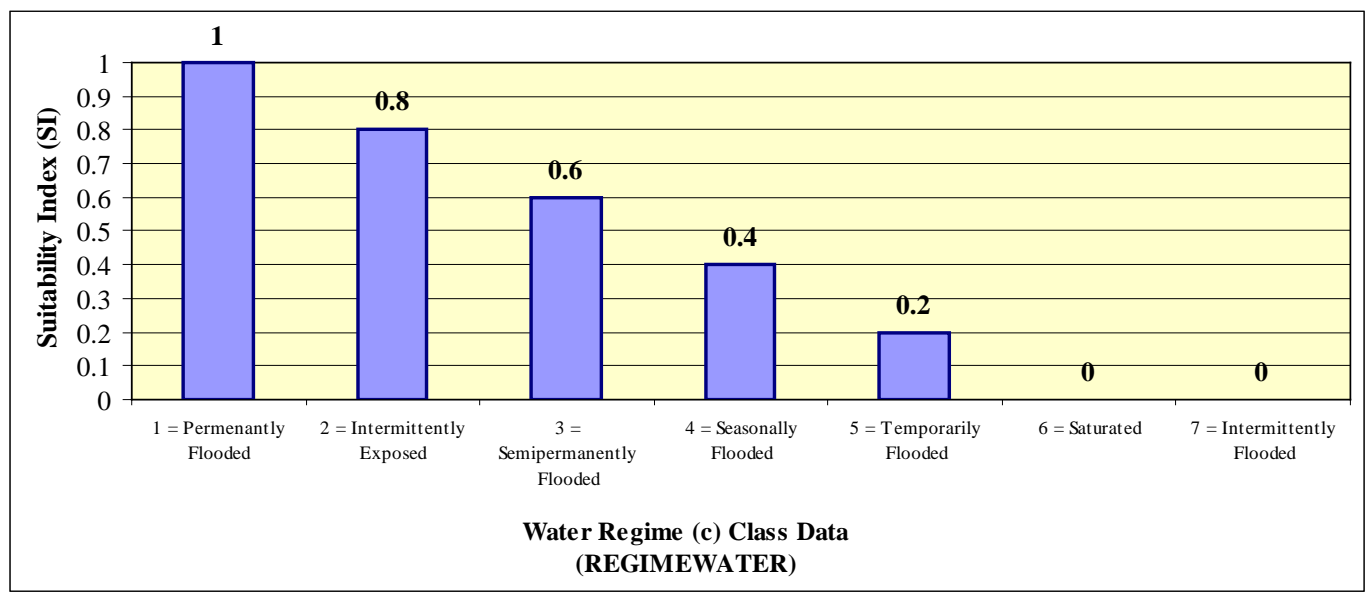

$\mathrm{X}, \mathrm{Y}$ coordinates in tabular fashion:

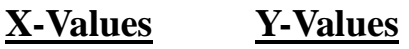

$\mathrm{X}, \mathrm{Y}$ coordinates in EXHEP syntax:

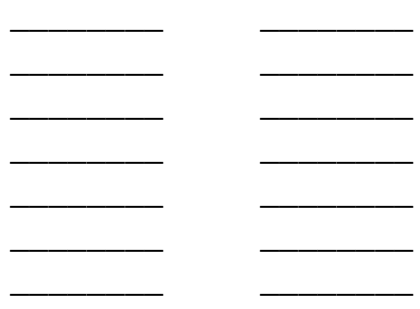


Here are the answers:

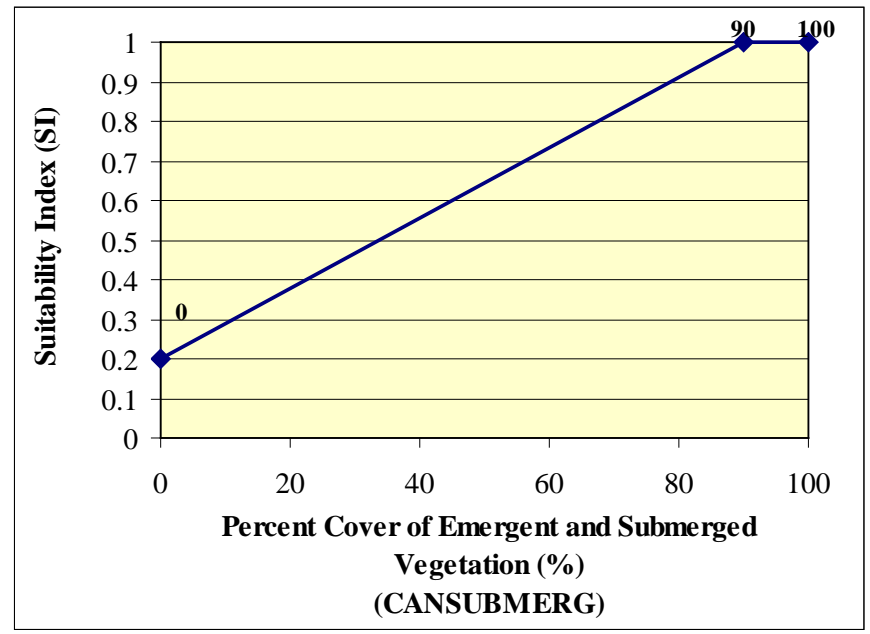

$\mathrm{X}, \mathrm{Y}$ coordinates in tabular fashion:

X-Values $\quad$ Y-Values

$\begin{array}{lll}0 & & 0.2 \\ 90 & & 1 \\ 100 & & 1\end{array}$

$\mathrm{X}, \mathrm{Y}$ coordinates in EXHEP syntax:

\section{$0,0.2,90,1,100,1$}

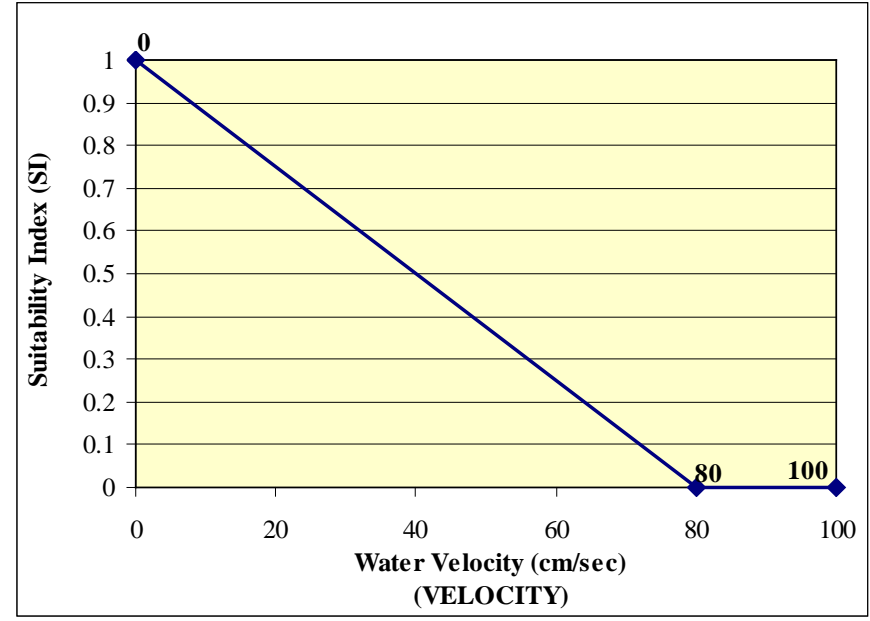

$\mathrm{X}, \mathrm{Y}$ coordinates in tabular fashion:

$\underline{\mathrm{X} \text {-Values }} \quad \underline{\text { Y-Values }}$

0

80

1

100

0

0

$\mathrm{X}, \mathrm{Y}$ coordinates in EXHEP syntax:

$\mathbf{0}, 1,80,0,100,0$ 


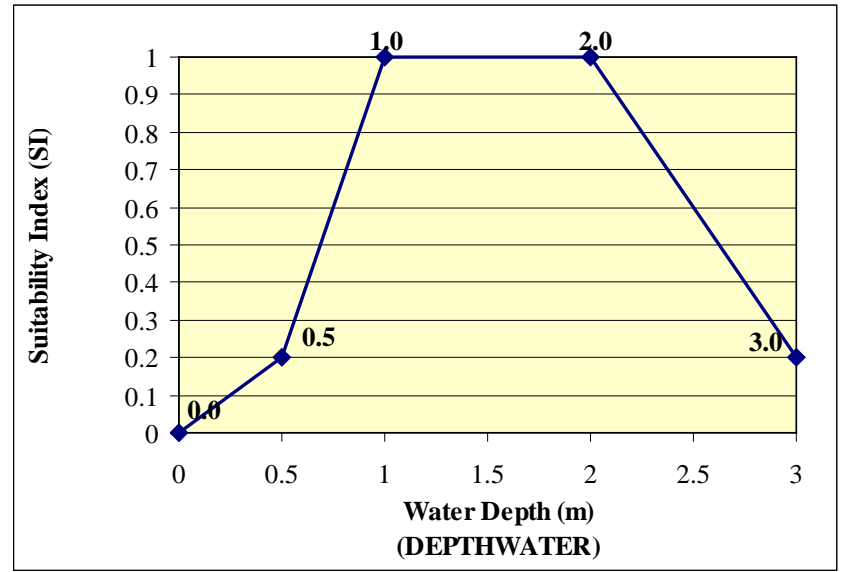

$\mathrm{X}, \mathrm{Y}$ coordinates in tabular fashion:

$\underline{X \text {-Values }} \quad \underline{\text { Y-Values }}$

0

0.5

0.2

1.0

0.2

2.0

1

3.0

0.2

$\mathrm{X}, \mathrm{Y}$ coordinates in

EXHEP syntax:

$\underline{0,0,0.5,0.2,1,1,2,1,3,0.2}$

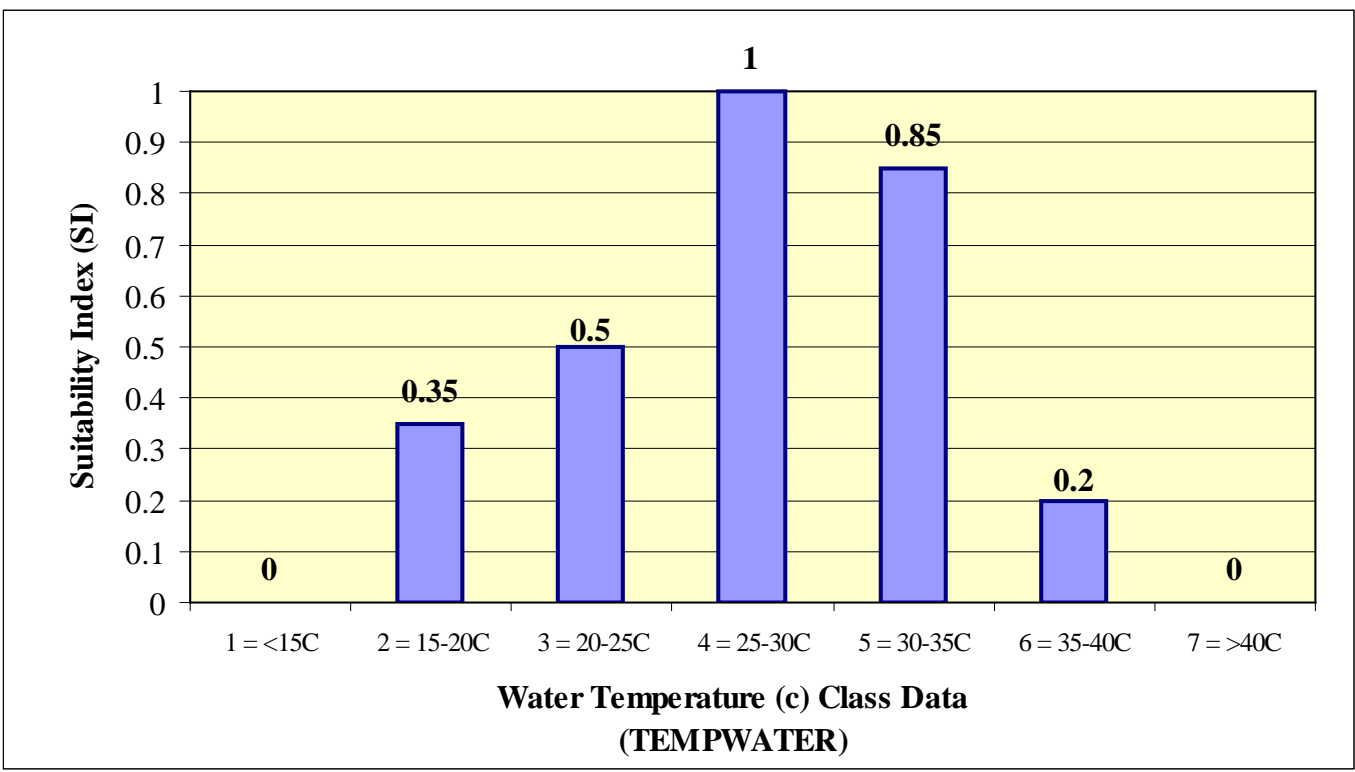

$\mathrm{X}, \mathrm{Y}$ coordinates in tabular fashion:

\begin{tabular}{ccc} 
X-Values & & Y-Values \\
\cline { 1 - 1 } 1 & & 0 \\
2 & & 0.35 \\
3 & & 0.5 \\
4 & 1 \\
5 & 0.85 \\
6 & 0.2 \\
7 & 0
\end{tabular}

$\mathrm{X}, \mathrm{Y}$ coordinates in EXHEP syntax:

$1,0,2,0.35,3,0.5,4,1,5,0.85,6,0.2,7,0$ 


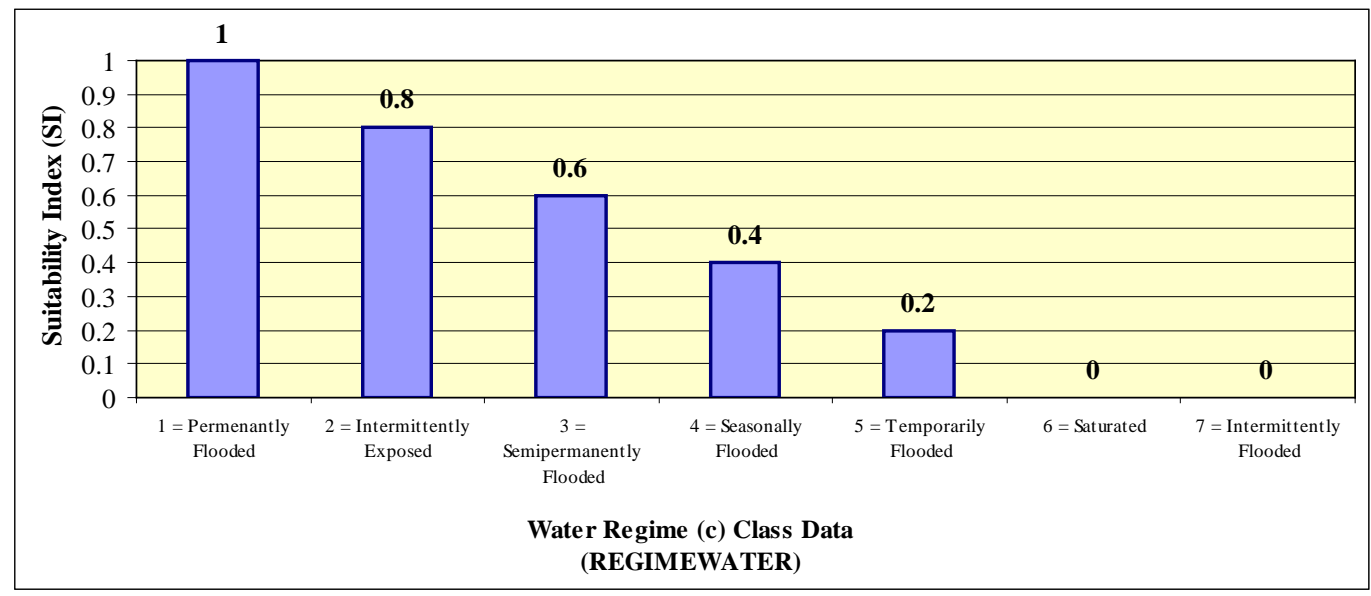

$\mathrm{X}, \mathrm{Y}$ coordinates in tabular fashion:

\begin{tabular}{|c|c|}
\hline$\underline{X \text {-Values }}$ & Y-Values \\
\hline 1 & 1 \\
\hline 2 & 0.8 \\
\hline 3 & 0.6 \\
\hline 4 & 0.4 \\
\hline 5 & 0.2 \\
\hline 6 & 0 \\
\hline 7 & 0 \\
\hline
\end{tabular}

$\mathrm{X}, \mathrm{Y}$ coordinates in EXHEP syntax:

$1,1,2,0.8,3,0.6,4,0.4,5,0.2,6,0,7,0$

The coordinates can now be entered into the software.

1. To begin, open the training datafile entitled Exercise 4.mdb and click on the Run button under the Multiple Formula Models section of the primary interface.

2. Double-click on the first option (1. Choose Working Model), select the Slider Turtle option from the pull-down list, and click Close.

3. Now double-click on the third option (3. Enter SI Graphs and Variable Data).

4. Select the STFOODCOVER life requisite from the pull-down box, and click $\mathbf{O K}$.

5. When the next screens open, click on the Cover Type drop-down box, and choose EMERGENT from the pull-down list.

6. Click on the Variable drop-down box, and choose CANSUBMERG from the pull-down list. 
7. Click onœ on the Graph Coordinates $(\mathbf{X}, \mathbf{Y})$ entry box, type in the

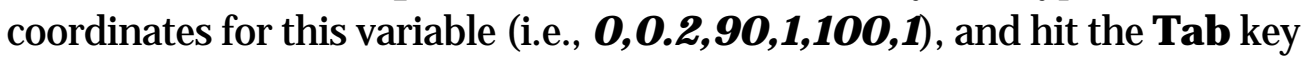
on the keyboard. If all is well, the cursor moves to the Variable Data entry window. Omitting a comma will result in an error message.

Copy this information to the remaining cover types in the model (i.e., NEWEMERG, NEWSCRUB, and SCRUBSHRUB).

8. Highlight the coordinates and press Ctrl C on the keyboard.

9. Now move up to the upper form (Step 1: Select Cover Types and Variable Combinations) and pull down NEWEMERG from the Cover Type pull-down box. Leave the variable selection as it is.

10. Move down to the lower form (Step 2: Enter Graph Coordinates and Variable Data) and left-click once in the Graph Coordinates $(\mathbf{X}, \mathbf{Y})$ box.

11. Press Ctrl V on the keyboard and Tab to accept the coordinates.

Since no other variables are used in this life requisite, another life requisite can be entered.

1. Click Close (returning to the primary interface).

2. Double-click on the third option (3. Enter SI Graphs and Variable Data).

3. Select the STTEMP life requisite from the pull-down box, and click $\mathbf{O K}$.

4. When the next screens open, click on the Cover Type drop-down box, and choose EMERGENT from the pull-down list.

5. Click on the Variable drop-down box, and choose TEMPWATER from the pull-down list.

6. Click once on the Graph Coordinates $(\mathbf{X}, \mathbf{Y})$ entry box, type in the coordinates for this variable (i.e., 1,0,2,0.35,3,0.5,4, 1,5,0.85, $\mathbf{6 , 0 . 2 , 7 , 0}$ ), and hit the Tab key on the keyboard. If all is well, the cursor moves to the Variable Data entry window. Omitting a comma will result in an error message.

7. Copy the coordinates to the remaining three cover types. 
Since no other variables are used in this life requisite, the last life requisite can be entered.

1. Click Close (returning to the primary interface).

2. Click on the third option (3. Enter SI Graphs and Variable Data),

3. Select the STWATER life requisite from the pull-down box.

4. Click on the Cover Type drop-down box, and choose EMERGENT from the pull-down list.

5. Click on the Variable drop-down box, choose DEPTHWATER from the pull-down list, and click $\mathbf{O K}$.

6. When the next screens open, click once on the Graph Coordinates $(\mathbf{X}, \mathbf{Y})$ entry box, type in the coordinates for this variable (i.e.,

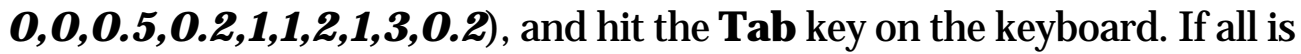
well, the cursor moves to the Variable Data entry window. Omitting a comma will result in an error message.

7. Copy the coordinates to the remaining three cover types.

8. There are two more variables for this life requisite (i.e., REGIMEWATR and VELOCITY). Systematically select the cover types for each variable, enter and copy the coordinates for each set, and hit the Close button when finished.

By now, there should be a total of 20 records - ( 5 variables x 4 cover types) (Table E9). 
Table E9. Record list for Exercise 4 after $\mathrm{X}, \mathrm{Y}$ coordinate entry into the multiple formula model.

\begin{tabular}{|c|c|c|c|c||}
\hline $\begin{array}{c}\text { Record } \\
\text { Count }\end{array}$ & Model & Cover Type & Variable & X,Y Coordinates \\
\hline 1 & SLIDTURTLE & EMERGENT & CANSUBMERG & $0,0.2,90,1,100,1$ \\
\hline 2 & SLIDTURTLE & NEWEMERG & CANSUBMERG & $0,0.2,90,1,100,1$ \\
\hline 3 & SLIDTURTLE & NEWSCRUB & CANSUBMERG & $0,0.2,90,1,100,1$ \\
\hline 4 & SLIDTURTLE & SCRUBSHRUB & CANSUBMERG & $0,0.2,90,1,100,1$ \\
\hline 5 & SLIDTURTLE & EMERGENT & DEPTHWATER & $0,0,0.5,0.2,1,1,2,1,3,0.2$ \\
\hline 6 & SLIDTURTLE & NEWEMERG & DEPTHWATER & $0,0,0.5,0.2,1,1,2,1,3,0.2$ \\
\hline 7 & SLIDTURTLE & NEWSCRUB & DEPTHWATER & $0,0,0.5,0.2,1,1,2,1,3,0.2$ \\
\hline 8 & SLIDTURTLE & SCRUBSHRUB & DEPTHWATER & $0,0,0.5,0.2,1,1,2,1,3,0.2$ \\
\hline 9 & SLIDTURTLE & EMERGENT & REGIMEWATR & $1,1,2,0.8,3,0.6,4,0.4,5,0.2,6,0,7,0$ \\
\hline 10 & SLIDTURTLE & NEWEMERG & REGIMEWATR & $1,1,2,0.8,3,0.6,4,0.4,5,0.2,6,0,7,0$ \\
\hline 11 & SLIDTURTLE & NEWSCRUB & REGIMEWATR & $1,1,2,0.8,3,0.6,4,0.4,5,0.2,6,0,7,0$ \\
\hline 12 & SLIDTURTLE & SCRUBSHRUB & REGIMEWATR & $1,1,2,0.8,3,0.6,4,0.4,5,0.2,6,0,7,0$ \\
\hline 13 & SLIDTURTLE & EMERGENT & TEMPWATER & $1,0,2,0.35,3,0.5,4,1,5,0.85,6,0.2,7,0$ \\
\hline 14 & SLIDTURTLE & NEWEMERG & TEMPWATER & $1,0,2,0.35,3,0.5,4,1,5,0.85,6,0.2,7,0$ \\
\hline 15 & SLIDTURTLE & NEWSCRUB & TEMPWATER & $1,0,2,0.35,3,0.5,4,1,5,0.85,6,0.2,7,0$ \\
\hline 16 & SLIDTURTLE & SCRUBSHRUB & TEMPWATER & $1,0,2,0.35,3,0.5,4,1,5,0.85,6,0.2,7,0$ \\
\hline 17 & SLIDTURTLE & EMERGENT & VELOCITY & $0,1,80,0,100,0$ \\
\hline 18 & SLIDTURTLE & NEWEMERG & VELOCITY & $0,1,80,0,100,0$ \\
\hline 19 & SLIDTURTLE & NEWSCRUB & VELOCITY & $0,1,80,0,100,0$ \\
\hline 20 & SLIDTURTLE & SCRUBSHRUB & VELOCITY & $0,1,80,0,100,0$ \\
\hline & & & & \\
\hline
\end{tabular}

Use the reports in EXHEP to check work and document analysis (Setup Reports button). The following report should be created as a result:

- Suitability Index Curves - Multiple Formula Models. 


\section{Exercise 5: Entering Data into EXHEP for Single Formula Models}

For users who need help entering data into EXHEP, assume the following data were gathered (through field collection, historical records, or GIS analyses) during the inventorying process for the study site (Table E10).

Table E10. Baseline data entry for the Exercise 5.

\begin{tabular}{|c|c|c|c|c|c|c|c|c|c|c|c|c|c|c|c|c|}
\hline \multirow[b]{2}{*}{ 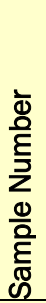 } & \multicolumn{4}{|c|}{ EMERGENT } & \multicolumn{4}{|c|}{ SCRUBSHRUB } & \multicolumn{4}{|c|}{ NEWEMERG } & \multicolumn{4}{|c|}{ NEWSCRUB } \\
\hline & 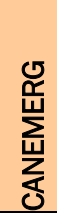 & $\begin{array}{l}8 \\
8 \\
8 \\
\vdots \\
\vdots \\
0\end{array}$ & 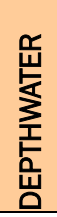 & 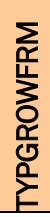 & 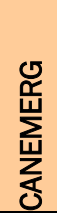 & 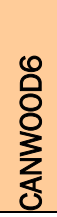 & 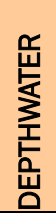 & 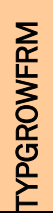 & 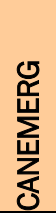 & 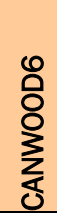 & 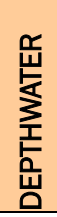 & 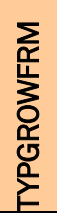 & 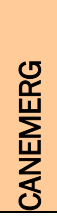 & 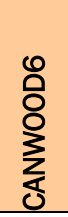 & 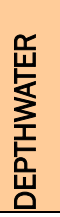 & 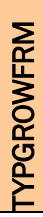 \\
\hline 1 & 40 & 20 & 50 & 1 & 10 & 80 & 10 & 3 & 0 & 0 & 0 & 0 & 0 & 0 & 0 & 0 \\
\hline 2 & 40 & 30 & 50 & & 15 & 70 & 10 & & & & & & & & & \\
\hline 3 & 40 & 40 & 30 & & 15 & 60 & 0 & & & & & & & & & \\
\hline 4 & 45 & 50 & 30 & & 10 & 50 & 0 & & & & & & & & & \\
\hline 5 & 45 & 60 & 50 & & 10 & 40 & 10 & & & & & & & & & \\
\hline
\end{tabular}

Although this is not a very robust data set, it is enough to calculate the baseline conditions at the site for the Marsh Wren in this exercise. There are two ways to enter the data into EXHEP: 1) manually enter data into the datafile, or 2) organize the data in a spreadsheet and copy it into the datafile. Exercises are provided below to practice both approaches.

\section{Part 1: Manual Data Entry}

1. To begin, open the training datafile entitled Exercise 5.mdb and click on the Run button under the Single Formula Models section of the primary interface.

2. Double-click on the first option (1. Choose Working Model), select the Marsh Wren option from the pull-down list, and click Close.

3. Now double-click on the third option (3. Enter SI Graphs and Variable Data).

4. Click on the Cover Type drop-down box, and choose EMERGENT from the pull-down list.

5. Click on the Variable drop-down box, and choose CANEMERG from the pull-down list. 
The first data set can now be entered. It is best to do this in a systematic fashion - either enter all the data for a single variable, or enter all the data for a single cover type. The first example enters all the data for this cover type.

1. Move down to the lower form and place the cursor in the "0" placeholder entry blank in the Variable Data entry form (on the right-hand side of the screen). Type the first data point (i.e., 40) and hit Enter on the keyboard.

2. Now type in the remaining data (i.e., 40, 40, 45, and 45) hitting Enter after each data point.

3. Go back to the top form, click on the Variable drop-down box, choose CANWOOD6 from the pull-down list, and enter the data for this second variable.

4. Repeat the process for DEPTHWATER and TYPEGROWFRM.

5. Data must be entered for all cover types associated with the model, so repeat the process for NEWEMERG and NEWSCRUB.

6. Click Close to finish the session.

Note: the " 0 " placeholder is not a data point. A zero must be entered into each of the variables for the two "newly developed" cover types that have no acres at baseline.

Now try entering the rest of the data electronically - go to the next section.

\section{Part 2: Electronic Data Entry}

The key to electronic data entry in EXHEP is formatting. The variable data must be arranged in a spreadsheet in column fashion (as done in the Exercise 5 Baseline Data - Marsh Wren.xls file in the Exercise 5 training folder).

1. Open this spreadsheet file now with MS Excel.

2. Return to the EXHEP interfaces and click on the Cover Type drop-down box again, but this time choose SCRUBSHRUB from the pull-down list.

3. Click on the Variable drop-down box, and choose CANEMERG from the pull-down list.

4. Go back to the external spreadsheet and highlight (i.e., block by holding down the left-mouse key and dragging down the column of data) the data in the CANEMERG column under the SCRUBSHRUB heading. 
Note: highlight only the data - do not block the column headings!!!

5. Press Ctrl $\mathbf{C}$ on the keyboard to copy the data to the clipboard.

6. Return to the EXHEP software and click the Import Data button above the Variable Data entry panel.

7. That's it! All five data points should have been entered into this screen. Now repeat the process for the remaining variables (i.e., CANWOOD6, DEPTHWATER and TYPEGROWFRM).

8. Click Close to finish the session.

Use the reports in EXHEP to check work and document analysis (Setup Reports button). The following report should be created as a result:

\section{- Variable Data - Single Formula Models}

The analysis can now be run. J ust double-click the fourth option (4. Perform Baseline (TY 0) Calculations* and the software calculates the baseline results for the study. Use the reports in EXHEP to check work and document analysis (Analysis Reports button). The following report should be created as a result:

\section{- Baseline (TY 0) Results}




\section{Exercise 6: Entering Data into EXHEP for Multiple Formula Models}

For users who need help entering data into EXHEP, assume that the following data were gathered (through field collection, historical records, or GIS analyses) during the inventorying process for the study site (Table E11).

Table E11. Baseline data entry for the Exercise 6.

\begin{tabular}{|c|c|c|c|c|c|c|c|c|c|c|c|c|c|c|c|c|c|c|c|c|}
\hline \multirow{2}{*}{ 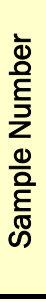 } & \multicolumn{5}{|c|}{ EMERGENT } & \multicolumn{5}{|c|}{ SCRUBSHRUB } & \multicolumn{5}{|c|}{ NEWEMERG } & \multicolumn{5}{|c|}{ NEWSCRUB } \\
\hline & 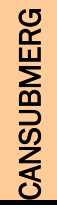 & 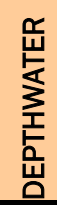 & 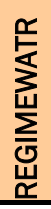 & 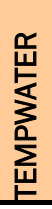 & $\begin{array}{l}\text { 등 } \\
\text { 얼 }\end{array}$ & 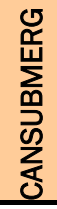 & 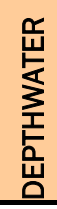 & 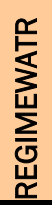 & 岾 & 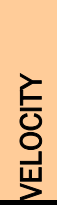 & 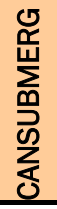 & 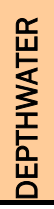 & 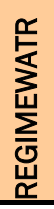 & 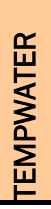 & $\begin{array}{l}\text { 릉 } \\
\text { 풀 }\end{array}$ & 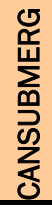 & $\begin{array}{l}\text { 㞻 } \\
\text { 站 } \\
\text { 号 } \\
\text { 岁 }\end{array}$ & 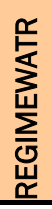 & 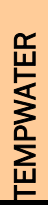 & $\begin{array}{l}\text { 등 } \\
\text { ய }\end{array}$ \\
\hline 1 & 45 & 0.5 & 3 & 4 & 20 & 5 & 0.1 & 5 & 5 & 40 & 0 & 0 & 0 & 0 & 0 & 0 & 0 & 0 & 0 & 0 \\
\hline 2 & 45 & 0.5 & & & 20 & 20 & 0.1 & & & 30 & & & & & & & & & & \\
\hline 3 & 45 & 0.3 & & & 20 & 20 & 0 & & & 30 & & & & & & & & & & \\
\hline 4 & 50 & 0.3 & & & 30 & 15 & 0 & & & 30 & & & & & & & & & & \\
\hline 5 & 50 & 0.5 & & & 20 & 15 & 0.1 & & & 20 & & & & & & & & & & \\
\hline
\end{tabular}

Although this is not a very robust data set, it is enough to calculate the baseline conditions at the site for the Slider Turtle in this exercise. There are two ways to enter the data into EXHEP: 1) manually enter data into the datafile, or 2) organize the data in a spreadsheet and copy it into the datafile. Exercises to practice both approaches are provided below.

\section{Part 1: Manual Data Entry}

1. To begin, open the training datafile entitled Exercise 6.mdb and click on the Run button under the Multiple Formula Models section of the primary interface.

2. Double-click on the first option (1. Choose Working Model), select the Slider Turtle option from the pull-down list, and click Close.

3. Now double-click on the thind option (3. Enter SI Graphs and Variable Data).

4. Select the STFOODCOVER life requisite from the pull-down box, and click $\mathbf{O K}$.

5. When the next screens open, click on the Cover Type drop-down box, and choose EMERGENT from the pull-down list.

6. Click on the Variable drop-down box, and choose CANSUBMERG from the pull-down list. 
The first data set can now be entered. It is best to do this in a systematic fashion - either enter all the data for a single variable, or enter all the data for a single cover type. Since there's only one variable in this life requisite, all of the data for the one variable will be entered.

1. Move down to the lower form and place your cursor in the " 0 " placeholder entry blank in the Variable Data entry form (on the right-hand side of the screen). Type the first data point (i.e., 45) and hit Enter on the keyboard.

2. Now type in the remaining data (i.e., 45, 45, 50, 50) hitting Enter after each data point.

3. Data must be entered for all cover types associated with the model, so repeat the process for NEWEMERG, NEWSCRUB, and SCRUBSHRUB.

4. Click Close to finish the session.

Note: the " 0 " placeholder is not a data point. A zero must be entered into each of the variables for the two "newly developed" cover types that have no acres at baseline.

To enter the rest of the data electronically, go to the next section.

\section{Part 2: Electronic Data Entry}

The key to electronic data entry in EXHEP is formatting. The variable data must be arranged in a spreadsheet in column fashion (as done in the Exercise 6 Baseline Data - Slider Turtle.xls file in the Exercise 6 training folder).

1. Open this spreadsheet file now with MS Excel.

2. Return back to the EXHEP interfaces and click on the Run button under the Multiple Formula Models section of the primary interface.

3. Double-click on the third option (Enter SI Graphs and Variable Data).

4. Select the STTEMP life requisite from the pull-down box, and click $\mathbf{O K}$.

5. When the next screens open, click on the Cover Type drop-down box, and choose EMERGENT from the pull-down list.

6. Click on the Variable drop-down box, and choose TEMPWATER from the pull-down list. 
7. Go back to the external spreadsheet and highlight the data in the TEMPATER column under the EMERGENT heading (i.e., highlight = block the data by holding down the left-mouse key and dragging down the column of data).

Note: highlight only the data - do not block the column headings!!!

8. Press Ctrl C on the keyboard to copy the data to the clipboard.

9. Return to the EXHEP software and click the Import Data button above the Variable Data entry panel.

10. That's it! The data point (i.e., 4) should have been entered into this screen. Now repeat the process for the remaining cover types (i.e., NEWEMERG, NEWSCRUB, and SCRUBSHRUB.).

11. Click Close to finish the session.

One more life requisite to go....

1. Double-click on the third option (3. Enter SI Graphs and Variable Data).

2. Select the STWATER life requisite from the pull-down box, and click $\mathbf{O K}$.

3. When the next screens open, click on the Cover Type drop-down box, and choose EMERGENT from the pull-down list.

4. Click on the Variable drop-down box, and choose DEPTHWATER from the pull-down list.

5. Go back to the external spreadsheet and highlight the data in the DEPTHWATER column under the EMERGENT heading (i.e., highlight $=$ block the data by holding down the left-mouse key and dragging down the column of data).

6. Press Ctrl C on the keyboard to copy the data to the clipboard.

7. Return to the EXHEP software and click the Import Data button above the Variable Data entry panel.

8. That's it! The data (i.e., $\mathbf{0 . 5}, \mathbf{0 . 5}, \mathbf{0 . 3}, \mathbf{0 . 3}$, and $\mathbf{0 . 5}$ ) should have been entered into this screen.

9. Now repeat the process for the remaining cover types (i.e., NEWEMERG, NEWSCRUB, and SCRUBSHRUB.). 
This process is not yet finished! There are still two more variables to fill in for this life requisite...

1. Click on the Cover Type drop-down box, and choose EMERGENT from the pull-down list.

2. Click on the Variable drop-down box, and choose REGIMEWATER from the pull-down list.

3. Go back to the external spreadsheet and highlight the data in the REGIMEWATER column under the EMERGENT heading (i.e., highlight = block the data by holding down theleft-mouse key and dragging down the column of data).

4. Press Ctrl C on the keyboard to copy the data to the clipboard.

5. Return to the EXHEP software and click the Import Data button above the Variable Data entry panel.

6. Now repeat the process for the remaining cover types (i.e., NEWEMERG, NEWSCRUB, and SCRUBSHRUB.).

7. Click Close to finish the session for this variable.

8. Now repeat the entire process one last time for the last variable: VELOCITY.

9. Click Close one last time to finish the entire process.

Use the reports in EXHEP to check work and document analysis (Setup Reports button). The following report should be created as a result:

\section{- Variable Data - Multiple Formula Models}

The analysis can now be run. J ust double-click the fourth option (4. Perform Baseline (TY 0) Calculations*) and the software calculates the baseline results for the study. Use the reports in EXHEP to check work and document analysis (Analysis Reports button). The following report should be created as a result:

\section{- Baseline (TY 0) Results}




\section{Exercise 7: Without-project Analyses in EXHEP}

To continue beyond the exercises above, assume that the "No Action" plan for the ecosystem restoration feasibility study must now be assessed. To begin, the evaluation team has established that three target years are necessary to assess the project's effect: baseline $\left(\mathrm{TY}_{0}\right)$, the year that construction is complete ( $\mathrm{TY}_{1}$ ), and the final "out-year" or TY 51 (i.e., the end of the project's life). Further assume that the owner of the property will in all likelihood drain the wetlands on the site and convert them to agricultural croplands over the next 50 years. As a result, the quality of the existing habitats will steadily decline over the life of the project and completely disappear in 50 years. Here are the trends the evaluation team generated as forecast conditions for the site:

Table E12. Without-project acre projections for Exercise 7.

\begin{tabular}{|c|c|c|c|c|c|}
\hline \multirow[b]{3}{*}{ No. } & \multirow[b]{3}{*}{ Code } & \multirow[b]{3}{*}{ Description } & \multicolumn{3}{|c|}{ Target Year } \\
\hline & & & 2000 & 2010 & 2060 \\
\hline & & & $T Y_{0}$ & $T Y_{1}$ & $T Y_{51}$ \\
\hline 1 & EMERGENT & Emergent Wetlands & 50 & 25 & 0 \\
\hline 2 & NEWEMERG & Newly Developed Emergent Wetlands & 0 & 0 & 0 \\
\hline 3 & SCRUBSHRUB & Scrub-Shrub Wetlands & 30 & 0 & 0 \\
\hline 4 & NEWSCRUB & Newly Developed Scrub-Shrub Wetlands & 0 & 0 & 0 \\
\hline 5 & AGCROP & Agricultural Croplands & 70 & 125 & 150 \\
\hline & & TOTALS: & 150 & 150 & 150 \\
\hline
\end{tabular}

Table E13. Without-project variable projections for the Marsh Wren HSI model in Exercise 7.

\begin{tabular}{|c|c|c|c|c|c|c|c|c|c|c|c|c|c|c|c|c|}
\hline \multirow[b]{3}{*}{ TY } & \multicolumn{16}{|c|}{ MARSH WREN } \\
\hline & \multicolumn{4}{|c|}{ EMERGENT } & \multicolumn{4}{|c|}{ SCRUBSHRUB } & \multicolumn{4}{|c|}{ NEWEMERG } & \multicolumn{4}{|c|}{ NEWSCRUB } \\
\hline & 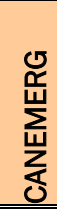 & $\begin{array}{l}0 \\
\text { ઠి } \\
\text { ㅇ } \\
\sum_{0} \\
\end{array}$ & 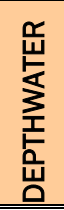 & 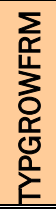 & 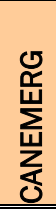 & $\begin{array}{l}0 \\
\text { ㅇํㅇ } \\
\text { ऐo } \\
\text { ऐo }\end{array}$ & 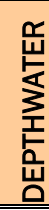 & 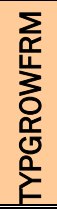 & 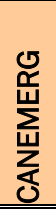 & $\begin{array}{l}0 \\
8 \\
8 \\
\sum_{0}^{2} \\
\sum_{0}\end{array}$ & 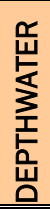 & 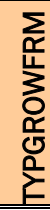 & 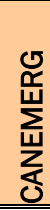 & $\begin{array}{l}0 \\
\text { Oे } \\
\text { ¿ } \\
\sum_{0}\end{array}$ & 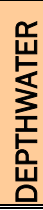 & 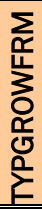 \\
\hline 0 & 42 & 40 & 42 & 1 & 12 & 60 & 6 & 3 & 0 & 0 & 0 & 0 & 0 & 0 & 0 & 0 \\
\hline 1 & 25 & 40 & 10 & 1 & 0 & 0 & 0 & 0 & 0 & 0 & 0 & 0 & 0 & 0 & 0 & 0 \\
\hline 51 & 0 & 0 & 0 & 0 & 0 & 0 & 0 & 0 & 0 & 0 & 0 & 0 & 0 & 0 & 0 & 0 \\
\hline
\end{tabular}


Table E14. Without-project variable projections for the Slider Turtle HSI model in Exercise 7.

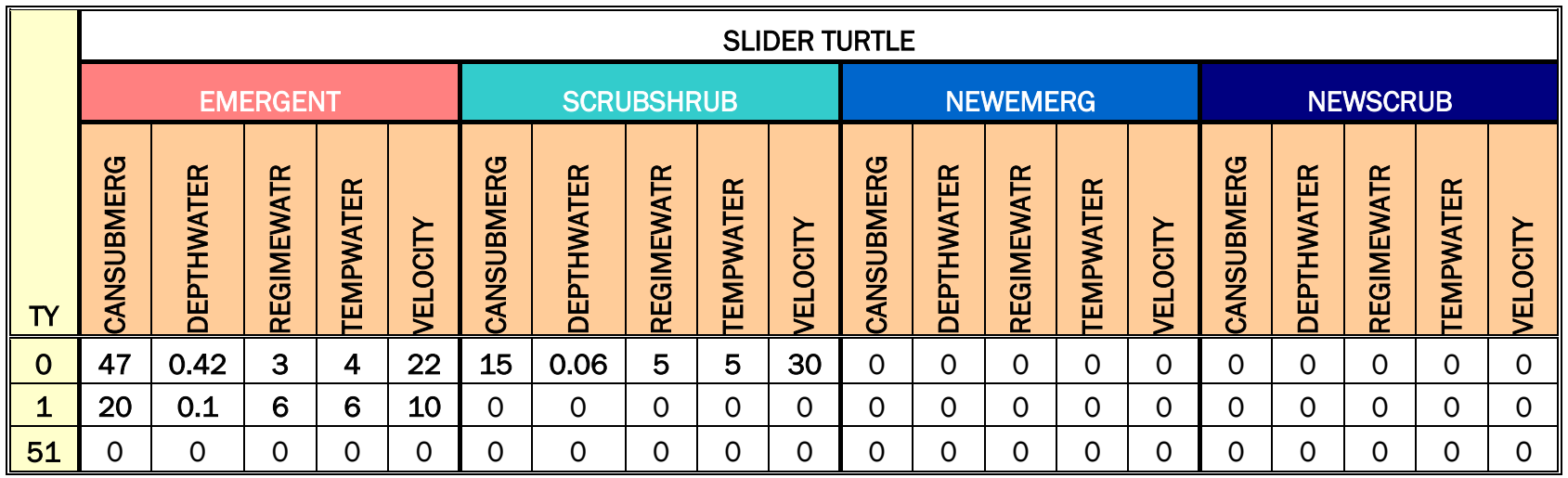

\section{Step 1: Target Year Entry}

1. First, set up the target years (i.e., $\mathbf{0}, \mathbf{1}$, and 51), by opening the training datafile entitled Exercise 7.mdb and clicking on the Run button under the Single Formula Models section of the primary interface.

2. Double-click on the first option (1. Choose Working Model), select the Marsh Wren option from the pull-down list, and click Close.

3. Now double-click on the fifth option (5. Define Target Years).

4. Enter each set of TY-Calendar Year combinations (i.e., $\mathbf{0}$ and 2000; 1 and 2010; 51 and 2060) and click Close when finished.

Use the reports in EXHEP to check work and document analysis (Setup Reports button). The following report should be created as a result:

\section{- Target Year List.}

\section{Step 2: Without-project Acres Entry}

1. Now double-click on the sixth option (6. Enter Without-Project Acres).

2. Click on the Cover Type pull-down menu and select the first cover type listed (i.e., AGCROP - Agricultural Croplands).

3. To the right in the acreage columns, input the acres for this cover type on a TY-by-TY basis. Each row represents one target year, so there will be three rows of data for this cover type (refer to TableE12 above). 
Note: the software automatically enters the first TY (TY $)_{0}$. This number cannot be changed in this screen - users must return to the setup menu to make changes to this year.

4. Now go back up to the Cover Type pull-down menu and select the second cover type (i.e., EMERGENT - Emergent Wetlands) and repeat the process.

5. Finish by entering the rest of the acres in each category. The software automatically assumes 0 acres, so only cover types that change from 0 must be filled in (but each oover type should be pulled down just to ensure that they have all been covered).

Note: the column to the immediate right of these acre projections keeps a running total of the acres put in per year. At the end of the effort, each cell in this section should carry the same numbers (i.e., 150 acres in this instance). If they are not equal, an error has occurred in "accounting" for one of the cover type's acreage shift in the analysis.

Because the Marsh Wren and Slider Turtle are both being used to assess the changes at this site, AND they share similar cover types, it is only necessary to enter the acres for one of the models - the acres are carried across all models in the analysis.

Use the reports in EXHEP to check work and document analysis (Setup Reports button). The following report should be created as a result:

\section{- Without-Project TY Acres}

\section{Step 3: Single Formula Model Without-project Data Entry}

Now that the acres have been input, it is time to focus on the variables.

1. To begin, click on the Run button under the Single Formula Models section of the primary interface.

2. Double-click on the first option (1. Choose Working Model), select the Marsh Wren option from the pull-down list, and click Close.

3. Now double-click on the seventh option (7. Enter Without-Project Projections*).

4. Say "Yes" to the initialization question, and the software forecasts the variable means forward into the future target years. 
If predicted forecasts deviate from the mean, new future projections must be entered.

5. Select EMERGENT from the Cover Type pull-down box, and select CANEMERG from the Variable pull-down box.

6. Highlight the TY 1 entry (i.e., 42) and type in the new value for theyear (i.e., 25) (see Table E13 above for these values).

7. Hit Tab on the keyboard and the software skips down to the next row (TY $\left.{ }_{51}\right)$. Highlight the $T Y_{51}$ entry (i.e., 42) and type in the new value for the year (i.e., $\mathbf{0}$ ).

8. Hit Tab on the keyboard. Data are now in for this variable.

9. Repeat the process for each variable (i.e., CANWOOD6, DEPTHWATER, and TYPGROWFRM) in this cover type.

10. Now switch to the next cover type (i.e., choose NEWEMERG from the Cover Type pull-down box). In most instances, the process would be repeated, but in this instance the "new" cover type is not being created under the without-project scenario, so the 0's in each cell are appropriate. The same is true for the next cover type (i.e., NEWSCRUB), so it can be skipped as well.

11. Pull down the last cover type (i.e., SCRUBSHRUB) and CANEMERG from the Variable pull-down box.

12. Highlight the $\mathrm{TY}_{1}$ value (i.e., 12), type in the new value for this year (i.e., 5), and hit Tab.

13. Finish entering data for this variable, and repeat the process for the remaining variables in this cover type.

14. When completely finished, click Close and click on Yes to save the data for later use.

Use the reports in EXHEP to check work and document analysis (Analysis Reports button). The following report should be created as a result:

\section{- Without-Project Projections}

\section{Step 4: Single Formula Model Without-project Analysis}

The analysis for the model can now be run.

1. Set the working model - double-click on the first option - 1. Choose Working Model, select the Marsh Wren option from the pull-down list, and click Close. 
2. Double-click on 4. Perform Baseline (TY 0) Calculations*, let the system run, and click $\mathbf{O K}$ when it is finished.

3. Double-click on 7. Enter Without-Project Projections*, click on the No button for initialization, click the Close button at the bottom of the interface, click Yes to save the data, and click $\mathbf{O K}$ when that save is affirmed.

4. Double-click on 8. Perform Without-Project Calculations*, let the system run, and click $\mathbf{O K}$ when it is finished.

Use the reports in EXHEP to check your work and document your analysis (Analysis Reports button). The following report should be created as a result:

\section{- Without-Project Results}

\section{Step 5: Multiple Formula Model Without-project Data Entry}

1. To begin, click on the Run button under the Multiple Formula Models section of the primary interface.

2. Double-click on the first option (1. Choose Working Model), select the Slider Turtle option from the pull-down list, and click Close.

3. Now double-click on the seventh option (7. Enter Without-Project Projections*).

4. Say "Yes" to the initialization question, and the software forecasts the variable means forward into the future target years.

If the predicted forecasts deviate from the mean, the new future projections must be input.

5. Select the STFOODCVR from the Life Requisite pull-down box, select EMERGENT from the Cover Type pull-down box, and select CANSUBMERG from the Variable pull-down box.

6. Highlight the TY 1 entry (i.e., 47) and type in the new value for theyear (i.e., 20) (look to Table E14 above for these values).

7. Hit Enter on the keyboard and the software skips down to the next row (TY51). Highlight the TY $Y_{51}$ entry (i.e., 47) and type in the new value for the year (i.e., 0). Hit Tab on the keyboard to finish it off.

8. The data are now input for this variable. Since only one variable is associated with this life requisite, go ahead and pull-down the remaining cover types (i.e., NEWEMERG, NEWSCRUB, and SCRUBSHRUB), and fill in the new variable values. 
9. Repeat the process for each life requisite-cover type-variable combination in the model (i.e., STTEMP and STWATER).

10. When completely finished, click Close and click on Yes to save the data for later use.

Use the reports in EXHEP to check work and document analysis

(Analysis Reports button). The following report should be created as a result:

\section{- Without-Project Projections}

\section{Step 6: Multiple Formula Model Without-project Analysis}

Now the analysis for the model can be run.

1. Set the working model - double-click on the first option - 1. Choose Working Model, select the Slider Turtle option from the pull-down list, and click Close.

2. Double-click on 4. Perform Baseline (TY 0) Calculations*, let the system run, and click $\mathbf{O K}$ when it is finished.

3. Double-click on 7. Enter Without-Project Projections*, click on the No button for initialization, click the Close button at the bottom of the interface, click Yes to save the data, and click $\mathbf{O K}$ when that save is affirmed.

4. Double-click on 8. Perform Without-Project Calculations*, let the system run, and click $\mathbf{O K}$ when it is finished.

Use the reports in EXHEP to check work and document analysis (Analysis Reports button). The following report should be created as a result:

\section{- Without-Project Results}




\section{Exercise 8: With-project Analyses in EXHEP}

Once familiar with "No Action" conditions, one of the restoration alternatives that the evaluation team devised to rehabilitate these wetlands can be assessed. There is no need to enter target years - the same series used in the without-project scenario are used in the with-project forecasting. Here are the trends the team generated under their first alternative formulation exercise:

Table E15. With-project acre projections for Exercise 8.

\begin{tabular}{|c|c|c|c|c|c|}
\hline \multirow[b]{3}{*}{ No. } & \multirow[b]{3}{*}{ Code } & \multirow[b]{3}{*}{ Description } & \multicolumn{3}{|c|}{ Target Year } \\
\hline & & & 2000 & 2010 & 2060 \\
\hline & & & TYo & $T Y_{1}$ & $T Y_{51}$ \\
\hline 1 & EMERGENT & Emergent Wetlands & 50 & 50 & 50 \\
\hline 2 & NEWEMERG & Newly Developed Emergent Wetlands & 0 & 35 & 35 \\
\hline 3 & SCRUBSHRUB & Scrub-Shrub Wetlands & 30 & 30 & 30 \\
\hline 4 & NEWSCRUB & Newly Developed Scrub-Shrub Wetlands & 0 & 35 & 35 \\
\hline 5 & AGCROP & Agricultural Croplands & 70 & 0 & 0 \\
\hline & & TOTALS: & 150 & 150 & 150 \\
\hline
\end{tabular}

Table E16. With-project variable projections for the Marsh Wren HSI model in Exercise 8.

\begin{tabular}{|c|c|c|c|c|c|c|c|c|c|c|c|c|c|c|c|c|}
\hline \multirow[b]{3}{*}{ TY } & \multicolumn{16}{|c|}{ MARSH WREN } \\
\hline & \multicolumn{4}{|c|}{ EMERGENT } & \multicolumn{4}{|c|}{ SCRUBSHRUB } & \multicolumn{4}{|c|}{ NEWEMERG } & \multicolumn{4}{|c|}{ NEWSCRUB } \\
\hline & 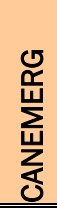 & $\begin{array}{l}0 \\
\text { ○े } \\
\text { ऐo } \\
\sum_{0} \\
\end{array}$ & 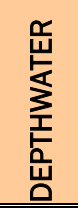 & 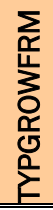 & 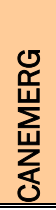 & 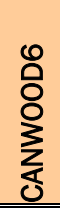 & 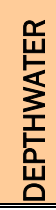 & 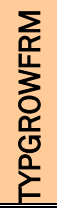 & 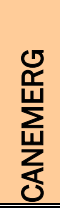 & 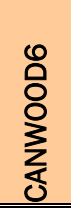 & 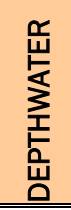 & 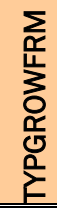 & 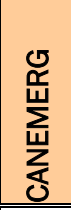 & $\begin{array}{l}0 \\
8 \\
8 \\
\sum_{0}\end{array}$ & 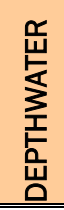 & 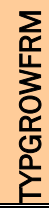 \\
\hline 0 & 42 & 40 & 42 & 1 & 12 & 60 & 6 & 3 & 0 & 0 & 0 & 0 & 0 & 0 & 0 & 0 \\
\hline 1 & 50 & 20 & 100 & 1 & 20 & 50 & 50 & 3 & 25 & 0 & 100 & 1 & 10 & 5 & 50 & 3 \\
\hline 51 & 80 & 10 & 100 & 1 & 50 & 30 & 50 & 3 & 80 & 10 & 100 & 1 & 50 & 30 & 50 & 3 \\
\hline
\end{tabular}

Table E17. With-project variable projections for the Slider Turtle HSI model in Exercise 8.

\begin{tabular}{|c|c|c|c|c|c|c|c|c|c|c|c|c|c|c|c|c|c|c|c|c|}
\hline & \multicolumn{20}{|c|}{ SLIDER TURTLE } \\
\hline & \multicolumn{5}{|c|}{ EMERGENT } & \multicolumn{5}{|c|}{ SCRUBSHRUB } & \multicolumn{5}{|c|}{ NEWEMERG } & \multicolumn{5}{|c|}{ NEWSCRUB } \\
\hline TY & 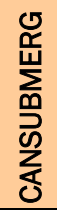 & $\begin{array}{l}\text { 営 } \\
\text { 站 } \\
\text { 岀 }\end{array}$ & 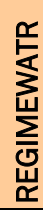 & 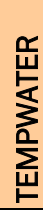 & 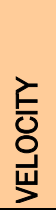 & 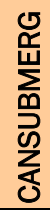 & 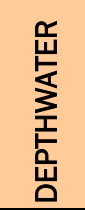 & 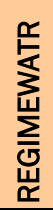 & 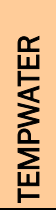 & 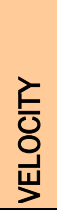 & 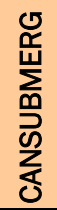 & 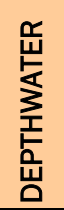 & 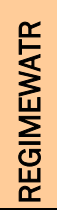 & 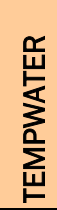 & 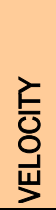 & 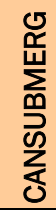 & 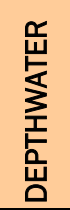 & 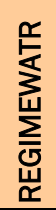 & 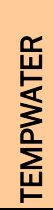 & 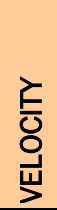 \\
\hline 0 & 47 & 0.42 & 3 & 4 & 22 & 15 & 0.06 & 5 & 5 & 30 & 0 & 0 & 0 & 0 & 0 & 0 & 0 & 0 & 0 & 0 \\
\hline 1 & 50 & 1.0 & 2 & 4 & 11 & 20 & 0.5 & 3 & 5 & 15 & 25 & 1.0 & 2 & 4 & 11 & 10 & 0.5 & 3 & 5 & 15 \\
\hline 51 & 90 & 1.0 & 2 & 4 & 11 & 50 & 0.5 & 3 & 5 & 15 & 90 & 1.0 & 2 & 4 & 11 & 50 & 0.5 & 3 & 5 & 15 \\
\hline
\end{tabular}




\section{Step 1: With-project Acres Entry}

1. To begin, open the training datafile entitled Exercise 8.mdb and click on the Run button under the Single Formula Models section of the primary interface.

2. Double-click on the ninth option (Enter With-Project Acres).

3. Click on the Cover Type pull-down menu and select the first cover type listed (i.e., AGCROP - Agricultural Croplands).

4. To the right in the acreage columns, input the acres for this cover type on a TY-by-TY basis. Each row represents one target year, so there will be three rows of data for this cover type when input is completed (refer to TableE15 above).

Note: the software automatically enters the first TY (TYo). This number cannot be changed in this screen - the user must return to the setup menu to make changes to this year.

5. Now go back up to the Cover Type pull-down menu and select the second cover type (i.e., EMERGENT - Emergent Wetlands) and repeat the process.

6. Finish by entering the rest of the acres in each category. The software automatically assumes 0 acres, so only cover types that change from 0 must be filled in (but each cover type should be pulled down just to ensure that they have all been covered).

Note: the column to the immediate right of these acre projections keeps a running total of the acres put in per year. At the end of the effort, each cell in this section should carry the same numbers (i.e., 150 acres in this instance). If they are not equal, one of the cover ty pe's acreage shift in the analysis has not been "accounted" for.

Because the Marsh Wren and Slider Turtle are both being used to assess the changes at this site, AND they share similar cover types, it is only necessary to enter the acres for one of the models - the acres are carried across all models in the analysis.

Use the reports in EXHEP to check work and document analysis (Setup Reports button). The following report should be created as a result: 


\section{Step 2: Single Formula Model With-project Data Entry}

Now that the acres have been input, it is time to focus on the variables.

1. To begin, click on the Run button under the Single Formula Models section of the primary interface.

2. Double-click on the first option (1. Choose Working Model), select the Marsh Wren option from the pull-down list, and click Close.

3. Now double-click on the tenth option (10. Enter With-Project Projections*).

4. Say "Yes" to the initialization question, and the software forecasts the variable means forward into the future target years.

If predicted forecasts deviate from the mean, then the new future projections must be input.

5. Select the EMERGENT from the Cover Type pull-down box, and select CANEMERG from the Variable pull-down box.

6. Highlight the TY 1 entry (i.e., 42) and type in the newvalue for theyear (i.e., 50) (see Table E16 for these values).

7. Hit Tab on the keyboard and the software skips down to the next row (TY $Y_{51}$. Highlight the $T Y_{51}$ entry (i.e., 42) and type in the new value for the year (i.e., 80).

8. Hit Tab on the keyboard. The data are now input for this variable.

9. Repeat the process for each variable (i.e., CANWOOD6, DEPTHWATER, and TYPGROWFRM) in this cover type.

10. Repeat the process for each variable under each cover type.

11. When completely finished, click Close and click on Yes to save the data for later use.

Use the reports in EXHEP to check work and document analysis (Analysis Reports button). The following report should be created as a result:

\section{- With-Project Projections}




\section{Step 3: Single Formula Model With-project Analysis}

The analysis for the model can now be run.

1. Set the working model - double-click on the first option - 1. Choose Working Model, select the Marsh Wren option from the pull-down list, and click Close.

2. Double-click on 4. Perform Baseline (TY 0) Calculations*, let the system run, and click $\mathbf{O K}$ when it is finished.

3. Double-click on 7. Enter Without-Project Projections*, click on the No button for initialization, click the Close button at the bottom of the interface, click Yes to save the data, and click $\mathbf{O K}$ when that save is affirmed.

4. Double-click on 8. Perform Without-Project Calculations*, let the system run, and click $\mathbf{O K}$ when it is finished.

5. Double-click on 10. Enter With-Project Projections*, click on theNo button for initialization, click the Close button at the bottom of the interface, click Yes to save the data, and click $\mathbf{O K}$ when that save is affirmed.

6. Double-click on 11. Perform With-Project Calculations*, let the system run, and click $\mathbf{O K}$ when it is finished.

Use the reports in EXHEP to check work and document analysis (Analysis Reports button). The following reports should be created as a result:

\section{- With-Project Results}

- With- vs. Without-Project Results

\section{- AAHU Summary.}

Remember, this last report (i.e., AAHU Summary) is overwritten each time a new working model is selected. This report should be exported to MS Word for safe-keeping before proceeding. 


\section{Step 4: Multiple Formula Model With-project Data Entry}

1. To begin, click on the Run button under the Multiple Formula Models section of the primary interface.

2. Double-click on the first option (1. Choose Working Model), select the Slider Turtle option from the pull-down list, and click Close.

3. Now double-click on the tenth option (10. Enter With-Project Projections*).

4. Say "Yes" to the initialization question, and the software forecasts the variable means forward into the future target years.

If predicted forecasts deviate from the mean, then the new future projections must be input.

5. Select the STFOODCVR from the Life Requisite pull-down box, select EMERGENT from the Cover Type pull-down box, and select CANSUBMERG from the Variable pull-down box.

6. Highlight the TY 1 entry (i.e., 47) and type in the new value for theyear (i.e., 50) (see Table E17 above for these values).

7. Hit Enter on the keyboard and the software skips down to the next row (TY51). Highlight the TY 51 entry (i.e., 47) and type in the new value for the year (i.e., 90). Hit Tab on the keyboard to finish.

8. The data are now input for this variable. Since only one variable is associated with this life requisite, go ahead and pull down the remaining cover types (i.e., NEWEMERG, NEWSCRUB, and SCRUBSHRUB) and fill in the new variable values.

9. Repeat the process for each life requisite-cover type-variable combination in the model (i.e., STTEMP and STWATER).

10. When completely finished, click Close and click on Yes to save the data for later use.

Use the reports in EXHEP to check work and document analysis (Analysis Reports button). The following report should be created as a result:

\section{- With-Project Projections}




\section{Step 5: Multiple Formula Model With-project Analysis}

Now the analysis for the model can berun.

1. Set the working model - double-click on the first option - 1. Choose Working Model, select the Slider Turtle option from the pull-down list, and click Close.

2. Double-click on 4. Perform Baseline (TY 0) Calculations*, let the system run, and click $\mathbf{O K}$ when it is finished.

3. Double-click on 7. Enter Without-Project Projections*, click on the No button for initialization, click the Close button at the bottom of the interface, click Yes to save the data, and click $\mathbf{O K}$ when that save is affirmed.

4. Double-click on 8. Perform Without-Project Calculations*, let the system run, and click $\mathbf{O K}$ when it is finished.

5. Double-click on 10. Enter With-Project Projections*, click on theNo button for initialization, click the Close button at the bottom of the interface, click Yes to save the data, and click $\mathbf{O K}$ when that save is affirmed.

6. Double-click on 11. Perform With-Project Calculations*, let the system run, and click $\mathbf{O K}$ when it is finished.

Use the reports in EXHEP to check work and document analysis (Analysis Reports button). The following reports should be created as a result:

- With-Project Results

- With- vs. Without-Project Results

- AAHU Summary

- Overall Net Results - All Models.

Remember, the third report (i.e., AAHU Summary) is overwritten each time a new working model is selected. This report should be exported to MS Word for safe-keeping before proceeding. 


\section{Exercise 9: Master File Creation in EXHEP}

Scenario A: Create a "master file" that can be used for new alternatives at the same site

Assume that the feasibility study requires the formulation and evaluation of multiple alternative designs at this site. Also assume that the evaluation team has taken the time to generate a series of forecasts to characterize the outcomes of these numerous scenarios. It will be time-consuming to start with a blank datafile and rebuild all the information previously entered in the above exercises for each alternative analysis. Instead, take one of those files and recycle it for the next design. Then changes unique to the new scenario can be made and the analyses can be run quickly.

Step 1: Copy the file and change the name

1. To begin, open the training datafile entitled Scenario A - Design $1 . \mathbf{m d b}$.

2. Click on the Other Activities button, and choose the Save Datafile Under New Name option.

3. Be sure to click once on the Data Control Check Box at the bottom of this screen to assure that the file becomes the "acting" file, and click on the Save button in the upper left-hand corner of this form.

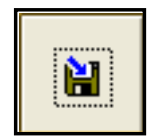

4. In the Save datafile as screen, identify the storage location via the Save in: window at the top of the interface. Name the file Scenario A Design 2.mdb in the File name: window. Now press Save and OK to finish the copying activity.

Step 2: Change the alternative name in the methods section

1. Click on the Setup button under the Single Formula Models section of the primary interface, and choose the first option (i.e., 1. Enter Project Methods).

2. Change the Alternative Name from Scenario A - Design 1 to Scenario A - Design 2, and click Close.

Assume that the following acres and variable trends have been projected for this design alternative: 
Table E18. With-project acre projections for “Design 2" in Exercise 9 (Scenario A).

\begin{tabular}{|c|c|c|c|c|c|}
\hline \multirow[b]{3}{*}{ No. } & \multirow[b]{3}{*}{ Code } & \multirow[b]{3}{*}{ Description } & \multicolumn{3}{|c|}{ Target Year } \\
\hline & & & 2000 & 2010 & 2060 \\
\hline & & & TYo & $T Y_{1}$ & $T Y_{51}$ \\
\hline 1 & EMERGENT & Emergent Wetlands & 50 & 50 & 50 \\
\hline 2 & NEWEMERG & Newly Developed Emergent Wetlands & 0 & 100 & 100 \\
\hline 3 & SCRUBSHRUB & Scrub-Shrub Wetlands & 30 & 0 & 0 \\
\hline 4 & NEWSCRUB & Newly Developed Scrub-Shrub Wetlands & 0 & 0 & 0 \\
\hline 5 & AGCROP & Agricultural Croplands & 70 & 0 & 0 \\
\hline & & TOTALS: & 150 & 150 & 150 \\
\hline
\end{tabular}

Table E19. With-project variable projections for the Marsh Wren HSI model in "Design 2" in Exercise 9 (Scenario A).

\begin{tabular}{|c|c|c|c|c|c|c|c|c|c|c|c|c|c|c|c|c|}
\hline \multirow[b]{3}{*}{$T Y$} & \multicolumn{16}{|c|}{ MARSH WREN } \\
\hline & \multicolumn{4}{|c|}{ EMERGENT } & \multicolumn{4}{|c|}{ SCRUBSHRUB } & \multicolumn{4}{|c|}{ NEWEMERG } & \multicolumn{4}{|c|}{ NEWSCRUB } \\
\hline & 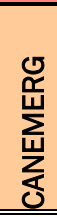 & $\begin{array}{l}0 \\
\text { o̊ } \\
\text { o } \\
\sum_{2} \\
0 \\
\end{array}$ & 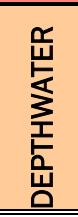 & 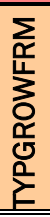 & 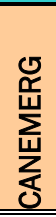 & 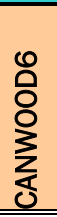 & 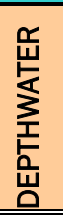 & 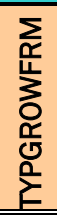 & 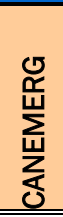 & 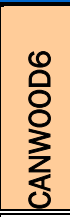 & 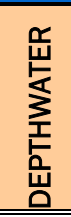 & 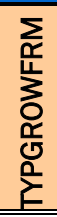 & 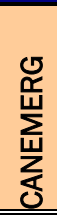 & 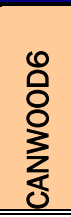 & 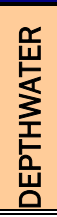 & 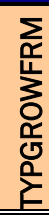 \\
\hline 0 & 42 & 40 & 42 & 1 & 12 & 60 & 6 & 3 & 0 & 0 & 0 & 0 & 0 & 0 & 0 & 0 \\
\hline 1 & 50 & 20 & 330 & 1 & 0 & 0 & 0 & 0 & 25 & 0 & 330 & 1 & 0 & 0 & 0 & 0 \\
\hline 51 & 80 & 10 & 330 & 1 & 0 & 0 & 0 & 0 & 80 & 10 & 330 & 1 & 0 & 0 & 0 & 0 \\
\hline
\end{tabular}

Table E20. With-project variable projections for the Slider Turtle HSI model in "Design 2" in Exercise 9 (Scenario A).

\begin{tabular}{|c|c|c|c|c|c|c|c|c|c|c|c|c|c|c|c|c|c|c|c|c|}
\hline \multirow[b]{3}{*}{ TY } & \multicolumn{20}{|c|}{ SLIDER TURTLE } \\
\hline & \multicolumn{5}{|c|}{ EMERGENT } & \multicolumn{5}{|c|}{ SCRUBSHRUB } & \multicolumn{5}{|c|}{ NEWEMERG } & \multicolumn{5}{|c|}{ NEWSCRUB } \\
\hline & 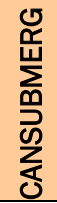 & $\begin{array}{l}\text { 営 } \\
\text { 䇈 } \\
\text { 点 } \\
\text { 岀 }\end{array}$ & 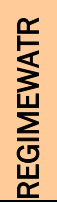 & 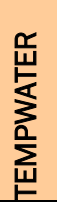 & 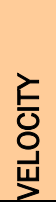 & 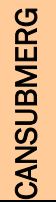 & $\begin{array}{l}\text { 営 } \\
\text { 点 } \\
\text { 点 } \\
\text { 岀 }\end{array}$ & 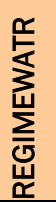 & 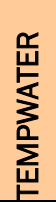 & 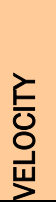 & 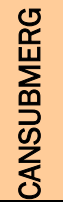 & $\begin{array}{l}\text { 営 } \\
\text { 点 } \\
\text { 岕 }\end{array}$ & 営 & 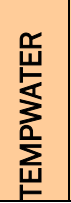 & $\begin{array}{l}\text { 穷 } \\
\text { 岁 }\end{array}$ & 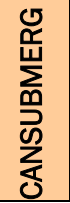 & 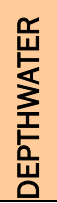 & 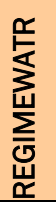 & 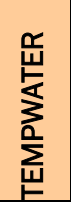 & $\begin{array}{l}\frac{\mathrm{c}}{\mathrm{O}} \\
\mathrm{O} \\
\mathrm{J}\end{array}$ \\
\hline 0 & 47 & 0.42 & 3 & 4 & 22 & 15 & 0.06 & 5 & 5 & 30 & 0 & 0 & 0 & 0 & 0 & 0 & 0 & 0 & 0 & 0 \\
\hline 1 & 50 & 3.3 & 2 & 4 & 11 & 0 & 0 & 0 & 0 & 0 & 25 & 3.3 & 2 & 4 & 11 & 0 & 0 & 0 & 0 & 0 \\
\hline 51 & 90 & 3.3 & 2 & 4 & 11 & 0 & 0 & 0 & 0 & 0 & 90 & 3.3 & 2 & 4 & 11 & 0 & 0 & 0 & 0 & 0 \\
\hline
\end{tabular}

Changes from Design 1's trends are bolded - these are the only numbers that need to be changed in the new file. 
Step 3: Change the with-project acres

1. Double-click on the ninth option (9. Enter With-Project Acres).

2. Only three of the five cover types changed from Design 1- NEWEMERG, SCRUBSHRUB, and NEWSCRUB (refer to Table E18 above). Pull down each cover type and enter in the new acreage trends.

3. Click Close when finished.

Because the Marsh Wren and Slider Turtle are both being used to assess the changes at this site, AND they share similar cover types, it is only necessary to enter the acres for one of the models - the acres are carried across all models in the analysis.

Use the reports in EXHEP to check work and document analysis (Setup Reports button). The following report should be created as a result:

\section{- With-Project TY Acres}

Step 4: Change the with-project data

Now that the acres have been input, it is time to focus on the variables.

1. To begin, click on the Run button under the Single Formula Models section of the primary interface.

2. Double-click on the first option (1. Choose Working Model), select the Marsh Wren option from the pull-down list, and click Close.

3. Now double-click on the tenth option (10. Enter With-Project Projections*).

4. Say "No" to the initialization question.

Only a few variables must be changed in EMERGENT and

NEWEMERG, so those changes will be made first.

5. Select EMERGENT from the Cover Type pull-down box, and select DEPTHWATER from the Variable pull-down box.

6. Highlight the TY $\mathrm{Y}_{1}$ entry (i.e., 100) and type in the new value for the year (i.e., 330) (see TableE19 above for these values).

7. Hit Tab on the keyboard and the software skips down to the next row (TY $\left.{ }_{51}\right)$. Highlight the $\mathrm{TY}_{51}$ entry (i.e., 100) and type in the new value for the year (i.e., 330).

8. Hit Tab on the keyboard to finish. The data are now input for this variable. 
9. Repeat the process for the NEWEMERG cover type and the DEPTHWATER variable.

Now focus on NEWSCRUB and SCRUBSHRUB.

10. Select NEWSCRUB from the Cover Type pull-down box, and select CANEMERG from the Variable pull-down box.

11. Highlight the TY 1 entry (i.e., 10) and type in the new value for the year (i.e., 0) (see Table E20 above for these values).

12. Hit Tab on the keyboard and the software skips down to the next row (TY ${ }_{51}$ ). Highlight the $T Y_{51}$ entry (i.e., 50) and type in the new value for the year (i.e., 0).

13. Hit Tab on the keyboard to finish. The data are now input for this variable.

14. Repeat the process for all the variables in the NEWSCRUB cover type (i.e., CANWOOD6, DEPTHWATER, and TYPGROWFRM).

15. Repeat the process for all variables in the SCRUBSHRUB cover type (i.e., CANEMERG, CANWOOD6, DEPTHWATER, and TYPGROWFRM).

16. When completely finished, click Close and click on Yes to save the data for later use.

Repeat the process for the multiple formula models.

17. Click on the Run button under the Multiple Formula Models section of the primary interface.

18. Double-click on the first option (1. Choose Working Model), select the Slider Turtle option from the pull-down list, and click Close.

19. Now double-click on the tenth option (10. Enter With-Project Projections*).

20. Say "No" to the initialization question.

Only a few variables must be changed in EMERGENT and

NEWEMERG; start with those changes first.

21. Select STWATER from the Life Requisite pull-down box, select EMERGENT from the Cover Type pull-down box, and select DEPTHWATER from the Variable pull-down box.

22. Highlight the TY 1 entry (i.e., 1) and type in the new value for the year (i.e., 3.3) (see Table E20 above for these values). 
23. Hit Enter on the keyboard and the software skips down to the next row (TY51). Highlight the TY 51 entry (i.e., 1) and type in the new value for the year (i.e., 3.3). Hit Tab on the keyboard to finish.

24. Repeat the process for the NEWEMERG cover type and the DEPTHWATER variable.

Now focus on NEWSCRUB and SCRUBSHRUB.

25. Select STFOODCVR from the Life Requisite pull-down box, select NEWSCRUB from the Cover Type pull-down box, and select CANSUBMERG from the Variable pull-down box.

26. Highlight the TY 1 entry (i.e., 10) and type in the new value for the year (i.e., 0) (see TableE20 above for these values).

27. Hit Enter on the keyboard and the software skips down to the next row (TY $Y_{51}$. Highlight the $\mathrm{TY}_{51}$ entry (i.e., 50) and type in the new value for the year (i.e., 0). Hit Tab on the keyboard to finish.

28. All the TY 1 and $\mathrm{TY}_{51}$ variable values for NEWSCRUB and

SCRUBSHRUB must be changed to zeroes in every life requisite, so systematically repeat the process to make these changes.

29. When completely finished, click Close and click on Yes to save the data for later use.

Use the reports in EXHEP to check work and document analysis (Analysis Reports button). The following reports should be created as a result:

- With-Project Projections - Single Formula Models

- With-Project Projections - Multiple Formula Models

Step 5: Re-run the analysis

Now run the analysis for the models.

1. Set the working model (i.e., double-click on the first option - 1. Choose Working Model, select the Marsh Wren option from the pull-down list, and click Close.

2. Double-click on 4. Perform Baseline (TY 0) Calculations*, let the system run, and click $\mathbf{O K}$ when it is finished. 
3. Double-click on 7. Enter Without-Project Projections*, click on the No button for initialization, click the Close button at the bottom of the interface, click Yes to save the data, and click $\mathbf{O K}$ when that save is affirmed.

4. Double-click on 8. Perform Without-Project Calculations*, let the system run, and click $\mathbf{O K}$ when it is finished.

5. Double-click on 10. Enter With-Project Projections*, click on the No button for initialization, click the Close button at the bottom of the interface, click Yes to save the data, and click $\mathbf{O K}$ when that save is affirmed.

6. Double-click on 11. Perform With-Project Calculations*, let the system run, and click $\mathbf{O K}$ when it is finished.

7. Go to the Analysis Reports button, open the AAHU Summary Report, and export it to MSWord.

Again, repeat the process for the multiple formula models.

8. Set the working model (i.e., double-click on the first option - 1. Choose Working Model, select the Slider Turtle option from the pull-down list, and click Close.

9. Double-click on 4. Perform Baseline (TY 0) Calculations*, let the system run, and click $\mathbf{O K}$ when it is finished.

10. Double-click on 7. Enter Without-Project Projections*, click on the No button for initialization, click the Close button at the bottom of the interface, click Yes to save the data, and click $\mathbf{O K}$ when that save is affirmed.

11. Double-click on 8. Perform Without-Project Calculations*, let the system run, and click $\mathbf{O K}$ when it is finished.

12. Double-click on 10. Enter With-Project Projections*, click on the No button for initialization, click the Close button at the bottom of the interface, click Yes to save the data, and click $\mathbf{O K}$ when that save is affirmed.

13. Double-click on 11. Perform With-Project Calculations*, let the system run, and click $\mathbf{O K}$ when it is finished.

14. Go to the Analysis Reports button, open the AAHU Summary Report, and export it to MSWord.

Use the reports in EXHEP to check work and document analysis (Analysis Reports button). The following reports should be created as a result: 
Single Formula Models:

\section{- AAHU Summary}

- With-Project Results

- With- vs. Without-Project Results.

Multiple Formula Models:

- AAHU Summary

- With-Project Results

- With- vs. Without-Project Results

Both Models Types:

\section{- Overall Net Results - All Models}

Which design was more productive? According to the outputs in Exercise 8 and the analysis that was just run, one can expect to see the following results:

Table E21. Results of the Scenario A Restoration Analyses (“Design 1" vs. “Design 2").

\begin{tabular}{|c|c|c|c|c|c|}
\hline \multirow[b]{2}{*}{ Model Name } & \multicolumn{2}{|c|}{ Scenario A - Design 1} & \multicolumn{2}{|c|}{ Scenario A - Design 2} & \multirow[b]{2}{*}{$\begin{array}{c}\text { Net } \\
\text { Difference }\end{array}$} \\
\hline & $\begin{array}{l}\text { Acres } \\
\text { Restored } \\
\text { and } \\
\text { Created }\end{array}$ & Net Gains & $\begin{array}{l}\text { Acres } \\
\text { Restored } \\
\text { and } \\
\text { Created }\end{array}$ & Net Gains & \\
\hline Marsh Wren & 150 & 60 & 150 & 92 & 32 \\
\hline Slider Turtle & 150 & 74 & 150 & 30 & 44 \\
\hline Average Gains: & 150 & 134 & 150 & 122 & 76 \\
\hline
\end{tabular}

The restoration design proposed under the first alternative (i.e., Design 1) is clearly more productive (i.e., on average 134 AAHUs vs. 122 AAHUs). The first design is more productive for the slider turtle, and the second design is more productive for the marsh wren. Design 1 only 
produces 60 and 74 AAHUs respectively. Design 2 produces 92 marsh wren AAHUs and $\mathbf{3 0}$ slider turtle AAHUs.

If time permits, Scenario A should be re-run a few more times - users should attempt to develop a restoration scheme to rehabilitate the habitat for these animals. Remember, acres can be incrementally increased or decreased, but the variables can also be changed to reflect different levels of restoration and management activities (which ultimately affect the cost of the project). Be sure to re-name these when moving through the process to keep track of the analyses.

\section{Scenario B: Create a "master file" that can be used for new sites}

Now assume that the feasibility study spans an entire watershed, and as such, several individual sites are the focus of the comprehensive restoration plan. A second type of "master file" is needed to assess each proposed action site - one that accounts for the new baseline and without-project conditions at each new location. This time, the models and their associations are retained, but the baseline data and future conditions (both with- and without-project) must be modified.

Step 1: Copy the file and change the name

1. To begin, open the training datafile entitled Scenario A Design 1.mdb.

2. Click on the Other Activities button, and choose the Save Datafile Under New Name option.

3. Be sure to click once on the Data Control Check Box at the bottom of this screen to assure that the file becomes the "acting" file when finished, and click on the Save button in the upper left-hand corner of this form.

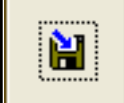

4. In the Save datafile as screen, identify the storage location via the Save in: window at the top of the interface. Name the file Scenario B Design 1.mdb in the File name: window. Now press Save and OK to finish the copying activity.

Step 2: Change the alternative name in the methods section

1. Click on the Setup button under the Single Formula Models section of the primary interface, and choose the first option (i.e., 1. Enter Project Methods). 


\section{Change the Alternative Name from Scenario A - Design 1 to Scenario B - Design 1, and click Close.}

Now assume that the site is purely agricultural cropland, waiting for hydrology to be restored and wetland vegetation to be re-planted on its newly sculpted topography. The following acres and variable trends have been projected for this design alternative:

Table E22. With-project acre projections for "Design 1" in Exercise 9 (Scenario B).

\begin{tabular}{|c|c|c|c|c|c|c|c|c|}
\hline \multirow[b]{4}{*}{ No. } & \multirow[b]{4}{*}{ Code } & \multirow[b]{4}{*}{ Description } & \multirow{2}{*}{\multicolumn{3}{|c|}{$\begin{array}{c}\text { Without-Project } \\
\text { Target Year }\end{array}$}} & \multirow{2}{*}{\multicolumn{3}{|c|}{$\begin{array}{l}\text { With-Project } \\
\text { Target Year }\end{array}$}} \\
\hline & & & & & & & & \\
\hline & & & 2000 & 2010 & 2060 & 2000 & 2010 & 2060 \\
\hline & & & TYO & TY1 & TY51 & TYO & TY1 & TY51 \\
\hline 1 & EMERGENT & Emergent Wetlands & 0 & 0 & 0 & 0 & 0 & 0 \\
\hline 2 & NEWEMERG & $\begin{array}{l}\text { Newly Developed Emergent } \\
\text { Wetlands }\end{array}$ & 0 & 0 & 0 & 0 & 100 & 100 \\
\hline 3 & SCRUBSHRUB & Scrub-Shrub Wetlands & 0 & 0 & 0 & 0 & 0 & 0 \\
\hline 4 & NEWSCRUB & $\begin{array}{l}\text { Newly Developed Scrub- } \\
\text { Shrub Wetlands }\end{array}$ & 0 & 0 & 0 & 0 & 100 & 100 \\
\hline 5 & AGCROP & Agricultural Croplands & 200 & 200 & 200 & 200 & 0 & 0 \\
\hline & & TOTALS & 200 & 200 & 200 & 200 & 200 & 200 \\
\hline
\end{tabular}

Table E23. With-project variable projections for the Marsh Wren HSI model in "Design 1" in Exercise 9 (Scenario B).

\begin{tabular}{|c|c|c|c|c|c|c|c|c|c|c|c|c|c|c|c|c|}
\hline & \multicolumn{16}{|c|}{ MARSH WREN } \\
\hline & \multicolumn{4}{|c|}{ EMERGENT } & \multicolumn{4}{|c|}{ SCRUBSHRUB } & \multicolumn{4}{|c|}{ NEWEMERG } & \multicolumn{4}{|c|}{ NEWSCRUB } \\
\hline TY & 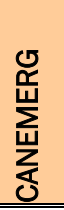 & $\begin{array}{l}0 \\
8 \\
8 \\
\sum_{0} \\
\text { 엉 }\end{array}$ & 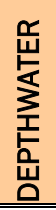 & 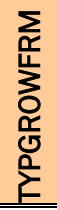 & 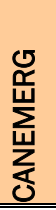 & $\begin{array}{l}0 \\
8 \\
8 \\
\sum_{0} \\
\text { ऐo }\end{array}$ & 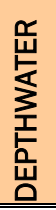 & 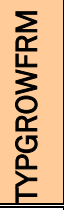 & 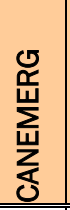 & $\begin{array}{l}0 \\
8 \\
8 \\
\sum_{0} \\
0\end{array}$ & 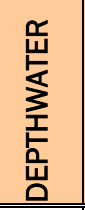 & 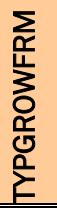 & 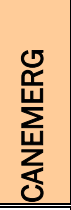 & $\begin{array}{l}0 \\
0 \\
8 \\
\vdots \\
\sum_{0}\end{array}$ & 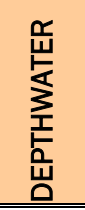 & 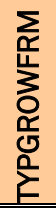 \\
\hline 0 & 0 & 0 & 0 & 0 & 0 & 0 & 0 & 0 & 0 & 0 & 0 & 0 & 0 & 0 & 0 & 0 \\
\hline 1 & 0 & 0 & 0 & 0 & 0 & 0 & 0 & 0 & 25 & 0 & 300 & 1 & 10 & 5 & 200 & 3 \\
\hline 51 & 0 & 0 & 0 & 0 & 0 & 0 & 0 & 0 & 80 & 10 & 300 & 1 & 50 & 30 & 200 & 3 \\
\hline
\end{tabular}


Table E24. With-project variable projections for the Slider Turtle HSI model in "Design 1" in Exercise 9 (Scenario B).

\begin{tabular}{|c|c|c|c|c|c|c|c|c|c|c|c|c|c|c|c|c|c|c|c|c|}
\hline & \multicolumn{20}{|c|}{ SLIDER TURTLE } \\
\hline & \multicolumn{5}{|c|}{ EMERGENT } & \multicolumn{5}{|c|}{ SCRUBSHRUB } & \multicolumn{5}{|c|}{ NEWEMERG } & \multicolumn{5}{|c|}{ NEWSCRUB } \\
\hline TY & 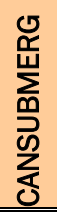 & 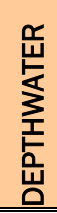 & 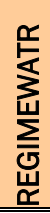 & 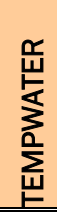 & 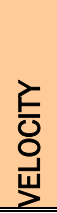 & 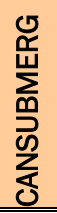 & 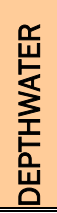 & 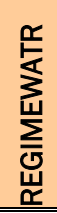 & 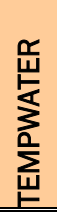 & 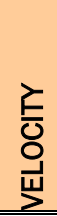 & 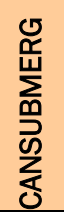 & 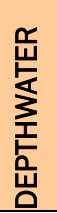 & 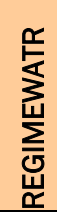 & 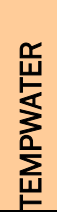 & $\begin{array}{l}\frac{\mathrm{c}}{\mathrm{O}} \\
\mathrm{d} \\
\mathrm{J}\end{array}$ & 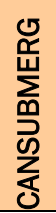 & 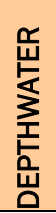 & 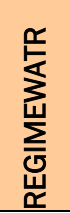 & 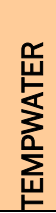 & $\begin{array}{l}\text { 등 } \\
\text { 일 }\end{array}$ \\
\hline 0 & 0 & 0 & 0 & 0 & 0 & 0 & 0 & 0 & 0 & 0 & 0 & 0 & 0 & 0 & 0 & 0 & 0 & 0 & 0 & 0 \\
\hline 1 & 0 & 0 & 0 & 0 & 0 & 0 & 0 & 0 & 0 & 0 & 25 & 3 & 2 & 4 & 11 & 10 & 2 & 3 & 5 & 15 \\
\hline 51 & 0 & 0 & 0 & 0 & 0 & 0 & 0 & 0 & 0 & 0 & 90 & 3 & 2 & 4 & 11 & 50 & 2 & 3 & 5 & 15 \\
\hline
\end{tabular}

Focus on the acres first, and then return to handle the variables.

Step 3: Change the acres

1. Click the Setup button under the Single Formula Models section, and double-click the third option (i.e., 3. Define Cover Types and Baseline Acres).

2. Zero out the first four cover types and enter $\mathbf{2 0 0}$ acres for the last cover type (i.e., AGCROP - Agricultural Croplands) so that this information matches the acres in the table above (Table E22).

3. Click Close when finished.

Now focus on the without-project acres.

4. Double-click on the sixth option (6. Enter Without-Project Acres).

5. Again, zero out all the cover type except AGCROP-Agricultural Croplands so that the information matches the without-project acreage trends in the table above (Table E22).

6. Click Close when finished.

Fianlly, deal with the with-project acres.

7. Double-click on the ninth option (9. Enter With-Project Acres).

8. Pull-down the cover types and fill in the with-project acreage trends as indicated in the table above (Table E22).

9. Click Close when finished. 
Because the Marsh Wren and Slider Turtle are both being used to assess the changes at this site, AND they share similar cover types, it is only necessary to enter the acres for one of the models - the acres are carried across all models in the analysis.

Use the reports in EXHEP to check work and document analysis (Setup Reports button). The following report should be created as a result:

\section{- Cover Types and Baseline (TY 0) Acres}

- Without-Project TY Acres

- With-Project TY Acres

Step 4: Change the baseline data

Now that the acres have been input, it is time to focus on the variables (refer to Table E23 above).

1. To begin, click on the Run button under the Single Formula Models section of the primary interface.

2. Double-click on the first option (1. Choose Working Model), select the Marsh Wren option from the pull-down list, and click Close.

3. Now double-click on the thind option (3. Enter SI Graphs and Variable Data).

4. Delete all the baseline data for every variable shown, and enter a zero for each variable in each cover type (i.e., delete the individual data points by clicking on the Record Indicator Bar to the immediate left of each data point and pressing Delete on the keyboard).

5. Click Close when finished.

Repeat the process for the multiple formula models (refer to Table E24 above).

6. Click on the Run button under the Multiple Formula Models section of the primary interface.

7. Double-click on the first option (1. Choose Working Model), select the Slider Turtle option from the pull-down list, and click Close.

8. Now double-click on the third option (3. Enter SI Graphs and Variable Data). 
9. Delete all the baseline data for every variable in each life requisite, and enter a zero for each variable in each cover type-life requisite combination (i.e., delete the individual data points by clicking on the Record

Indicator Bar to the immediate left of each data point while pressing Delete on the keyboard).

10. Click Close when finished.

Use the reports in EXHEP to check work and document analysis (Setup Reports button). The following reports should be created as a result:

\section{- Variable Data - Single Formula Models}

\section{- Variable Data - Multiple Formula Models}

Another way to check work is to run the baseline calculations for each model (the result should be 0.0 HSIs and $0.0 \mathrm{HUs}$ ). The following report should be created as a result:

- Baseline (TY 0) Results - Single Formula Models

- Baseline (TY 0) Results - Multiple Formula Models.

Step 5: Change the without-project data

The without-project condition is the "status quo" ( 0.0 quality and no acres associated with the models), the baseline data should be initialized (which will set all the values to zero), and then the projections should be saved (refer to Table E23 above).

1. To begin, click on the Run button under the Single Formula Models section of the primary interface.

2. Double-click on the first option (1. Choose Working Model), select the Marsh Wren option from the pull-down list, and click Close.

3. Now double-click on the seventh option (7. Enter Without-Project Projections*).

4. Say "Yes" to the initialization question. There is no need to change any of the data - they are all zeros; now the without-project analysis can begin.

5. Click Close, and say Yes to the save question.

Repeat the process for the multiple formula models (refer to Table E24 above). 
6. Click on the Run button under the Multiple Formula Models section of the primary interface.

7. Double-click on the first option (1. Choose Working Model), select the Slider Turtle option from the pull-down list, and click Close.

8. Now double-click on the seventh option (7. Enter Without-Project Projections*).

9. Say "Yes" to the initialization question. There is no need to change any of the data - they are all zeros; now the without-project analysis can begin.

10. Click Close, and say Yes to the save question.

Use the reports in EXHEP to check work and document analysis (Analysis Reports button). The following reports should be created as a result:

- Without-Project Projections - Single Formula Models

- Without-Project Projections - Multiple Formula Models

Step 6: Change the with-project data

The with-project condition is the restoration plan, and as such requires major changes in the variable trends.

1. To begin, click on the Run button under the Single Formula Models section of the primary interface.

2. Double-click on the first option (1. Choose Working Model), select the Marsh Wren option from the pull-down list, and click Close.

3. Now double-click on the tenth option (10. Enter With-Project Projections*).

4. Say "Yes" to the initialization question.

5. Enter the variable trends into this screen based on the information provided in Table E23 above. Refer to Exercise 8 above for a refresher on how to enter this information.

6. Click Close, and say Yes to the save question.

Again, repeat the process for the multiple formula models.

7. To begin, click on the Run button under the Multiple Formula Models section of the primary interface.

8. Double-click on the first option (1. Choose Working Model), select the Slider Turtle option from the pull-down list, and click Close. 
9. Now double-click on the tenth option (10. Enter With-Project Projections*).

10. Say "Yes" to the initialization question.

11. Enter the variable trends into this screen based on the information provided in TableE24 above. Refer to Exercise 8 above for a refresher on how to enter this information.

12. Click Close, and say Yes to the save question.

Use the reports in EXHEP to check work and document analysis (Analysis Reports button). The following reports should be created as a result:

- With-Project Projections - Single Formula Models

- With-Project Projections - Multiple Formula Models

Step 7: Re-run the analysis

Now the analysis for the models can be run.

1. Set the working model (i.e., double-click on the first option - 1. Choose Working Model, select the Marsh Wren option from the pull-down list, and click Close.

2. Double-click on 4. Perform Baseline (TY 0) Calculations*, let the system run, and click $\mathbf{O K}$ when it is finished.

3. Double-click on 7. Enter Without-Project Projections*, click on the No button for initialization, click the Close button at the bottom of the interface, click Yes to save the data, and click $\mathbf{O K}$ when that save is affirmed.

4. Double-click on 8. Perform Without-Project Calculations*, let the system run, and click $\mathbf{O K}$ when it is finished.

5. Double-click on 10. Enter With-Project Projections*, click on theNo button for initialization, click the Close button at the bottom of the interface, click Yes to save the data, and click $\mathbf{O K}$ when that save is affirmed.

6. Double-click on 11. Perform With-Project Calculations*, let the system run, and click $\mathbf{O K}$ when it is finished.

7. Go to the Analysis Reports button, open the AAHU Summary Report, and export it to MSWord. 
Now focus on the multiple formula models.

8. Set the working model (i.e., double-click on the first option - 1. Choose Working Model, select the Slider Turtle option from the pull-down list, and click Close).

9. Double-click on 4. Perform Baseline (TY 0) Calculations*, let the system run, and click $\mathbf{O K}$ when it is finished.

10. Double-click on 7. Enter Without-Project Projections*, click on the No button for initialization, click the Close button at the bottom of the interface, click Yes to save the data, and click $\mathbf{O K}$ when that save is affirmed.

11. Double-click on 8. Perform Without-Project Calculations*, let the system run, and click $\mathbf{O K}$ when it is finished.

12. Double-click on 10. Enter With-Project Projections*, click on the No button for initialization, click the Close button at the bottom of the interface, click Yes to save the data, and click $\mathbf{O K}$ when that save is affirmed.

13. Double-click on 11. Perform With-Project Calculations*, let the system run, and click $\mathbf{O K}$ when it is finished.

14. Go to the Analysis Reports button, open the AAHU Summary Report, and export it to MSWord.

Use the reports in EXHEP to check work and document analysis (Analysis Reports button). The following reports should be created as a result:

Single Formula Models:

- AAHU Summary

- Without-Project Results

- With-Project Results

- With- vs. Without-Project Results 
Multiple Formula Models:

\section{- AAHU Summary}

- Without-Project Results

- With-Project Results

- With- vs. Without-Project Results

Both Model Types:

\section{- Overall Net Results - All Models}

So which designs were more productive - Scenario A or B? According to the outputs in Exercise 8 and the analysis run so far, one can expect to see the following results:

Table E25. Results of the Scenario A Restoration Analyses (“Design 1" vs. “Design 2").

\begin{tabular}{|c|c|c|c|c|c|c|}
\hline \multirow[b]{2}{*}{ Model Name } & \multicolumn{2}{|c|}{ Scenario A - Design 1} & \multicolumn{2}{|c|}{ Scenario A - Design 2} & \multicolumn{2}{|c|}{ Scenario B - Design 1} \\
\hline & $\begin{array}{l}\text { Acres } \\
\text { Restored } \\
\text { and } \\
\text { Created }\end{array}$ & $\begin{array}{l}\text { Net } \\
\text { Gains }\end{array}$ & $\begin{array}{l}\text { Acres } \\
\text { Restored } \\
\text { and } \\
\text { Created }\end{array}$ & Net Gains & $\begin{array}{l}\text { Acres } \\
\text { Restored } \\
\text { and } \\
\text { Created }\end{array}$ & Net Gains \\
\hline Marsh Wren & 150 & 60 & 150 & 92 & 200 & 76 \\
\hline Slider Turtle & 150 & 74 & 150 & 30 & 200 & 75 \\
\hline Average Gains: & 150 & 134 & 150 & 122 & 200 & 151 \\
\hline
\end{tabular}

This latest design (i.e., Scenario B - Design 1) is now the most productive but not by much. A small increase in acreage at this second site (i.e., Scenario B - Design 2) might lead to an even more productive alternative. If time permits, Scenario B - Design 1 should be modified a few more times - users should try their hands at improving the rehabilitation scheme. Remember, acres can be incrementally increased or decreased, but variables can also be changed to reflect different levels of restoration and management activities (which ultimately affect the cost of the project). Be sure to re-name these when moving through the process in order to keep track of the analyses. 


\section{Exercise 10: Project Information and Model Setup in EXHGM}

Mr. Howard J ones, a savvy businessman and local farmer, owns a 250acre plot of ground just outside the town of Biloxi, MS called White Clouds Farm. The area immediately surrounding the town has recently experienced a dramatic increase in industrial growth, and development pressure has led Mr. J ones to explore the possibility of building an industrial complex on his land. Of the 250 acres currently under his control, 25 acres can be characterized as cypress pine savanna, 25 acres as switchcane, 50 as hardwoods, and an additional 150 acres can be classified as bunchgrass pine savanna (both have been further characterized as wet pine flats on mineral soils). Below is a table containing a breakdown of acres by partial wetland assessment area (PWAA) (Table E26).

Table E26. Baseline PWAAs and acreages for Exercise 10.

\begin{tabular}{|l|l|l|c||}
\hline No. & Code & Partial Wetland Assessment Area Descriptions & $\begin{array}{l}\text { Baseline } \\
\text { Acres (TYo) }\end{array}$ \\
\hline \hline 1 & BNCHGRPSAV & Bunchgrass/Pine Savanna & 150 \\
\hline 2 & CYPRESPSAV & Cypress/Pine Savanna & 25 \\
\hline 3 & SWTCHPSAV & Switchcane/Pine Savanna & 25 \\
\hline 4 & HARDWOODS & $\begin{array}{l}\text { Uplands, Hardwoods \& Other Non Applicable } \\
\text { Areas }\end{array}$ & 50 \\
\hline 5 & IMPACT & Area Impacted By Proposed Development & 0 \\
\hline \hline & & & TOTAL: \\
\hline
\end{tabular}

As one might expect, the area provides little economic benefit to Mr. J ones under his current operational scheme (i.e., the acres are too wet to farm), but once they are developed, Mr. J ones stands to turn a good profit. Being a conscientious farmer and a closet naturalist, Mr. J ones acknowledges that wetlands provide ecosystem services (i.e., flood control, water quality, aesthetics, recreation, etc.) to the region, and has offered to buy (and preserve in perpetuity) 200 acres of high quality wetlands adjacent to his farm to offset these losses. Mr. J ones has recently applied to the U.S. Army Corps of Engineers (USACE) for a permit, and is eagerly awaiting the reply.

In order to process the permit, a USACE regulator must determine:

1. The number of functional capacity units lost as a result of Mr. J ones' development proposal. 
2. The number of additional acres, of a specific quality, that must be purchased to mitigate for the losses not covered under Mr. J ones' mitigation plan.

An interagency team has been convened to participate in the permitting process. Their names include:

- Andrea Fisher and J oe Black (USACE District)

o J ohn Anderson (County Forest Preserve)

- Eric Brand (Natural Resources Conservation Service)

o William Malone and Chris White (State Department of Natural Resources)

o Cynthia Miller (U.S. Geological Survey)

o Sally Mengler (USFWS)

o Kelly Burks-Copes and Antisa Webb (ERDC Facilitators)

The team has participated in the intensive HGM model development process, and is now eager to apply the model on a real-world regulatory problem. Details regarding the model's functions, associated partial wetland assessment areas, and variables have been provided in a file on the installation CD (refer to the Wet Pine Flats Field Kit workbook). Use this information to set up the models in EXHGM.

1. To begin, open the Blank datafile.mdb with EXHGM (look at the Getting Started chapter to locate this file), name it something unique (say Exercise 10.mdb), and save it to the Exercise 10 folder under the Training Exercise subdirectory under Environmental Programs on the hard drive. Now, document the basic premises behind the study in the methods interface (click the Setup button) and choose the 1. Enter Project Methods option. The following information might be important to enter into the interface:

- Model references and support documentation.

- Model modifications (if any).

- List of evaluation team members.

- Goals and objectives.

- Data management strategies.

- Evaluation strategies (including tradeoff approaches).

- Field sampling team and metadata (include locations, assumptions, dates, etc.). 
At the absolute minimum, a Project Name (i.e., White Clouds Farm) and Alternative Name (i.e., Impact Analysis) must be entered in the spaces provided.

2. Now set up functions for the Pine Flats model by assigning names and descriptions in the function description interface (double-click the 2. Enter Function Descriptions option). Hint - look in the MS Excel "fieldkits" for the Function List worksheet to see details.

3. Define the PWAAs in the study area in the PWAA interface (double-click the 3. Define Partial Wetland Areas option). Hint - look in the MS Excel "fieldkits" for the PWAA worksheet to see details.

4. Now enter the variables (double-click the 4. Define Variables option). Hint - look in the MS Excel "fieldkits" for the Master Variables List worksheet to see details.

5. Use the reports in EXHGM to check work and document analysis (Setup Reports button). The following reports should be created as a result:

- Methods and Assumptions

- Function List

- PWAAs and Baseline (TY 0) Acres

- Variables List

The files on the hard drive are not formatted as they might appear in EXHGM (the actual EXHGM reports were exported to MS Word for purposes of distribution), but the information contained within is accurate. 


\section{Exercise 11: Formula Association and FCI Formula Writing in EXHGM}

For users who need help writing formulas in EXHGM, three helpful tools are provided in the software to make the process easier. The first tool is called a "Cheat Sheet." Just click on the Help button at the top of the Function Association screen (under Run, 2. Associate Parameters) to see:

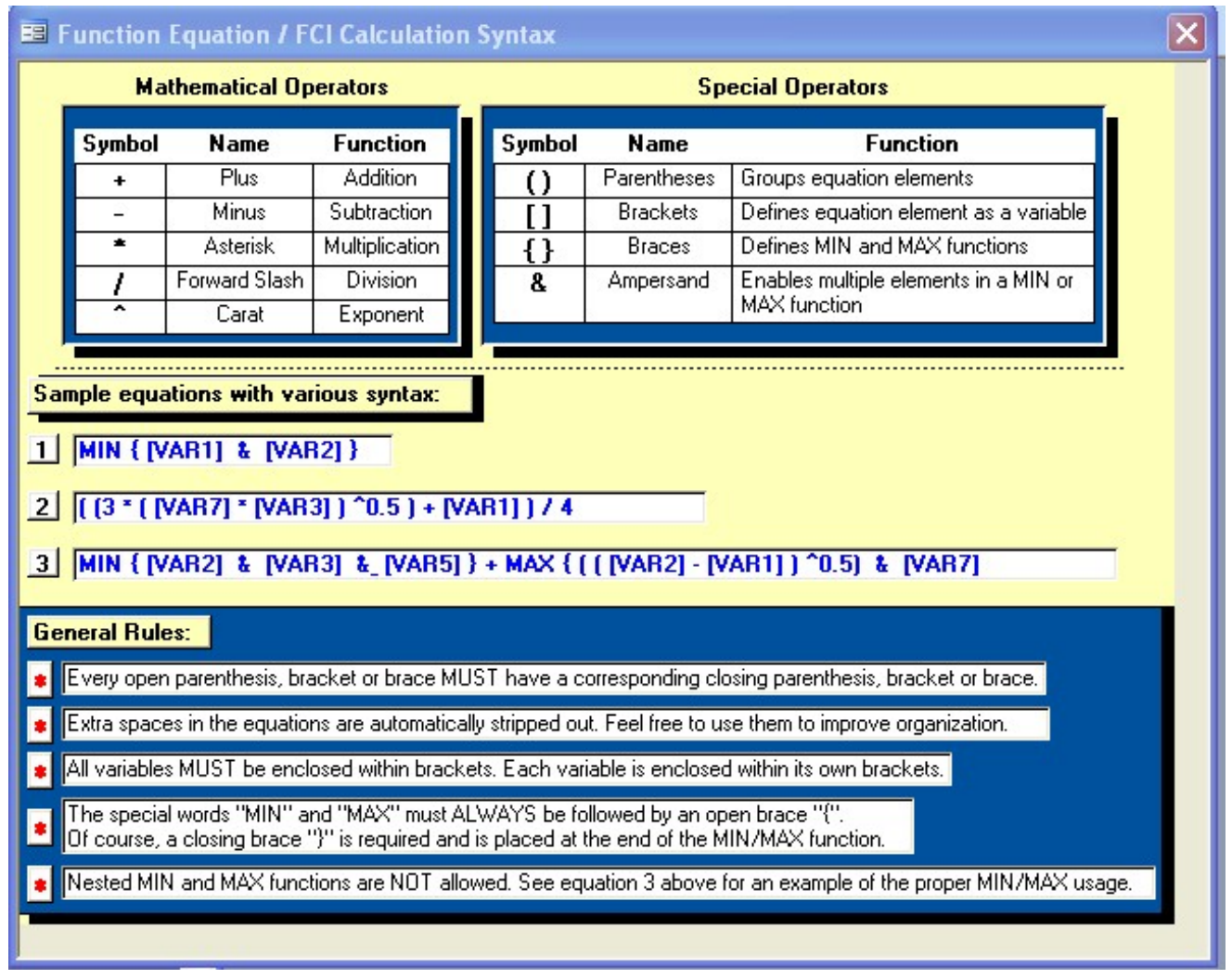

Now write the four FCI Formulas for the Wet Pine Flats HGM model using these syntax rules. 


\section{Wet Pine Flats - Fxn 1: Water Regime}

FCl WATEREGIME For all PWAAs $=$

$$
\text { Minimum of } V_{1} \text { or } V_{2} \text { or } V_{3} \text { or } V_{4} \text { or }\left(V_{5} \times \frac{V_{6}+V_{7}}{2}\right)^{1 / 2}
$$

Where:

$$
\begin{aligned}
& V_{1}=\text { Surface Flow of Water (SURFFLOW) } \\
& V_{2}=\text { Outflow of Water (OUTFLOW) } \\
& V_{3}=\text { Surface Water Storage (STORAGE) } \\
& V_{4}=\text { Importation of Water from Elsewhere (INFLOW) } \\
& V_{5}=\text { Evapotranspiration Potential (EVAPOTRANS) } \\
& V_{6}=\text { Microtopographic Features (MICRO) } \\
& V_{7}=\text { Soil Porosity (PORE) }
\end{aligned}
$$

Follow the syntax rules to enter the formula into the software (see next page to check the formula).

The Wet Pine Flats FCI formula for Fxn 1(Water Regime) for all PWAAs = 
Here is the correct answer:

Wet Pine Flats - Fxn 1: Water Regime

FCl $_{\text {WATEREGIME }}=$ MIN $\{[$ SURFFLOW $] \&[O U T F L O W] \&[$ STORAGE $] \&[$ INFLOW $] \&$

$\left.\left(([E V A P O T R A N S] *(([M I C R O]+[P O R E]) / 2))^{\wedge}(1 / 2)\right)\right\}$

Now try the second function - remember that some functions are written specifically for the PWAAs in which they are applied (as is the case with the Fxn 2: Maintain Characteristic Plant Community function in the Wet Pine Flats model).

Wet Pine Flats - Fxn 2: Maintain Characteristic Plant Community

1) FClpLANTCOMm for Bunchgrass/Pine (BNCHGRPSAV) =

Where:

$$
\text { Maximum of } \mathrm{V}_{1} \text { or } \mathrm{V}_{2}
$$

$V_{1}=$ Herbaceous Indicator Score (HERB)

$V_{2}=$ Percent Cover of Selected Native Bunchgrass (NBG)

2) FClplantcomm for Cypress/Pine Savanna (CYPRESPSAV) =

$$
\text { Maximum of } V_{1} \text { or } V_{2} \text { or }\left(V_{3} X \underline{V}_{4}+\frac{V_{5}}{2}\right)^{1 / 2}
$$

Where:

$\mathrm{V}_{1}=$ Herbaceous Indicator Score (HERB)

$\mathrm{V}_{2}=$ Percent Cover of Selected Native Bunchgrass (NBG)

$\mathrm{V}_{3}=$ Density of Pond Cypress in each of 3 size classes (CYPRESS).

$\mathrm{V}_{4}=$ Percent Cover of Sedges (SEDGES)

$\mathrm{V}_{5}=$ Sub-canopy Density per ha (SUBCAN)

3) FClplantcomm for Switchcane/Pine Savanna (SWTCHPSAV) $=$

$$
\left(v_{1} \times \underline{v_{2}}+\frac{v_{3}}{2}\right)^{1 / 2}
$$

Where:

$\mathrm{V}_{1}=$ Density of Longleaf and Pond Pines in the Canopy layer (LONGL)

$\mathrm{V}_{2}=$ Sub-canopy Density per ha (SUBCAN)

$\mathrm{V}_{3}=$ Pine Density per ha (PINES) 
Follow the syntax rules to enter the formula into the software (see next page to check formula).

The Wet Pine Flats FCI formula for Fxn 2 (Plant Community) for BNCHGRPSAV $=$

The Wet Pine Flats FCI formula for Fxn 2 (Plant Community) for CYPRESPSAV $=$

The Wet Pine Flats FCI formula for Fxn 2 (Plant Community) for SWTCHPSAV $=$ 
Here are the correct answers:

Wet Pine Flats - Fxn 2: Maintain Characteristic Plant Community

1) Bunchgrass/Pine (BNCHGRPSAV) =

FClPLANTCOMm $=\quad \operatorname{MAX}\{[\mathrm{HERB}] \&[\mathrm{NBG}]\}$

2) Cypress/Pine Savanna (CYPRESPSAV) =

FClPLANTCOMM $=\quad \operatorname{MAX}\{[\mathrm{HERB}] \&[\mathrm{NBG}] \&(([\mathrm{CYPRESS}]$ * $\left.\left.(([\mathrm{SEDGES}]+[\mathrm{SUBCAN}]) / 2))^{\wedge}(1 / 2)\right)\right\}$

3) Switchcane/Pine Savanna (SWTCHPSAV) =

FClPLANTCOMM $=([\text { LONGL }] *(([S U B C A N]+[\text { PINES }]) / 2))^{\wedge}(1 / 2)$

Now try the third function - again, this function is written specifically for the PWAAs in which it is applied, so there will be a new formula for each PWAA in Fxn 3: Maintain Characteristic Animal Community. 
Wet Pine Flats - Fxn 3: Maintain Characteristic Animal Community

1) $\mathrm{FCl}_{\text {ANIMALCOMM }}$ for Bunchgrass/Pine (BNCHGRPSAV) $=$

$$
\left[\left(\text { Maximum of } \mathrm{V}_{1} \text { or } \mathrm{V}_{2}\right) \times \mathrm{V}_{3}\right]^{1 / 2}
$$

Where:

$$
\begin{aligned}
& V_{1}=\text { Herbaceous Indicator Score (HERB) } \\
& V_{2}=\text { Percent Cover of Selected Native Bunchgrass (NBG) } \\
& V_{3}=\text { Area of Contiguous Fire-Maintained Landscape (LANDSCP) }
\end{aligned}
$$

2) $\mathrm{FCl}_{\text {ANIMALCOMm }}$ for Cypress/Pine Savanna (CYPRESPSAV) $=$

$$
\left\{\left[\text { Maximum of } \mathrm{V}_{1} \text { or } \mathrm{V}_{2} \text { or }\left(\mathrm{V}_{3} \times \underline{\mathrm{V}}_{4}+\frac{\mathrm{V}_{5}}{2}\right)^{1 / 2}\right] \times \mathrm{V}_{6}\right\}^{1 / 2}
$$

Where:

$$
\mathrm{V}_{1}=\text { Herbaceous Indicator Score (HERB) }
$$

$V_{2}=$ Percent Cover of Selected Native Bunchgrass (NBG)

$V_{3}=$ Density of Pond Cypress in each of three size classes (CYPRESS).

$\mathrm{V}_{4}=$ Percent Cover of Sedges (SEDGES)

$V_{5}=$ Sub-canopy Density per ha (SUBCAN)

$\mathrm{V}_{6}=$ Area of Contiguous Fire-Maintained Landscape (LANDSCP)

3) $\mathrm{FCl}_{\text {ANIMALCOMm }}$ for Switchcane/Pine Savanna $($ SWTCHPSAV) $=$

$$
\left[\left(v_{1} \times \underline{v}_{2}+\frac{v_{3}}{2}\right)^{1 / 2} \times v_{4}\right]^{1 / 2}
$$

Where:

$\mathrm{V}_{1}=$ Density of Longleaf and Pond Pines in the Canopy layer (LONGL)

$\mathrm{V}_{2}=$ Sub-canopy Density per ha (SUBCAN)

$\mathrm{V}_{3}=$ Pine Density per ha (PINES)

$\mathrm{V}_{4}=$ Area of Contiguous Fire-Maintained Landscape (LANDSCP)

Follow the syntax rules to enter the formula into the software (see next page to check formula). 
The Wet Pine Flats FCI formula for Fxn 3 (Animal Community) for BNCHGRPSAV $=$

The Wet Pine Flats FCI formula for Fxn 3 (Animal Community) for CYPRESPSAV $=$

The Wet Pine Flats FCI formula for Fxn 3 (Animal Community) for SWTCHPSAV $=$ 
Here are the correct answers:

Wet Pine Flats - Fxn 3: Maintain Characteristic Animal Community

1) Bunchgrass/Pine (BNCHGRPSAV) =

$$
\mathrm{FCl}_{\text {ANIMALCOMm }}=\quad((\mathrm{MAX}\{[\mathrm{HERB}] \&[\mathrm{NBG}]\}) *[\mathrm{LANDSCP}])^{\wedge}(1 / 2)
$$

2) Cypress/Pine Savanna (CYPRESPSAV) =

$$
\begin{aligned}
\mathrm{FCl}_{\text {ANIMALCOMM }}=\quad & ((\mathrm{MAX}\{[\mathrm{HERB}] \&[\mathrm{NBG}] \&(([\mathrm{CYPRESS}] \text { * } \\
& \left.\left.\left.(([\mathrm{SEDGES}]+[\mathrm{SUBCAN}]) / 2))^{\wedge}(1 / 2)\right)\right\}\right) \text { * } \\
& {[\text { LANDSCP] })^{\wedge}(1 / 2) }
\end{aligned}
$$

3) Switchcane/Pine Savanna (SWTCHPSAV) =

$$
\begin{aligned}
\mathrm{FCl}_{\text {ANIMALCOMM }}= & \left(\left(([\text { LONGL }] *(([S U B C A N]+[\text { PINES }]) / 2))^{\wedge}(1 / 2)\right)\right. \\
& *[\text { LANDSCP }])^{\wedge}(1 / 2)
\end{aligned}
$$

Now try the last function - again, this function is written specifically for the PWAAs in which it is applied, so there will be a new formula for each PWAA in Fxn 4: Maintain Characteristic Biogeochemical

\section{Processes.}


Wet Pine Flats - Fxn 4: Maintain Characteristic Biogeochemical Processes

1) $\mathrm{FCl}_{\text {BIOGEOCHEM }}$ for Bunchgrass/Pine (BNCHGRPSAV) $=$

$\left\{\right.$ Maximum of $\mathrm{V}_{1}$ or $\mathrm{V}_{2} \mathrm{X}$

Minimum of $V_{3}$ or $V_{4}$ or $V_{5}$ or $V_{6}$ or $\left.\left[\left(V_{7} X \underline{V}_{8}+V_{9}\right)^{1 / 2}\right]\right\}^{1 / 2}$

Where:

$\mathrm{V}_{1}=$ Herbaceous Indicator Score (HERB)

$V_{2}=$ Percent Cover of Selected Native Bunchgrass (NBG)

$\mathrm{V}_{3}=$ Surface Flow of Water (SURFFLOW)

$\mathrm{V}_{4}=$ Outflow of Water (OUTFLOW)

$\mathrm{V}_{5}=$ Surface Water Storage (STORAGE)

$\mathrm{V}_{6}=$ Importation of Water from Elsewhere (INFLOW)

$\mathrm{V}_{7}=$ Evapotranspiration Potential (EVAPOTRANS)

$\mathrm{V}_{8}=$ Microtopographic Features (MICRO)

$\mathrm{V}_{9}=$ Soil Porosity (PORE)

2) $\mathrm{FCl}_{\text {BIOGEOCHEM }}$ for Cypress/Pine Savanna (CYPRESPSAV) =

$\left\{\right.$ Maximum of $\mathrm{V}_{1}$ or $\mathrm{V}_{2}$ or $\left(\mathrm{V}_{3} \mathrm{X} \underline{\mathrm{V}}_{\underline{4}}+\frac{\mathrm{V}}{2} \underline{\mathrm{V}}^{1 / 2} \mathrm{X}\right.$

Minimum of $V_{6}$ or $V_{7}$ or $V_{8}$ or $V_{9}$ or $\left.\left[\left(V_{10} X \frac{V_{11}+V_{12}}{2}\right)^{1 / 2}\right]\right\}^{1 / 2}$

Where:

$\mathrm{V}_{1}=$ Herbaceous Indicator Score (HERB)

$\mathrm{V}_{2}=$ Percent Cover of Selected Native Bunchgrass (NBG)

$V_{3}=$ Density of Pond Cypress in each of three size classes (CYPRESS).

$\mathrm{V}_{4}=$ Percent Cover of Sedges (SEDGES)

$V_{5}=$ Sub-canopy Density per ha (SUBCAN)

$\mathrm{V}_{6}=$ Surface Flow of Water (SURFFLOW)

$\mathrm{V}_{7}=$ Outflow of Water (OUTFLOW)

$\mathrm{V}_{8}=$ Surface Water Storage (STORAGE)

$\mathrm{V}_{9}=$ Importation of Water from Elsewhere (INFLOW)

$\mathrm{V}_{10}=$ Evapotranspiration Potential (EVAPOTRANS)

$\mathrm{V}_{11}=$ Microtopographic Features (MICRO)

$\mathrm{V}_{12}=$ Soil Porosity (PORE) 
Wet Pine Flats - Fxn 4: Maintain Characteristic Biogeochemical Processes (Continued)

3) $\mathrm{FCl}_{\text {BIOGEOCHEM }}$ for Switchcane/Pine Savanna (SWTCHPSAV) =

$$
\left\{\left(V_{1} X \underline{v}_{2}+V_{3}\right)^{1 / 2} X\right.
$$

Minimum of $V_{4}$ or $V_{5}$ or $V_{6}$ or $V_{7}$ or $\left.\left[\left(V_{8} X \underline{V}_{9}+V_{10}\right)^{1 / 2}\right]\right\}^{1 / 2}$

Where:

$\mathrm{V}_{1}=$ Density of Longleaf and Pond Pines in the Canopy layer (LONGL)

$V_{2}=$ Sub-canopy Density per ha (SUBCAN)

$V_{3}=$ Pine Density per ha (PINES)

$\mathrm{V}_{4}=$ Surface Flow of Water (SURFFLOW)

$V_{5}=$ Outflow of Water (OUTFLOW)

$\mathrm{V}_{6}=$ Surface Water Storage (STORAGE)

$\mathrm{V}_{7}=$ Importation of Water from Elsewhere (INFLOW)

$\mathrm{V}_{8}=$ Evapotranspiration Potential (EVAPOTRANS)

$\mathrm{V}_{9}=$ Microtopographic Features (MICRO)

$\mathrm{V}_{10}=$ Soil Porosity (PORE)

Follow the syntax rules to enter the formula into the software (see next page to check formula). 
The Wet Pine Flats FCI formula for Fxn 4 (Biogeochemical Processes) for BNCHGRPSAV $=$

The Wet Pine Flats FCI formula for Fxn 4 (Biogeochemical Processes) for CYPRESPSAV $=$

The Wet Pine Flats FCI formula for Fxn 4 (Biogeochemical Processes) for SWTCHPSAV $=$ 
Here are the correct answers:

Wet Pine Flats - Fxn 4: Maintain Characteristic Biogeochemical Processes

1) Bunchgrass/Pine (BNCHGRPSAV) =

$$
\begin{aligned}
\text { FCl }_{\text {BIOGEOCHEM }=} & ((\text { MAX }\{[\text { HERB }] \&[N B G]\}) *(\text { MIN }\{[S U R F F L O W] \& \\
& {[\text { OUTFLOW]\& [STORAGE]\& [INFLOW]\& }} \\
& (([\text { EVAPOTRANS] * }(([\mathrm{MICRO}]+ \\
& {\left.\left.\left.\left.[\text { PORE }]) / 2))^{\wedge}(1 / 2)\right)\right\}\right)\right)^{\wedge}(1 / 2) }
\end{aligned}
$$

2) Cypress/Pine Savanna (CYPRESPSAV) $=$

$$
\begin{aligned}
\mathrm{FCl}_{\text {BIOGEOCHEM }=} & ((\mathrm{MAX}\{[\mathrm{HERB}] \&[\mathrm{NBG}] \&(([\mathrm{CYPRESS}] \text { * } \\
& \left.\left.\left.(([\mathrm{SEDGES}]+[\mathrm{SUBCAN}]) / 2))^{\wedge}(1 / 2)\right)\right\}\right) *(\mathrm{MIN} \\
& \{[\text { SURFFLOW]\& [OUTFLOW]\& }[\text { STORAGE }] \& \\
& {[\text { INFLOW]\& }(([\mathrm{EVAPOTRANS}] *(([\mathrm{MICRO}]+} \\
& {\left.\left.\left.\left.[\mathrm{PORE}]) / 2))^{\wedge}(1 / 2)\right)\right\}\right)\right)^{\wedge}(1 / 2) }
\end{aligned}
$$

3) Switchcane/Pine Savanna (SWTCHPSAV) =

$$
\begin{aligned}
& \mathrm{FCl}_{\text {BIOGEOCHEM }}=\quad\left(\left(([\mathrm{LONGL}] \text { * }(([\mathrm{SUBCAN}]+[\mathrm{PINES}]) / 2))^{\wedge}(1 / 2)\right)\right. \text { * } \\
& \text { (MIN }\{[S U R F F L O W] \& \text { [OUTFLOW]\& [STORAGE]\& } \\
& \text { [INFLOW]\& }(([\text { EVAPOTRANS] * }(([\mathrm{MICRO}]+ \\
& \left.\left.\left.\left.[\mathrm{PORE}]) / 2))^{\wedge}(1 / 2)\right)\right\}\right)\right)^{\wedge}(1 / 2)
\end{aligned}
$$

Now enter the formulas into the software.

1. To begin, open the training datafile entitled Exercise 11.mdb and click on the Run button.

2. Double-click on the first option (1. Choose Working Function), select the Fxn01: Water Level Regime option from the pull-down list, and click Close.

3. Now double-click on the second option (2. Associate Parameters).

4. Click on the PWAA drop-down box, and choose BNCHGRPSAV from the pull-down list.

5. Click once on the FCI Function Formula input box and verify that the cursor is to the immediate right of the " $1 *$ " placeholder.

6. Now move the cursor to the floating Variables Available keypad and scroll down through the list of variables. Single click on the variables SURFFLOW, OUTFLOW, STORAGE, INFLOW, EVAPOTRANS, MICRO, and finally PORE. 
7. Systematically place the cursor between each of the variable codes in the FCI Function Formula input box, and insert an operator (refer to the manual for these) using either the operator keys on the floating keypad, or typing them usingyour keyboard (look to the box above to check yourself).

8. Put the cursor between this first variable and the " $1 *$ " and backspace twice or put the cursor immediately before the " $1 *$ " and hit the Delete button on the floating keypad.

9. Click on the Equation Check button immediately to the right of the FCI Function Formula input box to check work.

10. Remove any phantom records if they exist.

Now duplicate the formula for all the PWAAs associated with the model.

1. To begin, click on the cover type drop-down box, and choose

\section{CYPRESPSAV.}

2. Click on the Back Record button, click on the Copy button, click on the Forward Record button, and click on the Paste button.

3. Click on the cover type drop-down box, choose SWTCHPSAV, and click on the Paste button once again.

4. Remove any phantom records if they exist.

5. Repeat this process for each function in the model.

By now, there should be three records for each function (12 records total)one for each PWAA in the model (Table E27). 
Table E27. Record list for Exercise 12 after formula entry.

\begin{tabular}{|c|c|c|c|}
\hline $\begin{array}{l}\text { Record } \\
\text { Count }\end{array}$ & Function & PWAA & FCI Formula \\
\hline \multicolumn{4}{|c|}{ Fxn 1: Water Regime } \\
\hline 1 & WATEREGIME & BNCHGRPSAV & $\begin{array}{l}\text { MIN \{[SURFFLOW]\& [OUTFLOW]\& [STORAGE]\& [INFLOW]\& } \\
\left(\left(\left[\text { EVAPOTRANS] * }(([\text { MICRO] + [PORE] }] / 2))^{\wedge}(1 / 2)\right)\right\}\right.\end{array}$ \\
\hline 2 & WATEREGIME & CYPRESPSAV & $\begin{array}{l}\text { MIN }\{[\text { SURFFLOW]\& [OUTFLOW]\& [STORAGE]\& [INFLOW]\& } \\
\left(\left(\left[\text { EVAPOTRANS] * }\left(([\text { MICRO] }+[\text { PORE] }] / 2))^{\wedge}(1 / 2)\right)\right\}\right.\right.\end{array}$ \\
\hline 3 & WATEREGIME & SWTCHPSAV & 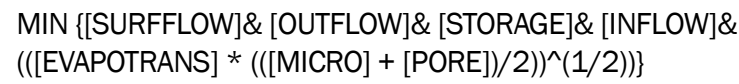 \\
\hline \multicolumn{4}{|c|}{ Fxn 2: Plants } \\
\hline 4 & PLANTCOMM & BNCHGRPSAV & $\operatorname{MAX}\{[\mathrm{HERB}] \&[\mathrm{NBG}]\}$ \\
\hline 5 & PLANTCOMM & CYPRESPSAV & $\begin{array}{l}\operatorname{MAX}\{[\text { HERB] \& [NBG] \& }(([\text { CYPRESS }] *(([S E D G E S]+ \\
\left.\left.[S U B C A N]) / 2))^{\wedge}(1 / 2)\right)\right\}\end{array}$ \\
\hline 6 & PLANTCOMM & SWTCHPSAV & $([\mathrm{LONGL}] *(([\mathrm{SUBCAN}]+[\mathrm{PINES}]) / 2))^{\wedge}(1 / 2)$ \\
\hline \multicolumn{4}{|c|}{ Fxn 3: Animals } \\
\hline 7 & ANIMALCOMM & BNCHGRPSAV & $((\operatorname{MAX}\{[\mathrm{HERB}] \&[\mathrm{NBG}]\}) *[\text { LANDSCP }])^{\wedge}(1 / 2)$ \\
\hline 8 & ANIMALCOMM & CYPRESPSAV & $\begin{array}{l}((\mathrm{MAX}\{[\mathrm{HERB}] \&[\mathrm{NBG}] \&(([\mathrm{CYPRESS}] *(([\mathrm{SEDGES}]+ \\
\left.\left.\left.[\mathrm{SUBCAN]}] / 2))^{\wedge}(1 / 2)\right)\right\}\right) *[\text { LANDSCP] })^{\wedge}(1 / 2)\end{array}$ \\
\hline 9 & ANIMALCOMM & SWTCHPSAV & $\begin{array}{l}\left(\left(([\mathrm{LONGL}] *(([\mathrm{SUBCAN}]+[\mathrm{PINES}]) / 2))^{\wedge}(1 / 2)\right) *\right. \\
{[\text { LANDSCP] })^{\wedge}(1 / 2)}\end{array}$ \\
\hline \multicolumn{4}{|c|}{ Fxn4: Biogeochemistry } \\
\hline 10 & BIOGEOCHEM & BNCHGRPSAV & $\begin{array}{l}((\operatorname{MAX}\{[\text { HERB]\& [NBG]\})* }(\text { MIN }\{[\text { SURFFLOW]\& } \\
{[\text { OUTFLOW]\& [STORAGE]\& [INFLOW]\& }(([\text { EVAPOTRANS] * }} \\
\left.\left.\left.\left.(([\mathrm{MICRO}]+[\text { PORE] }] / 2))^{\wedge}(1 / 2)\right\}\right\}\right)\right)^{\wedge}(1 / 2)\end{array}$ \\
\hline 11 & BIOGEOCHEM & CYPRESPSAV & $\begin{array}{l}((\text { MAX }\{[\text { HERB] } \&[\text { NBG] \& }(([\text { CYPRESS }] *(([S E D G E S]+ \\
\left.\left.\left.[\text { SUBCAN] }) / 2))^{\wedge}(1 / 2)\right)\right\}\right) *(\text { MIN }\{[\text { SURFFLOW]\& } \\
{[\text { OUTFLOW]\& [STORAGE]\& [INFLOW]\& }(([\text { EVAPOTRANS }] \text { * }} \\
\left.\left.\left.\left.(([\text { MICRO }]+[\text { PORE] }) / 2))^{\wedge}(1 / 2)\right)\right\}\right)\right)^{\wedge}(1 / 2)\end{array}$ \\
\hline 12 & BIOGEOCHEM & SWTCHPSAV & $\begin{array}{l}((([L O N G L] *(([S U B C A N]+[\text { PINES])/2))^(1/2))* }(\text { MIN } \\
\{[\text { SURFFLOW]\& [OUTFLOW]\& [STORAGE] \& [INFLOW]\& } \\
(([E V A P O T R A N S] *(([M I C R O]+ \\
\left.\left.\left.\left.[\text { PORE] }] / 2))^{\wedge}(1 / 2)\right)\right\}\right)\right)^{\wedge}(1 / 2)\end{array}$ \\
\hline
\end{tabular}

Use the reports in EXHGM to check work and document analysis (Setup Reports button). The following reports should be created as a result:

\section{- Formulas}

\section{- Associations}




\section{Exercise 12: Entering Curves into EXHGM}

For users who need help reading and converting SI curves into X,Y coordinates in EXHGM, a series of graphs taken from the Wet Pine Flats HGM documentation are provided. The Wet Pine Flats model has four functions, so the process below must be completed four times to fully populate the datafile in EXHGM. For now, the first function (Fxn 01: Water Level Regime) will be focused on. Look to the field kit to explore the remaining functions and their variable coordinates (Wet Pine Flats Field Kit). To begin, write out the $\mathrm{X}, \mathrm{Y}$ coordinates in tabular fashion, and then convert these to EXHGM syntax (i.e., $\mathrm{X}_{1}, \mathrm{Y}_{1}, \mathrm{X}_{2}, \mathrm{Y}_{2}, \mathrm{X}_{3}, \mathrm{Y}_{3}, \ldots \mathrm{X}_{\mathrm{n}}$ $\left.Y_{n}\right)$. When calculations have been completed, see the answers provided on the pages that follow.
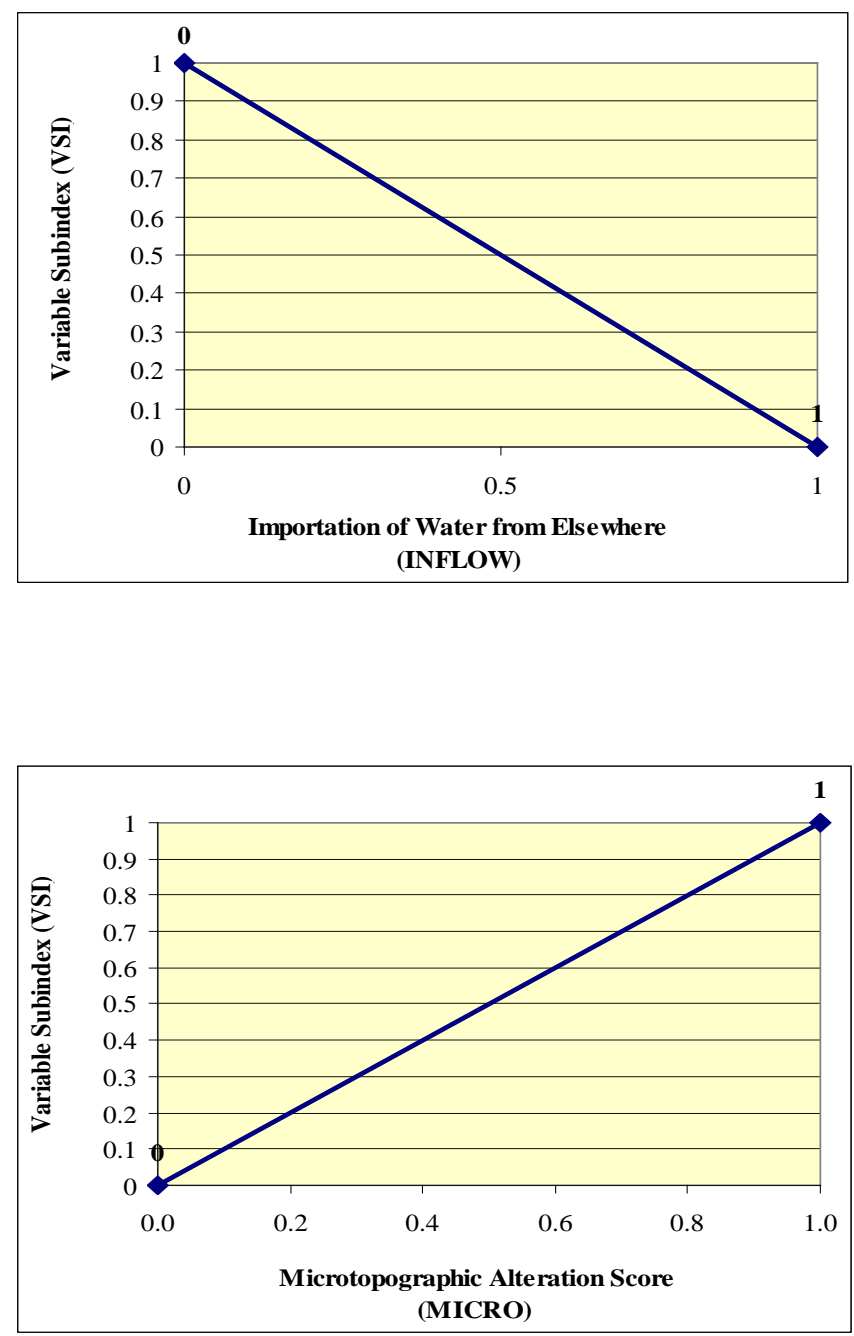

$\mathrm{X}, \mathrm{Y}$ coordinates in tabular fashion:

$\underline{\text { X-Values }} \underline{\text { Y-Values }}$

$\mathrm{X}, \mathrm{Y}$ coordinates in EXHGM syntax:

$\mathrm{X}, \mathrm{Y}$ coordinates in tabular fashion:

$\underline{\text { X-Values }} \quad \underline{\text { Y-Values }}$

$\mathrm{X}, \mathrm{Y}$ coordinates in EXHGM syntax: 


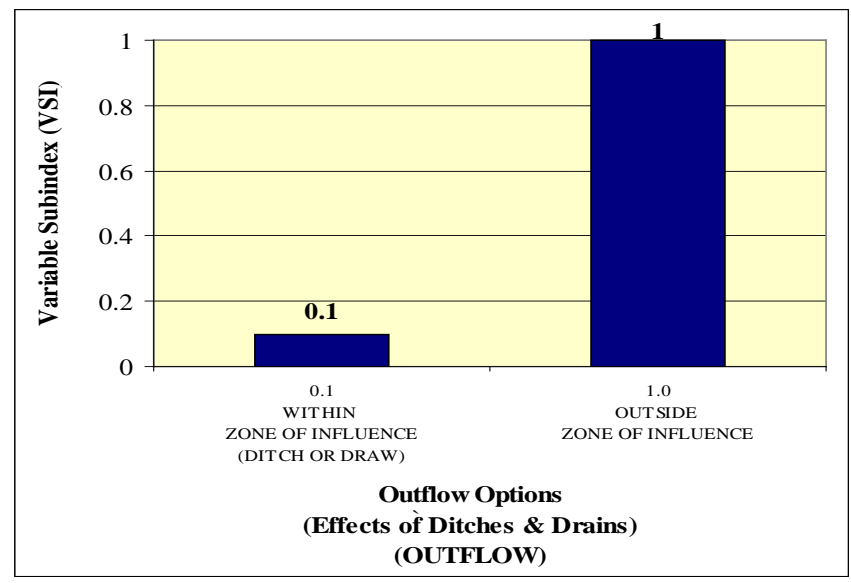

$\mathrm{X}, \mathrm{Y}$ coordinates in tabular fashion:

$\underline{\text { X-Values }} \underline{\text { Y-Values }}$

$\mathrm{X}, \mathrm{Y}$ coordinates in EXHGM syntax:

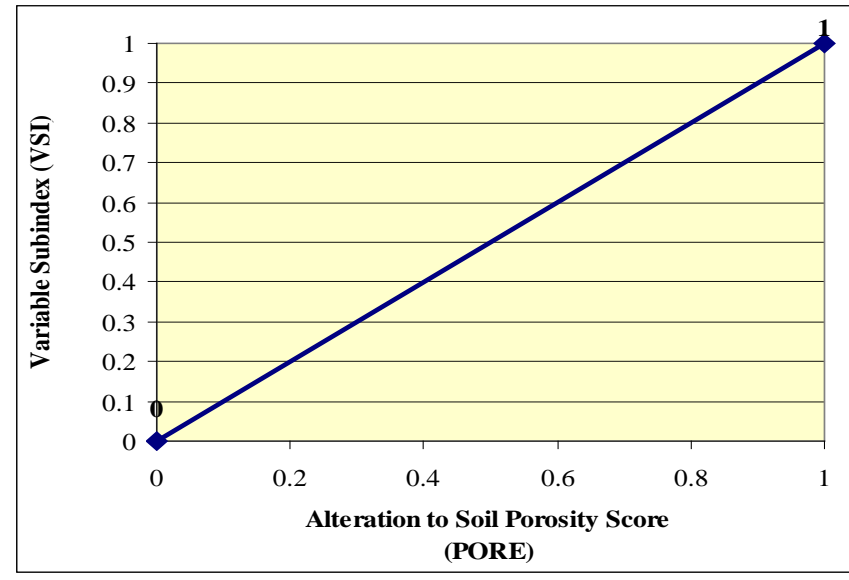

$\mathrm{X}, \mathrm{Y}$ coordinates in tabular fashion:

$\underline{\text { X-Values }} \quad \underline{\text { Y-Values }}$

X,Y coordinates in EXHGM syntax:

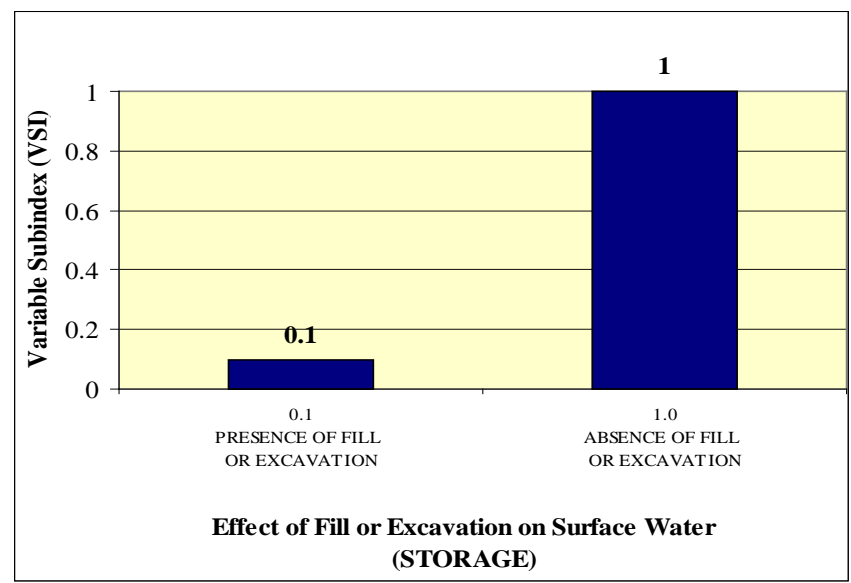

$\mathrm{X}, \mathrm{Y}$ coordinates in tabular fashion:

$\underline{\text { X-Values }} \underline{\text { Y-Values }}$

$\mathrm{X}, \mathrm{Y}$ coordinates in EXHGM syntax: (STORAGE) 

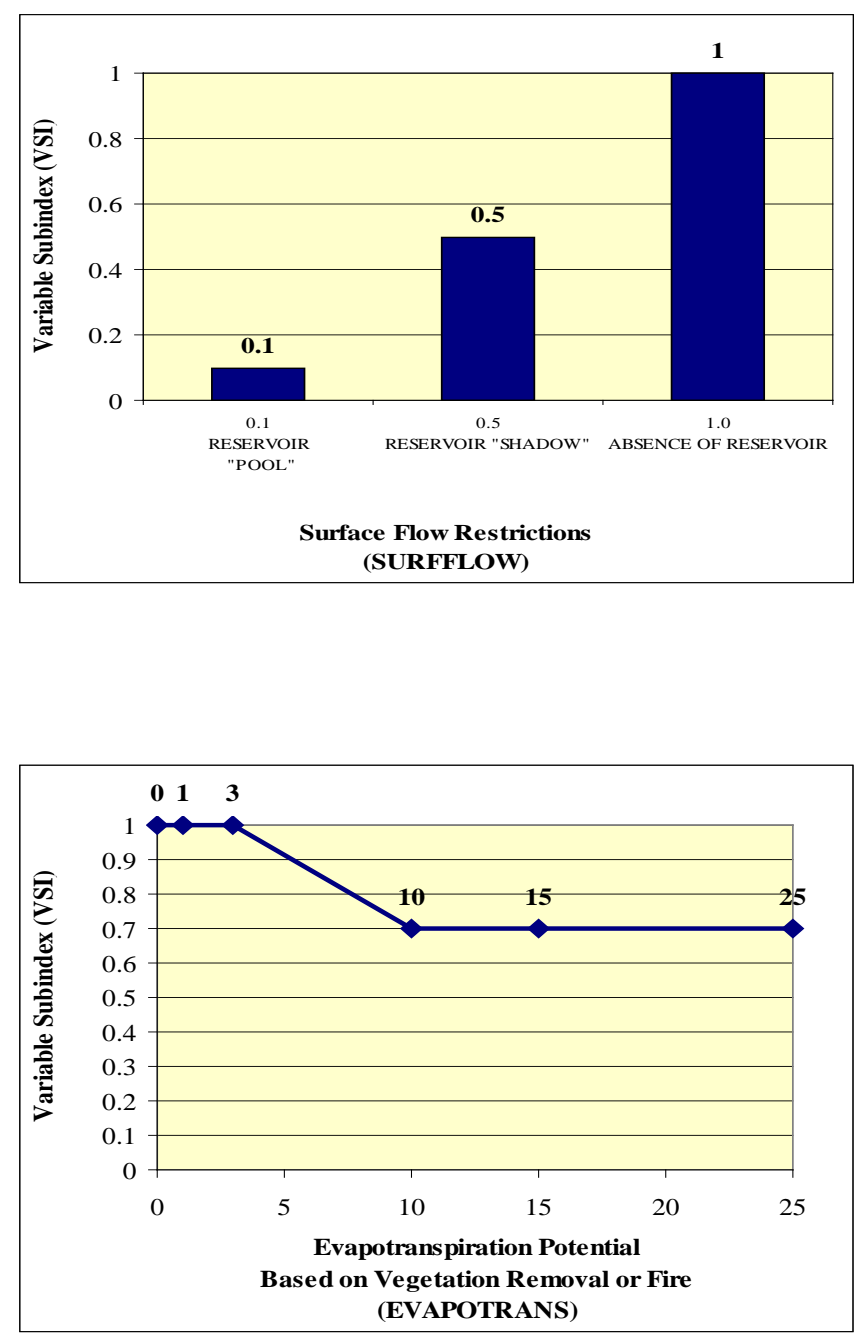

$\mathrm{X}, \mathrm{Y}$ coordinates in tabular fashion:

$\underline{\text { X-Values }} \underline{\text { Y-Values }}$

$\mathrm{X}, \mathrm{Y}$ coordinates in EXHGM syntax:

$\mathrm{X}, \mathrm{Y}$ coordinates in tabular fashion:

$\underline{\text { X-Values }} \quad \underline{\text { Y-Values }}$

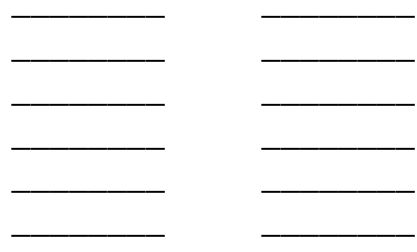

$\mathrm{X}, \mathrm{Y}$ coordinates in EXHGM syntax: 
Here are the answers:
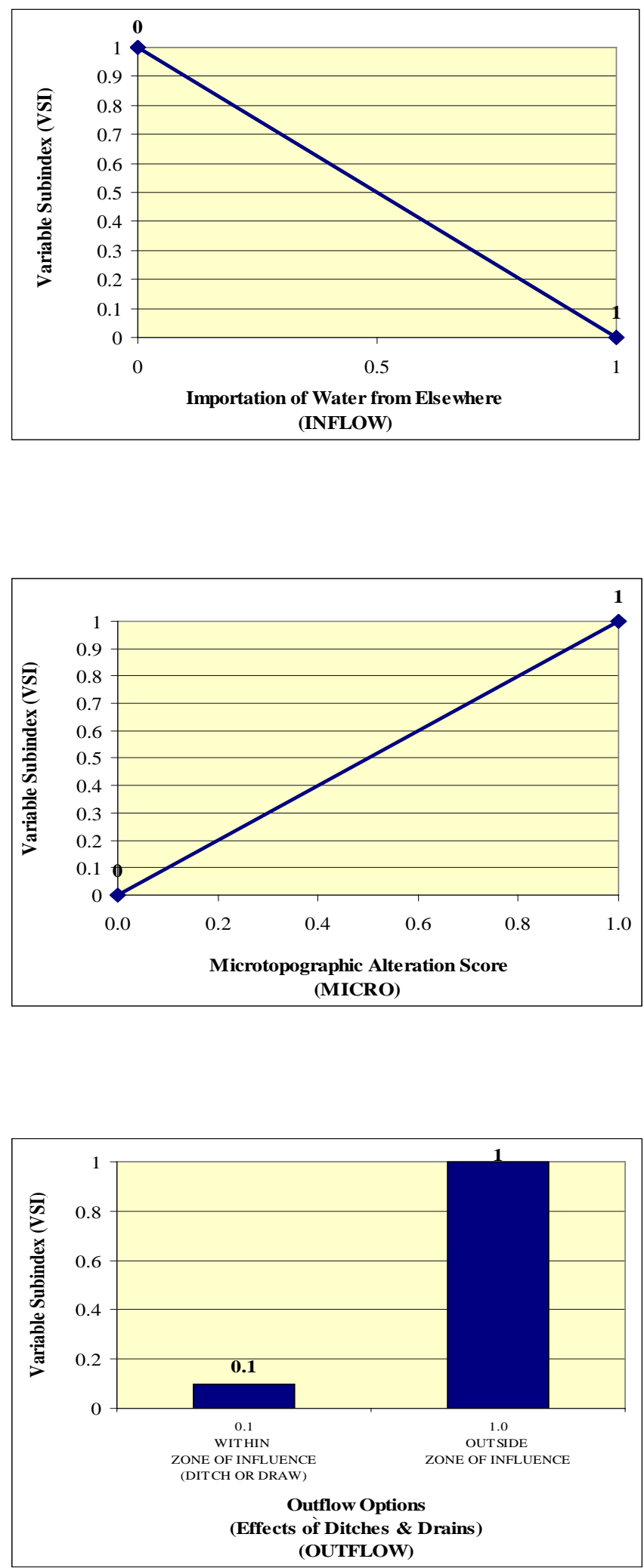

$\mathrm{X}, \mathrm{Y}$ coordinates in tabular fashion:

$\underline{X-V a l u e s} \quad \underline{\text { Y-Values }}$ 0 1 1 0

$\mathrm{X}, \mathrm{Y}$ coordinates in EXHGM syntax:

\section{$\mathbf{0 , 1 , 1 , 0}$}

$\mathrm{X}, \mathrm{Y}$ coordinates in tabular fashion:

$\begin{array}{cc}\frac{\text { X-Values }}{0} & \frac{\text { Y-Values }}{0} \\ 1 & 1\end{array}$

$\mathrm{X}, \mathrm{Y}$ coordinates in EXHGM syntax:

$\mathbf{0 , 0}, 1,1$

$\mathrm{X}, \mathrm{Y}$ coordinates in tabular fashion:

$\begin{array}{cc}\frac{\text { X-Values }}{0.1} & \frac{\text { Y-Values }}{0.1} \\ 1 & 1\end{array}$

$\mathrm{X}, \mathrm{Y}$ coordinates in EXHGM syntax:

\section{1,0.1,1,1}



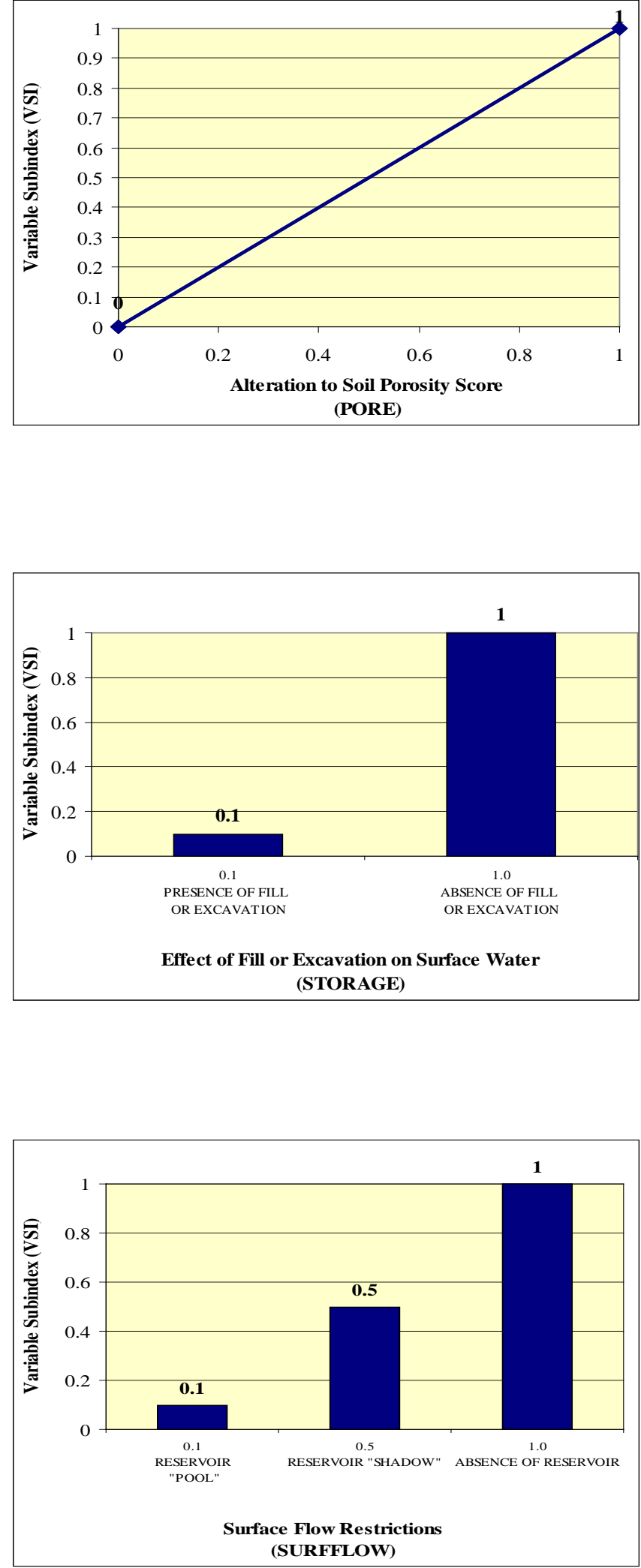

$\mathrm{X}, \mathrm{Y}$ coordinates in tabular fashion:

$\underline{\text { X-Values }}$

$\underline{\text { Y-Values }}$

0

1

1

$\mathrm{X}, \mathrm{Y}$ coordinates in

EXHGM syntax:

$\mathbf{0 , 0}, \mathbf{1}, 1$

$\mathrm{X}, \mathrm{Y}$ coordinates in tabular fashion:

$\begin{array}{cc}\frac{\text { X-Values }}{0.1} & \frac{\text { Y-Values }}{0.1} \\ 1 & 1\end{array}$

$\mathrm{X}, \mathrm{Y}$ coordinates in EXHGM syntax:

0.1,0.1,1,1

X,Y coordinates in tabular fashion:

\begin{tabular}{cc} 
X-Values & Y-Values \\
\hline 0.1 & 0.1 \\
0.5 & 0.5 \\
1 & 1
\end{tabular}

$\mathrm{X}, \mathrm{Y}$ coordinates in EXHGM syntax:

\section{1,0.1,0.5,0.5, 1,1}




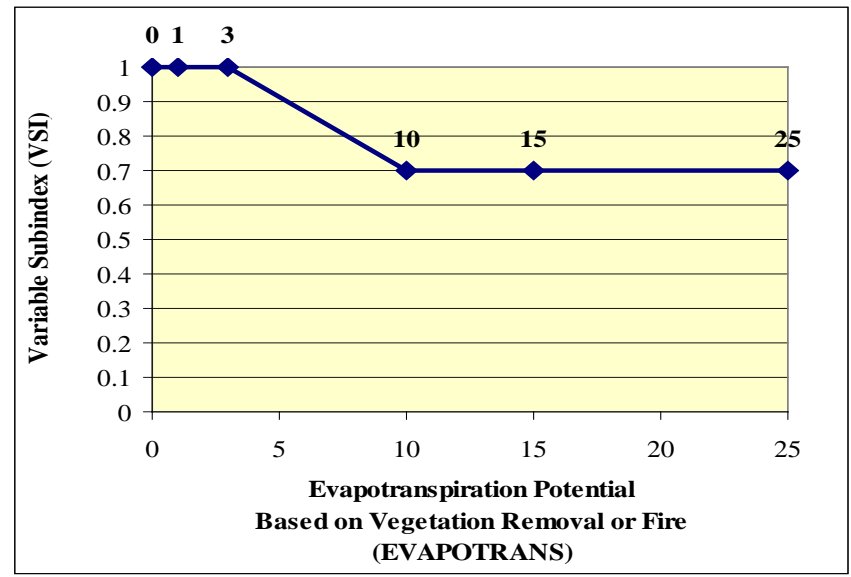

$\mathrm{X}, \mathrm{Y}$ coordinates in tabular fashion:

\begin{tabular}{cc} 
X-Values & Y-Values \\
\hline 0 & \\
1 & 1 \\
3 & 1 \\
10 & 0.7 \\
15 & 0.7 \\
25 & 0.7
\end{tabular}

$\mathrm{X}, \mathrm{Y}$ coordinates in

EXHGM syntax:

$\underline{0,1,1,1,3,1,10,0.7,15,0.7,25,0.7}$

Now enter the coordinates into the software.

1. To begin, open the training datafile entitled Exercise 12.mdb and click on the Run button.

2. Double-click on the first option (1. Choose Working Function), select the Fxn01: Water Level Regime option from the pull-down list, and click Close.

3. Now double-click on the third option (3. Enter VSI Graphs and Variable Data).

4. Click on the PWAA drop-down box, and choose BNCHGRPSAV from the pull-down list.

5. Click on the Variable drop-down box, and choose EVAPOTRANS from the pull-down list.

6. Click onœ on the Graph Coordinates $(\mathbf{X}, \mathbf{Y})$ entry box and type in the coordinates for this variable (i.e., $\mathbf{0}, \mathbf{1}, \mathbf{1}, \mathbf{1}, \mathbf{3}, \mathbf{1}, 10,0.7,15,0.7,25,0.7)$, and hit the Tab key on the keyboard. If all is well, the cursor moves to the Variable Data entry window. Omitting commas will generate an error message.

Copy this information to the remaining PWAAs in the model (i.e., CYPRESPSAV and SWTCHPSAV). 
1. Highlight the coordinates and press Ctrl C on the keyboard.

2. Now move up to the upper form (Step 1: Select Cover Types and Variable Combinations) and pull down CYPRESPSAV from the PWAA pull-down box. Leave the variable selection as it is.

3. Move down to the lower form (Step 2: Enter Graph Coordinates and Variable Data) and left-click once in the Graph Coordinates $(\mathbf{X}, \mathbf{Y})$ box.

4. Press Ctrl V on the keyboard and Tab to accept the coordinates.

5. Now repeat the process for the remaining PWAA (i.e., SWTCHPSAV).

Once that variable has been completed, there are still six variables that need coordinates, so repeat the entire process for INFLOW, MICRO, OUTFLOW, PORE, STORAGE, and SURFLOW.

At this point, there should be 21 records - (7 variables $x 3$ PWAAs) (Table E28).

Use the reports in EXHGM to check work and document analysis (Setup Reports button). The following report should be created as a result:

\section{- Variable Subindex Curves}

Reminder: There are three more functions in the Wet Pine Flats Model. Look to the documentation, and develop and enter the curves for each function, just as was done with the first. 
Table E28. Record list for Exercise 13 after X,Y coordinate entry.

\begin{tabular}{|c|c|c|c|c|}
\hline $\begin{array}{l}\text { Record } \\
\text { Count }\end{array}$ & Function & PWAA & Variable & $X, Y$ Coordinates \\
\hline 1 & Fxn 01: Water Level Regime & BNCHGRPSAV & EVAPOTRANS & $0,1,1,1,3,1,10,0.7,15,0.7,25,0.7$ \\
\hline 2 & Fxn 01: Water Level Regime & CYPRESPSAV & EVAPOTRANS & $0,1,1,1,3,1,10,0.7,15,0.7,25,0.7$ \\
\hline 3 & Fxn 01: Water Level Regime & SWTCHPSAV & EVAPOTRANS & $0,1,1,1,3,1,10,0.7,15,0.7,25,0.7$ \\
\hline 4 & Fxn 01: Water Level Regime & BNCHGRPSAV & INFLOW & $0,1,1,0$ \\
\hline 5 & Fxn 01: Water Level Regime & CYPRESPSAV & INFLOW & $0,1,1,0$ \\
\hline 6 & Fxn 01: Water Level Regime & SWTCHPSAV & INFLOW & $0,1,1,0$ \\
\hline 7 & Fxn 01: Water Level Regime & BNCHGRPSAV & MICRO & $0,0,1,1$ \\
\hline 8 & Fxn 01: Water Level Regime & CYPRESPSAV & MICRO & $0,0,1,1$ \\
\hline 9 & Fxn 01: Water Level Regime & SWTCHPSAV & MICRO & $0,0,1,1$ \\
\hline 10 & Fxn 01: Water Level Regime & BNCHGRPSAV & OUTFLOW & $0.1,0.1,1,1$ \\
\hline 11 & Fxn 01: Water Level Regime & CYPRESPSAV & OUTFLOW & $0.1,0.1,1,1$ \\
\hline 12 & Fxn 01: Water Level Regime & SWTCHPSAV & OUTFLOW & $0.1,0.1,1,1$ \\
\hline 13 & Fxn 01: Water Level Regime & BNCHGRPSAV & PORE & $0,0,1,1$ \\
\hline 14 & Fxn 01: Water Level Regime & CYPRESPSAV & PORE & $0,0,1,1$ \\
\hline 15 & Fxn 01: Water Level Regime & SWTCHPSAV & PORE & $0,0,1,1$ \\
\hline 16 & Fxn 01: Water Level Regime & BNCHGRPSAV & STORAGE & $0.1,0.1,1,1$ \\
\hline 17 & Fxn 01: Water Level Regime & CYPRESPSAV & STORAGE & $0.1,0.1,1,1$ \\
\hline 18 & Fxn 01: Water Level Regime & SWTCHPSAV & STORAGE & $0.1,0.1,1,1$ \\
\hline 19 & Fxn 01: Water Level Regime & BNCHGRPSAV & SURFLOW & $0.1,0.1,0.5,0.5,1,1$ \\
\hline 20 & Fxn 01: Water Level Regime & CYPRESPSAV & SURFLOW & $0.1,0.1,0.5,0.5,1,1$ \\
\hline 21 & Fxn 01: Water Level Regime & SWTCHPSAV & SURFLOW & $0.1,0.1,0.5,0.5,1,1$ \\
\hline
\end{tabular}




\section{Exercise 13: Entering Data into EXHGM}

For users who need help entering data into EXHGM, assume the following data were gathered (through field collection, historical records, or GIS analyses) during the inventorying process for the study site (Table E29).

Table E29. Baseline data entry for Exercise 14.

\begin{tabular}{|c|c|c|c|c|c|c|c|c|c|c|c|c|c|c|c|}
\hline \multirow[b]{2}{*}{ 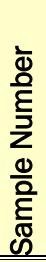 } & \multicolumn{15}{|c|}{ BNCHGRPSAV } \\
\hline & 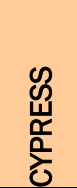 & 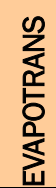 & $\begin{array}{l}\frac{m}{\widetilde{w}} \\
\text { 岌 }\end{array}$ & $\begin{array}{l}\text { zo } \\
\text { 는 } \\
\underline{\underline{u}}\end{array}$ & 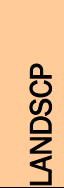 & 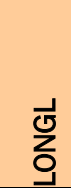 & $\frac{\text { 유 }}{\frac{0}{2}}$ & 品 & 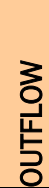 & 岕 & 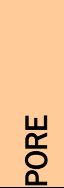 & $\begin{array}{l}\text { यू } \\
\text { Wू }\end{array}$ & 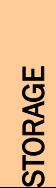 & 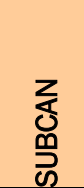 & 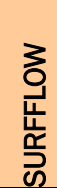 \\
\hline 1 & & 1 & 5 & 0.5 & 80 & & 1 & 50 & 1 & & 0.5 & & 1 & & 1 \\
\hline 2 & & 2 & 6 & 0.4 & 50 & & 1 & 50 & & & 0.6 & & & & \\
\hline 3 & & 3 & 7 & 0.3 & 60 & & 0.5 & 45 & & & 0.7 & & & & \\
\hline 4 & & 4 & 8 & 0.2 & 70 & & 0.5 & 40 & & & 0.8 & & & & \\
\hline 5 & & 5 & 8 & 0.1 & 100 & & 0.75 & 30 & & & 0.9 & & & & \\
\hline \multirow[b]{2}{*}{ 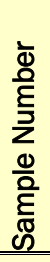 } & \multicolumn{15}{|c|}{ CYPRESPSAV } \\
\hline & 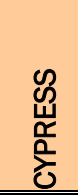 & 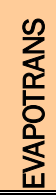 & $\begin{array}{l}\stackrel{m}{\tilde{\sim}} \\
\stackrel{\underline{I}}{\underline{I}} \\
\end{array}$ & 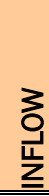 & 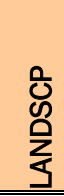 & गे & $\begin{array}{l}\text { O } \\
\text { U. } \\
\frac{1}{\Sigma}\end{array}$ & $\begin{array}{l}\text { 罗 } \\
\end{array}$ & 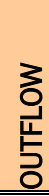 & 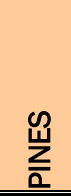 & 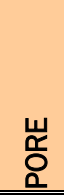 & $\begin{array}{l}\text { 岕 } \\
\text { 岕 }\end{array}$ & 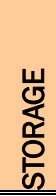 & 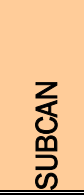 & 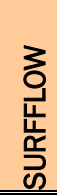 \\
\hline 1 & 0.75 & 2 & 5 & 0.5 & 80 & & 1 & 50 & 1 & & 0.5 & 50 & 1 & 4000 & 1 \\
\hline 2 & 0.75 & 3 & 6 & 0.4 & 50 & & 1 & 50 & & & 0.6 & 45 & & 5000 & \\
\hline 3 & 1 & 4 & 7 & 0.3 & 60 & & 0.5 & 45 & & & 0.7 & 60 & & 6500 & \\
\hline 4 & 1 & 5 & 5 & 0.2 & 70 & & 0.5 & 40 & & & 0.8 & 70 & & 4500 & \\
\hline 5 & 1 & 6 & 6 & 0.1 & 100 & & 0.75 & 30 & & & 0.9 & 40 & & 5500 & \\
\hline \multirow[b]{2}{*}{ 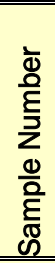 } & \multicolumn{15}{|c|}{ SWTCHPSAV } \\
\hline & 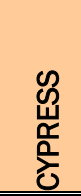 & 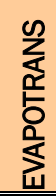 & $\begin{array}{l}\stackrel{m}{\widetilde{r}} \\
\text { 岌 }\end{array}$ & $\begin{array}{l}z \\
\text { ב⿱ } \\
\underline{\underline{u}}\end{array}$ & 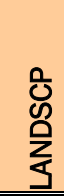 & $\begin{array}{l}\text { गे } \\
\text { Q }\end{array}$ & 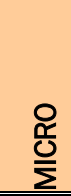 & $\begin{array}{l}\text { ! } \\
\text { ב }\end{array}$ & 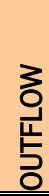 & 岕 & $\begin{array}{l}\text { 山्र } \\
\text { 임 }\end{array}$ & 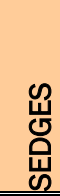 & 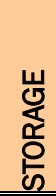 & $\begin{array}{l}z \\
\text { zo } \\
\text { ge } \\
\text { ch }\end{array}$ & $\begin{array}{l}3 \\
\text { 임 } \\
\text { 竞 } \\
\text { 年 } \\
\text { ⿹ }\end{array}$ \\
\hline 1 & & 1 & & 0.5 & 80 & 175 & 1 & & 1 & 100 & 0.5 & & 1 & 4000 & 1 \\
\hline 2 & & 3 & & 0.4 & 50 & 400 & 1 & & & 200 & 0.6 & & & 5000 & \\
\hline 3 & & 5 & & 0.3 & 60 & 300 & 0.5 & & & 300 & 0.7 & & & 6500 & \\
\hline 4 & & 7 & & 0.2 & 70 & 150 & 0.5 & & & 400 & 0.8 & & & 4500 & \\
\hline 5 & & 9 & & 0.1 & 100 & 500 & 0.75 & & & 500 & 0.9 & & & 5500 & \\
\hline
\end{tabular}


Although this is not a very robust data set, it is enough to calculate the baseline conditions at the site for the Wet Pine Flats in this exercise. There are two ways to enter the data into EXHGM: 1) manually enter data into the datafile, or 2) organize the data in a spreadsheet and copy it into the datafile. Exercises to practice both approaches are provided below.

\section{Part 1: Manual Data Entry}

1. To begin, open the training datafile entitled Exercise 13.mdb and click on the Rum button on the primary interface.

2. Double-click on the first option (1. Choose Working Function), select the Fxn01: Water Level Regime option from the pull-down list, and click Close.

3. Now double-click on the thind option (3. Enter VSI Graphs and Variable Data).

4. Click on the PWAA drop-down box, and choose BNCHGRPSAV from the pull-down list.

5. Click on the Variable drop-down box, and choose EVAPOTRANS from the pull-down list.

Now the first data set can be entered. It is best to do this in a systematic fashion - either enter all the data for a single variable, or enter all the data for a single PWAA. All the data for this PWAA will be entered as follows:

1. Move down to the lower form and put the cursor in the " $\mathbf{0}$ " placeholder entry blank in the Variable Data entry form (on the right-hand side of the screen). Type the first data point (i.e., 1) and hit Enter on the keyboard.

2. Type in the remaining data (i.e., 2, 3, 4, and 5) hitting Enter after each data point.

3. Go back to the top form, click on the Variable drop-down box, choose INFLOW from the pull-down list, and enter the data for this second variable.

4. Repeat the process for INFLOW, MICRO, OUTFLOW, PORE, STORAGE, and SURFLOW.

5. Data for all PWAAs associated with the model must be entered, so repeat the process for CYPRESPSAV and SWTCHPSAV.

6. Click Close to finish the session. That completes the first function.

Try entering the rest of the data electronically - go to the next section. 


\section{Part 2: Electronic Data Entry}

The key to electronic data entry in EXHGM is formatting. The variable data must be arranged in a spreadsheet in column fashion (which has been done in the Exercise 13 Baseline Data - Wet Pine Flats.xls file in the Exercise 13 training folder).

1. Open this spreadsheet file with MS Excel.

2. Return back to the EXHGM interface. The next function will be entered electronically, so click on the Run button on the primary interface.

3. Double-click on the first option (1. Choose Working Function), select the Fxn02: Plant Communities from the pull-down list, and click Close.

4. Now double-click on the third option (3. Enter VSI Graphs and Variable Data).

5. Click on the PWAA drop-down box, and choose BNCHGRPSAV from the pull-down list.

6. Click on the Variable drop-down box, and choose HERB from the pulldown list.

7. Go back to the external spreadsheet and highlight (i.e., block by holding down the left-mouse key and dragging down the column of data) the data in the HERB column under the BNCHGRPSAV heading.

Note: highlight only the data - do not block the column headings!!!

1. Press Ctrl C on the keyboard to copy the data to the clipboard.

2. Return to the EXHGM software and click the Import Data button above the Variable Data entry panel.

3. That's it! All five data points should have been entered into this screen. Now repeat the process for the remaining variable (NBGBI).

4. Two PWAAs (i.e., CYPRESPSAV and SWTCHPSAV) still need to be entered (with their numerous variables and data).

Note: the list of variables changes with the change in PWAA be sure to scroll all the way to top of the variable list when changing PWAAs ... the "CYPRESS" variable is hiding up at the top of the list!!!

5. Click Close to finish the session. That's it for the second function.

6. Repeat the process for the two remaining functions. 
Use the reports in EXHGM to check work and document analysis (Setup Reports button). The following report should be created as a result:

\section{- Variable Data}

The analysis can now be run. Double-click the fourth option (4. Perform Baseline (TY 0) Calculations* and the software calculates the baseline results for the study. Use the reports in EXHGM to check work and document analysis (Analysis Reports button). The following report should be created as a result:

\section{- Baseline (TY 0) Results}




\section{Exercise 14: Without-project Analyses in EXHGM}

Continue beyond the exercises above and assume that the "No Action" plan for the impact site must now be assessed. To begin, the evaluation team has established that three target years are necessary to assess the project's design: baseline $\left(\mathrm{TY}_{0}\right)$, the year that construction is complete ( $\left(Y_{1}\right)$, and the final "out-year" or TY 51 (i.e., the end of the project's life). Further assume that the owner of the property will in all likelihood maintain the "status quo" (i.e., he will not farm the wetlands because they are too wet to be productive). As a result, the quality of the existing habitats will demonstrate a slight improvement over the study period. Here are the trends the evaluation team generated as forecast conditions for the site:

Table E30. Without-project acre projections for Exercise 14.

\begin{tabular}{|c|c|c|c|c|c|}
\hline \multirow[b]{3}{*}{ No. } & \multirow[b]{3}{*}{ Code } & \multirow[b]{3}{*}{ Description } & \multicolumn{3}{|c|}{ Target Year } \\
\hline & & & 2000 & 2010 & 2060 \\
\hline & & & TYo & $T Y_{1}$ & $T Y_{51}$ \\
\hline 1 & BNCHGRPSAV & Bunchgrass/Pine Savanna & 150 & 150 & 150 \\
\hline 2 & CYPRESPSAV & Cypress/Pine Savanna & 25 & 25 & 25 \\
\hline 3 & SWTCHPSAV & Switchcane/Pine Savanna & 25 & 25 & 25 \\
\hline 4 & HARDWOODS & Uplands, Hardwoods \& Other Non Applicable Areas & 50 & 50 & 50 \\
\hline 5 & IMPACT & Area Impacted By Proposed Development & 0 & 0 & 0 \\
\hline & & TOTALS: & 250 & 250 & 250 \\
\hline
\end{tabular}

Table E31. Without-project variable projections for Exercise 14.

\begin{tabular}{|c|c|c|c|c|c|c|c|c|c|c|c|c|c|c|c|}
\hline TY & 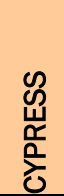 & 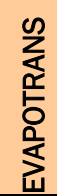 & 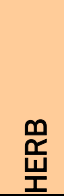 & $\begin{array}{l}\underline{z} \\
\text { 모 } \\
\underline{\underline{u}}\end{array}$ & $\begin{array}{l}\text { O্ } \\
0 \\
\text { ऐo }\end{array}$ & $\begin{array}{l}\text { ग } \\
\text { Dू }\end{array}$ & $\begin{array}{l}\text { O } \\
\frac{\pi}{0} \\
\frac{1}{\Sigma}\end{array}$ & 怘 & 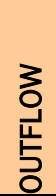 & 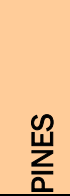 & 岕 & 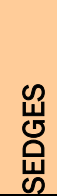 & 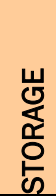 & 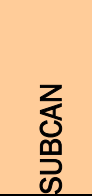 & $\begin{array}{l}3 \\
\frac{3}{0} \\
\frac{1}{4} \\
\frac{1}{5} \\
\text { क }\end{array}$ \\
\hline \multicolumn{16}{|c|}{ BNCHGRPSAV } \\
\hline 0 & & 3 & 6.8 & 0.3 & 72 & & 0.75 & 43 & 1 & & 0.7 & & 1 & & 1 \\
\hline 1 & & 3 & 7 & 0.3 & 100 & & 0.75 & 50 & 1 & & 0.7 & & 1 & & 1 \\
\hline 51 & & 3 & 8 & 0.3 & 100 & & 0.75 & 50 & 1 & & 0.7 & & 1 & & 1 \\
\hline \multicolumn{16}{|c|}{ CYPRESPSAV } \\
\hline 0 & 0.9 & 4 & 5.8 & 0.3 & 72 & & 0.75 & 43 & 1 & & 0.7 & 53 & 1 & 5100 & 1 \\
\hline 1 & 1 & 4 & 6 & 0.3 & 100 & & 0.75 & 50 & 1 & & 0.7 & 55 & 1 & 5100 & 1 \\
\hline 51 & 1 & 4 & 7 & 0.3 & 100 & & 0.75 & 50 & 1 & & 0.7 & 60 & 1 & 5100 & 1 \\
\hline \multicolumn{16}{|c|}{ SWITCHSAV } \\
\hline 0 & & 5 & & 0.3 & 72 & 305 & 0.75 & & 1 & 300 & 0.7 & & 1 & 5100 & 1 \\
\hline 1 & & 5 & & 0.3 & 100 & 305 & 0.75 & & 1 & 300 & 0.7 & & 1 & 5100 & 1 \\
\hline 51 & & 5 & & 0.3 & 100 & 305 & 0.75 & & 1 & 300 & 0.7 & & 1 & 5100 & 1 \\
\hline
\end{tabular}




\section{Step 1: Target Year Entry}

1. The first thing to do is to set up the target years (i.e., $\mathbf{0}, \mathbf{1}$, and 51), so open the training datafile entitled Exercise 14.mdb and click on the Run button on the primary interface.

2. Double-click on the first option (1. Choose Working Function), select the Fxn01: Water Level Regime option from the pull-down list, and click Close.

3. Now double-click on the fifth option (5. Define Target Years).

4. Enter each set of TY-Calendar Year combinations (i.e., $\mathbf{0}$ and 2000; 1 and 2010; 51 and 2060) and click Close when finished.

Use the reports in EXHGM to check work and document analysis (Setup Reports button). The following report should be created as a result:

\section{- Target Year List}

\section{Step 2: Without-project Acres Entry}

1. Now double-click on the sixth option (6. Enter Without-Project Acres).

2. Click on the PWAA pull-down menu and select the first PWAA listed (BNCHGRPSAV - Bunchgrass/Pine Savanna).

3. To the right in the acreage columns, input the acres for this PWAA on a TY-by-TY basis. Each row represents one target year, so there will be three rows of data for this PWAA when input is complete (refer to TableE30 above).

Note: the software automatically enters the first TY (TY 0 ). This number cannot be changed in this screen - users must return to the setup menu to make changes to this year.

4. Now go back up to the PWAA pull-down menu, select the second PWAA (i.e., CYPRESPSAV - Cypress/Pine Savanna), and repeat the process.

5. Finish by entering the rest of the acres in each category. The software automatically assumes 0 acres, so only the PWAAs that change from 0 need to be filled in (each PWAA should be pulled down just to ensure that they have all been covered). 
Note: the column to the immediate right of these acre projections keeps a running total of the acres input per year. At the end of the effort, each cell in this section should carry the same numbers (i.e., 250 acres in this instance). If they are not equal, one of the PWAA's acreage shifts has not been "accounted" for in the analysis.

Because all four functions are being used to assess the changes at this site, AND they share similar PWAAs, it is only necessary to enter the acres for one of the functions - the acres are carried across all functions in the analysis.

Use the reports in EXHGM to check work and document analysis (Setup Reports button). The following report should be created as a result:

\section{- Without-Project TY Acres}

\section{Step 3: Without-project Data Entry}

Now that the acres have been input, it is time to focus on the variables.

1. To begin, click on the Run button on the primary interface.

2. Double-click on the first option (1. Choose Working Function the Fxn01: Water Level Regime option from the pull-down list, and click Close.

3. Now double-click on the seventh option (7. Enter Without-Project Projections*).

4. Say "Yes" to the initialization question, and the software forecasts the variable means forward into the future target years.

If predicted forecasts deviate from the mean, new future projections must be input.

5. Select BNCHGRPSAV from the PWAA pull-down box. Only the variable means that change from the baseline condition must be entered they are shown in bold in Table E31 (i.e., HERB, LANDSCP, and NBG in each PWAA). So, select HERB from the Variable pull-down box.

6. Highlight the TY 1 entry (i.e., 6.8) and type in the new value for the year (i.e., 7). 
7. Hit Tab on the keyboard and the software skips down to the next row (TY51). Highlight the TY51 entry (i.e., 6.8) and type in the new value for the year (i.e., 8).

8. Hit Tab on the keyboard to finish. The data are now input for this variable.

9. Repeat the process for LANDSCP and NBG in this PWAA.

10. Now switch to the next PWAA (i.e., choose CYPRESPSAV from the PWAA pull-down box), and select HERB from the Variable pull-down box.

11. Highlight the $\mathrm{TY}_{1}$ value (i.e., 5.8), type in the new value for this year (i.e., 6), and hit Tab.

12. Finish entering data for this variable, and repeat the process for the remaining variables in this PWAA.

13. Repeat the process for the final PWAA - SWTCHPSAV.

14. When completely finished, click Close and click on Yes to save the data for later use.

Use the reports in EXHGM to check work and document analysis (Analysis Reports button). The following report should be created as a result:

\section{- Without-Project Projections}

\section{Step 4: Without-project Analysis}

Now run the analysis for the model.

1. Set the working function - double-click on the first option (1. Choose Working Function), select the Fxn01: Water Level Regime option from the pull-down list, and click Close.

2. Double-click on 4. Perform Baseline (TY 0) Calculations*, let the system run, and click $\mathbf{O K}$ when it is finished.

3. Double-click on 7. Enter Without-Project Projections*, click on the No button for initialization, click the Close button at the bottom of the interface, click Yes to save the data, and click $\mathbf{O K}$ when that save is affirmed.

4. Double-click on 8. Perform Without-Project Calculations*, let the system run, and click $\mathbf{O K}$ when it is finished.

5. Repeat steps 1-4 for each of the remaining three functions. 
Use the reports in EXHGM to check work and document analysis

(Analysis Reports button). The following report should be created as a result:

- Without-Project Results 


\section{Exercise 15: With-project Analyses in EXHGM}

Knowing what the "No Action" conditions look like, the impact scenario on the wetlands can be assessed. There is no need to enter target years - the same series used in the without-project scenario are used in the withproject forecasting. Here are the trends the team generated under the alternative formulation exercise:

Table E32. With-project acre projections for Exercise 15.

\begin{tabular}{|c|c|c|c|c|c|}
\hline \multirow[b]{3}{*}{ No. } & \multirow[b]{3}{*}{ Code } & \multirow[b]{3}{*}{ Description } & \multicolumn{3}{|c|}{ Target Year } \\
\hline & & & 2000 & 2010 & 2060 \\
\hline & & & TYo & $\mathrm{TY}_{1}$ & $\mathrm{TY}_{51}$ \\
\hline 1 & BNCHGRPSAV & Bunchgrass/Pine Savanna & 150 & 0 & 0 \\
\hline 2 & CYPRESPSAV & Cypress/Pine Savanna & 25 & 0 & 0 \\
\hline 3 & SWTCHPSAV & Switchcane/Pine Savanna & 25 & 0 & 0 \\
\hline 4 & HARDWOODS & Uplands, Hardwoods \& Other Non Applicable Areas & 50 & 0 & 0 \\
\hline 5 & IMPACT & Area Impacted By Proposed Development & 0 & 250 & 250 \\
\hline & & TOTALS: & 250 & 250 & 250 \\
\hline
\end{tabular}

Table E33. With-project variable projections for Exercise 15.

\begin{tabular}{|c|c|c|c|c|c|c|c|c|c|c|c|c|c|c|c|}
\hline TY & 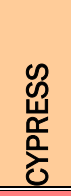 & 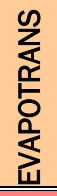 & 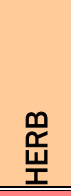 & $\begin{array}{l}\underline{3} \\
\text { 章 } \\
\underline{\underline{z}} \\
\end{array}$ & 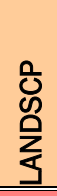 & $\begin{array}{l}\text { J } \\
\text { Zू } \\
\end{array}$ & $\begin{array}{l}\text { 옹 } \\
\frac{0}{\Sigma} \\
\end{array}$ & $\begin{array}{l}\text { g } \\
\text { Z }\end{array}$ & 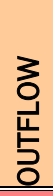 & $\stackrel{\mathscr{m}}{\frac{\mathfrak{L}}{\alpha}}$ & 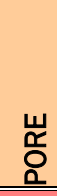 & 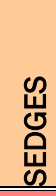 & 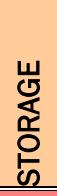 & $\begin{array}{l}z \\
\text { J } \\
\text { M } \\
\text { 心 }\end{array}$ & 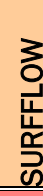 \\
\hline \multicolumn{16}{|c|}{ BNCHGRPSAV } \\
\hline 0 & & 3 & 6.8 & 0.3 & 72 & & 0.75 & 43 & 1 & & 0.7 & & 1 & & 1 \\
\hline 1 & & 0 & 0 & 0 & 0 & & 0 & 0 & 0 & & 0 & & 0 & & 0 \\
\hline 51 & & 0 & 0 & 0 & 0 & & 0 & 0 & 0 & & 0 & & 0 & & 0 \\
\hline \multicolumn{16}{|c|}{ CYPRESPSAV } \\
\hline 0 & 0.9 & 4 & 5.8 & 0.3 & 72 & & 0.75 & 43 & 1 & & 0.7 & 53 & 1 & 5100 & 1 \\
\hline 1 & 0 & 0 & 0 & 0 & 0 & & 0 & 0 & 0 & & 0 & 0 & 0 & 0 & 0 \\
\hline 51 & 0 & 0 & 0 & 0 & 0 & & 0 & 0 & 0 & & 0 & 0 & 0 & 0 & 0 \\
\hline \multicolumn{16}{|c|}{ SWTCHPSAV } \\
\hline 0 & & 5 & & 0.3 & 72 & 305 & 0.75 & & 1 & 300 & 0.7 & & 1 & 5100 & 1 \\
\hline 1 & & 0 & & 0 & 0 & 0 & 0 & & 0 & 0 & 0 & & 0 & 0 & 0 \\
\hline 51 & & 0 & & 0 & 0 & 0 & 0 & & 0 & 0 & 0 & & 0 & 0 & 0 \\
\hline
\end{tabular}




\section{Step 1: With-project Acres Entry}

1. To begin, open the training datafile entitled Exercise 15.mdb and click on the Run button on the primary interface.

2. Double-click on the ninth option (9. Enter With-Project Acres).

3. Click on the PWAA pull-down menu and select the first PWAA listed (i.e., BNCHGRPSAV - Bunchgrass/Pine Savanna).

4. To the right in the acreage columns, input the acres for this PWAA on a TY-by-TY basis. Each row represents one target year, so there will be three rows of data for this PWAA (refer to Table E32 above).

Note: the software automatically enters the first TY (TY $)_{0}$ ). This number cannot be changed in this screen - return to the setup menu to make changes to this year.

5. Now go back up to the PWAA pull-down menu and select the second PWAA (i.e., CYPRESPSAV - Cypress/Pine Savanna) and repeat the process.

6. Finish by entering the rest of the acres in each category. The software automatically assumes 0 acres, so only the PWAAs that change from 0 need to be filled in (pull down each PWAA just to ensure that they all have been covered).

Note: the column to the immediate right of these acre projections keeps a running total of the acres put in per year. At the end of the effort, each cell in this section should carry the same numbers (i.e., 250 acres in this instance). If they are not equal, one of the PWAA's acreage shifts has not been "accounted" for in the analysis.

Because all four functions are being used to assess the changes at this site, AND they share similar PWAAs, it is only necessary to enter the acres for one of the functions - the acres are carried across all functions in the analysis.

Use the reports in EXHGM to check work and document analysis (Setup Reports button). The following report should be created as a result:

\section{- With-Project TY Acres}




\section{Step 2: With-project Data Entry}

Now that the acres are input, it is time to focus on the variables.

1. To begin, click on the Run button on the primary interface.

2. Double-click on the first option (1. Choose Working Function), select the Fxn01: Water Level Regime option from the pull-down list, and click Close.

3. Now double-click on the tenth option (10. Enter With-Project Projections*).

4. Say "Yes" to the initialization question, and the software forecasts the variable means forward into the future target years.

If predicted forecasts deviate from the mean, the new future projections must be input.

5. Select the BNCHGRPSAV from the PWAA pull-down box. Under this scenario, each variable is driven to zero by $\mathrm{TY}_{1}$ and stays there for $\mathrm{TY}_{51}$ (refer to Table E33 above). Select EVAPOTRANS from the Variable pull-down box.

6. Highlight the TY 1 entry (i.e., 3) and type in the new value for the year (i.e., 0).

7. Hit Tab on the keyboard and the software skips down to the next row $\left(T Y_{51}\right)$. Highlight the TY 51 entry (i.e., 3) and type in the new value for the year (i.e., $\mathbf{0}$ ).

8. Hit Tab on the keyboard to finish. The data are now input for this variable.

9. Repeat the process for HERB, INFLOW, LANDSCP, MICRO, NBG, OUTFLOW, PORE, STORAGE, and SURFFLOW in this PWAA.

10. Repeat the process for the next two PWAAs - CYPRESPSAV and SWTCHPSAV.

11. When completely finished, click Close and click on Yes to save the data for later use.

Use the reports in EXHGM to check work and document analysis (Analysis Reports button). The following report should be created as a result:

\section{- With-Project Projections}




\section{Step 3: With-project Analysis}

Now the analysis for the model can be run.

1. Set the working function - double-click on the first option (1. Choose Working Function), select the Fxn01: Water Level Regime option from the pull-down list, and click Close.

a. Double-click on 4. Perform Baseline (TY 0) Calculations*, let the system run, and click $\mathbf{O K}$ when it is finished.

b. Double-click on 7. Enter Without-Project Projections*, click on the No button for initialization, click the Close button at the bottom of the interface, click Yes to save the data, and click OK when that save is affirmed.

c. Double-click on 8. Perform Without-Project Calculations*, let the system run, and click $\mathbf{O K}$ when it is finished.

d. Double-click on 10. Enter With-Project Projections*, click on the No button for initialization, click the Close button at the bottom of the interface, click Yes to save the data, and click OK when that save is affirmed.

e. Double-click on 11. Perform With-Project Calculations*, let the system run, and click $\mathbf{O K}$ when it is finished.

f. Go to the Analysis Reports button, open the AAFCU Summary Report, and export it out to MSWord.

2. Set the working function - double-click on the first option (1. Choose Working Function), select the Fxn02: Plant Communities option from the pull-down list, and click Close.

3. Now repeat steps a-f above.

4. Repeat for the two remaining functions.

Use the reports in EXHGM to check work and document analysis (Analysis Reports button). The following reports should be created as a result:

- Fxn 1AAFCU Summary

- Fxn 2 AAFCU Summary

- Fxn 3 AAFCU Summary

- Fxn 4 AAFCU Summary 
- With-Project Results

- With- vs. Without-Project Results

- Overall Net Results - All Functions 


\section{Exercise 16: Master File Creation in EXHGM}

Scenario A: Create a "master file" that can be used for new sites

Assume the impact analysis requires the formulation and evaluation of mitigation at another site. Also assume that the evaluation team has taken the time to generate a baseline, without-project and with-project design to mitigate for the impacts at this new site. It will be time-consuming to start with a blank datafile and rebuild all the information previously entered in the above exercises for this analysis. Instead, take one of those files and recycle it for the new site. Then changes can be made that are unique to the new location (and mitigation plan) and scenario and the analysis can be run quickly.

\section{Step 1: Copy the file and change the name}

1. To begin, open the training datafile entitled Impact Site.mdb.

2. Click on the Other Activities button, and choose the Save Datafile Under New Name option.

3. Be sure to click once on the Data Control Check Box at the bottom of this screen to assure that the file becomes the "acting" file when finished, and click on the Save button in the upper left-hand corner of this form.

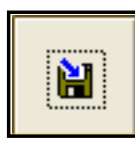

4. In the Save datafile as screen, identify the storage location via the Save in: window at the top of the interface. Name the file Mitigation Site A.mdb in the File name: window. Now press Save and OK to finish the copying activity.

Step 2: Change the alternative name in the methods section

1. Click on the Setup button on the primary interface, and choose the first option (i.e., 1. Enter Project Methods).

2. Change the Alternative Name from Impact Analysis to Scenario A: Mitigation Analysis (300 Acres), and click Close.

Now assume the following acres and variable trends have been projected for this new site: 
Table E34. With-project acre projections for "Scenario A" in Exercise 16.

\begin{tabular}{|c|c|c|c|c|c|c|c|c|}
\hline \multirow[b]{4}{*}{ No. } & \multirow[b]{4}{*}{ Code } & \multirow[b]{4}{*}{ Description } & \multirow{2}{*}{\multicolumn{3}{|c|}{$\begin{array}{c}\text { Without-Project } \\
\text { Target Year }\end{array}$}} & \multirow{2}{*}{\multicolumn{3}{|c|}{$\begin{array}{l}\text { With-Project } \\
\text { Target Year } \\
\end{array}$}} \\
\hline & & & & & & & & \\
\hline & & & 2000 & 2010 & 2060 & 2000 & 2010 & 2060 \\
\hline & & & TYO & TY1 & TY51 & TYO & TY1 & TY51 \\
\hline 1 & BNCHGRPSAV & Bunchgrass/Pine Savanna & 0 & 0 & 0 & 0 & 200 & 200 \\
\hline 2 & CYPRESPSAV & Cypress/Pine Savanna & 0 & 0 & 0 & 0 & 50 & 50 \\
\hline 3 & SWTCHPSAV & Switchcane/Pine Savanna & 0 & 0 & 0 & 0 & 50 & 50 \\
\hline 4 & HARDWOODS & $\begin{array}{l}\text { Uplands, Hardwoods \& Other } \\
\text { Non Applicable Areas }\end{array}$ & 0 & 0 & 0 & 0 & 0 & 0 \\
\hline 5 & IMPACT & $\begin{array}{l}\text { Area Impacted By Proposed } \\
\text { Development }\end{array}$ & 0 & 0 & 0 & 0 & 0 & 0 \\
\hline 6 & AGCROP & Agricultural Croplands & 300 & 300 & 300 & 300 & 0 & 0 \\
\hline & & TOTALS: & 300 & 300 & 300 & 300 & 300 & 300 \\
\hline
\end{tabular}

Table E35. With-project variable projections for "Scenario A" in Exercise 16.

\begin{tabular}{|c|c|c|c|c|c|c|c|c|c|c|c|c|c|c|c|}
\hline TY & 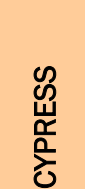 & 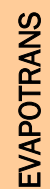 & 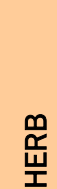 & $\begin{array}{l}3 \\
\text { 을 } \\
\text { 는 }\end{array}$ & 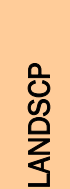 & $\begin{array}{l}\text { J } \\
\text { o }\end{array}$ & $\begin{array}{l}\text { O } \\
\frac{\mathbb{R}}{2}\end{array}$ & 品 & 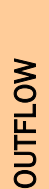 & 峁 & 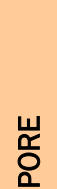 & 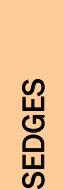 & 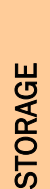 & 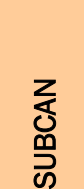 & 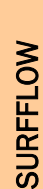 \\
\hline \multicolumn{16}{|c|}{ BNCHGRPSAV } \\
\hline 0 & & 0 & 0 & 0 & 0 & & 0 & 0 & 0 & & 0 & & 0 & & 0 \\
\hline 1 & & 1 & 5 & 0 & 300 & & 0.75 & 50 & 1 & & 1 & & 1 & & 1 \\
\hline 51 & & 1 & 8 & 0 & 300 & & 1 & 100 & 1 & & 1 & & 1 & & 1 \\
\hline \multicolumn{16}{|c|}{ CYPRESPSAV } \\
\hline 0 & 0 & 4 & 5.8 & 0.3 & 72 & & 0 & 0 & 0 & & 0 & 0 & 0 & 0 & 0 \\
\hline 1 & 0.25 & 1 & 5 & 0 & 300 & & 0 & 0 & 0 & & 1 & 50 & 1 & 1000 & 1 \\
\hline 51 & 1 & 1 & 7 & 0 & 300 & & 0 & 0 & 0 & & 1 & 100 & 1 & 2000 & 1 \\
\hline \multicolumn{16}{|c|}{ SWTCHPSAV } \\
\hline 0 & & 0 & & 0 & 0 & 0 & 0 & & 0 & 0 & 0 & & 0 & 0 & 0 \\
\hline 1 & & 1 & & 0 & 300 & 75 & 0.75 & & 1 & 75 & 1 & & 1 & 1000 & 1 \\
\hline 51 & & 1 & & 0 & 300 & 150 & 1 & & 1 & 150 & 1 & & 1 & 2000 & 1 \\
\hline
\end{tabular}

Focus on the acres first, and then return to handle the variables. The additional PWAA (i.e., AGCROP - Agricultural Croplands) should be noted in the acreage table. This coverage is not associated with the model, but is the target of the mitigation activities. The baseline functional capacity of this coverage is assumed to be zero until it has been changed (i.e., creation or restoration activities must be employed). Add this PWAA 
to the baseline tables, but no data are needed to quantify the baseline condition.

Step 3: Add the new PWAA and its baseline acres

1. Click on the Setup button, and double-click on the third option (i.e., 3. Define Partial Wetland Assessment Areas).

2. Go to the last row on the new interface, and enter the details for the new PWAA:

a. Numeric Code $=6$

b. Identification Code $=$ AGCROP

c. Description $=$ Agricultural Croplands

d. Acres $=300$.

3. Zero-out the other five PWAAs so that they match the table above (TableE34).

4. Click Close when finished.

Use the reports in EXHGM to check work and document analysis (Setup Reports button). The following report should be created as a result:

\section{- PWAAs and Baseline (TY 0) Acres}

Step 4: Change the without-project and with-project acres

1. Click on the Run button on the primary interface.

2. Double-click on the first option (1. Choose Working Function), select the Fxn01: Water Level Regime option from the pull-down list, and click Close.

3. Double-click on the sixth option (6. Enter Without-Project Acres), and change the acres to match the new information (Table E34). Refer to Exercise 14 above for a refresher on how to enter this information.

4. Double-click on the ninth option (9. Enter With-Project Acres), and change the acres to match the new information (Table E34). Refer to Exercise 15 above for a refresher on how to enter this information.

Use the reports in EXHGM to check work and document analysis (Setup Reports button). The following reports should be created as a result:

- Without-Project TY Acres

- With-Project TY Acres. 
Step 5: Change the baseline data

1. To begin, click on the Run button on the primary interface.

2. Double-click on the first option (1. Choose Working Function), select the Fxn01: Water Level Regime option from the pull-down list, and click Close.

3. Now double-click on the third option (3. Enter VSI Graphs and Variable Data).

4. Deleteall the baseline data for every variable shown, and enter a zero for each variable in each cover type (i.e., delete the individual data points by clicking on the Record Indicator Bar to the immediate left of each data point and pressing Delete on the keyboard) (refer to Table E35 above).

5. Click Close when finished.

6. Repeat for each function.

Use the reports in EXHGM to check work and document analysis (Setup Reports button). The following report should be created as a result:

\section{- Variable Data}

Another way to check work is to run the baseline calculations for each function (the result should be 0.0 FCI and 0.0 FCUs for all functions). The following report should be created as a result:

\section{- Baseline (TY 0) Results}

Step 6: Change the without-project data

The without-project condition is the "status quo" ( 0.0 quality and no acres associated with the functions), so the baseline data should be initialized (which will set all the values to zero), and the projections should be saved.

1. To begin, click on the Run button on the primary interface.

2. Double-click on the first option (1. Choose Working Function) the Fxn01: Water Level Regime option from the pull-down list, and click Close.

3. Now double-click on the seventh option (7. Enter Without-Project Projections*).

4. Say "Yes" to the initialization question. There is no need to change any of the data - they are all zeros (refer to TableE35 above), so the withoutproject analysis can begin. Click Close, and say Yes to the save question. 
Use the reports in EXHGM to check work and document analysis (Analysis Reports button). The following report should be created as a result:

\section{- Without-Project Projections}

Step 7: Change the with-project data

The with-project condition is the mitigation plan, and as such, there will be many changes in the variable trends.

1. To begin, click on the Run button on the primary interface.

2. Double-click on the first option (1. Choose Working Function) the Fxn01: Water Level Regime option from the pull-down list, and click Close.

3. Now double-click on the tenth option (10. Enter With-Project Projections*).

4. Say "Yes" to the initialization question.

5. Enter the variable trends into this screen based on the information provided in Table E35 above. Refer to Exercise 15 above for a refresher on how to enter this information.

6. Click Close, and say Yes to the save question.

Use the reports in EXHGM to check work and document analysis (Analysis Reports button). The following report should be created as a result:

\section{- With-Project Projections}

Step 8: Run the full analysis

The analysis for the model can now be run.

1. Set the working function - double-click on the first option (1. Choose Working Function), select the Fxn01: Water Level Regime option from the pull-down list, and click Close.

a. Double-click on 4. Perform Baseline (TY 0) Calculations*, let the system run, and click $\mathbf{O K}$ when it is finished.

b. Double-click on 7. Enter Without-Project Projections*, click on the No button for initialization, click the Close button at the bottom of 
the interface, click Yes to save the data, and click $\mathbf{O K}$ when that save is affirmed.

c. Double-click on 8. Perform Without-Project Calculations*, let the system run, and click $\mathbf{O K}$ when it is finished.

d. Double-click on 10. Enter With-Project Projections*, click on the No button for initialization, click the Close button at the bottom of the interface, click Yes to save the data, and click $\mathbf{O K}$ when that save is affirmed.

e. Double-click on 11. Perform With-Project Calculations*, let the system run, and click $\mathbf{O K}$ when it is finished.

f. Go to the Analysis Reports button, open the AAFCU Summary Report, and export it out to MSWord.

2. Set the working function - double-click on the first option (1. Choose Working Function), select the Fxn02: Plant Communities option from the pull-down list, and click Close.

3. Now repeat steps a-f above.

4. Repeat again for the two remaining functions.

Use the reports in EXHGM to check work and document analysis (Analysis Reports button). The following reports should be created as a result:

- Fxn 1AAFCU Summary

- Fxn 2 AAFCU Summary

- Fxn 3 AAFCU Summary

- Fxn 4 AAFCU Summary

- Without-Project Results

- With-Project Results

- With- vs. Without-Project Results

- Overall Net Results - All Functions 
Was the mitigation enough to compensate for the losses? Although this is a regulatory issue, and a lot of extemporary information would be taken into account to make the decision above and beyond this analysis, the quantitative results can be examined to respond to this question given the analysis. According to the outputs in Exercise 15 and this mitigation analysis, one can expect to see the following outputs:

Table E36. Results of the "Impact" and "Mitigation Analyses" ("Scenario A").

\begin{tabular}{|c|c|c|c|c|c|c|}
\hline \multirow[b]{2}{*}{ Function Name } & \multicolumn{2}{|c|}{ Impact Site } & \multicolumn{4}{|c|}{ Mitigation Scenario A } \\
\hline & $\begin{array}{c}\text { Acres } \\
\text { Impacted }\end{array}$ & $\begin{array}{c}\text { Net } \\
\text { Losses }\end{array}$ & $\begin{array}{c}\text { Acres } \\
\text { Restored }\end{array}$ & $\begin{array}{l}\text { Net } \\
\text { Gains }\end{array}$ & $\begin{array}{c}\text { Mitigation } \\
\text { Deficit or } \\
\text { Excess }\end{array}$ & $\begin{array}{c}\text { Mitigation } \\
\text { Ratio }\end{array}$ \\
\hline Fxn 01: Water Level Regime & -200 & -139 & 300 & 286 & 147 & 1.5 to 1 \\
\hline Fxn 02: Plant Communities & -200 & -198 & 300 & 296 & 98 & \\
\hline Fxn 03: Animal Communities & -200 & -198 & 300 & 296 & 98 & \\
\hline Fxn 04: Biogeochemical Processes & -200 & -166 & 300 & 292 & 126 & \\
\hline Average Losses: & -200 & -176 & 300 & 293 & 117 & \\
\hline Maximum Lost and Offset: & -200 & -198 & 300 & 296 & 98 & \\
\hline
\end{tabular}

The compensation generated by the proposed Scenario A mitigation plan exceeds the units (AAFCUs) necessary to offset the losses in all functions (i.e., mitigation excess is $>0$ ), and cumulatively (i.e., averaged across the functions the outputs exceed the losses) at a mitigation ratio of 1.5 to 1 (300 acres were purchased/restored and $\mathbf{2 0 0}$ acres were impacted). In other words, it is enough - in fact it is too much (i.e., in excess of $\mathbf{9 8}$ AAFCUs for each function, and an average of $\mathbf{1 1 7} \mathrm{AAFCUS}$ over and above the mitigation required).

The question now becomes can another mitigation scenario be generated that uses the same restoration plan, but perhaps requires fewer acres? The goal is still to mitigate all functional losses, but the plan will likely cost less because there will be fewer acres to purchase and restore. To do this, take the mitigation site file, recycle it, and re-run the analysis with fewer acres (say 250). Refer to Scenario B below for directions. 
Scenario B: Create a "master file" that can be used for new alternatives at the same site

Step 1: Copy the file and change the name

1. To begin, open the training datafile entitled Mitigation Site -A.mdb.

2. Click on the Other Activities button, and choose the Save Datafile Under New Name option.

3. B sure to click once on the Data Control Check Box at the bottom of this screen to assure that the file becomes the "acting" file when finished, and click on the Save button in the upper left-hand corner of this form.

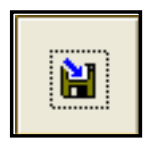

4. In the Save datafile as screen, identify the storage location via the Save in: window at the top of the interface. Name the file Mitigation Site B.mdb in the File name: window. Now press Save and OK to finish the copying activity.

Step 2: Change the alternative name in the methods section

1. Click on the Setup button on the primary interface, and choose the first option (i.e., 1. Enter Project Methods).

2. Change the Alternative Name from Scenario A: Mitigation Analysis (300 Acres) to Scenario B: Mitigation Analysis (250 Acres), and click Close.

Assume the only thing that changes is site size. The evaluation team made new projections for these acres: 
Table E37. With-project acre projections for "Scenario B" in Exercise 16.

\begin{tabular}{|c|c|c|c|c|c|c|c|c|}
\hline \multirow[b]{4}{*}{ No. } & \multirow[b]{4}{*}{ Code } & \multirow[b]{4}{*}{ Description } & \multirow{2}{*}{\multicolumn{3}{|c|}{$\begin{array}{c}\text { Without-Project } \\
\text { Target Year }\end{array}$}} & \multirow{2}{*}{\multicolumn{3}{|c|}{$\begin{array}{l}\text { With-Project } \\
\text { Target Year }\end{array}$}} \\
\hline & & & & & & & & \\
\hline & & & 2000 & 2010 & 2060 & 2000 & 2010 & 2060 \\
\hline & & & TYO & TY1 & TY51 & TYO & TY1 & TY51 \\
\hline 1 & BNCHGRPSAV & Bunchgrass/Pine Savanna & 0 & 0 & 0 & 0 & 150 & 150 \\
\hline 2 & CYPRESPSAV & Cypress/Pine Savanna & 0 & 0 & 0 & 0 & 50 & 50 \\
\hline 3 & SWTCHPSAV & Switchcane/Pine Savanna & 0 & 0 & 0 & 0 & 50 & 50 \\
\hline 4 & HARDWOODS & $\begin{array}{l}\text { Uplands, Hardwoods \& Other } \\
\text { Non Applicable Areas }\end{array}$ & 0 & 0 & 0 & 0 & 0 & 0 \\
\hline 5 & IMPACT & $\begin{array}{l}\text { Area Impacted By Proposed } \\
\text { Development }\end{array}$ & 0 & 0 & 0 & 0 & 0 & 0 \\
\hline 6 & AGCROP & Agricultural Croplands & 250 & 250 & 250 & 250 & 0 & 0 \\
\hline & & TOTALS & 250 & 250 & 250 & 250 & 250 & 250 \\
\hline
\end{tabular}

Step 3: Change the acres

First, handle the baseline acres (refer to Table E37).

1. Click on the Setup button, and double-click on the third option (i.e., 3. Define Partial Wetland Assessment Areas).

2. Go to the last row on the new interface and change the AGCROP acres from 300 to 250.

3. Click Close when finished.

Now handle the without-project and with-project acres (refer to Table E37 above).

1. Click on the Run button on the primary interface.

2. Double-click on the first option (1. Choose Working Function), the Fxn01: Water Level Regime option from the pull-down list, and click Close.

3. Double-click on the sixth option (6. Enter Without-Project Acres), and change the AGCROP acres from 300 to 250 in all the TYs. Refer to Exercise 14 above for a refresher on how to enter this information.

4. Double-click on the ninth option (9. Enter With-Project Acres), and change the acres to match the new information. Refer to Exercise 15 above for a refresher on how to enter this information. 
Use the reports in EXHGM to check work and document analysis (Setup Reports button). The following reports should be created as a result:

\section{- PWAAs and Baseline (TY 0) Acres}

\section{- Without-Project TY Acres}

\section{- With-Project TY Acres}

Step 4: Change the With-project data

Normally, a change in site size wouldn't have much effect on the future variable trends if the same mitigation plan was followed. However, one variable (i.e., LANDSCP) is dependent on patch size, so the reduction in size directly affects this variable's future condition. In other words, this variable in the with-project projections will need to be changed. Here are the projections for this variable:

Table E38. With-project variable projections for "Scenario B" in Exercise 16.

\begin{tabular}{|c|c|c|c|}
\hline \multirow{3}{*}{ TY } & BNCHGRPSAV & CYPRESPSAV & SWTCHPSAV \\
\cline { 2 - 4 } & LANDSCP & LANDSCP & LANDSCP \\
\hline \hline 0 & 0 & 0 & 0 \\
\hline 1 & 250 & 250 & 250 \\
\hline 51 & 250 & 250 & 250 \\
\hline
\end{tabular}

Make this change now.

1. To begin, click on the Run button on the primary interface.

2. Double-click on the first option (1. Choose Working Function), the Fxn01: Water Level Regime option from the pull-down list, and click Close.

3. Now double-click on the tenth option (10. Enter With-Project Projections*).

4. Say "No" to the initialization question.

5. For each PWAA (i.e., BNCHGRPSAV, CYPRESPSAV, and SWTCHPSAV), select the LANDSCP variable from the Variable pulldown box, and change all occurrences of $\mathbf{3 0 0}$ to $\mathbf{2 5 0}$ (refer to TableE38 above).

6. Click Close, and say Yes to the save question. 
Use the reports in EXHGM to check work and document analysis (Analysis Reports button). The following report should be created as a result:

\section{- With-Project Projections}

Step 5: Run the full analysis

Now the analysis for the model can be run.

1. Set the working function - double-click on the first option (1. Choose Working Function), select the Fxn01: Water Level Regime option from the pull-down list, and click Close.

a. Double-click on 4. Perform Baseline (TY 0) Calculations*, let the system run, and click $\mathbf{O K}$ when it is finished.

b. Double-click on 7. Enter Without-Project Projections*, click on the No button for initialization, click the Close button at the bottom of the interface, click Yes to save the data, and click $\mathbf{O K}$ when that save is affirmed.

c. Double-click on 8. Perform Without-Project Calculations*, let the system run, and click $\mathbf{O K}$ when it is finished.

d. Double-click on 10. Enter With-Project Projections*, click on the No button for initialization, click the Close button at the bottom of the interface, click Yes to save the data, and click $\mathbf{O K}$ when that save is affirmed.

e. Double-click on 11. Perform With-Project Calculations*, let the system run, and click $\mathbf{O K}$ when it is finished.

f. Go to the Analysis Reports button, open the AAFCU Summary Report, and export it out to MSWord.

2. Set the working function - double-click on the first option (1. Choose Working Function), select the Fxn02: Plant Communities option from the pull-down list, and click Close.

3. Now repeat steps a-f above.

4. Repeat again for the two remaining functions.

Use the reports in EXHGM to check work and document analysis (Analysis Reports button). The following reports should be created as a result: 


\section{- Fxn 1AAFCU Summary}

- Fxn 2 AAFCU Summary

- Fxn 3 AAFCU Summary

- Fxn 4 AAFCU Summary

- Baseline (TY 0) Results

- Without-Project Results

- With-Project Results

- With- vs. Without-Project Results

- Overall Net Results - All Functions

Was the mitigation enough to compensate for the losses? The answer is "Yes," but again, the outputs were in excess of the losses (Table E39).

Table E39. Results of the "Impact" and "Mitigation Analyses" ("Scenario B").

\begin{tabular}{|c|c|c|c|c|c|c|}
\hline \multirow[b]{2}{*}{ Function Name } & \multicolumn{2}{|c|}{ Impact Site } & \multicolumn{4}{|c|}{ Mitigation Scenario B } \\
\hline & $\begin{array}{c}\text { Acres } \\
\text { Impacted }\end{array}$ & $\begin{array}{c}\text { Net } \\
\text { Losses }\end{array}$ & $\begin{array}{c}\text { Acres } \\
\text { Restored }\end{array}$ & $\begin{array}{l}\text { Net } \\
\text { Gains }\end{array}$ & $\begin{array}{c}\text { Mitigation } \\
\text { Deficit or } \\
\text { Excess }\end{array}$ & $\begin{array}{c}\text { Mitigation } \\
\text { Ratio }\end{array}$ \\
\hline Fxn 01: Water Level Regime & -200 & -139 & 250 & 239 & 100 & 1.25 to 1 \\
\hline Fxn 02: Plant Communities & -200 & -198 & 250 & 247 & 48 & \\
\hline Fxn 03: Animal Communities & -200 & -198 & 250 & 247 & 48 & \\
\hline Fxn 04: Biogeochemical Processes & -200 & -166 & 250 & 243 & 77 & \\
\hline Average Losses: & -200 & -176 & 250 & 244 & 68 & \\
\hline Maximum Lost and Offset: & -200 & -198 & 300 & 296 & 98 & \\
\hline
\end{tabular}

If time permits, re-run Scenario B as many times as necessary to tighten up the outputs to meet the "no net loss" goal and reduce the costs to the permittee. Try increments of acres, such as $\mathbf{2 1 0}, \mathbf{2 1 5}, \mathbf{2 2 5}$, etc. Remember to re-name these when moving through the process to keep track of analyses. 


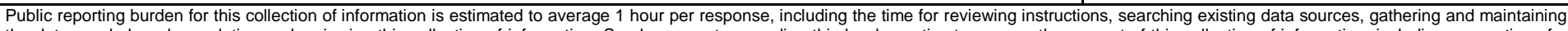

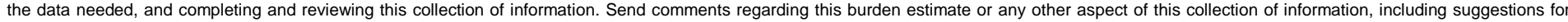

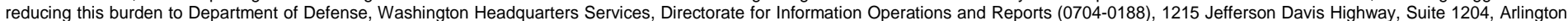

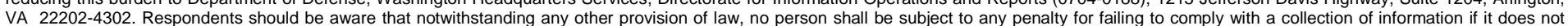
display a currently valid OMB control number. PLEASE DO NOT RETURN YOUR FORM TO THE ABOVE ADDRESS.

\begin{tabular}{l|c}
$\begin{array}{l}\text { 1. REPORT DATE (DD-MM-YYYY) } \\
\text { December } 2012\end{array}$ & $\begin{array}{c}\text { 2. REPORT TYPE } \\
\text { Final report }\end{array}$ \\
\hline
\end{tabular}

4. TITLE AND SUBTITLE

HEAT - Habitat Evaluation and Assessment Tools for Effective Environmental

Evaluations: User's Guide

3. DATES COVERED (From - To)

5a. CONTRACT NUMBER

5b. GRANT NUMBER

5c. PROGRAM ELEMENT NUMBER

5d. PROJECT NUMBER

6. AUTHOR(S)

Kelly A. Burks-Copes, Antisa C. Webb, Michael F. Passmore,

and Sheila D. McGee-Rosser

5e. TASK NUMBER

5f. WORK UNIT NUMBER

8. PERFORMING ORGANIZATION REPORT

NUMBER

U.S. Army Engineer Research and Development Center, Environmental Laboratory,

3909 Halls Ferry Road, Vicksburg, MS 39180-6199;

ERDC/EL SR-12-1

Applied Research Associates, Inc., 4300 San Mateo Blvd. NE, Suite A-220

Albuquerque, NM 87110

9. SPONSORING I MONITORING AGENCY NAME(S) AND ADDRESS(ES)

U.S. Army Corps of Engineers

Washington, DC 20314-1000

10. SPONSOR/MONITOR'S ACRONYM(S)

11. SPONSOR/MONITOR'S REPORT NUMBER(S)

\section{DISTRIBUTION I AVAILABILITY STATEMENT}

Approved for public release, distribution unlimited.

\section{SUPPLEMENTARY NOTES}

An installation CD for the Habitat Evaluation and Assessment Tools (HEAT) program can be obtained by contacting Kelly BurksCopes at 601-634-2290 (Kelly.A.Burks-Copes@usace.army.mil).

\section{ABSTRACT}

Rapidly assessing changing habitat conditions and the evaluation of the effects these changes have on species, communities and ecosystems must be determined by planners, resource managers, and biologists when comparing environmental design alternatives. Many techniques (e.g., population assessments, qualitative matrices, life-history modeling, and habitat evaluation techniques) have been developed to investigate and predict environmental impacts on ecological systems at numerous scales with varying degrees of success. Advances in technology have led many agencies to automate and distribute automated environmental evaluation tools to users. The value and validity of these packages depends greatly on their objectivity, repeatability, and efficiency. To guarantee their consistent use, these systems must be easy to apply, cost-effective, and instantly responsive. The Habitat Evaluation and Assessment Tools (HEAT) software was developed to provide a user-friendly (intuitive), flexible, and efficient means to conduct Habitat Evaluation Procedures (HEP) and the Hydrogeomorphic Approach to Wetland Assessments (HGM), using Microsoft ${ }^{\circledR}$ Windows programming capabilities. HEAT operates on a personal computer with a 486 (or better) processor, using readily available commercial software. Two modules within HEAT, namely EXHEP: EXpert Habitat Evaluation Procedures and EXHGM: EXpert HydroGeoMorphic Approach to Wetland Assessments, calculate outputs for with-project conditions (comparing these to baseline and without-project conditions). HEAT accommodates a variety of data input and output files; incorporates a broad range of user-specified index models; allows customization of outputs; and dramatically reduces computation time. This document is a User's Guide, intended to provide both a stepby-step protocol for completing a HEP and/or an HGM analysis using the software, and an illustration of this software's effectiveness in the evaluation of contemporary index models. In addition, this report includes a brief description of the hardware and software requirements of the system, and provides directions for the installation of the program.

$\begin{array}{lll}\text { 15. SUBJECT TERMS } & \text { EXHEP } & \text { Functional Capacity Index (FCI) } \\ \text { Habitat Evaluation Procedures (HEP) } & \text { EXHGM } & \text { Environmental Impact Assessment } \\ \text { Hydrogeomorphic Wetland Assessment (HGM) } & \text { HEAT } & \text { Ecosystem Restoration Assessment }\end{array}$

$\begin{array}{ll}\text { Hydrogeomorphic Wetland Assessment (HGM) } & \text { HEAT } \\ & \text { Habitat Suitability Index (HSI) }\end{array}$

16. SECURITY CLASSIFICATION OF:
\begin{tabular}{l|l|l|}
\hline a. REPORT & b. ABSTRACT & c. THIS PAGE \\
UNCLASSIFIED & UNCLASSIFIED & UNCLASSIFIED \\
\hline
\end{tabular}

17. LIMITATION OF ABSTRACT
18. NUMBER OF PAGES

472 19a. NAME OF RESPONSIBLE PERSON

19b. TELEPHONE NUMBER (include area code) 